MISCELLANEOUS PAPER M-77-1

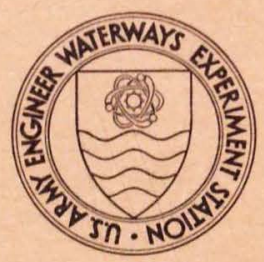

\title{
MOBILITY PERFORMANCE OF TOWED AND SELF-PROPELLED ARTILLERY AND RELATED VEHICLES
}

by

Donald D. Randolph, James H. Robinson

Mobility and Environmental Systems Laboratory

U. S. Army Engineer Waterways Experiment Station

P. O. Box 631, Vicksburg, Miss. 39180

January 1977

Final Report

Approved For Public Release; Distribution Unlimited

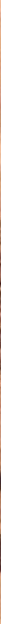

Prepared for U. S. Army Training and Doctrine Command

Fort Monroe, Virginia 2365I 
inimiming 
Unclassified

SECURITY CLASSIFICATION OF THIS PAGE (When Data Entorod)

\begin{tabular}{|c|c|}
\hline REPORT DOCUMENTATION PAGE & $\begin{array}{l}\text { READ INSTRUCTIONS } \\
\text { BEFORE COMPLETING FORM }\end{array}$ \\
\hline $\begin{array}{l}\text { 1. REPORT NUMBER } \\
\qquad \begin{array}{l|l|}\text { 2. GOVT ACCESSION NO. } \\
\end{array}\end{array}$ & 3. RECIPIENT'S CATALOG NUMBER \\
\hline $\begin{array}{l}\text { 4. TITLE (and Subtitio) } \\
\text { MOBILITY PERFORMANCE OF TOWED AND SELF-PROPELLED }\end{array}$ & $\begin{array}{l}\text { 5. TYPE OF REPORT a PERIOD COVERED } \\
\text { Final report }\end{array}$ \\
\hline ARTILLERY AND RELATED VEHICLES & 6. PERFORMING ORG. REPORT NUMBER \\
\hline $\begin{array}{l}\text { 7. AUTHOR(o) } \\
\text { Donald D. Randolph } \\
\text { James H. Robinson }\end{array}$ & 8. CONTRACT OR GRANT NUMBER(*) \\
\hline $\begin{array}{l}\text { 9. PERFORMING ORGANIZATION NAME AND ADDRESS } \\
\text { U. S. Army Engineer Waterways Experiment Station } \\
\text { Mobility and Environmental Systems Laboratory } \\
\text { P. O. Box 631, Vicksburg, Miss. } 39780\end{array}$ & $\begin{array}{l}\text { 10. PROGRAMELEMENT,PROJECT. TASK } \\
\text { AREA \& WORK UNIT NUMEESS }\end{array}$ \\
\hline $\begin{array}{l}\text { 11. CONTROLLING OFFICE NAME AND ADDRESS } \\
\text { U. S. Army Training and Doctrine Command. }\end{array}$ & $\begin{array}{l}\text { 12. REPORT DATF } \\
\text { January } 1977\end{array}$ \\
\hline Fort Monroe, Va. 23651 & $\begin{array}{l}\text { 13. NUMBER OF PAGES } \\
278\end{array}$ \\
\hline 14. MONITORING AGENCY NAME A ADDRESS(If difforont from Controlling Offico) & $\begin{array}{l}\text { 15. SECURITY CLASS. (of thie toport) } \\
\text { Unclassified }\end{array}$ \\
\hline & $\begin{array}{l}\text { 15a. DECLASSIFICATION/DOWNGRADING } \\
\text { SCHEDULE }\end{array}$ \\
\hline \multicolumn{2}{|l|}{$\begin{array}{l}\text { 16. DISTRIBUTION STATEMENT (of thla Roport) } \\
\text { Approved for public release; distribution unlimited. }\end{array}$} \\
\hline \multicolumn{2}{|l|}{ 17. DISTRIBUTION STATEMENT (of the abotract ontered in Block 20 , If different from Roport) } \\
\hline \multicolumn{2}{|l|}{ 18. SUPPLEMENTARY NOTES } \\
\hline \multirow{2}{*}{\multicolumn{2}{|c|}{ 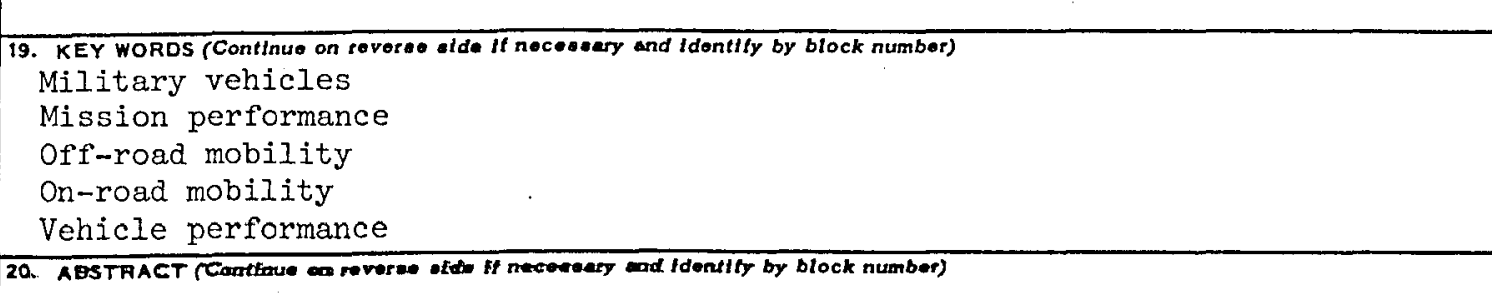 }} \\
\hline & \\
\hline $\begin{array}{l}\text { This study establishes a mobility performance } \\
\text { towed and self-propelled artillery and related veh } \\
\text { and weather conditions using the Army mobility mod } \\
\text { for making Go/NoGo predictions termed the JIFFY mo } \\
\text { predictions are in terms of (a) percentage of a col } \\
\text { ability of Go"; (b) speed profile and statistical } \\
\text { roads, secondary roads, trails, and off-road; }\end{array}$ & $\begin{array}{l}\text { prediction data base for } \\
\text { icles for several terrain } \\
\text { el (AMM) and a simple model } \\
\text { bility model. Performance } \\
\text { untry with a given "prob- } \\
\text { diagnostics for primary } \\
\text { (Continued) }\end{array}$ \\
\hline
\end{tabular}




\section{ABSTRACT (Continued)}

(c) performance index; (d) average speed for supply network; (e) selected mobility level rating speed; $(f)$ vehicle group performance; and $(g)$ time to complete special artillery missions.

This study does not attempt to analyze the mobility performance predictions data base, but does suggest methods or parameters which are considered as best for comparing the relative mobility of $(a)$ the prime movers, ( $b$ ) the prime mover-towed artillery and self-propelled artillery, and (c) group performance of selected vehicles.

Appendix A describes the JIFFY mobility model; Appendix B presents the vehicle characteristics; Appendix $C$ includes the results of experimental vehicle dynamics tests; Appendix $D$ describes the generalized terrain data; Appendix $E$ contains the basic performance data for HIMO West Germany and Mideast study areas; Appendix $F$ discusses the selection of tactical high as the suggested mobility level for comparing study vehicles; Appendix G shows the computation of mission-oriented speed based on statistical mission definition and vehicle performance statistics for an area and condition. 
THE CONTENTS OF THIS REPORT ARE NOT TO BE USED FOR ADVERTISING, PUBLICATION, OR PROMOTIONAL PURPOSES. CITATION OF TRADE NAMES DOES NOT CONSTITUTE AN OFFICIAL ENDORSEMENT OR APPROVAL OF THE USE OF SUCH COMMERCIAL PRODUCTS. 
PREFACE

Personnel of the U. S. Army Engineer Waterways Experiment Station (WES) conducted the study described herein during the period December 1975-July 1976 for the U. S. Army Training and Doctrine Command (TRADOC) under Intra-Army Order for Reimbursable Services No. CD-81-76, dated 19 December 1975 and Change Order No. C1, dated 10 June 1976.

The study was conducted under the general supervision of Messrs. W. G. Shockley, Chief, Mobility and Environmental Systems Laboratory (MESL), A. A. Rula, Chief, Mobility Systems Division (MSD), C. J. Nuttall, Jr., Chief, Mobility Research and Methodology Branch (MRMB), and Mr. E. S. Rush, Mobility Investigations Branch (MIB). Messrs. D. D. Randolph, MRMB, and J. H. Robinson, MIB, directed the overall study and prepared this report. Messrs. Robinson, J. N. Peacock, L. M. Lewis, and S. M. Hodge of MIB, and Mr. B. G. Palmertree, Instrumentation Services DIvision, Operations Branch, WES, conducted the dynamics test program at Fort Sill, Oklahoma. Mr. R. G. Temple, MRMB, prepared artillery route network maps from job overlay maps, and Mr. C. D. Currie, MIB, digitized the route network maps for computer use. Messrs. R. P. Smith, B. R. Wright, and R. B. Ahlvin, Data Handling Branch (DHB), MSD, prepared the vehicle performance predictions for this study. Messrs. Temple, Peacock, and Currie, and Mrs. S. B. Anglin (DHB) assembled the vehicle characteristics data and prepared the data tables. Personnel of the Science and Technology Division, U. S. Army Tank-Automotive Research and Development Command (TARADCOM), exercised the Army mobility model (AMM) to predict vehicle performance in linear terrain. Supporting activities of TARADCOM were under the direction of Dr. J. G. Parks, Chief, Engineering Sciences Division, and Mr. Z. J. Janosi, Chief, Methodology Function Directorate.

COL G. H. Hilt, CE, and COL J. L. Cannon, CE, were Directors of WES during the conduct of the study and preparation of the report. Mr. F. R. Brown was Technical Director. 
CONTENTS

Page

PREFACE . . . . . . . . . . . . . . . . . . . . 2

LIST OF TABLES . . . . . . . . . . • . . . . . . . . . . 4

CONVERSION FACTORS, METRIC (SI) TO U. S. CUSTOMARY AND

U. S. CUSTOMARY TO METRIC (SI) UNITS OF MEASUREMENT . . • . • 6

PART I: INTRODUCTION . . . . . . . . . . . . . . . 7

Background ..................... 7

Objective of LEGAL MIX V Study ............. 8

Objective of WES Support ............... 8

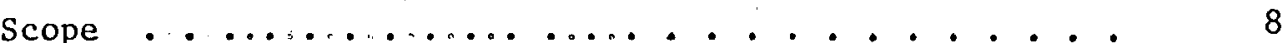

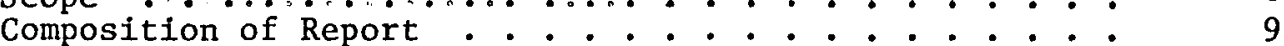

PART II: DESCRIPTION OF VEHICLES, TERRAIN DATA, AND SEASONAL CONDITIONS USED IN THIS STUDY . . . . . . . . . . 10

Vehicles . . . . . . . . . . . . . . 10

Terrain and Seasonal Conditions . . . . . . . . . 11

PART III: MOBILITY PREDICTIONS . . . . . . . . . . . . . 14

Performance Prediction over Generalized Terrain . . . 15

Performance Predictions over HIMO Detalled Terrain . . , 16

Performance Predictions for Artillery Missions . . . . 20

PART IV: RELATIVE MOBILITY OF CANDIDATE VEHICLES . . . . . . 21

Suggested Mobility Comparisons Based on Generalized

Terrain Data . . . . . . . . . . . . . .

Suggested Mobility Comparisons Based on HIMO

Methodology and Detailed Terrain Data . . . . . . . 22

Suggested Method for Ranking of Vehicle Groups . . . . 26

REFERENCES . . . . . . . . . . . . . . . . . . 34

APPENDIX A: JIFFY MOBILITY MODEL . . . . . . . . . . . . Al

APPENDIX B: DATA USED TO CHARACTERIZE STUDY VEHICLES . . . . B1

APPENDIX C: RESULTS OF EXPERIMENTAL DYNAMICS TESTS . . . . . $\quad$ C1

APPENDIX D: GENERALIZED TERRAIN DATA . . . . . . . . . $\quad$ D1

APPENDIX E: BASIC PERFORMANCE PREDICTIONS FOR HIMO WEST

GERMANY AND MID-EAST STUDY AREAS . . . . . . . E1

APPENDIX F: SELECTION OF TACTICAL HIGH AS MOBILITY LEVEL

FOR COMPARING STUDY VEHICLES . . . . . . . . . F1

APPENDIX G: COMPUTATION OF MISSION-ORIENTED AVERAGE SPEED

BASED ON STÄTISTICAL MISSION DEFINITION AND

VEHICLE PERFORMANCE STATISTICS FOR AN AREA

AND CONDITION ................

G1 
1 List of Study Vehicles . . . . . . . . . . . 35

2 Principal Characteristics of Study Vehicles . . . . 36

3 Characteristics of As-Is Route Networks . . . . . 38

4 Performance Predictions for Study Prime Mover-Towed Artillery Combinations in the Countries Described by Generalized Terrain Data . . . . . . . . .

5 Performance Predictions for Study Self-Propelled Artillery and Reference Vehicles in the Countries Described by Generalized Terrain Data . . . . .

$6 \mathrm{~V}_{90}$ Off-Road Performance Indicies for Study Prime Mover-Towed Artillery Combinations in the HIMO Study Areas ...................

$7 \quad \mathrm{~V}_{90}$ Off-Road Performance Indicies for Study SelfPropelled Artillery and Reference Vehicles in

the HIMO Study Areas ................

$8 \mathrm{~V}_{100}$ Speeds for Study Prime Mover-Towed Artillery Combinations Over Primary and Secondary Roads and Trails in the HIMO Study Areas . . . . . . .

$9 \mathrm{~V}_{100}$ Speeds for Study Self-Propelled Artillery Secondary Roads and Trails in the HIMO Study Areas . . . . . . . . . . . . . . . .

10 Percent NOGO for Study Prime Mover-Towed Artillery Combinations on Primary and Secondary Roads and Trails in the HIMO Study Areas . . . . . . . . .

11 Percent NOGO on Primary and Secondary Roads and Trails for Study Self-Propelled and Reference Vehicles in the HIMO Study Areas... . . . . . .

12 Percent NOGO for Study Prime Mover-Towed Artillery Combinations in off-Road Terrain in the HIMO Study Areas .................. .

13 Percent NOGO for Self-Propelled Artillery and Reference Vehicles in Off-Road Terrain in the HIMO Study Areas..................

14 Average Link Speeds (mph) for the Study Prime Mover-Towed Artillery Combinations in the HIMO Study Areas . . . . . . . . . . . . . . . 
15 Average Link Speeds (mph) for the Study Self-Propelled and Reference Vehicles in the HIMO Study Areas...

16 Percentage of the Total Time for Traversing Links That Study Prime Mover-Towed Artillery Combinations Spent Crossing Linear Features in the HIMO Study Areas ......................

17 Percentage of the Total Time for Traversing Links That Study Self-Propelled Artillery and Reference Vehicles Spent in Crossing Linear Features in the HIMO Study Areas . . . . . . . . . . . . . . . .

18 Preliminary Quantification of WHEELS Study Definitions of Tactical Mobility . . . . . . . . . . . .

19 High and High-High Levels of Tactical Mobility Performance of Study Prime Mover-Towed Artillery Combinations in the HIMO Study Areas . . . . . . .

20 High and High-High Levels of Tactical Mobility Performance of Study Self-Propelled Artillery and Reference Vehicles in the HIMO Study Areas ...

21 Comparison of Performance of Individual Vehicles and Selected Vehicle grouped on Primary Roads, Secondary Roads, Trails, and Off-Road in the HIMO Study Areas . . . . . . . . . . . . . . . .

22 Relative Mobility Performance of Study Prime Movers Based on Performance in Countries Described by the Generalized Terrain Data . . . . . . . . .

23 Comparison of Selected Prime Mover-Towed Artillery with Self-Propelled M109A1 Based on Performance in Countries Described by the Generalized Terrain Data ....................

24 Relative Mobility of Prime Movers Based on Tactical High Mobility Level in HIMO Study Areas . . . . . .

Comparison of Selected Prime Mover-Towed Artillery and the MI09Al at Two Levels of Mobility in the HIMO Study Areas

26 Ranking of Selected Vehicle Groups Based on Performance in the HIMO Study Areas . . . . . . . 
CONVERSION FACTORS, METRIC (SI) TO U. S. CUSTOMARY AND

U. S. CUSTOMARY TO METRIC (SI) UNITS OF MEASUREMENT

Units of measurement used in this report can be converted as follows:

\begin{tabular}{|c|c|c|}
\hline Multiply & By & To obtain \\
\hline \multicolumn{3}{|c|}{ Metric (SI) to U. S. Customary } \\
\hline millimetres & 0.03937007 & Inches \\
\hline square kilometres & 0.3861 & $\begin{array}{l}\text { square miles } \\
\text { (U. S. statute) }\end{array}$ \\
\hline \multicolumn{3}{|c|}{ U. S. Customary to Metric (SI) } \\
\hline Inches & 0.0254 & metres \\
\hline feet & 0.3048 & metres \\
\hline miles (U. S. statute) & 1.609344 & kilometres \\
\hline square inches & $6.4516 \times 10^{-4}$ & square metres \\
\hline pounds (force) & 4.448222 & newtons \\
\hline kips (force) & 4448.222 & newtons \\
\hline tons (force) & 8896.444 & newtons \\
\hline $\begin{array}{l}\text { pounds per square } \\
\text { Inch }\end{array}$ & 6.894757 & kilopascals \\
\hline $\begin{array}{l}\text { miles (U. S. statute) } \\
\text { per hour }\end{array}$ & 1.609344 & $\begin{array}{l}\text { kilometres per } \\
\text { hour }\end{array}$ \\
\hline degrees (angular) & 0.01745329 & radians \\
\hline horsepower per ton & 83.82 & watts per kilonewton \\
\hline
\end{tabular}




\section{MOBILITY PERFORMANCE OF TOWED AND SELF-PROPELLED ARTILLERY AND RELATED VEHICLES}

PART I: INTRODUCTION

\section{Background}

1. As part of Force Artillery Mix-1976 (FAM-76), the U. S. Army Engineer Waterways Experiment Station (WES) conducted a study in 1972 for the U. S. Army Combat Development Command Field Artillery Agency (USACDFAA). In this study WES used the Army mobility model AMC-71 ${ }^{1}$ to determine the on- and off-road mobility performances of a group of selfpropelled artillery and prime mover-towed artillery combinations, with generalized and detailed terrain descriptions representing a variety of operational environments.

2. As a result of recent development in applying mobility methodology to and analyzing detailed terrain data from two study areas in the U. S. Army Training and Doctrine Command (TRADOC) study "Special Analysis of High-Mobility Vehicles" (short title, "HIMO Study") ${ }^{2}$ and related studies, TRADOC asked WES to conduct similar mobility determinations in support of another study, LEGAL MIX V. For this study, WES used the updated Army mobility mode1, AMC- $74 \mathrm{X}^{2,3,4}$ and the terrain data base developed for the HIMO Study to determine performances of selfpropelled artillery, prime mover-towed artillery combinations, and other artillery support and reference vehicles. In addition, a simple model to make GO/NOGO predictions termed the "JIFFY mobility model"* was also used to make predictions for nine countries in which terrain was described with less detail than the HIMO data base. The JIFFY mobility model was designed for use with terrain data described in less detall than required for $\mathrm{AMC}-74 \mathrm{X}$. The $\mathrm{AMC}-74 \mathrm{X}$ was not used to make predictions for the generalized terrain data because the terrain was not described in sufficlent detail.

* Described in Appendix A. 


\section{Objective of LEGAL MIX V Study}

3. The objective of the LEGAL MIX V Study is to evaluate the relative effectiveness of current and projected towed and self-propelled artillery and of various mixes in specific artillery missions and operational environments.

\section{Objective of WES Support}

4. The objective of the WES support was to provide specific predictions of on- and off-road mobility performances of varfous study vehicles, i.e. self-propelled artillery, prime mover-towed artillery combinations, and other related vehicles in selected terrains or road combinations, and use these mobility predictions as a data base to suggest methods for analyzing the relative mobility effectiveness of candidate vehicles.

\section{Scope}

5. The scope of activities necessary to achieve the study objective were:

a. The JIFFY mobility model (Appendix A) was used to predict the probability of off-road GO performances of 60 study vehicles in 9 countries described by generalized terrain data (Part III).

b. The AMC-74X mobility model (AMM) (paragraph 20) and HIMO methodology were used to predict off-road and on-road performances of 13 selected study vehicles in the HIMO West Germany and Mid-East study areas described by detalled terrain data. Performance predictions in each study area included the following:

(1) Speed profiles for primary roads, secondary roads, trails, and off-road and related indices $\left(\mathrm{V}_{90}, \mathrm{~V}_{100}\right.$ ' percent NOGO) (Part III).

(2) Diagnostic statistics for primary roads, secondary roads, trails, and off-road (Part III).

(3) Link statistics for "as-is" and off-road links (Part IV). 
(4) Ranking of vehicles for tactical high and tactical high-high missions (Part IV).

(5) Relative ranking of selected groups of vehicles (Part IV).

(6) Time to complete selected artillery-related missions in West Germany (Part IV).

Items $1,2,4$, and 5 were developed using the same representative sample of the terrain for the two HIMO study areas as used in the HIMO analyses. The West Germany sample terrain contained 16 percent of the study area and the Mid-East sample terrain contained 13 percent of the study area.

c. A limited number of experimental ride and shock tests were conducted to establish ride dynamics relations for some of the study vehicles.

\section{Composition of Report}

6. This report contains a main text and 8 appendices: Appendix A describes the JIFFY mobility model, Appendix B presents the vehicle characteristics used in this study, Appendix $\mathrm{C}$ includes the results of experimental vehicle dynamic tests, Appendix D describes the generalized terrain data, Appendix E contains the basic performance data for HIMO West Germany and Mid-East study areas, Appendix F discusses the selection of tactical high as suggested mobility level for comparing study vehicles, Appendix G'shows the computation of mission-oriented speed based on statistical mission definition and vehicle performance statistics for a given area and condition. 
PART II: DESCRIPTION OF VEHICLES, TERRAIN DATA, AND SEASONAL CONDITIONS USED IN THIS STUDY

\section{Vehicles}

7. Sixty study vehicles were selected by the LEGAL MIX V Study Group, 47 prime mover-towed artillery combinations, 3 self-propelled artillery, and 10 reference vehicles. A list of the study vehicles is given in Table 1 .

Vehicle characteristics

8. All study vehicles were characterized as carrying their rated payloads. Principal characteristics of all of the study vehicles are listed in Table 2. Vehicle data as required for input to the AMM and JIFFY mobility model are given in Appendix $B$.

Ride and shock tests

9. Both field experience and simulations have shown that dynamic responses of vehicles traversing rough terrain and crossing minor obstacles often significantly influence speed. AMM is so structured that values for these critical vehicle characteristics can be obtained by simulations or from experimental data.

10. Experimental ride and shock data were available from previous test programs ${ }^{5}$ for most of the prime movers, but not for the towed artillery. Therefore, a test program was conducted to determine if the towing of an artillery piece by a prime mover would result in significant changes in the ride and shock responses of the prime mover, and to fill the voids in the existing data base. Also, data were collected for two self-propelled artillery vehicles. The results of these tests are presented in Appendix $C$.

11. It was concluded from this spot-check test program that the towed artillery had no significant effect on the vertical ride and shock characteristics of the prime movers at the driver's station. These characteristics are important inputs to the mobility model. It was also noted that additional limits to those used limiting performance due to ride and shock responses might be required to protect tires and rims 
of the towed artillery from physical damage. The limited scope of this test program did not produce sufficient data to define such additional limits, however, and none were used in evaluations made in the mobility mode1.

\section{Terrain and Seasonal Conditions}

\section{Terrain}

12. Generalized terrain data for nine countries and detailed terrain data for portions of two countries were used in this study.

13. Generalized terrain. The generalized terrain data were prepared from maps with scales ranging from $1: 825,000$ to $1: 3,200,000$ and other limited data. Because the maps are classified, the countries are referred to herein as countries A, B, C, D, E, F, G, H, and I. These countries are identified in Reference 6 . The generalized terrain data use 5 parameters (soil type, soil strength, slope, obstacle height, and vegetation stem size and spacing) to describe the off-road terrain (Appendix D). No linear terrain features or roads are described for the generalized terrain data base.

14. Detailed terrain. The detailed terrain data used in this study are for the HIMO West Germany and Mid-East study areas and were prepared from maps at a scale of 1:50,000. In the West Germany study area, predictions were made for a condition in which the terrain was uniformly covered with $10 \mathrm{in.} \mathrm{of} \mathrm{dry} \mathrm{snow,} \mathrm{which} \mathrm{is} \mathrm{a} \mathrm{reasonably} \mathrm{maximum}$ average depth for the actual area. In the Mid-East study area, predictions were made for a condition in which the terrain was arbitrarily covered with clean sand common in sand dunes. These special terrain conditions are described in Reference 2.

15. Each HIMO study area contairs about $3000 \mathrm{~km}^{2}$.* The terrain descriptions inc:ilde areal, linear, and road-network data; 22 factors describe a patch of areal terrain, 10 a segment. of linear terrain, and

* A table of factors for converting metric (SI) units of measurements to U. S. customary units, and vice versa, is given on page 6 . 
8 a segment of road. The HIMO road-network data were derived by connecting the mission road and points (units and supply points or successive unit positions). Since this network was made up primarily of routes in connection with tactical support, the LEGAL MIX V Study Group selected a group of 37 missions within the West Germany HIMO study area related to artillery movement (identified as tactical movements 1-37 in Figure 1), and this network was used in this study. No routes were selected by the LEGAL MIX V Study Group for Mid-East terrain. The percentages of primary and secondary roads, trails, and off-road terrain in the HIMO and artillery routes in West Germany and the HIMO Mid-East routes are given in Table 3 . This distribution shows that the artillery route network contains more trail and off-road travel than does the HIMO network in West Germany.

Seasonal weather conditions

16. Generalized terrain. Vehicle performance was predicted for the 9 countries for both wet and dry surface conditions using the soil strength associated with these two conditions (Appendix D). The wet condition is decribed as wet with associated reduced soil strengths but surface not considered to be slippery.

17. Detailed terrain. Vehicle performance was predicted for each HIMO study area for an excessive wet period and still raining, hereafter called wet condition. The wet condition for the detailed terrain data base is generally worse from the standpoint of vehicle mobility than the wet condition described for the generalized terrain because potential slipperiness of soil surfaces was considered in addition to reduced soil strengths due to high moisture contents of soil. During the wet condition, the soil surface of trails, primary roads, and secondary roads in the detailed terrain data base were considered to be slippery. 


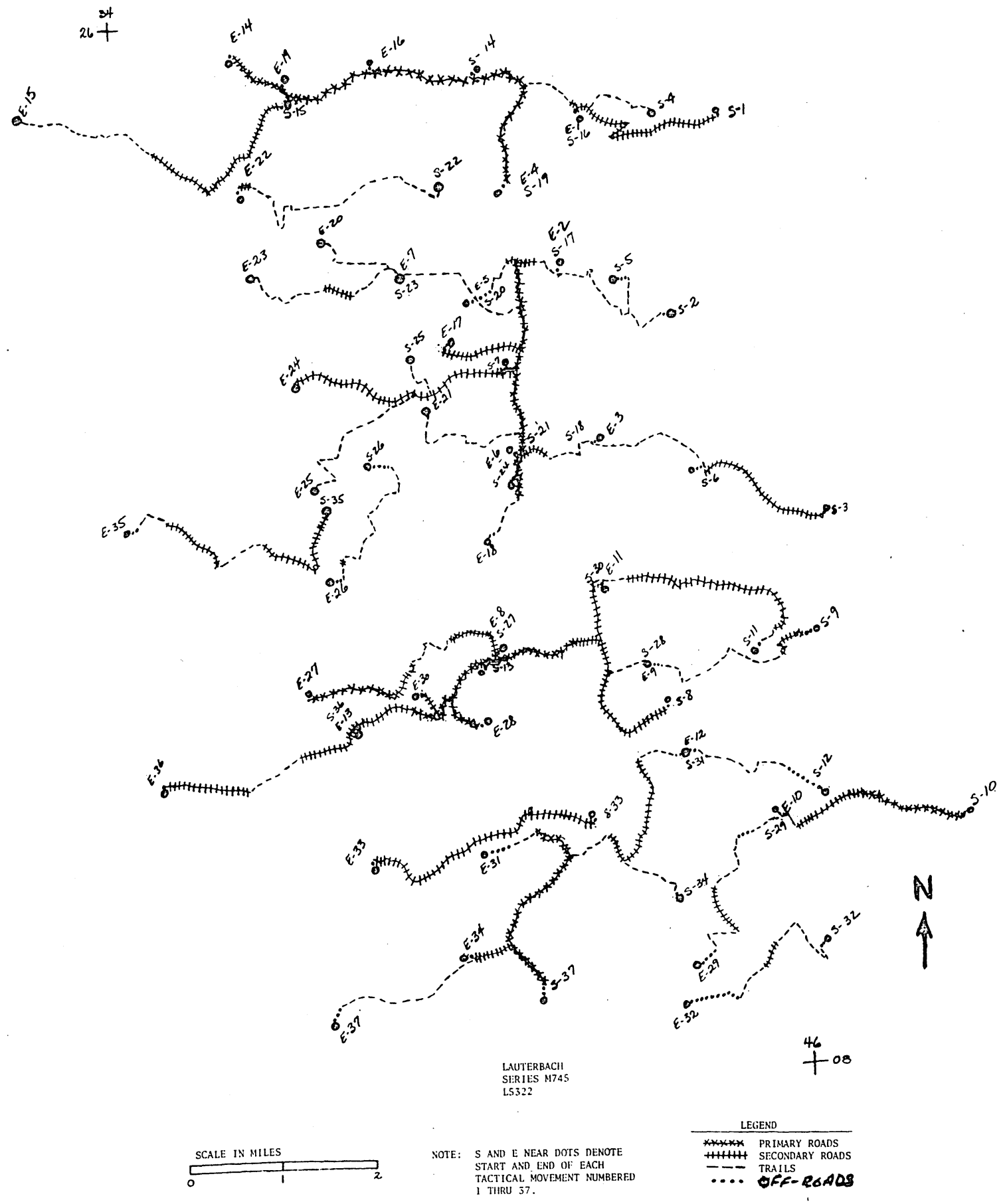

Figure 1. Mission network map 
18. The JIFFY mobility model (Appendix A) is a very simple model designed for use with generalized terrain data. It was used to predict GO/NOGO for all the study vehicles in the nine countries described by generalized terrain data.

19. The AMC mobility model (AMM) was used to predict on-road and off-road speed performance and time to cross linear terrain features for 43 selected study vehicles in the two HIMO areas and for selected artillery routes. The version of the AMM (AMC-74X) used in this study was the first-generation AMC-71 with a number of significant improvements in the predictive algorithms that have since been incorporated in the second-generation version (AMC-74) published in 1975. A brief description is given in Appendix $A$ of Reference 2.

20. Most of the performance data for prime movers towing artillery are presented in the form of matrices to make it easy to compare the relative mobility of the candidate prime movers. The towed artillery were also grouped according to weight as follows: the 3340-1b M102 and the 4775-1b XM204 were referred to as lightweight artillery; the 12,7001b. M114A1, the 15,250-1b XM198, and the 16,590-1b XM(130-mm) were referred to as medium-weight artillery; and the 19,250-1b FH70 was designated heavyweight artillery.

21. To reduce cost, performance predictions over the detailed HIMO terrain and artillery routes were not made for 17 of the prime movertowed artillery pieces, but performances of these 17 vehicles are referenced to similar prime mover-towed artillery combinations for which predictions were made.

22. Performance predictions for the study vehicles over the generalized terrain, the detailed terrain, and the special artillery routes are discussed in the following paragraphs. 


\section{Performance Predictions over Generalized Terrain}

23. Since the generalized terrain data were prepared from smallscale maps and other limited available data (paragraph 12), quantitative terrain factors were mapped in broad class intervals. In the basic GO/NOGO predictions from the JIFFY mobility model (Appendix A), the midclass value could be used as most representative of each terrain factor. However, endless possibilities exist, within the original description, for each value independently to lie at other points within the range, some of which may be more favorable for vehicle progress, others less so. Therefore, for this study GO/NOGO predictions were made with the JIFFY model for maximum, middle, and minimum values of soil, slope, and obstacle parameters (27 combinations) allowed by the class designators for each terrain unit (Appendix D). On the assumption that each of the three levels of each parameter had an equal likelihood of occurrence, and that the three parameters were independent, the total number of Go cases divided by the full 27 possibilities was taken as an expression of "probability of GO" for the given vehicle in a terrain unit.

24. For this study probabilities of GO were then grouped in three classes and assigned mobility levels as follows:

$\frac{\text { Probability of GO }}{0-33}$
$>33-66$
$>66-100$

Mobility Leve1

NOGO to marginal GO

$\mathrm{GO}$, but with some route selection required

GO with relative freedom of movement

25. Probability of $G 0$ predictions were made for each of the study vehicles during the wet and dry conditions over each of the countries described by generalized terrain data. The percentage of each country that a given study vehicle could negotiate in that country at a given mobility level is given for each study vehicle in Tables 4 and 5 . 


\section{Performance Predictions Over HIMO Detalled Terrain}

26. Performance predictions for the HIMO detailed terrain data are described in the following paragraphs.

Speed profiles

27. A principal means of displaying area-wide off-road performance in areal terrain is an off-road speed profile. The off-road speed profile for a given vehicle, terrain, and seasonal weather condition shows the average speed the vehicle can sustain, as a function of the percentage of the total area under consideration that it avoids, under the assumption that it avoids areas posing the greatest impediment to its motion. AMM areal terrain speed predictions were run for all vehicles and two conditions (wet and snow conditions for West Germany and wet and sand conditions for the Mid-East) in the link traverse sample of the areal terrain characterized for one full topographic map sheet in each study area. The single map sheet selected for each area, covering 16 percent of the total West Germany study area and 13 percent of the Mid-East study area, is considered to be representative of the entire area and was the same sample used for the HIMO study. An example of an off-road profile derived from the AMM predictions is given in Figure 2.

28. The performances of the 43 selected study vehicles on primary roads, secondary roads, and trails can be displayed in speed profiles similar to the off-road speed profiles. Tables E1-E43 (Appendix E) 1ist the speed profiles data in tabulated form (same as tabulated form shown in bottom right corner of Figure 2) for each of the study vehicles in the HIMO study areas for two selected conditions on primary roads, secondary roads, trails, and off-road.

Performance indices

29. Four performance indices $\left(V_{90}\right.$ for off-road areal terrain and $v_{100}$ for primary and secondary roads and trails) were derived directly from speed profiles. All were used to make discriminations in vehicle performance in the Department of the Army (DA) WHEELS Study. ${ }^{7} \mathrm{v}_{90}$ is the average areal terrain speed when the vehicle is avoiding the 10 
JORDAN

VEHICLE: MII3AI

CONDITION WET

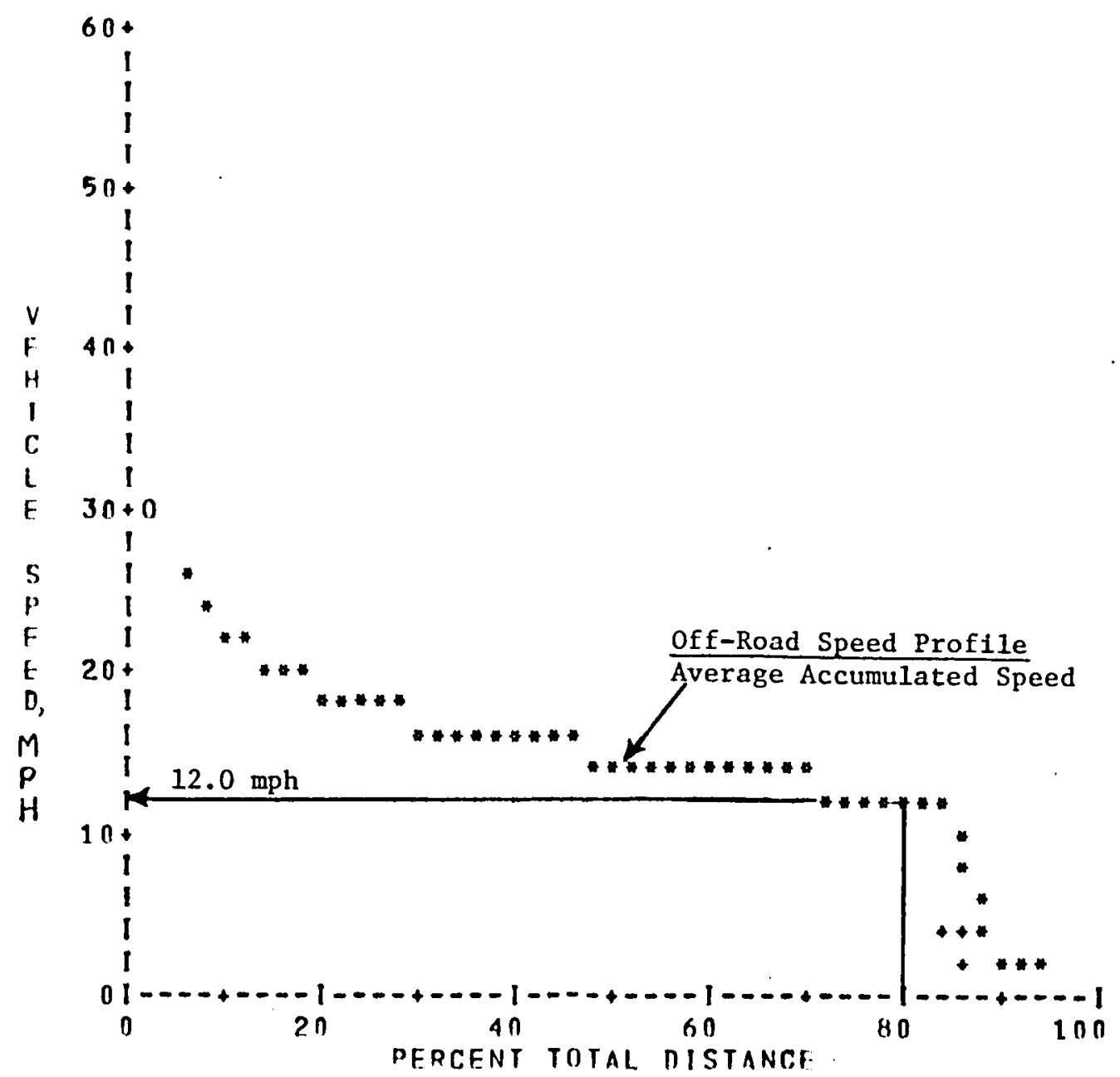

PERCENT MISTANCE "NO-GO" $=14.1$.

\section{PERCENT TOTAL DISTANCE}

\begin{tabular}{|c|c|c|c|c|c|}
\hline $\begin{array}{r}x \\
1 x \\
2 x\end{array}$ & $\begin{array}{l}x=0 \\
31.9 \\
22.3 \\
18.7\end{array}$ & $\begin{array}{c}2 \\
3 \cap .1 \\
21.5 \\
18.3\end{array}$ & $\begin{array}{c}4 \\
28.1 \\
20.9 \\
18.0\end{array}$ & $\begin{array}{c}6 \\
25.2 \\
20.0 \\
17.6\end{array}$ & $\begin{array}{c}8 \\
23.6 \\
19.3 \\
17.2\end{array}$ \\
\hline $\begin{array}{l}3 x \\
4 x\end{array}$ & $\begin{array}{l}16.9 \\
15.6\end{array}$ & $\begin{array}{l}16.6 \\
15.4\end{array}$ & $\begin{array}{l}16.3 \\
15.3\end{array}$ & $\begin{array}{l}16.1 \\
15.1\end{array}$ & \\
\hline $\begin{array}{l}5 x \\
6 x \\
7 x \\
8 x \\
9 x\end{array}$ & $\begin{array}{r}14.7 \\
13.9 \\
13.0 \\
\frac{12.0}{1.9}\end{array}$ & $\begin{array}{r}14.6 \\
13.8 \\
12.8 \\
11.7 \\
1.4\end{array}$ & $\begin{array}{r}14.4 \\
13.6 \\
12.6 \\
11.3 \\
1.1\end{array}$ & $\begin{array}{r}14.3 \\
13.4 \\
12.1 \\
10.7 \\
0.9\end{array}$ & $\begin{array}{r}14.1 \\
13.2 \\
13.2 \\
3.2 \\
0.8\end{array}$ \\
\hline & 0. & & & & \\
\hline
\end{tabular}

Figure 2. Off-Road speed profile data 
percent of the areal terrains that is the most difficult. $v_{1}$ is the average speed $\left(\mathrm{V}_{100}\right)$ when the vehicle is traversing all primary roads, $\mathrm{V}_{2}$ is the average speed when it is traversing all secondary roads, and $\mathrm{V}_{3}$ is the average speed when it is traversing all trails. $v_{90}$ speed for each study vehicle is given in Tables 6 and $7 . v_{1}, v_{2}$, and $v_{3}$ for each study vehicle is given in Tables 8 and 9 .

3). Other indices that have been used to make discriminations in vehicle performance are "percent NOGO" for primary roads, secondary roads, trails, and off-road (DA WHEELS Study used "percent GO"). "Percent NOGO" for primary roads, secondary roads, and trails is given in Tables 10 and 11; and "Percent NOGO" for off-road is given in Tables 12 and 13.

Reasons for NOGO's or speed limits

31. Concurrent with the assembly of the speed profiles, the data were analyzed to obtain diagnostic statistics that show the relative occurrence of reasons for NOGO's and the factors limiting speed in the GO situations. Also included in these statistics is the average speed of the vehicle when controlled by a given factor. Tables E44-E87 (Appendix E) give the diagnostic statistics for the study vehicles for two selected conditions in the HIMO study areas. These diagnostic data characterize the two areas in terms of the type of mobility problems posed, as well as indicate for specific vehicles the design areas in which changes might benefit performance.

Link predictions

32. When predictions were made for the composite route network of a11 roads and trails in the study area, plus such off-road traverses as might be required for mission completion under reasonably favorable combat conditions ( 0.1 percent off-road in the West Germany study area; 1.1 percent in the Mid-East study area), the predictions were referred to as the "as-is link predictions." When all links are treated as off- road traverses, the route network becomes a substantial scenariooriented sample of all off-road areal and linear terrain throughout the study area and is referred to as "off-road link predictions."

33. As-is and off-road predictions of average speed over all links 
were made for each of the study vehicles for two selected conditions in both of the HIMO West German and Mid-East study areas. These link speed prediction data are given in Tables 14 and 15 . The percentages of the total time for traversing links that the vehicles spent crossing linear features are given in Tables 16 and 17. Tactical mobility levels rating speeds

34. The link prediction data and the methodology described in the HIMO report were used to obtain the performance of the study vehicles at the high and high-high (see Table 18 for definition of "high-high") mobility levels. These data are given in Tables 19 and 20. Tactical mobility levels are discussed in more detail in paragraphs 45 and 46. Vehicle group performance

35. Self-propelled artillery and prime mover-towed artillery are often accompanied by other vehicles. When artillery and other vehicles travel together, the speed of the slowest vehicle in each terrain patch crossed will control the overall group speed in that patch. Since the same vehicle is not always the slowest vehicle in every patch, the vehicle group speed for a complete traverse through a study area will in general be slower than that of any one of the vehicles singly. On roads (but not trails), limiting speeds are largely determined by vehicle power levels, so that in road operations the speed of a mixed vehicle group will be very close to that of the slowest vehicle in the group.

36. Four vehicle groups were selected by LEGAL MIX V Study Group as representative of the types of vehicle groups as follows:

\begin{aligned} Group & $\begin{array}{c}\text { Self-Prope1led, Prime Mover-Towed Artillery, and } \\ \text { Accompanying Vehicles }\end{array} \\$\cline { 2 - 2 } I & M109A1, M548E1, M577A1 \\ II & M813-XM198, M561 \\ III & M548E1-XM198, M577A1 \\ IV & M548E1-XM198, M561 \end{aligned}

37. Using the speed predictions for the individual vehicles in the group and selecting the vehicle with the lowest speed as controlling the speed of the vehicle group, speed profiles were prepared for each 
vehicle group for two selected conditions on primary roads, secondary roads, trails, and off-road for the HIMO West Germany and Mid-East study areas. The speed profiles for the vehicle groups are given in Tables E88-E91 (Appendix E).

38. The speed profiles for the vehicle groups were used to obtain $\mathrm{V}_{100}$ and percent NOGO for the primary roads, secondary roads, and trails; and $V_{90}$ for off-road. These data and similar data for individual vehicles traveling alone are presented in Table 21 for comparison. Also shown in Table 21 is the percentage of the traverse distance that each vehicle limited the overall performance of the group. When two or more vehicles were controlling at the same time in a given terrain patch due to identical speeds in that patch, the percentage of traverse distance was included for each vehicle; therefore, the total distance of all vehicles traveling together is greater than 100 percent.

\section{Performance Predictions for Artillery Missions}

39. Performance predictions in terms of time for one-way trave1 between two points indicated as start and end of each mission were made for each of the study vehicles over each of the missions in the West German study area identified by the LEGAL MIX V Study Group. These performance predictions are given in Tables E92 and E93 (Appendix E). Also included in the tables are the mission distances from which, when coupled with the times, the average speeds over the routes can be computed. 
40. This study does not analyze the mobility predictions for the generalized and detailed terrain data presented in Part III but does suggest methods of establishing (a) the relative mobility of candidate prime movers for towing lightweight, medium-weight, and heavyweight artillery, (b) the relative mobility of candidate self-propelled artillery, and (c) the mobility of selected prime mover-towed artillery relative to that of self-propelled artillery. The mobility predictions for the detailed terrain data were also used to suggest methods for establishing the relative mobility for each selected group of vehicles traveling together.

\section{Suggested Mobility Comparisons Based on Generalized Terrain Data}

41. The performance parameter selected for comparing the study vehicles in each of the nine countries described by generalized terrain data was the percentage of the country with a $>66-100$ probability of $G 0$ (Tables 4 and 5).

Relative mobility of

candidate prime movers

42. The data in Table 4 giving the percentage of country with >66-100 percent probability of GO were used to determine the relative mobility of the prime movers towing lightwelght artillery (group 1), prime movers towing medium-welght artillery (group 2), and prime movers towing heavyweight artillery (group 3 ) in each of the nine countries (Table 22). The relative mobility of each vehicle in a group is expressed as a percent of the "best vehicle", 1.e. that vehicle that can negotiate the largest percentage of a country.

Relative mobility of self-propelled artillery

43. Table 5 shows very few differences in the performances of the self-propelled artillery in terms of percent of country with $>66-100$ percent probability of GO, except in country B during dry conditions 
where the M107 had about a 20 percent increase in GO area over the MIIOE2 and MI09Al. The self-propelled artillery always equaled or exceeded the performance of the M60A2 and M113A1 reference vehicles. Mobility of prime mover-towed artillery combinations relative to test of self-propelled artillery

44. Three prime movers towing one of each weight class of artillery (1ightweight, medium-weight, and heavyweight) were selected for comparing their mobility performances with the mobility performance of a selected self-propelled artillery piece. The percentages of each country. (for two conditions) that the prime mover-towed artillery could negotiate with a $>66-100$ probability of GO are compared to similar performance data for the self-propelled M109A1 in Table 23. These data are also plotted in the form of bar graphs in Figures 3 and 4. Sufficient data are listed in Tables 4 and 5 for making similar comparisons between any of the other prime mover-towed artillery combinations and self-propelled artillery.

\section{Suggested Mobility Comparisons Based on HIMO}

Methodology and Detailed Terrain Data

45. The mobility of the study vehicles can be compared by using any of the single vehicle performance parameters presented in Part III. The WHEELS study ${ }^{7}$ used the $v_{90}$ off-road speeds (Tables 6 and 7 ), the $\mathrm{V}_{100}$ speeds for primary and secondary roads and trails (Tables 8 and 9), and the percent of off-road terrain NOGO (Tables 12 and 13). The average link speeds (Tables 14 and 15) and the percentage of link time vehicles spent crossing linear features (Tables 16 and 17), developed for the HIMO study, also represent performance data for assessing mobility of the study vehicles. These mobility performance data are all useful in comparing study vehicles with vehicles used in other studies; however, vehicle comparisons based on the WHEELS mobility-level definitions seem to be the most useful since they define in general terms the missions that vehicles are required to complete. The WHEELS study 


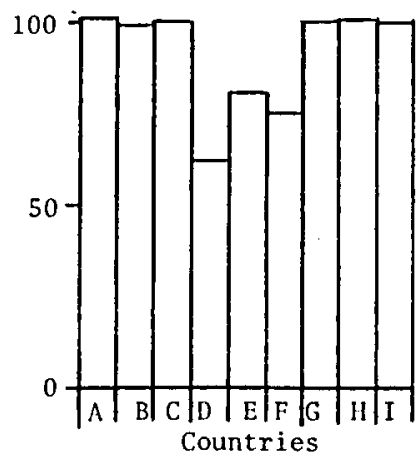

a. ASV-XM204

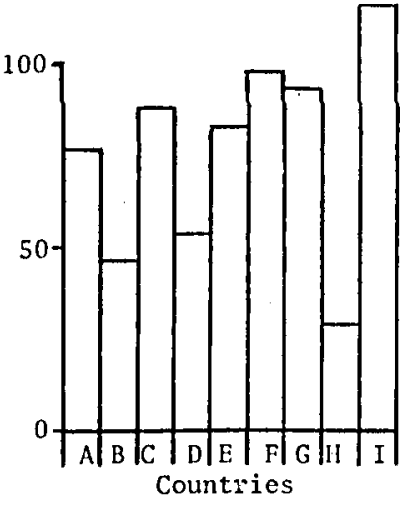

b. TDW901-XM204

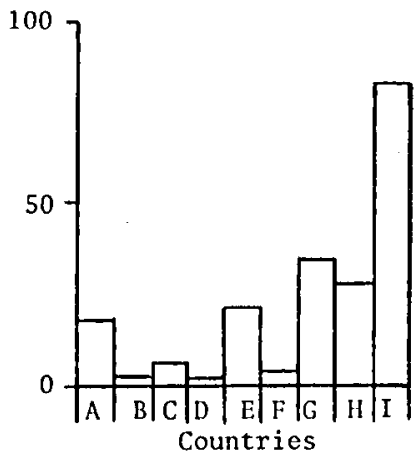

c. M561-XM204

0
0
0
0
0
0
4
0
0
0
0
0
0
0
2
4
0
0
0
0
0
0
0
0

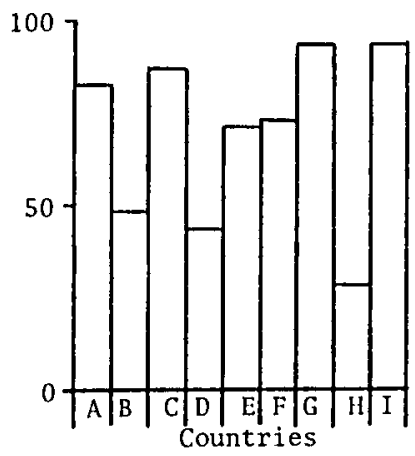

d. ASV $-\mathrm{XM} 198$

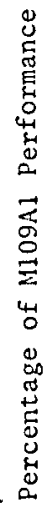

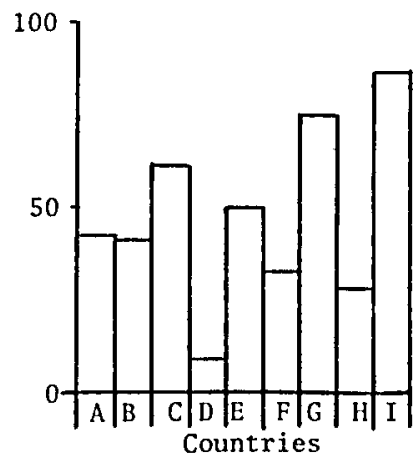

g. ASV-FH70

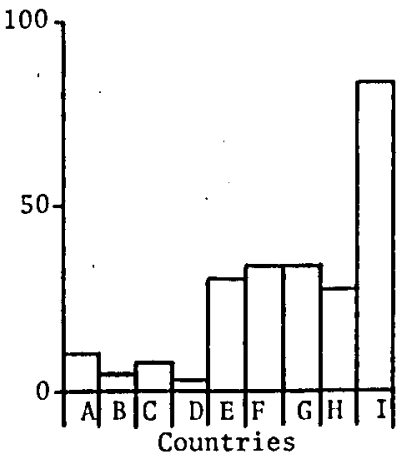

h. M125E1-FH70

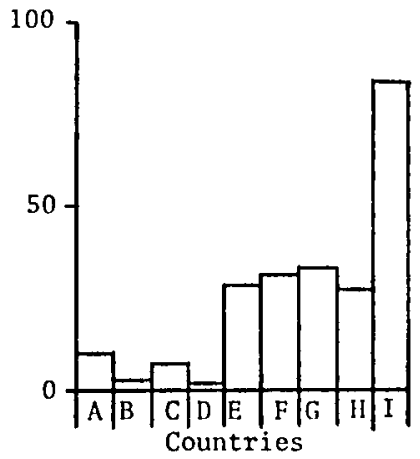

i. M520E1-FH70

NOTE: XM204 is considered lightweight artillery, XM198 considered medium-weight artillery, and FH7O considered heavyweight artillery.

Figure 3. Comparisons of performances of the selected prime movers towing artillery of different weight classes with performance of self-propelled Ml09Al in countries A-1 during wet conditions 


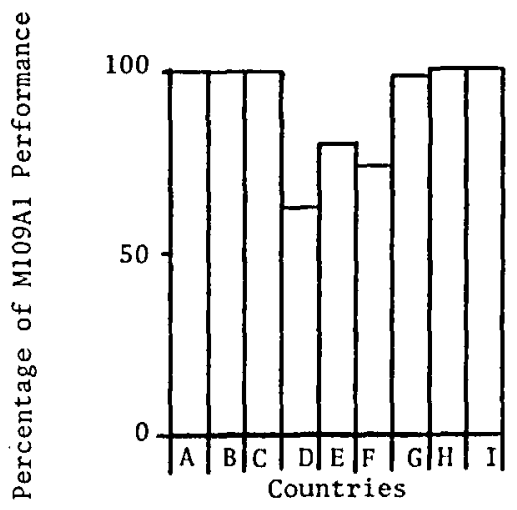

a. ASV-XM204

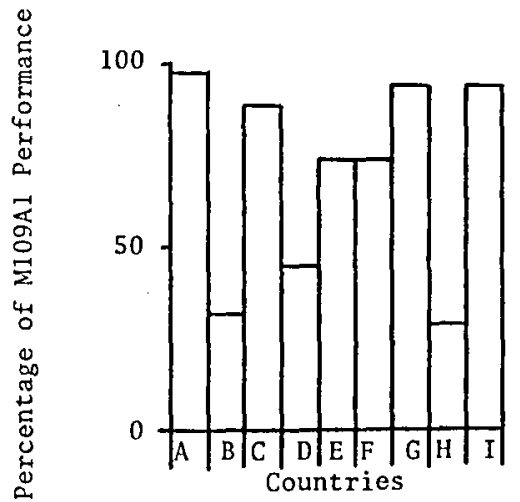

d. ASV-XM198

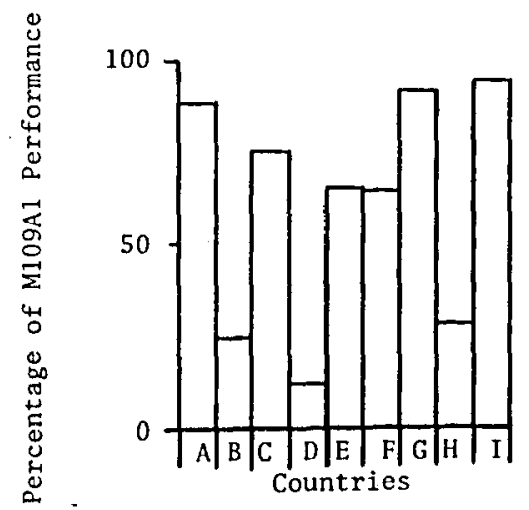

g. ASV-FH70

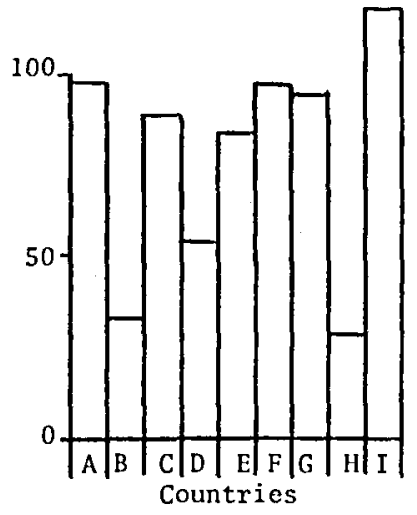

b. TDW901-XM204

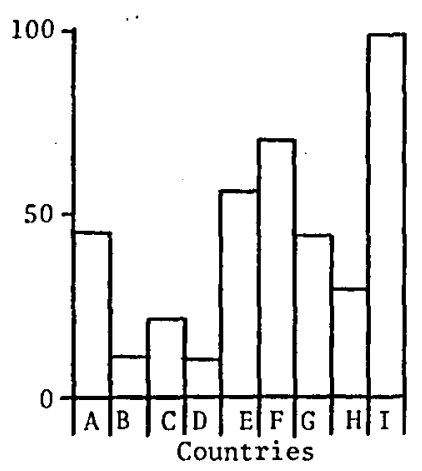

e. TDW901-XM198

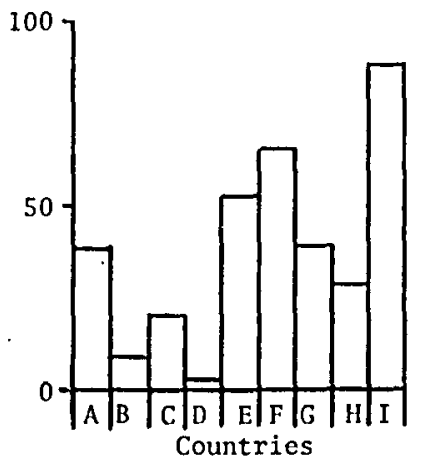

h. M125E1-FH70

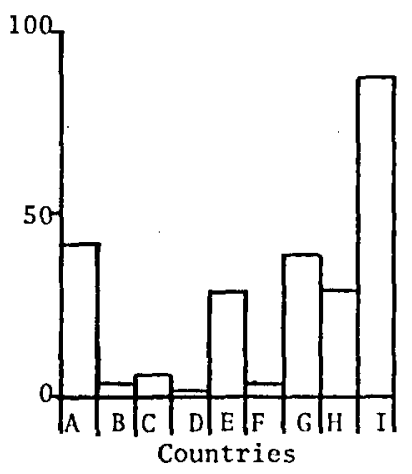

c. M561-XM204

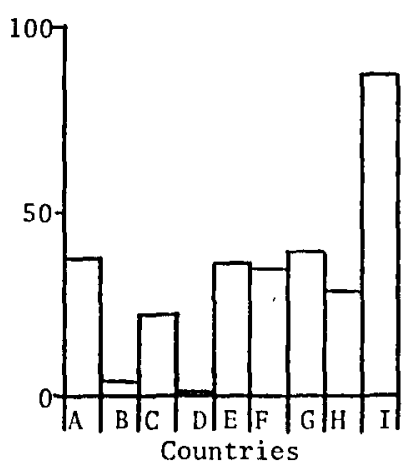

f. M656-XM198

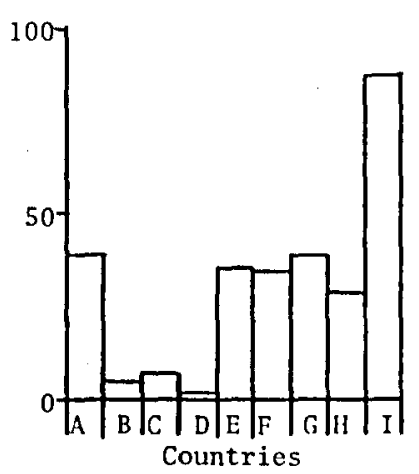

i. M520E1-FH70

NOTE: XM204 is considered lightweight artillery, XM198 considered medium-weight artillery, and FH70 considered heavyweight artillery.

Figure 4. Comparisons of the performances of selected prime movers towing artillery of different weight classes with the performance of the self-propelled MI09Al in countried A-I during dry conditions 
defined three levels of tactical mobility. These are given in Table 18, along with the identification of two additional mobility levels (highhigh and on-road mobility), which were developed for the HIMO study to complete the range.

46. The tactical high mobllity level was felt to more closely fit the missions of the towed and self-propelled artillery (see Appendix F) and is suggested as the overall parameter for comparing the mobility of the study vehicles. High-high mobility was also computed to show what effect more off-road travel would have on the study vehicles. These data are given in Tables 19 and 20. It should also be noted that the basic data are available in this report for determining other levels of mobility (on-road, support, and standard) using the equations presented in Appendix $G$ and the definitions used for the mobility levels in Table 18.

Relative mobility of candidate prime movers

47. Table 24 shows the relative mobility of the prime movers for towing lightweight, medium-weight, and heavyweight artillery based on the tactical high mobility level. The M35A2 ranks first in towing the lightweight artillery in the wet condition in West Germany followed closely by the ASV. The ASV ranks first in towing the lightweight artillery in the snow condition in West Germany and both wet and sand conditions of the Mid-East. The ASV and UET rank first in towing the medium-weight artillery in the wet condition in West Germany. The ASV ranks first in towing the medium-weight artillery in the snow condition of West Germany and the wet and sand conditions of the Mid-East. The ASV ranks first in towing the heavyweight artillery in the snow condition of West Germany and the sand condition of the Mid-East. None of the prime movers are acceptable for towing the heavyweight artillery in the wet conditions of the West Germany and Mid-East study areas. The prime movers towing the heavyweight artillery have better performance in the snow condition than in the wet condition because the snow condition is described as $10 \mathrm{in}$. of snow on frozen ground; whereas, the wet condition is described as a wet and slippery surface. 
Relative mobility of

self-propelled artillery

48. Table 20 shows very little difference in the performances of the self-propelled artillery at the tactical high mobility level. The high-high mobility level shows a decrease in performance of all vehicles and reflects the NOGO performance of the study vehicles in off-road terrain.

Mobility of prime mover-towed artillery relative to that of self-propelled artillery

49. The performances of four selected prime movers towing 1ightweight (group 1), medium-weight (group 2), and heavyweight (group 3) artillery were compared with that of the MI09Al self-propelled artillery piece for both the tactical high and high-high mobility levels (Table 25) for both wet and snow conditions in West Germany and wet and sand conditions in the Mid-East. Each group of prime movers selected for comparison contained the ASV (tracked) and the TDW901 (wheeled), which are not currently artillery prime movers, and the M548El (tracked) and the M561 towing group 1, M656 towing group 2, and M520E1 towing group 3 (wheeled) currently used as prime movers for towing artillery. The comparisons shown in Table 25 are also shown in Figures 5-10 in the form of bar graphs.

50. A minimum acceptable level of mobility has not been established for towed or self-propelled artillery. However, it is noted that in the HIMO study, the minimum acceptable level of mobility for support vehicles was described as seventy-five percent of the M60A2 mobility rating speed. This percentage or some other percentage of the M109Al mobility rating speed might be a good method of defining the minimum acceptable level of mobility for prime movers towing artillery.

\section{Suggested Method for Ranking of Vehicle Groups}

51. The data presented in Table 21 show that for primary roads, secondary roads, and trails, there is only generally a little difference between the speed of the slowest vehicle in a group and the group speed. However, off-road group performance shows that the NOGO percentages of 
vehicles traveling together often add up to more than that of any individual and, therefore, have a greater effect (paragraphs 35 and 38).

52. Table 26 shows the ranking of the vehicle groups for the two selected conditions in the West Germany and the Mid-East on primary roads, secondary roads, trails, and off-road. 


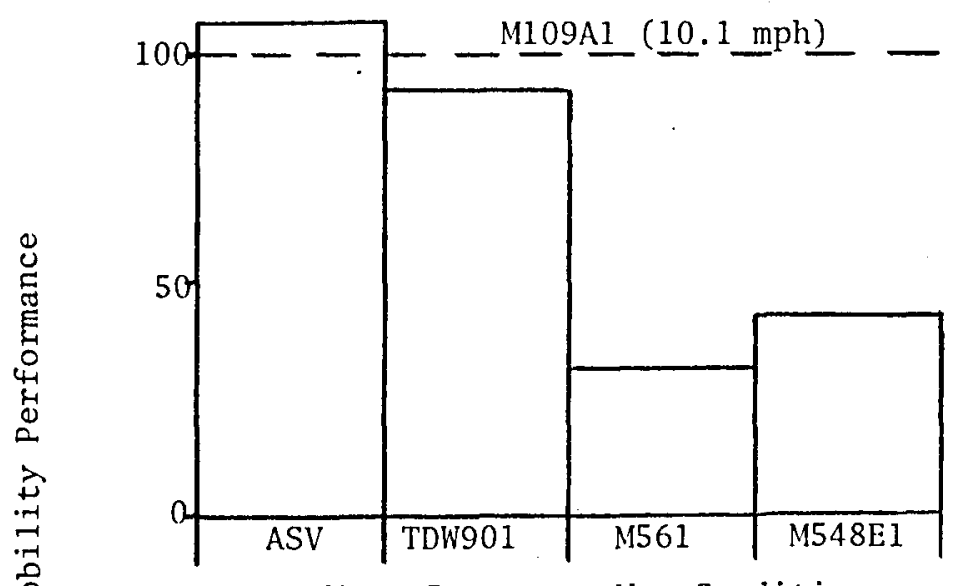

a. West Germany - Wet Condition

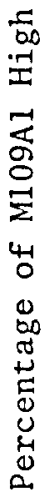

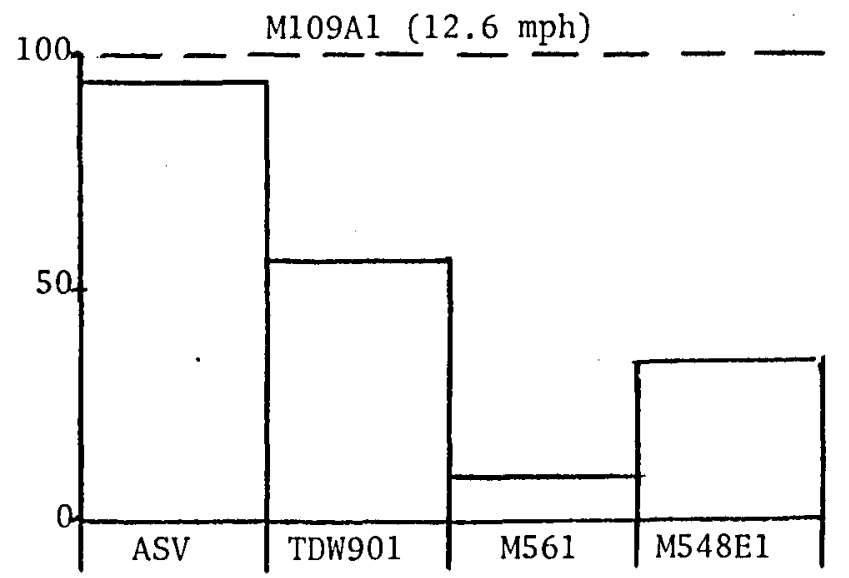

c. Mid-East - Wet Condition

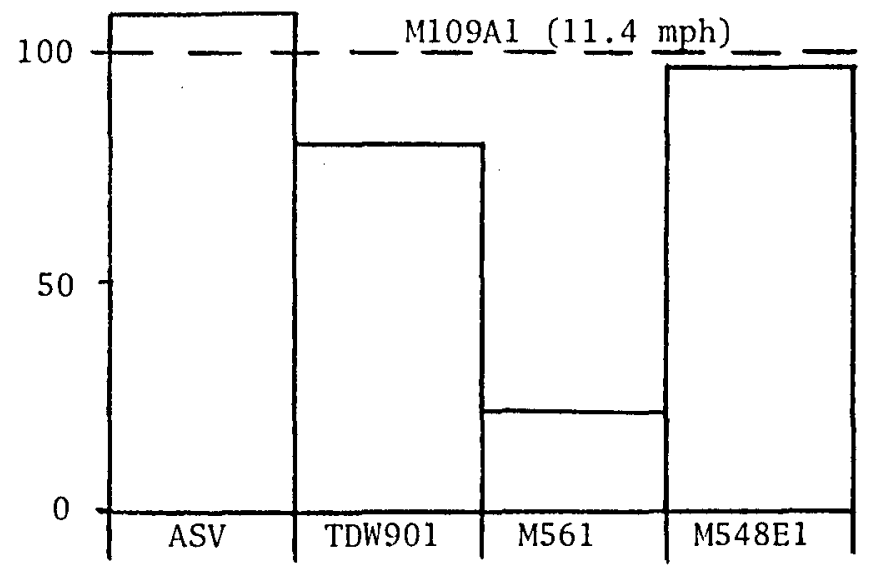

b. West Germany - Snow Condition

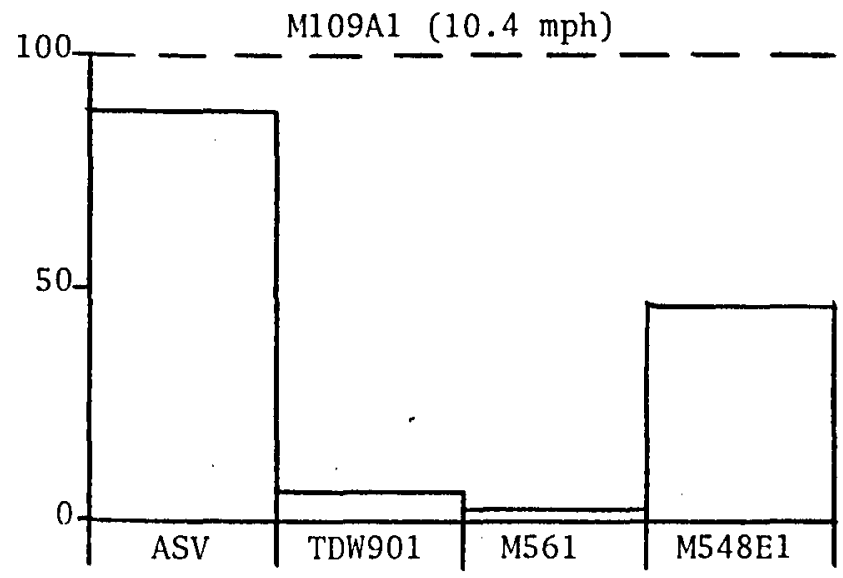

d. Mid-East - Sand Condition

Figure 5. Comparisons of the performances of selected prime movers towing lightweight artillery with the performance of self-propelled artillery at the tactical high mobility level 

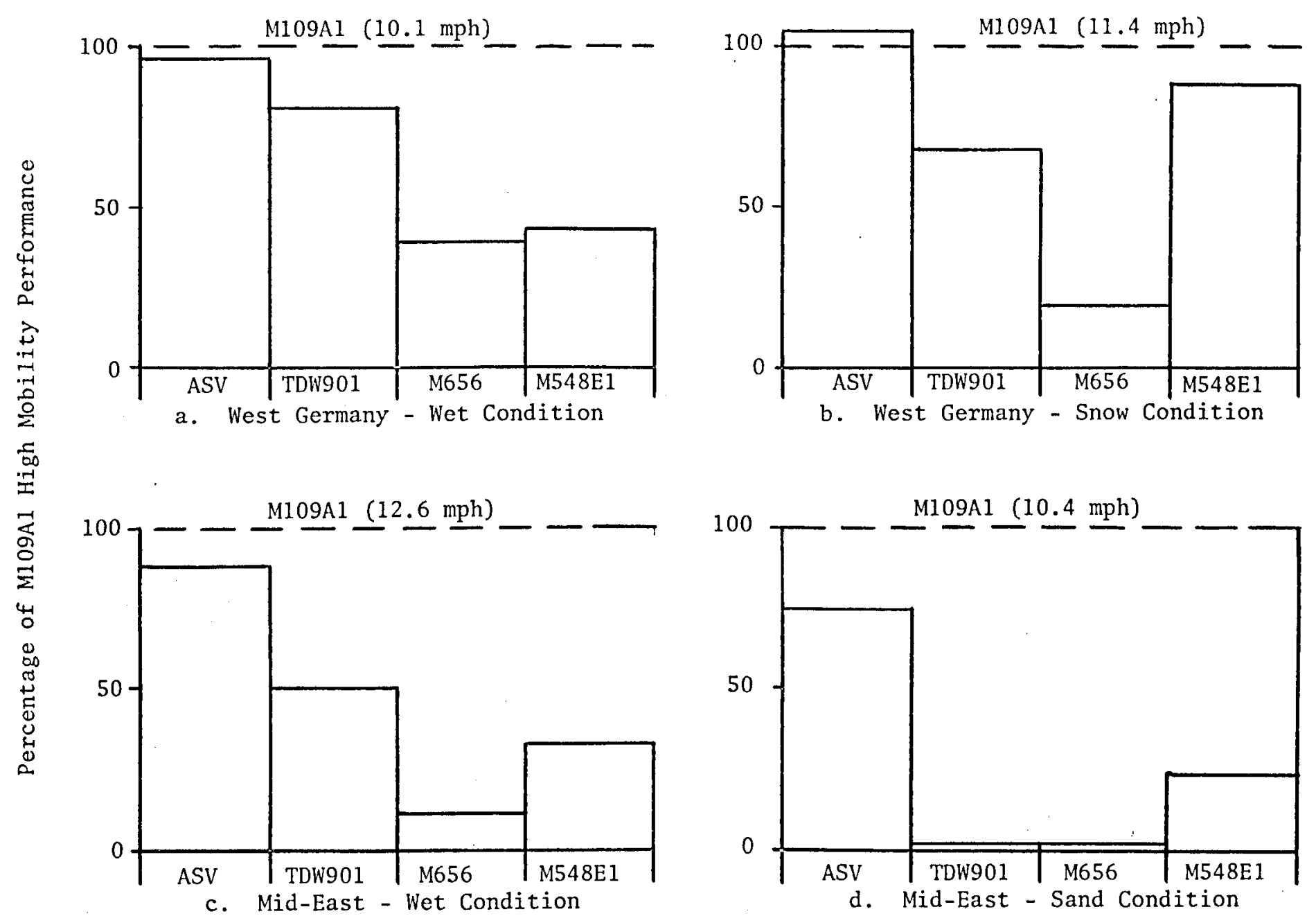

Figure 6. Comparisons of the performances of selected prime movers towing medium-weight artillery with the performance of self-propelled artillery at the tactical high mobility level 


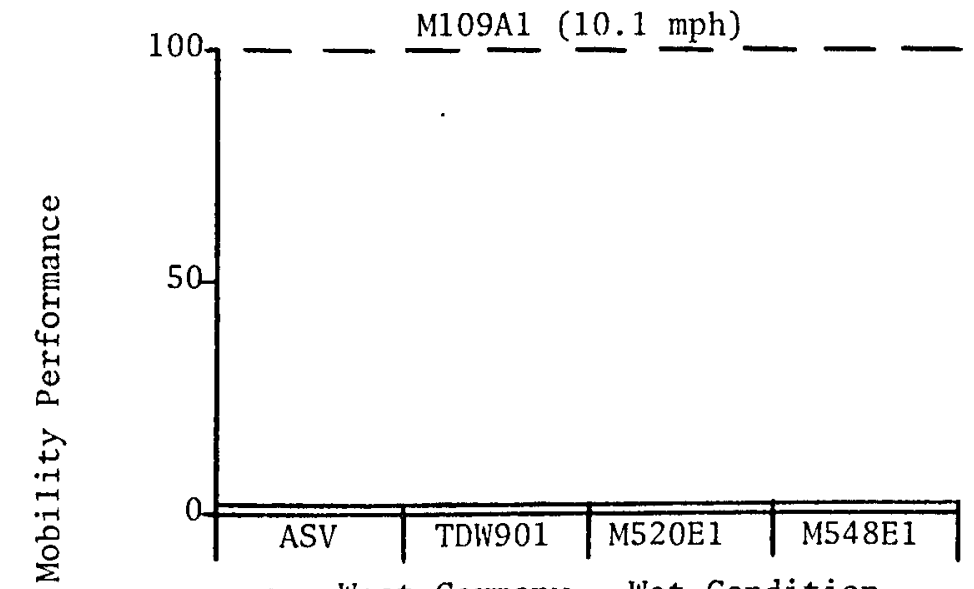

a. West Germany - Wet Condition

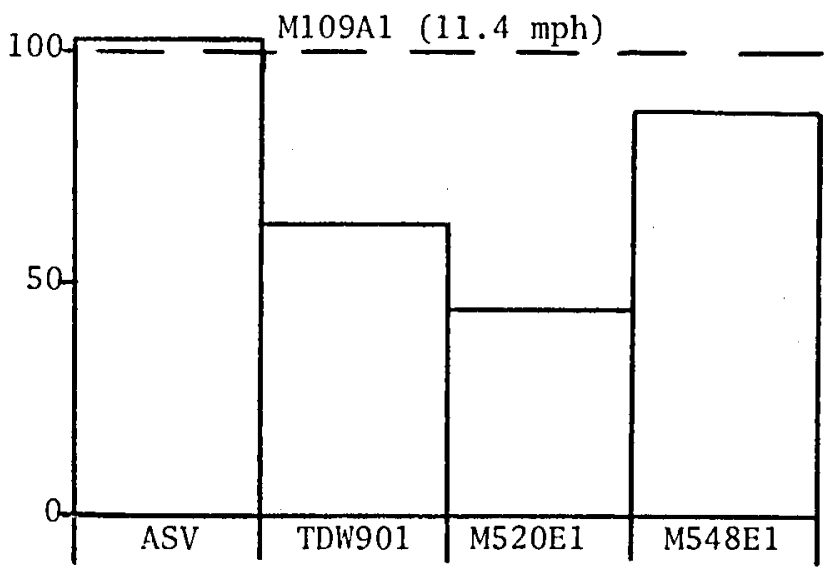

b. West Germany - Snow Condition

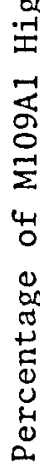
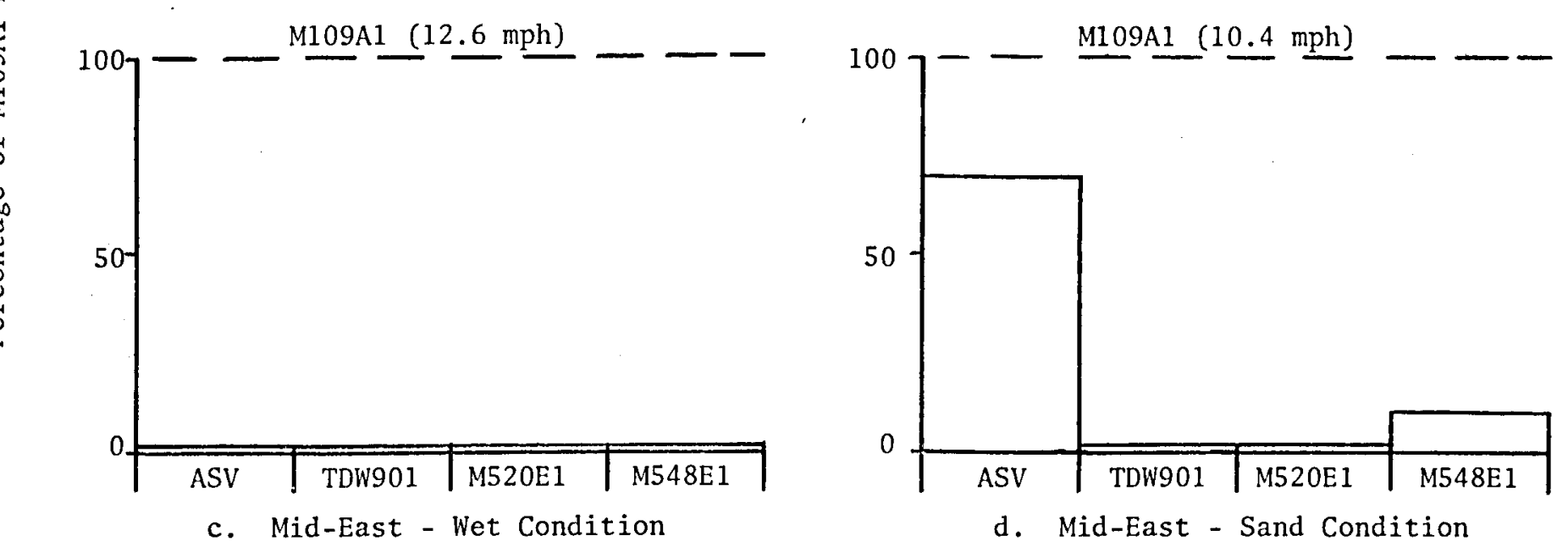

Figure 7. Comparisons of the performances of selected prime movers towing heavyweight artillery with the performance of self-propelled artillery at the tactical high performance level 

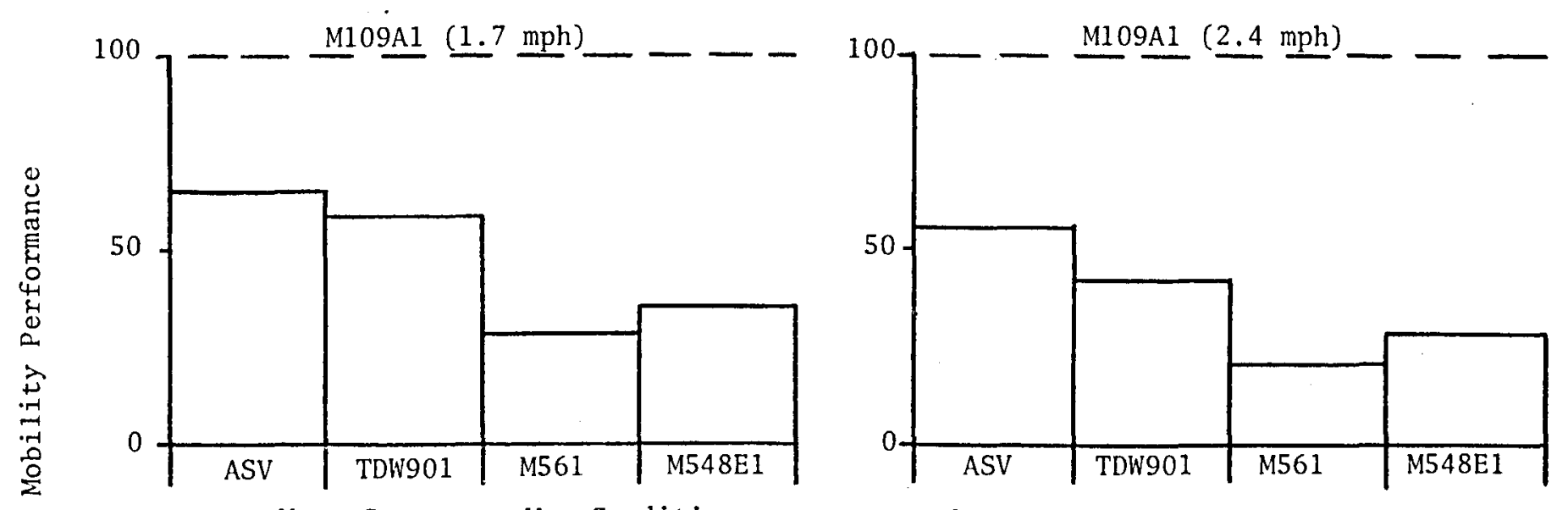

a. West Germany - Wet Condition

b. West Germany - Snow Condition

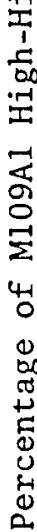
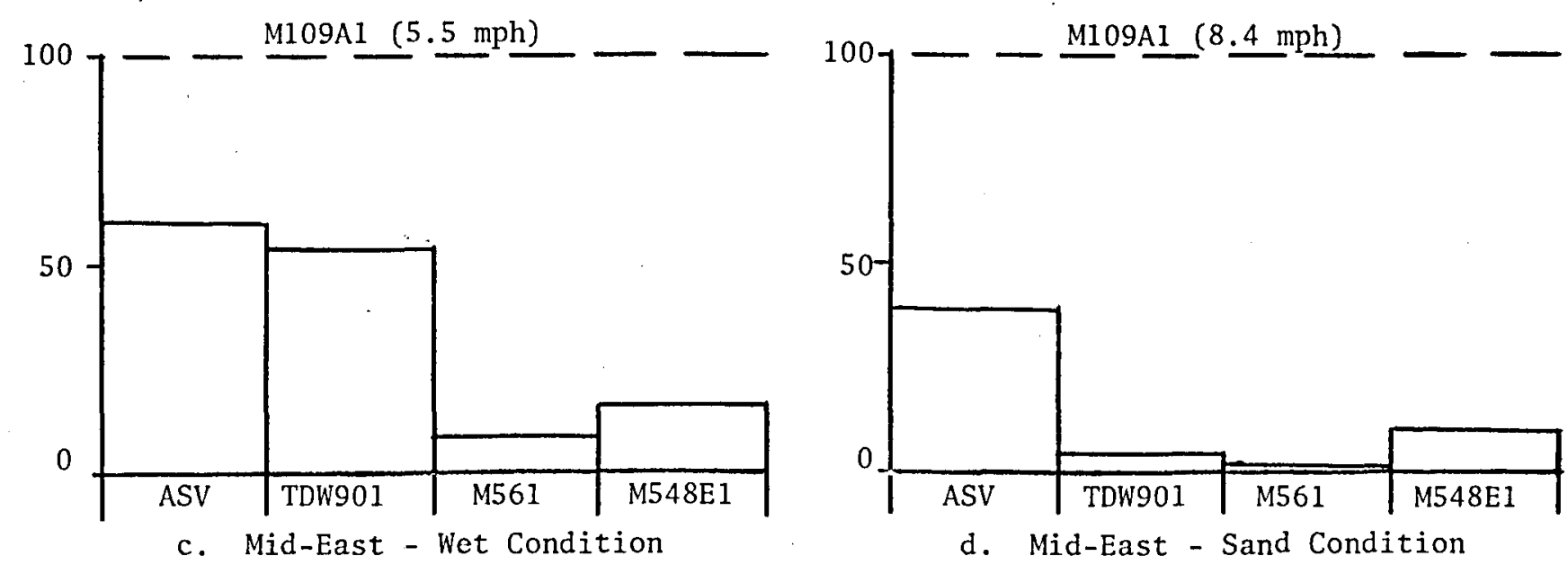

Figure 8. Comparisons of the performances of selected prime movers towing lightweight artillery with the performance of self-propelled artillery at the highhigh mobility leve1 

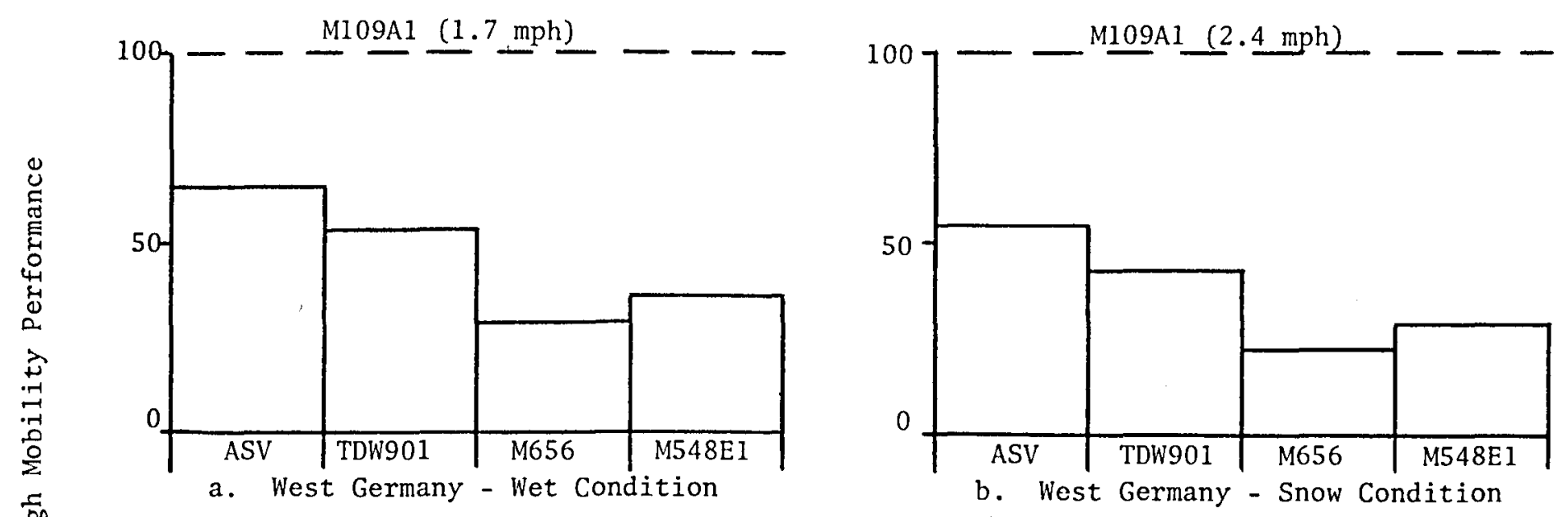

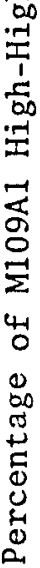

a. West Germany - Wet Condition

b. West Germany - Snow Condition
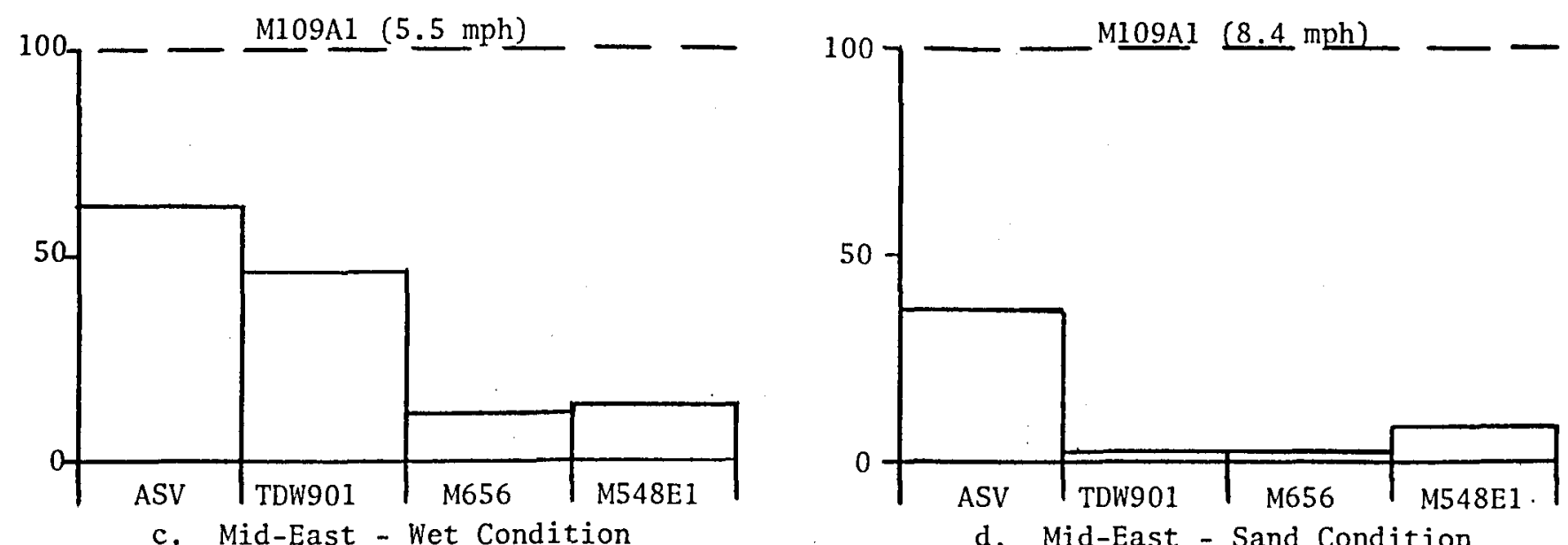

Figure 9. Comparisons of the performances of selected prime movers towing mediumweight artillery with the performance of self-propelled artillery at the high-high mobility level 

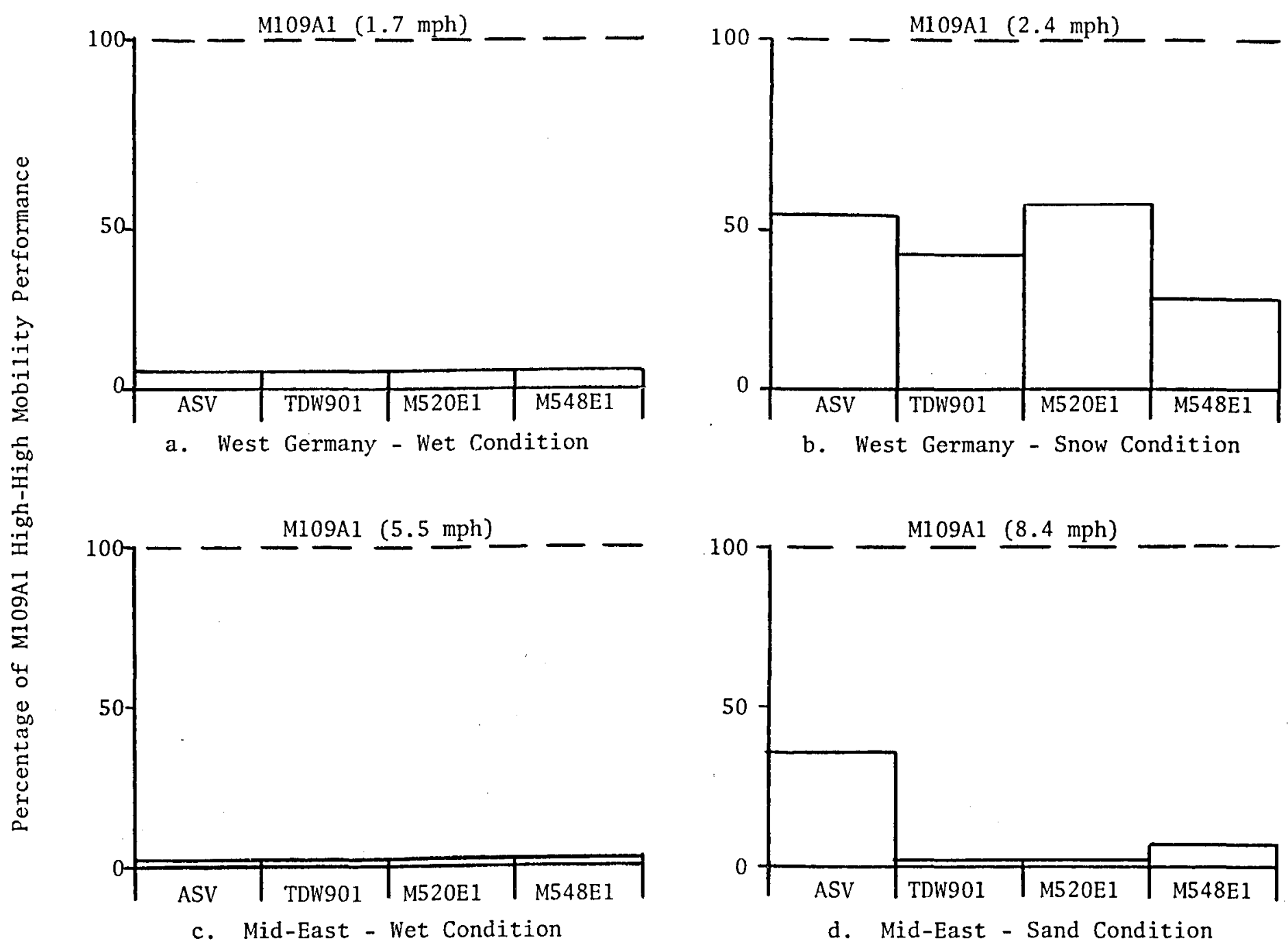

Figure 10. Comparisons of the performances of selected prime movers towing heavyweight artillery with the performance of self-propelled artillery at the high-high mobility level 


\section{REFERENCES}

.1. Jurkat, M. Peter, Nutta11, C. J., Jr., and Haley, Peter, "The AMC-71 Mobility Mode1," Technical Report No. 11789 (LL 143), Vols I and II, Jul 1973, U. S. Army Tank-Automotive Command, Warren, Mich.

2. Nutta11, C. J. and Randolph, D. D., "Mobility Analyses of Standardand High-Mobility Tactical Support Vehicles (HIMO Study)," Technical Report M-76-3, Feb 1976, U. S. Army Engineer Waterways Experiment Station, $\mathrm{CE}$, Vicksburg, Miss.

3. Nutta11, C. J., Jr., Rula, A. A., and Dugoff, H. J., "Computer Model for Comprehensive Evaluation of Cross-Country Vehicle Mobility," Paper No. 740426 presented at Society of Automotive Engineers Earthmoving Industry Conference, Apr 1974.

4. Murphy, N. R., Jr., and Ahlvin, R. B., "The AMC-74 Vehicle Dynamics Module," Technical Report M-76-1, Jan 1976, U. S. Army Engineer Waterways Experiment Station, CE, Vicksburg, Miss.

5. Robinson, J. H., Randolph, D. D., and Murphy, N. R., Jr., "Comparison of the Ride and Mobility Characterisitcs of Advanced Concept Vehicle-Cargo (ACF-V) and Selected Currently Authorized Vehicles," Nov 1974, U. S. Army Engineer Waterways Experiment Station, CE, Vicksburg, Miss.

6. Classified reference.*

7. Rula, A. A., Nuttall, C. J., Jr., and Dugoff, H., "Vehicle Mobility Assessment for Project WHEELS Study Group," Technical Report M-73-1, Apr 1973, U. S. Army Engineer Waterways Experiment Station, CE, Vicksburg, Miss.

8. Pradko, F., Richard, L., and Victor, Kaluza, "Theory of Human Vibration Response," presented at the Winter Annual Meeting and Energy Systems Exposition of the American Society of Mechanical Engineers, New York, N.Y., Nov 27-Dec 1, 1966.

9. Lins, W. F., "Human Vibration REsponse Measurement," Technical Report 11t1, Jun 1972, U. S. Army Tank-Automotive Command, Warren, Mich.

* Bibliographic material for the classified reference will be furnished to qualified agencies on request. 
10. Robinson, J. H., and Murphy, N. R., Jr., "Comparison of the Ride and Mobility Characteristics of Advanced Concept Vehicle-Support (ACV-S) and Selected Currently Authorized Vehicles," unpublished report. 
$\checkmark$ 
Table 1

Study Vehicles

\begin{tabular}{|c|c|c|c|c|c|c|c|c|}
\hline Vehicle/Prime Mover & \multicolumn{6}{|c|}{ Towed Artillery } & $\begin{array}{c}\text { Self- } \\
\text { Propelled } \\
\text { Artillery }\end{array}$ & $\begin{array}{c}\text { Other } \\
\text { Reference } \\
\text { Vehicles }\end{array}$ \\
\hline \multicolumn{9}{|l|}{ Wheeled Prime Movers } \\
\hline M561, 1-1/4-ton GAMA GOAT, $6 \times 6$ & $\mathrm{x}$ & $x$ & & $x$ & $x$ & & & \\
\hline M35A2, 2-1/2-ton truck, cargo, $6 \times 6$ & $x$ & $x$ & $x$ & $x$ & $x$ & $x$ & & \\
\hline M813, 5-ton truck, cargo, $6 \times 6$ & & $x$ & $\mathrm{x}$ & $x$ & $\mathrm{x}$ & $x$ & & \\
\hline M656, 5-ton truck, cargo, $8 \times 8$ & & $x$ & $x$ & $x$ & $x$ & $\mathrm{x}$ & & \\
\hline $\begin{array}{l}\text { Twister Dragon Wagon } 901 \text { (TDW901), } \\
8 \text {-ton cargo, } 8 \times 8\end{array}$ & & $\mathrm{x}$ & $\mathrm{x}$ & $\mathrm{x}$ & $x$ & $x$ & & \\
\hline$\underset{4 \times 4}{\text { M520E } 1 \text {, GOER, 8-ton truck, cargo, }}$ & & $\mathrm{x}$ & $\mathrm{x}$ & $\mathrm{x}$ & $\mathrm{x}$ & $\mathrm{x}$ & & \\
\hline M125E1, 10-ton truck, cargo, $6 \times 6$ & & & $x$ & $\mathrm{x}$ & $\mathrm{x}$ & & & \\
\hline \multicolumn{9}{|l|}{ Tracked Prime Movers } \\
\hline M548E1, 5-ton carrier, cargo, tracked & $x$ & $x$ & $x$ & $\mathrm{x}$ & $x$ & $\mathrm{x}$ & & \\
\hline UET, Universa1 Engineer Tractor & & & $x$ & $x$ & & $\mathrm{x}$ & & \\
\hline ASV, Artillery Support Vehicle & & $x$ & $\mathrm{x}$ & $\mathrm{x}$ & $x$ & $x$ & & \\
\hline \multicolumn{9}{|l|}{ Self-Propelled (SP) Artillery } \\
\hline M109Al, $155 \mathrm{~mm}$ & & & & & & & $\mathrm{x}$ & \\
\hline $\mathrm{M107}, 175 \mathrm{~mm}$ & & & & & & & $x$ & \\
\hline $\mathrm{M} 10 \mathrm{E} 2,8 \mathrm{in}$. & & & & & & & $\mathrm{x}$ & \\
\hline \multicolumn{9}{|l|}{ Other Vehicles } \\
\hline M113 Armored Personnel Carrier (APC) & & & & & & & & $x$ \\
\hline M60A2, tank, combat, full tracked & & & & & & & & $\mathrm{x}$ \\
\hline M548E1, 5-ton carrier, cargo, tracked & & & & & & & & $\mathrm{x}$ \\
\hline M577A1, carrier, Command Post (CP) & & & & & & & & $x$ \\
\hline $\begin{array}{l}\text { XM723, Mechanized Infantry Combat } \\
\text { Vehicle (MICV) }\end{array}$ & & & & & & & & $\mathrm{x}$ \\
\hline XM1, tank, combat, full tracked & & & & & & & & $x$ \\
\hline ASV, Artillery Support Vehicle & & & & & & & & $x$ \\
\hline M561, 1-1/4-ton GAMA GOAT, $6 \times 6$ & & & & & & & & $x$ \\
\hline M813, 5-ton truck, cargo, $6 \times 6$ & & & & & & & & $x$ \\
\hline UET, Universal Engineer Tractor & & & & & & & & $\mathrm{x}$ \\
\hline
\end{tabular}


Table 2

Principal Characteristics of Study Vehicles

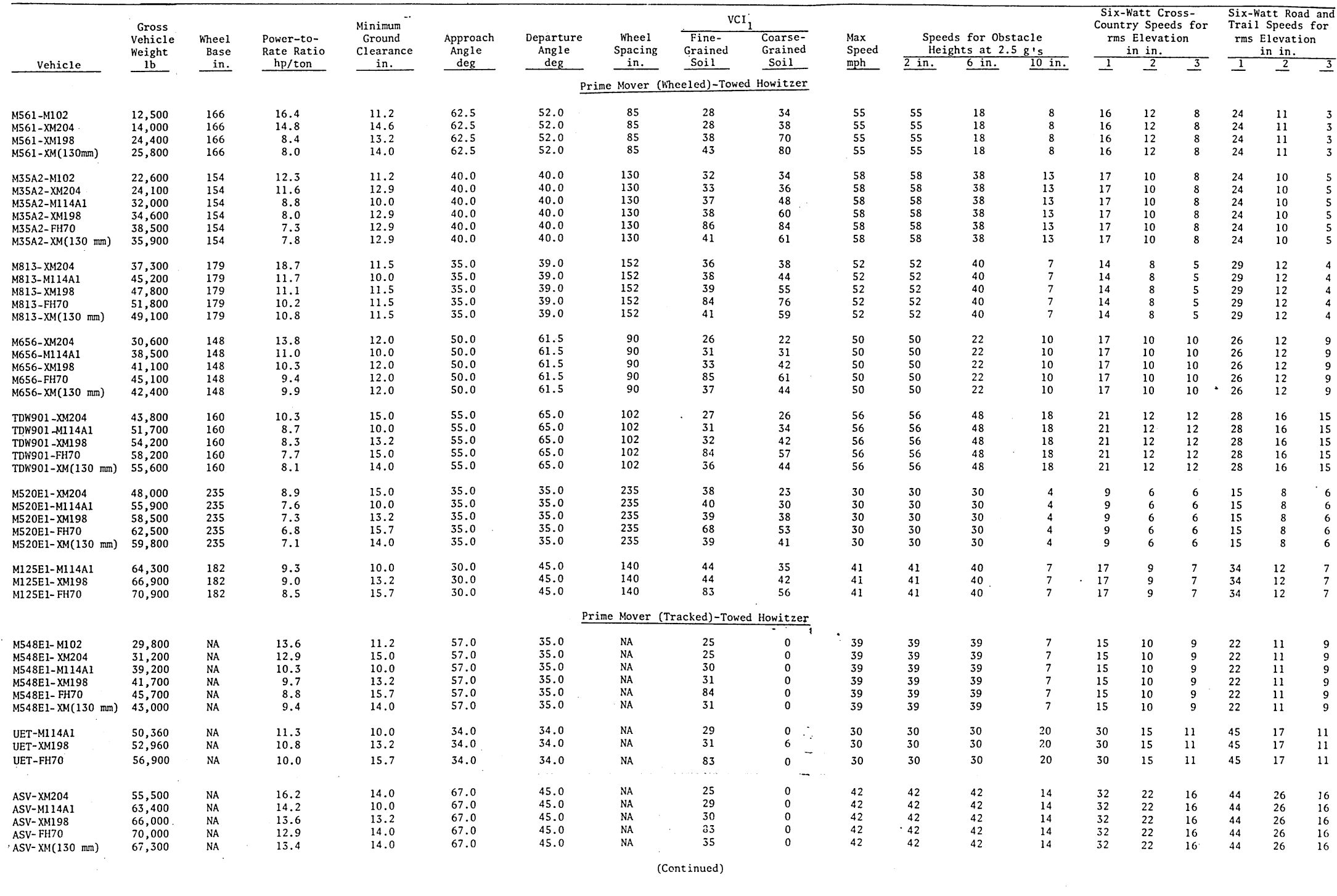


Table 2 (Concluded)

\begin{tabular}{|c|c|c|c|c|c|c|c|c|c|c|c|c|c|c|c|c|c|c|c|}
\hline \multirow[b]{3}{*}{ Vehicle } & \multirow{3}{*}{$\begin{array}{c}\text { Gross } \\
\text { Vehicle } \\
\text { Weight } \\
\text { lbt } \\
\end{array}$} & \multirow{3}{*}{$\begin{array}{c}\text { Whee1 } \\
\text { Base } \\
\text { in. }\end{array}$} & \multirow{3}{*}{$\begin{array}{l}\text { Power-to- } \\
\text { Rate Ratio } \\
\text { hp/ton } \\
\end{array}$} & \multirow{3}{*}{$\begin{array}{l}\text { Minimum } \\
\text { Ground } \\
\text { Clearance } \\
\text { in. } \\
\end{array}$} & \multirow{3}{*}{$\begin{array}{c}\text { Approach } \\
\text { Angle } \\
\text { deg }\end{array}$} & \multirow{3}{*}{$\begin{array}{c}\text { Departure } \\
\text { Angle } \\
\text { deg } \\
\end{array}$} & \multirow{3}{*}{$\begin{array}{r}\text { Wheel } \\
\text { Spacing } \\
\text { in. } \\
\end{array}$} & \multicolumn{2}{|c|}{$\mathrm{VCI}_{3}$} & \multirow{3}{*}{$\begin{array}{l}\text { Max } \\
\text { Speed } \\
\text { mph } \\
\end{array}$} & \multirow{2}{*}{\multicolumn{3}{|c|}{ Speeds for Obstacle }} & \multirow{2}{*}{\multicolumn{3}{|c|}{$\begin{array}{l}\text { Six-Watt Cross- } \\
\text { Country Speeds for } \\
\text { rms Elevation } \\
\text { in in. } \\
\end{array}$}} & \multirow{2}{*}{\multicolumn{3}{|c|}{$\begin{array}{l}\text { Six-Watt Road and } \\
\text { Trail Speeds for } \\
\text { rms Elevation } \\
\text { in in. }\end{array}$}} \\
\hline & & & & & & & & \multirow{2}{*}{$\begin{array}{c}\text { Fine- } \\
\text { Grained } \\
\text { Soil } \\
\end{array}$} & \multirow{2}{*}{$\begin{array}{l}\text { Coarse- } \\
\text { Grained } \\
\text { Soil } \\
\end{array}$} & & & & & & & & & & \\
\hline & & & & & & & & & & & \multicolumn{3}{|c|}{ 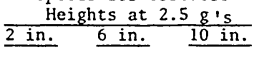 } & 1 & 2 & 3 & 1 & 2 & 3 \\
\hline & \multicolumn{19}{|c|}{ Self-Propelled Artillery } \\
\hline M109A1 & 53,100 & $\mathrm{NA}$ & 15.7 & 17.7 & 75.0 & 80.5 & $\mathrm{NA}$ & 25 & 0 & 35 & 35 & 17 & 12 & 30 & 16 & 14 & 36 & 16 & 14 \\
\hline \multirow{2}{*}{ M110E2 } & $\begin{array}{l}62,100 \\
62,100\end{array}$ & $\begin{array}{l}\mathrm{NA} \\
\mathrm{NA}\end{array}$ & $\begin{array}{l}13.0 \\
13.7\end{array}$ & $\begin{array}{l}14.8 \\
18.4\end{array}$ & $\begin{array}{l}90.0 \\
90.0\end{array}$ & $\begin{array}{l}43.5 \\
44.5\end{array}$ & $\begin{array}{l}\mathrm{NA} \\
\mathrm{NA}\end{array}$ & $\begin{array}{l}22 \\
22\end{array}$ & $\begin{array}{l}0 \\
0\end{array}$ & $\begin{array}{l}34 \\
34\end{array}$ & $\begin{array}{l}34 \\
34\end{array}$ & $\begin{array}{l}17 \\
17\end{array}$ & $\begin{array}{l}12 \\
12\end{array}$ & $\begin{array}{l}30 \\
30\end{array}$ & $\begin{array}{l}16 \\
16\end{array}$ & $\begin{array}{l}14 \\
14\end{array}$ & $\begin{array}{l}36 \\
36\end{array}$ & $\begin{array}{l}16 \\
16\end{array}$ & $\begin{array}{l}14 \\
14\end{array}$ \\
\hline & \multicolumn{19}{|c|}{ Other Vehicles } \\
\hline M113A1 & 23,400 & $\mathrm{NA}$ & 17.9 & 16.0 & 70.0 & 40.0 & $\mathrm{NA}$ & 16 & 0 & 40 & 40 & 40 & 10 & 24 & 13 & 10 & 34 & 15 & 10 \\
\hline $\begin{array}{lll}M 60 \mathrm{~A} 2 & \\
M & \end{array}$ & $\begin{array}{l}25,400 \\
104,000\end{array}$ & NA & 15.0 & 18.0 & 90.0 & 42.5 & $\mathrm{NA}$ & 21 & 0 & 30 & 30 & 30 & 12 & 44 & 20 & 13 & 44 & 20 & 13 \\
\hline M548E1 & 26,500 & $\mathrm{NA}$ & 15.3 & 16.0 & 57.0 & 35.0 & $\mathrm{NA}$ & 16 & 0 & 40 & 40 & 40 & 7 & 15 & 10 & 9 & 22 & 11 & 9 \\
\hline M577A1 & 24,400 & NA & 17.9 & 16.0 & 70.0 & 40.0 & NA & 16 & 0 & 40 & 40 & 40 & 10 & 24 & 13 & 10 & 34 & 15 & 10 \\
\hline XM723 & 40,400 & $\mathrm{NA}$ & 22.3 & 18.0 & 83.0 & 78.0 & $\mathrm{NA}$ & 12 & 0 & 48 & 48 & 48 & 14 & 32 & 22 & 16 & 44 & 26 & 16 \\
\hline $\mathrm{XM1}$ & 115,400 & NA & 26.0 & 19.0 & 77.0 & 68.0 & NA & 26 & 0 & 48 & 48 & 48 & 48 & 52 & 24 & 14 & 52 & 24 & 14 \\
\hline ASV & 50,700 & $\mathrm{NA}$ & 17.7 & 14.0 & 67.0 & 45.0 & NA & 15 & 0 & 42 & 42 & 42 & 14 & 32 & 22 & 16 & 44 & 26 & 16 \\
\hline M561 & 9,170 & 166 & 22.5 & 14.6 & 62.5 & 52.0 & 85 & 19 & 13 & 55 & 55 & 18 & 8 & 16 & 12 & 8 & 24 & 11 & 3 \\
\hline M813 & 32,540 & 179 & 16.3 & 11.5 & 35.0 & 39.0 & 152 & 35 & 26 & 52 & 52 & 40 & 7 & 14 & 8 & 5 & 29 & 12 & 4 \\
\hline UET & 37,700 & NA & 15.1 & 18.0 & 34.0 & 34.0 & $\mathrm{NA}$ & 18 & 0 & 30 & 30 & 30 & 20 & 36 & 15 & 11 & 45 & 17 & 11 \\
\hline
\end{tabular}


Table 3

Characteristics of As-Is* Route Networks

\begin{tabular}{|c|c|c|c|c|}
\hline Network Type & $\begin{array}{l}\text { Primary } \\
\text { Roads, \% }\end{array}$ & $\begin{array}{l}\text { Secondary } \\
\text { Roads, } \%\end{array}$ & $\begin{array}{c}\text { Trails } \\
\frac{\%}{\%} \\
\end{array}$ & $\begin{array}{c}\text { Off-Road } \\
\frac{\%}{0} \\
\end{array}$ \\
\hline HIMO routes, West Germany** & 20.3 & 64.5 & 15.2 & 0.0 \\
\hline Artillery routes, West Germanyt & 14.8 & 33.1 & 42.0 & 10.1 \\
\hline HIMO routes, Mid-East $+\dagger$ & 1.1 & 16.1 & 51.3 & 31.5 \\
\hline
\end{tabular}

* See paragraph 33 for definitions of "as-is."

* Based on a total distance of 309.7 miles of network routes.

+ Based on a total distance of 84.7 miles of network routes.

t+ Based on a total distance of 84.8 miles of network routes. 
Table 4

Performance Predictions for Prime Mover-Towed Artillery Combinations in the Countries Described by Generalized Terrain Data

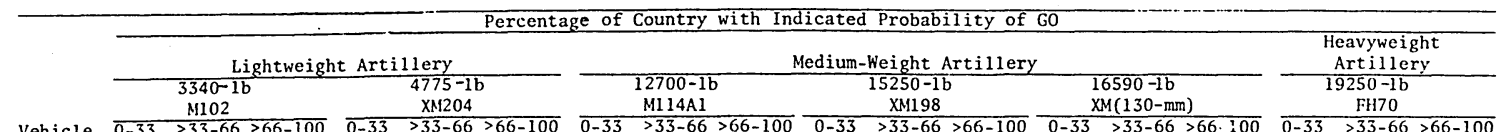

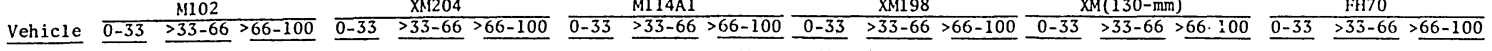
Country A - Wet Condition

\begin{tabular}{|c|c|c|c|c|c|c|c|c|c|c|c|c|c|c|c|c|c|c|}
\hline M561 & 45.0 & 22.8 & 32.2 & 63.5 & 19.5 & 17.0 & & & & 100.0 & 0.0 & 0.0 & 100.0 & 0.0 & 0.0 & & & \\
\hline M35A2 & 28.4 & 31.0 & 40.6 & 38.0 & 31.5 & 30.5 & 83.0 & 14.2 & 2.8 & 96.5 & 3.5 & 0.0 & 98.3 & 1.7 & 0.0 & 99.0 & 1.0 & 0.0 \\
\hline M813 & & & & 28.4 & 30.5 & 41.1 & 58.9 & 15.0 & 26.0 & 69.1 & 21.0 & 9.8 & 69.5 & 20.6 & 9.8 & 90.2 & 7.0 & 2.9 \\
\hline M656 & & & & 8.0 & 30.0 & 62.0 & 59.3 & 14.7 & 26.0 & 74.0 & 16.2 & 9.8 & 74.1 & 23.2 & 2.8 & 90.2 & 9.8 & 0.0 \\
\hline TDW901 & & & & 7.7 & 20.7 & 71.6 & 18.9 & 44.0 & 37.1 & 32.5 & 35.5 & 32.0 & 42.1 & 26.0 & 32.0 & 76.6 & 19.3 & 4.2 \\
\hline M520E 1 & & & & 32.8 & 23.1 & 44.1 & 48.6 & 27.7 & 23.7 & 64.9 & 16.3 & 18.8 & 64.9 & 16.4 & 18.8 & 76.3 & 13.9 & 9.8 \\
\hline M125E1 & & & & & & & 32.5 & 36.7 & 30.7 & 33.1 & 41.2 & 25.6 & & & & 60.3 & 29.8 & 9.8 \\
\hline $\mathrm{M} 548 \mathrm{E} 1$ & 3.1 & 3.2 & 93.7 & 3.3 & 3.6 & 93.1 & 6.3 & 35.8 & 57.9 & 10.9 & 52.3 & 36.8 & 10.9 & 56.6 & 32.5 & 50.6 & 31.5 & 17.8 \\
\hline UET & & & & & & & 5.1 & 15.9 & 79.0 & 5.2 & 23.3 & 71.5 & & & & 33.0 & 43.3 & 23.7 \\
\hline ASV & & & & 1.5 & 3.3 & 95.2 & 3.9 & 10.0 & 86.1 & 5.1 & 16.0 & 78.9 & 5.1 & 18.6 & 76.3 & 30.4 & 29.6 & 40.0 \\
\hline
\end{tabular}

\begin{tabular}{|c|c|c|c|c|c|c|c|c|c|c|c|c|c|c|c|c|c|c|}
\hline \multirow[b]{2}{*}{ M561 } & \multicolumn{18}{|c|}{ Country A - Dry Condition } \\
\hline & 40.6 & 13.8 & 45.6 & 44.8 & 15.1 & 40.2 & & & & 100.0 & 0.0 & 0.0 & 100.0 & 0.0 & 0.0 & & . & \\
\hline M35A2 & 4.5 & 36.8 & 58.7 & 7.8 & 37.2 & 55.0 & 59.8 & 24.3 & 15.9 & 82.1 & 17.9 & 0.0 & 93.1 & 6.9 & 0.0 & 95.1 & 4.9 & 0.0 \\
\hline M813 & & & & 4.5 & 36.7 & 58.8 & 41.2 & 14.4 & 44.4 & 44.6 & 17.9 & 37.6 & 45.1 & 17.4 & 37.6 & 62.4 & 21.4 & 16.2 \\
\hline M656 & & & & 3.7 & 4.1 & 92.2 & 41.3 & 14.3 & 44.4 & 55.6 & 6.9 & 37.5 & 55.6 & 28.6 & 15.9 & 62.5 & 37.5 & 0.0 \\
\hline TDW901 & & & & 3.4 & 1.0 & 95.5 & 3.7 & 40.6 & 55.7 & 4.5 & 50.8 & 44.7 & 7.8 & 47.5 & 44.7 & 47.4 & 21.1 & 31.4 \\
\hline M520EI & & & & 4.2 & 6.5 & 89.3 & 8.1 & 39.3 & 52.6 & 41.5 & 16.7 & 41.8 & 41.5 & 16.7 & 41.8 & 47.4 & 15.0 & 37.6 \\
\hline M125E1 & & & & & & & 4.5 & 40.1 & 55.4 & 4.5 & 41.2 & 54.3 & & & & 14.1 & 48.4 & 37.6 \\
\hline M548E1 & 2.3 & 1.4 & 96.3 & 2.3 & 1.4 & 96.3 & 3.7 & 14.3 & 82.0 & 3.7 & 41.0 & 55.2 & 3.7 & 50.7 & 45.6 & 13.3 & 45.7 & 40.9 \\
\hline UFT & & & & & & & 3.5 & 1.0 & 95.5 & 3.7 & 0.8 & 95.5 & & & & 6.6 & 40.8 & 52.6 \\
\hline ASV & & & & 0.0 & 2.3 & 97.7 & 2.3 & 1.5 & 96.3 & 2.5 & 2.0 & 95.5 & 3.5 & 1.0 & 95.2 & 6.6 & 7.4 & 85.9 \\
\hline \multicolumn{19}{|c|}{ Country B - Wet Condition } \\
\hline M561 & 96.1 & 2.1 & 1.8 & 96.1 & 3.4 & 0.5 & & & & 100.0 & 0.0 & 0.0 & 100.0 & 0.0 & 0.0 & & & \\
\hline $\mathrm{M} 35 \mathrm{~A} 2$ & 87.3 & 8.8 & 3.9 & 87.4 & 10.2 & 2.4 & 98.8 & 1.2 & 0.0 & 100.0 & 0.0 & 0.0 & 100.0 & 0.0 & 0.0 & 100.0 & 0.0 & 0.0 \\
\hline M813 & & & & 87.3 & 8.8 & 3.9 & 96.1 & 2.1 & 1.8 & 96.1 & 3.4 & 0.5 & 96.1 & 3.4 & 0.5 & 98.8 & 1.2 & 0.0 \\
\hline M656 & & & & 86.1 & 1.3 & 12.6 & 96.1 & 2.1 & 1.8 & 96.1 & 3.4 & 0.5 & 96.1 & 3.9 & 0.0 & 98.8 & 1.2 & 0.0 \\
\hline TDW901 & & & & 84.3 & 1.3 & 14.5 & 85.5 & 10.5 & 3.9 & 85.5 & 12.1 & 2.4 & 87.4 & 10.2 & 2.4 & 97.6 & 1.9 & 0.5 \\
\hline M520E 1 & & & & 85.5 & 3.5 & 11.1 & 87.4 & 10.2 & 2.4 & 96.1 & 2.1 & 1.8 & 96.1 & 2.1 & 1.8 & 97.6 & 1.9 & 0.5 \\
\hline $\mathrm{M} 25 \mathrm{E} 1$ & & & & & & & 87.3 & 10.3 & 2.4 & 87.3 & 10.3 & 2.4 & & & & 87.4 & 11.4 & 1.2 \\
\hline $\mathrm{M} 548 \mathrm{EI}$ & 65.7 & 14.1 & 20.2 & 65.7 & 14.2 & 20.1 & 79.8 & 7.3 & 12.9 & 83.6 & 12.8 & 3.6 & 84.4 & 12.0 & 3.6 & 87.1 & 9.3 & 3.6 \\
\hline UET & & & & & & & 77.2 & 9.2 & 13.6 & 77.3 & 10.4 & 12.3 & & & & 85.0 & 11.4 & 3.6 \\
\hline ASV & & & & 20.6 & 48.2 & 31.2 & 65.9 & 14.9 & 19.6 & 68.6 & 16.3 & 15.1 & 77.0 & 7.9 & 15.1 & 77.8 & 9.3 & 12.9 \\
\hline
\end{tabular}

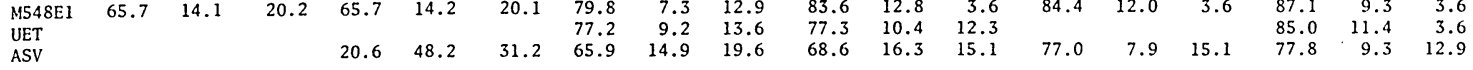

\begin{tabular}{|c|c|c|c|c|c|c|c|c|c|c|c|c|c|c|c|c|c|c|}
\hline \\
\hline M561 & 91.0 & 5.0 & 4.0 & 91.0 & 6.1 & 2.9 & & & & 100.0 & 0.0 & 0.0 & 100.0 & 0.0 & 0.0 & & & \\
\hline M35A2 & 75.6 & 15.4 & 9.0 & 79.5 & 12.1 & 8.4 & 92.7 & 7.3 & 0.0 & 100.0 & 0.0 & 0.0 & 100.0 & 0.0 & 0.0 & 100.0 & 0.0 & 0.0 \\
\hline$M 813$ & & & & 75.6 & 15.4 & 9.0 & 91.0 & 5.0 & 4.0 & 91.0 & 6.1 & 2.9 & 91.0 & 6.1 & 2.9 & 92.7 & 7.3 & 0.0 \\
\hline M656 & & & & 74.9 & 4.7 & 20.5 & 91.0 & 5.0 & 4.0 & 91.0 & 6.1 & 2.9 & 91.0 & 9.0 & 0.0 & 92.7 & 7.3 & 0.0 \\
\hline TDW901 & & & & 74.9 & 1.1 & 24.8 & 76.0 & 15.0 & 9.0 & 76.0 & 15.6 & 8.4 & 79.5 & 12.1 & 8.4 & 91.6 & 5.5 & 2.9 \\
\hline M520E 1 & & & & 75.6 & 4.5 & 19.9 & 79.5 & 12.1 & 8.4 & 91.0 & 5.0 & 4.0 & 91.0 & 5.0 & 4.0 & 91.6 & 5.5 & 2.9 \\
\hline M125E1 & & & & & & & 75.6 & 16.0 & 8.4 & 76.0 & 15.6 & 8.4 & & & & 79.5 & 13.2 & 7.3 \\
\hline M548E1 & 21.5 & 53.3 & 25.1 & 21.5 & 53.6 & 24.8 & 74.9 & 4.4 & 20.7 & 75.2 & 20.8 & 4.0 & 75.2 & 20.8 & 4.0 & 80.1 & 15.9 & 4.0 \\
\hline UET & & & & & & & 74.9 & 1.0 & 24.2 & 75.2 & 5.7 & 19.1 & & & & 75.9 & 20.1 & 4.0 \\
\hline ASV & & & & 0.1 & 22.3 & 77.6 & 21.9 & 53.3 & 24.8 & 22.3 & 52.9 & 24.8 & 74.8 & 0.4 & 24.8 & 75.6 & 4.5 & 19.9 \\
\hline \multicolumn{19}{|c|}{ Country C - Wet Condition } \\
\hline M561 & 44.7 & 47.9 & 7.4 & 50.0 & 44.3 & 5.7 & & & & 100.0 & 0.0 & 0.0 & 100.0 & 0.0 & 0.0 & & & \\
\hline M35A2 & 12.4 & 37.6 & 50.0 & 14.2 & 15.3 & 32.5 & 92.7 & 7.3 & 0.0 & 95.3 & 4.7 & 0.0 & & & & 95.3 & 4.7 & 0.0 \\
\hline$M 813$ & & & & 12.4 & 37.6 & 50.0 & 50.0 & 42.7 & 7.3 & 50.1 & 44.5 & 5.4 & 51.9 & 42.7 & 5.4 & 92.9 & 7.1 & 0.0 \\
\hline M656 & & & & 12.4 & 1.8 & 85.8 & 44.7 & 48.0 & 7.3 & 73.6 & 21.0 & 5.4 & 73.6 & 26.4 & 0.0 & 92.9 & 7.1 & 0.0 \\
\hline TDW901 & & & & 7.8 & 4.7 & 87.5 & 12.5 & 37.5 & 50.0 & 12.5 & 78.5 & 9.0 & 19.5 & 71.5 & 9.0 & 69.6 & 29.7 & 0.7 \\
\hline M520E I & & & & 13.2 & 24.1 & 62.7 & 19.6 & 50.0 & 30.4 & 50.0 & 42.9 & 7.1 & 50.0 & 42.9 & 7.0 & 69.6 & 25.0 & 5.4 \\
\hline M12SE1 & & & & & & & 17.7 & 49.8 & 32.5 & 17.8 & 51.8 & 30.4 & & & & 21.7 & 71.2 & 7.1 \\
\hline M548E1 & 0.0 & 7.8 & $92.2^{\prime}$ & 0.0 & 12.5 & 87.5 & 12.4 & 22.9 & 24.7 & 12.5 & 56.6 & 30.9 & 12.5 & 58.4 & 29.1 & 39.1 & 53.8 & 7.1 \\
\hline UET & & & & & & & 7.8 & 27.5 & 64.7 & 12.5 & 24.4 & 63.1 & & & & 18.1 & 53.1 & 28.8 \\
\hline ASV & & & & 0.0 & 0.1 & 99.9 & 0.0 & 12.5 & 87.5 & 0.1 & 12.4 & 87.5 & 7.8 & 4.7 & 87.5 & 13.5 & 25.6 & 60.9 \\
\hline \multicolumn{19}{|c|}{ Country C - Dry Condition } \\
\hline M561 & 44.7 & 47.9 & 7.4 & 45.7 & 48.4 & 5.9 & & & & 100.0 & 0.0 & 0.0 & 100.0 & 0.0 & 0.0 & & & \\
\hline M35A2 & 12.4 & 33.3 & 54.3 & 13.8 & 40.9 & 45.4 & 79.6 & 18.9 & 1.5 & & 4.9 & 0.0 & & & & 95.1 & 4.9 & 0.0 \\
\hline $\mathrm{M} 813$ & & & & 12.4 & 33.3 & 54.3 & 45.7 & 46.9 & 7.4 & 45.7 & 48.5 & 5.8 & 46.9 & 47.3 & 5.8 & 79.7 & 18.8 & 1.5 \\
\hline M656 & & & & 12.4 & 1.4 & 86.2 & 44.7 & 47.9 & 7.4 & 69.2 & 24.9 & 21.8 & 69.2 & 29.3 & 1.5 & 79.7 & 20.3 & 0.0 \\
\hline TDW901 & & & & 7.8 & 4.7 & 87.5 & 12.5 & 33.2 & 54.3 & 12.5 & 65.7 & 21.8 & 14.8 & 63.4 & 21.8 & 55.9 & 41.6 & 2.4 \\
\hline M520E1 & & & & 8.9 & 15.0 & 76.1 & 14.8 & 41.1 & 44.1 & 45.7 & 47.0 & 7.3 & 45.7 & 47.0 & 7.3 & 55.9 & 38.2 & 5.8 \\
\hline M125E1 & & & & & & & 13.4 & 41.2 & 45.4 & 13.5 & 42.4 & 44.1 & & & & 16.1 & 63.6 & 20.3 \\
\hline M548E1 & 0.0 & 7.8 & 92.2 & 0.0 & 12.5 & 87.5 & 12.4 & 10.1 & 77.5 & 12.5 & 56.6 & 30.9 & 12.5 & 57.7 & 29.8 & 25.0 & 67.7 & 7.3 \\
\hline UET & & & & & & & 7.8 & 14.7 & 77.5 & 12.5 & 24.4 & 63.1 & & & & 13.6 & 56.7 & 29.7 \\
\hline & & & & 0.0 & 0.0 & 0.0 & & & 87.5 & 0.0 & 12.5 & 87.5 & 7.8 & 4.7 & 87.5 & & 16.0 & 75.0 \\
\hline
\end{tabular}

(Continued) 
Table 4 (Continued)

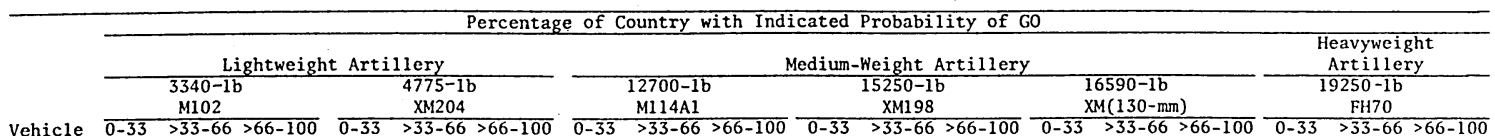

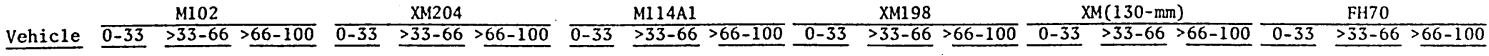
Country D - Wet Condition

\begin{tabular}{|c|c|c|c|c|c|c|c|c|c|c|c|c|c|c|c|c|c|c|}
\hline M561 & 77.0 & 21.9 & 1.2 & 90.2 & 9.0 & 0.9 & & & & 100.0 & 0.0 & 0.0 & 100.0 & 0.0 & 0.0 & 1000 & & \\
\hline $\begin{array}{l}\text { M35A2 } \\
M 813\end{array}$ & 65.6 & 18.6 & 15.8 & 66.7 & 26.8 & 6.5 & 98.2 & 1.8 & $\begin{array}{l}0.0 \\
7.6\end{array}$ & $\begin{array}{l}99.5 \\
83.6\end{array}$ & 0.5 & 0.0 & 99.5 & $\begin{array}{r}0.5 \\
10.6\end{array}$ & 0.0 & $\begin{array}{r}100.0 \\
98.5\end{array}$ & $\begin{array}{l}0.0 \\
1.5\end{array}$ & $\begin{array}{l}0.0 \\
0.0\end{array}$ \\
\hline $\begin{array}{l}\text { M813 } \\
\text { M656 }\end{array}$ & & & & 59.0 & $\begin{array}{l}25.0 \\
29.7\end{array}$ & $\begin{array}{l}16.0 \\
33.4\end{array}$ & $\begin{array}{l}83.5 \\
70.7\end{array}$ & $\begin{array}{r}8.9 \\
22.2\end{array}$ & $\begin{array}{l}7.6 \\
7.1\end{array}$ & $\begin{array}{l}83.6 \\
88.5\end{array}$ & $\begin{array}{l}15.4 \\
10.5\end{array}$ & $\begin{array}{l}1.0 \\
1.0\end{array}$ & $\begin{array}{l}88.4 \\
88.9\end{array}$ & $\begin{array}{l}10.6 \\
11.1\end{array}$ & $\begin{array}{l}1.0 \\
0.0\end{array}$ & $\begin{array}{l}98.5 \\
98.5\end{array}$ & $\begin{array}{l}1.5 \\
1.5\end{array}$ & 0.0 \\
\hline $\begin{array}{l}\text { M6so } \\
\text { TDW901 }\end{array}$ & & & & 27.0 & 31.7 & 41.3 & 36.2 & 47.4 & 16.5 & 36.7 & 55.1 & 8.2 & 72.4 & 19.4 & 8.2 & 92.1 & 7.4 & \\
\hline MS2OEI & & & & 49.2 & 27.2 & 23.5 & 72.8 & 19.4 & 7.9 & 83.6 & 9.1 & 7.3 & 83.6 & 9.1 & 7.3 & 92.1 & 6.9 & 1.0 \\
\hline M125El & & & & & & & 49.4 & 37.6 & 13.0 & 49.8 & 37.7 & 12.5 & & & & 77.9 & 20.6 & \\
\hline $548 \mathrm{EI}$ & 56.7 & 8.7 & 34.6 & 56.7 & 15.1 & 28.2 & 71.1 & 16.9 & 12.0 & 71.8 & 22.1 & 6.0 & 71.8 & 26.9 & 1.2 & 94.2 & 5.0 & 0.8 \\
\hline JET & & & & & & & 29.9 & 51.6 & 18.5 & 36.2 & 45.9 & 17.9 & & & & 72.1 & 20.6 & \\
\hline & & & & 21 & 30.8 & 47.7 & 21.6 & 43.5 & 34.8 & 45 & 19 & 34.7 & 49.3 & 16.0 & 4.7 & 78.6 & 15.3 & \\
\hline
\end{tabular}

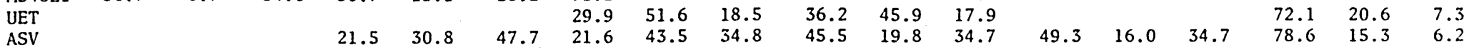
Country D - Dry Condition

\begin{tabular}{|c|c|c|c|c|c|c|c|c|c|c|c|c|c|c|c|c|c|c|}
\hline M561 & 77.0 & 21.9 & 1.2 & 90.2 & 9.0 & 0.9 & & & & 100.0 & 0.0 & 0.0 & 100.0 & 0.0 & 0.0 & & & \\
\hline M35A2 & 65.6 & 18.6 & 15.8 & 66.7 & 26.8 & 6.5 & 98.2 & 1.8 & 0.0 & 99.5 & 0.5 & 0.0 & 99.5 & 0.5 & 0.0 & 100.0 & .0 .0 & 0.0 \\
\hline M813 & & & & 58.1 & 25.9 & 16.0 & 83.5 & 8.9 & 7.6 & 83.6 & 15.4 & 1.0 & 86.1 & 12.9 & 1.0 & 98.5 & 1.5 & 0.0 \\
\hline$M 656$ & & & & 36.9 & 29.7 & 33.4 & 70.7 & 22.2 & 7.1 & 88.5 & 10.5 & 1.0 & 88.9 & 11.1 & 0.0 & 98.5 & 1.5 & 0.0 \\
\hline TDW901 & & & & 26.9 & 30.9 & 42.2 & 36.2 & 47.4 & 16.5 & 36.7 & 55.1 & 8.2 & 71.5 & 20.3 & 8.2 & 89.8 & 9.7 & 0.5 \\
\hline M520E1 & & & & 46.3 & 29.2 & 24.5 & 71.9 & 17.9 & 10.2 & 83.6 & 9.1 & 7.3 & 83.6 & 9.1 & 7.3 & 89.8 & 9.3 & 1.0 \\
\hline M1 25E1 & & & & & & & 49.4 & 37.6 & 13.0 & 49.8 & 37.7 & 12.5 & & & & 74.6 & 23.9 & 1.5 \\
\hline MS48E1 & 56.7 & 8.7 & 34.7 & 56.7 & 15.1 & 28.2 & 71.1 & 16.9 & 12.0 & 71.8 & 22.1 & 6.0 & 71.8 & 24.6 & 3.6 & 91.8 & 7.4 & 0.8 \\
\hline UET & & & & & & & 29.8 & 51.7 & 18.5 & 36.2 & 45.9 & 17.9 & & & & 71.2 & 19.1 & 9.7 \\
\hline ASV & & & & 21.5 & 29.6 & 48.9 & 21.6 & 43.5 & 34.8 & 43.4 & 21.9 & 34.7 & 48.4 & 17.0 & 34.7 & 75.7 & 15.8 & 8.6 \\
\hline \multicolumn{19}{|c|}{ Country E - Wet Condition } \\
\hline 561 & 56.7 & 19.0 & 24.2 & 70.7 & 12.0 & 17.3 & & & & 100.0 & 0.0 & 0.0 & 100.0 & 0.0 & 0.0 & & & \\
\hline M35A2 & 41.3 & 19.3 & 39.3 & 43.4 & 31.9 & 24.7 & 75.3 & 21.7 & 3.0 & 94.5 & 5.3 & 0.2 & 96.0 & 3.8 & 0.2 & 99.7 & 0.3 & 0.0 \\
\hline M813 & & & & 32.5 & 28.2 & 39.3 & 58.1 & 8.0 & 33.9 & 58.1 & 18.2 & 23.7 & 58.5 & 17.8 & 23.7 & 76.1 & 21.0 & 2.9 \\
\hline M656 & & & & 25.0 & 15.8 & 59.2 & 49.5 & 14.1 & 36.4 & 58.7 & 17.7 & 23.6 & 58.7 & 38.3 & 3.0 & 76.2 & 23.8 & 0.0 \\
\hline TDW901 & & & & 24.6 & 7.8 & 67.6 & 25.0 & 33.3 & 41.6 & 30.2 & 35.6 & 34.2 & 43.0 & 22.9 & 34.2 & 66.3 & 12.7 & 21.0 \\
\hline MS20E1 & & & & 41.4 & 9.5 & 49.1 & 45.6 & 20.6 & 33.7 & 60.8 & 6.0 & 33.2 & 60.8 & 6.0 & 33.2 & 66.3 & 10.2 & 23.6 \\
\hline M125E I & & & & & & & 38.8 & 26.6 & 34.6 & 38.8 & 27.0 & 34.2 & & & & 46.5 & 29.5 & 23.9 \\
\hline M548E1 & 26.1 & 20.2 & 53.7 & 34.0 & 12.3 & 53.7 & 39.2 & 33.9 & 26.9 & 46.3 & 29.3 & 24.4 & 46.3 & 29.6 & 24.1 & 62.3 & 14.4 & 23.3 \\
\hline UET & & & & & & & 27.8 & 26.9 & 45.3 & 27.8 & 27.1 & 45.1 & & & & 39.3 & 27.4 & 33.3 \\
\hline ASV & & & & 14.3 & 21.2 & 64.5 & 22.6 & 13.7 & 63.6 & 29.1 & 12.4 & 58.4 & 29.6 & 12.1 & 58.3 & 45.7 & 14.7 & 39.7 \\
\hline \multicolumn{19}{|c|}{ Country E - Dry Condition } \\
\hline 561 & 52.7 & 23.0 & 24.3 & 57.8 & 18.4 & 23.9 & & & & 99.9 & 0.1 & 0.0 & 99.9 & 0.1 & 0.0 & & & \\
\hline M35A2 & 37.1 & 10.9 & 52.0 & 37.6 & 23.1 & 39.3 & 54.4 & 40.2 & 5.4 & 94.1 & 5.7 & 0.2 & 94.1 & 5.7 & 0.2 & 98.7 & 1.3 & 0.1 \\
\hline M813 & & & & 28.2 & 19.7 & 52.0 & 45.4 & 20.5 & 34.1 & 45.4 & 23.9 & 30.7 & 45.5 & 23.8 & 30.7 & 54.8 & 39.6 & 5.6 \\
\hline M656 & & & & 20.9 & 14.1 & 65.0 & 45.4 & 18.1 & 36.6 & 46.0 & 23.3 & 30.7 & 46.0 & 48.6 & 5.4 & & & \\
\hline TDIF901 & & & & 20.6 & 7.7 & 71.8 & 21.0 & 24.4 & 54.6 & 26.0 & 25.4 & 48.6 & 28.6 & 22.7 & 48.6 & 51.2 & 18.6 & 30.2 \\
\hline M520E1 & & & & 28.6 & 5.9 & 65.6 & 31.2 & 20.0 & 48.8 & 48.0 & 18.2 & 33.8 & 48.0 & 18.2 & 33.8 & 51.2 & 18.1 & 30.7 \\
\hline M125E1 & & & & & & & 26.0 & 24.9 & 49.1 & 26.1 & 24.8 & 49.0 & & & & 31.5 & 23.2 & 45.2 \\
\hline M548E1 & 25.5 & 16.6 & 57.9 & 33.9 & 8.4 & 57.8 & 34.9 & 23.8 & 41.2 & 42.2 & 33.2 & 24.5 & 42.2 & 33.2 & 24.5 & 45.8 & 30.5 & 23.7 \\
\hline UET & & & & & & & 23.6 & 16.7 & 59.7 & 23.7 & 31.1 & 45.2 & & & & 26.4 & 39.6 & 34.0 \\
\hline ASV & & & & 14.2 & 17.2 & 68.6 & 22.6 & 9.7 & 67.7 & 25.0 & 12.4 & 62.6 & 25.5 & 12.0 & 62.5 & 32.7 & 11.0 & 56.4 \\
\hline \multicolumn{19}{|c|}{ Country F - Wet Condition } \\
\hline 561 & 72.4 & 24.5 & 3.0 & 81.2 & 16.5 & 2.2 & & & & 100.0 & 0.0 & 0.0 & 100.0 & 0.0 & 0.0 & & & \\
\hline M35A2 & 42.2 & 12.4 & 45.4 & 44.8 & 51.1 & 4.1 & 74.2 & 25.8 & 0.0 & & 0. & 0. & 99.8 & 0.1 & 0.0 & 100.0 & 0.0 & 0.0 \\
\hline$M 813$ & & & & 23.1 & 31.0 & 46.0 & 51.6 & 18.9 & 29.5 & 54.1 & 21.4 & 24.5 & 54.3 & 21.2 & 24.5 & 73.8 & 26.1 & 0.1 \\
\hline M656 & & & & 21.0 & 21.1 & 57.9 & 42.7 & 27.7 & 29.6 & 54.3 & 21.3 & 24.4 & 54.3 & 45.0 & 0.0 & 73.9 & 26.1 & 0.0 \\
\hline TDW901 & & & & 20.8 & 2.4 & 76.7 & 21.7 & 29.9 & 48.4 & 21.9 & 44.4 & 33.7 & 32.3 & 34.1 & 33.7 & 69.0 & 6.7 & 24.4 \\
\hline M520E1 & & & & 30.6 & 19.0 & 50.4 & 35.0 & 34.0 & 31.0 & 54.2 & 16.4 & 29.4 & 54.2 & 16.4 & 29.4 & 69.0 & 6.6 & 24.4 \\
\hline M125 & & & & & & & 30.6 & 38.2 & 31.3 & 30.9 & 37.9 & 31.2 & & & & 35.2 & 38.6 & 26.2 \\
\hline M548 & 47.7 & 23.4 & 28.9 & 48.0 & 23.4 & 28.6 & 48.8 & 46.8 & 4.5 & 71.7 & 25.2 & 3.0 & 71.7 & 25.3 & 3.0 & 95.5 & 2.0 & 2.5 \\
\hline UET & & & & & & & 21.3 & 44.2 & 34.4 & 22.1 & 45.1 & 32.8 & & & & 35.2 & 35.7 & 29.2 \\
\hline ASV & & & & 20.1 & 21.4 & 58.5 & 21.2 & 21.1 & 57.7 & 22.6 & 19.8 & 57.6 & 22.8 & 21.9 & 55.3 & 53.7 & 19.2 & 27.1 \\
\hline \multicolumn{19}{|c|}{ Country F-Dry Condition } \\
\hline M561 & 72.4 & 24.5 & 3.0 & 72.4 & 24.7 & 2.9 & & & & 99. & 0. & 0.0 & 99.9 & 0.1 & 0.0 & & & \\
\hline M35A2 & 41.6 & 20 & 56.4 & 42.4 & 31.1 & 26.5 & 49.5 & 50.3 & 0.2 & & 0. & 0.1 & 99.7 & 0.2 & 0.1 & 99.8 & 0.2 & 0.0 \\
\hline M813 & & & & 22.2 & 20.6 & 57.2 & 42.6 & 25.2 & 32.2 & 42 & 30.0 & 27.3 & 42.8 & 29.9 & 27.3 & 48.8 & 50.9 & 0.3 \\
\hline M656 & & & & 20.3 & 21.8 & 57.9 & 42.6 & 25.2 & 32.1 & & 30.0 & 27.1 & 42.9 & 56.9 & 0.2 & 48.9 & 51.1 & 0.0 \\
\hline TDW901 & & & & 20.7 & 2.2 & 77.1 & 21.4 & 21.2 & 57.3 & 21.6 & 22.4 & 55.9 & 22.9 & 21.2 & 55.9 & 44.0 & 28.9 & 27.1 \\
\hline M520E1 & & & & 20.8 & 3.4 & 75.9 & 22.9 & 21.1 & 56.0 & 42.6 & 25.2 & 32.2 & 42.6 & 25.2 & 32.2 & 44.0 & 28.8 & 27.2 \\
\hline M125E1 & & & & & & & 21.0 & 23.0 & 56.1 & 21.7 & 22.3 & 56.0 & & & & 23.0 & 25.9 & 51.2 \\
\hline M548E & 45.8 & 24.8 & 29.5 & 46.1 & 25.2 & 28.7 & 46.3 & 27.2 & 26.5 & 71.7 & 25.2 & 3.1 & 71.7 & 25.2 & 3.0 & 72.9 & 24.1 & 3.0 \\
\hline UET & & & & & & & 20.9 & 22.6 & 56.6 & 21.8 & 45.4 & 32.8 & & & & 23.2 & 45.0 & 31.9 \\
\hline ASV & & & & 19.9 & 21.4 & 58.6 & 20.9 & 21.4 & 57.7 & 22.2 & 20.1 & 57.7 & 22.4 & 20.0 & 57.6 & 41.7 & 6.6 & 51.8 \\
\hline
\end{tabular}


Table 4 (Concluded)

\begin{tabular}{|c|c|c|c|c|c|c|c|c|c|c|c|c|c|c|c|c|c|c|}
\hline \multirow[b]{4}{*}{ Vehicle } & \multicolumn{18}{|c|}{ Percentage of Country with Indicated Probability of $\mathrm{GO}$} \\
\hline & \multicolumn{6}{|c|}{ Lightweight Artillery } & \multicolumn{9}{|c|}{ Medium-Weight Artillery } & \multicolumn{3}{|c|}{$\begin{array}{l}\text { Heavyweight } \\
\text { Artillery }\end{array}$} \\
\hline & & $\begin{array}{l}3340-1 \mathrm{~b} \\
\mathrm{M1O2}\end{array}$ & & & $\begin{array}{r}4775-1 \\
X N 1204\end{array}$ & & & $\begin{array}{l}12700-11 \\
\text { M1 } 14 \mathrm{Al}\end{array}$ & & & $\begin{array}{c}15250-1 \mathrm{~b} \\
\text { XM199 }\end{array}$ & & & $\begin{array}{l}6590-1 \mathrm{~b} \\
\mathrm{M}(13 n-\mathrm{mm}\end{array}$ & & & $19250-1 \mathrm{~b}$ & \\
\hline & $\underline{0-33}$ & $>33-66$ & $>\underline{66-100}$ & $\underline{0-33}$ & $\geq 33-66$ & $>66-100$ & $\underline{0-33}$ & $\geq 33-66$ & $>\underline{66-100}$ & $0-33$ & $\geq 33-66$ & $>\underline{66-100}$ & $\underline{0-33}$ & $\geq 33-66$ & $>\underline{66-100}$ & $0-33$ & $\geq 33-66$ & $>\underline{66-100}$ \\
\hline & & & & & & & & ountry $\mathrm{C}$ & G - Wet $\mathrm{C}$ & Conditio & & & & & & & & \\
\hline M561 & 35.8 & 14.9 & 49.2 & 38.3 & 28.7 & 33.0 & & & & 100.0 & 0.0 & 0.0 & 100.0 & 0.0 & 0.0 & & & \\
\hline M35A2 & 6.2 & 30.1 & 63.7 & 10.0 & 28.3 & 61.7 & 67.0 & 21.4 & 11.6 & 86.8 & 13.2 & 0.0 & 86.9 & 13.1 & 0.0 & 88.4 & 11.6 & 0.0 \\
\hline M813 & & & & 6.2 & 30.1 & 63.7 & 36.3 & 21.1 & 42.6 & 38.3 & 28.7 & 33.0 & 38.4 & 28.6 & 33.0 & 67.0 & 21.4 & 11.6 \\
\hline M656 & & & & 5.7 & 4.3 & 90.0 & 36.3 & 21.1 & 42.6 & 57.4 & 9.6 & 33.0 & 57.4 & 31.0 & 11.6 & 67.0 & 33.0 & 0.0 \\
\hline TDW901 & & & & 2.7 & 3.5 & 93.8 & 5.8 & 32.5 & 61.7 & 6.2 & 51.2 & 42.6 & 10.0 & 47.3 & 42.6 & 50.8 & 17.9 & 31.4 \\
\hline M520E 1 & & & & 6.2 & 3.9 & 90.0 & 10.0 & 40.7 & 49.2 & 36.3 & 21.1 & 42.6 & 36.3 & 211 & 42.6 & 50.8 & 16.2 & 33.0 \\
\hline M125E1 & & & & & & & 6.2 & 30.1 & 63.7 & 6.2 & 32.1 & 61.7 & & & & 24.6 & 42.4 & 33.0 \\
\hline $\mathrm{M} 548 \mathrm{El}$ & 0.0 & 2.7 & 97.3 & 0.0 & 5.7 & 94.3 & 5.7 & 0.4 & 93.8 & 5.7 & 32.6 & 61.7 & 5.7 & 45.0 & 49.2 & 24.1 & 33.2 & 42.6 \\
\hline UET & & & & & & & 2.7 & 3.5 & 93.8 & 5.7 & 0.4 & 93.8 & & & & 5.7 & 45.0 & 49.2 \\
\hline ASV & & & & 0.0 & 0.7 & 99.5 & 0.0 & 5.8 & 94.2 & 0.8 & 5.4 & 93.8 & 2.7 & 3.5 & 93.8 & 5.7 & 18.8 & 75.4 \\
\hline & & & & & & & & ountry $\mathrm{C}$ & - Dry $C$ & Conditio & & & & & & & & \\
\hline M561 & 35.8 & 2.7 & 61.4 & 36.3 & 24.5 & 39.2 & & & & 100.0 & 0.0 & 0.0 & 100.0 & 0.0 & 0.0 & & & \\
\hline M35A2 & 6.2 & 30.1 & 63.7 & 8.9 & 27.4 & 63.7 & 60.8 & 26.1 & 13.1 & 86.8 & 13.2 & 0.0 & 86.9 & 13.1 & 0.0 & 86.9 & 1.3 .1 & 0.0 \\
\hline$M 813$ & & & & 6.2 & 30.1 & 63.7 & 36.3 & 21.1 & 42.6 & 36.3 & 24.5 & 39.2 & 36.3 & 24.5 & 39.2 & 60.8 & 26.1 & 13.1 \\
\hline M656 & & & & 5.7 & 3.2 & 91.1 & 36.3 & 21.1 & 42.6 & 57.4 & 3.4 & 39.2 & 57.4 & 29.5 & 13.1 & 60.8 & 39.2 & 0.0 \\
\hline TDW901 & & & & 2.7 & 3.5 & 93.8 & 5.8 & 30.6 & 63.7 & 6.2 & 51.2 & 42.6 & 8.9 & 48.4 & 42.6 & 36.5 & 24.4 & 39.1 \\
\hline M520E1 & & & & 3.2 & 5.8 & 91.1 & 8.9 & 27.5 & 63.5 & 36.3 & 21.1 & 42.6 & 36.3 & 21.1 & 42.6 & 36.5 & 24.3 & 39.2 \\
\hline M125E1 & & & & & & & 6.2 & 30.1 & 63.7 & 6.2 & 30.1 & 63.7 & & & & 9.2 & 51.6 & 39.2 \\
\hline M548E1 & 0.0 & 2.7 & 97.3 & 0.0 & 5.7 & 94.3 & 5.7 & 0.4 & 93.8 & 5.7 & 30.6 & 63.7 & 5.7 & 32.7 & 61.5 & 8.8 & 48.6 & 42.6 \\
\hline UET & & & & & & & 2.7 & 3.7 & 93.8 & 5.7 & 0.4 & 93.8 & & & & 5.7 & 30.8 & 63.5 \\
\hline ASV & & & & 0.0 & 0.6 & 99.4 & 0.0 & 5.8 & 94.2 & 0.6 & 5.6 & 93.8 & 2.7 & 3.5 & 93.8 & 2.7 & 6.4 & 90.8 \\
\hline & & & & & & & & ountry $\mathrm{H}$ & 1 - Wet $c$ & Conditio & & & & & & & & \\
\hline 4561 & 70.8 & 0.0 & 29.2 & 70.8 & 0.5 & 28.7 & & & & 100.0 & 0.0 & 0.0 & 100.0 & 0.0 & 0.0 & & & \\
\hline $5 \mathrm{~A} 2$ & 70.8 & 0.0 & 29.2 & 70.8 & 0.5 & 28.7 & 71.3 & 28.5 & 0.2 & 99.8 & 0.2 & 0.0 & 99.8 & 0.2 & 0.0 & 99.8 & 0.2 & 0.0 \\
\hline M813 & & & & 70.8 & 0.0 & 29.2 & 70.8 & 0.5 & 28.7 & 71.3 & 1.1 & 27.6 & 71.3 & 1.1 & 27.6 & 72.4 & 27.4 & 0.2 \\
\hline M656 & & & & 70.8 & 0.0 & 29.2 & 70.8 & 0.5 & 28.7 & 71.3 & 1.1 & 27.6 & 71.3 & 28.5 & 0.2 & 72.4 & 27.6 & 0.0 \\
\hline TDW901 & & & & 70.8 & 0.0 & 29.2 & 70.8 & 0.0 & 29.2 & 70.8 & 0.0 & 29.2 & 70.8 & 0.0 & 29.2 & 72.4 & 0.0 & 27.6 \\
\hline M520E1 & & & & 70.8 & 1.6 & 27.6 & 71.3 & 1.1 & 27.6 & 71.3 & 1.1 & 27.6 & 71.3 & 1.1 & 27.6 & 72.4 & 0.0 & 27.6 \\
\hline M125E1 & & & & & & & 70.8 & 0.5 & 28.7 & 70.8 & 0.5 & 28.7 & & & & 72.4 & 0.0 & 27.6 \\
\hline $\mathrm{M} 548 \mathrm{E} 1$ & 0.0 & 70.8 & 29.2 & 0.0 & 70.8 & 29.2 & 70.8 & 0.0 & 29.2 & 70.8 & 0.0 & 29.2 & 70.8 & 0.0 & 29.2 & 72.4 & 0.0 & 27.6 \\
\hline UET & & & & & & & 70.8 & 0.0 & 29.2 & 70.8 & 0.0 & 29.2 & & & & 72.4 & 0.0 & 27.6 \\
\hline ASV & & & & 0.0 & 0.0 & 100.0 & 0.0 & 70.8 & 29.2 & 0.0 & 70.8 & 29.2 & 70.8 & 0.0 & 29.2 & 72.4 & 0.0 & 27.6 \\
\hline & & & & & & & & ountry $H$ & 1 - Dry C & Conditio & & & & & & & & \\
\hline M561 & 70.8 & 0.0 & 29.2 & 70.8 & 0.5 & 28.7 & & & & 100.0 & 0.0 & 0.0 & 100.0 & 0.0 & 0.0 & & & \\
\hline M35A2 & 70.8 & 0.0 & 29.2 & 70.8 & 0.5 & 28.7 & 71.3 & 28.5 & 0.2 & 99.8 & 0.2 & 0.0 & 99.8 & 0.2 & 0. & 9.8 & 0.2 & 0.0 \\
\hline M813 & & & & 70.8 & 0.0 & 29.2 & 70.8 & 0.5 & 28.7 & 71.3 & 1.1 & 27.6 & 71.3 & 1.1 & 27 & 4 & 27.4 & 0.2 \\
\hline M656 & & & & 70.8 & 0.0 & 29.2 & 70.8 & 0.5 & 28.7 & 71.3 & 1.1 & 27.6 & 71.3 & 28.5 & 0. & 72.4 & 27.6 & 0.0 \\
\hline TDW90 & & & & 70.8 & 0.0 & 29.2 & 70.8 & 0.0 & 29.2 & 70.8 & 0.0 & 29.2 & 70.8 & 0.0 & 29.2 & 72.4 & 0.0 & 27.6 \\
\hline M520E 1 & & & & 70.8 & 1.6 & 27.6 & 71.3 & 1.1 & 27.6 & 71.3 & 1.1 & 27.6 & 71.3 & 1.1 & 27.6 & 72.4 & 0.0 & 27.6 \\
\hline M12SE1 & & & & & & & 70.8 & 0.5 & 28.7 & 70.8 & 0.5 & 28.7 & & & & 72.4 & 0.0 & 27.6 \\
\hline MS48E I & 0.0 & 70.8 & 29.2 & 0.0 & 70.8 & 29.2 & 70.8 & 0.0 & 29.2 & 70.8 & 0.0 & 29.2 & & & & 72.4 & 0.0 & 27.6 \\
\hline UET & & & & & & & 70.8 & 0.0 & 29.2 & 70.8 & 0.0 & 29.2 & & & & 72.4 & 0.0 & 27.6 \\
\hline ASV & & & & 0.0 & 0.0 & 100.0 & 0.0 & 70.8 & 29.2 & 0.0 & 70.8 & 29.2 & 70.8 & 0.0 & 29.2 & 72.4 & 0.0 & 27.6 \\
\hline & & & & & & & & untry & - Wet & Conditis & & & & & & & & \\
\hline$M$ & 20.6 & 9.1 & 70.2 & 21.0 & 12.2 & 66.8 & & & & 81.3 & 18.7 & 0.0 & 81.3 & 18.7 & 0.0 & & & \\
\hline M35A2 & 6.7 & 22.5 & 70.8 & 9.3 & 22.1 & 68.5 & 3.1 & 48.1 & 18.8 & 81.0 & 0.3 & 18.7 & 81.0 & 0.3 & & 3 & 0.0 & 18.7 \\
\hline M813 & & & & 6.4 & 14.3 & 79. & 20.6 & 11.5 & 67.9 & 31.4 & 1.9 & 66.7 & 31.4 & 1.9 & 66 & 2 & 48.1 & 18.8 \\
\hline M656 & & & & 6.4 & 3.3 & 90.4 & 20.7 & 11.5 & 67.9 & 32.1 & 1.2 & & $32: 1$ & 49.2 & & 2 & 48.0 & 18.7 \\
\hline $\mathrm{TL}$ & & & & 5.4 & 1.0 & 93.5 & 6.4 & 14.6 & 79.0 & 6.5 & 15.2 & 78.3 & 9.4 & 20.8 & 69.8 & 2 & 10.4 & 66.4 \\
\hline MS20EI & & & & 5.4 & 6.0 & 88.6 & 11.3 & 11.9 & 76.8 & 22.6 & 1.1 & 76.3 & 22.6 & 9.6 & 67.8 & & 10.1 & 66.7 \\
\hline M125E1 & & & & & & & 6.3 & 24.8 & 68.9 & 6.4 & 25.1 & 68.5 & & & & 8 & 21.4 & 66.8 \\
\hline M548 & 1.0 & 23.6 & 75.4 & 19.8 & 5.1 & 75.2 & 24.8 & 0.7 & 74.5 & 24.8 & 15.0 & 60.2 & 24.8 & 15.1 & 60.0 & 30.8 & 11.6 & 57.5 \\
\hline UET & & & & & & & 24.2 & 0.4 & 75.4 & 24.5 & 1.0 & 74.4 & & & & 26.6 & 15.3 & 58.0 \\
\hline ASV & & & & 1.0 & 19.5 & 79.5 & 20.1 & 4.5 & 75.5 & 20.5 & 4.3 & 75.2 & 24.2 & 0.6 & 75.2 & 26.4 & 4.4 & 69.2 \\
\hline & & & & & & & & ntry & - Dry C & Conditic & & & & & & & & \\
\hline & 20.6 & 9.0 & 70. & 20.6 & 2 & 69.8 & & & & 3 & 18.7 & 0.0 & 81.3 & 18.7 & 0.0 & & & \\
\hline M35A2 & 6.7 & 22.5 & 70.9 & 6.4 & 22.8 & 70.8 & 30.1 & 50.8 & 19.1 & & 0.4 & & & 0 & & & 0. & 18.7 \\
\hline M813 & & & & 6.4 & 14.3 & 79.4 & 20.6 & 9. & 69.8 & 29.1 & 1.1 & 69.8 & 29.1 & 1. & & & 50.8 & 19.1 \\
\hline M656 & & & & 6.4 & 0. & 93.2 & 20.6 & 9.6 & 69.8 & 30.2 & 0.1 & 69.8 & 30.2 & 50.8 & & 2 & 51.1 & 18.7 \\
\hline TDW901 & & & & 5.4 & 1.0 & 93.5 & 6.4 & 14.3 & 79.3 & 6.5 & 15.2 & 78.4 & 6.5 & 23.7 & 69.8 & 20.7 & 9.6 & 69.8 \\
\hline M520E 1 & & & & 5.4 & 1.0 & 93.5 & 6.4 & 14.3 & 79.3 & 20.7 & 1.0 & 78.3 & 20.7 & 9.5 & 69.8 & 20.7 & 9.5 & 69.8 \\
\hline M125E1 & & & & & & & 6.3 & 22.8 & 70.9 & 6.4 & 22.8 & 70.8 & & & & 6.4 & 23.8 & 69.8 \\
\hline M548E1 & 1.0 & 23.6 & 75.4 & 19.8 & 5.1 & 75.2 & 24.8 & 0.7 & 74.5 & 24.8 & 14.7 & 60.5 & 24.8 & 15.0 & 60.1 & 25.5 & 15.0 & 59.5 \\
\hline UET & & & & & & & 24.2 & 0.3 & 75.5 & 24.5 & 1.0 & 74. & & & & 24.5 & 15.0 & 60.5 \\
\hline ASV & & & & 1.0 & 19.1 & 79.9 & 20.1 & 4.5 & 75.5 & 20.1 & 4.8 & 75.2 & 24.2 & 0.6 & 75.2 & 24.3 & 1.2 & 74.5 \\
\hline
\end{tabular}


Table 5

Performance Predictions for Self-Propelled Artillery and Reference Vehicles

in the Countries Described by Generalized Terrain Data

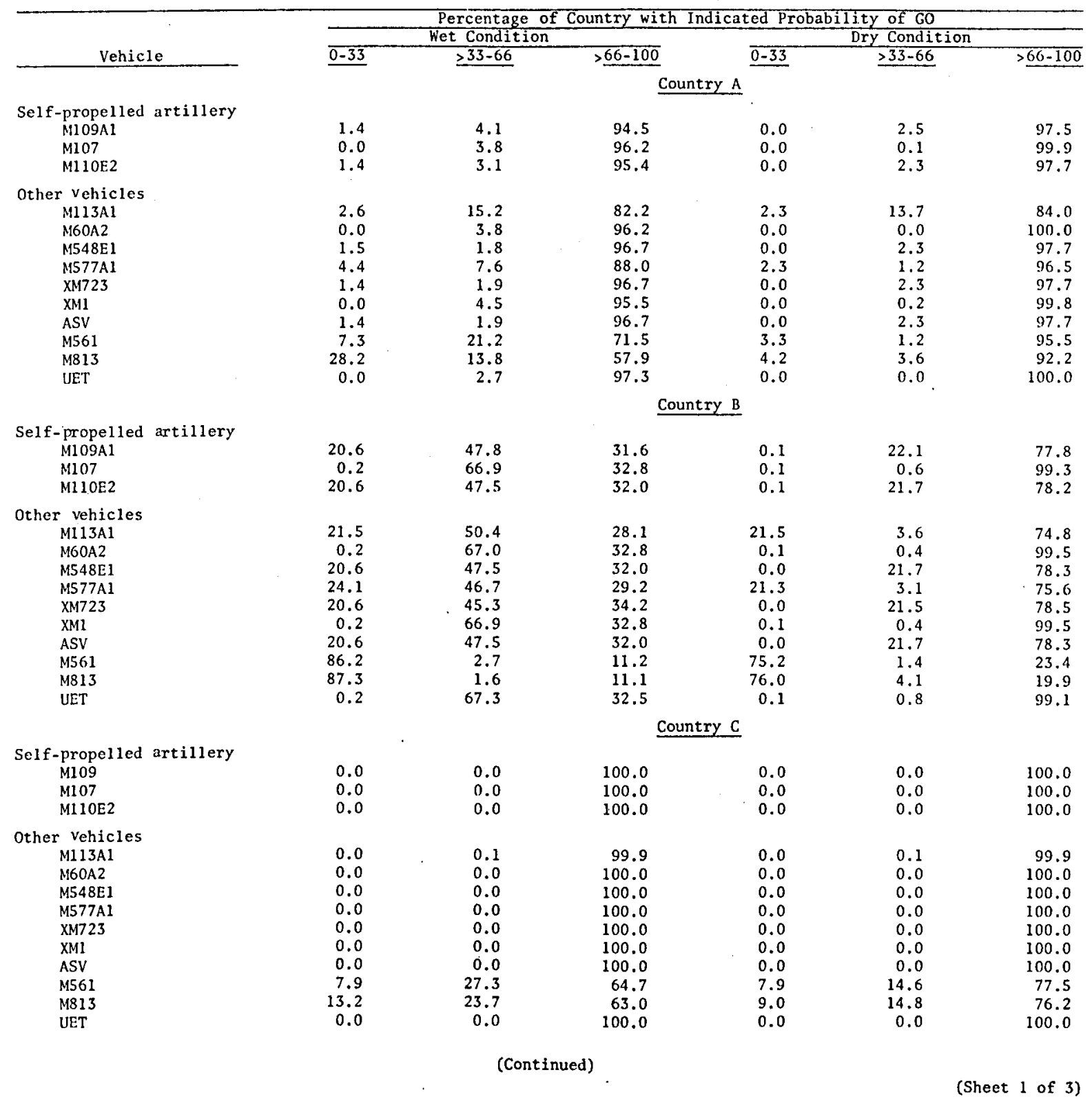


Table 5 (Continued)

\begin{tabular}{|c|c|c|c|c|c|c|}
\hline \multirow[b]{3}{*}{ Vehicle } & \multicolumn{6}{|c|}{ Percentage of Country with Indicated Probability of $\mathrm{GO}$} \\
\hline & \multirow{2}{*}{\multicolumn{3}{|c|}{$\frac{\text { Wet Condition }}{>33-66}$}} & \multicolumn{3}{|c|}{ Dry Condition } \\
\hline & & & & $0-33$ & $333-66$ & $>66-100$ \\
\hline & \multicolumn{6}{|c|}{ Country D } \\
\hline \multicolumn{7}{|l|}{ Self-propelled artillery } \\
\hline M109A1 & 21.5 & 0.4 & 78.1 & 21.5 & 0.4 & 78.1 \\
\hline M107 & 21.5 & 0.0 & 78.5 & 21.5 & 0.0 & 78.5 \\
\hline M110E2 & 21.5 & 0.0 & 78.5 & 21.5 & 0.0 & 78.5 \\
\hline \multicolumn{7}{|l|}{ Other vehicles } \\
\hline M113Al & 57.1 & 3.3 & 39.6 & 57.1 & 3.3 & 39.6 \\
\hline $\mathrm{M} 60 \mathrm{~A} 2$ & 21.5 & 22.5 & 56.1 & 21.5 & 21.6 & 56.9 \\
\hline M548E1 & 56.5 & 0.6 & 42.9 & 56.5 & 0.6 & 42.9 \\
\hline M577Al & 57.0 & 0.5 & 42.5 & 57.0 & 0.5 & 42.5 \\
\hline XM723 & 50.5 & 0.2 & 49.3 & 50.5 & 0.2 & 49.3 \\
\hline$X M 1$ & 21.4 & 22.5 & 56.1 & 21.4 & 21.6 & 56.9 \\
\hline M561 & 65.5 & 22.5 & 12.0 & 65.5 & 22.5 & 12.0 \\
\hline$M 813$ & 43.4 & 32.9 & 23.7 & 43.4 & 32.0 & 24.6 \\
\hline UET & 21.5 & 3.2 & 75.4 & 21.5 & 3.2 & 75.4 \\
\hline & \multicolumn{6}{|c|}{ Country E } \\
\hline \multicolumn{7}{|l|}{ Self-propelled artillery } \\
\hline M109Al & 14.3 & 4.6 & 81.0 & 14.1 & 0.6 & 85.3 \\
\hline M107 & 11.6 & 7.1 & 81.3 & 11.6 & 3.1 & 85.3 \\
\hline M1 10E2 & 11.6 & 7.1 & 81.3 & 11.6 & 3.1 & 85.3 \\
\hline \multicolumn{7}{|l|}{ Other vehicles } \\
\hline M113Al & 32.9 & 12.9 & 54.2 & 32.9 & 8.8 & 58.2 \\
\hline M60A2 & 11.6 & 9.4 & 79.0 & 11.6 & 5.3 & 83.1 \\
\hline M548E1 & 23.0 & 22.3 & 54.7 & 22.9 & 18.4 & 58.7 \\
\hline M577A1 & 40.9 & 4.5 & 54.6 & 40.7 & 0.6 & 58.7 \\
\hline XM723 & 20.2 & 11.1 & 68.7 & 20.2 & 11.1 & 68.7 \\
\hline XM1 & 11.6 & 9.3 & 79.1 & 11.6 & 5.0 & 83.3 \\
\hline ASV & 11.6 & 9.6 & 78.8 & 11.6 & 5.3 & 83.1 \\
\hline M561 & 46.0 & 18.3 & 35.7 & 41.9 & 7.9 & 50.1 \\
\hline M813 & 38.8 & 11.4 & 49.8 & 26.1 & 8.0 & 65.9 \\
\hline \multirow[t]{2}{*}{ UET } & 14.5 & 4.7 & 80.0 & 14.5 & 0.6 & 85.0 \\
\hline & \multicolumn{6}{|c|}{ Country F } \\
\hline \multicolumn{7}{|l|}{ Self-propelled artillery } \\
\hline M109Al & 20.2 & 0.9 & 78.8 & 20.1 & 0.6 & 79.3 \\
\hline M107 & 19.9 & 1.1 & 79.0 & 19.9 & 0.8 & 79.3 \\
\hline M1 10E2 2 & 19.9 & 1.1 & 79.0 & 19.9 & 0.8 & 79.3 \\
\hline \multicolumn{7}{|l|}{ Other Vehicles } \\
\hline MI13A1 & 70.2 & 1.4 & 28.4 & 70.2 & 1.4 & 28.4 \\
\hline $\mathrm{M} 60 \mathrm{~A} 2$ & 19.9 & 2.4 & 77.6 & 19.9 & 2.0 & 78.0 \\
\hline M548E1 & 47.2 & 23.1 & 29.6 & 45.3 & 25.0 & 29.6 \\
\hline MS77A1 & 70.0 & 0.7 & 29.3 & 69.9 & 0.6 & 29.6 \\
\hline$X M 723$ & 40.6 & 3.1 & 56.3 & 40.6 & 0.9 & 58.5 \\
\hline $\mathrm{XM1}$ & 20.3 & 1.9 & 77.7 & 20.0 & 1.8 & 78.2 \\
\hline ASV & 19.9 & 2.4 & 77.6 & 19.9 & 2.0 & 78.0 \\
\hline M561 & 71.3 & 23.5 & 5.2 & 71.2 & 1.5 & 27.2 \\
\hline M813 & 30.8 & 16.2 & 53.1 & 21.6 & 2.6 & 75.8 \\
\hline UET & 20.4 & 0.9 & 78.8 & 20.3 & 0.5 & 79.2 \\
\hline
\end{tabular}


Table 5 (Concluded)

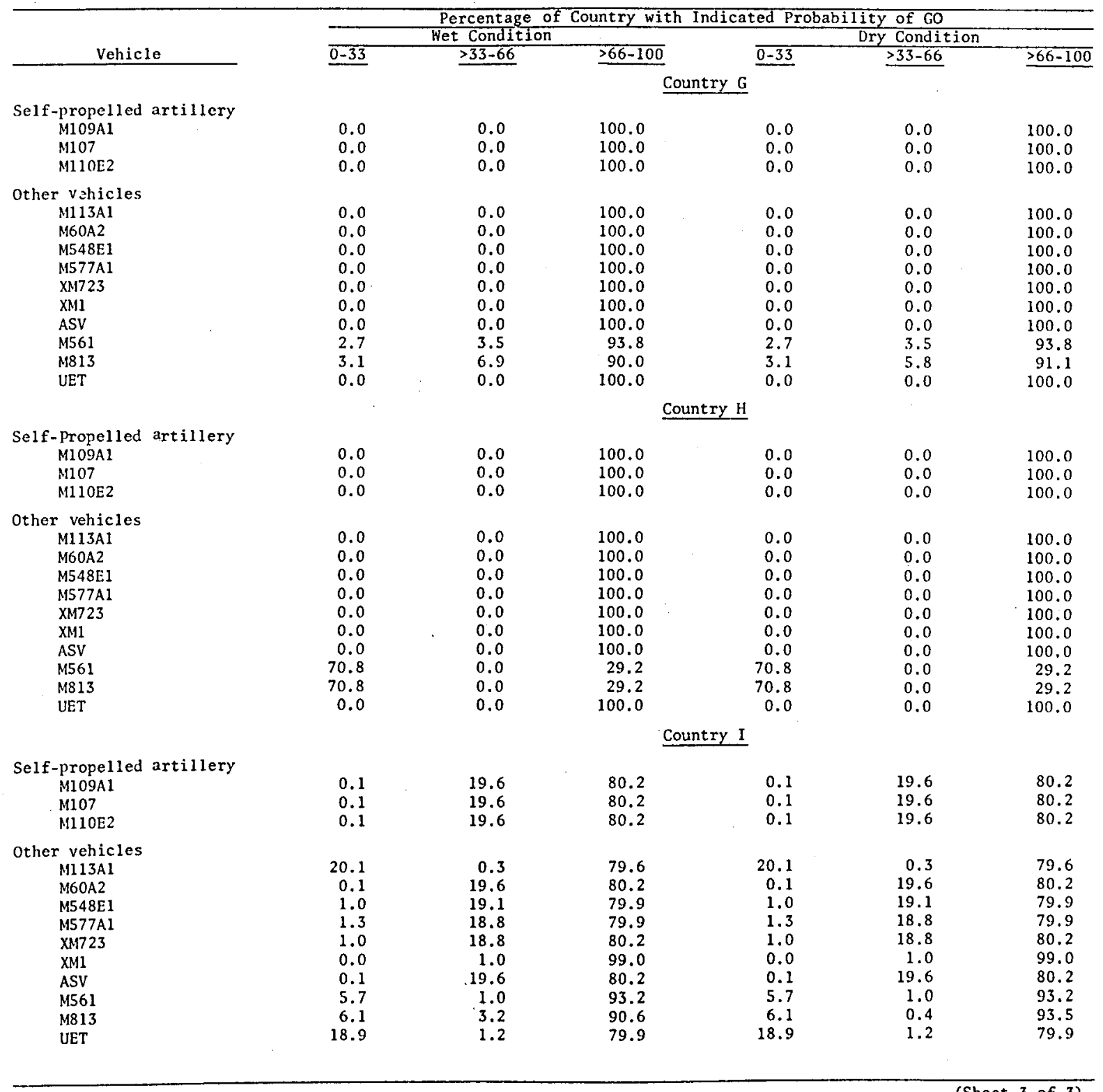


Table 6

$\mathrm{V}_{90}$ Off-Road Performance Indices for Prime Mover-Towed Artillery Combinations

in the HIMO Study Areas

\begin{tabular}{|c|c|c|c|c|c|c|}
\hline \multirow[b]{4}{*}{ Prime Mover } & \multicolumn{6}{|c|}{$\mathrm{V}_{90}$ Speed, mph } \\
\hline & \multicolumn{2}{|c|}{ Lightweight Artillery } & \multicolumn{3}{|c|}{ Medium-Weight Artillery } & \multirow{3}{*}{$\begin{array}{l}\text { Heavyweight } \\
\text { Artillery } \\
19250^{-1 b} \\
\text { FH70 }\end{array}$} \\
\hline & $3340-1 b$ & $4775-1 b$ & $\overline{12700-1 b}$ & $15250-1 \mathrm{~b}$ & $16590-1 b$ & \\
\hline & $\mathrm{M102}$ & $\mathrm{XM} 204$ & M114A1 & $\mathrm{XM198}$ & $X M(130-\mathrm{mm})$ & \\
\hline \multicolumn{7}{|c|}{ West Germany - Wet Condition } \\
\hline M561 & 5.4 & 2.7 & & 0.5 & * & $\cdot$ \\
\hline M35A2 & 10.9 & 10.8 & * & 2.0 & * & ** \\
\hline$M 813$ & & 9.6 & 8.3 & 6.5 & 6.2 & 0.1 \\
\hline M656 & & 11.3 & * & 3.4 & $\star$ & $\star *$ \\
\hline TDW901 & & 10.6 & 9.3 & 8.7 & 8.4 & 0.1 \\
\hline M520EI & & 6.6 & $\star$ & 5.7 & $\star$ & 0.1 \\
\hline MI25E1 & & & * & 8.1 & & 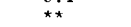 \\
\hline M548E1 & $t$ & 3.5 & * & 3.5 & * & 0.1 \\
\hline UET+t & & & * & 10.0 & & 0.1 \\
\hline ASV & & 11.2 & * & 10.1 & * & 0.1 \\
\hline \multicolumn{7}{|c|}{ West Germany - Snow Condition } \\
\hline M561 & 10.7 & 2.2 & & 0.2 & * & \\
\hline M35A2 & 1.3 & 1.0 & * & 0.1 & * & $\star \star$ \\
\hline M813 & & 2.8 & 0.6 & 0.5 & 0.4 & 0.3 \\
\hline M656 & & 11.2 & $\star$ & 1.7 & * & $\star \star$ \\
\hline TDW901 & & 11.1 & 9.8 & 9.1 & $8.9^{\circ}$ & 8.6 \\
\hline M52OE1 & & 6.6 & * & 5.7 & * & 5.5 \\
\hline M1 25E1 & & & * & 1.4 & & $\star \star$ \\
\hline M548E 1 & + & 11.6 & * & 10.4 & * & 10.3 \\
\hline UET+t & & & * & 12.7 & & 12.5 \\
\hline ASV & & 14.7 & * & 13.9 & * & 13.7 \\
\hline \multicolumn{7}{|c|}{ Mid-East - Wet Condition } \\
\hline M561 & 1.2 & 1.0 & & 0.5 & * & \\
\hline M35A2 & 1.8 & 1.8 & * & 1.2 & * & $\star *$ \\
\hline M813 & & 3.9 & 3.8 & 1.8 & 3.4 & 0.1 \\
\hline M656 & & 2.5 & * & 1.5 & * & $\star \star$ \\
\hline TDW901 & & 10.3 & 9.3 & 8.6 & 8.3 & 0.1 \\
\hline M520E 1 & & 5.4 & * & 4.9 & $\star$ & 0,1 \\
\hline M12SE1 & & & * & 5.8 & & $\star * \star 1$ \\
\hline M548E 1 & $t$ & 2.6 & * & 2.6 & * & 0.1 \\
\hline UETtt & & & * & 5.4 & & 0.1 \\
\hline ASV & & 11.5 & * & 10.5 & * & 0.1 \\
\hline \multicolumn{7}{|c|}{ Mid-East - Sand Condition } \\
\hline M561 & 0.2 & 0.1 & & 0.1 & * & \\
\hline M35A2 & 0.1 & 0.1 & * & 0.1 & * & $\star \star \star$ \\
\hline M813 & 0.1 & 0.1 & 0.1 & 0.1 & 0.1 & 0.1 \\
\hline$M 656$ & & 0.2 & $\star$ & 0.1 & * & $\star \star \star *$ \\
\hline TDW901 & & 0.5 & 0.2 & 0.1 & 0.1 & 0.1 \\
\hline M520E1 & & 0.6 & * & 0.1 & * & 0.1 \\
\hline M125E1 & & & * & 0.1 & & $\star \star \star \star^{2}$ \\
\hline M548E 1 & + & 3.1 & * & 1.8 & * & 0.8 \\
\hline UET+t & & & * & 4.8 & & \\
\hline ASV & & 9.1 & * & 7.9 & * & 7.4 \\
\hline
\end{tabular}

* Performance predictions not made for indicated prime mover-M114Al and prime mover-XM(130-mm) on detailed terrain data in order to reduce cost, but vehicle performance should be very similar to performance for prime mover-XM198.

** Performance predictions not made for prime mover-FH70 on detailed terrain data in order to reduce cost, but performance should be very similar to performance of M813-FH70.

+ Performance predictions not made for MS48E1-M102 on detailed terrain data in order to reduce cost, but performance should be very similar to performance for the M548E1-XM204.

tt Values of $\mathrm{v}_{90}$ were computed using $0.5 \mathrm{mph}$ for obstacle NOGO to allow for advantages of the UET's earthmoving capabilities. NOTE: When no performance data or explanation given, prime mover-towed artillery combination was not evaluated. 
Table 7

$\mathrm{V}_{90}$ Off-Road Performance Indices for Self-Propelled Artillery and Reference

Vehicles in the HIMO Study Areas

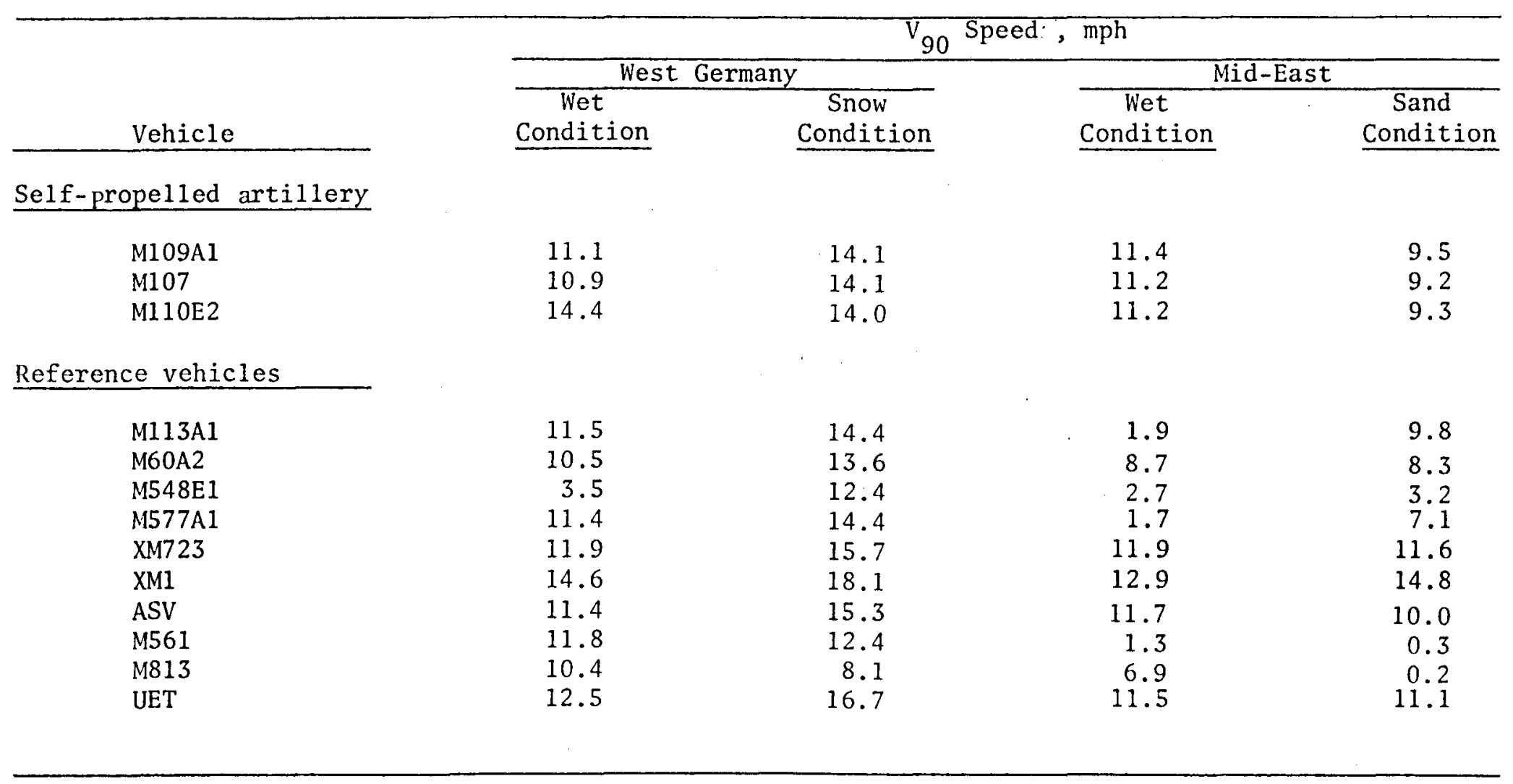


Table 8

$v_{100}$ Speeds for Prime Mover-Towed Artillery Combinations Over Primary and Secondary Roads

and Trails in the HIMO Study Areas

\begin{tabular}{|c|c|c|c|c|c|c|c|c|c|c|c|c|c|c|c|c|c|c|}
\hline \multirow{4}{*}{ Vehicle } & \multicolumn{18}{|c|}{$\mathrm{V}_{100}$ Speed, mph } \\
\hline & \multicolumn{6}{|c|}{ Lightweight Artillery } & \multicolumn{9}{|c|}{ Medium-Weight Artillery } & \multirow{2}{*}{\multicolumn{3}{|c|}{$\begin{array}{c}\text { Heavyweight Arti11ery } \\
19250-1 \mathrm{~b} \\
\text { FH70 }\end{array}$}} \\
\hline & \multicolumn{3}{|c|}{$\begin{array}{l}3340-1 b \\
M 102\end{array}$} & \multicolumn{3}{|c|}{$\begin{array}{c}4775-1 \mathrm{~b} \\
\times M 204\end{array}$} & \multicolumn{3}{|c|}{$\begin{array}{l}12700-1 \mathrm{~b} \\
M 114 \mathrm{~A} 1\end{array}$} & \multicolumn{3}{|c|}{$\begin{array}{c}15250-1 \mathrm{~b} \\
\times \mathrm{M} 198 \\
\end{array}$} & \multicolumn{3}{|c|}{$\begin{array}{l}16590-1 \mathrm{~b} \\
\mathrm{XM}(130-\mathrm{mm}) \\
\end{array}$} & & & \\
\hline & $\begin{array}{l}\text { Primary } \\
\text { Roads }\end{array}$ & $\begin{array}{l}\text { M102 } \\
\text { Secondary } \\
\text { Roads }\end{array}$ & Trails & $\begin{array}{l}\text { Primary } \\
\text { Roads }\end{array}$ & $\begin{array}{l}\text { Mecout } \\
\text { Secondary } \\
\text { Roads } \\
\end{array}$ & Trails & $\begin{array}{l}\text { Primary } \\
\text { Roads } \\
\end{array}$ & $\begin{array}{l}\text { Secondary } \\
\text { Soads } \\
\end{array}$ & Trails & $\begin{array}{l}\text { Primary } \\
\text { Roads } \\
\end{array}$ & $\begin{array}{c}\text { Secondary } \\
\text { Roads } \\
\end{array}$ & Trails & $\begin{array}{l}\text { Primary } \\
\text { Roads }\end{array}$ & $\begin{array}{l}\text { Secondary } \\
\text { Roads }\end{array}$ & Trails & $\begin{array}{l}\text { Primary } \\
\text { Roads } \\
\end{array}$ & $\begin{array}{l}\text { Secondary } \\
\text { Roads }\end{array}$ & $\overline{\text { Trails }}$ \\
\hline & \multicolumn{18}{|c|}{ West Germany - Wet Condition } \\
\hline M561 & 28.2 & 22.9 & 8.8 & 28.0 & 22.4 & 3.8 & & & & 21.3 & 17.1 & 1.0 & * & * & * & & & \\
\hline M35A2 & 28.9 & 23.7 & 13.6 & 28.6 & 23.2 & $\begin{array}{r}3.0 \\
13.3\end{array}$ & $*$ & $\star$ & * & 24.7 & 19.1 & 1.9 & * & * & * & $\star *$ & $\star \star$ & $\star \star *$ \\
\hline$M 813$ & & & 10.0 & 27.4 & 22.6 & 3.9 & 25.2 & 20.6 & 3.9 & 24.6 & 20.2 & 3.9 & 24.1 & 19.9 & 3.9 & 23.8 & 19.2 & 0.1 \\
\hline $\begin{array}{l}\text { M656 } \\
\text { TDW901 }\end{array}$ & & & & $\begin{array}{l}26.7 \\
22.1\end{array}$ & $\begin{array}{l}22.0 \\
17.5\end{array}$ & $\begin{array}{l}12.0 \\
12.6\end{array}$ & 19.6 & 15.5 & 11.2 & $\begin{array}{l}24.3 \\
18.9\end{array}$ & $\begin{array}{l}19.6 \\
15.0\end{array}$ & $\begin{array}{r}4.0 \\
10.7\end{array}$ & 18.5 & 14.8 & 4.1 & $\begin{array}{ll}\star * \\
17.7\end{array}$ & 14.4 & $0_{0.1}^{*}$ \\
\hline MS20E1 & & & & 20.7 & 16.0 & 3.6 & $\star$ & $\star$ & 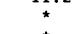 & 18.7 & 14.2 & 3.5 & +0 & $*$ & $* *$ & 18.1 & 13.6 & 0.1 \\
\hline M125E1 & & & & & & & * & * & * & 21.0 & 18.0 & 4.0 & $*$ & $\star$ & $*$ & & & ** \\
\hline M548E1 & + & + & + & 25.9 & 21.5 & 11.2 & * & * & * & $\begin{array}{l}25.1 \\
22.6\end{array}$ & 19.4 & 10.2 & * & * & * & 23.5 & 18.6 & 0.1 \\
\hline \multirow[t]{2}{*}{$\begin{array}{l}\text { UET } \\
\text { ASV }\end{array}$} & & & & 26.4 & 22.6 & 15.6 & * & * & * & $\begin{array}{l}25.6 \\
25.8\end{array}$ & $\begin{array}{l}19.9 \\
21.3\end{array}$ & $\begin{array}{l}13.8 \\
13.8\end{array}$ & * & * & * & 25.5 & 20.8 & $\begin{array}{l}0.1 \\
0.1\end{array}$ \\
\hline & \multicolumn{18}{|c|}{ West Germany - Snow Condition } \\
\hline M561 & & 17.5 & 8.9 & 19.6 & 10.1 & 6.2 & & & & 0.5 & 0.3 & 0.2 & * & * & * & & & \\
\hline & 12.5 & 4.5 & $\begin{array}{l}0.9 \\
3.9\end{array}$ & $\begin{array}{l}11.0 \\
11.8\end{array}$ & 2.6 & $\begin{array}{l}1.6 \\
1.7\end{array}$ & * & * & * & 0.4 & 0.2 & 0.2 & * & * & * & ** & $\star \star *$ & 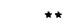 \\
\hline MS13 & 12.5 & & & 11.2 & $\begin{array}{l}7.5 \\
7.5\end{array}$ & 5.6 & 8.9 & 1.6 & 1.3 & 7.4 & 1.3 & 1.2 & 7.3 & 1.3 & 1.2 & 7.2 & 0.5 & 0.4 \\
\hline M656 & & & & 19.7 & 16.5 & 11.6 & $\star$ & & * & 15.2 & 4.8 & 3.9 & $\therefore$ & $\stackrel{*}{*}$ & $\star$ & 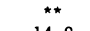 & 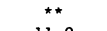 & $\star \star \star *$ \\
\hline TDW901 & & & & 16.7 & 14.4 & 12.4 & 15.2 & 13.0 & 11.4 & 14.7 & 12.6 & 11.0 & 14.5 & 12.4 & 10.8 & 14.0 & 11.9 & 10.3 \\
\hline MS20EI & & & & 14.4 & 12.1 & 7.7 & $*$ & $\star$ & $\star$ & 11.9 & 10.2 & 7.0 & & & & 11.5 & 9.8 & 6.8 \\
\hline M125E1 & & & & & & & * & & * & 9.5 & $\begin{array}{r}4.0 \\
\end{array}$ & 3.3 & $\star$ & $\star$ & $\star$ & $\ddot{* *}$ & $\ddot{18} 3$ & $\ddot{~}$ \\
\hline M548E1 & + & + & ${ }^{+}$ & 25.7 & 19.7 & 11.9 & * & * & * & 25.1 & 18.7 & 11.6 & * & * & * & $\begin{array}{l}24.9 \\
22.9\end{array}$ & 18.3 & 11.4 \\
\hline \multirow[t]{2}{*}{ UET } & & & & 26.4 & 20.3 & 18.4 & * & * & * & 25.9 & $\begin{array}{l}18.7 \\
19.7\end{array}$ & $\begin{array}{l}17.8 \\
17.8\end{array}$ & * & * & * & 25.8 & $\begin{array}{l}18.5 \\
19.5\end{array}$ & $\begin{array}{l}\frac{14.6}{17.6} \\
17.0\end{array}$ \\
\hline & & & & & & & & & & & & & & & & & & . \\
\hline M561 & 32.0 & 24.8 & 4.2 & 31.0 & 24.0 & 1.2 & & & & 23.7 & 20.3 & 0.6 & * & * & * & & & \\
\hline M35A2 & 31.6 & 26.0 & 15.1 & 30.8 & 25.7 & 13.7 & * & * & * & 26.6 & 22.6 & 1.1 & * & * & * & $\star \star \star$ & 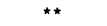 & 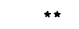 \\
\hline$M 813$ & & & & 29.8 & 25.9 & 11.4 & 27.9 & 24.0 & 10.2 & 27.2 & 23.6 & 9.6 & 26.8 & 23.2 & 9.4 & 25.9 & 22.4 & 0.1 \\
\hline M656 & & & & 29.4 & 24.6 & 12.0 & $\star$ & $\stackrel{ }{*}$ & $\star *$ & 26.4 & 22.9 & 1.3 & * & * & * & $* *$ & $* \star *$ & $* \star *$ \\
\hline TDW901 & & & & 23.2 & 20.2 & 13.4 & 20.5 & 17.8 & 11.0 & 19.8 & 17.4 & 10.3 & 19.4 & 17.1 & 9.9 & 18.6 & 16.7 & 0.1 \\
\hline M520E1 & & & & 21.5 & 19.0 & 8.4 & * & * & * & 19.6 & 17.6 & 7.2 & & & & 19.1 & 17.0 & 0.1 \\
\hline M125E1 & & & & & & & * & * & * & $\begin{array}{l}22.5 \\
\end{array}$ & 21.1 & 11.0 & 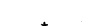 & 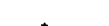 & 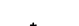 & 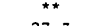 & $\stackrel{\star *}{*}$ & ** \\
\hline $\begin{array}{l}\text { M548E1 } \\
\text { JFT }\end{array}$ & $\dagger$ & t & + & 28.9 & 23.7 & 11.0 & * & * & * & $\begin{array}{l}27.7 \\
25.0\end{array}$ & $\begin{array}{l}23.5 \\
23.0\end{array}$ & 11.0 & * & * & * & 27.3 & 23.4 & 0.1 \\
\hline $\begin{array}{l}\text { UET } \\
\text { ASV }\end{array}$ & & & & 29.4 & 26.0 & 15.9 & * & * & * & $\begin{array}{l}25.0 \\
28.6\end{array}$ & $\begin{array}{l}23.0 \\
25.6\end{array}$ & $\begin{array}{l}14.3 \\
15.9\end{array}$ & * & * & * & $\begin{array}{l}28.9 \\
28.3\end{array}$ & $\begin{array}{l}25.9 \\
25.4\end{array}$ & $\begin{array}{l}0.1 \\
0.1\end{array}$ \\
\hline \multicolumn{19}{|c|}{ Mid-East - Sand Condition } \\
\hline & & & 0.3 & 30.3 & 20.2 & 0.3 & & & & 23.3 & 18.0 & 0.1 & * & * & * & & & \\
\hline M35A2 & $\begin{array}{l}1.2 \\
30.9\end{array}$ & 21.2 & 0.2 & 30.1 & 21.2 & 0.2 & $\star$ & ${ }^{*}$ & $*$ & 26.1 & 19.4 & 0.1 & 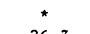 & $\star *$ & $\star$ & $\approx *$ & $\star \star *$ & $\star * *$ \\
\hline M813 & & & & 29.2 & 21.1 & 0.2 & 27.3 & 20.3 & 0.1 & 26.7 & 20.0 & 0.1 & 26.3 & 19.8 & 0.1 & 25.5 & 19.3 & 0.1 \\
\hline M656 & & & & 28.8 & 20.7 & 0.3 & $*$ & $*$ & * & 25.9 & 19.7 & 0.1 & $\stackrel{*}{*}$ & $\star$ & 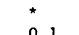 & $\star \star *$ & $\approx \star \star \star *$ & $* *$ \\
\hline TDWO01 & & & & 22.8 & 17.9 & 0.6 & 20.3 & 16.1 & 0.5 & 19.6 & 15.8 & 0.1 & 19.2 & 15.6 & 0.1 & 18.4 & 15.2 & 0.1 \\
\hline MS20E1 & & & & 21.2 & 17.0 & 0.6 & * & * & * & $\begin{array}{l}19.3 \\
22.2\end{array}$ & $\begin{array}{l}16.0 \\
18.5\end{array}$ & $\begin{array}{l}0.6 \\
0.1\end{array}$ & & & & 18.9 & 15.5 & 0.1 \\
\hline M125E1 & $\ddagger$ & $t$ & + & 28.3 & 20.3 & 9.1 & 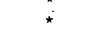 & * & * & $\begin{array}{l}22.2 \\
27.2\end{array}$ & $\begin{array}{l}18.5 \\
20.1\end{array}$ & $\begin{array}{l}0.1 \\
3.0\end{array}$ & * & * & * & 26.8 & 20.1 & 1.0 \\
\hline $\begin{array}{l}\text { M548E1 } \\
\text { UET }\end{array}$ & 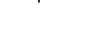 & & & 20.0 & 20.3 & 9.1 & t & * & * & 24.6 & 19.9 & 9.1 & & & & 24.5 & 19.8 & 8.5 \\
\hline ASV & & & & 28.7 & 21.3 & 10.5 & * & * & * & 28.0 & 21.1 & 8.7 & * & * & * & 27.7 & 21.0 & 8.1 \\
\hline
\end{tabular}

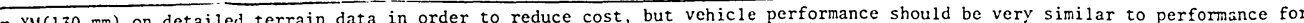

prime mover - Mid-East - Wet Condition

** Performance predictions not made for prime mover-FH70 on detailed terrain data in order to reduce cost, but perme should be very similar to performance for the M548E1-XM204.

WOTE: When no performance data or explanation given, prime mover-towed artillery combination was not evaluated. 
Table 9

$\mathrm{V}_{100}$ Speeds for Self-Propelled Artillery and Reference Vehicles Over Primary and Secondary Roads

and Trails in the HIMO Study Areas

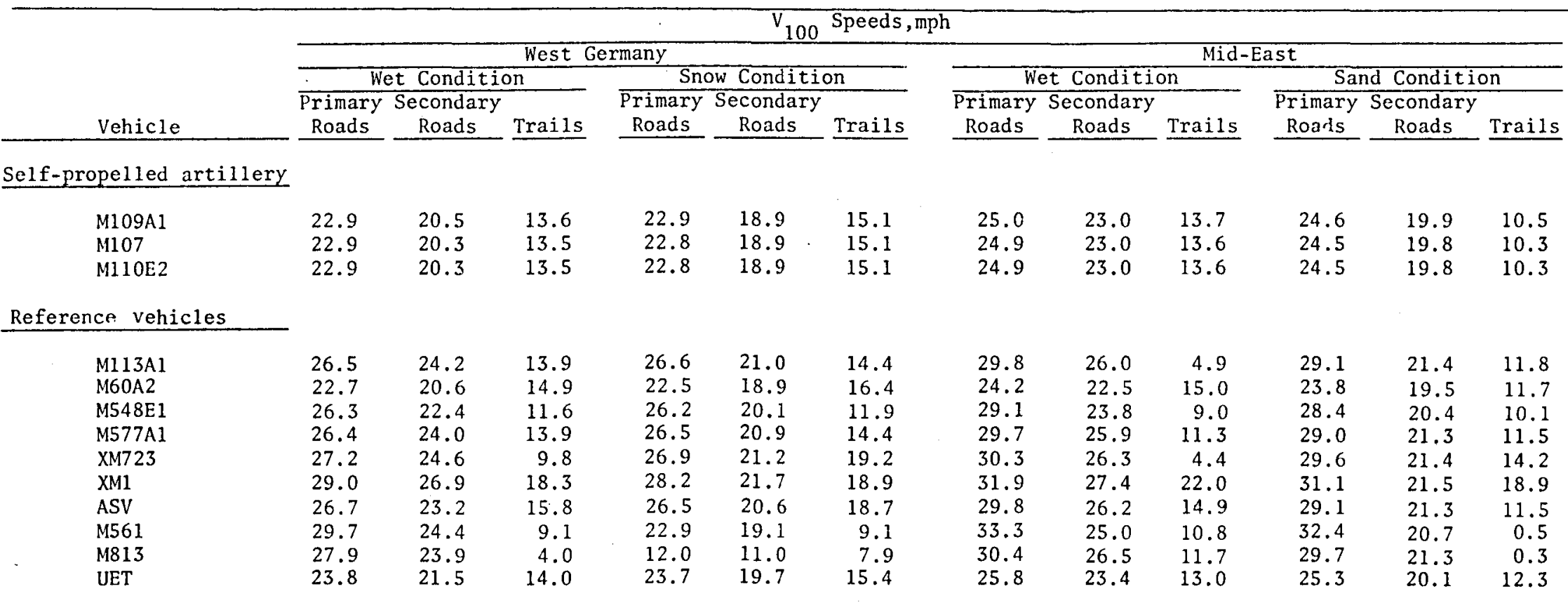


Table 10

Percent NOGO for Prime Mover-Towed Artillery Combinations on Primary and Secondary Roads and Trails in the HIMO Study Areas

\begin{tabular}{|c|c|c|c|c|c|c|c|c|c|c|c|c|}
\hline \multirow[b]{4}{*}{ Prime Nover } & \multicolumn{12}{|c|}{$\%$ NOGO * } \\
\hline & \multicolumn{4}{|c|}{ Lightweight Artillery } & \multicolumn{6}{|c|}{ Medium-Weight Artillery } & \multirow{2}{*}{\multicolumn{2}{|c|}{$\begin{array}{c}\text { Heavyweight } \\
\text { Artillery } \\
19250-1 \mathrm{~b} \\
\text { FH70 }\end{array}$}} \\
\hline & \multicolumn{2}{|c|}{$\begin{array}{l}3340-1 \mathrm{~b} \\
\mathrm{M} 102\end{array}$} & \multicolumn{2}{|c|}{$\begin{array}{c}4775-1 \mathrm{~b} \\
\mathrm{XM} 204\end{array}$} & \multicolumn{2}{|c|}{$\begin{array}{r}12700-1 \mathrm{~b} \\
\mathrm{M} 114 \mathrm{Al} \\
\end{array}$} & \multicolumn{2}{|c|}{$\begin{array}{c}15250-1 \mathrm{~b} \\
\text { XM198 }\end{array}$} & \multicolumn{2}{|c|}{$\begin{array}{l}16590-1 \mathrm{~b} \\
X M(130-\mathrm{mm})\end{array}$} & & \\
\hline & $\begin{array}{l}\text { Secondary } \\
\text { Roads } \\
\end{array}$ & Trails & $\begin{array}{l}\text { Secondary } \\
\text { Roads }\end{array}$ & Trails & $\begin{array}{l}\text { Secondary } \\
\text { Roads } \\
\end{array}$ & Trails & $\begin{array}{l}\text { Secondary } \\
\text { Roads } \\
\end{array}$ & Trails & $\begin{array}{l}\text { Secondary } \\
\text { Roads } \\
\end{array}$ & Trails & $\begin{array}{l}\text { Secondary } \\
\text { Roads }\end{array}$ & Trails \\
\hline & & & & & est Germany & - Wet C & ondition & & & & & \\
\hline M561 & 0 & 0 & 0 & 1.5 & & & 0 & 8.6 & ** & $\star \star$ & & \\
\hline M35A2 & 0 & 0 & 0 & 0 & $\star \star$ & $\star \star$ & 0 & 4.4 & ** & ** & $t$ & t \\
\hline M813 & & & 0 & 1.5 & 0 & 1.5 & 0 & 1.5 & 0 & 1.5 & 0 & 96.2 \\
\hline M656 & & & 0 & 0 & ** & $\star \star$ & 0 & 1.5 & $\star \star$ & $\star \star$ & + & $t$ \\
\hline TDW901 & & & 0 & 0 & 0 & 0 & 0 & 0 & 0 & 1.5 . & 0 & 96.2 \\
\hline M520E 1 & & & 0 & 1.5 & ** & ** & 0 & 1.5 & ** & ** & 0 & 96.2 \\
\hline M125E 1 & & & & & ** & $\star \star$ & 0 & 1.5 & & & $t$ & \\
\hline $\mathrm{M} 548 \mathrm{E} 1$ & t+ & $t+$ & 0 & 0 & $\star \star$ & ** & 0 & 0 & ** & ** & 0 & 96.2 \\
\hline UET & & & & & $\star \star$ & $\star \star$ & 0 & 0 & & & 0 & 96.2 \\
\hline \multirow[t]{2}{*}{ ASV } & & & 0 & 0 & ** & ** & 0 & 0 & ** & ** & 0 & 96.2 \\
\hline & \multicolumn{10}{|c|}{ West Germany - Snow Condition } & & \\
\hline M561 & 0 & 0 & 0.4 & 0.5 & & & 32.1 & 44.5 & $\star \star *$ & ** & & \\
\hline$M 55 A 2$ & 1.3 & 1.5 & 2.9 & 4.7 & ** & $\star \star$ & 39.4 & 54.8 & $\star \star$ & ** & + & + \\
\hline M813 & & & 0.4 & 0.5 & 5.2 & 6.1 & 6.1 & 6.5 & 6.5 & 7.0 & 18.1 & 25.9 \\
\hline M656 & & & 0 & 0 & $\star \star$ & $\star \star$ & 1.3 & 1.5 & $\star \star$ & $\star \star$ & + & $t$ \\
\hline TDW901 & & & 0 & 0 & 0 & 0 & 0 & 0 & 0 & 0 & 0 & 0 \\
\hline M520E 1 & & & 0 & 0 & $\star *$ & ** & 0 & 0 & $\star \star$ & ** & 0 & 0 \\
\hline M125E1 & & & & & $\star \star$ & ** & 1.3 & 1.5 & & & $t$ & + \\
\hline $\mathrm{M} 548 \mathrm{E} 1$ & $+t$ & $t+$ & 0 & 0 & ** & ** & 0 & 0 & ** & ** & 0 & 0 \\
\hline UET & & & & & $\star *$ & $\star \star$ & 0 & 0 & & & 0 & 0 \\
\hline \multirow[t]{2}{*}{ ASV } & & & 0 & 0 & ** & ** & 0 & 0 & $\star \star$ & ** & 0 & 0 \\
\hline & \multicolumn{10}{|c|}{ Mid-East - Wet Condition } & & \\
\hline M561 & 0 & 1.4 & 0 & 7.2 & & & 0 & 14.7 & $\star \star$ & $\star *$ & & \\
\hline M35A2 & 0 & 0 & 0 & 0.1 & $\star \star$ & $\star \star$ & 0 & 8.2 & $\star \star$ & $\star \star *$ & + & + \\
\hline$M 813$ & & & 0 & 0 & 0 & 0.1 & 0 & 0.1 & 0 & 0.1 & 0 & 98.7 \\
\hline M656 & & & 0 & 0.1 & ** & $\star \star$ & 0 & 7.2 & ** & $\star \star \star$ & + & + \\
\hline TDW901 & & & 0 & 0 & 0 & 0.1 & 0 & 0.1 & 0 & 0.1 & 0 & 98.7 \\
\hline M520E1 & & & 0 & 0 & ** & ** & 0 & 0.1 & ** & $\star \star$ & 0 & 98.7. \\
\hline M125El & & & & & $\star \star$ & ** & 0 & 0 & & & + & + \\
\hline $\mathrm{M} 548 \mathrm{E} 1$ & $+t$ & $t+$ & 0 & 0.1 & $\star \star *$ & 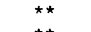 & 0 & 0.1 & ** & ** & 0 & 98.7 \\
\hline UET & & & & & $\star \star$ & $\star \star \star$ & 0 & 0.1 & & & 0 & 98.7 \\
\hline \multirow[t]{2}{*}{ ASV } & & & 0 & 0.1 & ** & ** & 0 & 0.1 & ** & ** & 0 & 98.7 \\
\hline & \multicolumn{10}{|c|}{ Mid-East - Sand Condition } & & \\
\hline M561 & 0 & 29.5 & 0 & 29.5 & & & 0 & 100.0 & $\star \star$ & $\star \star \star$ & & \\
\hline M35A2 & 0 & 49.3 & 0 & 49.3 & $\star \star$ & ** & 0 & 99.6 & **. & ** & $t$ & + \\
\hline M813 & & & 0 & 49.3 & 0 & 99.6 & 0 & 99.6 & 0 . & 99.6 & 0 & 100.0 \\
\hline M656 & & & 0 & 29.5 & $\star \star$ & $\star \star$ & 0 & 99.6 & ** & $\star \star *$ & $t$ & + \\
\hline TDW901 & & & 0 & 15.5 & 0 & 20.3 & 0 & 99.6 & 0 & 99.6 & 0 & 99.6 \\
\hline M520E I & & & 0 & 14.7 & $\star \star$ & $\star \star$ & 0 & 15.5 & $\star \star$ & ** & 0 & 100.0 \\
\hline M125EI & & & & & $\star \star *$ & ** & 0 & 99.6 & & & $t$ & $t$ \\
\hline M548E 1 & $t+$ & $t+$ & 0 & 0 & ** & $\star \star$ & 0 & 1.8 & $\star \star \star$ & $\star \star \star$ & 0 & 8.2 \\
\hline UET & & & & & ** & $\star \star \star$ & 0 & 0 & & & 0 & 0 \\
\hline ASV & & & 0 & 0 & ** & ** & 0 & 0 & $\star \star \star$ & $\star \star *$ & 0 & 0 \\
\hline
\end{tabular}

* The M561-XM198 had 19.1 percent NOGO and the M35A2-XM198 had 22.3 percent NOGO on primary roads in West Germany with snow condition. No other prime mover-towed artillery combination had any NOGO on primary roads.

** Performance predictions not made for indicated prime mover-Ml14Al and prime mover-XM9130-mn) on detailed terrain data in order to to ace cost, but vehicle performance should be very similar to performance for prime mover-XM198.

+ Performance predictions not made for prime mover-FH70 on detailed terrain data in order to reduce cost, but performance should be very similar to performance of M813-FH70 and TDW901-FH70.

t+ Performance predictions not made for M548E1-M102 on detailed terrain data in order to reduce cost, but performance should be very similar to performance for the M548E1-XM204.

NOTE: When no performance data or explanation given, prime mover-towed artillery combination was not evaluated. 
Percent NOGO on Primary and Secondary Roads* and Trails for SelfPropelled and Reference Vehicles in the HIMO Study Areas

\begin{tabular}{lcc}
\hline & \multicolumn{2}{c}{ Trails** } \\
\cline { 2 - 3 } Vehicles & $\begin{array}{c}\text { West Germany } \\
\text { Wet Condition }\end{array}$ & $\begin{array}{c}\text { Mid-East } \\
\text { Wet Condition }\end{array}$ \\
\cline { 2 - 3 } Self-propelled artillery & & \\
M109A1 & 0.0 & 0.1 \\
M107 & 0.0 & 0.1 \\
M110E2 & 0.0 & 0.1 \\
Reference vehicles & & \\
M113A1 & 0.0 & 1.4 \\
M60A2 & 0.0 & 0.1 \\
M548E1 & 0.0 & 0.1 \\
M577Al & 0.0 & 0.1 \\
XM723 & 0.5 & 1.8 \\
XM1 & 0.0 & 0.0 \\
ASV & 0.0 & 0.1 \\
M561 & 0.0 & 0.0 \\
M813 & 1.5 & 0.0 \\
UET & 0.0 & 0.1
\end{tabular}

* All self-propelled and reference vehicles were GO on 100 percent of primary and secondary roads in West Germany for both wet and snow conditions and in the Mid-East for both wet and sand conditions.

** All self-propelled and reference vehicles were $G 0$ on trails in West Germany snow condition, and all were GO on Mid-East sand condition except the M813 with 32.5 percent NOGO and M561 with 20.3 percent NOGO. 
Table 12

Percent NOGO for Prime Mover-Towed Artillery Combinations in Off-Road Terrain in the HIMO Study Areas

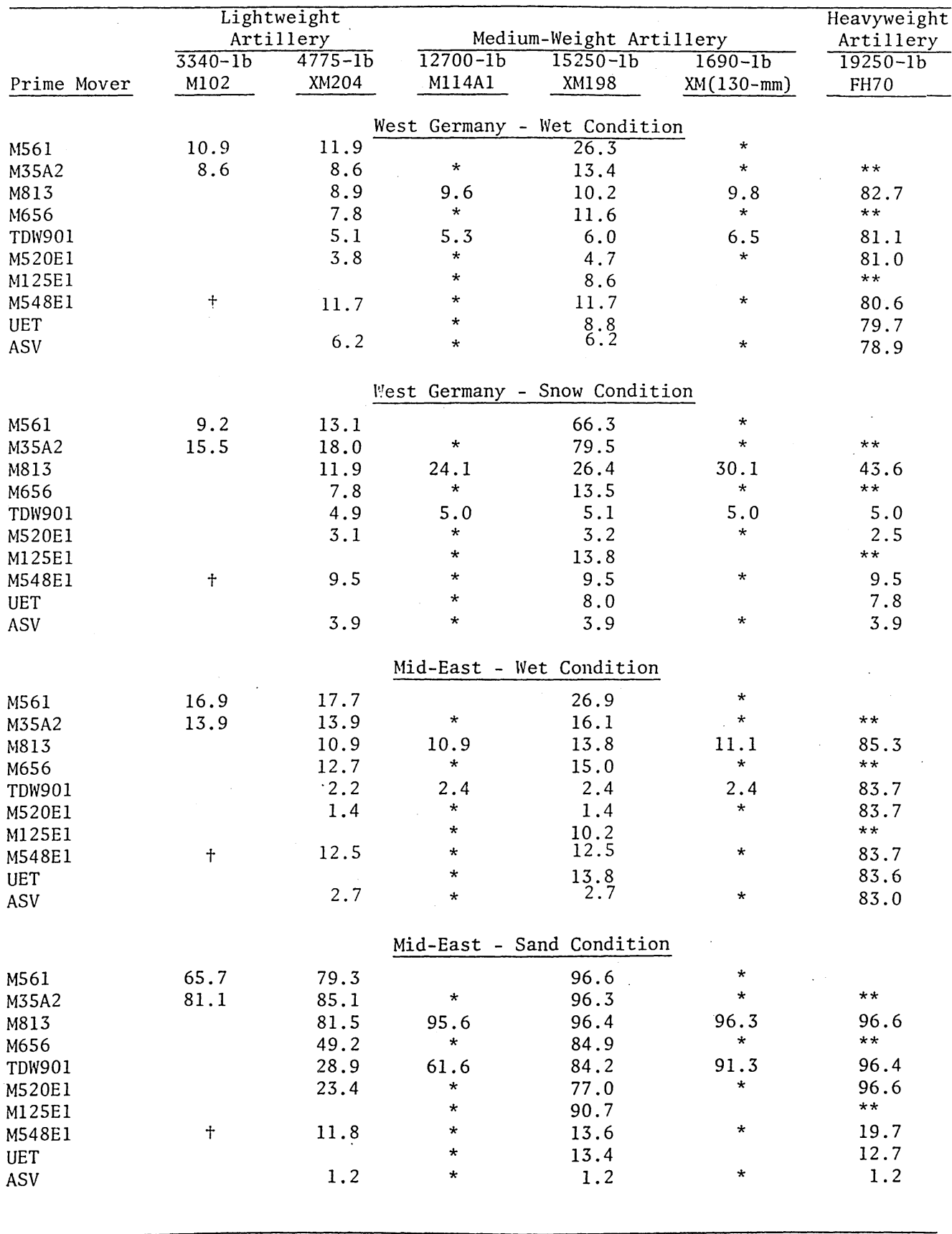

* Performance predictions not made for indicated prime mover-M114Al and prime, mover-XM(130-mm) on detailed terrain data in order to reduce cost, but vehicle: performance should be very similar to performance for prime mover-XM198.

** Performance predictions not made for prime mover-FH70 on detailed terrain data in order to reduce cost but performance should be very similar to performance of M813-FH70 and TDW901-FH70.

+ Performance predictions not made for M548E1-M102 on detailed terrain data in order to reduce cost, but performance should be very similar to performance for the M548E1-XM204.

NOTE: When no performance data or explanation given, prime mover-towed artillery combination were not evaluated 
Table 13

Percent NOGO for Self-Propelled Artillery and Reference Vehicles in Off-Road Terrain in the HIMO Study Areas

\begin{tabular}{|c|c|c|c|c|}
\hline \multirow[b]{2}{*}{ Vehicle } & \multicolumn{2}{|c|}{ West Germany } & \multicolumn{2}{|c|}{ Mid-East } \\
\hline & $\begin{array}{c}\text { Wet } \\
\text { Condition }\end{array}$ & $\begin{array}{c}\text { Snow } \\
\text { Condition }\end{array}$ & $\begin{array}{c}\text { Wet } \\
\text { Condition }\end{array}$ & $\begin{array}{c}\text { Sand } \\
\text { Conditio }\end{array}$ \\
\hline \multicolumn{5}{|c|}{ Self-propelled artillery } \\
\hline M109A1 & 2.6 & 1.6 & 0.8 & 0.0 \\
\hline M107 & 4.6 & 3.2 & 6.6 & 6.3 \\
\hline M1 10E2 & 2.0 & 1.6 & 0.8 & 0.5 \\
\hline \multicolumn{5}{|c|}{ Reference vehicles } \\
\hline$M 113 A 1$ & 8.7 & 6.6 & 13.9 & 9.1 \\
\hline M60A2 & 0.5 & 2.9 & 9.3 & 0.5 \\
\hline M548E 1 & 11.7 & 9.5 & 12.5 & 11.8 \\
\hline M577A1 & 8.8 & 6.6 & 14.6 & 10.3 \\
\hline XM723 & 5.4 & 2.4 & 7.9 & 1.2 \\
\hline$X M 1$ & 3.1 & 2.3 & 4.0 & 4.0 \\
\hline ASV & 6.2 & 3.9 & 2.7 & 1.2 \\
\hline M561 & 9.2 & 9.2 & 16.3 & 42.6 \\
\hline M813 & 8.0 & 7.8 & 10.0 & 62.9 \\
\hline UET & 6.5 & 5.0 & 9.7 & 9.7 \\
\hline
\end{tabular}


Table 14

Average Link Speeds (mph) for the Prime Mover-Towed Artillery Combinations in the HIMO Study Areas

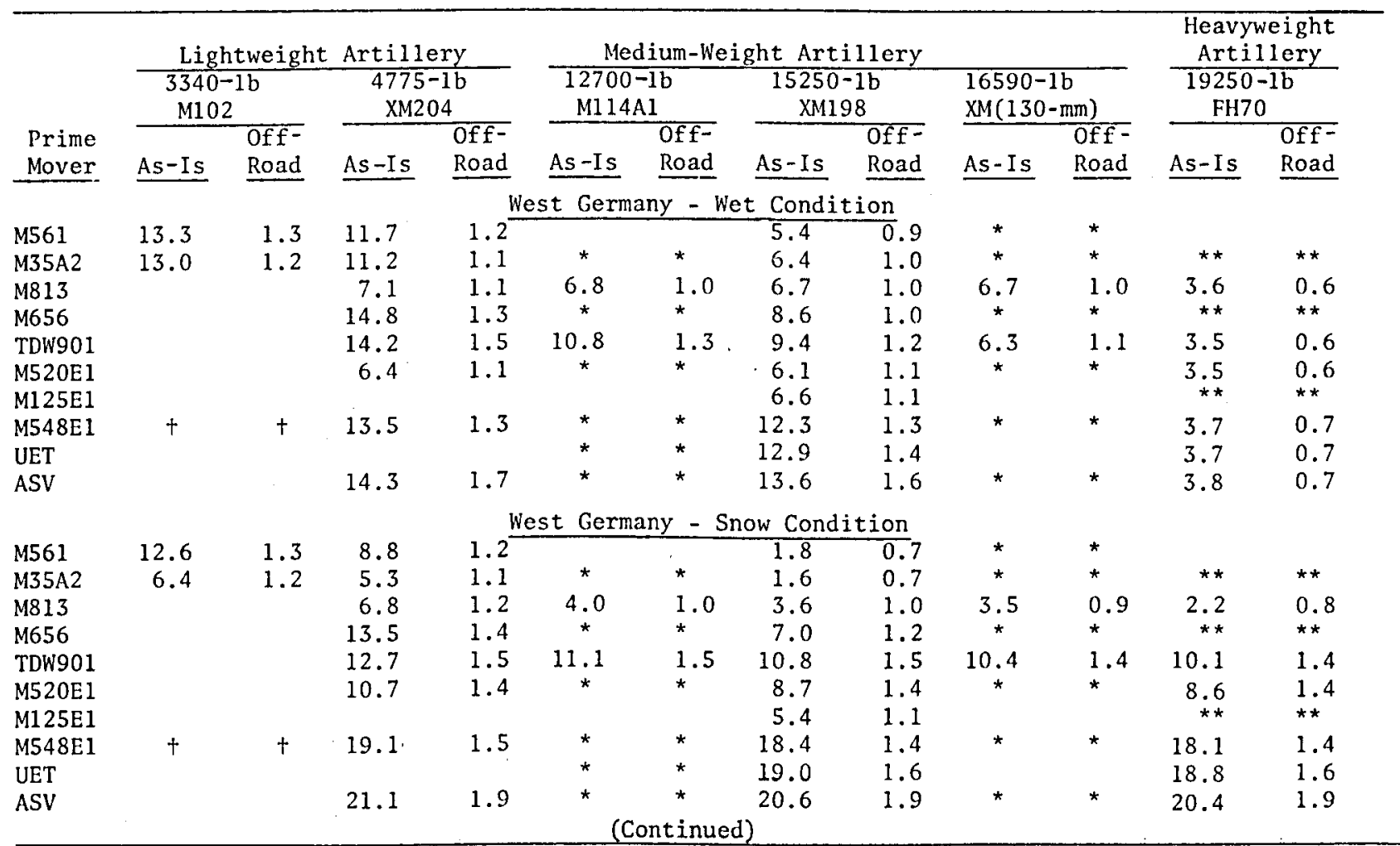

* Performance predictions not made for indicated prime mover-M114A1 and prime mover-XM(130-min) on detailed terrain data in order to reduce cost, but vehicle nerformance should be very similar to performance for prime mover-XM198.

* Performance predictions not made for prime mover-FH70 on detailed terrain data in order to reduce cost, but performance should be very similar to performance of M813-FH70 and TDW901-FH70. + Performance predictions not made for M548E1-M102 on detailed terrain data in order to reduce cost, but performance should be very similar to performance for the M548E1-XM204.

NOTE: When no performance data or explanation given, prime mover-towed artillery combination was not evaluated. 
Table 14 (Concluded)

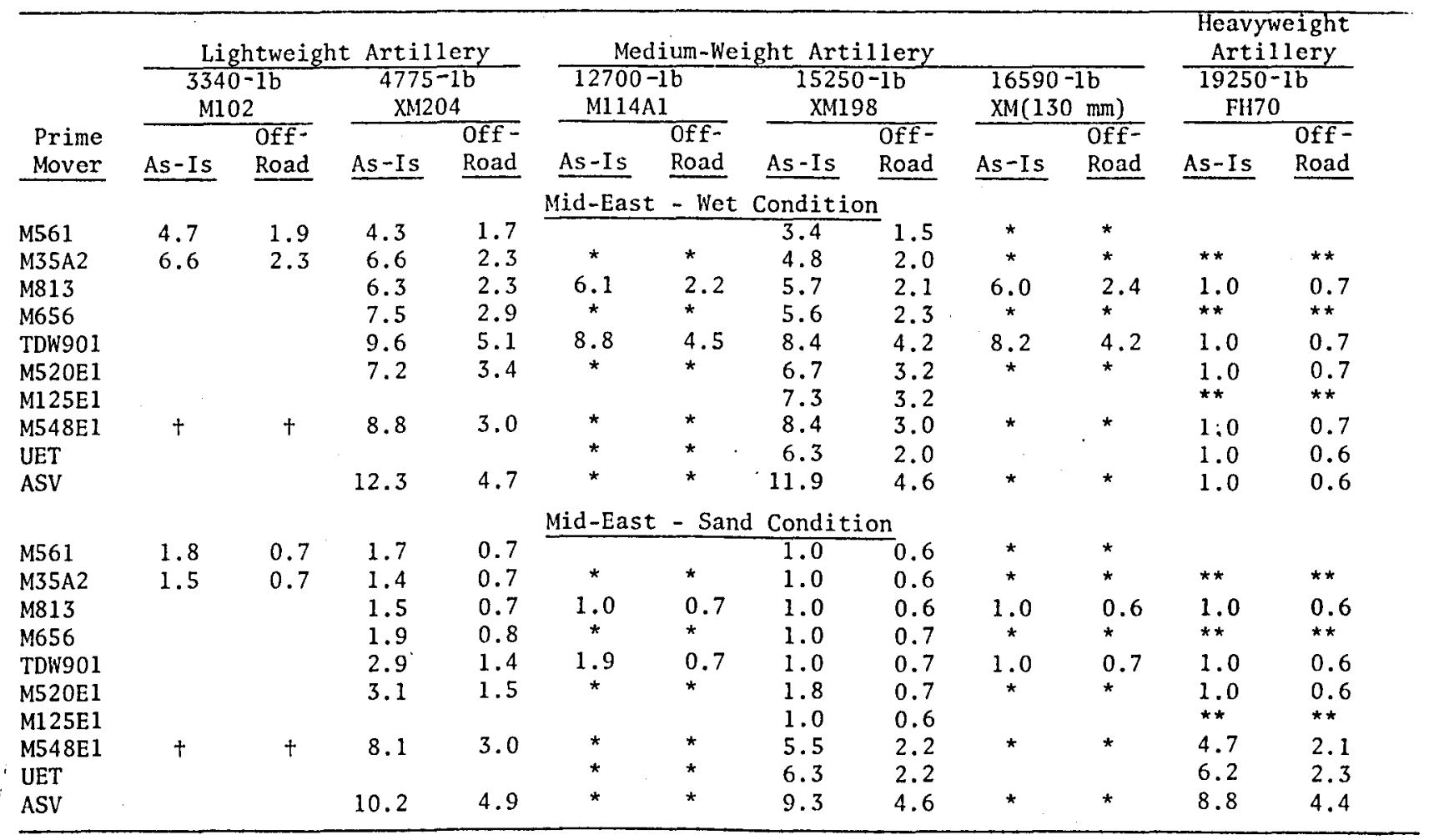

* Performance predictions not made for indicated prime mover-M114A1 and prime mover-XM(130-mm) on detailed terrain data in order to reduce cost, but vehicle performance should be very. similar to performance for prime mover-XM198.

** Performance predictions not made for prime mover-FH70 on detailed terrain data in order to reduce cost, but performance should be very similar to performance of M813-FH70 and TDW901-FH70.

+ Performance predictions not made for M548E1-M102 on detailed terrain data in order to reduce cost, but performance should be very similar to performance for the M548E1-XM204. 
Table 15

Average Link Speeds (mph) for the Self-Propelled and Reference Vehicles in the HIMO Study Areas

\begin{tabular}{|c|c|c|c|c|c|c|c|c|}
\hline & & West G & rmany & & & Mid & ast & \\
\hline Vehicles & $\frac{\text { Wet }}{\text { As-Is }}$ & $\frac{\text { Condition }}{\text { Off-Road }}$ & Snow & $\begin{array}{r}\text { Condition } \\
\text { Off-Road }\end{array}$ & $\begin{array}{r}\text { Wet } \\
\text { As-Is }\end{array}$ & $\begin{array}{c}\text { Condition } \\
\text { Off-Road }\end{array}$ & Sand & $\begin{array}{c}\text { Condition } \\
\text { Off-Road }\end{array}$ \\
\hline Self-propelled & lery & & & & & & & \\
\hline M109A1 & 13.3 & 1.8 & 19.1 & 2.1 & 13.5 & 6.7 & 12.4 & 7.9 \\
\hline M107 & 13.3 & 1.7 & 19.0 & 1.9 & 11.7 & 5.2 & 10.2 & 5.1 \\
\hline M110E2 & 13.3 & 3.7 & 19.0 & 2.1 & 13.1 & 6.3 & 11.6 & 7.1 \\
\hline Reference veh & & & & & & & & \\
\hline$M 113 A 1$ & 14.5 & 1.5 & 20.7 & 1.7 & 6.4 & 2.2 & 9.5 & 3.8 \\
\hline M60A2 & 13.6 & 2.1 & 19.2 & 2.8 & 9.9 & 3.5 & 11.0 & 5.6 \\
\hline M548E1 & 13.9 & 1.8 & 19.3 & 2.0 & 8.2 & 2.9 & 8.5 & 3.3 \\
\hline M577Al & 14.6 & 1.5 & 20.7 & 1.7 & 6.2 & 2.1 & 9.0 & 3.3 \\
\hline XM723 & 14.6 & 1.7 & 21.8 & 2.1 & 9.1 & 4.3 & 13.9 & 7.8 \\
\hline XM1 & 16.0 & 1.7 & 22.2 & 2.0 & 12.8 & 4.5 & 12.7 & 5.5 \\
\hline ASV & 14.5 & 1.6 & 21.3 & 1.9 & 10.7 & 5.1 & 12.5 & 7.0 \\
\hline M561 & 18.5 & 1.8 & 16.3 & 1.8 & 5.5 & 1.9 & 2.4 & 0.9 \\
\hline M813 & 7.5 & 1.4 & 9.7 & 1.7 & 8.1 & 2.9 & 1.8 & 0.7 \\
\hline UET & 13.9 & 1.6 & 19.6 & 1.8 & 8.5 & 3.2 & 8.4 & 3.3 \\
\hline & & & & & & & & \\
\hline & & & & & & & & \\
\hline & & & & & & & & \\
\hline & & & & . & & & & \\
\hline & & & & & & & & \\
\hline
\end{tabular}


Table 16

Percentage of the Total Time for Traversing Links That Prime Mover-

Towed Artillery Combinations Spent Crossing Linear Features in the HIMO Study Areas

\begin{tabular}{|c|c|c|c|c|c|c|}
\hline \multirow[b]{4}{*}{ Prime Mover } & \multicolumn{6}{|c|}{ Time, min } \\
\hline & \multicolumn{2}{|c|}{$\begin{array}{l}\text { Lightweight } \\
\text { Artillery }\end{array}$} & \multicolumn{3}{|c|}{ Medium-Weight Artillery } & \multirow{3}{*}{$\begin{array}{l}\text { Heavyweight } \\
\text { Artillery } \\
19250-1 \mathrm{~b} \\
\text { FH70 } \\
\end{array}$} \\
\hline & $\frac{1}{3340-1 b}$ & $4775-1 b$ & $12700-1 b$ & $15250-1 b$ & $16590-1 b$ & \\
\hline & $\mathrm{M} 102$ & $\mathrm{XM} 204$ & $\mathrm{M} 114 \mathrm{Al}$ & $\mathrm{XM198}$ & $\mathrm{XM}(130 \mathrm{~mm})$ & \\
\hline & \multicolumn{5}{|c|}{ West Germany - Wet Condition } & \\
\hline M561 & 54.8 & 52.6 & & 39.0 & * & \\
\hline M35A2 & 51.6 & 50.3 & * & 44.1 & * & ** \\
\hline M813 & & 48.1 & 46.5 & 45.7 & 45.8 & 28.2 \\
\hline M656 & & 59.6 & * & 46.8 & * & ** \\
\hline TDW901 & & 65.4 & 57.3 & 52.3 & 49.4 & 28.2 \\
\hline M520E1 & & 49.1 & * & 47.5 & * & 28.1 \\
\hline $\mathrm{M} 125 \mathrm{E} 1$ & & & * & 47.7 & & $* *$ \\
\hline M548E1 & + & 45.2 & * & 44.5 & * & 23.7 \\
\hline UET & & & * & 50.6 & & 23.8 \\
\hline \multirow[t]{2}{*}{ ASV } & & 58.4 & * & 57.4 & * & 24.1 \\
\hline & \multicolumn{5}{|c|}{ West Germany - Snow Condition } & \\
\hline M561 & 57.9 & 53.5 & & 31.4 & * & \\
\hline M35A2 & 51.9 & 49.3 & * & 29.5 & * & ** \\
\hline M813 & & 53.4 & 45.4 & 42.9 & 39.6 & 34.2 \\
\hline M656 & & 61.2 & * & 52.8 & * & $* *$ \\
\hline TDW901 & & 67.7 & 66.1 & 65.2 & 64.8 & 64.4 \\
\hline M520E1 & & 63.3 & * & 61.1 & * & 60.6 \\
\hline M125E1 & & & * & 51.1 & & 50.1 \\
\hline M548E1 & + & 49.6 & * & 50.1 & * & 56.8 \\
\hline UET & & & * & 57.0 & & 56.0 \\
\hline \multirow[t]{2}{*}{ ASV } & & 67.6 & * & 67.4 & * & 67.3 \\
\hline & \multicolumn{5}{|c|}{ Mid-East - Wet Condition } & \\
\hline M561 & 3.0 & 2.7 & & 2.4 & * & \\
\hline M35A2 & 3.4 & 3.5 & * & 3.2 & * & $* *$ \\
\hline M813 & & 1.6 & 1.6 & 1.5 & 1.7 & 0.5 \\
\hline M656 & & 2.1 & * & 1.7 & * & $\star \star$ \\
\hline TDW901 & & 7.3 & 7.4 & 6.8 & 6.8 & 1.1 \\
\hline M520E1 & & 2.5 & * & 2.3 & * & 0.5 \\
\hline M125E1 & & & * & 2.3 & & $\star \star$ \\
\hline M548E1 & $t$ & 2.4 & * & 2.6 & * & 0.6 \\
\hline UET & & & * & 12.5 & & 3.9 \\
\hline \multirow[t]{2}{*}{ ASV } & & 28.4 & * & 28.2 & * & 3.9 \\
\hline & \multicolumn{5}{|c|}{ Mid-East - Sand Condition } & \\
\hline M561 & 0.5 & 0.5 & & 0.5 & * & \\
\hline M35A2 & 0.5 & 0.5 & * & 0.5 & * & $* *$ \\
\hline M813 & & 0.5 & 0.5 & 0.5 & 0.5 & 0.5 \\
\hline M656 & & 0.6 & * & 0.5 & * & $* *$ \\
\hline TDW901 & & 1.0 & 0.5 & 0.5 & 0.5 & 0.5 \\
\hline M520E1 & & 1.1 & 0.5 & 0.5 & * & 0.5 \\
\hline $\mathrm{M} 125 \mathrm{E} 1$ & & & $*$ & 0.5 & & $\star *$ \\
\hline M548E1 & 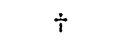 & 0.5 & * & 0.4 & * & 0.3 \\
\hline UET & & & * & 11.7 & & 12.0 \\
\hline ASV & & 25.9 & * & 23.9 & * & 22.9 \\
\hline
\end{tabular}

* Performance predictions not made for indicated prime mover-MI14Al and prime mover-XM9130-mm) on detailed terrain data in order to reduce cost, but vehicle performance should be very similar to performance for prime mover-XM198.

* * Performance predictions not made for prime mover-FH70 on detailed terrain data in order to reduce cost, but performance should be very similar to performance of M813-FH70 and TDW901-FH70.

+ Performance predictions not made for M548E1-M102 on detailed terrain data in order to reduce cost, but performance should be very similar to performance for the M548E1-XM204.

NOTE: When no performance data or explanation given, prime mover-towed artillery combination was not evaluated. 
Table 17

Percentage of the Total Time for Traversing Links That Spent Crossing

Linear Features Self-Propelled Artillery and Reference Vehicles

in the HIMO Study Areas

\begin{tabular}{|c|c|c|c|c|}
\hline \multirow[b]{3}{*}{ Vehicles } & \multicolumn{4}{|c|}{ Time, min. } \\
\hline & \multicolumn{2}{|c|}{ West Germany } & \multicolumn{2}{|c|}{ Mid-East } \\
\hline & $\begin{array}{c}\text { Wet } \\
\text { Condition }\end{array}$ & $\begin{array}{c}\text { Snow } \\
\text { Condition }\end{array}$ & $\begin{array}{c}\text { Wet } \\
\text { Condition }\end{array}$ & $\begin{array}{c}\text { Sand } \\
\text { Condition }\end{array}$ \\
\hline \multicolumn{5}{|c|}{ Self-propelled artillery } \\
\hline M109A1 & 62.7 & 73.8 & 4.8 & 1.7 \\
\hline M107 & 58.6 & 67.8 & 0.9 & 0.7 \\
\hline M110E2 & 62.0 & 73.1 & 4.3 & 1.0 \\
\hline \multicolumn{5}{|c|}{ Reference vehicles } \\
\hline M113A1 & 50.2 & 58.6 & 6.1 & 5.2 \\
\hline M60A2 & 39.6 & 51.9 & 2.8 & 0.8 \\
\hline M548E1 & 26.9 & 31.0 & 2.6 & 0.6 \\
\hline M577A1 & 50.3 & 58.6 & 5.5 & 1.6 \\
\hline XM723 & 57.1 & 71.4 & 2.5 & 1.1 \\
\hline XM1 & 66.2 & 76.7 & 3.1 & 2.1 \\
\hline ASV & 56.6 & 68.1 & 3.6 & 1.5 \\
\hline M561 & 45.2 & 45.5 & 3.2 & 0.7 \\
\hline$M 813$ & 38.7 & 47.4 & 2.1 & 0.5 \\
\hline UET & 53.2 & 62.7 & 19.3 & 16.6 \\
\hline
\end{tabular}


Table 18

Preliminary Quantification of WHEELS Study Definitions of Tactical Mobility ${ }^{1}$

\begin{tabular}{|c|c|c|c|c|}
\hline Mobility Level & \multicolumn{2}{|c|}{$\begin{array}{l}\text { Operating } \\
\text { Distance }\end{array}$} & $\begin{array}{l}\frac{\text { Severity of }}{\text { Off-Road* }} \\
\frac{\text { Terrain }}{\text { Challenged }} \\
\% \\
\end{array}$ & $\begin{array}{c}\text { Operation } \\
\text { On-Road } \\
\text { Trails } \\
\text { Included } \\
\% \\
\end{array}$ \\
\hline \multicolumn{5}{|l|}{ High-high mobility** } \\
\hline All off-road operation & 100 & 0 & 100 & - \\
\hline \multicolumn{5}{|l|}{ Tactical high mobility } \\
\hline $\begin{array}{l}\text { The highest level of mobility designating the } \\
\text { requirement for extensive cross-country } \\
\text { maneuverability characteristic of operations in } \\
\text { the ground-gaining and fire-support environment. }\end{array}$ & 50 & 50 & 90 & 100 \\
\hline \multicolumn{5}{|l|}{ Tactical standard mobility } \\
\hline $\begin{array}{l}\text { The second highest level of mobility designating } \\
\text { the requirement for occasional cross-country } \\
\text { movement. }\end{array}$ & 15 & 85 & 80 & 100 \\
\hline \multicolumn{5}{|l|}{ Tactical support mobility } \\
\hline $\begin{array}{l}\text { A level of mobility designating the requirement } \\
\text { for infrequent off-road operations over selected } \\
\text { terrain with the preponderance of movement on } \\
\text { primary and secondary roads. }\end{array}$ & 5 & 95 & 50 & 50 \\
\hline \multicolumn{5}{|l|}{ On-road mobility** } \\
\hline $\begin{array}{l}\text { All on superhighways, primary and secondary roads, } \\
\text { and the best tertiary roads and trails. }\end{array}$ & 0 & 100 & - & 10 \\
\hline
\end{tabular}

* In terms of percentage of best off-road terrain to be challenged (off-road speed profile). $* *$ Not a WHEELS study definition. 
Table 19

High and High-High Levels of Tactical Mobility Performance of Prime Mover-Towed Artillery Combinations in the HIMO Study Areas

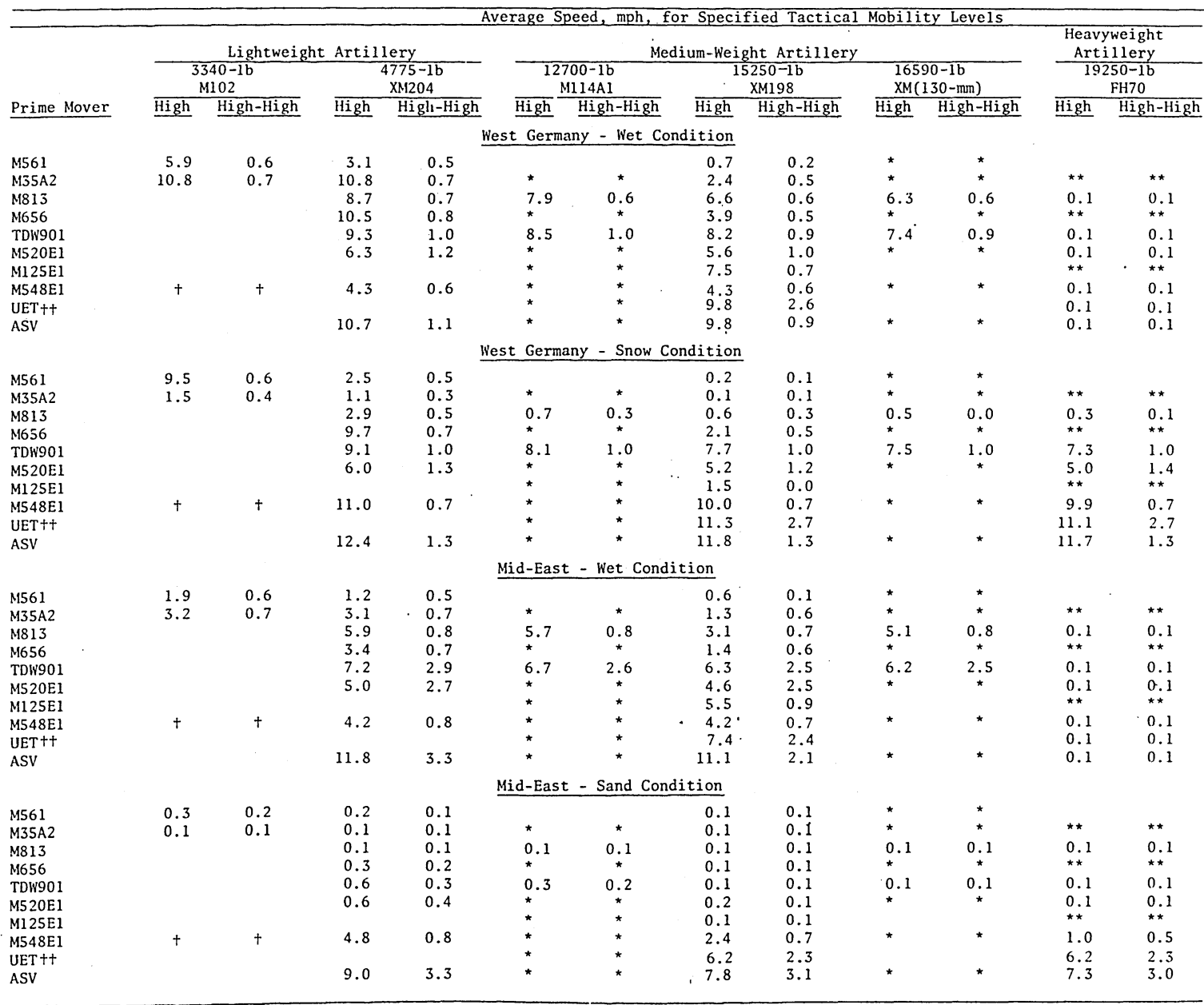

* Performance predictions not made for indicated prime mover-M114Al and prime mover-XM(130-mm) on detailed terrain data in order to reduce cost, but vehicle performance should be very similar to performance for prime mover-XM198.

** Performat be very similar to performance of $\mathrm{M} 813$-FH70.

( very similar to performance for the M548E1-XM204.

t+ High and high-high rating speeds were computed using $0.5 \mathrm{mph}$ for obstacle NOGO to allow for advantages of the UET's earthmoving capabilities.

NOTE: WHEELS study definitions in Table 18. 
Table 20

High and High-High Levels of Tactical Mobility Performance of Self-Propelled Artillery and Reference Vehicles in the HIMO Study Areas

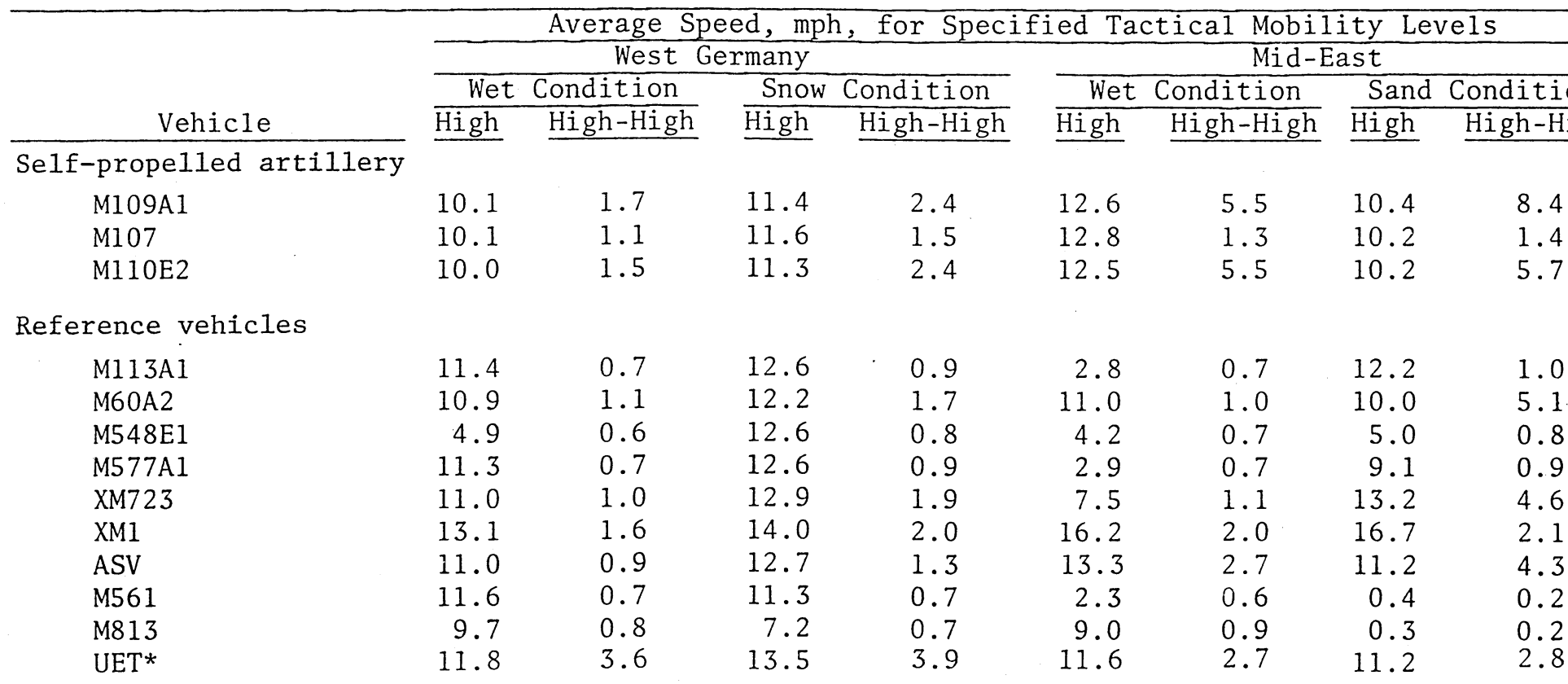

* High and high-high rating speeds were computed using $0.5 \mathrm{mph}$ for obstacle NOGO to allow for advantages of the UET's earthmoving capabilities.

NOTE: WHEELS study definitions quantified per Table 18. 
Table 21

Comparison of Performances of Individual Vehicles and Selected Vehicle Groups on Primary Roads, Secondary Roads, Trails and off-Road in the HIMO Study Areas

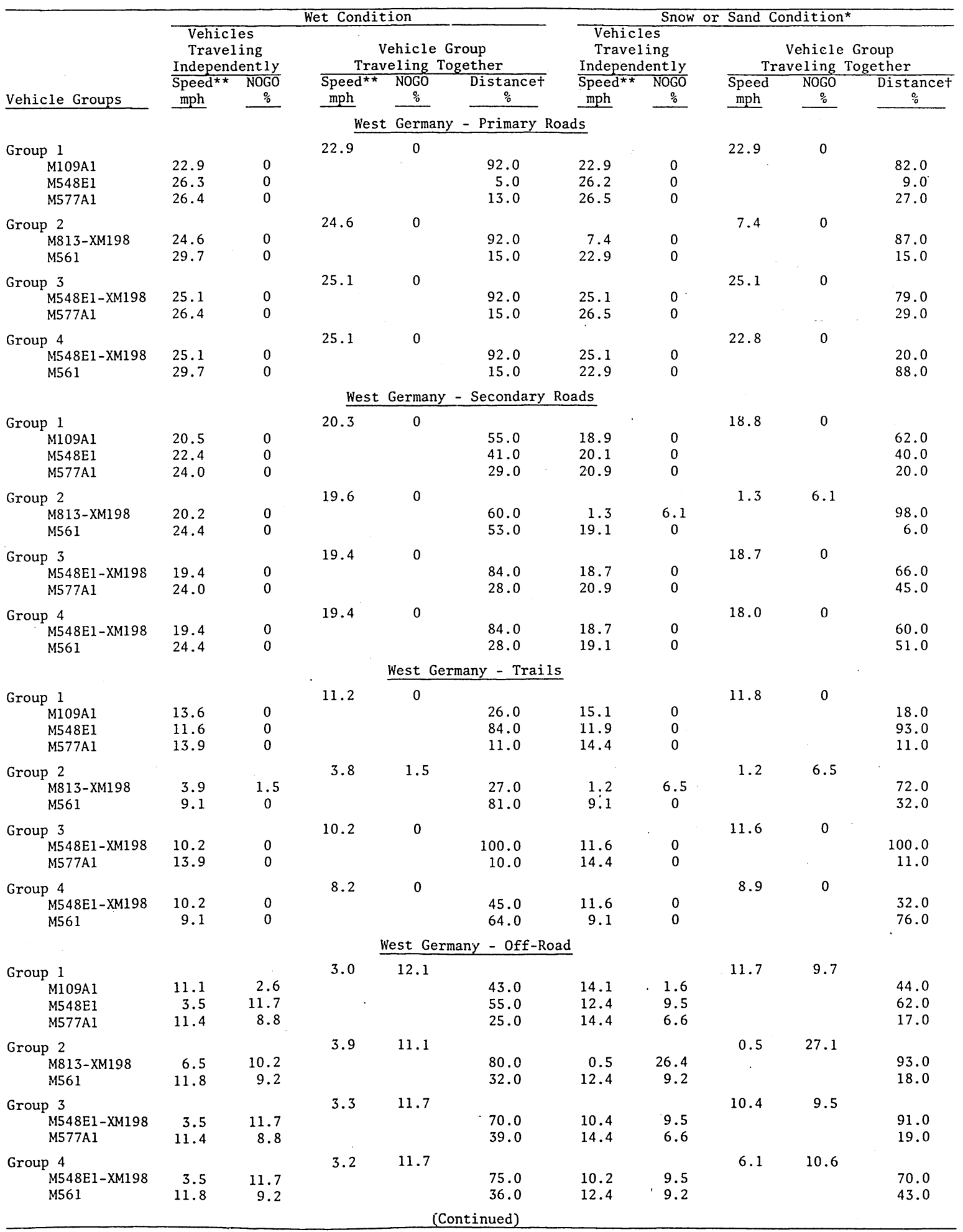

* West Germany - snow; Mid-East - sand.

** Speed of vehicle on primary and secondary roads and trails is $V_{100}$, and off-road is $V_{90}$.

+ Percentage of total traverse limited by indicated vehicle. 
Table 21 (Concluded)

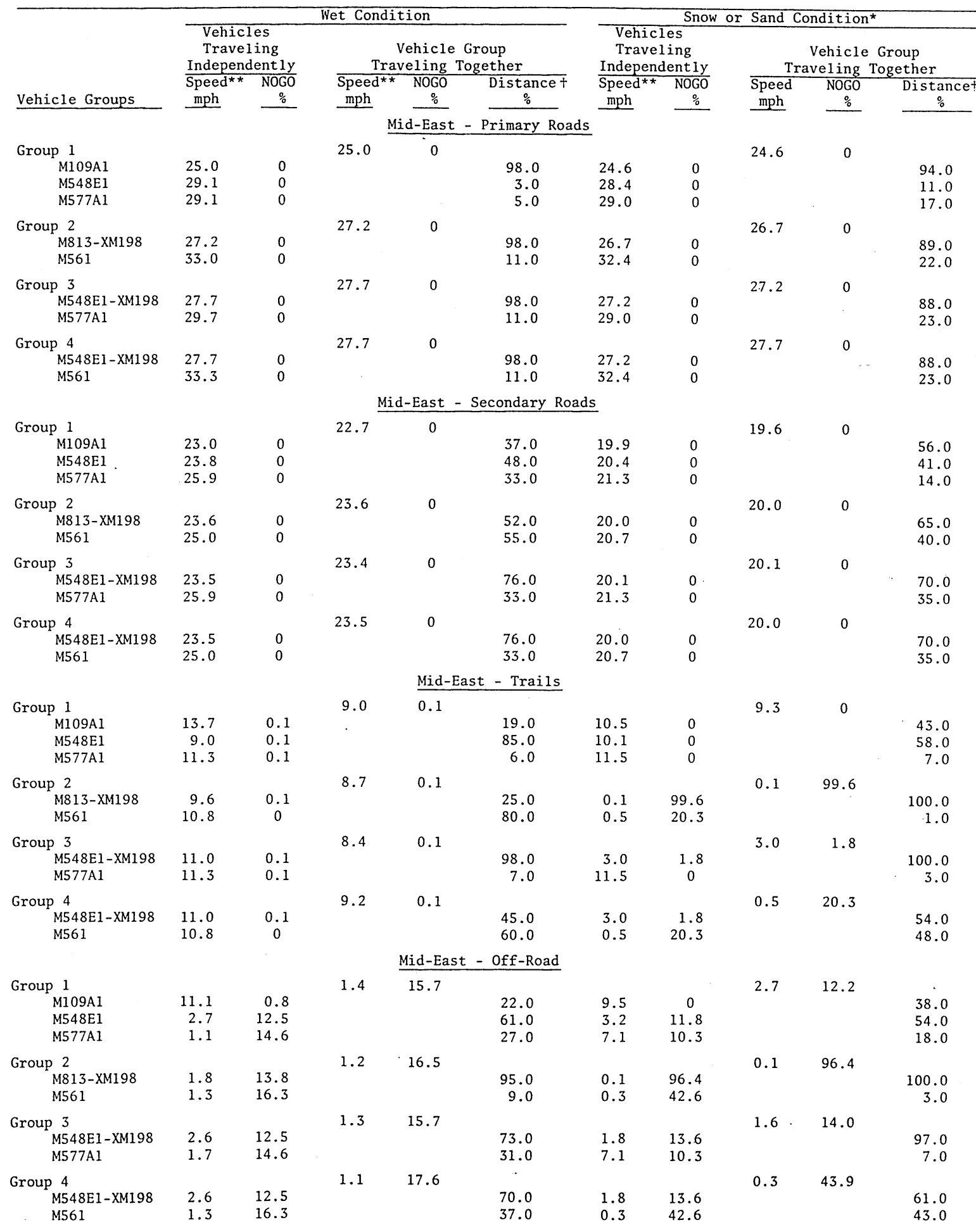


Table 22

Relative Mobility Performance of Prime Movers Based on Performance in Countries Described by the Generalized Terrain Data

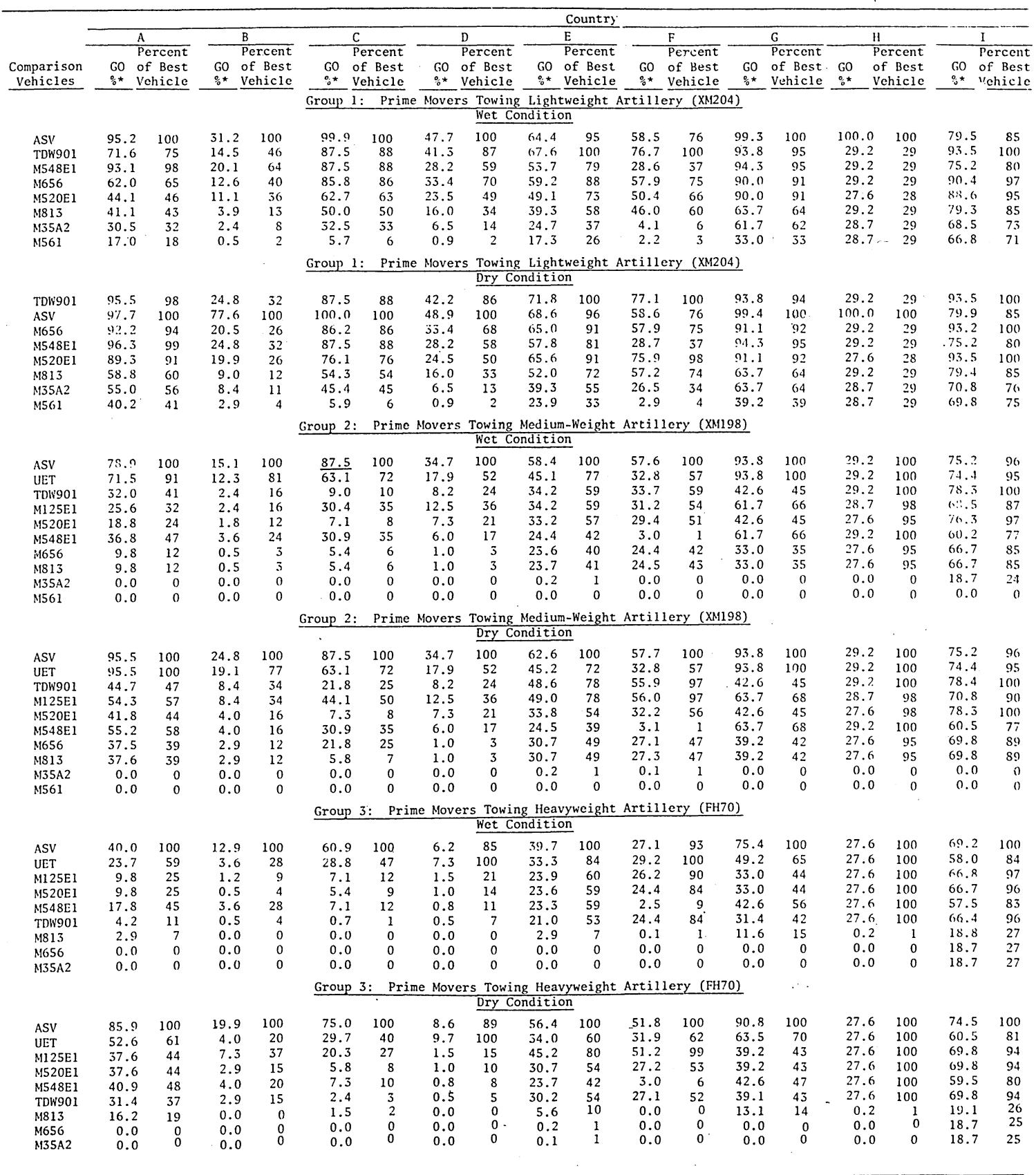

* Percentage of country with $>66-100$ percent probability of 60.
$\star *$ Vehicle that can negotiate the largest percentage of country is considered "Best Vehicle". 
Table 23

Comparisons of Selected Prime Mover-Towed Artillery with Self-Propelled M109A1

Based on Performance in Countries Described by the Generalized Terrain Data

\begin{tabular}{|c|c|c|c|c|c|c|c|c|c|c|c|c|c|c|c|c|c|c|}
\hline \multirow[b]{3}{*}{$\begin{array}{l}\text { Comparison } \\
\text { Vehicles }\end{array}$} & \multicolumn{18}{|c|}{ Country } \\
\hline & \multicolumn{2}{|c|}{$\bar{A}$} & \multicolumn{2}{|c|}{$\bar{B}$} & \multicolumn{2}{|c|}{$\mathrm{C}$} & \multicolumn{2}{|l|}{$\mathrm{D}$} & \multicolumn{2}{|c|}{$E$} & \multicolumn{2}{|l|}{$\bar{F}$} & \multicolumn{2}{|c|}{$\bar{G}$} & \multicolumn{2}{|c|}{$\mathrm{H}$} & \multicolumn{2}{|c|}{$\mathrm{I}$} \\
\hline & G0 & 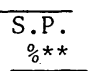 & $\begin{array}{r}\mathrm{GO} \\
\%\end{array}$ & $\begin{array}{c}\text { S.P. } \\
\frac{\%}{0}\end{array}$ & $\begin{array}{r}\text { G0 } \\
\%\end{array}$ & $\begin{array}{c}\mathrm{S.P} . \\
\frac{0}{0}\end{array}$ & $\begin{array}{r}\mathrm{GO} \\
\% \\
\end{array}$ & $\begin{array}{c}\text { S.P. } \\
\frac{0}{0}\end{array}$ & $\begin{array}{l}\mathrm{GO} \\
\%\end{array}$ & $\underset{\frac{0}{0}}{\mathrm{S.P}}$ & $\begin{array}{r}\mathrm{GO} \\
\%\end{array}$ & $\underset{\frac{\sigma}{0}}{\text { S.P. }}$ & $\begin{array}{c}\mathrm{GO} \\
\%\end{array}$ & $\frac{\text { S.P. }}{\%}$ & $\begin{array}{r}\mathrm{GO} \\
\%\end{array}$ & $\frac{\text { S.P. }}{\%}$ & $\begin{array}{r}\mathrm{GO} \\
\%\end{array}$ & $\begin{array}{c}\text { S.P. } \\
\% \\
\%\end{array}$ \\
\hline \multicolumn{19}{|c|}{$\frac{\text { Prime Movers Towing Lightweight Artillery }}{\text { Wet Condition }}$} \\
\hline ASV-XM204 & 95.2 & 101.0 & 31.2 & 99.0 & 99.0 & 100.0 & 47.7 & 61.0 & 64.4 & 80.0 & 58.5 & 74.0 & 99.3 & 99.0 & 100.0 & 100.0 & 79.5 & 99.0 \\
\hline TDW901 - XM204 & 71.6 & 76.0 & 14.5 & 46.0 & 87.5 & 88.0 & 41.3 & 53.0 & 67.6 & 83.0 & 76.7 & 97.0 & 93.8 & 94.0 & 29.2 & 29.0 & 93.5 & 117.0 \\
\hline M561-XM204 & 17.0 & 18.0 & 0.5 & 2.0 & 5.7 & 6.0 & 0.9 & 1.0 & 17.3 & 21.0 & 2.2 & 3.0 & 33.0 & 33.0 & 28.7 & 29.0 & 66.8 & 83.0 \\
\hline \multicolumn{19}{|c|}{$\frac{\text { Prime Movers Towing Medium-Weight Artillery }}{\text { Wet Condition }}$} \\
\hline ASV-XM198 & 78.9 & 83.0 & 15.1 & 48.0 & 87.5 & 88.0 & 34.7 & 44.0 & 58.4 & 72.0 & 57.6 & 73.0 & 93.8 & 94.0 & 29.2 & 29.0 & 75.2 & 94.0 \\
\hline TDW901-XM1 98 & 32.0 & 34.0 & 2.4 & 8.0 & 9.0 & 9.0 & 8.2 & 10.0 & 34.2 & 42.0 & 33.7 & 43.0 & 42.6 & 43.0 & 29.2 & 29.0 & 78.3 & 98.0 \\
\hline M656-XM198 & 9.8 & 10.0 & 0.5 & 2.0 & 5.4 & 5.0 & 1.0 & 1.0 & 23.6 & 29.0 & 24.4 & 31.0 & 33.0 & 33.0 & 27.6 & 28.0 & 66.7 & 83.0 \\
\hline \multicolumn{19}{|c|}{$\frac{\text { Prime Movers Towing Heavyweight Artillery }}{\text { Wet Condition }}$} \\
\hline ASV-FH70 & 40.0 & 42.0 & 12.9 & 41.0 & 60.9 & 61.0 & 6.2 & 8.0 & 39.7 & 49.0 & 27.1 & 34.0 & 75.4 & 75.0 & 27.6 & 28.0 & 69.2 & 86.0 \\
\hline $\mathrm{M} 125 \mathrm{E} 1-\mathrm{FH} 70$ & 9.8 & 10.0 & 1.2 & 4.0 & 7.1 & 7.0 & 1.5 & 2.0 & 23.9 & 30.0 & 26.2 & 33.0 & 33.0 & 33.0 & 27.6 & 28.0 & 66.8 & 83.0 \\
\hline M520E1-FH70 & 9.8 & 10.0 & 0.5 & 2.0 & 5.4 & 5.0 & 1.0 & 1.0 & 23.6 & 29.0 & 24.4 & 31.0 & 33.0 & 33.0 & 27.6 & 28.0 & 67.7 & 84.0 \\
\hline \multicolumn{19}{|c|}{$\frac{\text { Prime Movers Towing Lightweight Artillery }}{\text { Dry Condition }}$} \\
\hline ASV-XM204 & 97.7 & 100.0 & 77.6 & 100.0 & 100.0 & 100.0 & 48.9 & 63.0 & 68.6 & 80.0 & 58.6 & 74.0 & 99.4 & 99.0 & 100.0 & 100.0 & 79.9 & 100.0 \\
\hline TDW901-XM204 & 95.5 & 98.0 & 24.8 & 32.0 & 87.5 & 88.0 & 42.2 & 54.0 & 71.8 & 84.0 & 77.1 & 97.0 & 93.8 & 94.0 & 29.2 & 29.0 & 93.5 & 117.0 \\
\hline M561-XM204 & 40.2 & 41.0 & 2.9 & 4.0 & 5.9 & 6.0 & 0.9 & 1.0 & 23.9 & 28.0 & 2.9 & 4.0 & 39.2 & 39.0 & 28.7 & 29.0 & 69.8 & 87.0 \\
\hline \multicolumn{19}{|c|}{$\frac{\text { Prime Movers Towing Medium-Weight Artillery }}{\text { Dry Condition }}$} \\
\hline ASV-XM198 & 95.5 & 98.0 & 24.8 & 32.0 & 87.5 & 88.0 & 34.7 & 44.0 & 62.6 & 73.0 & 57.7 & 73.0 & 93.8 & 94.0 & 29.2 & 29.0 & 75.2 & 94.0 \\
\hline TDW901-XM198 & 44.7 & 46.0 & 8.4 & 11.0 & 21.8 & 22.0 & 8.2 & 10.0 & 48.6 & 57.0 & 55.9 & 70.0 & 42.6 & 43.0 & 29.2 & 29.0 & 78.4 & 98.0 \\
\hline M656-XM198 & 37.5 & 38.0 & 2.9 & 4.0 & 21.8 & 22.0 & 1.0 & 1.0 & 30.7 & 36.0 & 27.1 & 34.0 & 39.2 & 39.0 & 27.6 & 28.0 & 69.8 & 87.0 \\
\hline \multicolumn{19}{|c|}{$\frac{\text { Prime Movers Towing Heavyweight Artillery }}{\text { Dry Condition }}$} \\
\hline ASV-FH70 & 85.9 & 88.0 & 19.9 & 26.0 & 75.0 & 75.0 & 8.6 & 11.0 & 56.4 & 66.0 & 51.8 & 65.0 & 90.8 & 91.0 & 27.6 & 28.0 & 74.5 & 93.0 \\
\hline $\mathrm{M} 125 \mathrm{E} 1-\mathrm{FH} 70$ & 37.6 & 39.0 & 7.3 & 9.0 & 20.3 & 20.0 & 1.5 & 2.0 & 45.2 & 53.0 & 51.2 & 65.0 & 39.2 & 39.0 & 27.6 & 28.0 & 69.8 & 87.0 \\
\hline M520E1-FH70 & 37.6 & 39.0 & 2.9 & 4.0 & 5.8 & 6.0 & 1.0 & 1.0 & 30.7 & 36.0 & 27.2 & 34.0 & 39.2 & 39.0 & 27.6 & 28.0 & 69.8 & 87.0 \\
\hline
\end{tabular}

* Percentage of country with $>66-100$ percent probability of GO.

* Performance of prime mover-towed artillery compared with self-propelled M109Al in percent. 
Table 24

Relative Mobility of Prime Movers Based on Tactical High Mobility Level in HIMO Study Areas

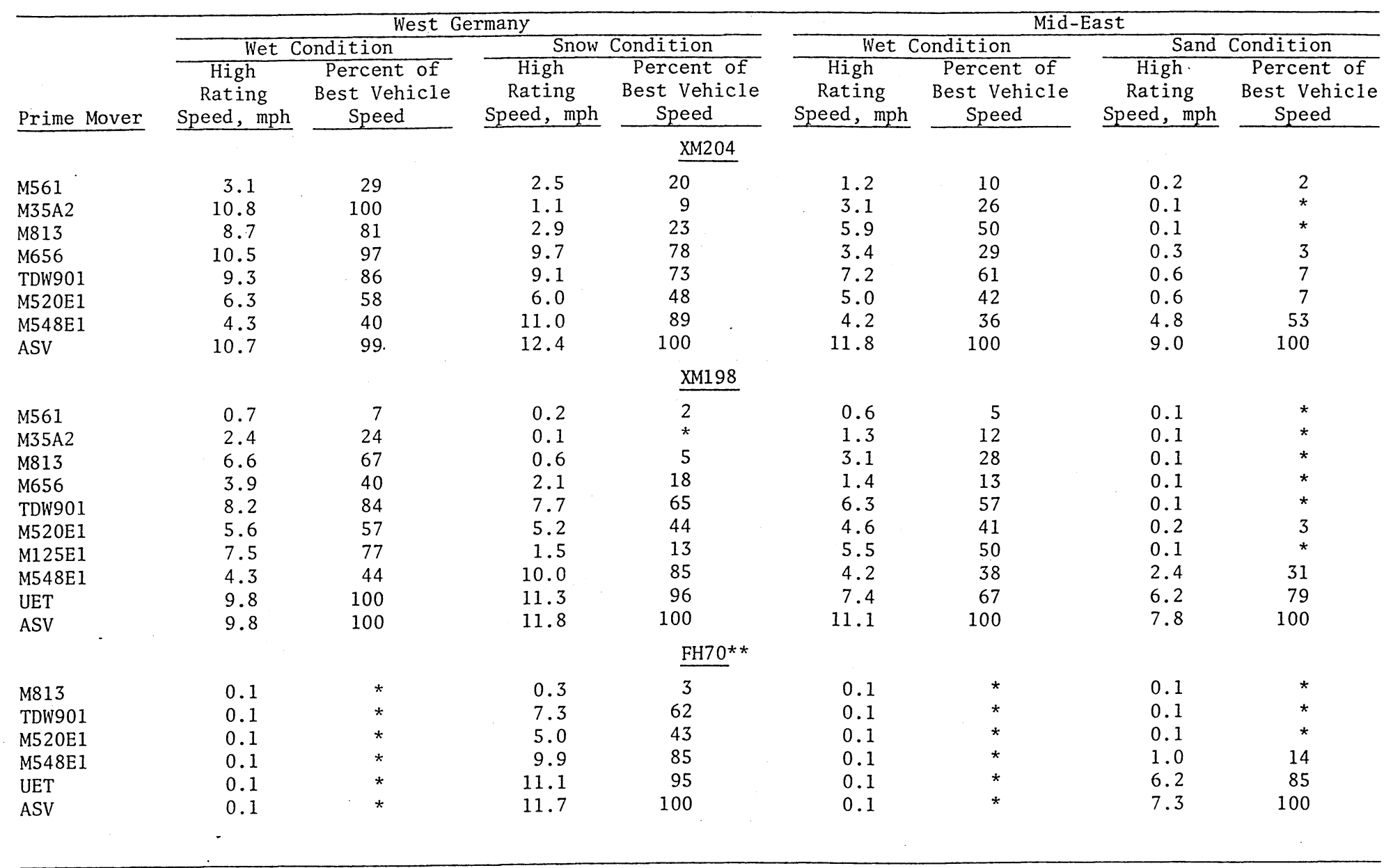

* A 0.1-mph speed indicates that this prime mover is completely inadequate for towing this given artillery in given country and condition.

** No performance predictions were made for the M35A2 and the M125E1 towing the FH70, but they should have performance similar to that of the M813 towing the FH70. 
Table 25

Comparisons of Selected Prime Mover-Towed Artillery and the M109A1 at Two Levels of Mobility

in the HIMO Study Areas

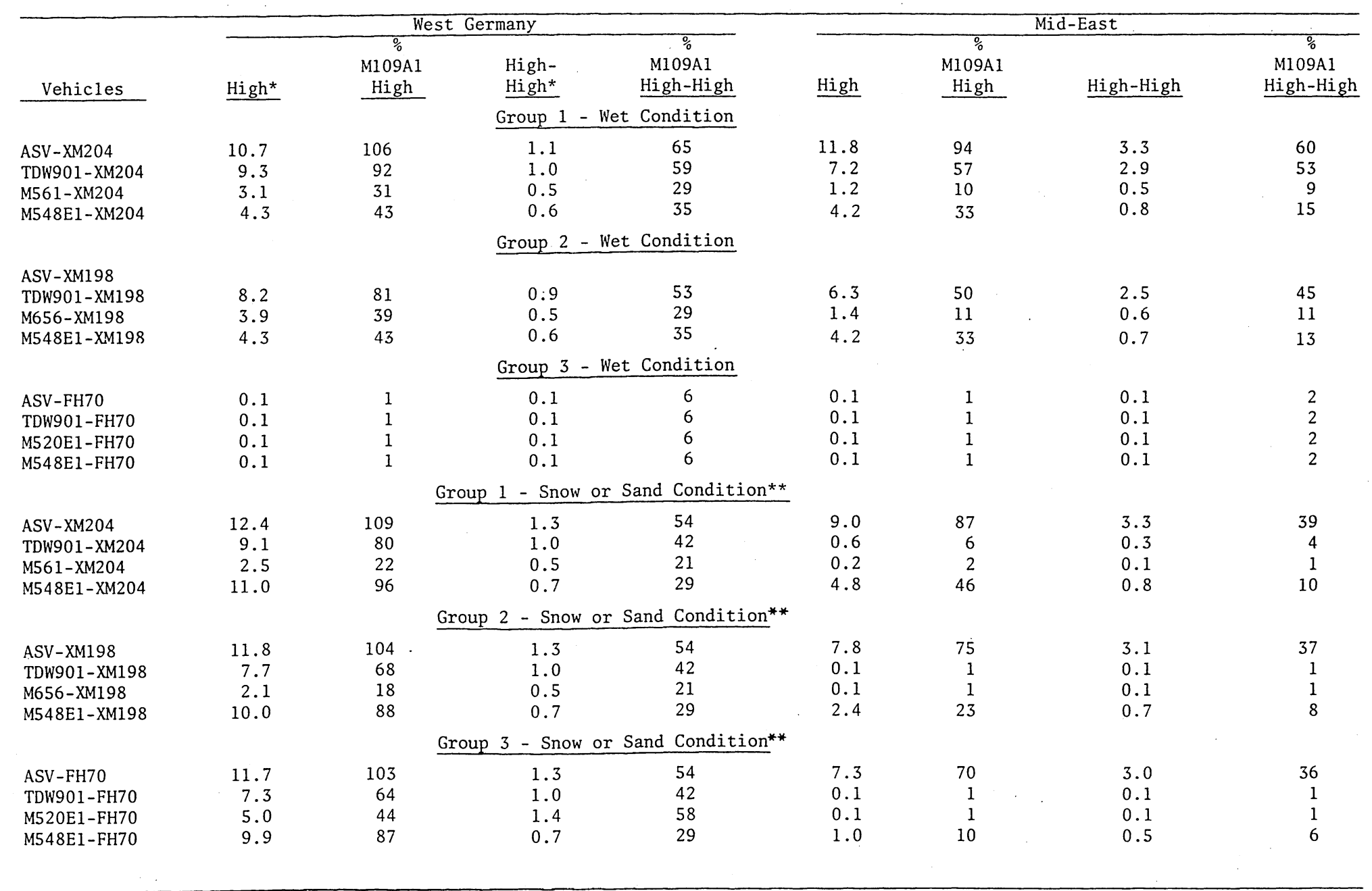

* WHEELS study definitions in Table 18, and mph.

** West Germany snow condition; Mid-East sand condition. 
Table 26

Ranking of Selected Vehicle Groups Based on Performance in the HIMO Study Areas

\begin{tabular}{|c|c|c|c|c|c|c|c|c|c|}
\hline \multirow[b]{3}{*}{$\begin{array}{c}\text { Surface } \\
\text { Type } \\
\end{array}$} & \multirow[b]{3}{*}{ Group No.* } & \multicolumn{4}{|c|}{ West Germany } & \multicolumn{4}{|c|}{ Mid-East } \\
\hline & & \multicolumn{2}{|c|}{ Wet } & \multicolumn{2}{|c|}{ Snow } & \multicolumn{2}{|c|}{ Wet } & \multicolumn{2}{|c|}{ Sand } \\
\hline & & $\begin{array}{l}\text { Group**} \\
\text { Speed } \\
\text { mph }\end{array}$ & Rankt & $\begin{array}{l}\text { Group } \\
\text { Speed } \\
\text { mph } \\
\end{array}$ & Rank & $\begin{array}{c}\text { Group } \\
\text { Speed } \\
\text { mph } \\
\end{array}$ & Rank & $\begin{array}{l}\text { Group } \\
\text { Speed } \\
\text { mph }\end{array}$ & Rank \\
\hline Primary roads & 1 & 22.9 & 4 & 22.9 & 2 & 25.0 & 4 & 24.6 & 4 \\
\hline & 2 & 24.6 & 3 & 7.4 & 4 & 27.2 & 3 & 26.7 & 3 \\
\hline & 3 & 25.1 & 1 & 25.1 & 1 & 27.7 & 1 & 27.2 & 1 \\
\hline & 4 & 25.1 & 1 & 22.8 & 3 & 27.7 & 1 & 27.2 & 1 \\
\hline Secondary roads & 1 & 20.3 & 1 & 18.8 & 1 & 22.7 & 4 & 19.6 & 4 \\
\hline & 2 & 19.6 & 2 & 1.3 & 4 & 23.6 & 1 & 20.0 & 2 \\
\hline & 3 & 19.4 & 3 & 18.7 & 2 & 23.4 & 3 & 20.1 & 1 \\
\hline & 4 & 19.4 & 3 & 18.0 & 3 & 23.5 & 2 & 20.0 & 2 \\
\hline Trails & 1 & 11.2 & 1 & 11.8 & 1 & 9.0 & 2 & 9.3 & 1 \\
\hline & 2 & 3.8 & 4 & 1.2 & 4 & 8.7 & 3 & 0.1 & 4 \\
\hline & 3 & 10.2 & 2 & 11.6 & 2 & 8.4 & 4 & 3.0 & 2 \\
\hline & 4 & 8.2 & 3 & 8.9 & 3 & 9.2 & 1 & 0.5 & 3 \\
\hline off-roads & 1 & 3.0 & 4 & 11.7 & 1 & 1.4 & 1 & 2.7 & 1 \\
\hline & 2 & 3.9 & 1 & 0.5 & 4 & 1.2 & 3 & 0.1 & 4 \\
\hline & 3 & 3.3 & 2 & 10.4 & 2 & 1.3 & " 2 & 1.6 & 2 \\
\hline & 4 & 3.2 & 3 & 6.1 & 3 & 1.1 & 4 & 0.3 & 3 \\
\hline
\end{tabular}

* Group $1=$ M109A1, M548E1, M577Al

Group $2=$ M813-XM198, M561

Group $3=$ M548E1-XM198, M577A1

Group $4=$ M548E1-XM198, M561

** Group speed for primary and secondary roads and trails based on $V_{100}$ and for off-road on $\mathrm{V}_{90^{\circ}}$

+ Ranked 1-4, best to worst 
APPENDIX A: JIFFY MOBILITY MODEL

1. The JIFFY mobility model was developed for use with generalized terrain data because a limited amount of detail with respect to terrain data does not warrant the use of a more complex model, such as the Army mobility model (AMM). The terrain-vehicle relations used by the JIFFY mobility model to predict GO/NOGO vehicle performance are soil-vehicle, soil strength-slope-vehicle, obstacle-vehicle gometry, and vegetation-vehicle. These relations and the method used to determine GO/NOGO by the JIFFY model are discussed briefly in this appendix.

\section{Terrain-Vehicle Relations}

\section{Soil-vehicle relations}

2. Vehicle performance varies as a function of soll type therefore, separate relations for surface material strength versus vehicle parameters were used for fine-grained soils (clays and silts) and coarsegrained soils (sands). Fine-grained soil-vehicle relations are also used for rock surfaces. Fine-grained soil was the predominant surface material in the nine countries considered in this study. Coarse-grained soil occurred in relatively few of the terrain units.

3. The soll-vehicle relations used by the JIFFY model to predict vehicle performance include the minimum soil strength required for a vehicle to complete one pass and the first-pass maximum tractive force (drawbar pul1).

4. $\mathrm{VCI}_{1}$. For fine-grained soils, $\mathrm{VCI}_{1}$ is defined as the minimum soil strength in terms of rating cone index (RCI) that will permit one pass. The generalized terrain data describe soil strength only in terms of cone index; therefore, it is assumed that rating cone index and cone index are the same. For coarse-grained soils, $\mathrm{VCI}_{1}$ is computed and defined as the soil strength in terms of cone index at which zero net drawbar pull occurs. The procedures for computing VCI 1 for both finegrained and coarse-grained soils are the same as those used in the AMC-74X mobility model. ${ }^{2}$ 
5. $\mathrm{VCI}_{1}$ for vehicles towing artillery can be computed by adding the increase in motion resistance offered by the artillery for a given soil strength, and then determining the increase in soil strength required to offset the motion resistance, using the soil strengthdrawbar pull relations.

6. Traction force-soil strength relations. The maximum force a vehicle can develop on level ground is primarily a function of soil strength, and vehicle type (wheeled or tracked), ground pressure, and slip. Tractive force versus excess rating cone index (RCI minus VCI ${ }_{1}$ ) relations for tracked and wheeled vehicles operating in fine-grained soils are shown in Figure Al.

7. For this study, tractive force in fine-grained soil for wheeled vehicles was predicted for the sol1 strength values $(150,112$, 60 , and 22) representative of the surface composition classes given in Table D1 (Appendix D). Strength of coarse-grained soil has little or no effect on tractive performance, but the type of track on a vehicle does affect tractive performance. For the vehicles in this study with flexible tracks, a tractive force coefficient of 0.70 was assigned; for vehicles with girderized tracks, a tractive force coefficient of 0.57 was assigned. Although this may appear somewhat arbitrary, previous studies have shown these values to be reasonable.

Soil strength-slope-vehicle relations

8. Vehicle performance on a slope depends largely on the magnitude of the slope and the excess traction that the vehicle can develop, which, in turn, depends on soil strength. For a given soil strength the maximum slope negotiable (expressed in percent) is equal to the tractive force (expressed as percent of vehicle weight) that a vehicle can develop on level soil of the same strength. Thus, the tractive force-soil strength relations for fine-grained soils (Figure A1) and and the computational form for determing tractive force for coarse-grained soils (Figure A2) are used to determine performance on slopes. Obstacle-vehicle geometry relations

9. The only obstacle geometry characteristics available in the generalized terrain data are the heights of the obstacles. By using 
pertinent obstacle-vehicle geometry, the maximum attitude angle that the vehicle would attain if it were to cross the obstacle and the traction required are determined.

10. To use limited available data to predict traction required by wheeled vehicles for obstacle crossing, the obstacle approach angle (A) was arbitrarily assumed to vary with obstacle height as follows:

$$
A=90-\frac{\mathrm{OH}}{2}, \mathrm{deg}
$$

where $\mathrm{OH}=$ obstacle height, in.

This was done on the basis that, as discrete natural obstacles become larger, they tend under the influence of both their origin and subsequent history to become less abrupt. The algorithm allows small obstacles to present essentially vertical faces to a vehicle approach,' whereas, the obstacle approach angle drops to about $70 \mathrm{deg}$ for the largest vertical obstacles considered. This is still formidable.

11. Tractive requirements for wheeled vehicles are computed on the following assumptions:

a. The obstacle is rigid.

b. The obstacle is approached head-on at very low speed.

c. Load is equally distributed among the driving axles, and load transfer due to vehicle attitude is ignored.

d. Tractive coefficient is the same under each axle and on the obstacle face.

The resulting simple static equilibrium equations lead to an equation for required tractive coefficient $\left(T_{R}\right)$ as follows:

$$
\mathrm{T}_{\mathrm{R}}=\sqrt{\lambda^{2}+\frac{1}{\mathrm{n}-1}}-\lambda
$$

where

$$
\begin{aligned}
\lambda & =\frac{1}{2(\mathrm{n}-1)} \times \cot \alpha \\
\mathfrak{n} & =\text { total number of driven axles } \\
\alpha & =\text { effect1ve wheel contact angle } \\
& =A \text { or } A_{1}, \text { whichever is least } \\
A_{1} & =90-\operatorname{arc} \sin \left(\frac{R W O H}{R W}\right), \text { deg }
\end{aligned}
$$


$\mathrm{RW}=$ wheel radius, in.

12. For tracked vehicles the traction required to surmount the obstacles that can be negotiated is computed with the following equations:

Traction required (\% of vehicle weight) $=\tan ($ MPA)

where

$$
\begin{aligned}
\text { MPA }= & \text { maximum pitch angle of vehicle (deg) } \\
= & \text { arc sin }[M V \div(\mathrm{LT}-\mathrm{DS})] \\
\mathrm{MV}= & \text { obstacle height, in. } \\
\mathrm{LT}= & \text { length of tractive element in contact with ground, in. } \\
\mathrm{DS}= & \text { distance between center of first road wheel and center of } \\
& \text { road wheel just forward of cneter of gravity of the } \\
& \text { vehicle, in. }
\end{aligned}
$$

The values determined in this manner for tractive force required are computed as percent of vehicle weight.

Vegetation-vehicle relations

13. Although both woody and nonwoody vegetation are deterrents to vehicle performance by restricting the driver's visibility or by causing the vehicle to slow down to maneuver around or override single and multiple stems, only the effects of overriding and maneuvering are considered by this model. For vehicle performance in vegetation, the peak and average forces developed in overriding a given array of vegetation are determined. These forces are then considered in terms of tractive and impact forces.

14. When the mean spacing of tree stems that must be overridden is less than 1.5 times the vehicle width, the vehicle cannot maneuver to circumvent them. To obtain the maximum stem size to be overridden; a value of 1.5 times the vehicle width is determined, and the stem diameter having that spacing is required to be overridden.

15. The peak impact force $\left(F_{h}\right)$ in pounds is computed by the following equation. 


$$
F_{h}=\left(40-\frac{H_{p}}{2}\right) d_{s}^{3}
$$

where

$$
\begin{aligned}
& H_{p}=\text { pushbar or height of leading edge of vehicle, in. } \\
& d_{S}^{3}=\text { stem diameter, in. }
\end{aligned}
$$

Peak impact forces are computed for the maximum stem diameters to be overridden. These values are used to determine whether the peak or impact force will exceed the force that the leading edge of the vehicle can withstand or whether the horizontal acceleration will exceed $2 \mathrm{~g}$ 's, the driver's tolerance limit. If either limit is exceeded, NOGO is predicted.

16. If a vehicle can override the maximum stem diameter to be overridden without exceeding the driver tolerance and leading edge limits, average tractive force $\left(F_{0}\right)$, in pounds, required to override the vegetation is computed by

$$
F_{0}=\frac{56 d_{s}^{3}}{5.8}
$$

17. Off-Road predictions for this study. Off-road vehicle performance in terms of GO/NOGO was predicted for the vehicles considered in this study by determining the tractive force available for a given surface composition factor classes for fine-and coarse-grained soils, and subtracting from the tractive force available the traction force required for the vehicle to negotiate the factor classes of slope, surface geometry, and vegetation. The difference was identified as excess traction. If the excess traction for a given terrain unit was positive, go performance was predicted; and if the excess traction was negative, NOGO performance was predicted. 

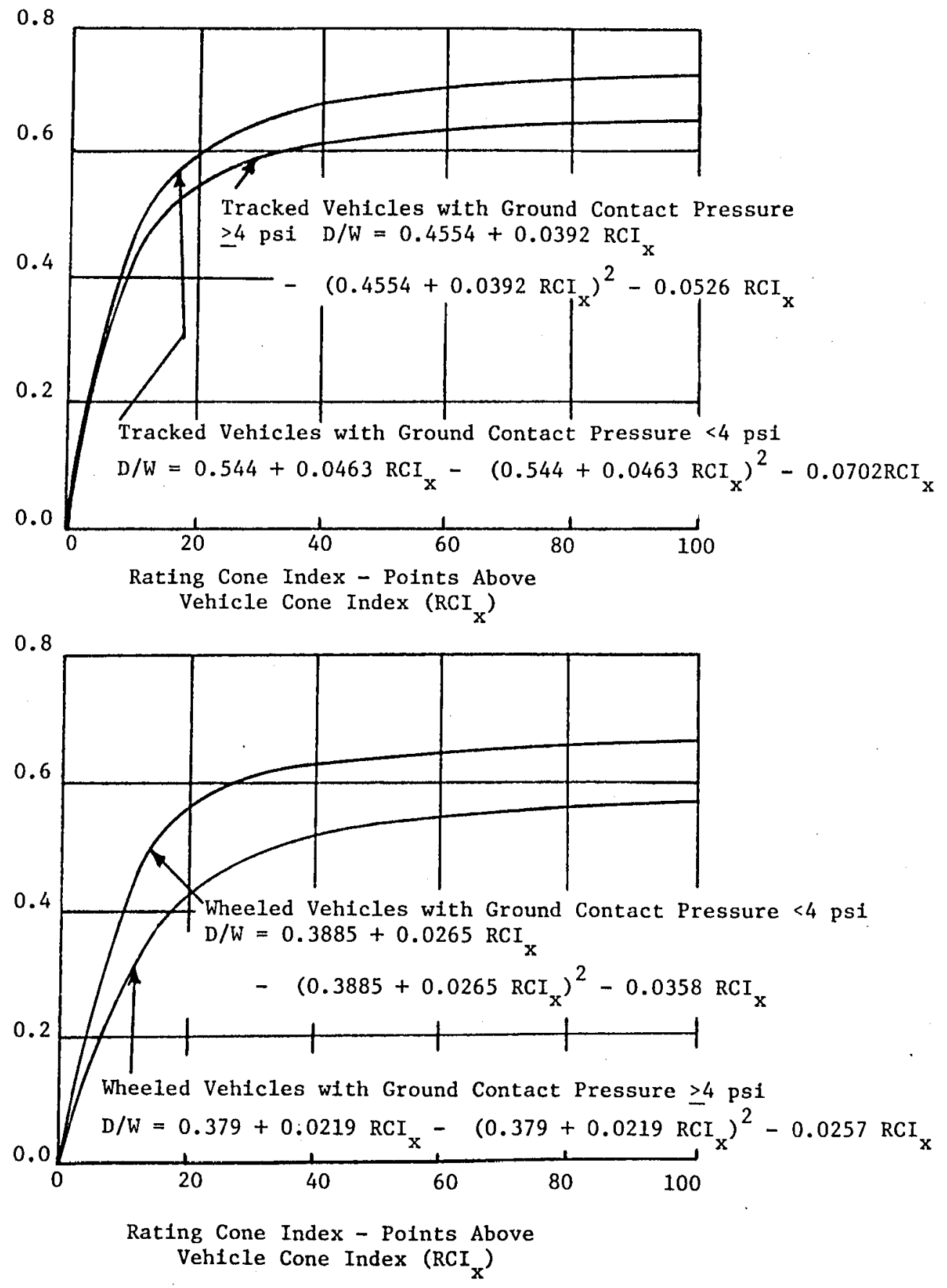

Figure Al. First-pass net maximum drawbar pu11, maximum slope negotiablesoil strength relations for tracked and wheeled vehicles operating on fine-grained soils. 
Vehicle

Basic Equations

$\mathrm{DP}_{\max }(\%$ of vehicle wt $)=28.87\left(\mathrm{X}_{1}\right)+10.10\left(\mathrm{X}_{2}\right)-1.52\left(\mathrm{X}_{3}\right)-0.61\left(\mathrm{X}_{4}\right)-\left(\mathrm{X}_{5}\right)=$

$S_{\max }(\%)=28.87\left(\mathrm{X}_{1}\right)+10.10\left(\mathrm{X}_{2}\right)-1.52\left(\mathrm{x}_{3}\right)-0.61\left(\mathrm{X}_{4}\right)-\left(\mathrm{X}_{6}\right)=$

Vehicle Characteristics and Cone Index

1. Gross wt, $1 b$

2. Nomfnal tire width, in.

3. Rim diameter, in.

4. No. of powered tires

5. Tire ply rating

6. Tire inflation pressure, psi

7. Cone index of 0 - to 6-in. layer

\section{Factors}

$\mathrm{x}_{1}=$ strength factor $=\log (7) *=$

$x_{2}=$ contact area factor $=\log \left[\frac{(1)}{\left(x_{a}\right)}\right]=$

$\mathrm{X}_{\mathrm{a}}=$ contact pressure factor $=0.607 \times$

(6) $+1.35\left[\frac{117.0 \times(5)}{\left(\mathrm{x}_{\mathrm{b}}\right)}\right]-4.93=$

$\mathrm{X}_{\mathrm{b}}=$ wheel diameter factor $=5.0 \times(2)+(3)=$

$x_{3}=$ same as (4)

$\mathrm{X}_{4}=$ same as (6)

$\mathrm{X}_{5}=43.82$ for maximum net drawbar pull computations

$X_{6}=45.82$ for maximum slope negotiable

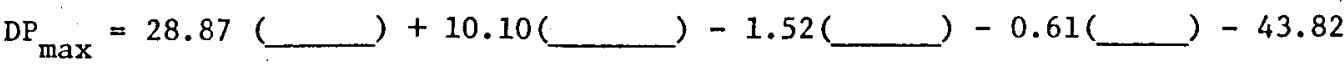

$=$

$S_{\max }=$

$=28.87(\quad)+10.10($ $-1.52($ )-0.61($ $-45.82$

* Number in parentheses indicates the vehicle characteristic, cone index, or $\mathrm{X}$ factor to use.

F1gure A2. DaĖं form for computing maximum net drawbar pull (DP ${ }_{\max }$ ) and maximum slope negotiable $\left(S_{\max }\right)$ for wheeled vehicles in sands 


\section{APPENDIX B: DATA USED TO CHARACTERIZE \\ STUDY VEHICLES}

1. Extensive data are required to characterize a vehicle to predict its performance with the Army mobility model (AMM). These data for each of the 60 study vehicles are given in Table B1 through B6. All vehicles are described as carrying their rated payloads, with tires at recommended inflations and corresponding deflections. Reduced inflation pressures allowable for operation in sand terrain are also specified.

2. Tractive force-speed relations are from Aberdeen Proving Ground engineering test data for all vehicles except the TDW901, the $\mathrm{ASV}$, and the XM723. For these vehicles, the tractive force-speed was computed from engine and power train data.

3. Both field experience and simulations have shown that dynamic responses of a vehicle crossing minor obstacles have a strong influence on actual vehicle performance. AMM is so structured that values used in it for critical vehicle characteristics may be those determined from dynamics simulation or from experimental data. Since experimental data were available for most of the prime movers in this study, a small' dynamics field test program was conducted to determine the effect on dynamic response when a prime mover was towing artillery. In addition, experimental dynamics data were collected on two pieces of self-propelled artillery, since no data on vehicles with similiar characteristics were available. The results of the dynamics test program are discussed in Appendix $C$, and the appropriate data have been incorporated in the tables in this appendix. 
Table B1

Values of Vehicle Characteristics

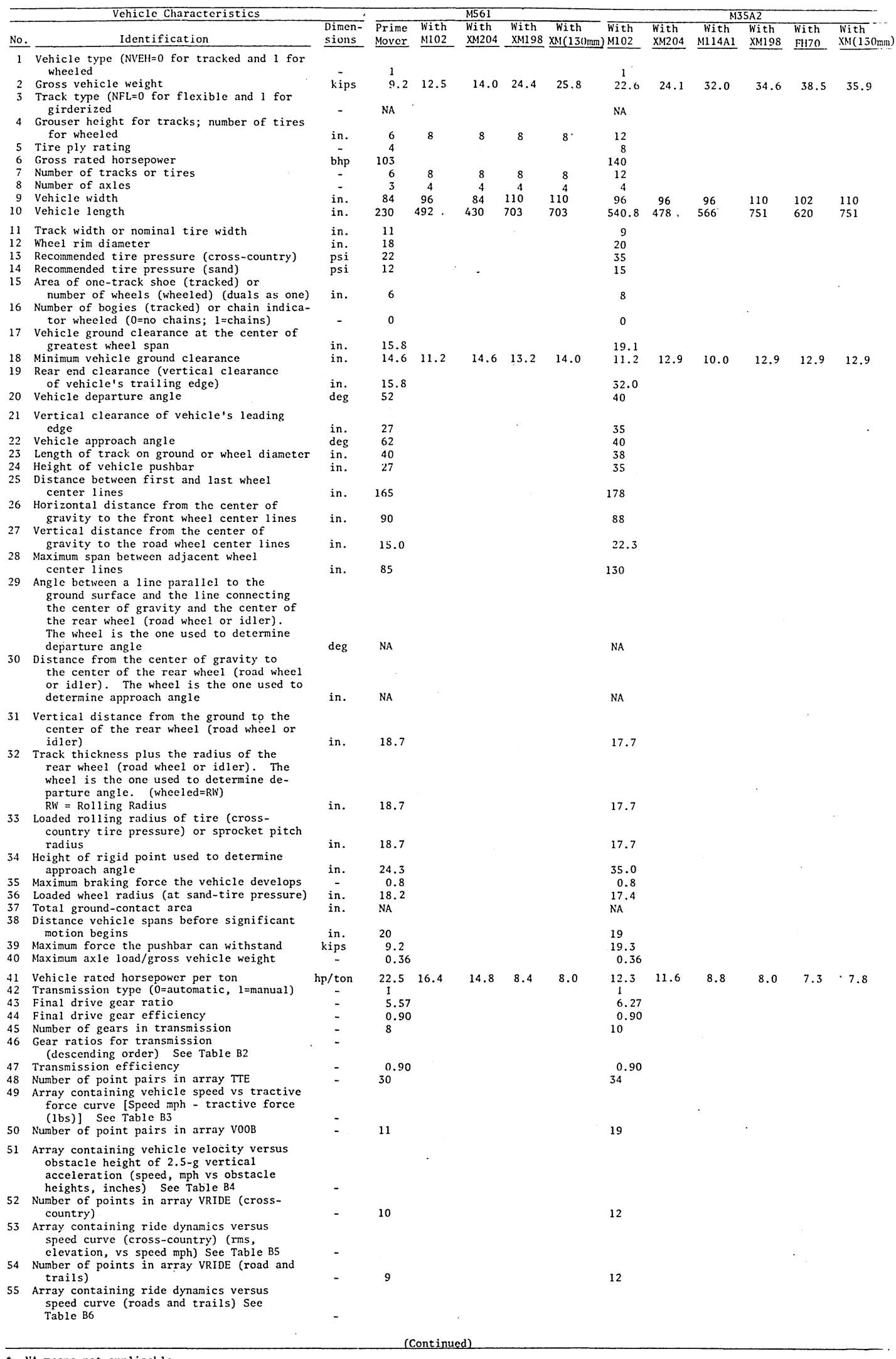

* NA means not applicable.

(Sheet 1 of 6 )

NOTE: Values were not repeated for additional towed artillery unless a change occurred. 
Table B1 (Continued)

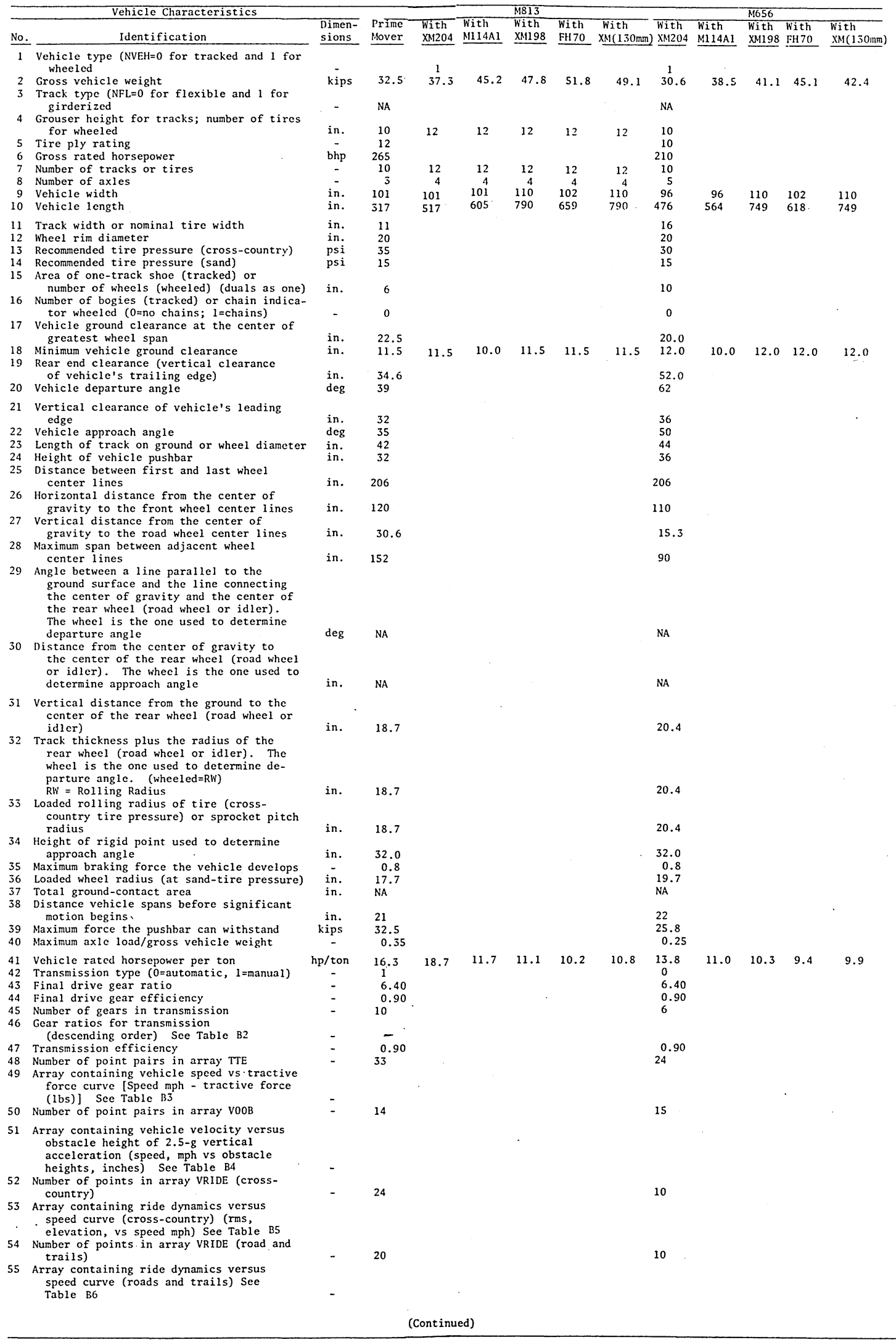

\footnotetext{
* NA means not applicable.
} 
Table B1 (Continued)

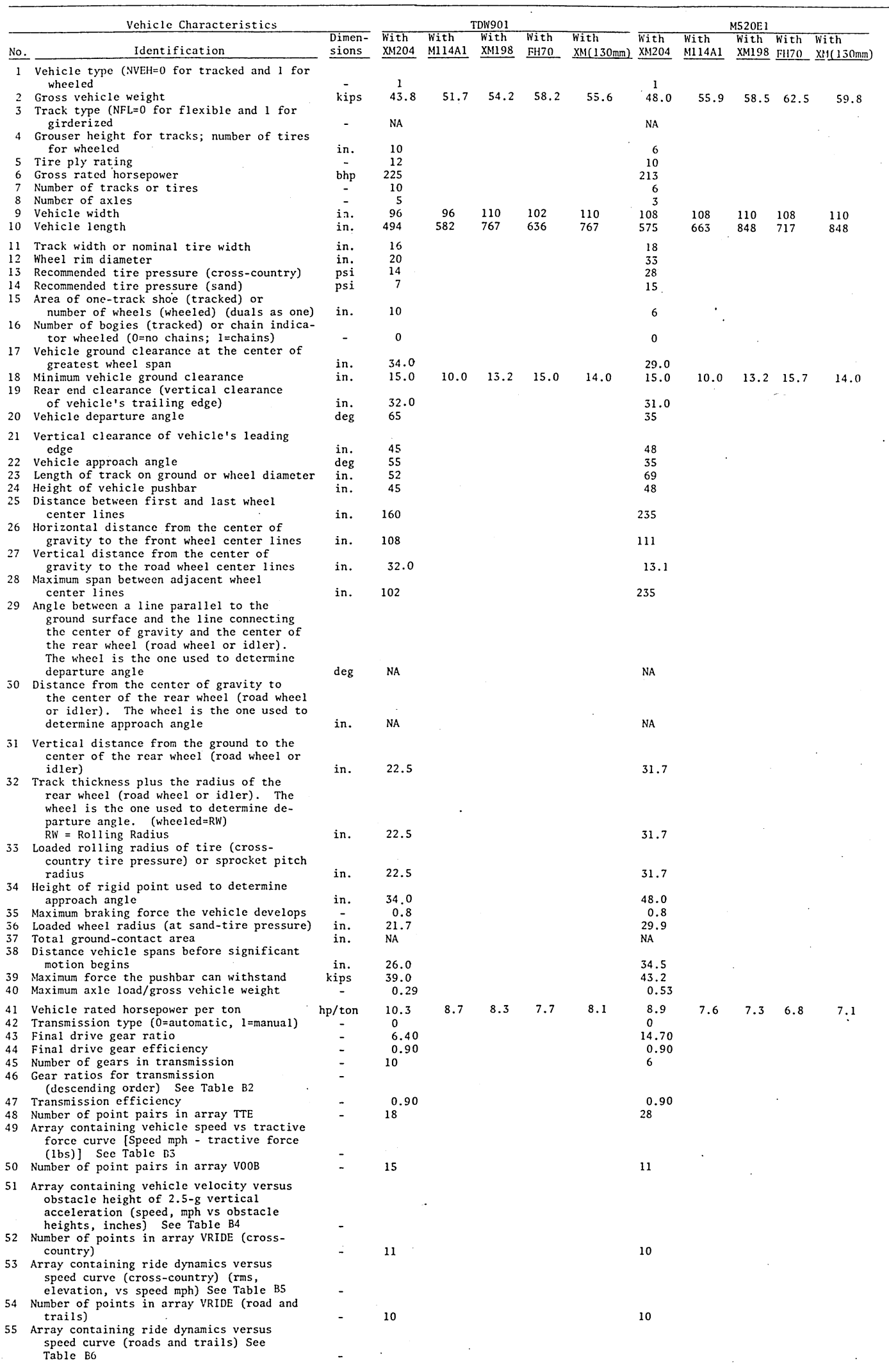


Table B1 (Continued)

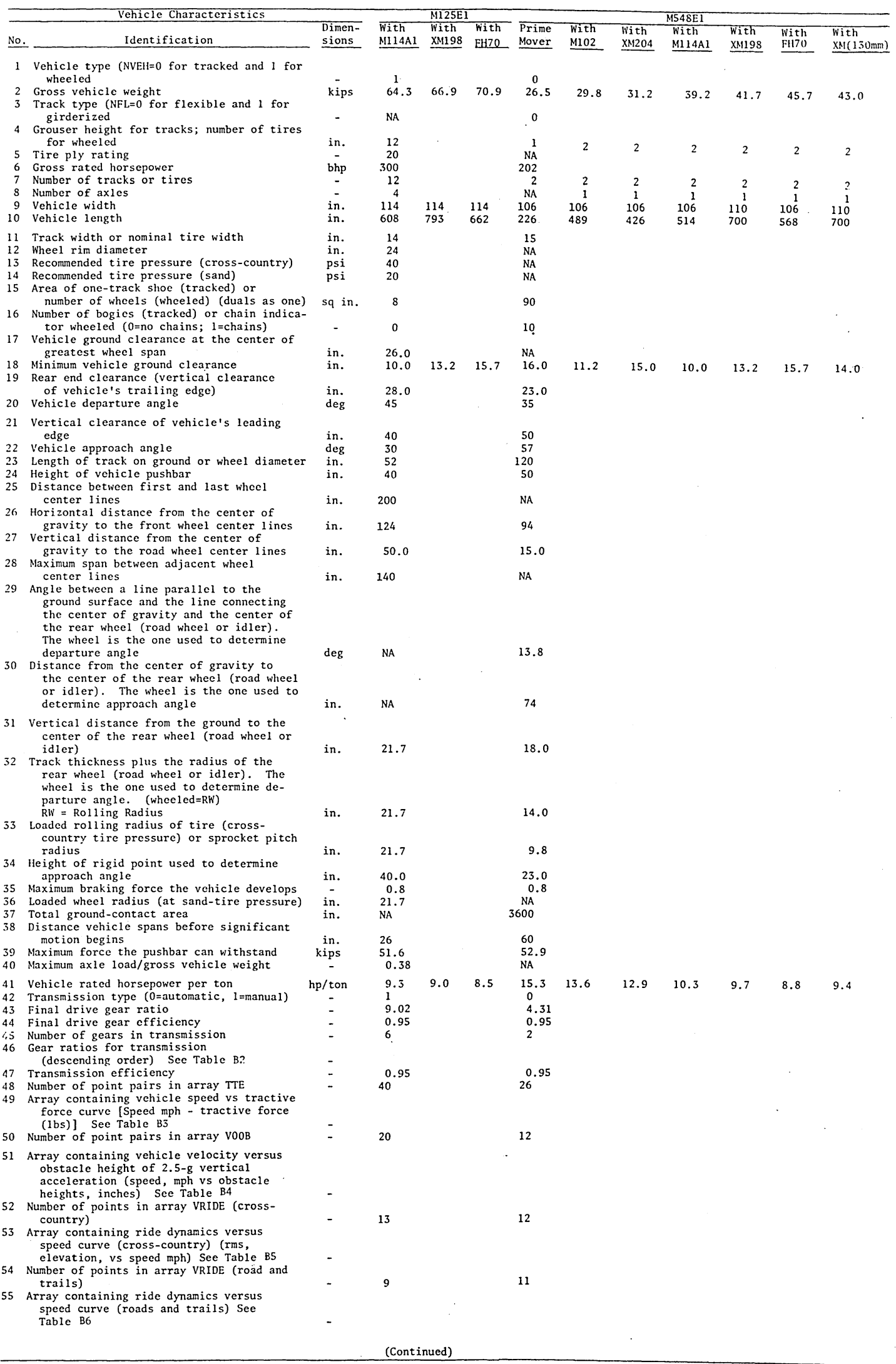

* NA means not applicable. 


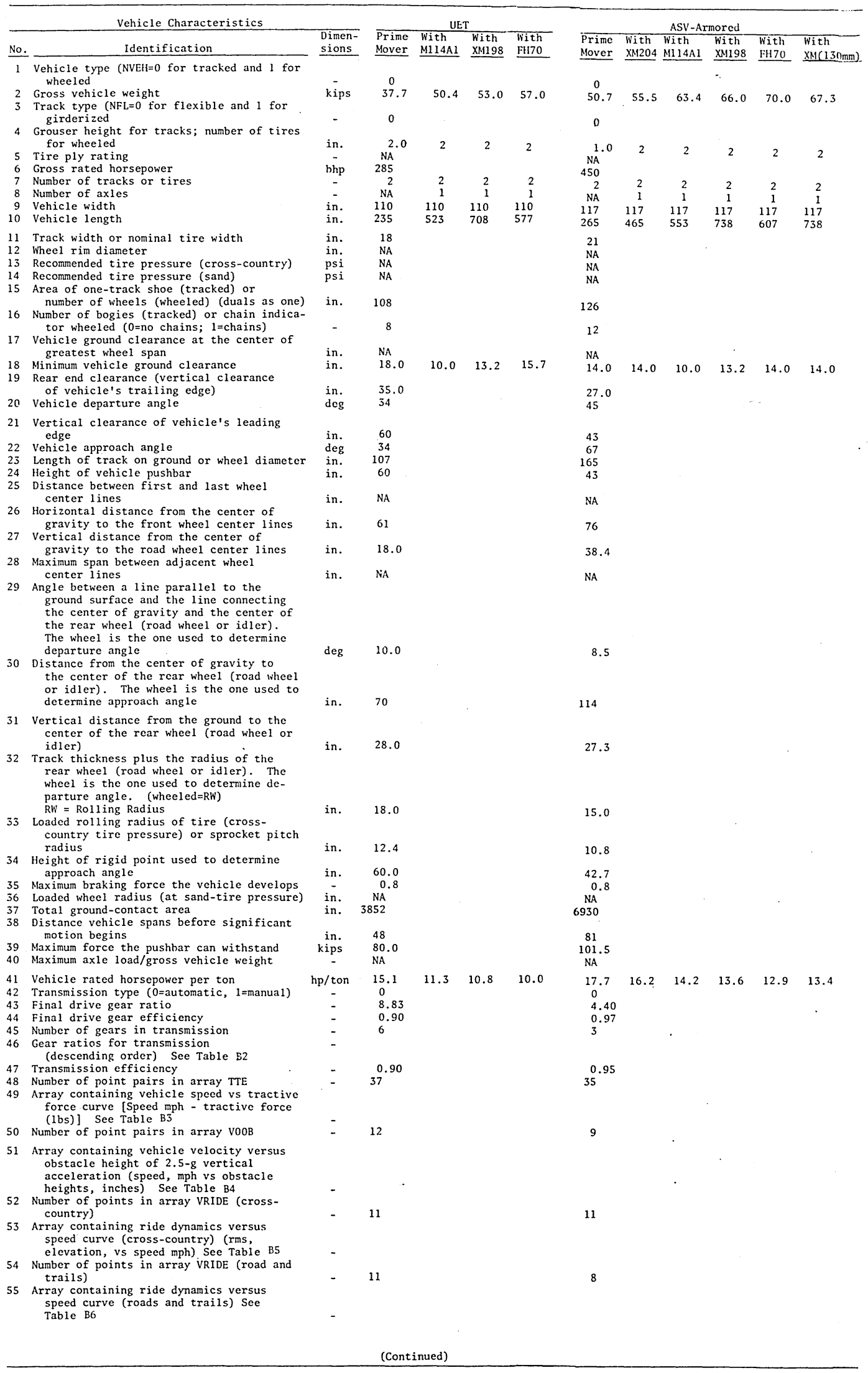

\footnotetext{
* NA means not applicable.
} 
Table BI (Concluded)

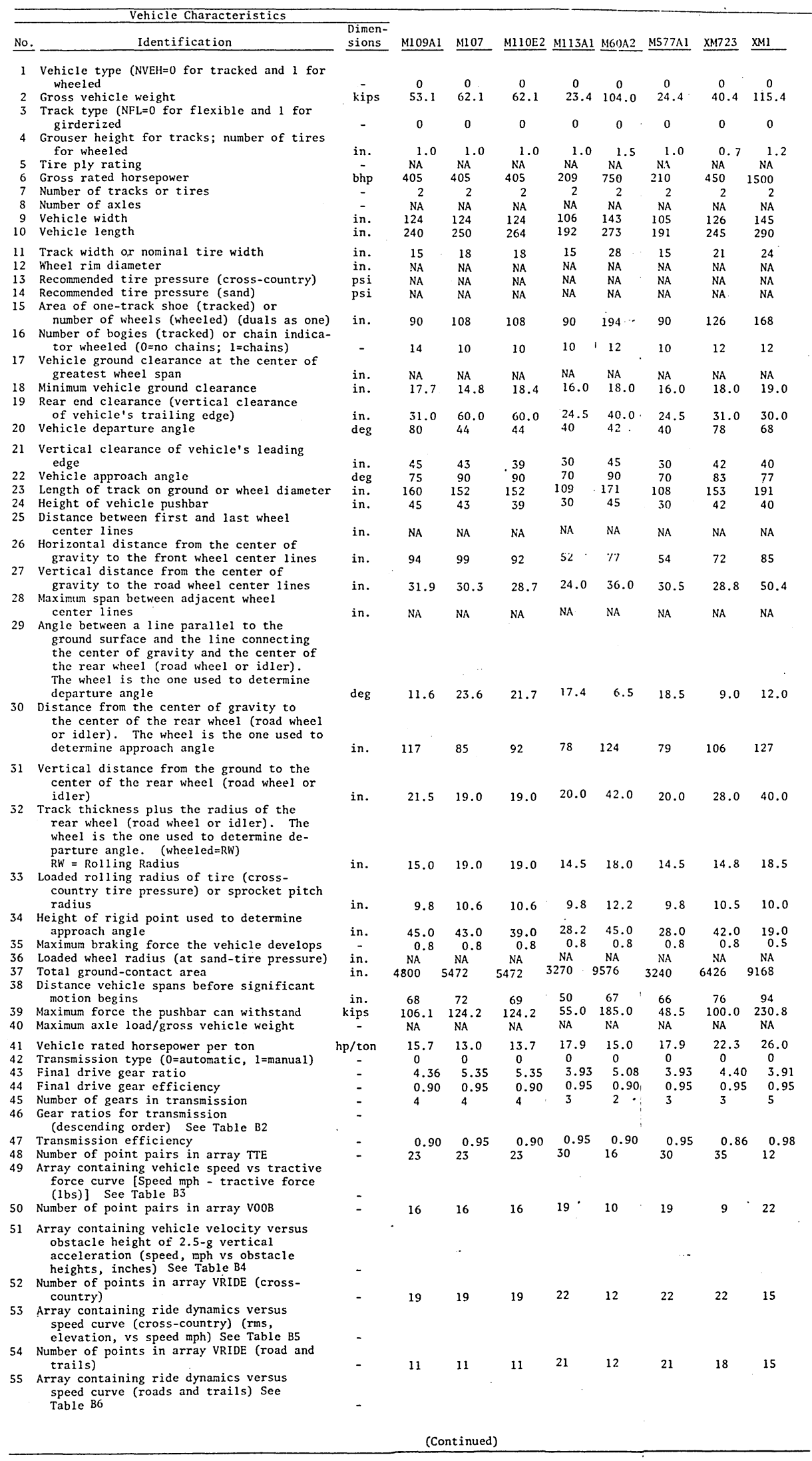

* NA'means not applicable.

(Sheet 6 of 6) 
Table B2

Gear Ratios for Study Vehicles

VEHICLE CHARACTERISTIC No. 46 in Table Bil

\begin{tabular}{|c|c|c|c|c|c|c|c|c|c|c|}
\hline \multirow{2}{*}{$\frac{\text { Vehicle }}{\text { M561 }}$} & \multicolumn{10}{|c|}{ Gear Ratios for Transmission } \\
\hline & 12.64 & 7.06 & 6.41 & 3.58 & 3.06 & 1.79 & 1.71 & 1.00 & & \\
\hline M35A2 & 9.94 & 5.50 & 5.02 & 3.21 & 2.78 & 1.98 & 1.62 & 1.56 & 1.00 & 0.79 \\
\hline M813 & 12.29 & 6.88 & 6.07 & 3.62 & 3.40 & 2.02 & 1.79 & 1.58 & 1.00 & 0.78 \\
\hline M656 & 5.49 & 3.95 & 2.79 & 2.01 & 1.44 & 1.04 & & & & \\
\hline TDW901 & 11.18 & 8.04 & 4.98 & 3.58 & 2.91 & 2.09 & 1.93 & 1.39 & 1.39 & 1.00 \\
\hline M520E 1 & 5.22 & 3.01 & 2.30 & 1.73 & 1.33 & 1.00 & & & & \\
\hline M125E1 & 4.00 & 2.28 & 2.00 & 1.41 & 1.00 & 0.71 & & & & \\
\hline $\mathrm{M} 548 \mathrm{E} 1$ & 3.81 & 1.94 & 1.00 & & & & & & & \\
\hline UET & 4.00 & 2.82 & 2.00 & 1.91 & 1.00 & & & & & \\
\hline ASV-ARMORED & - & - & - & & & & & & & \\
\hline M109A1 & 4.69 & 3.18 & 1.59 & 0.79 & & & & & & \\
\hline M107 & 4.69 & 3.18 & 1.59 & 0.79 & & & & & & \\
\hline M110E2 & 4.69 & 3.18 & 1.59 & 0.79 & & & & & & \\
\hline $\mathrm{M} 113 \mathrm{Al}$ & 3.81 & 1.94 & 1.00 & & & & & & & \\
\hline M60A2 & 3.50 & 1.26 & & & & & & & & \\
\hline M577A1 & 3.81 & 1.94 & 1.00 & & & & & & & \\
\hline XM723 & - & - & - & & & & & & & \\
\hline $\mathrm{XMI}$ & - & - & - & & & & & & & \\
\hline
\end{tabular}


TabTe B3

Tractive Force-Speed Relations for Vehicle Characteristic No. 49 in Table B1

\begin{tabular}{|c|c|c|c|c|c|c|c|c|c|c|c|}
\hline \multicolumn{2}{|c|}{ M561 } & \multicolumn{2}{|c|}{ M35A2 } & \multicolumn{2}{|c|}{ M813 } & \multicolumn{2}{|c|}{ M656 } & \multicolumn{2}{|c|}{ TDW901 } & \multicolumn{2}{|c|}{ M520E1 } \\
\hline $\begin{array}{l}\text { Vehicle } \\
\text { Speed } \\
\text { mph }\end{array}$ & $\begin{array}{c}\text { Tractive } \\
\text { Force } \\
\text { 1b }\end{array}$ & $\begin{array}{c}\text { Vehicle } \\
\text { Speed } \\
\text { mph } \\
\end{array}$ & $\begin{array}{l}\text { Tractive } \\
\text { Force } \\
\text { lb } \\
\end{array}$ & $\begin{array}{c}\text { Vhicle } \\
\text { Speed } \\
\text { mph } \\
\end{array}$ & $\begin{array}{l}\text { Tractive } \\
\text { Force } \\
\text { lb } \\
\end{array}$ & $\begin{array}{c}\text { Vhicle } \\
\text { Speed } \\
\text { mph } \\
\end{array}$ & $\begin{array}{l}\text { Tractive } \\
\text { Force } \\
\text { 1b } \\
\end{array}$ & $\begin{array}{c}\text { Vhicle } \\
\text { Speed } \\
\text { mph } \\
\end{array}$ & $\begin{array}{c}\text { Tractive } \\
\text { Force } \\
1 \mathrm{~b} \\
\end{array}$ & $\begin{array}{c}\text { Vehicle } \\
\text { Speed } \\
\text { mph } \\
\end{array}$ & $\begin{array}{c}\text { Tractive } \\
\text { Force } \\
1 \mathrm{~b} \\
\end{array}$ \\
\hline 0.0 & 8,520 & 0.0 & 14,013 & 0.0 & 25,540 & 0.0 & 21,600 & 0.0 & 25,000 & 0.0 & 38,500 \\
\hline 3.4 & 8,510 & 2.2 & 13,899 & 2.0 & 25,440 & 1.0 & 20,460 & 1.5 & 21,000 & 0.4 & 36,500 \\
\hline 4.0 & 8,160 & 3.2 & 12,991 & 2.4 & 25,190 & 2.6 & 16,460 & 2.5 & 17,300 & 0.9 & 32,500 \\
\hline 4.4 & 7,660 & 4.3 & 11,174 & 2.6 & 24,440 & 4.0 & 13,500 & 4.0 & 12,000 & 1.2 & 28,500 \\
\hline 4.5 & 6,940 & 4.4 & 7,824 & 3.1 & 21,440 & 6.2 & 9,710 & 5.0 & 10,400 & 1.6 & 24,500 \\
\hline 4.6 & 4,770 & 5.4 & 7,484 & 3.2 & 14,590 & 6.3 & 8,000 & 7.5 & 7,000 & 2.1 & 20,500 \\
\hline 5.2 & 4,760 & 6.6 & 6,973 & 4.0 & 14,540 & 7.2 & 7,920 & 10.0 & 5,000 & 2.9 & 16,500 \\
\hline 6.3 & 4,565 & 8.0 & 6,325 & 4.9 & 13,540 & 7.6 & 7,420 & 12.5 & 4,000 & 3.9 & 12,500 \\
\hline 7.0 & 4,375 & 8.1 & 6,098 & 5.5 & 12,750 & 7.7 & 6,820 & 15.0 & 3,300 & 4.7 & 9,700 \\
\hline 7.3 & 4,030 & 8.6 & 5,769 & 5.6 & 11,750 & 10.0 & 5,840 & 20.0 & 2,250 & 5.6 & 8,570 \\
\hline 7.8 & 3.660 & 8.7 & 4,633 & 6.3 & 10,860 & 12.2 & 5,050 & 25.0 & 2,000 & 7.4 & 6,830 \\
\hline 8.3 & 3,085 & 10.6 & 4,486 & 6.4 & 7,860 & 12.3 & 4,150 & 30.0 & 1,750 & 8.4 & 5,940 \\
\hline 8.4 & 2,500 & 12.2 & 4,168 & 7.6 & 7,830 & 13.8 & 4,050 & 35.0 & 1,600 & 10.0 & 5,750 \\
\hline 11.7 & 2,490 & 13.8 & 3,736 & 9.0 & 7,500 & 16.0 & 3,860 & 40.0 & 1,500 & 11.1 & 5,550 \\
\hline 12.8 & 2,470 & 13.9 & 3,566 & 11.0 & 6,730 & 16.1 & 3,260 & 45.0 & 1,250 & 12.1 & 5,060 \\
\hline 14.2 & 2,315 & 15.9 & 3,248 & 11.1 & 6,630 & 20.0 & 3,180 & 50.0 & 1,200 & 12.6 & 4,370 \\
\hline 15.0 & 2,130 & 16.0 & 2,850 & 11.6 & 6,340 & 22.0 & 2,990 & 55.0 & 1,000 & 14.0 & 4,280 \\
\hline 16.0 & 2,035 & 18.4 & 2,714 & 11.7 & 4,690 & 24.0 & 2,600 & 55.0 & - & 15.0 & 4,100 \\
\hline 16.9 & 1,930 & 21.1 & 2,521 & 13.7 & 4,670 & 27.9 & 2,280 & & & 16.0 & 3.810 \\
\hline 17.8 & 1,730 & 23.1 & 2,351 & 16.0 & 4,570 & 33.0 & 1,850 & & & 16.9 & 3,220 \\
\hline 17.9 & 1,270 & 23.2 & 2,181 & 19.7 & 4,060 & 33.1 & 1,700 & & & 18.6 & 3,140 \\
\hline 24.4 & 1,265 & 25.0 & 2,124 & 19.8 & 3,960 & 38.7 & 1,650 & & & 20.0 & 2,850 \\
\hline 27.5 & 1,170 & 28.2 & 1,840 & 22.5 & 3,600 & 50.0 & 1,200 & & & 21.7 & 2,560 \\
\hline 30.0 & 1,095 & 28.3 & 1,783 & 22.6 & 3,500 & 50.0 & -- & & & 24.0 & 2,480 \\
\hline 31.4 & 1,010 & 29.1 & 1,726 & 25.4 & 3,220 & & & & & 26.0 & 2,300 \\
\hline 31.5 & 740 & 29.2 & 1,385 & 25.5 & 2,420 & & & & & 28.0 & 1,940 \\
\hline 42.0 & 730 & 36.0 & 1,374 & 28.6 & 2,410 & & & . & & 30.0 & 1,600 \\
\hline 47.0 & 685 & 42.0 & 1,272 & 35.4 & 2,210 & & & & & 30.0 & -- \\
\hline 55.0 & 640 & 45.0 & 1,102 & 40.3 & 2,010 & & & & & & \\
\hline 55.0 & -- & 45.1 & 1,090 & 40.4 & 1,860 & & & & & & \\
\hline & & 51.0 & 1,034 & 45.4 & 1,730 & & & & & & \\
\hline & & 53.0 & 1,011 & 50.0 & 1,610 & & & & & & \\
\hline & & 58.0 & 919 & 50.0 & - & & & & & & \\
\hline & & 58.0 & & & & & & & & & \\
\hline
\end{tabular}

(Continued)

(Sheet 1 of 3 ) 
Table B3 (Continued)

\begin{tabular}{|c|c|c|c|c|c|c|c|c|c|c|c|}
\hline \multicolumn{2}{|c|}{$\mathrm{M} 125 \mathrm{El}$} & \multicolumn{2}{|c|}{$\mathrm{M} 548 \mathrm{El}$} & \multicolumn{2}{|c|}{ UET } & \multicolumn{2}{|c|}{ ASV $-\Lambda R M O R E D$} & \multicolumn{2}{|c|}{ MI09A1 } & \multicolumn{2}{|c|}{ M107 } \\
\hline $\begin{array}{c}\text { Vehicle } \\
\text { Speed } \\
\text { mph }\end{array}$ & $\begin{array}{c}\text { Tractive } \\
\text { Force } \\
\text { lb } \\
\end{array}$ & $\begin{array}{c}\text { Vehicle } \\
\text { Speed } \\
\text { mph } \\
\end{array}$ & $\begin{array}{l}\text { Tractive } \\
\text { Force } \\
\text { Ib } \\
\end{array}$ & $\begin{array}{c}\text { Vehicle } \\
\text { Speed } \\
\text { mph } \\
\end{array}$ & $\begin{array}{l}\text { Tractive } \\
\text { Force } \\
\text { lb } \\
\end{array}$ & $\begin{array}{c}\text { Vehicle } \\
\text { Speed } \\
\text { mph } \\
\end{array}$ & $\begin{array}{l}\text { Tractive } \\
\text { Force } \\
\text { 1b. } \\
\end{array}$ & $\begin{array}{c}\text { Vohiclo } \\
\text { Speed } \\
\text { mph } \\
\end{array}$ & $\begin{array}{l}\text { Tractive } \\
\text { Force } \\
1 \mathrm{~b} \\
\end{array}$ & $\begin{array}{c}\text { Vehicle } \\
\text { Speed } \\
\text { mph } \\
\end{array}$ & $\begin{array}{c}\text { Tractive } \\
\text { Force } \\
1 b \\
\end{array}$ \\
\hline 0.0 & 32,545 & 0.0 & 19,750 & 0.0 & 47,328 & 0.0 & 49,801 & 0.0 & 41,104 & 0.0 & 46,440 \\
\hline 1.9 & 32,345 & 0.8 & 19,650 & 0.5 & 41,961 & 0.1 & 49,801 & 0.8 & 41,015 & 0.8 & 46,340 \\
\hline 2.2 & 31,945 & 1.5 & 17,050 & 1.0 & 35,624 & 0.3 & 43,350 & 1.3 & 37,033 & 1.3 & 41,840 \\
\hline 2.4 & 30,545 & 2.6 & 13,050 & 1.5 & 29,367 & 0.5 & 42,689 & 2.2 & 28,182 & 2.2 & 31,840 \\
\hline 2.8 & 28,045 & 3.3 & 11,050 & 2.0 & 22,872 & 0.7 & 39,842 & 3.2 & 20,782 & 3.2 & 23,480 \\
\hline 3.2 & 25,545 & 4.2 & 9,050 & 2.5 & 21,471 & 0.9 & 38,308 & 3.7 & 20,711 & 3.7 & 23,400 \\
\hline 3.3 & 24,245 & 5.5 & 6,580 & 3.0 & 20,096 & 1.2 & 36,001 & 4.3 & 19,932 & 4.3 & 22,520 \\
\hline 3.4 & 19,045 & 6.4 & 6,565 & 3.5 & 17,492 & 1.5 & 32,260 & 5.5 & 15,015 & 5.5 & 16,965 \\
\hline 3.6 & 18,945 & 7.2 & 6,470 & 4.0 & 15,513 & 2.1 & 27,889 & 5.6 & 14,312 & 5.6 & 16,170 \\
\hline 4.0 & 18,545 & 8.0 & 6,080 & 4.5 & 15,658 & 2.8 & 23,704 & 6.4 & 13,356 & 6.4 & 15,090 \\
\hline 4.3 & 18,045 & 9.4 & 5,190 & 5.0 & 15,373 & 3.2 & 22,133 & 7.4 & 11,532 & 7.4 & 13,030 \\
\hline 5.0 & 16,445 & 10.0 & 4,700 & 5.5 & 14,554 & 3.7 & 20,074 & 8.2 & 10,312 & 8.2 & 11,650 \\
\hline 5.6 & 14,755 & 10.1 & 3,800 & 6.0 & 10,550 & 4.3 & 17,929 & 8.3 & 8,634 & 8.3 & 9,755 \\
\hline 5.7 & 11,860 & 13.3 & 3,775 & 6.5 & 11,188 & 5.2 & 15,172 & 10.3 & 6,780 & 10.3 & 7,660 \\
\hline 6.0 & 11,655 & 14.8 & 3,740 & 7.0 & 10,899 & 6.3 & 12,712 & 12.0 & 6,718 & 12.0 & 7,590 \\
\hline 6.6 & 11,575 & 16.0 & 3,650 & 7.5 & 10,515 & 6.5 & 10,261 & 14.1 & 6,293 & 14.1 & 7,110 \\
\hline 7.4 & 11,085 & 18.0 & 3,270 & 8.0 & 10,070 & 7.4 & 9,669 & 16.0 & 5,155 & 16.0 & 5,825 \\
\hline 8.1 & 10,595 & 19.3 & 3,030 & 8.5 & 7,508 & 8.6 & 8,723 & 16.1 & 4,452 & 16.1 & 5,030 \\
\hline 8.9 & 9,605 & 21.8 & 2,605 & 9.0 & 7,828 & 9.9 & 7,968 & 20.2 & 3,363 & 20.2 & 3,800 \\
\hline 9.5 & 9,115 & 24.0 & 2,310 & 10.0 & 7,686 & 11.4 & 7,295 & 24.0 & 3,355 & 24.0 & 3,790 \\
\hline 10.0 & 8,625 & 26.0 & 2,160 & 11.0 & 7,277 & 14.2 & 6,177 & 28.2 & 3,142 & 28.2 & 3,550 \\
\hline 10.1 & 7,625 & 28.0 & 2,110 & 11.5 & 7,036 & 17,0 & 5,313 & 32.0 & 2,576 & 32.0 & 2,910 \\
\hline 10.6 & 7,535 & 32.0 & 2,010 & 12.0 & 6,738 & 21.3 & 4,314 & 32.0 & - & 32.0 & -- \\
\hline 11.6 & 7,355 & 36.0 & 1,985 & 12.5 & 4,907 & 23.3 & 3,960 & & & & \\
\hline 13.0 & 6,985 & 40.0 & 1,960 & 13.5 & 4,539 & 25.6 & 3,597 & & & & \\
\hline 14.2 & 6,510 & 40.0 & -- & 14.5 & 4,220 & 27.5 & 3,306 & & & & \\
\hline 15.0 & 6,230 & & & 15.5 & 3,995 & 29.8 & 2,986 & & & & \\
\hline 16.6 & 5,360 & & & 17.5 & 3,834 & 31.4 & 2,928 & & & & \\
\hline 16.7 & 4,360 & & & 20.5 & 3,794 & 34.1 & 2,780 & & $\therefore$ & & \\
\hline 18.0 & 4,290 & & & 22.0 & 3,639 & 35.5 & 2,694 & & & & \\
\hline 20.0 & 4,030 & & & 23.0 & 3,518 & 36.9 & 2,589 & & & & \\
\hline 22.7 & 3,580 & & & 23.5 & 2,641 & 38.3 & 2,484 & & & & \\
\hline 25.6 & 3,095 & & & 25.0 & 2,751 & 41.2 & 2,255 & & & & \\
\hline 27.3 & 2,095 & & & 28.0 & 2,736 & 43.7 & 2,025 & & & & \\
\hline 27.4 & 2,425 & & & 31.0 & 2,582 & 43.7 & -- & & i & & \\
\hline 30.0 & 2,420 & & & 32.5 & 2,492 & & & & & & \\
\hline 34.0 & 2,415 & & & 32.5 & -- & & & & & & \\
\hline 38.0 & 2,410 & & & & & & & & & & \\
\hline 41.2 & 2,380 & & & & & & & & & (Sheet & 2 of 3 ) \\
\hline 41.2 & -- & & & & & & & & & & \\
\hline
\end{tabular}


Table BJ (Concluded)

\begin{tabular}{|c|c|c|c|c|c|c|c|c|c|c|c|}
\hline \multicolumn{2}{|c|}{ M110E2 } & \multicolumn{2}{|c|}{ MI 13AI } & \multicolumn{2}{|c|}{ M60A2 } & \multicolumn{2}{|c|}{ M577A } & \multicolumn{2}{|c|}{$\mathrm{XM723}$} & \multicolumn{2}{|c|}{ XMI } \\
\hline $\begin{array}{c}\text { Vehicle } \\
\text { Speed } \\
\text { mph } \\
\end{array}$ & $\begin{array}{l}\text { Tractive } \\
\text { Force } \\
\text { lb } \\
\end{array}$ & $\begin{array}{c}\text { Vehicle } \\
\text { Speed } \\
\text { mph }\end{array}$ & $\begin{array}{l}\text { Tractive } \\
\text { Force } \\
\text { Ib } \\
\end{array}$ & $\begin{array}{c}\text { Vehicle } \\
\text { Speed } \\
\text { mph }\end{array}$ & $\begin{array}{c}\text { Tractive } \\
\text { Force } \\
1 \mathrm{~b} \\
\end{array}$ & $\begin{array}{c}\text { Vehicle } \\
\text { Speed } \\
\text { mph } \\
\end{array}$ & $\begin{array}{l}\text { Tractive } \\
\text { Force } \\
1 \mathrm{~b} \\
\end{array}$ & $\begin{array}{c}\text { Vehicle } \\
\text { Speed } \\
\text { mph } \\
\end{array}$ & $\begin{array}{l}\text { Tractive } \\
\text { Force } \\
1 \mathrm{~b} \\
\end{array}$ & $\begin{array}{c}\text { Vehicle } \\
\text { Speed } \\
\text { mph }\end{array}$ & $\begin{array}{l}\text { Tractive } \\
\text { Force } \\
\text { lb } \\
\end{array}$ \\
\hline $\begin{array}{r}0.0 \\
0.8 \\
1.3 \\
2.2 \\
3.2 \\
3.7 \\
4.3 \\
5.5 \\
5.6 \\
6.4 \\
7.4 \\
8.2 \\
8.3 \\
10.3 \\
12.0 \\
14.1 \\
16.0 \\
16.1 \\
20.2 \\
24.0 \\
28.2 \\
32.0 \\
32.0\end{array}$ & $\begin{array}{c}46,440 \\
46,340 \\
41,840 \\
31,840 \\
23,480 \\
23,400 \\
22,520 \\
16,965 \\
16,170 \\
15,090 \\
13,030 \\
11,650 \\
9,755 \\
7,660 \\
7,590 \\
7,110 \\
5,825 \\
5,030 \\
3,800 \\
3,790 \\
3,550 \\
2,910 \\
-.\end{array}$ & $\begin{array}{r}0.0 \\
1.0 \\
1.5 \\
1.9 \\
1.9 \\
2.0 \\
2.5 \\
3.2 \\
3.9 \\
4.8 \\
5.8 \\
5.9 \\
7.5 \\
8.0 \\
9.5 \\
10.8 \\
10.9 \\
12.0 \\
13.1 \\
15.0 \\
17.1 \\
19.2 \\
21.3 \\
21.4 \\
25.3 \\
29.0 \\
33.0 \\
37.1 \\
42.0 \\
42.0\end{array}$ & $\begin{array}{r}17,950 \\
16,330 \\
15,850 \\
15,800 \\
14,250 \\
12,750 \\
11,250 \\
9,750 \\
8,770 \\
8,030 \\
7,380 \\
6,990 \\
6,975 \\
6,650 \\
6,050 \\
5,300 \\
4,100 \\
3,700 \\
3,500 \\
3,450 \\
3,300 \\
3,000 \\
2,500 \\
1,850 \\
1,815 \\
1,785 \\
1,710 \\
1,550 \\
1,300 \\
--\end{array}$ & $\begin{array}{l}0.0 \\
1.4 \\
2.3 \\
3.5 \\
4.5 \\
5.5 \\
6.8 \\
8.0 \\
12.0 \\
16.0 \\
20.0 \\
24.0 \\
26.0 \\
28.0 \\
30.0 \\
30.0\end{array}$ & $\begin{array}{c}72,790 \\
62,800 \\
52,850 \\
42,910 \\
38,000 \\
33,020 \\
28,100 \\
23,200 \\
14,600 \\
10,800 \\
9,100 \\
7,100 \\
6,700 \\
6,000 \\
3,000 \\
--\end{array}$ & $\begin{array}{r}0.0 \\
1.0 \\
1.5 \\
1.9 \\
1.9 \\
2.0 \\
2.5 \\
3.2 \\
3.9 \\
4.8 \\
5.8 \\
5.9 \\
7.5 \\
8.0 \\
9.5 \\
10.8 \\
10.9 \\
12.0 \\
13.1 \\
15.0 \\
17.1 \\
19.2 \\
21.3 \\
21.4 \\
25.3 \\
29.0 \\
33.0 \\
37.1 \\
42.0 \\
42.0\end{array}$ & $\begin{array}{r}17,950 \\
16,330 \\
15,850 \\
15,800 \\
14,250 \\
12,750 \\
11,250 \\
9,750 \\
8,770 \\
8,030 \\
7,380 \\
6,990 \\
6,975 \\
6,650 \\
6,050 \\
5,300 \\
4,100 \\
3,700 \\
3,500 \\
3,450 \\
3,300 \\
3,000 \\
2,500 \\
1,850 \\
1,815 \\
1,785 \\
1,710 \\
1,550 \\
1,300 \\
--\end{array}$ & $\begin{array}{r}0.0 \\
0.1 \\
0.3 \\
0.5 \\
0.7 \\
0.9 \\
1.2 \\
1.5 \\
2.1 \\
2.8 \\
3.2 \\
3.7 \\
4.3 \\
5.2 \\
6.3 \\
6.5 \\
7.3 \\
8.6 \\
9.9 \\
11.4 \\
14.2 \\
17.0 \\
21.3 \\
23.3 \\
25.6 \\
27.5 \\
29.8 \\
31.4 \\
34.1 \\
35.5 \\
36.9 \\
38.3 \\
41.2 \\
43.7 \\
43.7\end{array}$ & $\begin{array}{r}49,801 \\
49,801 \\
43,350 \\
42,689 \\
39,842 \\
38,308 \\
36,001 \\
32,260 \\
27,889 \\
23,704 \\
22,133 \\
20,074 \\
17,929 \\
15,172 \\
12,712 \\
10,261 \\
9,669 \\
8,723 \\
7,968 \\
7,295 \\
6,177 \\
5,313 \\
4,314 \\
3,960 \\
3,597 \\
3,306 \\
2,986 \\
2,928 \\
2,780 \\
2,694 \\
2,589 \\
2,484 \\
2,255 \\
2,025 \\
--\end{array}$ & $\begin{array}{r}0.0 \\
4.0 \\
8.0 \\
12.0 \\
16.0 \\
20.0 \\
24.0 \\
28.0 \\
32.0 \\
40.0 \\
48.0 \\
48.0\end{array}$ & $\begin{array}{r}103,968 \\
75,240 \\
51,984 \\
30,436 \\
25,650 \\
17,784 \\
17,100 \\
15,048 \\
13,680 \\
10,944 \\
9,576 \\
--\end{array}$ \\
\hline
\end{tabular}


Table B4

Obstacle Height-Speed Relations for Vehicle Characteristic No. 51 in Table Bl

\begin{tabular}{|c|c|c|c|c|c|c|c|c|c|c|c|}
\hline \multicolumn{2}{|c|}{ M561 } & \multicolumn{2}{|c|}{ M35A2 } & \multicolumn{2}{|c|}{ M813 } & \multicolumn{2}{|c|}{ M656 } & \multicolumn{2}{|c|}{ TDW901 } & \multicolumn{2}{|c|}{$\mathrm{M} 520 \mathrm{E} 1$} \\
\hline $\begin{array}{l}\text { Obstacle } \\
\text { Height } \\
\text { in. } \\
\end{array}$ & $\begin{array}{c}\text { Vehicle } \\
\text { Speed } \\
\text { mph } \\
\end{array}$ & $\begin{array}{c}\text { Obstacle } \\
\text { Height } \\
\text { in. } \\
\end{array}$ & $\begin{array}{c}\text { Vehicle } \\
\text { Speed } \\
\text { mph } \\
\end{array}$ & $\begin{array}{c}\text { Obstacle } \\
\text { Height } \\
\text { in. } \\
\end{array}$ & $\begin{array}{c}\text { Vehicle } \\
\text { Speed } \\
\text { mph } \\
\end{array}$ & $\begin{array}{c}\text { Obstacle } \\
\text { Height } \\
\text { in. } \\
\end{array}$ & $\begin{array}{c}\text { Vehicle } \\
\text { Speed } \\
\text { mph } \\
\end{array}$ & $\begin{array}{c}\text { Obstacle } \\
\text { Height } \\
\text { in. } \\
\end{array}$ & $\begin{array}{c}\text { Vehicle } \\
\text { Speed } \\
\text { mph } \\
\end{array}$ & $\begin{array}{c}\text { Obstacle } \\
\text { Height } \\
\text { in. } \\
\end{array}$ & $\begin{array}{c}\text { Vehicle } \\
\text { Speed } \\
\text { mph } \\
\end{array}$ \\
\hline 0.0 & 100.0 & 0.0 & 100.0 & 0.0 & 100.0 & 0.0 & 50.0 & 0.0 & 55.0 & 0.0 & 100.0 \\
\hline 4.5 & 100.0 & 3.7 & 100.0 & 4.5 & 100.0 & 0.1 & 50.0 & 0.1 & 55.0 & 5.5 & 100.0 \\
\hline 4.5 & 40.0 & 4.2 & 80.0 & 5.0 & 80.0 & 4.4 & 50.0 & 5.9 & 55.0 & 5.5 & 60.0 \\
\hline 5.0 & 30.0 & 4.8 & 56.0 & 5.5 & 60.0 & 4.6 & 40.0 & 6.0 & 48.0 & 6.0 & 34.0 \\
\hline 6.0 & 18.0 & 5.0 & 52.0 & 6.0 & 40.0 & 5.0 & 33.0 & 6.4 & 40.0 & 6.5 & 20.0 \\
\hline 8.0 & 10.0 & 5.8 & 40.0 & 7.0 & 22.0 & 5.6 & 26.0 & 7.0 & 33.0 & 7.0 & 16.0 \\
\hline 10.0 & 8.0 & 6.7 & 30.0 & 8.0 & 16.0 & 6.0 & 22.3 & 7.4 & 30.0 & 8.0 & 8.0 \\
\hline 15.0 & 5.0 & 8.4 & 20.0 & 9.0 & 10.0 & 7.0 & 17.2 & 8.0 & 26.0 & 10.0 & 4.0 \\
\hline 20.0 & 4.0 & 10.0 & 13.0 & 10.0 & 7.0 & 8.0 & 14.0 & 9.0 & 22.0 & 15.0 & 2.0 \\
\hline 30.0 & 3.0 & 11.5 & 10.0 & 12.5 & 4.0 & 10.0 & 9.7 & 10.0 & 18.2 & 25.0 & 2.0 \\
\hline 50.0 & 3.0 & 15.0 & 6.0 & 15.0 & 2.8 & 12.0 & 7.2 & 12.0 & 13.0 & 50.0 & 1.0 \\
\hline & & 20.0 & 3.2 & 20.0 & 2.0 & 14.0 & 5.7 & 14.0 & 9.8 & & \\
\hline & & 25.0 & 2.0 & 25.0 & 1.0 & 16.0 & 4.7 & 16.0 & 8.7 & & \\
\hline & & 50.0 & 0.6 & 50.0 & 1.0 & 18.0 & 3.8 & 18.0 & 8.0 & & \\
\hline & & & & & & 50.0 & 0.6 & 50.0 & 0.6 & & \\
\hline
\end{tabular}


Table B4 (Continued)

\begin{tabular}{|c|c|c|c|c|c|c|c|c|c|c|c|}
\hline \multicolumn{2}{|c|}{ M125E I } & \multicolumn{2}{|c|}{$\mathrm{M} 548 \mathrm{E} 1$} & \multicolumn{2}{|c|}{ UET } & \multicolumn{2}{|c|}{ ASV-ARMORED } & \multicolumn{2}{|c|}{ M109Al } & \multicolumn{2}{|c|}{ M107 } \\
\hline $\begin{array}{l}\text { Obstacle } \\
\text { Height } \\
\text { in. }\end{array}$ & $\begin{array}{c}\text { Vehicle } \\
\text { Speed } \\
\text { mph }\end{array}$ & $\begin{array}{c}\text { Obstacle } \\
\text { Height } \\
\text { in. } \\
\end{array}$ & $\begin{array}{c}\text { Vehicle } \\
\text { Speed } \\
\text { mph }\end{array}$ & $\begin{array}{c}\text { Obstacle } \\
\text { Height } \\
\text { in. } \\
\end{array}$ & $\begin{array}{c}\text { Vehicle } \\
\text { Speed } \\
\text { mph } \\
\end{array}$ & $\begin{array}{c}\text { Obstacle } \\
\text { Height } \\
\text { in. } \\
\end{array}$ & $\begin{array}{c}\text { Vehicle } \\
\text { Speed } \\
\text { mph } \\
\end{array}$ & $\begin{array}{c}\text { Obstacle } \\
\text { Height } \\
\text { in. } \\
\end{array}$ & $\begin{array}{c}\text { Vehicle } \\
\text { Speed } \\
\text { mph } \\
\end{array}$ & $\begin{array}{c}\text { Obstacle } \\
\text { Height } \\
\text { in. } \\
\end{array}$ & $\begin{array}{c}\text { Vehicle } \\
\text { Speed } \\
\text { mph } \\
\end{array}$ \\
\hline 0.0 & 100.0 & 0.0 & 100.0 & 0.0 & 100.0 & 0.0 & 100.0 & 0.0 & 100.0 & 0.0 & 100.0 \\
\hline 4.1 & 100.0 & 5.5 & 100.0 & 12.8 & 30.0 & 7.4 & 48.0 & 4.1 & 100.0 & 4.1 & 100.0 \\
\hline 5.0 & 86.0 & 5.8 & 60.0 & 13.0 & 25.0 & 7.8 & 40.0 & 4.1 & 35.0 & 4.1 & 35.0 \\
\hline 5.2 & 70.0 & 6.0 & 40.0 & 13.6 & 20.0 & 8.4 & 30.0 & 4.2 & 30.0 & 4.2 & 30.0 \\
\hline 5.4 & 60.0 & 6.5 & 20.0 & 14.9 & 15.0 & 9.2 & 20.0 & 4.3 & 27.5 & 4.3 & 27.5 \\
\hline 5.7 & 50.0 & 7.5 & 11.0 & 16.0 & 11.8 & 10.0 & 13.5 & 4.6 & 24.5 & 4.6 & 24.5 \\
\hline 6.0 & 40.0 & 8.0 & 9.0 & 17.0 & 10.0 & 12.0 & 10.0 & 4.9 & 21.5 & 4.9 & 21.5 \\
\hline 6.5 & 30.0 & 9.0 & 8.5 & 17.7 & 9.6 & 20.0 & 8.0 & 5.3 & 19.5 & 5.3 & 19.5 \\
\hline 7.5 & 20.0 & 10.0 & 7.0 & 20.0 & 9.0 & 50.0 & 2.0 & 5.7 & 18.0 & 5.7 & 18.0 \\
\hline 8.0 & 15.0 & 20.0 & 5.0 & 25.0 & 8.0 & & & 6.0 & 17.0 & 6.0 & 17.0 \\
\hline 9.0 & 10.0 & 30.0 & 4.0 & 30.0 & 7.3 & & & 7.0 & 15.5 & 7.0 & 15.5 \\
\hline 10.0 & 7.0 & 50.0 & 3.0 & 50.0 & 5.9 & & & 8.2 & 14.0 & 8.2 & 14.0 \\
\hline 11.0 & 6.0 & & & & & & & 9.4 & 13.0 & 9.4 & 13.0 \\
\hline 13.0 & 5.0 & & & & & . & & 10.2 & 12.5 & 10.2 & 12.5 \\
\hline 15.0 & 4.0 & & & & & & & 12.0 & 11.0 & 12.0 & 11.0 \\
\hline 20.0 & 3.0 & & & & & & & 50.0 & 0.0 & 50.0 & 0.0 \\
\hline 25.0 & 2.0 & & & & & & & & & & \\
\hline 30.0 & 1.5 & & & & & & & & & & \\
\hline 35.0 & 1.0 & & & & & & & & & & \\
\hline 50.0 & 0.6 & & & & & & & & & & \\
\hline
\end{tabular}


Table 34 (Concluded)

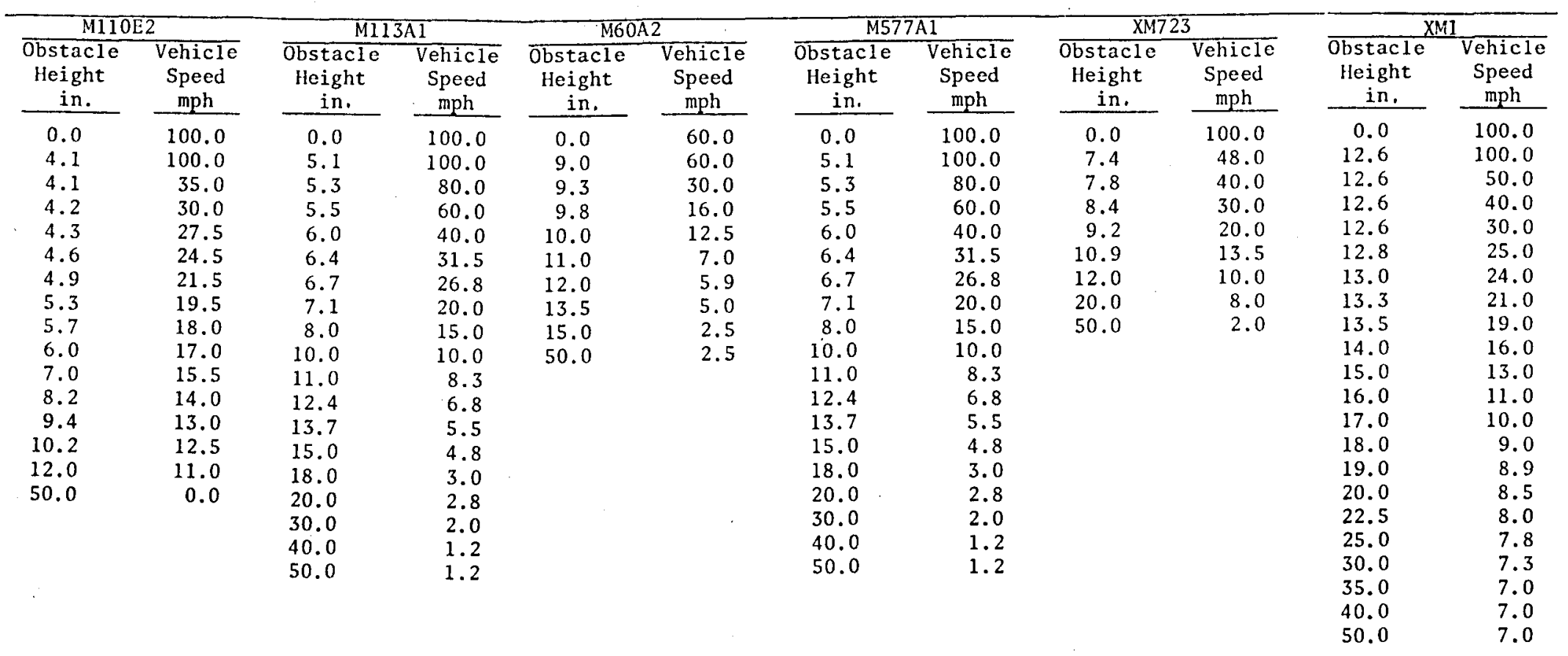


Table B5

Cross-Country Ride Versus Speed Relations for Vehicle Characteristic No. 53 in Table Bl

\begin{tabular}{|c|c|c|c|c|c|c|c|c|c|c|c|c|c|}
\hline \multicolumn{2}{|c|}{ M561 } & \multicolumn{2}{|c|}{ M35A2 } & \multicolumn{2}{|c|}{ M813 } & \multicolumn{2}{|c|}{ M656 } & \multicolumn{2}{|c|}{ TDW901 } & \multicolumn{2}{|c|}{ M520E1 } & \multicolumn{2}{|c|}{ M125E1 } \\
\hline $\begin{array}{l}\text { Ele- } \\
\text { vation } \\
\text { rms } \\
\text { in. } \\
\end{array}$ & $\begin{array}{c}\text { Speed } \\
\text { mph }\end{array}$ & $\begin{array}{l}\text { Ele- } \\
\text { vation } \\
\text { rms } \\
\text { in. } \\
\end{array}$ & $\begin{array}{c}\text { Speed } \\
\text { mph }\end{array}$ & $\begin{array}{l}\text { Ele- } \\
\text { vation } \\
\text { rms } \\
\text { in. } \\
\end{array}$ & $\begin{array}{c}\text { Speed } \\
\mathrm{mph}\end{array}$ & $\begin{array}{l}\text { Ele- } \\
\text { vation } \\
\text { rms } \\
\text { in. } \\
\end{array}$ & $\begin{array}{c}\text { Speed } \\
\text { mph }\end{array}$ & $\begin{array}{l}\text { Ele- } \\
\text { vation } \\
\text { rms } \\
\text { in. } \\
\end{array}$ & $\begin{array}{c}\text { Speed } \\
\mathrm{mph}\end{array}$ & $\begin{array}{l}\text { Ele- } \\
\text { vation } \\
\text { rms } \\
\text { in. } \\
\end{array}$ & $\begin{array}{c}\text { Speed } \\
\text { mph }\end{array}$ & $\begin{array}{l}\text { Ele- } \\
\text { vation } \\
\text { rms } \\
\text { in. } \\
\end{array}$ & $\begin{array}{c}\text { Speed } \\
\mathrm{mph}\end{array}$ \\
\hline 0 & 100.0 & 0 & 100.0 & 0 & 100.0 & 0 & 100.0 & 0 & 100.0 & 0 & 100.0 & 0 & 100.0 \\
\hline 0.15 & 60.0 & 0.23 & 80.0 & 0.20 & 100.0 & 0.24 & 80.0 & 0.24 & 80.0 & 0.10 & 100.0 & 0.18 & 100.0 \\
\hline 0.17 & 40.0 & 0.24 & 60.0 & 0.20 & 60.0 & 0.25 & 50.0 & 0.25 & 54.0 & 0.20 & 40.0 & 0.20 & 60.0 \\
\hline 0.25 & 30.0 & 0.25 & 40.0 & 0.23 & 50.0 & 0.34 & 30.0 & 0.30 & 40.0 & 0.30 & 24.0 & 0.30 & 38.0 \\
\hline 0.50 & 20.0 & 0.35 & 30.0 & 0.25 & 40.0 & 0.50 & 22.5 & 0.50 & 29.2 & 0.35 & 20.0 & 0.40 & 31.0 \\
\hline 1.00 & 15.5 & 0.46 & 25.0 & 0.28 & 35.0 & 0.75 & 19.6 & 0.75 & 24.1 & 0.50 & 14.0 & 0.60 & 24.0 \\
\hline 1.50 & 13.0 & 0.72 & 20.0 & 0.31 & 30.0 & 1.00 & 17.3 & 1.00 & 20.7 & 0.80 & 10.0 & 0.80 & 20.0 \\
\hline 2.00 & 11.5 & 1.00 & 16.6 & 0.34 & 28.0 & 2.00 & 10.0 & 1.50 & 15.5 & 1.20 & 8.0 & 1.05 & 16.0 \\
\hline 3.00 & 7.5 & 1.17 & 15.0 & 0.38 & 25.0 & 3.00 & 10.0 & 2.00 & 12.0 & 2.00 & 6.0 & 1.20 & 14.0 \\
\hline 8.00 & 6.5 & 2.00 & 10.0 & 0.47 & 22.0 & 8.00 & 10.0 & 3.00 & 12.0 & 8.00 & 5.5 & 1.70 & 10.0 \\
\hline & & 3.00 & 8.0 & 0.50 & 21.0 & & & 8.00 & 12.0 & & & 2.20 & 8.0 \\
\hline & & 8.00 & 6.0 & 0.55 & 20.0 & & & & & & & 3.00 & 7.0 \\
\hline & & & & 0.75 & 17.0 & & & & & & & 8.00 & 6.0 \\
\hline & & & & 0.85 & 16.0 & & & & & & & & \\
\hline & & & & 1.00 & 14.0 & & & & & & & & \\
\hline & & & & 1.25 & 12.0 & & & & & & & & \\
\hline & & & & 1.50 & 10.0 & & & & & & & & \\
\hline & & & & 1.75 & . 9.0 & & & & & & & & \\
\hline & & & & 2.00 & 8.0 & & & & & & & & \\
\hline & & & & 2.25 & 7.0 & & & & & & & & \\
\hline & & & & 2.50 & 6.5 & & & & & & & & \\
\hline & & & & 3.00 & 5.0 & & & & & & & & \\
\hline & & & & 4.00 & 5.0 & & & & & & & & \\
\hline & & & & 8.00 & 5.0 & & & & & & & & \\
\hline & & & & & & (Cont) & & & & & & (Sheet & of 3 ) \\
\hline
\end{tabular}


Table B5 (Continued)

\begin{tabular}{|c|c|c|c|c|c|c|c|c|c|c|c|}
\hline \multicolumn{2}{|c|}{ vis 4001} & \multicolumn{2}{|c|}{ UET } & \multicolumn{2}{|c|}{ ASV-Armorei } & \multicolumn{2}{|c|}{ M109Al } & \multicolumn{2}{|c|}{ M107 } & \multicolumn{2}{|c|}{ M110E2 } \\
\hline $\begin{array}{l}\text { Ele- } \\
\text { vation } \\
\text { rms } \\
\text { in. }\end{array}$ & $\begin{array}{l}\text { Speed } \\
\text { mph }\end{array}$ & $\begin{array}{c}\text { vation } \\
\text { rms } \\
\text { in. }\end{array}$ & $\begin{array}{c}\text { Speed } \\
\text { mph }\end{array}$ & $\begin{array}{l}\text { Ele- } \\
\text { vation } \\
\text { rms } \\
\text { in. }\end{array}$ & $\begin{array}{c}\text { Speed } \\
\mathrm{mph} \\
\end{array}$ & $\begin{array}{l}\text { Ele- } \\
\text { vation } \\
\text { rms } \\
\text { in. } \\
\end{array}$ & $\begin{array}{l}\text { Speed } \\
\text { mph } \\
\end{array}$ & $\begin{array}{l}\text { Ele- } \\
\text { vation } \\
\text { rms } \\
\text { in. } \\
\end{array}$ & $\begin{array}{c}\text { Speed } \\
\text { mph }\end{array}$ & $\begin{array}{l}\text { Ele- } \\
\text { vation } \\
\text { rms } \\
\text { in. } \\
\end{array}$ & $\begin{array}{c}\text { Speed } \\
\text { mph }\end{array}$ \\
\hline 0 & 100.0 & 0 & 100.0 & 0 & 100.0 & 0 & 80.0 & 0 & 80.0 & 0 & 80.0 \\
\hline 0.34 & 80.0 & 1.07 & 30.0 & 0.54 & 100.0 & 0.33 & 60.0 & 0.33 & 60.0 & 0.33 & 60.0 \\
\hline 0.35 & 60.0 & 1.13 & 25.0 & 0.54 & 60.0 & 0.50 & 51.0 & 0.50 & 51.0 & 0.50 & 51.0 \\
\hline 0.36 & 40.0 & 1.25 & 20.0 & 0.60 & 50.0 & 0.72 & 40.0 & 0.72 & 40.0 & 0.72 & 40.0 \\
\hline 0.40 & 30.0 & 1.97 & 16.0 & 0.64 & 45.0 & 0.90 & 33.0 & 0.90 & 33.0 & 0.90 & 33.0 \\
\hline 0.45 & 25.0 & 2.50 & 13.0 & 0.75 & 38.0 & 1.00 & 30.0 & 1.00 & 30.0 & 1.00 & 30.0 \\
\hline 0.62 & 20.0 & 3.00 & 11.3 & 0.87 & 35.0 & 1.15 & 26.0 & 1.15 & 26.0 & 1.15 & 26.0 \\
\hline 1.00 & 15.0 & 4.00 & 8.5 & 1.00 & 31.5 & 1.30 & 21.0 & 1.30 & 21.0 & 1.30 & 21.0 \\
\hline 1.46 & 12.0 & 5.00 & 7.1 & 1.20 & 29.0 & 1.41 & 20.0 & 1.41 & 20.0 & 1.41 & 20.0 \\
\hline 2.00 & 10.0 & 6.00 & 6.3 & 1.50 & 25.8 & 1.50 & 19.0 & 1.50 & 19.0 & 1.50 & 19.0 \\
\hline 3.00 & 9.0 & 8.00 & 5.4 & 2.00 & 21.5 & 1.60 & 18.0 & 1.60 & 18.0 & 1.60 & 18.0 \\
\hline \multirow[t]{11}{*}{8.00} & 6.0 & & & 2.50 & 18.3 & 1.75 & 17.0 & 1.75 & 17.0 & 1.75 & 17.0 \\
\hline & & & & 3.00 & 15.8 & 1.80 & 16.5 & 1.80 & 16.5 & 1.80 & 16.5 \\
\hline & & & . & 3.50 & 13.5 & 2.00 & 16.0 & 2.00 & 16.0 & 2.00 & 16.0 \\
\hline & & & & 4.00 & 11.9 & 2.25 & 15.0 & 2.25 & 15.0 & 2.25 & 15.0 \\
\hline & & & & 4.50 & 10.7 & 2.50 & 14.8 & 2.50 & 14.8 & 2.50 & 14.8 \\
\hline & & & & 5.00 & 10.0 & 2.75 & 14.0 & 2.75 & 14.0 & 2.75 & 14.0 \\
\hline & & & & 5.50 & 9.5 & 3.00 & 13.8 & 3.00 & 13.8 & 3.00 & 13.8 \\
\hline & & -..... & & 6.00 & $9: 0$ & 8.00 & 13.5 & 8.00 & 13.5 & 8.00 & 13.5 \\
\hline & & & & 6.50 & 8.8 & & & & & & \\
\hline & & & & 7.00 & 8.5 & & & & & & \\
\hline & & & & 8.00 & 8.0 & & & & & & \\
\hline
\end{tabular}


Table B5 (Concluded)

\begin{tabular}{|c|c|c|c|c|c|c|c|c|c|}
\hline \multicolumn{2}{|c|}{ M113A1 } & \multicolumn{2}{|c|}{ M60A2 } & \multicolumn{2}{|c|}{$\mathrm{M} 577 \mathrm{Al}$} & \multicolumn{2}{|c|}{$\mathrm{XM723}$} & \multicolumn{2}{|c|}{ XMI } \\
\hline $\begin{array}{l}\text { Ele- } \\
\text { vation } \\
\text { rms } \\
\text { in. } \\
\end{array}$ & $\begin{array}{c}\text { Speed } \\
\text { mph }\end{array}$ & $\begin{array}{l}\text { Ele- } \\
\text { vation } \\
\text { rms } \\
\text { in. } \\
\end{array}$ & $\begin{array}{c}\text { Speed } \\
\text { mph }\end{array}$ & $\begin{array}{l}\text { Ele- } \\
\text { vation } \\
\text { rms } \\
\text { in. } \\
\end{array}$ & $\begin{array}{l}\text { Speed } \\
\text { mph }\end{array}$ & $\begin{array}{l}\text { Ele- } \\
\text { vation } \\
\text { rms } \\
\text { in. } \\
\end{array}$ & $\begin{array}{c}\text { Sneed } \\
\text { mph }\end{array}$ & $\begin{array}{l}\text { Ele- } \\
\text { vation } \\
\text { rms } \\
\text { in. } \\
\end{array}$ & $\begin{array}{c}\text { Speed } \\
\mathrm{mph}\end{array}$ \\
\hline 0 & 100.0 & 0 & 65.0 & 0 & 100.0 & 0 & 100.0 & 0 & 52.0 \\
\hline 0.40 & 100.0 & 0.50 & 65.0 & 0.40 & 100.0 & 0.54 & 100.0 & 1.40 & 52.0 \\
\hline 0.40 & 80.0 & 1.00 & 44.0 & 0.40 & 80.0 & 0.54 & 60.0 & 1.50 & 52.0 \\
\hline 0.40 & 70.0 & 1.50 & 30.0 & 0.40 & 70.0 & 0.60 & 50.0 & 1.62 & 37.0 \\
\hline 0.43 & 60.0 & 2.00 & 20.0 & 0.43 & 60.0 & 0.64 & 45.0 & 1.72 & 30.0 \\
\hline 0.48 & 50.0 & 2.50 & 16.0 & 0.48 & 50.0 & 0.75 & 38.0 & 2.00 & 23.5 \\
\hline 0.50 & 43.0 & 2.75 & 14.0 & 0.50 & 43.0 & 0.87 & 35.0 & 2.50 & 17.0 \\
\hline 0.55 & 40.0 & 3.00 & 13.0 & 0.55 & 40.0 & 1.00 & 31.5 & 2.80 & 14.5 \\
\hline 0.62 & 35.0 & 4.00 & 11.5 & 0.62 & 35.0 & 1.20 & 29.0 & 3.00 & 13.5 \\
\hline 0.75 & 30.0 & 5.00 & 10.5 & 0.75 & 30.0 & 1.50 & 25.8 & 3.50 & 11.5 \\
\hline 1.00 & 24.0 & 6.00 & 10.0 & 1.00 & 24.0 & 2.00 & 21.5 & 4.00 & 10.0 \\
\hline 1.23 & 20.0 & 8.00 & 9.0 & 1.23 & 20.0 & 2.50 & 18.3 & 5.00 & 8.5 \\
\hline 1.50 & 16.0 & & & 1.50 & 16.0 & 3.00 & 15.8 & 6.00 & 8.0 \\
\hline 1.80 & 14.0 & & & 1.80 & 14.0 & 3.50 & 13.5 & 7.00 & 7.5 \\
\hline 2.00 & 13.0 & & & 2.00 & 13.0 & 4.00 & 11.9 & 8.00 & 7.0 \\
\hline 2.50 & 11.0 & & & 2.50 & 11.0 & 4.50 & 10.7 & & \\
\hline 3.00 & 10.0 & & & 3.00 & 10.0 & 5.00 & 10.0 & & \\
\hline 3.50 & 9.0 & & & 3.50 & 9.0 & 5.50 & 9.5 & & \\
\hline 4.00 & 8.0 & & & 4.00 & 8.0 & 6.00 & 9.0 & & \\
\hline 5.00 & 7.0 & & & 5.00 & 7.0 & 6.50 & 8.8 & & \\
\hline 6.00 & 6.0 & & & 6.00 & 6.0 & 7.00 & 8.5 & & \\
\hline 8.00 & 5.0 & & & 8.00 & 5.0 & 8.00 & 8.0 & & \\
\hline
\end{tabular}

(Sheet 3 of 3 ) 
Table $B 6$

Roads and Trails Ride-Speed Relations for Vehicle Characteristic No. 55 in Table Bl

\begin{tabular}{|c|c|c|c|c|c|c|c|c|c|c|c|c|c|}
\hline \multicolumn{2}{|c|}{ M1561 } & \multicolumn{2}{|c|}{ M35A2 } & \multicolumn{2}{|c|}{$\mathrm{M} 813$} & \multicolumn{2}{|c|}{ M656 } & \multicolumn{2}{|c|}{ MDW901 } & \multicolumn{2}{|c|}{ M520E I } & \multicolumn{2}{|c|}{ M125E } \\
\hline $\begin{array}{l}\text { Ele- } \\
\text { vation } \\
\text { rms } \\
\text { in. } \\
\end{array}$ & $\begin{array}{c}\text { Speed } \\
\text { mph }\end{array}$ & $\begin{array}{l}\text { Ele- } \\
\text { vation } \\
\text { rms } \\
\text { in. } \\
\end{array}$ & $\begin{array}{c}\text { Speed } \\
\text { mph } \\
\end{array}$ & $\begin{array}{l}\text { Ele- } \\
\text { vation } \\
\text { rms } \\
\text { in. } \\
\end{array}$ & $\begin{array}{c}\text { Speed } \\
\text { mph }\end{array}$ & $\begin{array}{l}\text { Ele- } \\
\text { vation } \\
\text { rms } \\
\text { in. } \\
\end{array}$ & $\begin{array}{c}\text { Speed } \\
\text { mph }\end{array}$ & $\begin{array}{l}\text { Ele- } \\
\text { vation } \\
\text { rms } \\
\text { in. } \\
\end{array}$ & $\begin{array}{c}\text { Speed } \\
\mathrm{mph}\end{array}$ & $\begin{array}{l}\text { Ele- } \\
\text { vation } \\
\text { rms } \\
\text { in. } \\
\end{array}$ & $\begin{array}{c}\text { Speed } \\
\text { mph }\end{array}$ & $\begin{array}{l}\text { Ele- } \\
\text { vation } \\
\text { rms } \\
\text { in. } \\
\end{array}$ & $\begin{array}{c}\text { Speed } \\
\text { mph }\end{array}$ \\
\hline 0 & 100.0 & 0 & 100.0 & 0 & 100.0 & 0 & 100.0 & 0 & 100.0 & 0 & 100.0 & 0 & 100.0 \\
\hline 0.20 & 60.0 & 0.49 & 80.0 & 0.20 & 100.0 & 0.24 & 80.0 & 0.45 & 80.0 & 0.10 & 100.0 & 0.18 & 100.0 \\
\hline 0.50 & 36.0 & 0.50 & 60.0 & 0.20 & 80.0 & 0.25 & 50.0 & 0.50 & 50.0 & 0.20 & 40.0 & 0.30 & 68.0 \\
\hline 0.66 & 30.0 & 0.54 & 50.0 & 0.25 & 70.0 & 0.50 & 37.5 & 0.75 & 32.5 & 0.30 & 32.0 & 0.55 & 55.0 \\
\hline 1.00 & 23.5 & 0.61 & 40.0 & 0.37 & 60.0 & 0.75 & 30.5 & 1.00 & 27.9 & 0.40 & 28.0 & 0.80 & 40.0 \\
\hline 1.50 & 15.5 & 0.77 & 30.0 & 0.50 & 50.0 & 1.00 & 26.2 & 1.50 & 20.7 & 0.65 & 20.0 & 1.47 & 22.0 \\
\hline 2.00 & 10.6 & 0.93 & 25.0 & 0.68 & 40.0 & 2.00 & 11.8 & 2.00 & 16.3 & 1.00 & 15.0 & 2.20 & 10.0 \\
\hline 3.00 & 3.0 & 1.00 & 23.5 & 0.80 & 35.0 & 2.30 & 9.2 & 2.30 & 15.3 & 1.60 & 10.0 & 2.80 & 6.8 \\
\hline \multirow[t]{12}{*}{8.00} & 2.9 & 1.50 & 15.0 & 0.95 & 30.0 & 3.00 & 9.2 & 3.00 & 15.3 & 2.30 & 6.0 & 8.00 & 6.0 \\
\hline & & 2.00 & 10.5 & 1.21 & 24.0 & 8.00 & 9.2 & 8.00 & 15.3 & 8.00 & 5.5 & & \\
\hline & & 3.00 & 4.8 & 1.42 & 20.0 & & & & & & & & \\
\hline & & 8.00 & 2.0 & 1.75 & 15.0 & & & & & & & & \\
\hline & & & & 2.00 & 11.8 & & & & & & & & \\
\hline & & & & 2.13 & 10.0 & & & & & & & & \\
\hline & & & & 2.22 & 9.0 & & & & & & & & \\
\hline & & & & 2.45 & 7.0 & & & & & & & & \\
\hline & & & & 2.60 & 6.0 & & & & & & & & \\
\hline & & & & 2.80 & 5.0 & & & & & & & & \\
\hline & & & & 3.00 & 4.5 & & & & & & & & \\
\hline & & & & 8.00 & 4.5 & & & & & & & & \\
\hline
\end{tabular}


Table B6 (Continued)

\begin{tabular}{|c|c|c|c|c|c|c|c|c|c|c|c|}
\hline \multicolumn{2}{|c|}{ M548El } & \multicolumn{2}{|c|}{ UET } & \multicolumn{2}{|c|}{ ASV-Armored } & \multicolumn{2}{|c|}{ M109A1 } & \multicolumn{2}{|c|}{ M107. } & \multicolumn{2}{|c|}{ Ml10E2 } \\
\hline $\begin{array}{l}\text { Ele- } \\
\text { vation } \\
\text { rms } \\
\text { in. } \\
\end{array}$ & $\begin{array}{c}\text { Speed } \\
\mathrm{mph}\end{array}$ & $\begin{array}{l}\text { E1e- } \\
\text { vation } \\
\text { rms } \\
\text { in. } \\
\end{array}$ & $\begin{array}{c}\text { Speed } \\
\text { mph }\end{array}$ & $\begin{array}{l}\text { Ele- } \\
\text { vation } \\
\text { rms } \\
\text { in. } \\
\end{array}$ & $\begin{array}{c}\text { Speed } \\
\text { mph } \\
\end{array}$ & $\begin{array}{l}\text { E1e- } \\
\text { vation } \\
\text { rms } \\
\text { in. } \\
\end{array}$ & $\begin{array}{c}\text { Speed } \\
\text { mph } \\
\end{array}$ & $\begin{array}{l}\text { Ele- } \\
\text { vation } \\
\text { rms } \\
\text { in. } \\
\end{array}$ & $\begin{array}{c}\text { Speed } \\
\text { rms }\end{array}$ & $\begin{array}{l}\text { Ele- } \\
\text { vation } \\
\text { rms } \\
\text { in. } \\
\end{array}$ & $\begin{array}{c}\text { Speed } \\
\text { mph }\end{array}$ \\
\hline 0 & 100.0 & 0 & 100.0 & 0 & 100.0 & 0 & 100.0 & 0 & 100.0 & 0 & 100.0 \\
\hline 0.41 & 80.0 & 1.07 & 42.0 & 0.54 & 100.0 & 0.45 & 100.0 & 0.45 & 100.0 & 0.45 & 100.0 \\
\hline 0.42 & 60.0 & 1.13 & 35.0 & 0.54 & 66.0 & 0.75 & 60.0 & 0.75 & 60.0 & 0.75 & 60.0 \\
\hline 0.43 & 40.0 & 1.25 & 27.0 & 0.75 & 55.0 & 0.85 & 50.0 & 0.85 & 50.0 & 0.85 & 50.0 \\
\hline 0.50 & 33.1 & 1.97 & 17.6 & 1.00 & 44.0 & 0.93 & 40.0 & 0.93 & 40.0 & 0.93 & 40.0 \\
\hline 0.79 & 25.0 & 2.50 & 13.0 & 1.50 & 34.0 & 1.15 & 28.0 & 1.15 & 28.0 & 1.15 & 28.0 \\
\hline 1.00 & 21.8 & 3.00 & 11.3 & 2.10 & 24.0 & 1.35 & 19.0 & 1.35 & 19.0 & 1.35 & 19.0 \\
\hline 1.60 & 15.0 & 4.00 & 8.5 & 2.50 & 18.3 & .1 .50 & 18.0 & 1.50 & 18.0 & 1.50 & 18.0 \\
\hline 2.00 & 11.2 & 5.00 & 7.1 & 3.00 & 15.8 & 2.00 & 16.0 & 2.00 & 16.0 & 2.00 & 16.0 \\
\hline 3.00 & 9.0 & 6.00 & 6.3 & 3.50 & 13.5 & 3.00 & 14.5 & 3.00 & 14.5 & 3.00 & 14.5 \\
\hline \multirow[t]{8}{*}{8.00} & 7.5 & 8.00 & 5.4 & 4.00 & 11.9 & 8.00 & 8.0 & 8.00 & 8.0 & 8.00 & 8.0 \\
\hline & & & & 4.50 & 10.7 & & & & & & \\
\hline & & & & 5.00 & 10.0 & & & & & & \\
\hline & & & & 5.50 & 9.5 & & & & & & \\
\hline & & & & 6.00 & 9.0 & & & & & & \\
\hline & & & & 6.50 & 8.8 & & & & & & \\
\hline & & & & 7.00 & 8.5 & & & & & & \\
\hline & & & & 8.00 & 8.5 & & & & & & \\
\hline
\end{tabular}


Table B6 (Concluded)

\begin{tabular}{|c|c|c|c|c|c|c|c|c|c|}
\hline \multicolumn{2}{|c|}{$\mathrm{M} 113 \mathrm{Al}$} & \multicolumn{2}{|c|}{ M6UA2 } & \multicolumn{2}{|c|}{ MS77A1 } & \multicolumn{2}{|c|}{$X M 723$} & \multicolumn{2}{|c|}{$X: 11$} \\
\hline $\begin{array}{l}\text { Ele- } \\
\text { vation } \\
\text { rms } \\
\text { in. }\end{array}$ & $\begin{array}{c}\text { Speed } \\
\text { mph }\end{array}$ & $\begin{array}{l}\text { Ele- } \\
\text { vation } \\
\text { rms } \\
\text { in. } \\
\end{array}$ & $\begin{array}{l}\text { Speed } \\
\text { mph }\end{array}$ & $\begin{array}{c}= \\
\text { Ele- } \\
\text { vation } \\
\text { rms } \\
\text { in. } \\
\end{array}$ & $\begin{array}{c}\text { Speed } \\
\mathrm{mph} \\
\end{array}$ & $\begin{array}{l}\text { Ele- } \\
\text { vation } \\
\text { rms } \\
\text { in. } \\
\end{array}$ & $\begin{array}{c}\text { Speed } \\
\text { mph }\end{array}$ & $\begin{array}{l}\text { Ele- } \\
\text { vation } \\
\text { rms } \\
\text { in. } \\
\end{array}$ & $\begin{array}{c}\text { Speed } \\
\mathrm{mph}\end{array}$ \\
\hline 0 & 100.0 & 0 & 65.0 & 0 & 100.0 & 0 & 100.0 & 0 & 52.0 \\
\hline 0.40 & 100.0 & 0.50 & 65.0 & 0.40 & 100.0 & 0.54 & 100.0 & 1.40 & 52.0 \\
\hline 0.40 & 80.0 & 1.00 & 44.0 & 0.40 & 80.0 & 0.54 & 66.0 & 1.50 & 52.0 \\
\hline 0.43 & $73.5^{\circ}$ & 1.50 & 30.0 & 0.43 & 73.5 & 0.75 & 55.0 & 1.62 & 37.0 \\
\hline 0.48 & 66.8 & 2.00 & 20.0 & 0.48 & 66.8 & 1.00 & 44.0 & 1.72 & 30.0 \\
\hline 0.53 & 60.0 & 2.50 & 16.0 & 0.53 & 60.0 & 1.50 & 34.0 & 2.00 & 23.5 \\
\hline 0.65 & 50.0 & 2.75 & 14.0 & 0.65 & 50.0 & 2.10 & 24.0 & 2.50 & 17.0 \\
\hline 0.82 & 40.0 & 3.00 & 13.0 & 0.82 & 40.0 & 2.50 & 18.3 & 2.80 & 14.5 \\
\hline 1.00 & 33.5 & 4.00 & 11.5 & 1.00 & 33.5 & 3.00 & 15.8 & 3.00 & 13.5 \\
\hline 1.22 & 27.5 & 5.00 & 10.5 & 1.22 & 27.5 & 3.50 & 13.5 & 3.50 & 11.5 \\
\hline 1.43 & 23.2 & 6.00 & 10.0 & 1.43 & 23.2 & 4.00 & 11.9 & 4.00 & 10.0 \\
\hline 1.66 & 20.0 & 8.00 & 9.0 & 1.66 & 20.0 & 4.50 & 10.7 & 5.00 & 8.5 \\
\hline 1.83 & 17.2 & & & 1.83 & 17.2 & 5.00 & 10.0 & 6.00 & 8.0 \\
\hline 2.00 & 15.0 & & & 2.00 & 15.0 & 5.50 & 9.5 & 7.00 & 7.5 \\
\hline 2.50 & 11.5 & & & 2.50 & 11.5 & 6.00 & 9.0 & 8.00 & 7.0 \\
\hline 3.00 & 10.0 & & & 3.00 & 10.0 & 6.50 & 8.8 & & \\
\hline 3.50 & 9.0 & & & 3.50 & 9.0 & 7.00 & 8.5 & & \\
\hline 4.00 & 8.0 & & & 4.00 & 8.0 & 8.00 & 8.5 & & \\
\hline 5.00 & 7.0 & & & 5.00 & 7.0 & & & & \\
\hline 6.00 & 6.0 & & & 6.00 & 6.0 & & & & \\
\hline 8.00 & 5.0 & & & 8.00 & 5.0 & & & & \\
\hline
\end{tabular}




\section{Test Objectives and Scope}

1. It was necessary to conduct a few experimental tests to fill certain voids in the existing vehicle ride and shock data base. As a result, ride and shock tests were conducted at Fort Sill, Oklahoma, with a selected group of vehicle-artillery configurations and two selfpropelled artillery pieces over selected secondary roads, trails and cross-country courses.

2. Since experimental data were already available on most of the prime movers of interest, the main purpose of the tests was to determine the effects of the towed load on the ride quality of the prime movers and to develop ride and shock relations for the two self-propelled artillery pieces for which no data existed.

\section{Vehicle Configurations}

3. Vehicle breakdowns, maintenance requirements, and adverse weather and time constraints limited the testing to the following seven configurations:

$$
\begin{array}{ll}
\text { a. } & \text { M35A2-XM198 } \\
\text { b. } & \text { M35A2-M102 } \\
\text { c. } & \text { M813-XM198 } \\
\text { d. } & \text { M520E1-XM198 } \\
\text { e. } & \text { M561-M102 } \\
\text { f. } & \text { M109A1 } \\
\text { g. } & \text { M110E2 } \\
\text { h. } & \text { M813 }
\end{array}
$$

The vehicle configurations, except $\underline{h}$ above, are shown in Figure $c 1$. 


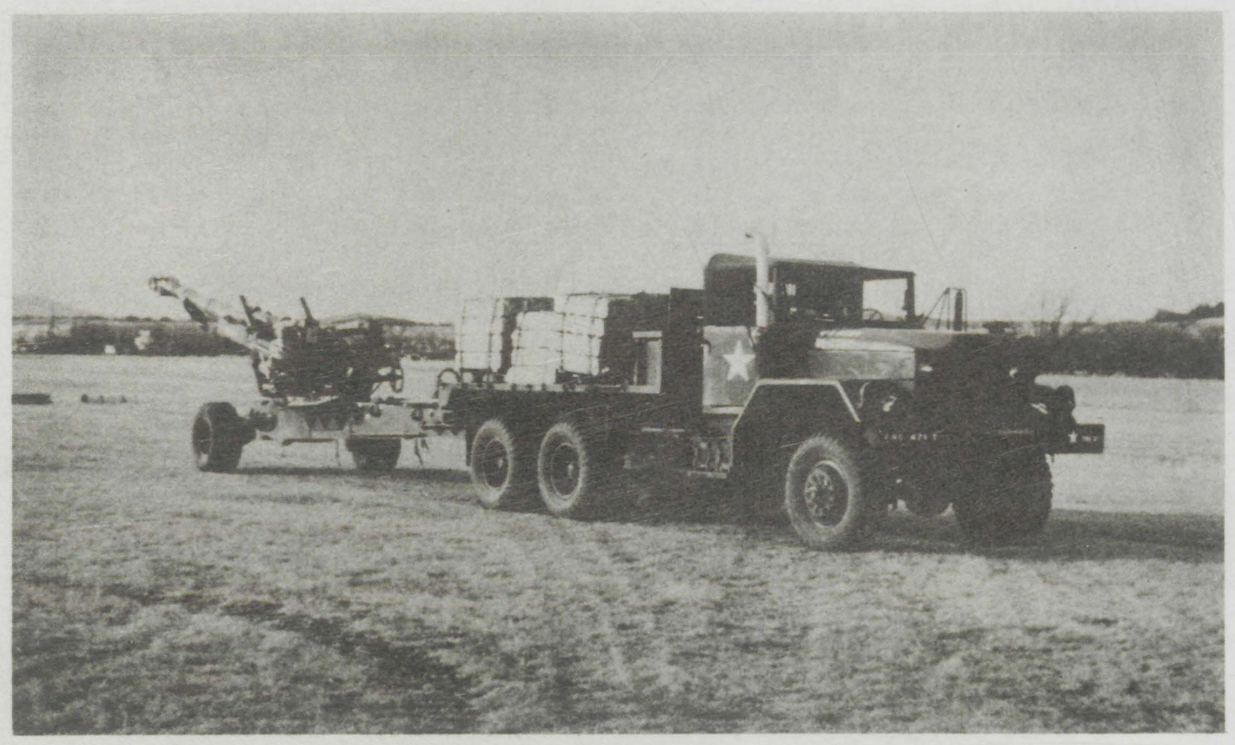

a. $\mathrm{M} 35 \mathrm{~A} 2-\mathrm{XM} 198$

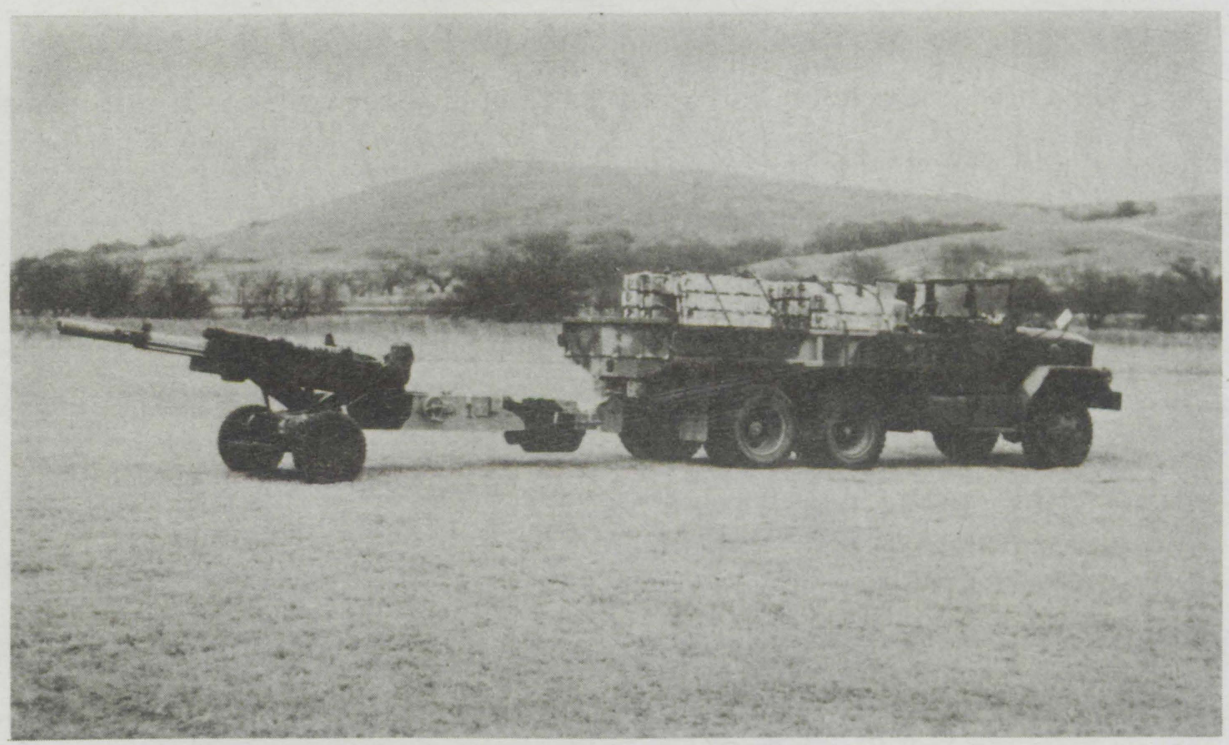

b. $\mathrm{M} 35 \mathrm{~A} 2-\mathrm{M} 102$

Figure C1. Test vehicles (sheet 1 of 4 ) 


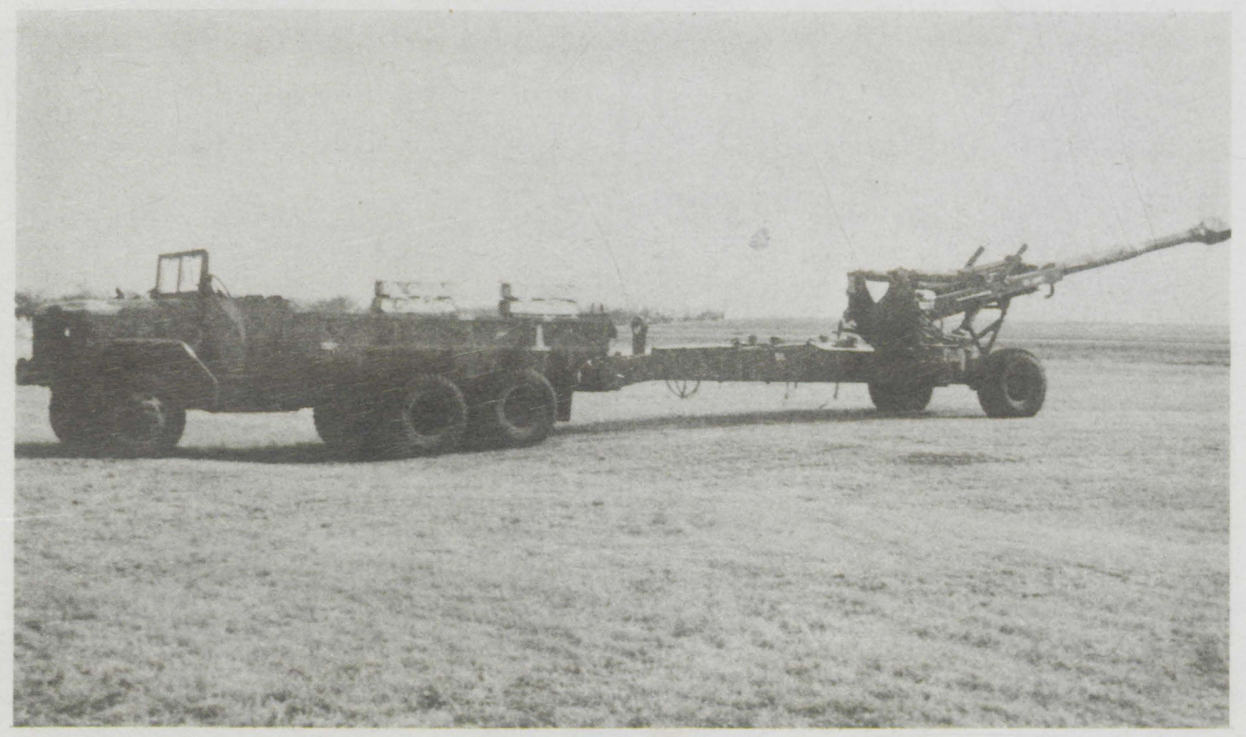

c. $\mathrm{M} 813-\mathrm{XM} 198$

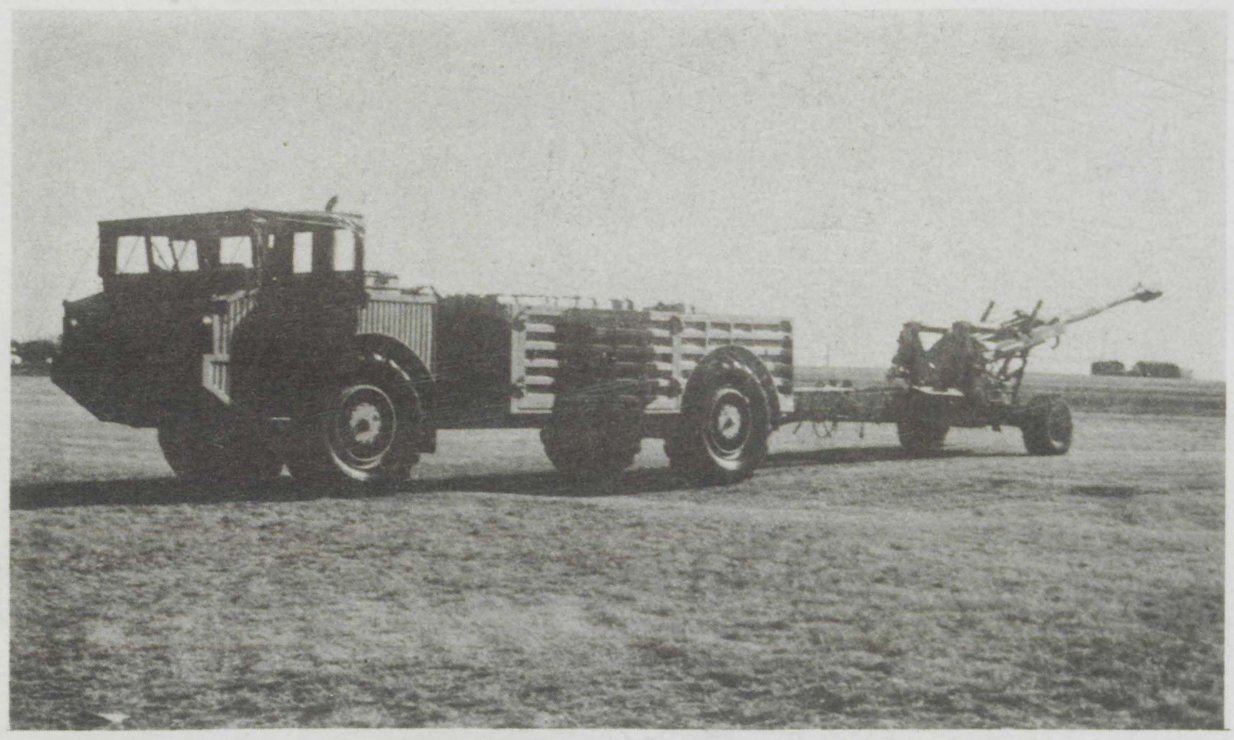

d. M520E1-XM198

Figure C1 (sheet 2 of 4 ) 


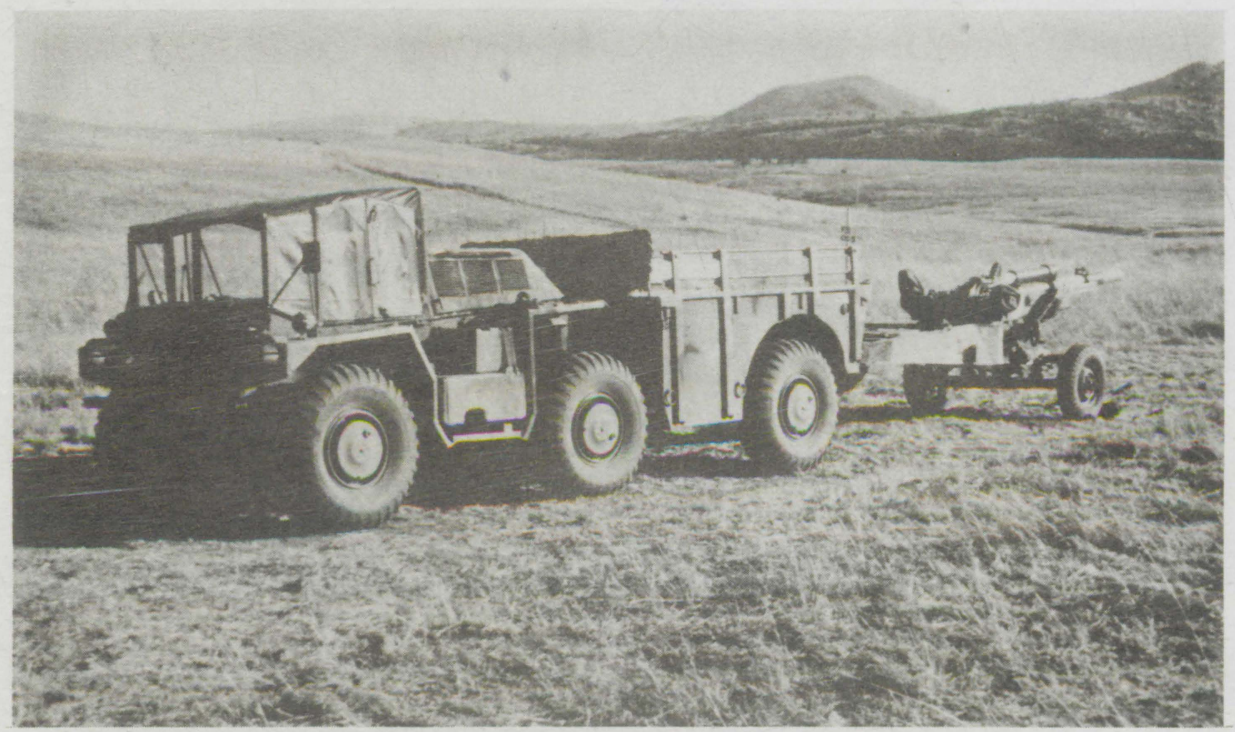

e. M561-M102

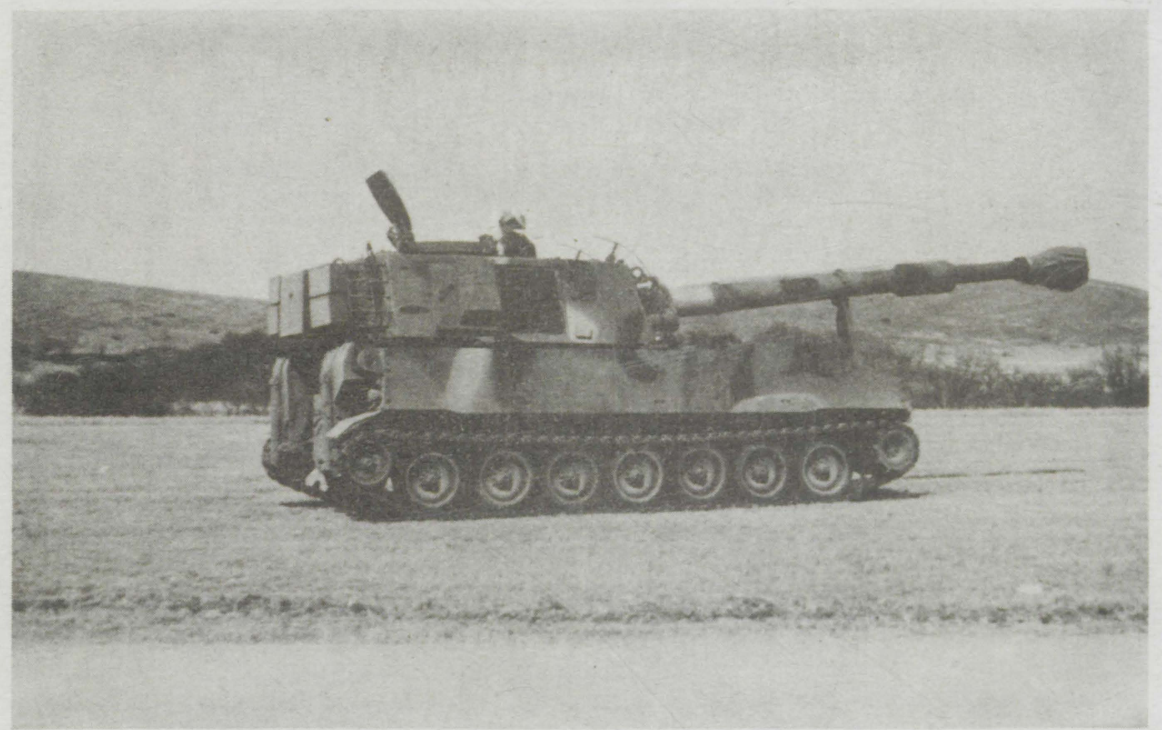

f. M109A1

Figure C1 (sheet 3 of 4 ) 


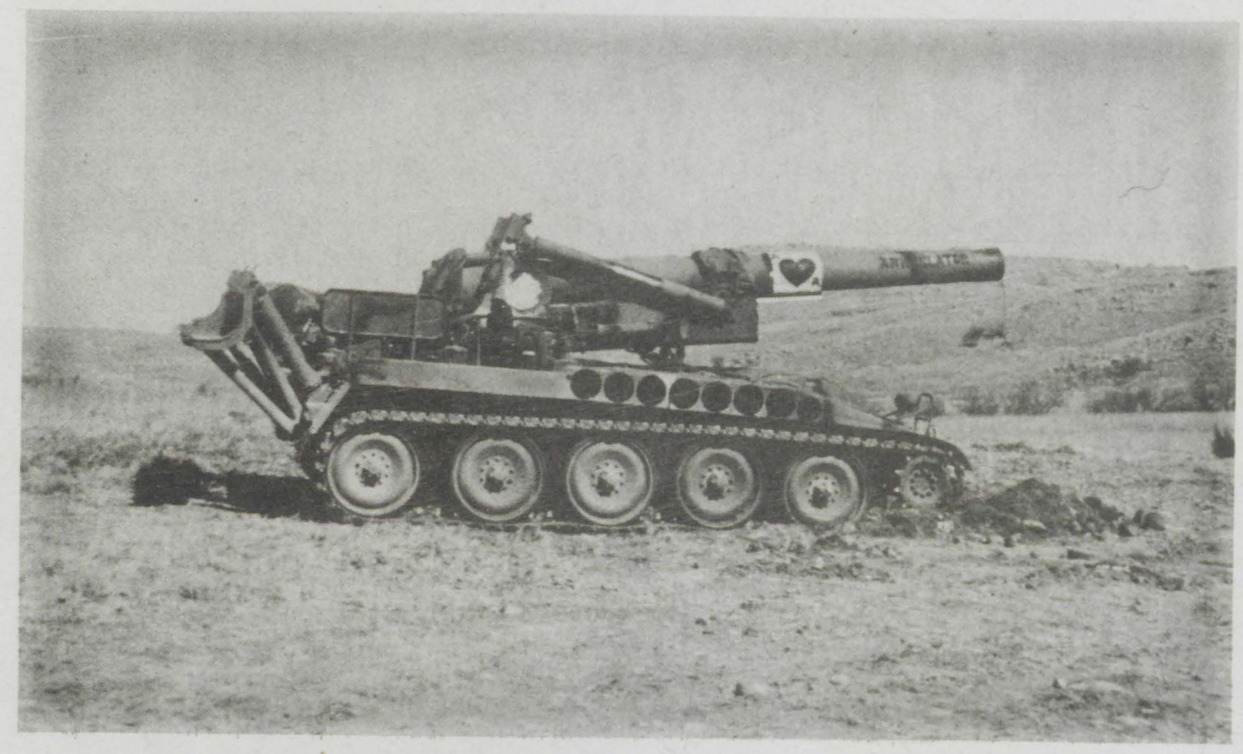

g. M110E2

Figure Cl (sheet 4 of 4 ) 
Instrumentation for Measuring Ride and Shock Responses

4. The instrumentation for measuring ride and shock responses consisted of: (a) three orthogonally positioned linear accelerometers and two angular accelerometers mounted near the geometric center of the cargo area to measure the bounce, fore-to-aft, side-to-side, and pitchand-roll accelerations in the cargo area; (b) three orthogonally positioned linear accelerometers mounted on the driver's seat and connected to a portable ride meter to measure the driver's absorbed power; (c) one vertically oriented accelerometer mounted on the floor beneath the driver's seat; and (d) one vertically oriented accelerometer mounted on each towed artillery piece. For the two self-propelled artillery vehicles, the cargo instrumentation was placed as near as possible to the vehicle's center of gravity. All signals were recorded on FM magnetic tape by a 14-channel heavy-duty recorder and its associated signal processor and $30-\mathrm{V}$ battery power source, which were also mounted on the vehicle (Figures $\mathrm{C} 2-\mathrm{C5}$ ). The ride meter converted the acceleration signals at the driver's seat to absorbed power. In addition to being recorded on tape, absorbed power was displayed continuously on a meter for visual observation of the responses occurring during each test. Also, the ride meter contained a peak counter that detected and counted the number of peak accelerations falling within six distinct intervals. The intervals are variable and are selected on the basis of the range of accceleration expected. The elapsed time and time-averaged absorbed power were obtained from a digital meter at the end of each test.

5. Absorbed power is the measure of the rate at which vibrational energy is absorbed by a human and is the quantity currently used to determine human tolerance to vibration when a vehicle is negotiating rough terrain. Ride quality is currently based on the vertical absorbed power at the driver's seat. The results of a comprehensive laboratory test program led to the establishment of a criterion of six watts as the human tolerance limit. The details of its development can be found in References 8 and 9. Experience from past tests based on comments of the 


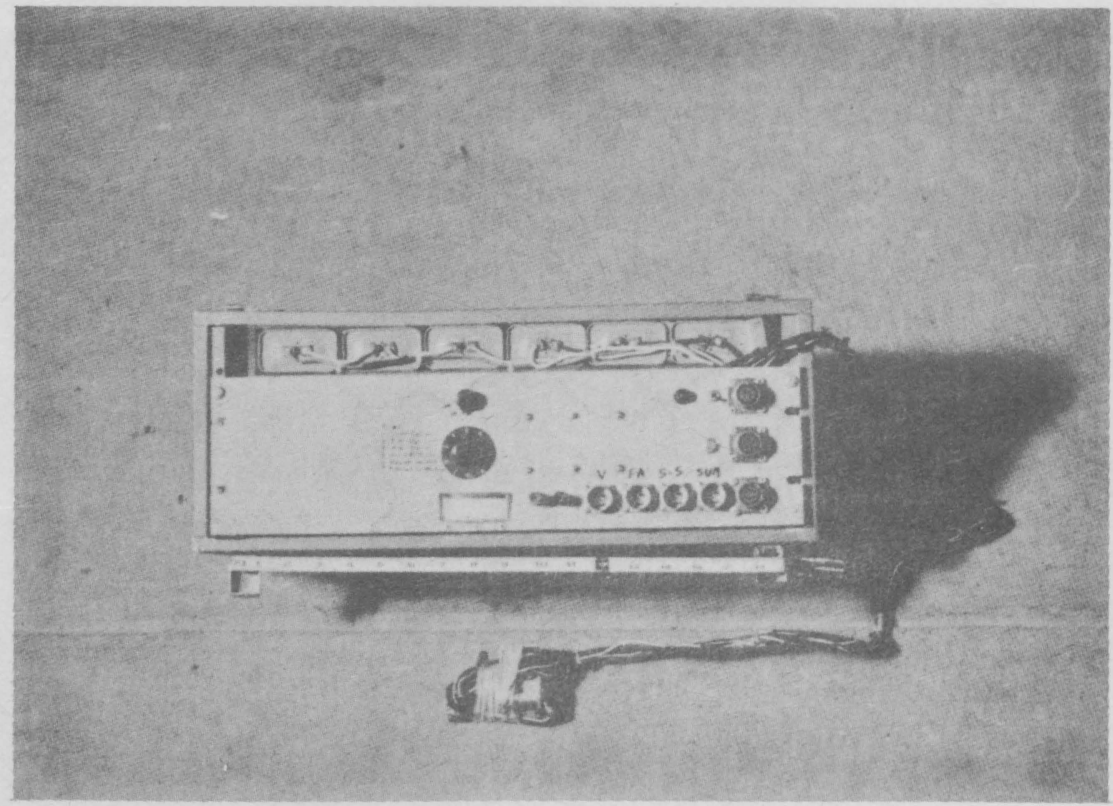

Figure C2. Portable ride meter and associated accelerometers

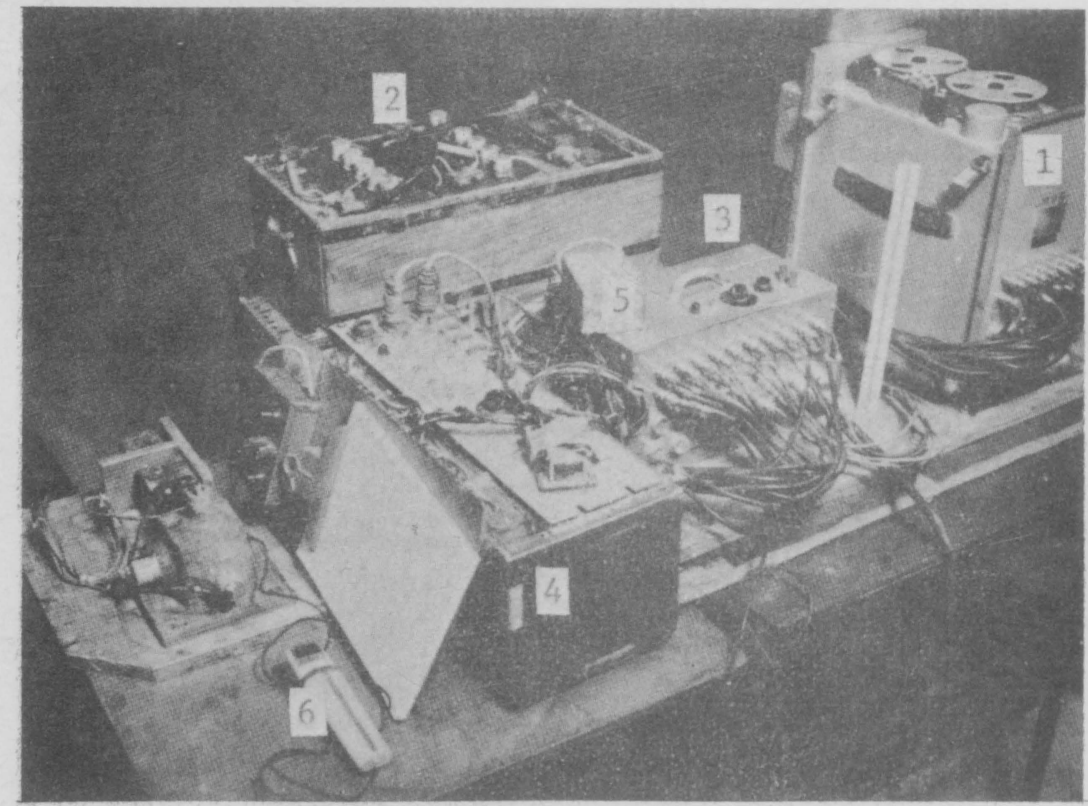

Figure C3. Basic instrumentation recording components

\section{LEGEND:}

(1) Tape recorder

(2) $30-v$ power source

(3) Signal controller

(4) Ride meter

(5) Absorbed power display

(6) Voltmeter with averaging circuit 


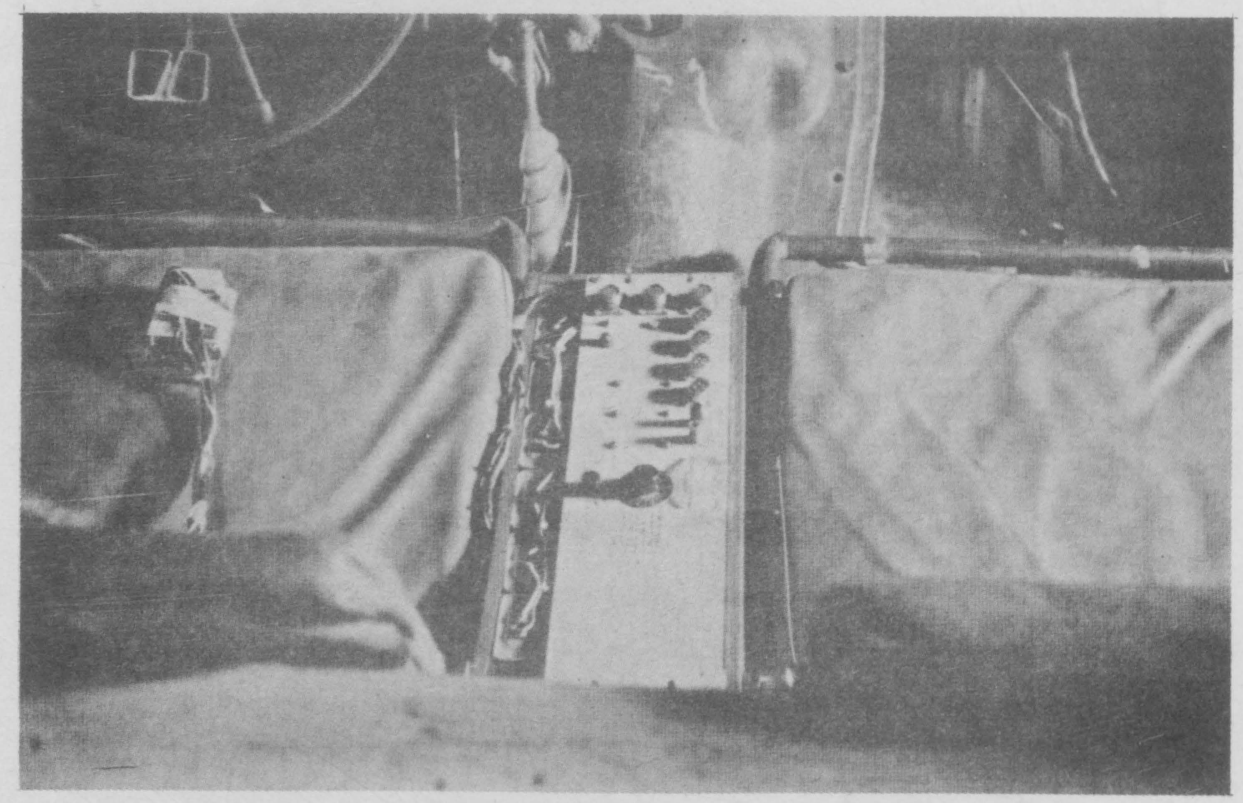

Figure C4. Ride meter installed for vehicle test

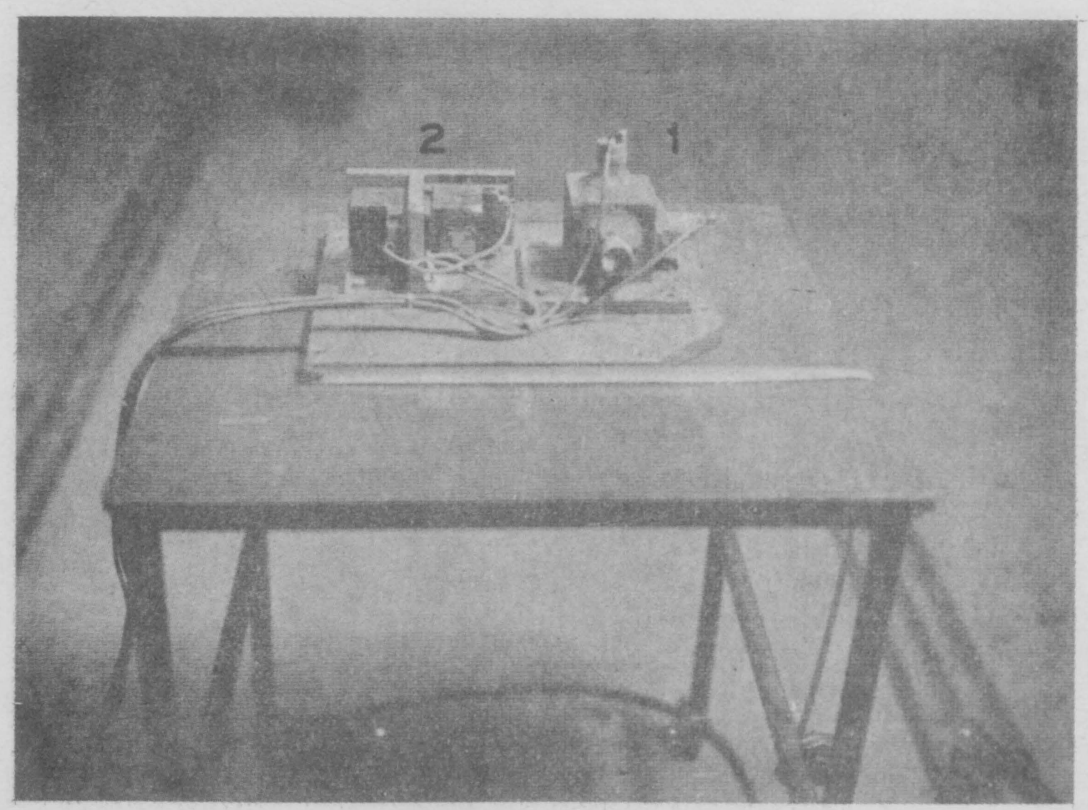

Figure C5. Accelerometer mount for cargo area

\section{Legend}

1 - Linear accelerometers

2 - Rotational (angular) accelerometers 
vehicle drivers and occupants has indicated that the vibrational environment affecting ride quality is influenced significantly by horizontal motion. Studies are being made to ascertain this influence and develop relations that include both vertical and horizontal motions. It has been observed that horizontal motions, particularly the fore-andaft, are more predominant in vehicles pulling heavy towed loads. However, because there are as of now no usable relations that include horizontal motions, ride quality for this study was defined in terms of vertical absorbed power.

\section{General Descriptin of Terrain and Climate} of Fort Sill, Oklahoma

6. The Fort Sill Reservation lies within Comanche County, Oklahoma, and occupies almost 6 of the county's $36 \mathrm{~km}^{2}$. About 51 percent of the land consists of sloping prairies and plains having less than 3 percent slope; about 20 percent is rolling with slopes from 3 to 5 percent; and 29 percent has slopes more than 5 percent, which includes Granite and Limestone Mountains. About 52 percent of Fort Sill is within the East Coche Creek watershed, 40 percent lies within the West Coche Creek watershed, and 8 percent is in the Big Beaver Creek watershed.

7. The geologic formations of Fort Sill consist of igneous and sedimentary rocks. Most of the soils are underlain by sedimentary rocks composed of fragments of older rocks.

8. The Fort Sill area has a temperate, continential climate of dry, subhumid type. The weather patterns that influence this area are sustained by the alternate movement of warm, moist air from the Gulf of Mexico and of either contrasting cooler, modified marine air from the West Coast or colder, dry air from around the Arctic Circle. Rapid changes are common and result in distinct fluctuations of temperature, humidity, cloudiness, wind, and precipitation. 


\section{Ride Test Courses}

\section{Location}

9. All courses were in the same general area of the Fort Sill Military Reservation near the west gate. Geographic coordinates of the area and location of the test sites are given in Figure C6. To provide a representative group of surface conditions, test courses were selected as follows:

a.. One secondary road (designated as FSSR1)

b. Four trails (designated FST1 through FST4)

c. Five cross-country courses (destgnaged as FSCC2 through FSCC6)

Course FSCC1 was determined unsuitable and eliminated from the program. Photographs of the courses are shown in Figure C7.

10. A profile of each course was measured with rod and level at 1-ft intervals, and surface roughness (rms elevation) was determined from these profiles using current procedures which filter out frequency components having wavelengths greater than $60 \mathrm{ft}$. The course lengths and rms elevations are listed in the tabulation below.

\begin{tabular}{lcc} 
Test Course & Length, ft & Elevation, rms in \\
\cline { 2 - 3 } FSSR1 & 400 & 0.09 \\
FST1 & 300 & 1.24 \\
FST2 & 300 & 0.51 \\
FST3 & 250 & 1.01 \\
FST4 & 250 & 1.44 \\
FSCC2 & 400 & 1.74 \\
FSCC3 & 300 & 0.92 \\
FSCC4 & 400 & 0.69 \\
FSCC5 & 300 & 0.87 \\
FSCC6 & 300 & 1.29
\end{tabular}

\section{Obstacle-Impact Test Course}

11. Rigid, semicircular obstacles 6,8 , and 12 in. high were positioned in a line on a level, hard surface. A 100-ft distance was measured perpendicular to the obstacles, and stakes, used to determine 


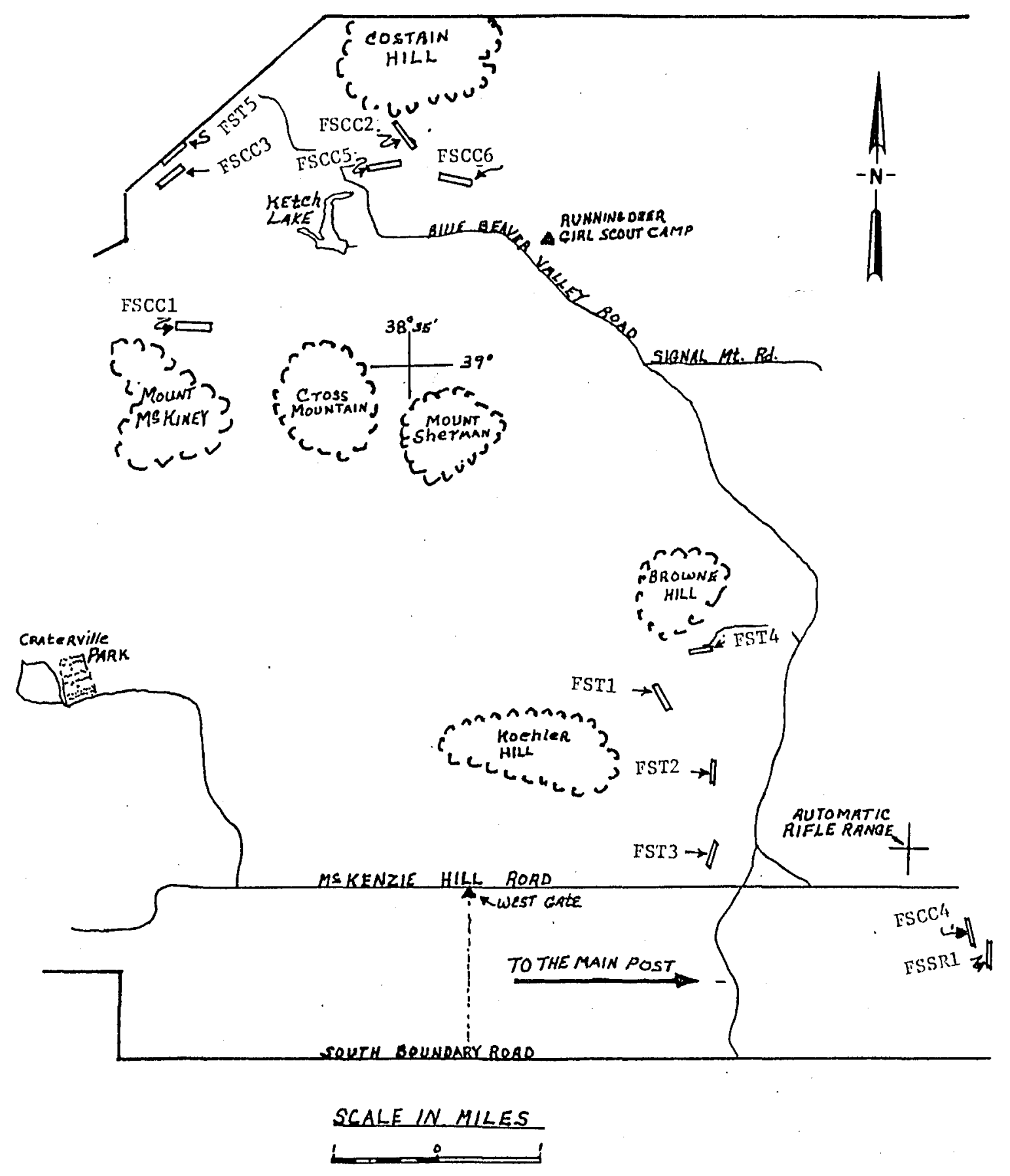

Figure C6. Location of ride test courses 


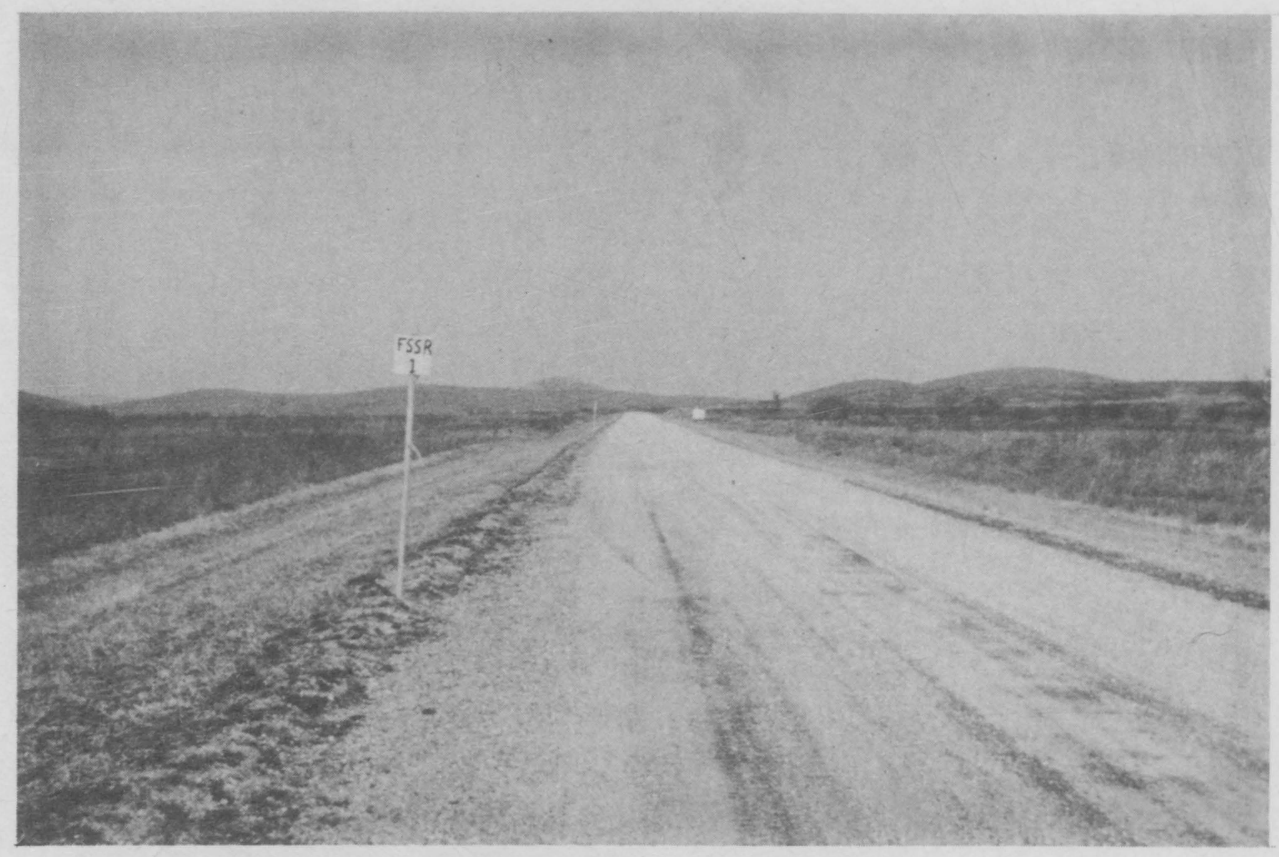

a. Fort Sill secondary road 1

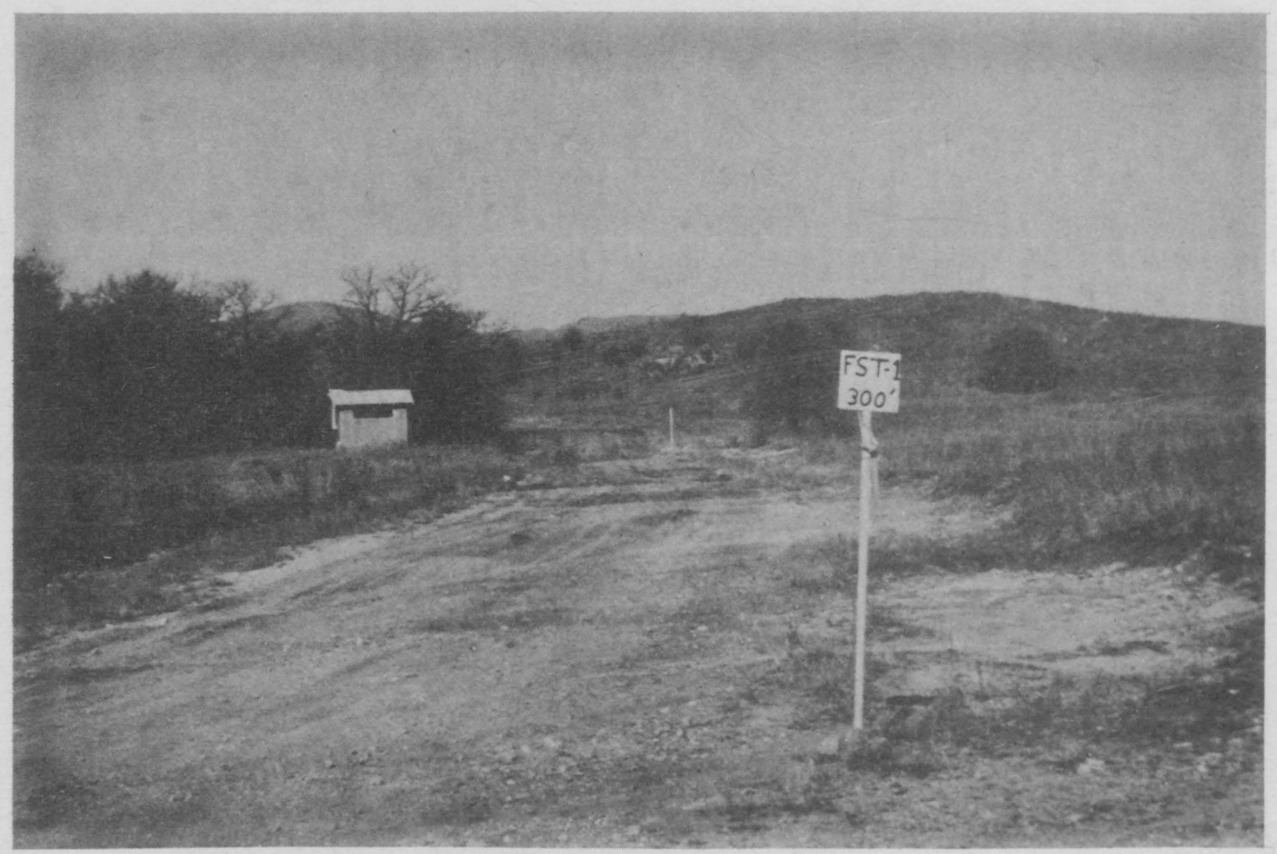

b. Fort Sill trail 1

Figure C7. Test courses (sheet 1 of 5 ) 


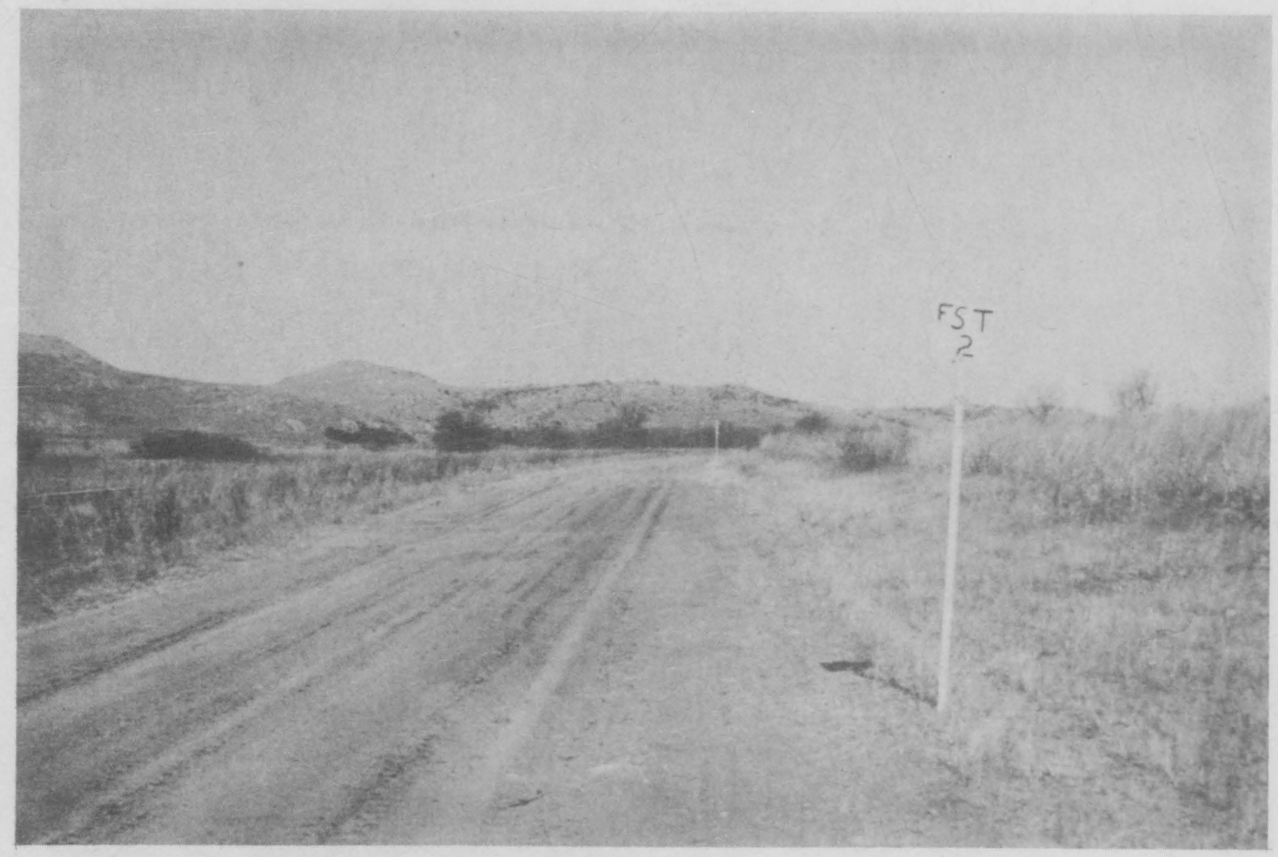

c. Fort Sill trail 2

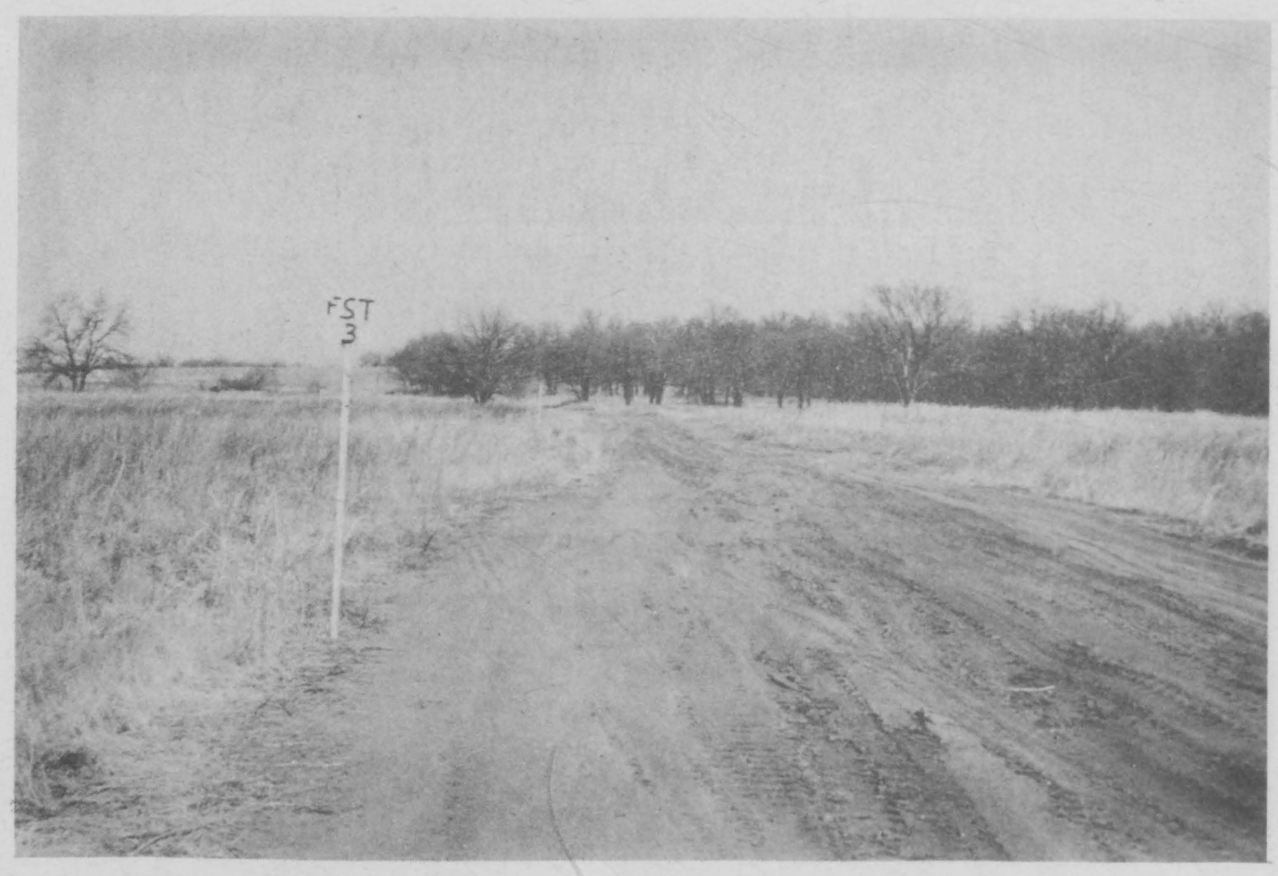

d. Fort Sill trail 3

Figure C7 (sheet 2 of 5) 


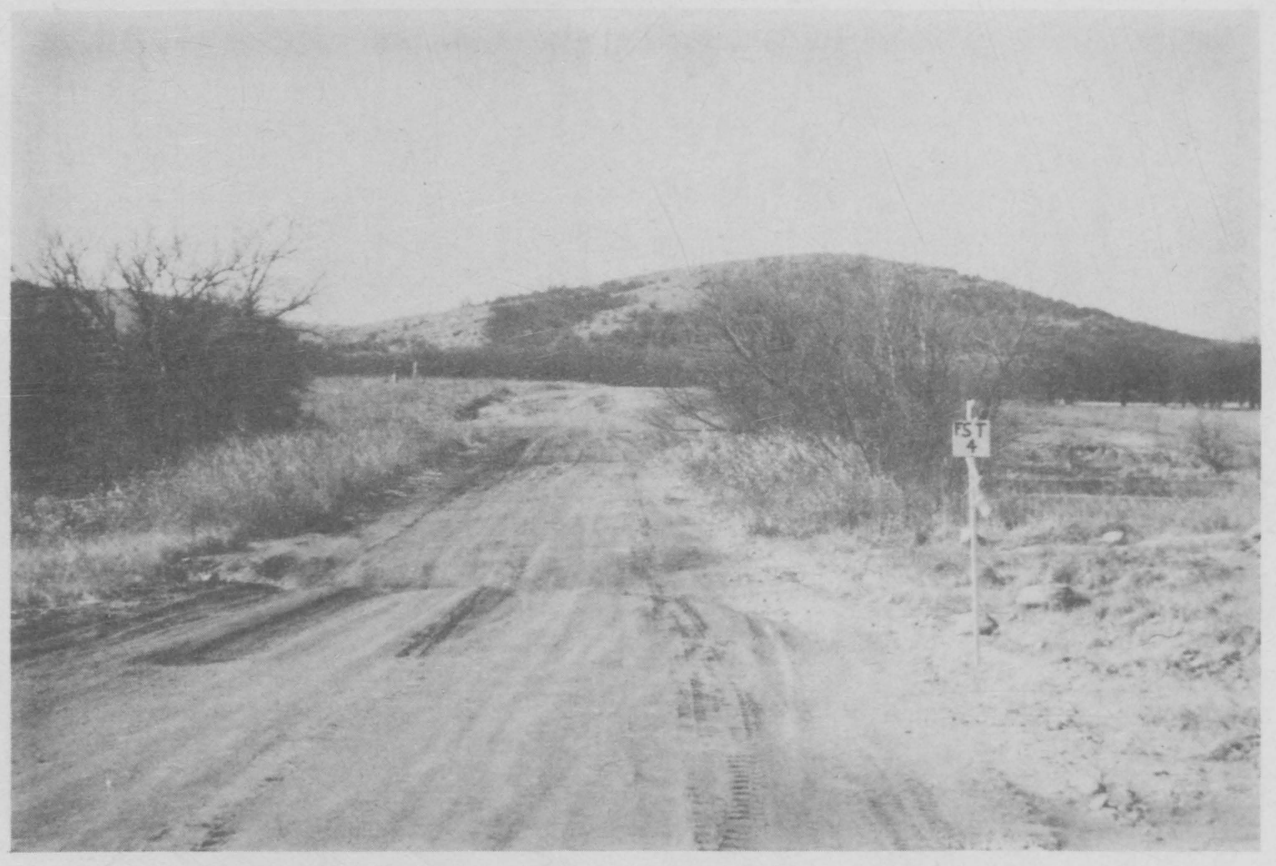

e. Fort Sill trail 4

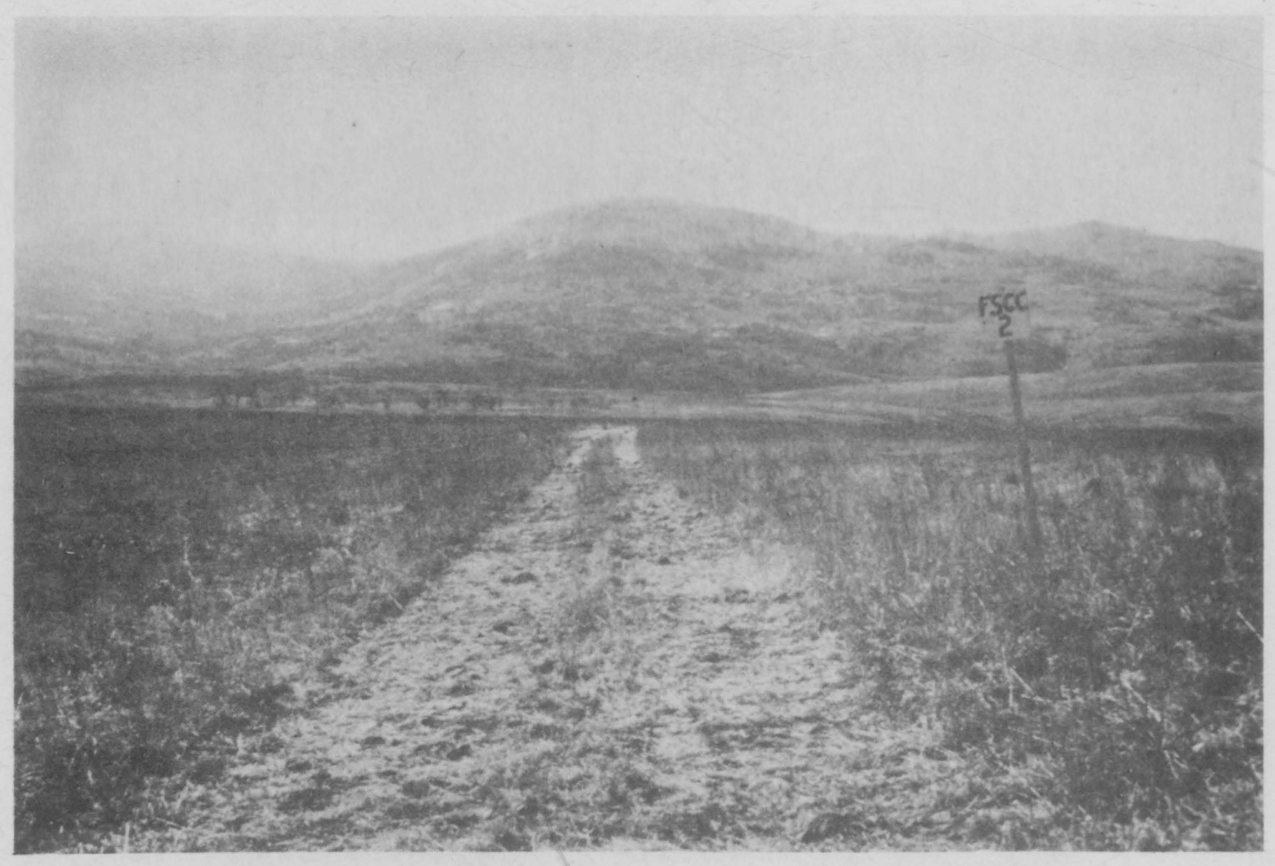

f. Fort Sill cross-country 2

Figure C7 (sheet 3 of 5) 


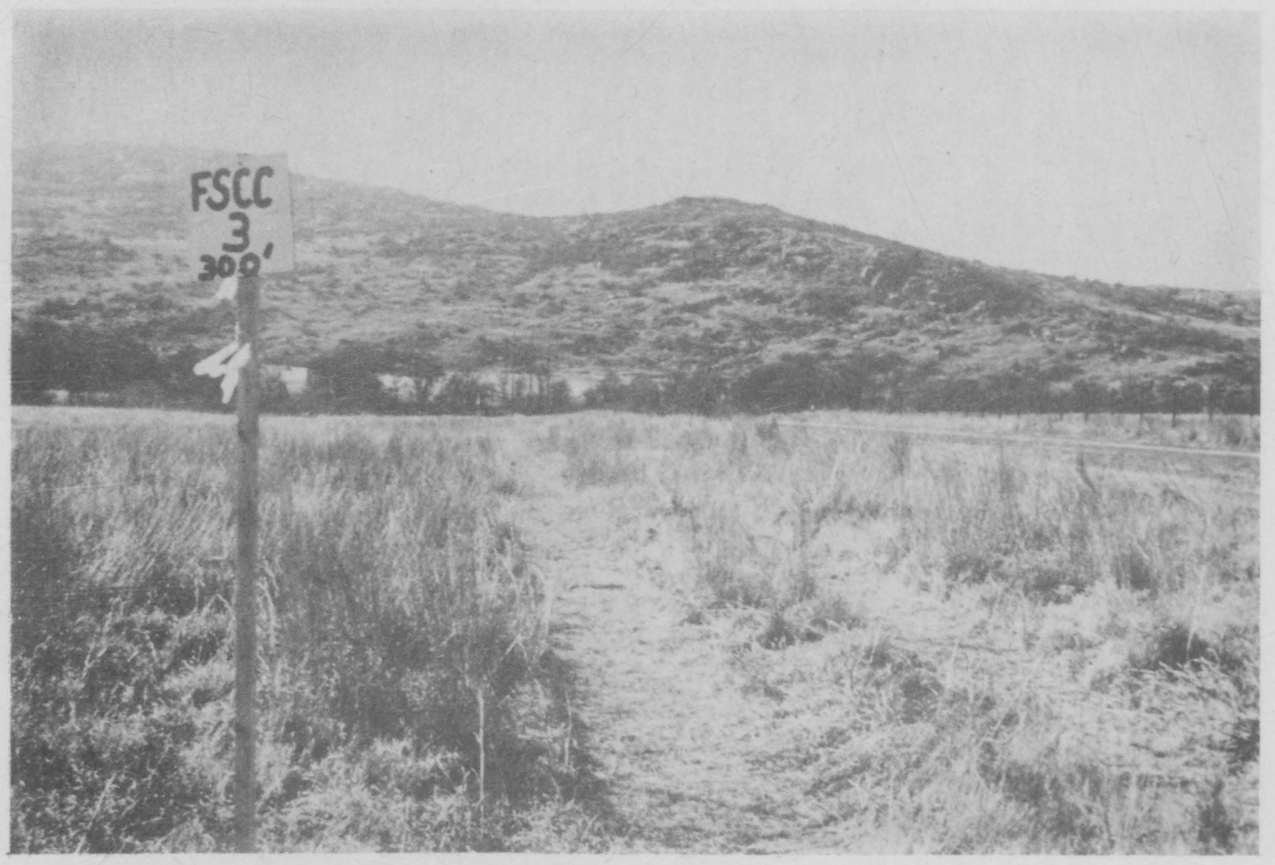

g. Fort Sill cross-country 3

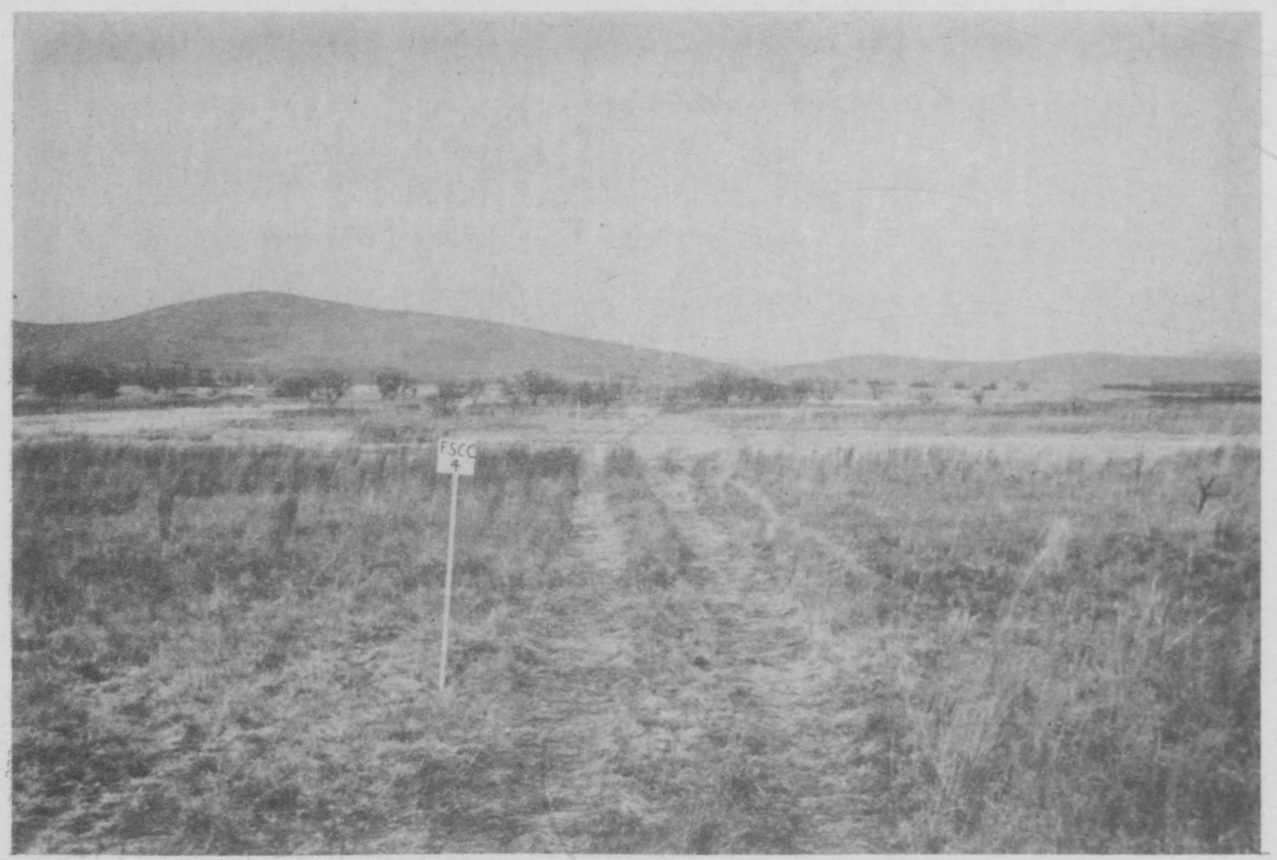

h. Fort Sill cross-country 4

Figure C7 (sheet 4 of 5) 


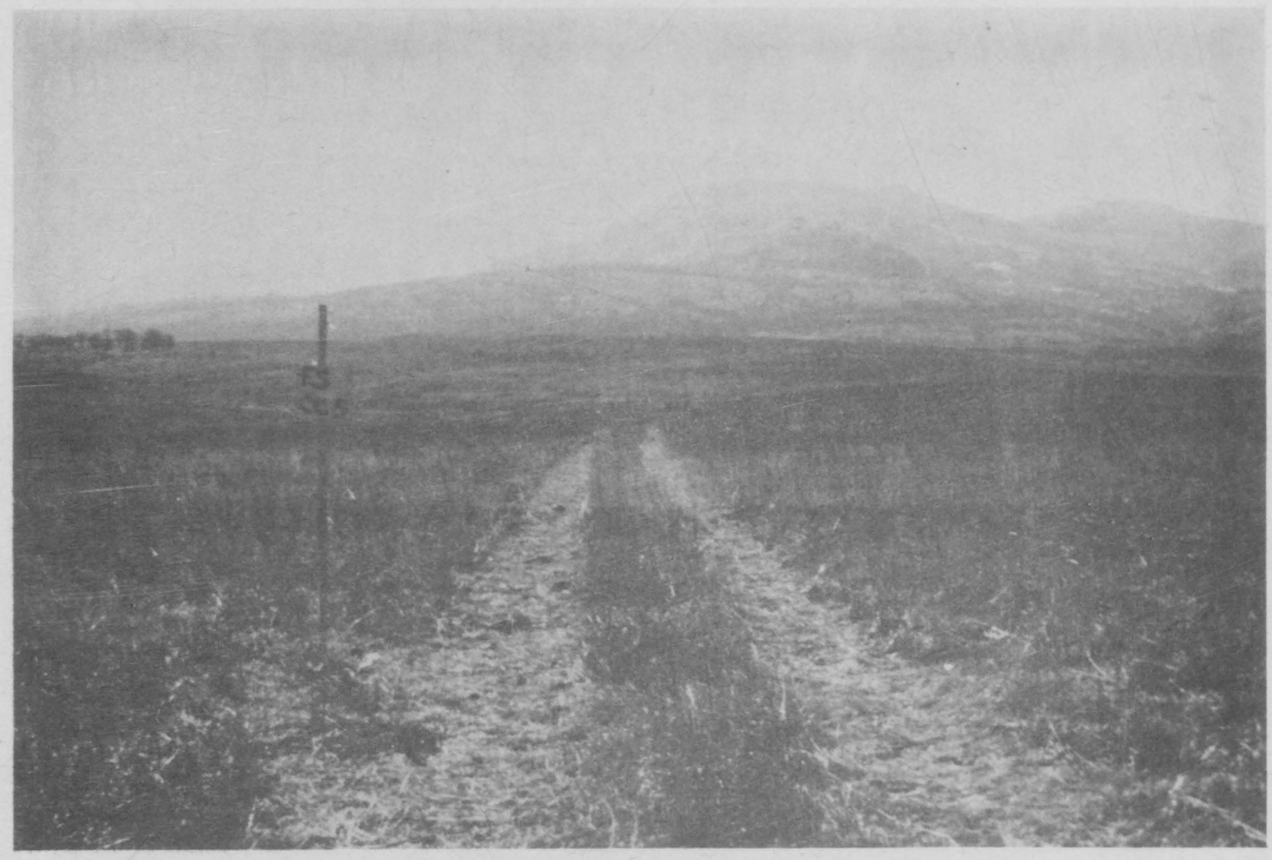

i. Fort Sill cross-country 5

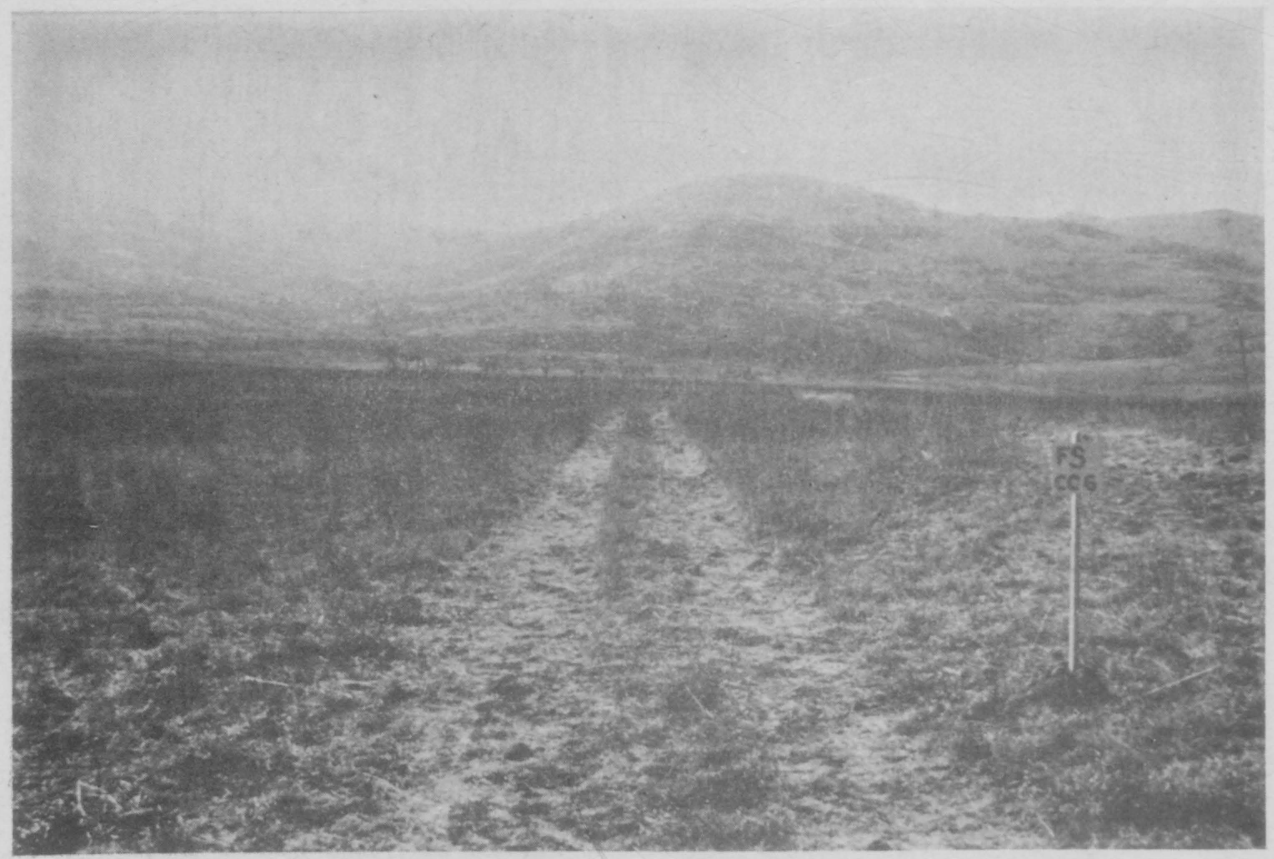

j. Fort Sill cross-country 6

Figure C7 (sheet 5 of 5) 
the impact speed by timing, were driven into the ground at that point. A sketch of the obstacle course is shown in Figure C8.

\section{Test Procedures}

Preparation of vehicles

for testing

12. The vehicles were serviced and checked before testing to ensure peak mechanical performance during all tests. When major mechanical problems developed, the vehicle was repaired or was replaced with a similar vehicle. Tests under loaded conditions were conducted at the recommended cross-country payloads or as near those payloads as could be determined using field loading techniques; boxes filled with soll were used as payload. Tires were inflated and maintained at the recommended cross-country tire pressures. Safety devices, such as seat belts, were used if the test vehicles were equipped with such devices.

Ride tests

13. Several tests were conducted with each vehicle configuration over each of the ride test courses at relatively constant speeds ranging from $5 \mathrm{mph}$ to the maximum safe speed, usually in about 5-mph increments. The objective was to reach and, if possible, surpass the 6-watt ride limit. However, on occasion the combination of short approaches to the test courses and insufficient horsepower precluded reaching the 6-watt limit.

14. With a few exceptions, each test began with the vehicle positioned a sufficient distance from the beginning of the test course to enable the driver to reach the desired test speed before entering the test course. This speed was then maintained at a relatively constant level (using the vehicle's speedometer) throughout the length of the course. An observer rode in the vehicle during each test to operate and monitor the output of the ride meter and narrate the pertinent activities. This narrative was recorded on the voice channel of the magnetic tape. At the end of the test, the driver's comments were also recorded. Measurements and test procedures were designed to be completely 


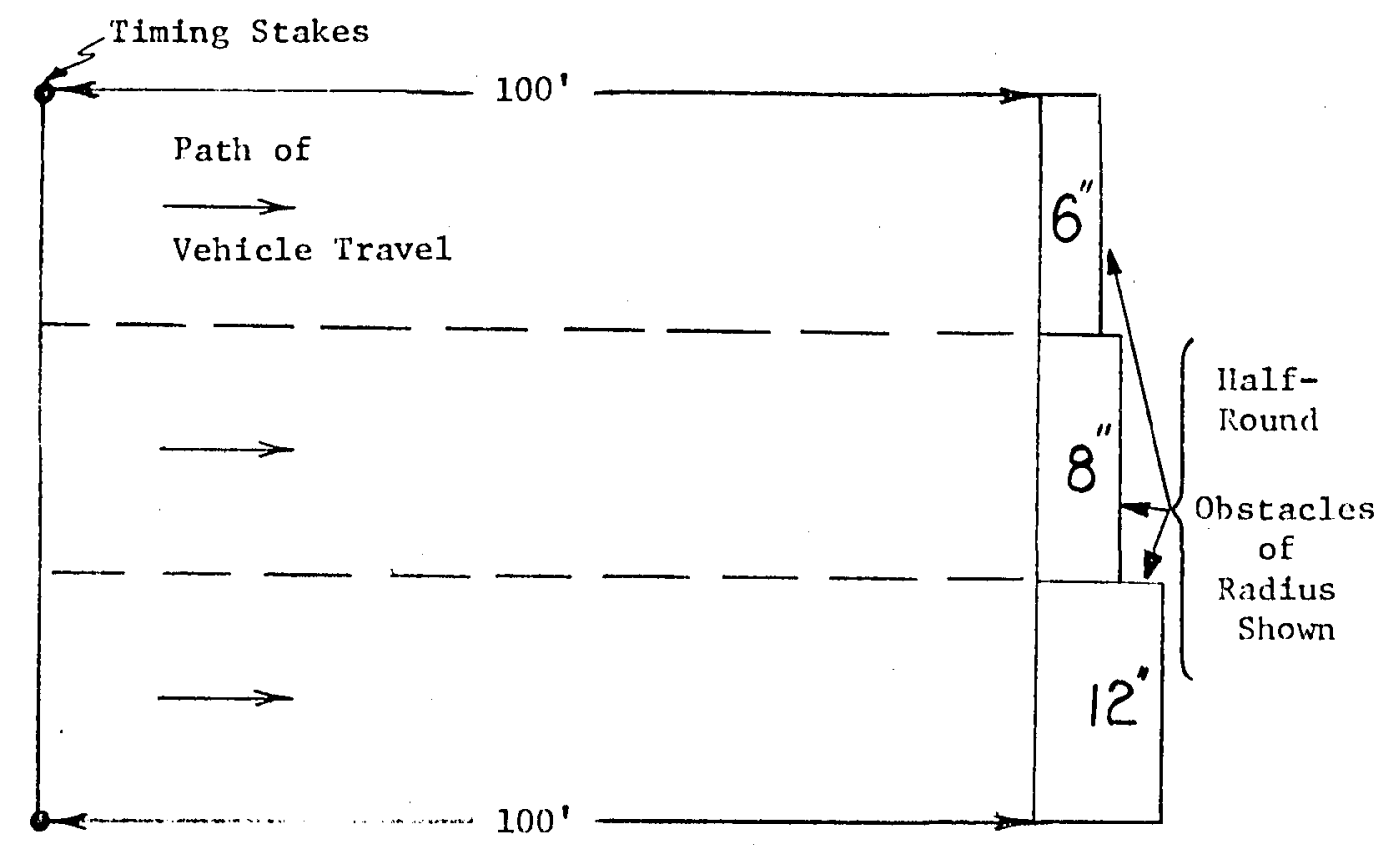

a. Plan View

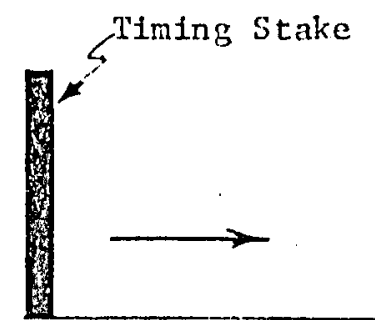

3 llalf-Round

Obstacles

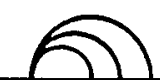

b. Profile View

Figure C8. Schematic of obstacle impact course 
independent of driver's judgment and provide a direct, objective measure of the vehicles' ride and vibration characterisitcs with changes in speed and surface roughness.

Shock tests

15. Four or five tests were conducted with each vehicle over each obstacle (6-, 8-, and 12-1n. helghts) at relatively constant speeds from $5 \mathrm{mph}$ to the maximum safe speed, usually in increments of about $5 \mathrm{mph}$.

16. Each test began by positioning the test vehicle a sufficient distance from the 100-ft timing stake (see Figure c8) so that the driver could obtain the desired test speed before reaching the stake. He then maintained that speed to the best of his ability (using the vehicle's speedometer) until the vehicle had completely crossed the obstacle. The vehicle impact speed when contacting the obstacles was computed by dividing the 100-ft distance by the time elapsed between passage of the stakes and obstacle contact. An observer rode in the vehicle during each test to operate and monitor the output of the peak counter and narrate the pertinent activities.

\section{Test Data Collected}

17. For this study the principal data for the ride tests consisted of vertical absorbed power at the driver's location. However, absorbed power was calculated for side-to-side and fore-to-aft motions. The same data recorded for the ride tests were recorded also for the discreteobstacle tests, but only the peak values of vertical accelerations beneath the driver's seat were considered in the shock analysis. The elapsed time and average speeds were determined for each ride and shock test. Although not used in the analysis, linear and angular accelerations were measured in the cargo area of vehicles with towed artillery and at the center of gravity of the two self-propelled pieces. The vertical accelerations of each towed plece were also measured. All measurements were recorded on magnetic tape. 


\section{$\underline{\text { Resu1ts }}$}

\section{Ride tests}

18. The Fort Sill Reservation did not offer the most desirable, terrain for ride quality evaluations. The level areas that were best suited for ride courses were either relatively smooth, cluttered with boulders, pitted with craters from artillery bursts, or lined with erosion features. Although they were representative of that particular geographic region, they did not fit the pattern of virgin terrain representative of many areas of the world in which ride and shock test data have been collected. 'The lack of this general degree of random, uniformity in the surface composition and a sultable range of terrain roughness restricted the use of the results of these tests in developing correlations with existing ride and shock relations. The tests, however, did serve the purpose for which they were intended and that was to show the effects of towed artillery on the ride quality of the prime movers.

19. The rationale for the analysis was to use the results of two recently completed experimental programs for investigating the ride and shock qualities of a number of standard and advanced-concept vehicles, which included pulling a variety of trailers with solid and liquid payloads. ${ }^{5,10}$ The results indicated that, for the most part, the towed load did not produce any significant influence on the ride quality of the prime mover.

20. A special test sequence was conducted to confirm these previously defined relations. Tests were conducted with the M35A2, 21/2-ton, cargo truck pulling the M102 and the XM198. The weight of the XM198 was nearly five times that of the M102 (15,250-1b and 3,338-1b respectively) and produced artillery-to-prime mover weight ratios of 0.83 and 0.18 . The M813, 5-ton, cargo truck was tested without a towed load and with the XM198, which produced artillery-to-prime mover weight ratios of 0.0 and 0.47 , respectively. It was felt that this range of conditions would suitably reflect any effects of towed load on ride quality for standard cargo vehicles. The results followed the pattern of the previous study, i.e., there was no distinguishable influence on 
ride quality by the towed load. This is reflected by the absorbed power-speed plots in Figures $\mathrm{C} 9$ through $\mathrm{Cl2}$, which illustrate representative relations for the $\mathrm{M} 35 \mathrm{~A} 2$ and $\mathrm{M} 813$ configurations on two crosscountry courses. The intermingling of the data substantiate that ride quality was not influenced by the towed load.

21. The next sequence of tests concerned two advanced-concept vehicles; the M520E1 towing the XM198 and the M561 towing the M102. The intent of these tests was to provide ride data with towed artillery to compare with ride data obtained from other tests with the prime movers operating without towed loads.

22. Figure C13 shows cross-country absorbed power-speed relations for the M520El with and without the towed artillery. The open symbols represent data obtained at Fort Sill with the XM198. The closed symbols represent data obtained in a previous program at Fort Hood, Texas, with the M520E1 operating without a towed load. The intermingling of the data again reflects that, as with the standard cargo vehicles, there is no effect of the towed load on the prime mover. Two separate relations are illustrated by the two lines drawn through the data. One relation is for data on the courses with surface roughness values ranging from 0.87 to $2.0 \mathrm{in.}$ (rms) and the other for those with surface roughness values of 0.50 and $0.69 \mathrm{in.}$ ( $\mathrm{rms}$ ).

23. The results of the M561 tests were not as well defined as those for the M35A2, M813, and M520E1 configurations. The absorbed power-speed data for the cross-country courses are plotted in Figure C14. Unfortunately, because of rain and vehicle breakdowns, tests with the M561 were restricted to only three of the six cross-country courses. The data reflect those obtained in the Fort Sill tests with the M561 pulling the M102 and those obtained in a previous program at Fort Hood with the M561 operating without a towed load. The data separate by surface roughness. Since the surface roughnesses of the courses at Fort Hood were different from those at Fort Sill, direct comparisons could not be made on the basis of the absorbed power-speed relations. Consequently, comparisons were made on the basis of the ride-limiting speed (the speed at which the 6-watt absorbed power level occurred) 


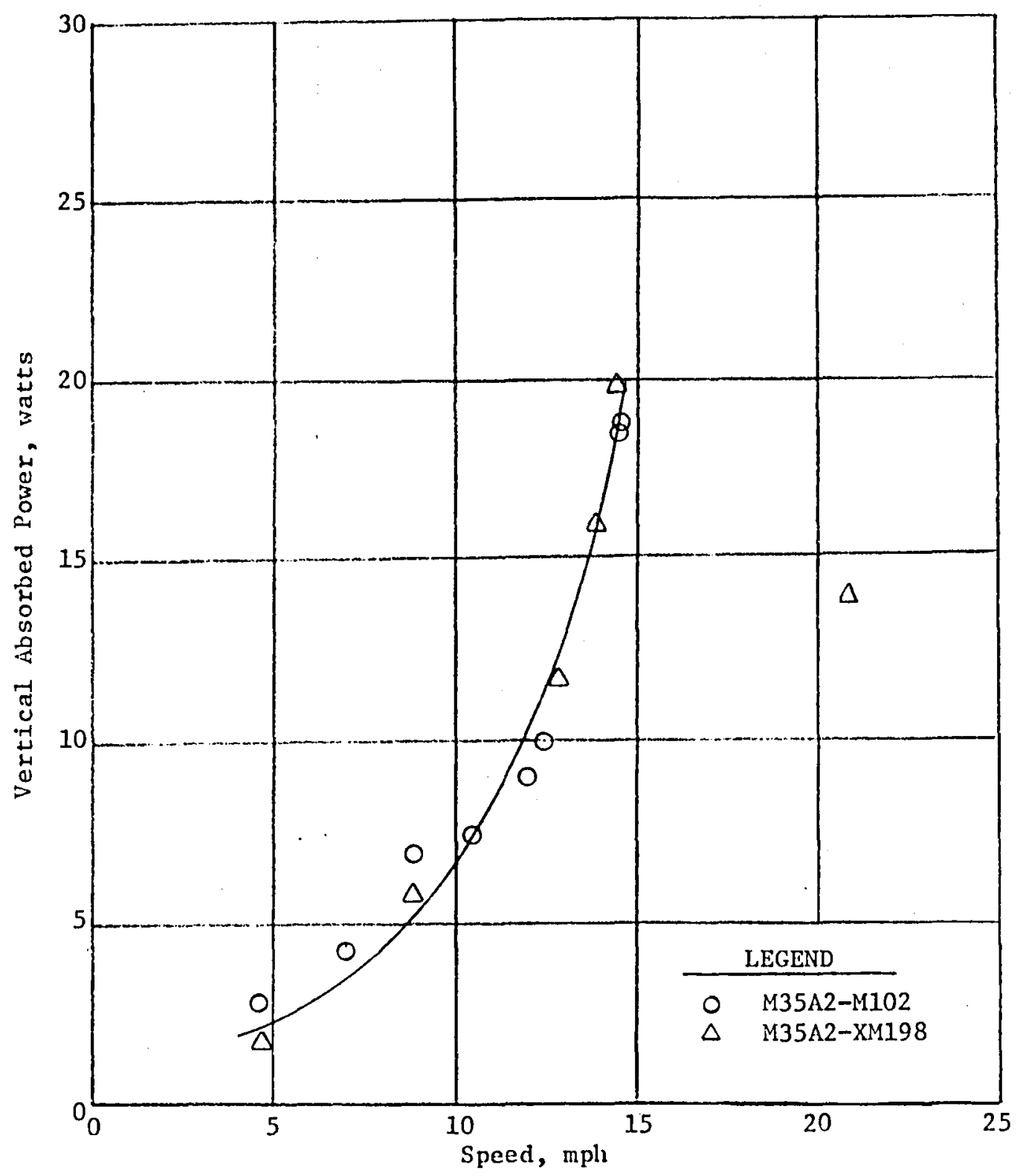

Figure C9. Vertical absorbed power-speed relations for $135 \mathrm{~A} 2$ configurations on course FSCC2 


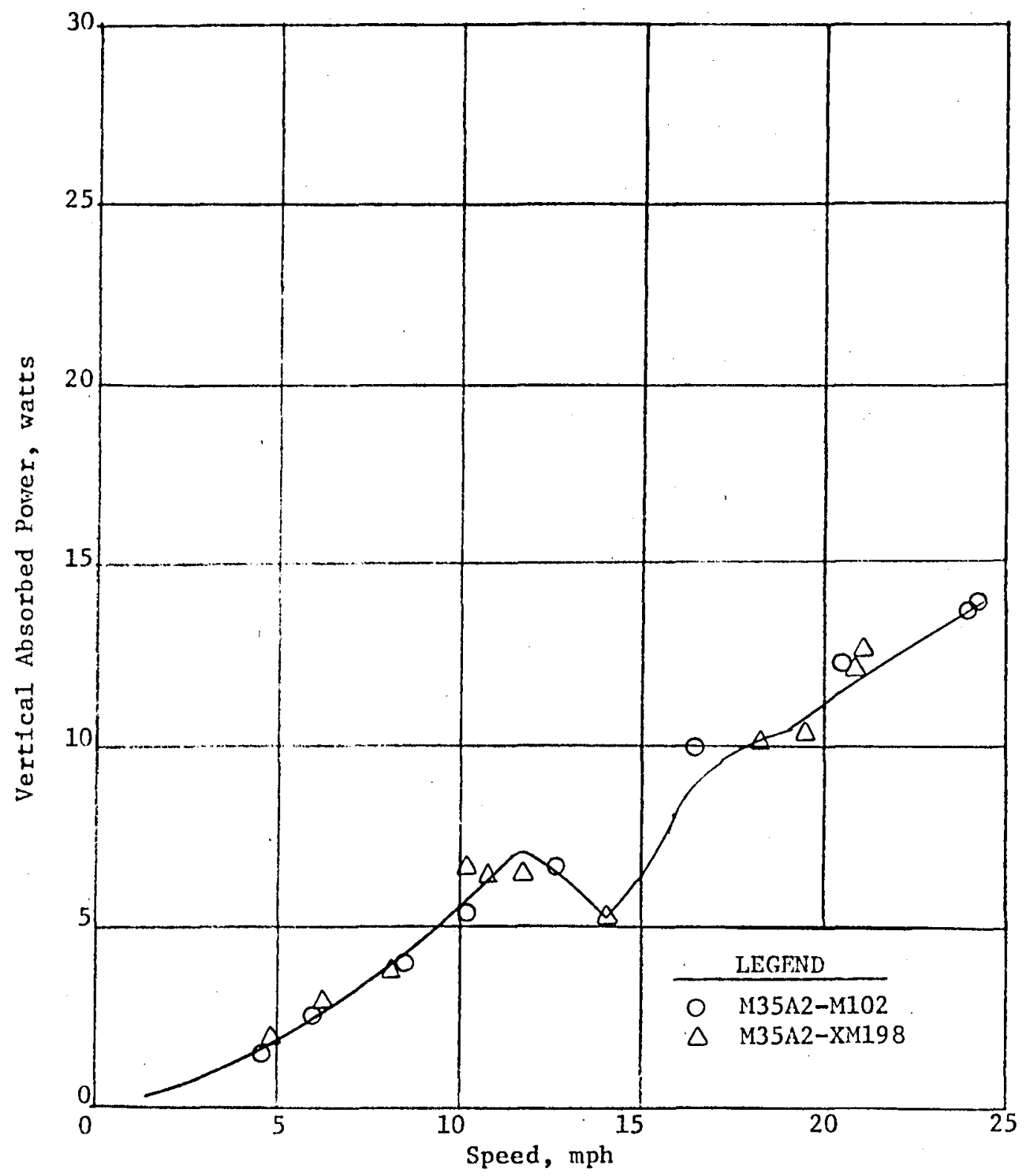

Figure C10. Vertical absorbed power-speed relations for $M 35 \Lambda 2$ configurations on course FSCC3 


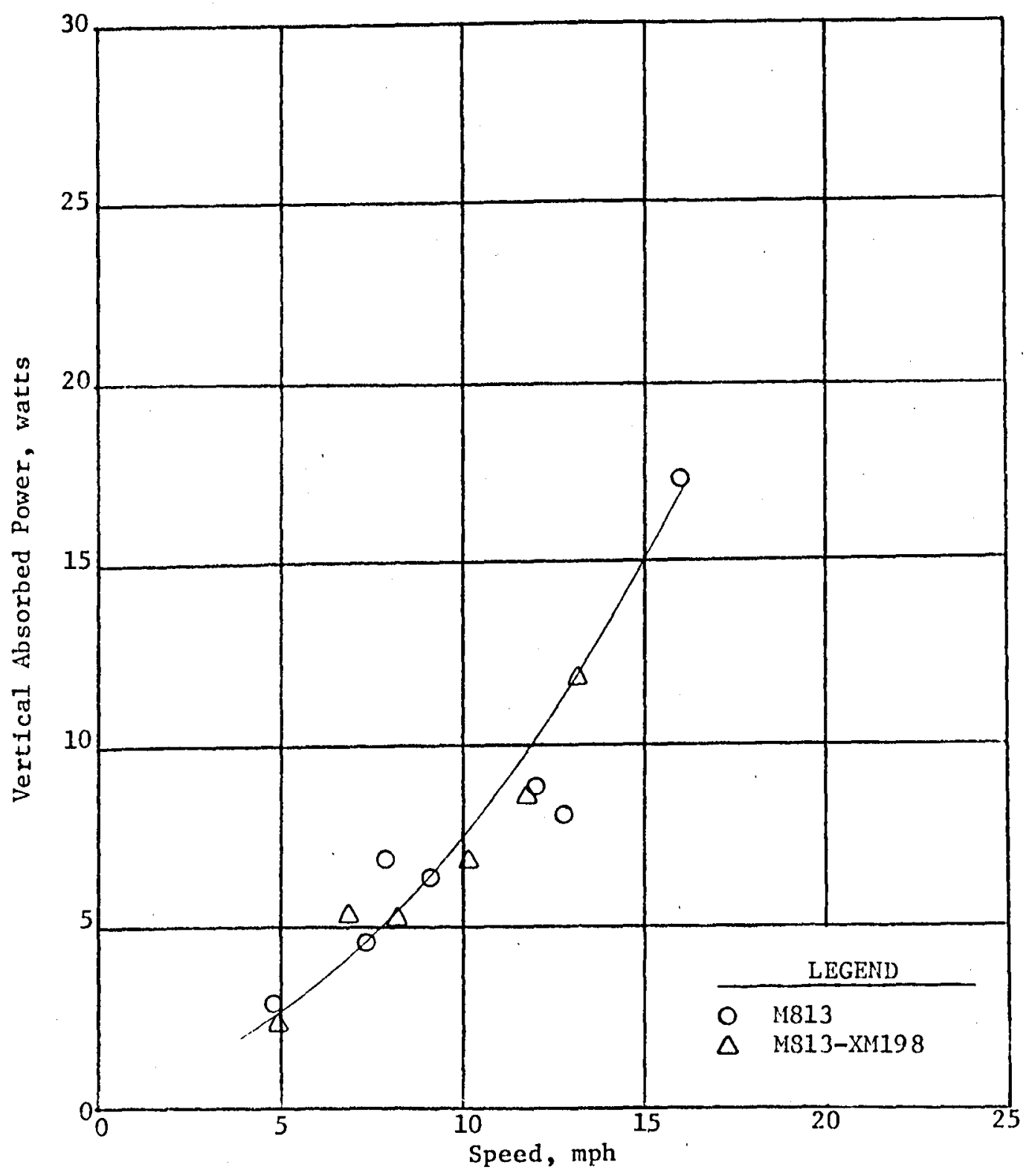

Figure C11. Vertical absorbed power-speed relations for M813 configurations on course FSCC2 


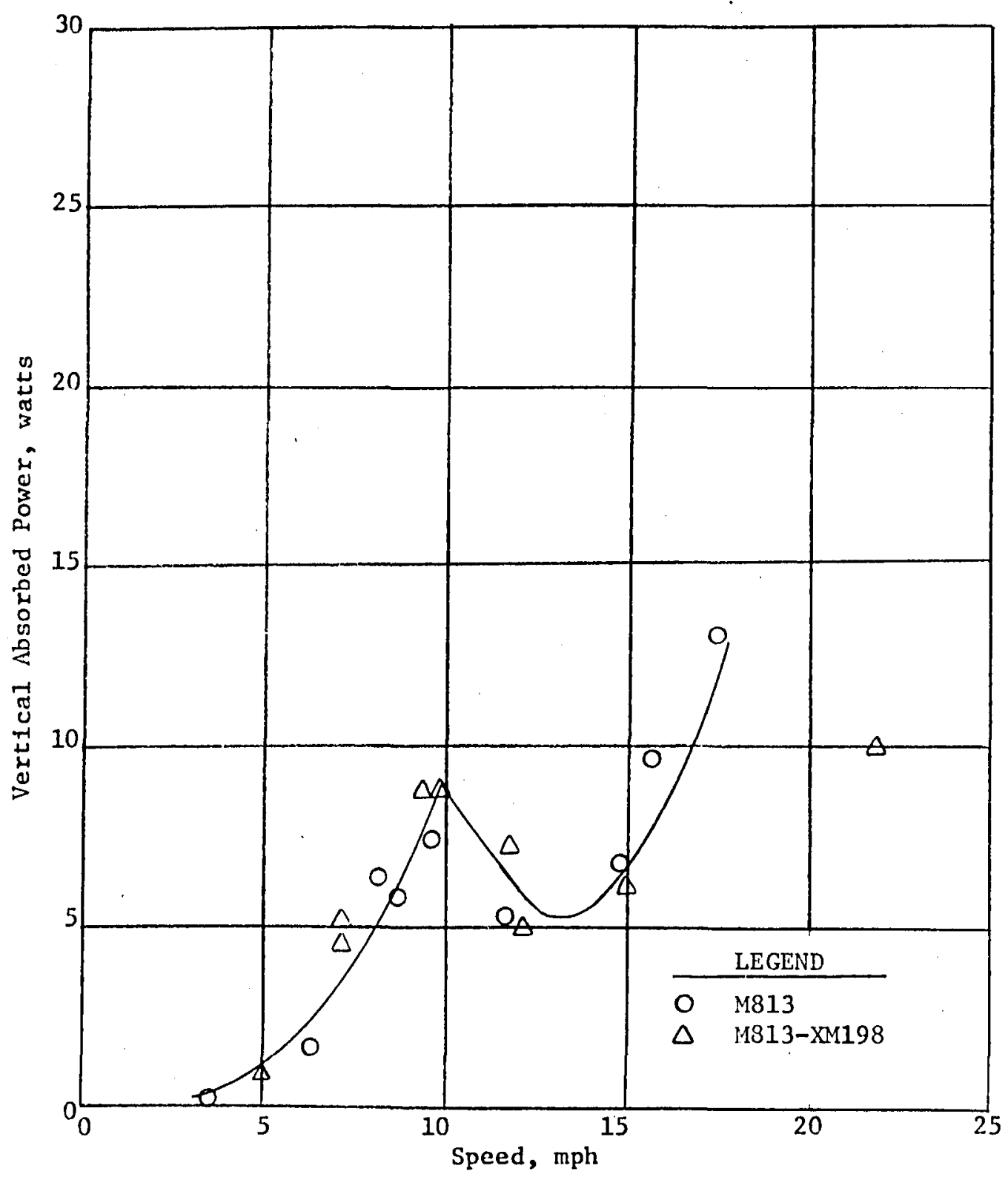

Figure C12. Vertical absorbed power-speed relations for M813 configurations on course FSCC3 


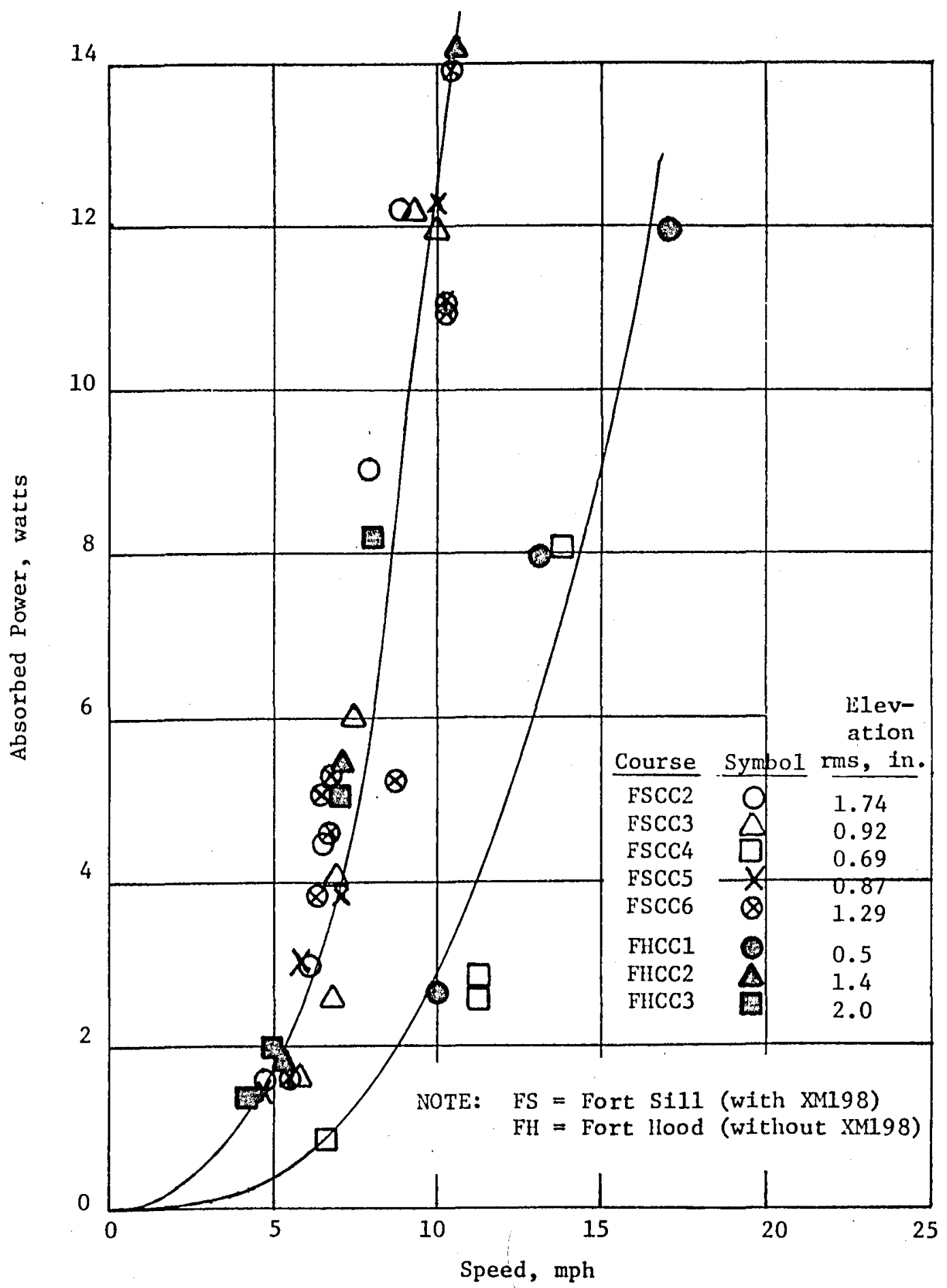

Figure C13. Cross-country absorbed power-speed relations for M520E1 with and without the XM198 


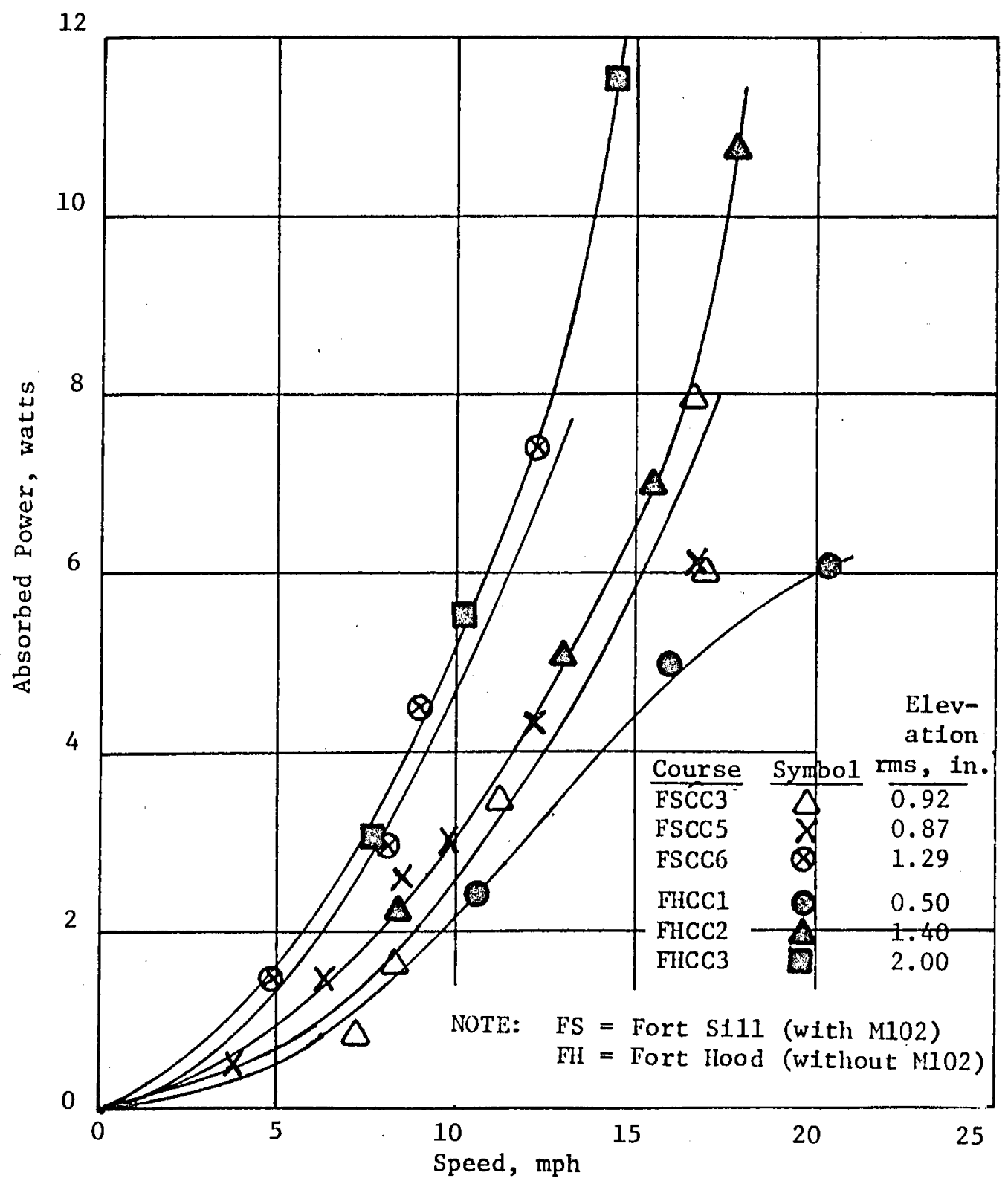

Figure C14. Absorbed power-speed relation for M561 with and without the towed M102 
versus surface roughness relation (Figure C15). Only two points were available from the Fort Sill tests since two of the courses were of about the same level of roughness and produced similar ride responses (see Figure $\mathrm{C14}$ ). These two points, along with the three obtained from the Fort Hood tests, are plotted with the curve representing the established ride relation for the M561 operating without a towed load. This curve was established from the results of the Fort Hood tests. A deviation from the curve of less than $2 \mathrm{mph}$ is noted for one of the Fort Sill test coordinates; the other falls very close to the curve. This deviation is within the accepted data scatter since it has been established from other test data that variations of $2 \mathrm{mph}$ are not significant. Although the data for this configuration are meager, it is felt justified to assume there is no effect of the towed load on ride quality.

24. Experimental ride data are completely non-existent for selfpropelled artillery. Consequently, a rather extensive testing program was anticipated with the M109A1 and M110E1 self-propelled pieces to define ride relations for those type of vehicles. Although a substantial number of tests were conducted with each of these vehicles, the results were not as definitive as had been desired. On most courses, the 6-watt tolerance limit was not reached because the approaches 1imited the vehicles' top speeds. Both the data and comments of the drivers confirmed that the two vehicles had similar ride characteristics. The similarity in the ride of the two vehicles is illustrated in Figure C16 by the absorbed power-speed plots which represent the results for the two vehicles operating on a cross-country and two trail courses. These are the only courses that produced any significant levels of absorbed power. By assuming that the vehicles had the same ride characteristics, ride relations were developed by extrapolating the data and using judgment based on past experience. The resulting relations for crosscountry and trails are shown in Figure $\mathrm{C} 17$. The ride relations for the other vehicles tested in this program are shown in Figures C18-C21. A distinction is made between cross-country courses and trail courses, and separate relations are drawn because past experience with ride tests on 


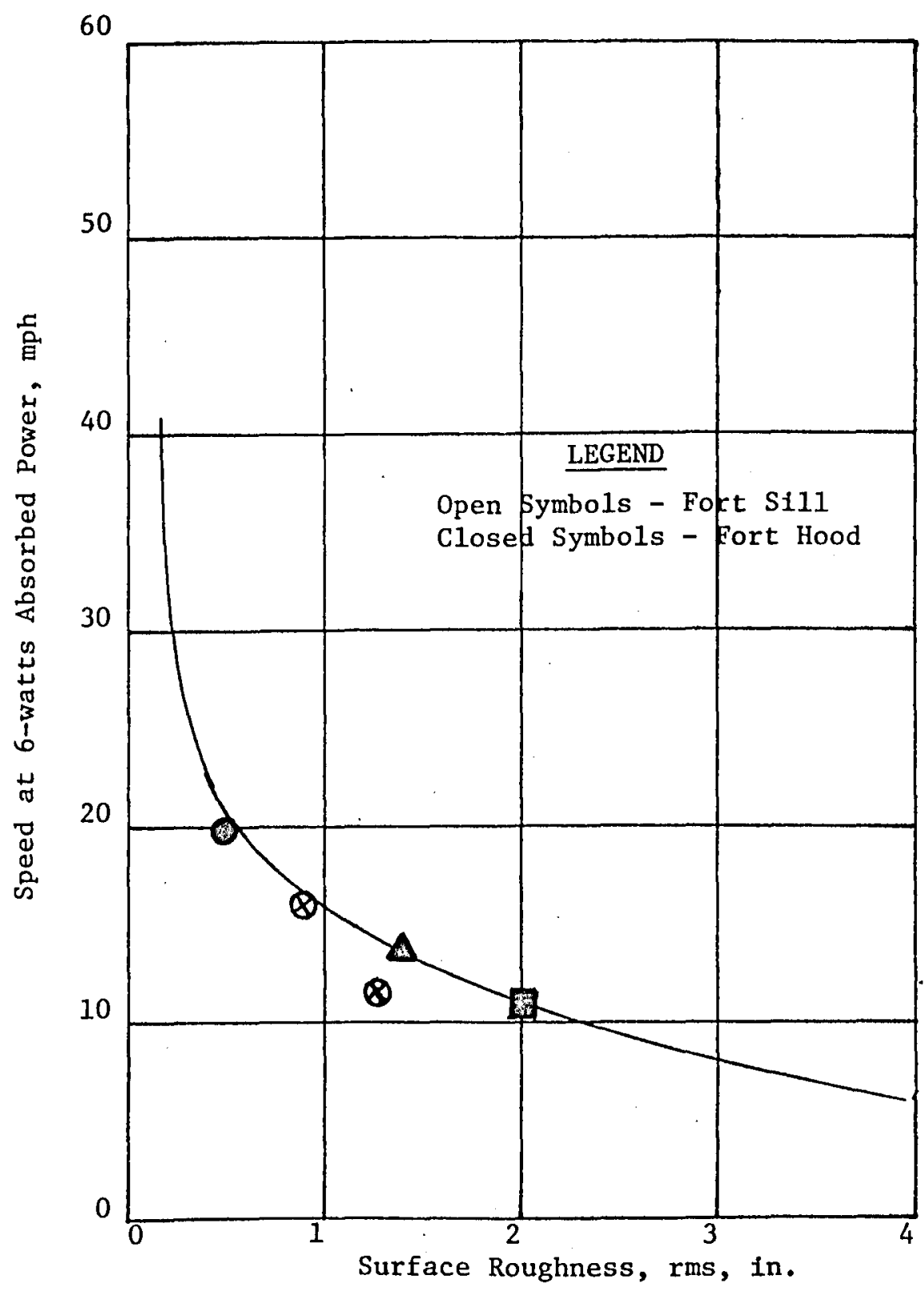

Figure C15. Ride limiting speed-surface roughness relation for M561 

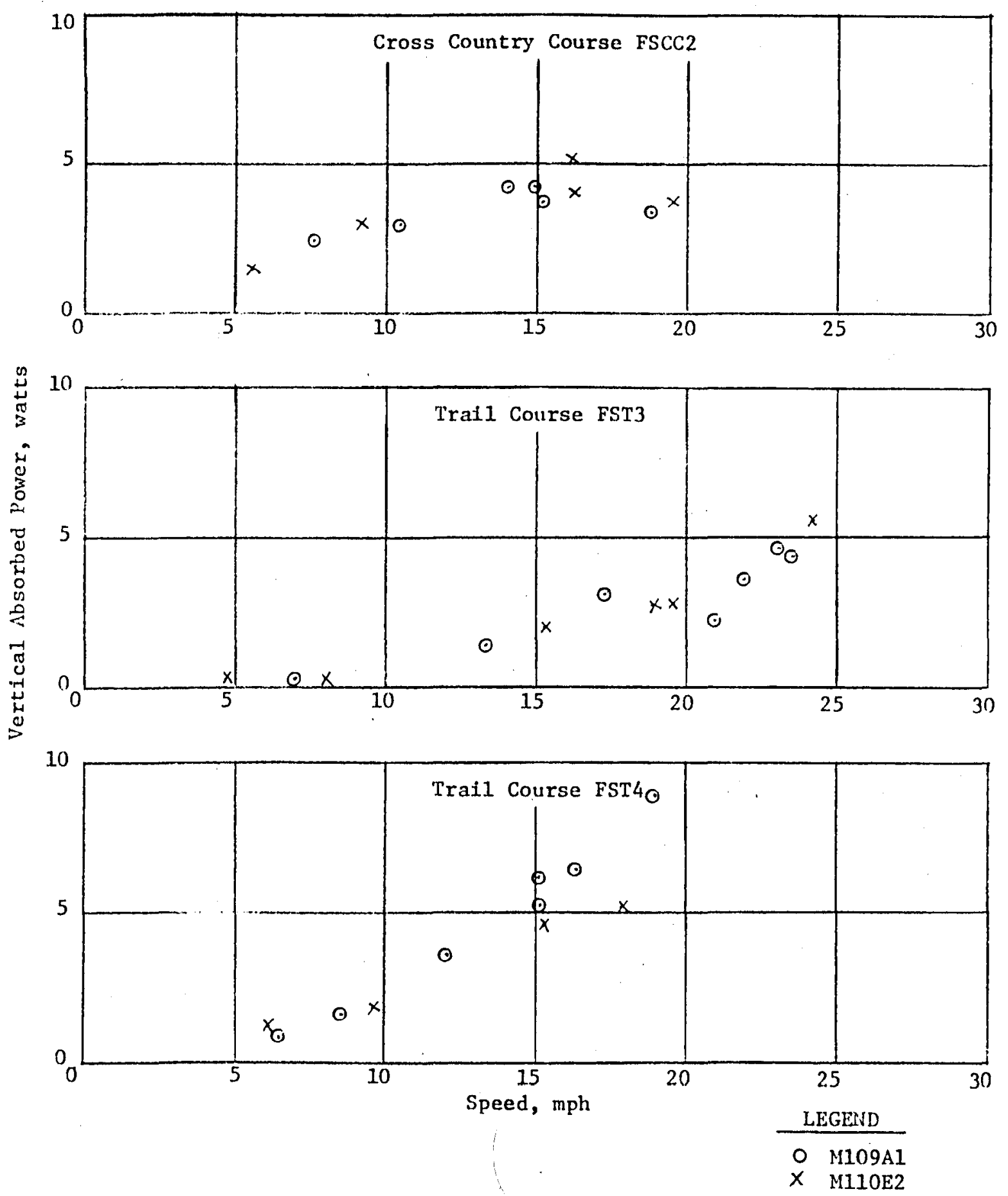

Figure C16. Absorbed power-speed relations for the M109A1 and M110E2 selfpropelled artillery on selected cross-country and trail courses 


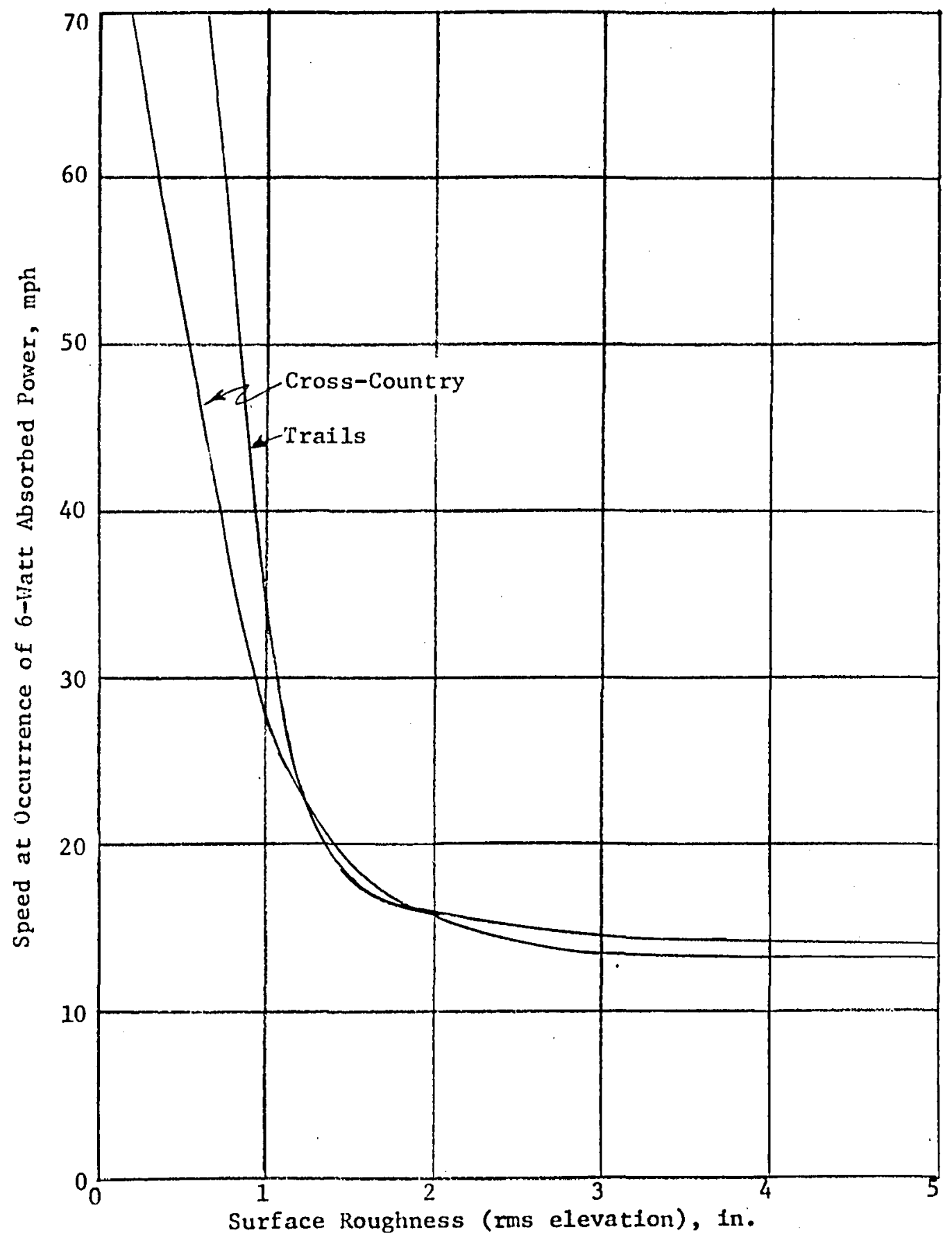

Figure C17. Ride limiting speed-surface roughness relations for M109A1 and MIIOE2 self-propelled artillery 


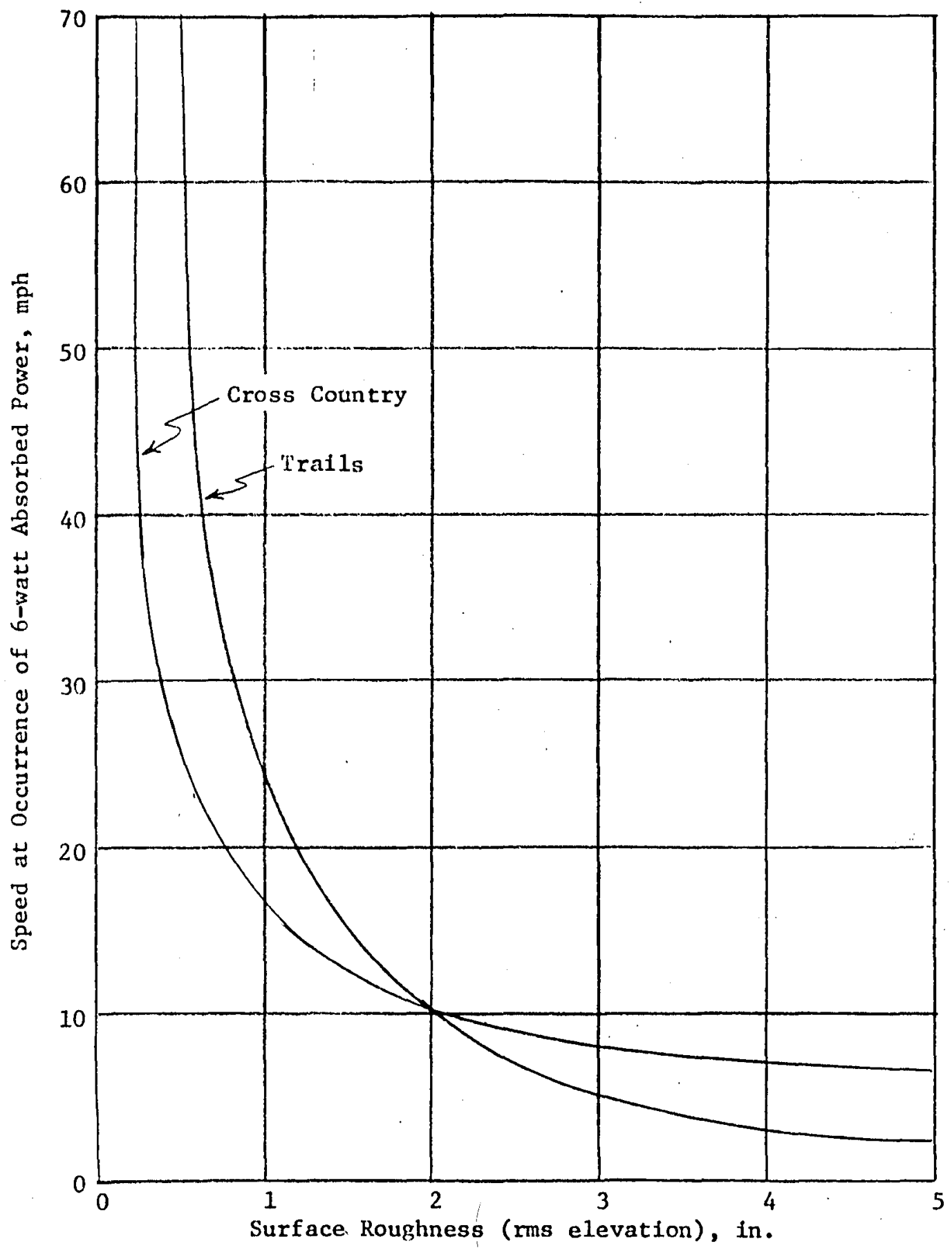

Figure C18. Ride limiting speed-surface roughness relations for M35A2 


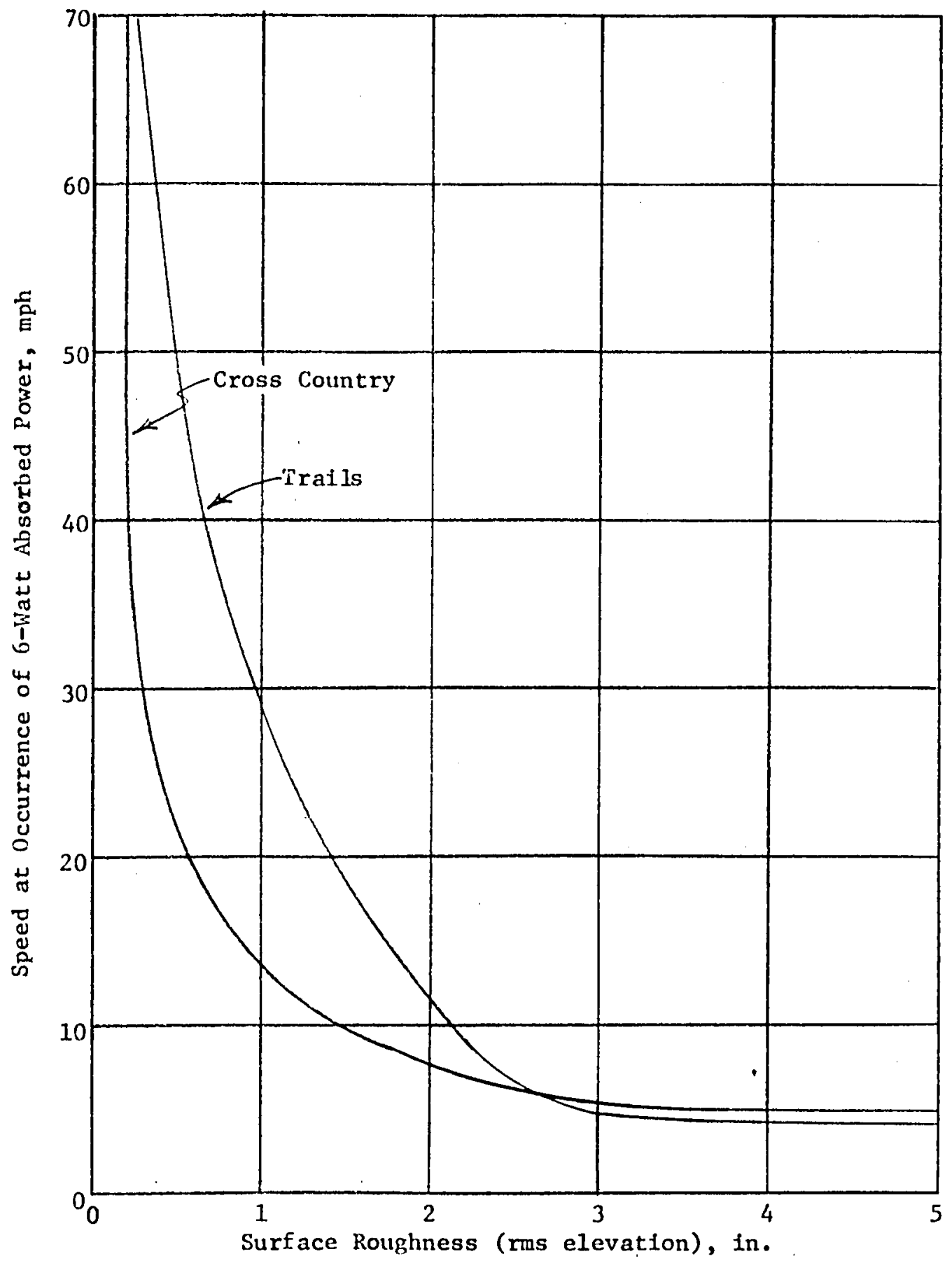

Figure C19. Ride limiting speed-surface roughness relations for M813 


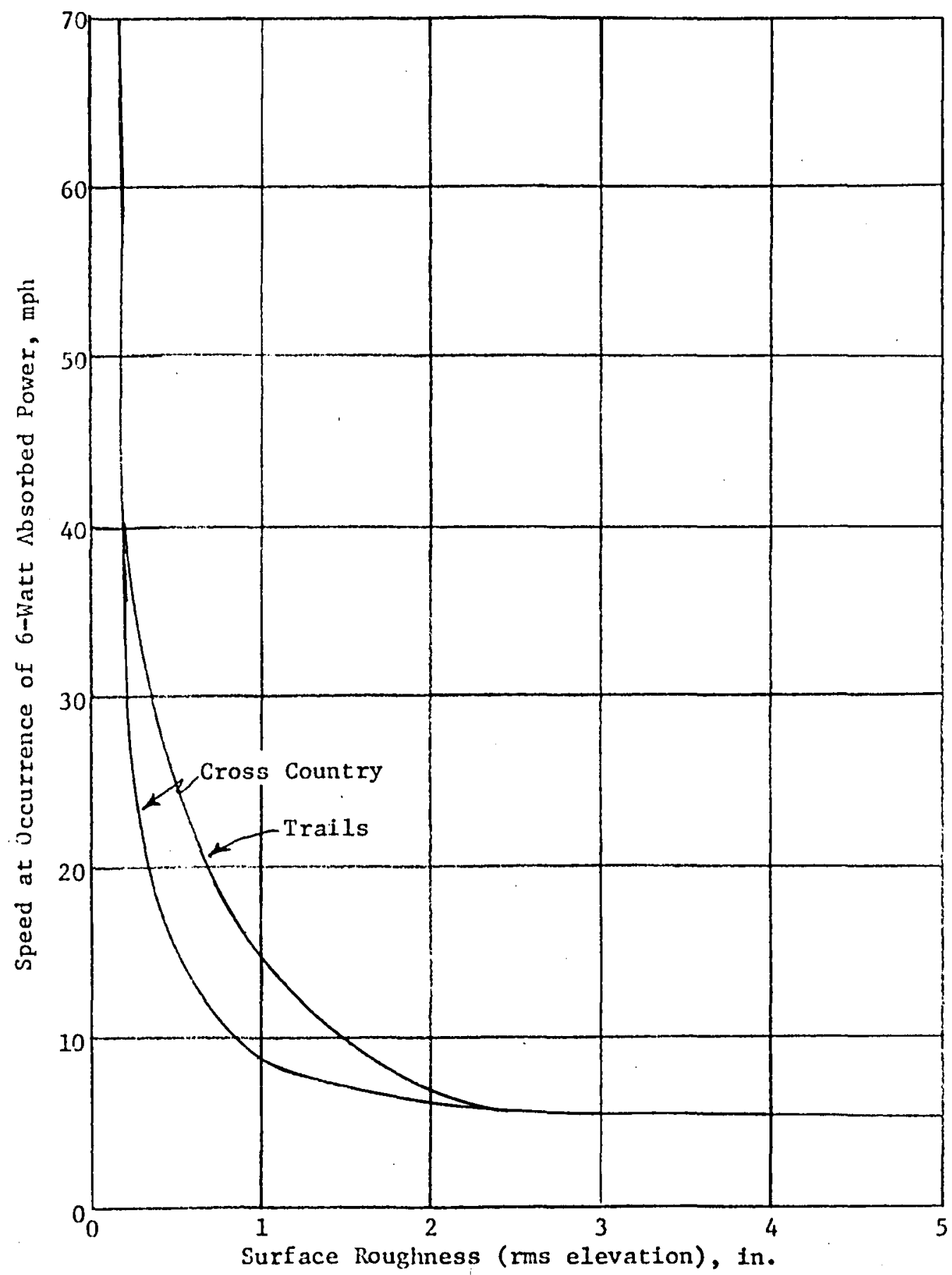

Figure $\mathrm{C20}$. Ride limiting speed-surface roughness relations for M520E1 


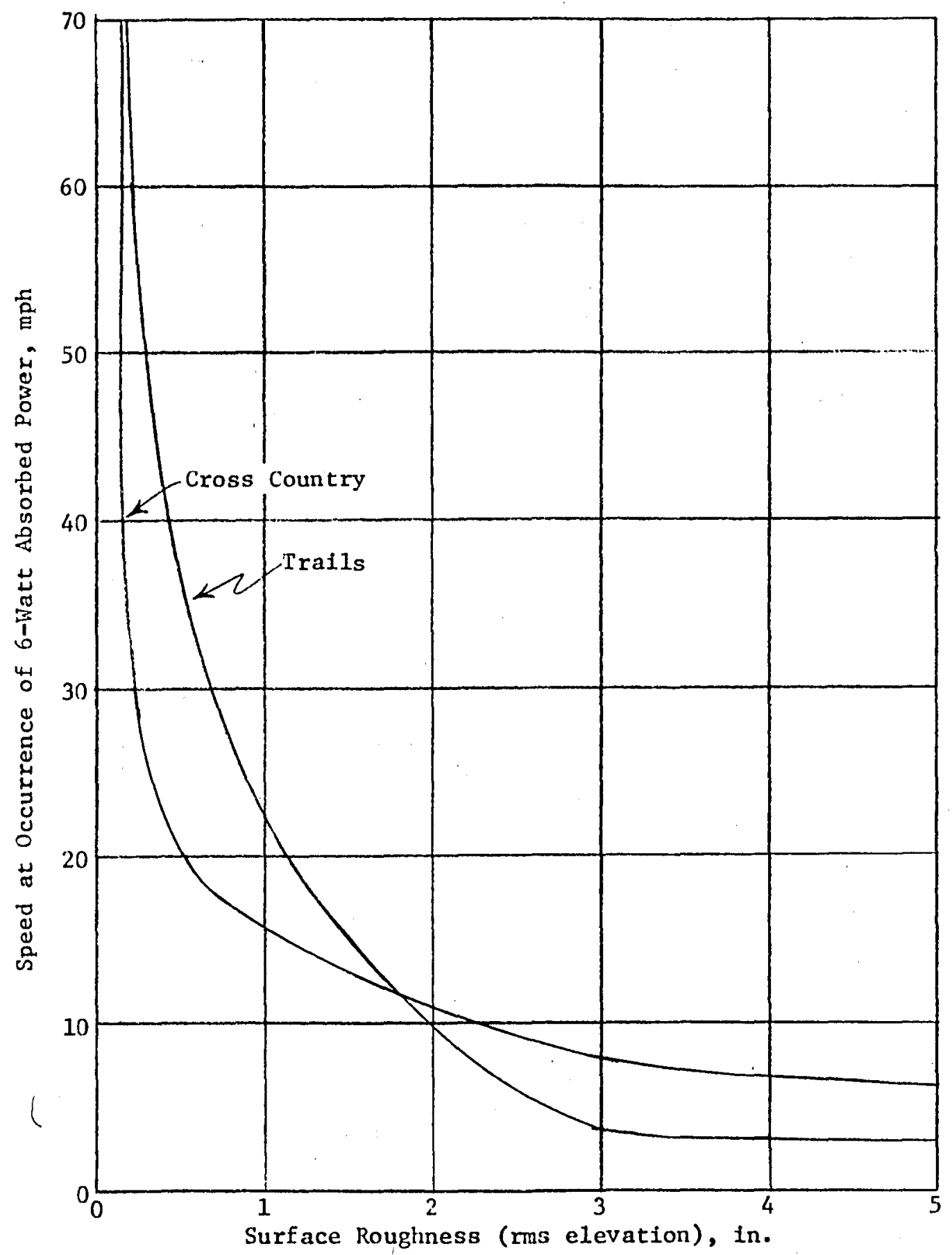

Figure C21. Ride limiting speed-surface roughness relations for M561 
cross-country and trail courses, or other such surfaces conditioned by repetitive vehicular traffic, has revealed that trail courses generally permit higher speeds for corresponding levels of ride and surface roughness. Repetitive vehicular traffic on trails, particularly that of heavy track-laying vehicles, tends to smooth out the high-frequency components in the terrain surface, which excite the vehicle suspensions in their sensitive frequency regions and constitute a large portion of the vibrational energy transmitted to the vehicle's main frame. The relations chosen by the Army Mobility Model in mobility predictions depend on the type of surface over which the vehicle is traveling. A complete listing of the ride relations for the various configurations considered in this study are listed for cross-country, and roads and trails, respectively in Table $\mathrm{B} 5-\mathrm{B} 6$ of Appendix $\mathrm{B}$. The ride data collected during this study are listed for each vehicle on each course tested in Table $\mathrm{Cl}$. The side-to-side, fore-to-aft, and total absorbed power are listed also.

25. Since these tests confirmed there were no significant influences on ride quality due to the towed loads, the ride relations established for the prime movers from the large bank of existing data were used in this study because they represent a broader range of terrain conditions.

\section{Shock tests}

26. The shock tests were limited by the Inability of both the M102 and the XM198 to cross the obstacles without damage. The data collected are listed in Table $\mathrm{C} 2$. These data indicate only the number of peak accelerations falling within six selected intervals ranging from 1 to $4 \mathrm{~g}$. Only rarely was the $2.5 \mathrm{~g}$ shock limit reached because the speeds were limited by the towed artillery. These data are not sufficient to draw any reliable conclusions concerning shock responses. Consequently, the shock relations characterized for the prime movers from previous data bases were used in this study. Shock responses for the self-propelled artillery were judiciously determined from existing shock relations of similar vehicles. The relations used in this study are listed in Table $B 4$. 
27. Tire and rim damage were predominant problems with the towed artillery. Over the rough cross-country and trail courses thetr severe motion limited speeds to not more than $15 \mathrm{mph}$. These limits were not considered in the mobility predictions, but it is suggested that more testing should be considered to develop relations for these limits in terms of speed and surface roughness. 
Table Cl

Ride Data

\begin{tabular}{|c|c|c|c|c|c|c|c|}
\hline \multirow[b]{2}{*}{$\begin{array}{c}\text { Course } \\
\text { No. } \\
\end{array}$} & \multirow[b]{2}{*}{$\begin{array}{c}\text { Test } \\
\text { No. } \\
\end{array}$} & \multirow[b]{2}{*}{$\begin{array}{c}\text { Roughness } \\
\text { rms } \\
\end{array}$} & \multirow[b]{2}{*}{$\begin{array}{c}\text { Speed } \\
\text { mph }\end{array}$} & \multicolumn{3}{|c|}{$\begin{array}{c}\text { Average Absorbed } \\
\text { Power, watts }\end{array}$} & \multirow{2}{*}{$\begin{array}{c}\text { Total } \\
\text { Average } \\
\text { Absorbed } \\
\text { Power, } \\
\text { watts } \\
\end{array}$} \\
\hline & & & & Vertical & $\begin{array}{l}\text { Side- } \\
\text { to-Side }\end{array}$ & $\begin{array}{l}\text { Fore- } \\
\text { to-Aft }\end{array}$ & \\
\hline & & & M35A & -XM198 & & & \\
\hline \multirow[t]{6}{*}{$\mathrm{CC}-2$} & 0104 & 1.74 & 8.8 & 5.8 & 3.3 & 3.8 & 12.9 \\
\hline & 0105 & & 20.8 & 14.0 & 3.9 & 6.9 & 24.8 \\
\hline & 0106 & & 13.8 & 16.1 & 6.7 & 11.3 & 34.1 \\
\hline & 0107 & & 12.9 & 11.7 & 5.2 & 7.5 & 24.4 \\
\hline & 0108 & & 14.5 & 19.9 & 4.8 & 13.7 & 38.4 \\
\hline & 0109 & & 4.8 & 1.8 & 2.9 & 1.6 & 6.3 \\
\hline \multirow[t]{15}{*}{$\mathrm{CC}-3$} & 0001 & 0.92 & * & * & * & * & * \\
\hline & 0002 & & 10.2 & 6.7 & 4.5 & 3.1 & 14.3 \\
\hline & 0003 & & 8.2 & 3.6 & 4.5 & 1.9 & 10.0 \\
\hline & 0004 & & 14.3 & 10.1 & 3.6 & 4.5 & 18.2 \\
\hline & 0005 & & $\star$ & * & $\star$ & $*$ & * \\
\hline & 0006 & & 4.8 & 0.8 & 2.0 & 0.7 & 3.5 \\
\hline & 0007 & & 19.5 & 10.3 & 7.6 & 5.4 & 23.3 \\
\hline & 0008 & & 6.3 & 2.7 & 2.9 & 2.6 & 8.2 \\
\hline & 0009 & & 21.1 & 12.7 & 7.6 & 7.0 & 27.3 \\
\hline & 0010 & & 10.8 & 6.5 & 4.1 & 2.8 & 13.4 \\
\hline & 0011 & & 20.9 & 12.2 & 5.5 & 6.4 & 24.3 \\
\hline & 0012 & & 11.8 & 6.6 & 3.3 & 2.6 & 12.5 \\
\hline & 0013 & & 18.3 & 9.9 & 6.4 & 4.8 & 21.1 \\
\hline & 0014 & & $\star$ & * & * & * & $\star$ \\
\hline & 0015 & & * & * & * & * & * \\
\hline \multirow[t]{6}{*}{$\mathrm{CC}-4$} & 0115 & 0.69 & 14.6 & 4.8 & 1.7 & 2.5 & 9.0 \\
\hline & 0116 & & 10.7 & 3.9 & 1.5 & 2.0 & 7.4 \\
\hline & 0117 & & 8.7 & 2.7 & 1.3 & 1.8 & 5.8 \\
\hline & 0118 & & 20.4 & 6.3 & 2.1 & 7.8 & 16.2 \\
\hline & 0119 & & 7.0 & 2.0 & 1.2 & 1.5 & 4.7 \\
\hline & 0120 & & 4.9 & 0.9 & 1.1 & 0.7 & 2.7 \\
\hline \multirow[t]{6}{*}{$\mathrm{CC}-5$} & 0090 & 0.87 & 8.9 & 3.6 & 1.4 & 2.4 & 7.4 \\
\hline & 0091 & & 7.5 & 3.8 & 2.3 & 2.8 & 8.9 \\
\hline & 0092 & & 15.0 & 5.0 & 2.2 & 2.9 & 10.1 \\
\hline & 0093 & & 4.8 & 1.7 & 2.1 & 1.4 & 5.2 \\
\hline & 0094 & & 15.9 & 5.0 & 2.2 & 4.0 & 11.2 \\
\hline & 0095 & & 21.1 & 9.0 & 2.8 & 5.5 & 17.3 \\
\hline
\end{tabular}

* Data voided on these tests.

(Sheet 1 of 16) 
Table C1 (Continued)

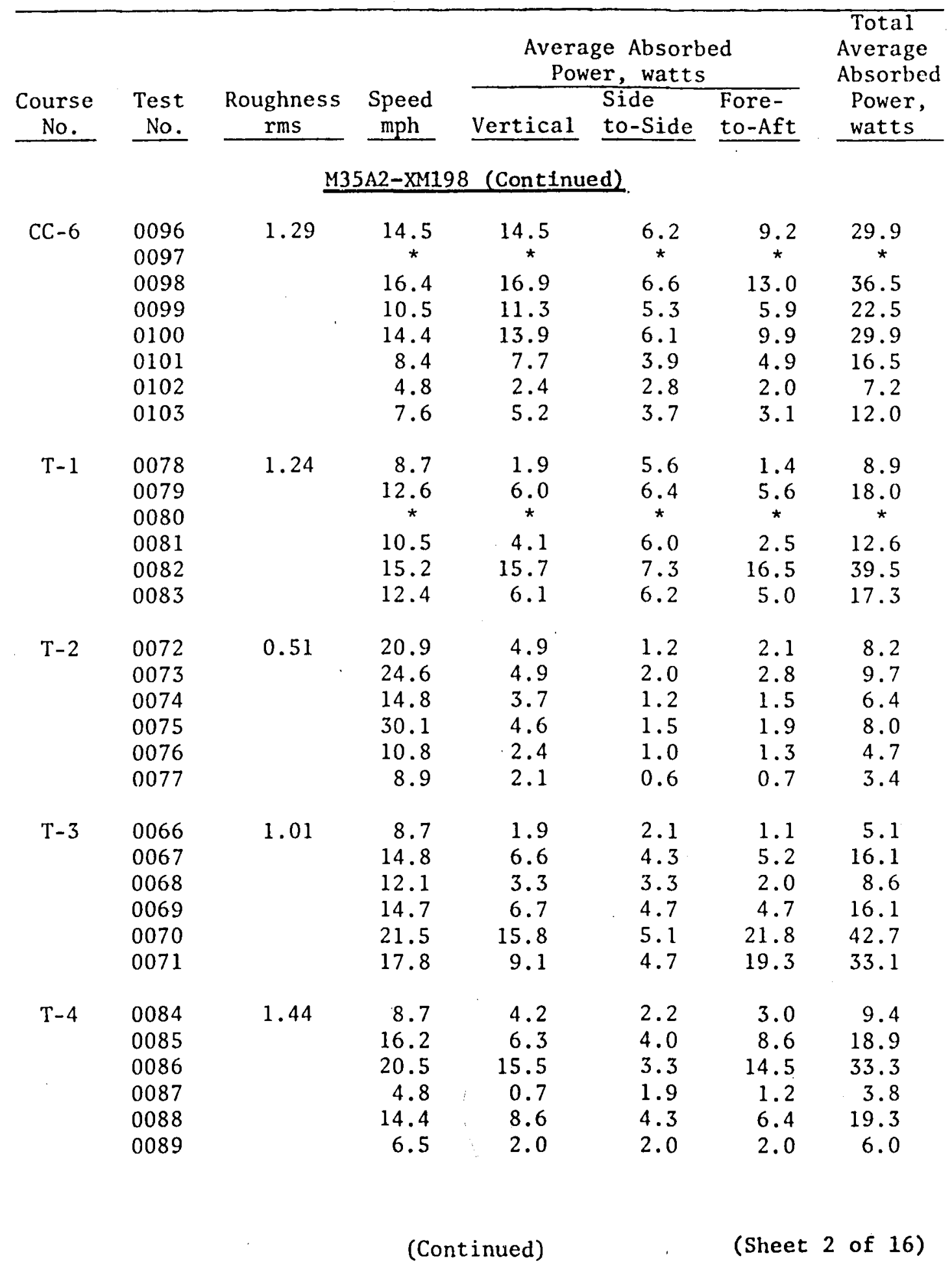


Table C1 (Continued)

\begin{tabular}{|c|c|c|c|c|c|c|c|}
\hline \multirow[b]{2}{*}{$\begin{array}{c}\text { Course } \\
\text { No. } \\
\end{array}$} & \multirow[b]{2}{*}{$\begin{array}{c}\text { Test } \\
\text { No. }\end{array}$} & \multirow[b]{2}{*}{$\begin{array}{c}\text { Roughness } \\
\text { rms }\end{array}$} & \multirow[b]{2}{*}{$\begin{array}{c}\text { Speed } \\
\mathrm{mph} \\
\end{array}$} & \multicolumn{3}{|c|}{$\begin{array}{c}\text { Average Absorbed } \\
\text { Power, watts }\end{array}$} & \multirow{2}{*}{$\begin{array}{l}\text { Total } \\
\text { Average } \\
\text { Absorbed } \\
\text { Power, } \\
\text { watts }\end{array}$} \\
\hline & & & & Vertical & $\begin{array}{l}\text { Side } \\
\text { to-Side }\end{array}$ & $\begin{array}{l}\text { Fore- } \\
\text { to-Aft }\end{array}$ & \\
\hline \multicolumn{8}{|c|}{ M35A2-XM198 (Continued) } \\
\hline \multirow[t]{6}{*}{$\mathrm{SR}-1$} & 0110 & 0.09 & 33.3 & 0.6 & 0.0 & 0.0 & 0.6 \\
\hline & 0111 & & 21.5 & 0.9 & 0.0 & 0.3 & 1.2 \\
\hline & 0112 & & 37.9 & 0.6 & 0.1 & 0.0 & 0.7 \\
\hline & 0113 & & 17.7 & 0.8 & 0.0 & 0.1 & 0.9 \\
\hline & 0114 & . & 10.7 & 0.4 & 0.0 & 0.0 & 0.4 \\
\hline & \multicolumn{6}{|c|}{$\mathrm{M} 35 \mathrm{~A} 2-\mathrm{M} 102$} & \\
\hline \multirow[t]{9}{*}{$\mathrm{CC}-2$} & 0025 & 1.74 & * & * & * & * & * \\
\hline & 0026 & & 4.7 & 2.9 & 3.5 & 1.6 & 8.0 \\
\hline & 0027 & & 8.7 & 7.0 & 4.2 & 3.5 & 14.7 \\
\hline & 0028 & & 10.4 & 7.4 & 3.8 & 2.9 & 14.1 \\
\hline & 0029 & & 14.7 & 18.7 & 4.7 & 7.6 & 31.0 \\
\hline & 0030 & & 12.6 & 10.0 & 3.6 & 3.7 & 17.3 \\
\hline & 0031 & & 12.0 & 8.9 & 3.2 & 3.3 & 15.4 \\
\hline & 0032 & & 7.1 & 4.3 & 3.6 & 2.6 & 10.5 \\
\hline & 0033 & & 14.7 & 18.8 & 4.9 & 7.0 & 30.7 \\
\hline \multirow[t]{9}{*}{$\mathrm{CC}-3$} & 0016 & 0.92 & 12.5 & 6.6 & 3.7 & 1.8 & 12.1 \\
\hline & 0017 & & 8.5 & 4.0 & 3.9 & 1.7 & 9.6 \\
\hline & 0018 & & 16.5 & 10.0 & 6.2 & 4.0 & 20.2 \\
\hline & 0019 & & 4.7 & 0.6 & 2.1 & 0.3 & 3.0 \\
\hline & 0020 & & 20.5 & 12.3 & 6.3 & 3.9 & 22.5 \\
\hline & 0021 & & 6.0 & 1.1 & 2.7 & 0.4 & 4.2 \\
\hline & 0022 & & 24.1 & 13.8 & 3.5 & 3.5 & 20.8 \\
\hline & 0023 & & 10.2 & 5.5 & 3.8 & 1.8 & 11.1 \\
\hline & 0024 & & 24.4 & 13.9 & 3.2 & 3.6 & 20.7 \\
\hline \multirow[t]{6}{*}{$\mathrm{CC}-4$} & 0146 & 0.69 & 14.5 & 3.6 & 1.4 & 1.1 & 6.1 \\
\hline & 0147 & & 21.0 & 5.1 & 2.8 & 2.4 & 10.3 \\
\hline & 0148 & & 21.8 & 6.6 & 1.9 & 3.0 & 11.5 \\
\hline & 0149 & & 24.4 & 5.3 & 2.2 & 2.5 & 10.0 \\
\hline & 0150 & & 11.2 & 2.9 & 1.1 & 1.1 & 5.1 \\
\hline & 0151 & & 18.1 & 5.0 & 2.3 & 2.6 & 9.9 \\
\hline \multirow{5}{*}{ CC -5} & 0047 & 0.87 & * & * & * & * & * \\
\hline & 0048 & & 8.7 & 4.1 & 2.6 & 1.7 & 8.4 \\
\hline & 0049 & & 15.2 & 6.2 & 1.3 & 1.8 & 9.3 \\
\hline & 0050 & & 12.1 & 6.4 & 1.4 & 2.1 & 9.9 \\
\hline & 0051 & & 12.9 & 5.1 & 1.3 & 1.4 & 7.8 \\
\hline
\end{tabular}

(Continued)

(Sheet 3 of 16) 
Table C1 (Continued)

\begin{tabular}{|c|c|c|c|c|c|c|c|}
\hline \multirow[b]{2}{*}{$\begin{array}{c}\text { Course } \\
\text { No. }\end{array}$} & \multirow[b]{2}{*}{$\begin{array}{l}\text { Test } \\
\text { No. }\end{array}$} & \multirow[b]{2}{*}{$\begin{array}{l}\text { Roughness } \\
\text { rms }\end{array}$} & \multirow[b]{2}{*}{$\begin{array}{c}\text { Speed } \\
\mathrm{mph}\end{array}$} & \multicolumn{3}{|c|}{$\begin{array}{c}\text { Average Absorbed } \\
\text { Power, watts }\end{array}$} & \multirow{2}{*}{$\begin{array}{c}\text { Total } \\
\text { Average } \\
\text { Absorbed } \\
\text { Power, } \\
\text { watts } \\
\end{array}$} \\
\hline & & & & Vertical & $\begin{array}{l}\text { Side } \\
\text { to-Side } \\
\end{array}$ & $\begin{array}{l}\text { Fore- } \\
\text { to-Aft }\end{array}$ & \\
\hline \multicolumn{8}{|c|}{ M35A2-M102 (Continued) } \\
\hline $\mathrm{CC}-5$ & $\begin{array}{l}0052 \\
0053\end{array}$ & 0.87 & $\begin{array}{r}17.3 \\
4.8\end{array}$ & $\begin{array}{l}6.1 \\
1.7\end{array}$ & $\begin{array}{l}1.5 \\
2.3\end{array}$ & $\begin{array}{l}2.3 \\
1.1\end{array}$ & $\begin{array}{l}9.9 \\
5.1\end{array}$ \\
\hline $\mathrm{CC}-6$ & $\begin{array}{l}0034 \\
0035 \\
0036 \\
0037 \\
0038 \\
0039 \\
0040 \\
0041 \\
0042 \\
0043 \\
0044\end{array}$ & . & $\begin{array}{r}4.8 \\
8.4 \\
10.8 \\
6.5 \\
14.5 \\
12.2 \\
10.1 \\
- \\
- \\
13.1 \\
9.8\end{array}$ & $\begin{array}{r}2.8 \\
9.5 \\
7.8 \\
4.7 \\
13.4 \\
14.6 \\
8.3 \\
- \\
- \\
11.7 \\
7.1\end{array}$ & $\begin{array}{c}3.1 \\
5.0 \\
4.1 \\
3.5 \\
4.9 \\
4.3 \\
- \\
- \\
- \\
5.2 \\
4.5\end{array}$ & $\begin{array}{c}1.6 \\
2.6 \\
2.5 \\
2.1 \\
5.5 \\
4.3 \\
- \\
- \\
- \\
4.4 \\
2.7\end{array}$ & $\begin{array}{c}7.5 \\
17.1 \\
14.4 \\
10.3 \\
23.8 \\
23.2 \\
- \\
- \\
- \\
21.3 \\
14.3\end{array}$ \\
\hline $\mathrm{T}-1$ & $\begin{array}{l}0139 \\
0140 \\
0141 \\
0142 \\
0143 \\
0144 \\
0145\end{array}$ & 1.24 & $\begin{array}{c}- \\
14.7 \\
8.9 \\
- \\
12.5 \\
- \\
-\end{array}$ & $\begin{array}{c}- \\
9.8 \\
2.4 \\
- \\
5.5 \\
- \\
-\end{array}$ & $\begin{array}{c}- \\
0.6 \\
5.0 \\
- \\
5.9 \\
- \\
-\end{array}$ & $\begin{array}{c}- \\
5.6 \\
1.1 \\
- \\
2.0 \\
- \\
-\end{array}$ & $\begin{array}{c}- \\
25.0 \\
8.5 \\
- \\
13.4 \\
- \\
-\end{array}$ \\
\hline $\mathrm{T}-2$ & $\begin{array}{l}0060 \\
0061 \\
0062 \\
0063 \\
0064 \\
0065\end{array}$ & 0.51 & $\begin{array}{c}14.9 \\
- \\
24.1 \\
31.0 \\
19.5 \\
27.3\end{array}$ & $\begin{array}{l}1.8 \\
- \\
4.6 \\
3.3 \\
4.6 \\
4.1\end{array}$ & $\begin{array}{l}0.7 \\
- \\
2.4 \\
1.5 \\
1.9 \\
1.9\end{array}$ & $\begin{array}{l}0.3 \\
- \\
1.8 \\
0.9 \\
2.1 \\
1.6\end{array}$ & $\begin{array}{l}2.8 \\
- \\
8.8 \\
5.7 \\
8.6 \\
7.6\end{array}$ \\
\hline $\mathrm{T}-3$ & $\begin{array}{l}0054 \\
0055 \\
0056 \\
0057 \\
0058 \\
0059\end{array}$ & 1.01 & $\begin{array}{r}8.8 \\
15.0 \\
12.5 \\
20.7 \\
16.9 \\
25.3\end{array}$ & $\begin{array}{r}1.8 \\
5.9 \\
3.4 \\
12.4 \\
7.6 \\
18.1\end{array}$ & $\begin{array}{l}1.7 \\
2.9 \\
2.5 \\
3.9 \\
3.1 \\
4.1\end{array}$ & $\begin{array}{r}0.7 \\
2.1 \\
1.5 \\
6.8 \\
3.8 \\
10.0\end{array}$ & $\begin{array}{r}4.2 \\
10.9 \\
7.4 \\
23.1 \\
14.5 \\
32.2\end{array}$ \\
\hline $\mathrm{T}-4$ & $\begin{array}{l}0133 \\
0134 \\
0135\end{array}$ & 1.44 & $\begin{array}{r}15.0 \\
8.9 \\
- \\
\text { (Cc }\end{array}$ & $\begin{array}{r}9.0 \\
3.7 \\
- \\
\text { inued) }\end{array}$ & $\begin{array}{c}6.1 \\
2.4 \\
-\end{array}$ & $\begin{array}{c}4.5 \\
3.0 \\
- \\
\text { (She }\end{array}$ & $\begin{array}{c}19.6 \\
9.1 \\
- \\
4 \text { of } 16)\end{array}$ \\
\hline
\end{tabular}


Table C1 (Continued)

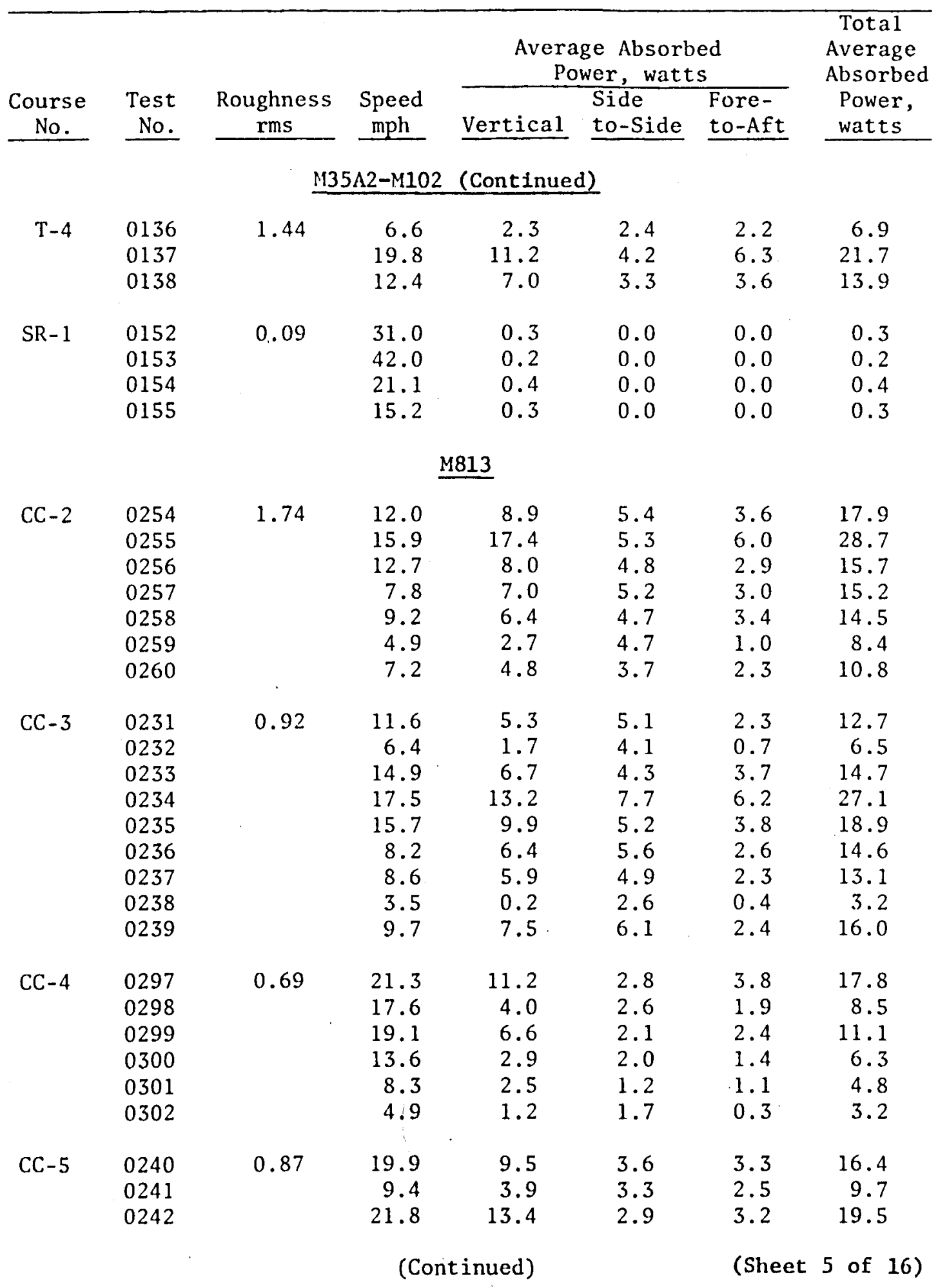


Table Cl (Continued)

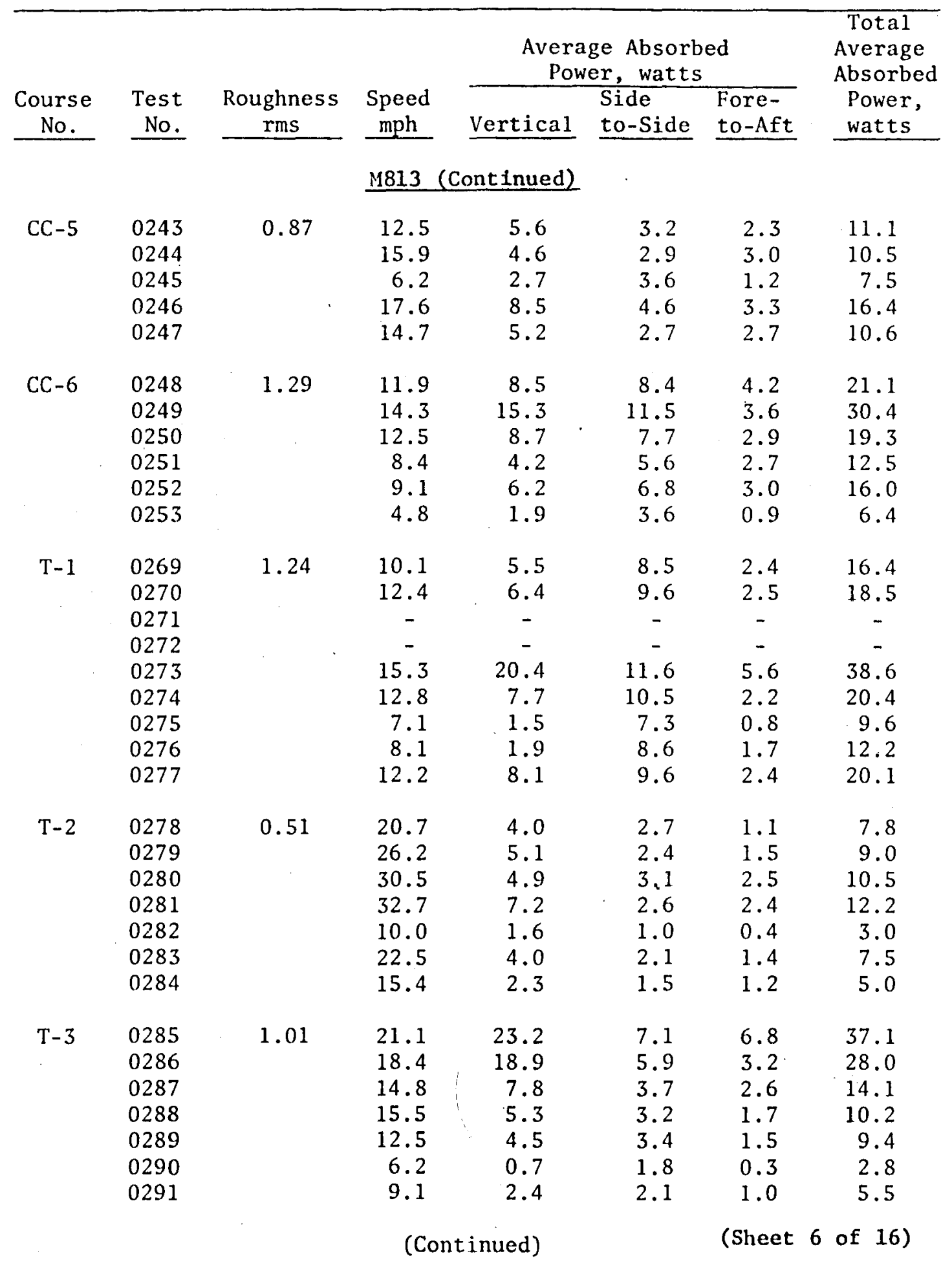


Table $\mathrm{Cl}$ (Continued)

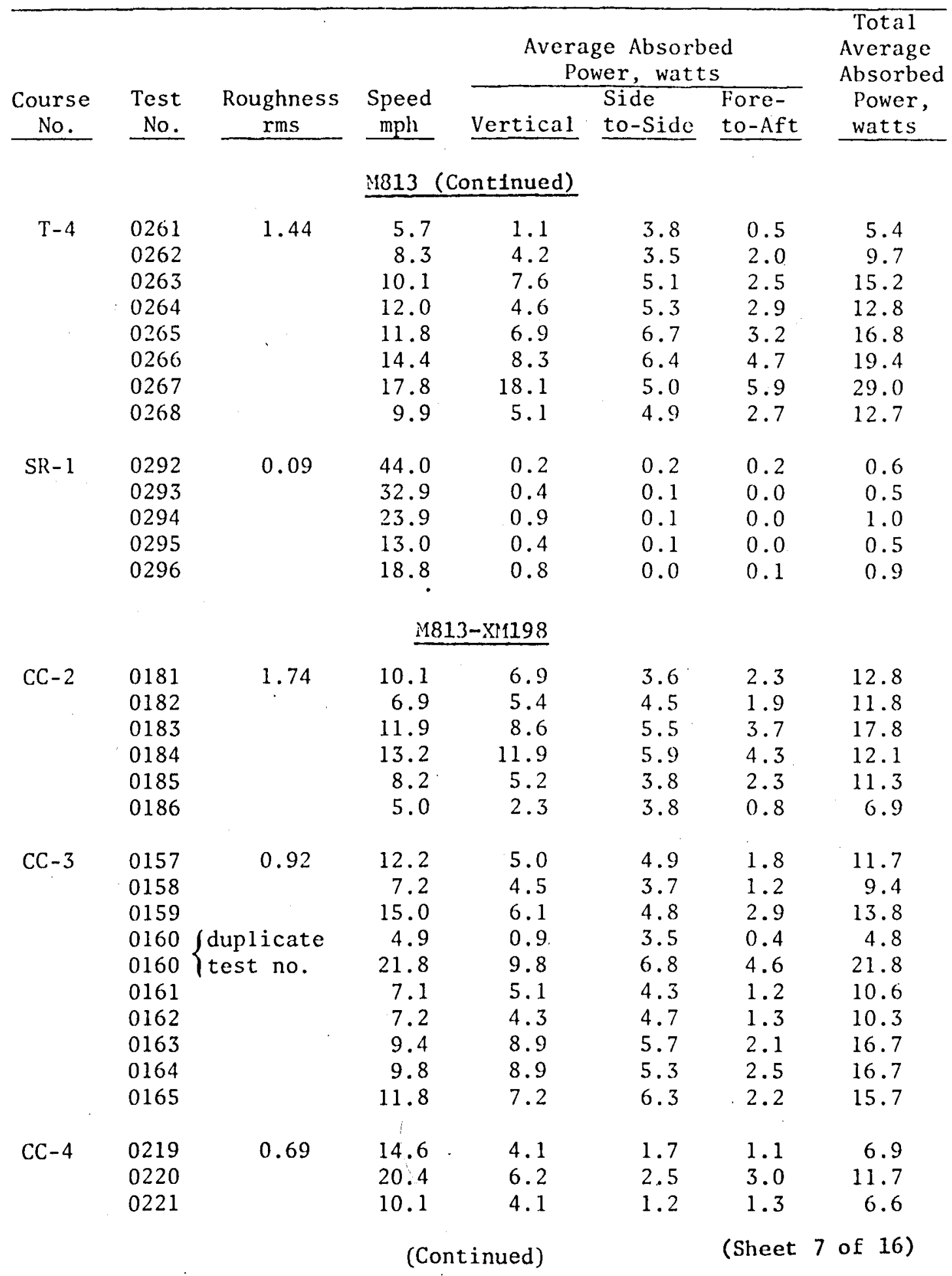


Table C1 (Continued)

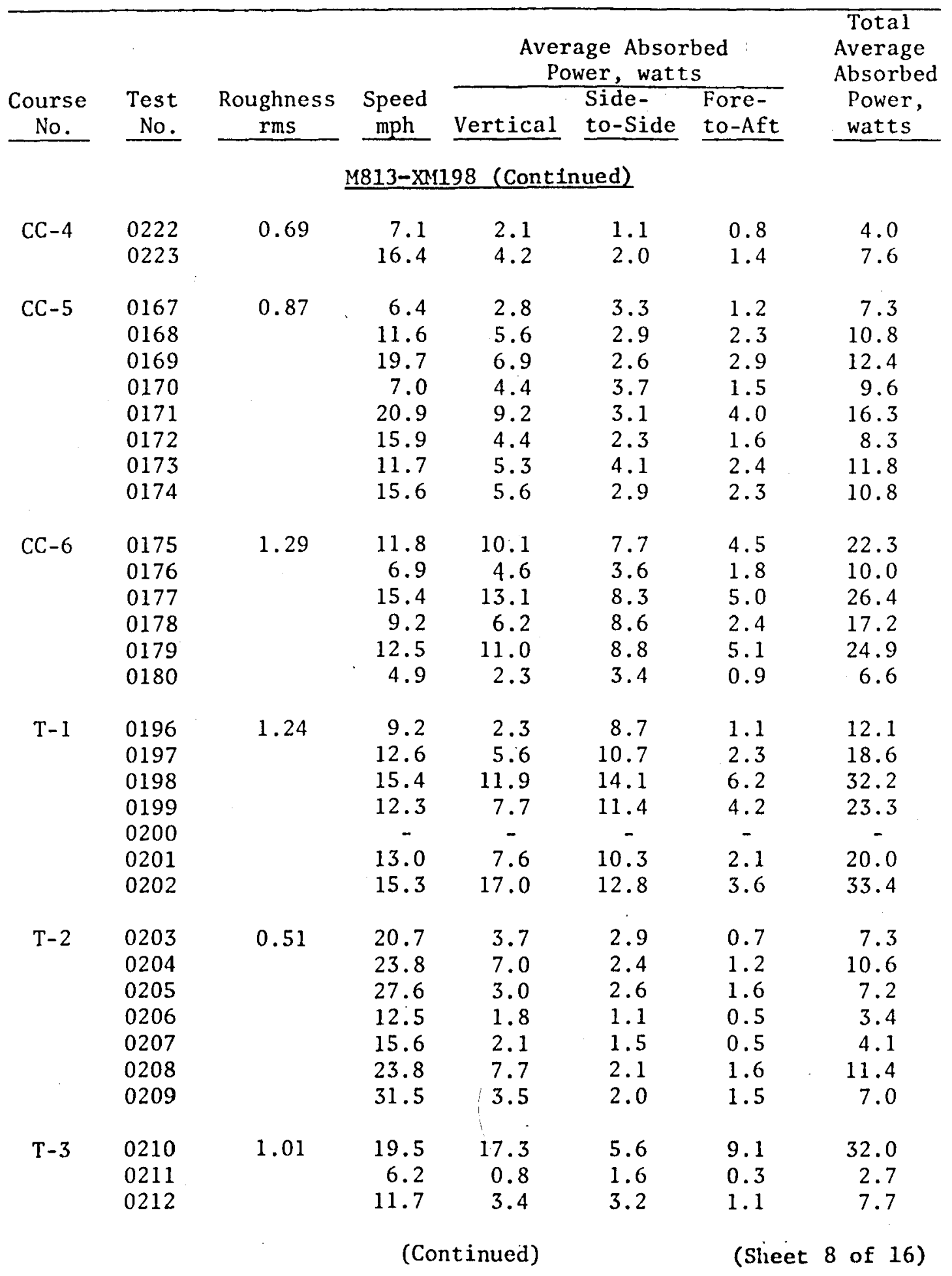


Table $\mathrm{Cl}$ (Continued)

\begin{tabular}{|c|c|c|c|c|c|c|c|}
\hline \multirow[b]{2}{*}{$\begin{array}{c}\text { Course } \\
\text { No. } \\
\end{array}$} & \multirow[b]{2}{*}{$\begin{array}{l}\text { Test } \\
\text { No. }\end{array}$} & \multirow[b]{2}{*}{$\begin{array}{c}\text { Roughness } \\
\text { rms } \\
\end{array}$} & \multirow[b]{2}{*}{$\begin{array}{c}\text { Speed } \\
\mathrm{mph}\end{array}$} & \multicolumn{3}{|c|}{$\begin{array}{c}\text { Average Absorbed } \\
\text { Power, watts } \\
\end{array}$} & \multirow{2}{*}{$\begin{array}{l}\text { Total } \\
\text { Average } \\
\text { Absorbed } \\
\text { Power, } \\
\text { watts } \\
\end{array}$} \\
\hline & & & & Vertical & $\begin{array}{l}\text { Side- } \\
\text { to-Side }\end{array}$ & $\begin{array}{l}\text { Fore- } \\
\text { to-Aft }\end{array}$ & \\
\hline \multicolumn{8}{|c|}{ M813-XM198 (Cont inued) } \\
\hline $\mathrm{T}+3$ & $\begin{array}{l}0213 \\
0214\end{array}$ & 1.01 & $\begin{array}{l}14.9 \\
18.1\end{array}$ & $\begin{array}{r}6.7 \\
11.9\end{array}$ & $\begin{array}{l}4.1 \\
5.3\end{array}$ & $\begin{array}{l}2.2 \\
5.0\end{array}$ & $\begin{array}{l}13.0 \\
22.2\end{array}$ \\
\hline $\mathrm{T}-4$ & $\begin{array}{l}0187 \\
0188 \\
0189 \\
0190 \\
0191 \\
0192 \\
0193 \\
0194 \\
0195\end{array}$ & 1.44 & $\begin{array}{r}9.4 \\
11.1 \\
12.7 \\
6.3 \\
14.6 \\
7.6 \\
9.6 \\
10.9 \\
8.2\end{array}$ & $\begin{array}{l}6.6 \\
5.4 \\
6.0 \\
2.6 \\
7.5 \\
4.8 \\
9.5 \\
4.6 \\
6.9\end{array}$ & $\begin{array}{l}5.7 \\
4.8 \\
6.5 \\
2.7 \\
7.0 \\
2.7 \\
4.7 \\
5.7 \\
3.8\end{array}$ & $\begin{array}{l}3.0 \\
2.2 \\
2.8 \\
1.1 \\
2.9 \\
1.7 \\
2.4 \\
2.1 \\
2.1\end{array}$ & $\begin{array}{r}15.3 \\
12.4 \\
15.3 \\
6.4 \\
17.4 \\
9.2 \\
16.6 \\
12.4 \\
12.8\end{array}$ \\
\hline SR-1 & $\begin{array}{l}0215 \\
0216 \\
0217 \\
0218\end{array}$ & 0.09 & $\begin{array}{l}43.3 \\
30.3 \\
24.6 \\
19.3\end{array}$ & $\begin{array}{l}0.5 \\
0.8 \\
0.9 \\
0.4\end{array}$ & $\begin{array}{l}0.0 \\
0.1 \\
0.0 \\
0.1\end{array}$ & $\begin{array}{l}0.2 \\
0.0 \\
0.1 \\
0.1\end{array}$ & $\begin{array}{l}0.7 \\
0.9 \\
1.0 \\
0.6\end{array}$ \\
\hline \multicolumn{8}{|c|}{ M520-XM198 } \\
\hline $\mathrm{CC}-2$ & $\begin{array}{l}0348 \\
0349 \\
0350 \\
0351 \\
0352 \\
0353\end{array}$ & 1.74 & $\begin{array}{l}8.9 \\
7.9 \\
6.5 \\
6.0 \\
5.1 \\
4.7\end{array}$ & $\begin{array}{r}12.2 \\
9.1 \\
4.5 \\
3.0 \\
2.0 \\
1.6\end{array}$ & $\begin{array}{r}9.9 \\
11.7 \\
9.2 \\
10.3 \\
6.2 \\
9.0\end{array}$ & $\begin{array}{r}17.0 \\
10.7 \\
5.1 \\
4.4 \\
1.7 \\
1.6\end{array}$ & $\begin{array}{r}39.1 \\
31.5 \\
18.8 \\
17.7 \\
9.9 \\
12.2\end{array}$ \\
\hline $\mathrm{CC}-3$ & $\begin{array}{l}0312 \\
0313 \\
0314 \\
0315 \\
0316 \\
0317 \\
0318 \\
0319\end{array}$ & 0.92 & $\begin{array}{c}6.8 \\
- \\
9.3 \\
7.4 \\
- \\
5.7 \\
9.9 \\
6.9\end{array}$ & $\begin{array}{c}2.6 \\
- \\
12.2 \\
6.0 \\
- \\
1.6 \\
12.0 \\
4.1\end{array}$ & $\begin{array}{c}4.1 \\
- \\
3.6 \\
10.4 \\
- \\
5.8 \\
5.9 \\
9.8\end{array}$ & $\begin{array}{c}9.2 \\
- \\
24.5 \\
12.9 \\
- \\
3.5 \\
17.7 \\
10.6\end{array}$ & $\begin{array}{c}15.9 \\
- \\
40.3 \\
29.3 \\
- \\
10.9 \\
35.6 \\
24.5\end{array}$ \\
\hline $\mathrm{CC}-4$ & $\begin{array}{l}0339 \\
0340 \\
0341\end{array}$ & 0.69 & $\begin{array}{r}11.2 \\
6.7 \\
17.6 \\
\quad(\mathrm{C} C\end{array}$ & $\begin{array}{r}2.6 \\
0.9 \\
13.6 \\
\text { tinued) }\end{array}$ & $\begin{array}{l}3.9 \\
2.8 \\
5.5\end{array}$ & $\begin{array}{c}3.0 \\
0.9 \\
8.3 \\
\text { (Sheet }\end{array}$ & $\begin{array}{r}9.5 \\
4.6 \\
27.4 \\
\text { of } 16)\end{array}$ \\
\hline
\end{tabular}


Table Cl (Continued)

\begin{tabular}{|c|c|c|c|c|c|c|c|}
\hline \multirow[b]{2}{*}{$\begin{array}{c}\text { Course } \\
\text { No. }\end{array}$} & \multirow[b]{2}{*}{$\begin{array}{l}\text { Test } \\
\text { No. }\end{array}$} & \multirow[b]{2}{*}{$\begin{array}{c}\begin{array}{c}\text { Roughness } \\
\text { rms }\end{array} \\
\end{array}$} & \multirow[b]{2}{*}{$\begin{array}{c}\text { Speed } \\
\text { mph }\end{array}$} & \multicolumn{3}{|c|}{$\begin{array}{c}\text { Average Absorbed } \\
\text { Power, watts }\end{array}$} & \multirow{2}{*}{$\begin{array}{c}\text { Total } \\
\text { Average } \\
\text { Absorbed } \\
\text { Power } \\
\text { watts } \\
\end{array}$} \\
\hline & & & & Vertical & $\begin{array}{l}\text { Side- } \\
\text { to-Side }\end{array}$ & $\begin{array}{l}\text { Fore- } \\
\text { to-Aft }\end{array}$ & \\
\hline \multicolumn{8}{|c|}{ M520-XM198 (Continued) } \\
\hline $\mathrm{CC}-4$ & $\begin{array}{l}0342 \\
0343\end{array}$ & 0.69 & $\begin{array}{l}13.8 \\
11.2\end{array}$ & $\begin{array}{l}8.1 \\
2.9\end{array}$ & $\begin{array}{l}4.7 \\
3.2\end{array}$ & $\begin{array}{l}9.8 \\
3.5\end{array}$ & $\begin{array}{r}22.6 \\
9.6\end{array}$ \\
\hline $\mathrm{CC}-5$ & $\begin{array}{l}0321 \\
0322 \\
0323 \\
0324 \\
0325 \\
0326 \\
0327\end{array}$ & 0.87 & $\begin{array}{c}4.7 \\
7.0 \\
5.9 \\
- \\
9.9 \\
- \\
8.0\end{array}$ & $\begin{array}{c}1.5 \\
3.8 \\
3.0 \\
- \\
12.1 \\
- \\
5.8\end{array}$ & $\begin{array}{c}5.3 \\
3.9 \\
5.0 \\
- \\
5.7 \\
- \\
5.3\end{array}$ & $\begin{array}{c}2.3 \\
6.4 \\
5.1 \\
- \\
22.0 \\
- \\
9.6\end{array}$ & $\begin{array}{c}9.1 \\
14.1 \\
13.1 \\
- \\
39.8 \\
- \\
20.7\end{array}$ \\
\hline $\mathrm{CC}-6$ & $\begin{array}{l}0328 \\
0329 \\
0330 \\
0331 \\
0332 \\
0333\end{array}$ & 1.29 & $\begin{array}{r}6.7 \\
6.3 \\
6.5 \\
10.2 \\
6.8 \\
10.2\end{array}$ & $\begin{array}{r}5.3 \\
3.8 \\
5.1 \\
10.9 \\
4.6 \\
8.9\end{array}$ & $\begin{array}{c}- \\
12.2 \\
- \\
- \\
- \\
-\end{array}$ & $\begin{array}{l}- \\
6.5 \\
- \\
- \\
- \\
-\end{array}$ & $\begin{array}{c}- \\
22.5 \\
- \\
- \\
- \\
-\end{array}$ \\
\hline $\mathrm{CC}-6$ & $\begin{array}{l}0344 \\
0345 \\
0346 \\
0347\end{array}$ & 1.29 & $\begin{array}{r}10.5 \\
8.9 \\
10.1 \\
5.6\end{array}$ & $\begin{array}{r}14.0 \\
5.2 \\
11.0 \\
1.6\end{array}$ & $\begin{array}{r}14.3 \\
9.4 \\
13.5 \\
8.7\end{array}$ & $\begin{array}{r}14.9 \\
8.4 \\
9.4 \\
1.6\end{array}$ & $\begin{array}{l}43.2 \\
23.0 \\
33.9 \\
11.9\end{array}$ \\
\hline $\mathrm{T}-1$ & $\begin{array}{l}0363 \\
0364 \\
0365 \\
0366 \\
0367 \\
0368 \\
0369\end{array}$ & 1.24 & $\begin{array}{r}11.8 \\
12.0 \\
9.6 \\
9.5 \\
10.9 \\
5.5 \\
7.2\end{array}$ & $\begin{array}{r}9.0 \\
13.4 \\
2.8 \\
11.6 \\
5.5 \\
0.5 \\
0.9\end{array}$ & $\begin{array}{l}22.4 \\
29.1 \\
18.9 \\
25.8 \\
19.1 \\
15.2 \\
12.9\end{array}$ & $\begin{array}{r}11.6 \\
11.1 \\
3.1 \\
17.8 \\
4.7 \\
1.2 \\
1.6\end{array}$ & $\begin{array}{l}43.0 \\
53.6 \\
24.8 \\
55.2 \\
29.3 \\
16.9 \\
15.4\end{array}$ \\
\hline $\mathrm{T}-2$ & $\begin{array}{l}0371 \\
0372 \\
0373 \\
0374 \\
0375 \\
0376 \\
0377 \\
0378 \\
0379\end{array}$ & 0.51 & $\begin{array}{l}10.8 \\
13.3 \\
12.0 \\
15.6 \\
14.3 \\
17.0 \\
17.3 \\
20.9 \\
24.6\end{array}$ & $\begin{array}{r}1.4 \\
9.6 \\
4.0 \\
7.5 \\
6.2 \\
6.5 \\
7.4 \\
9.8 \\
11.9\end{array}$ & $\begin{array}{l}1.9 \\
3.8 \\
2.2 \\
3.5 \\
3.0 \\
4.1 \\
4.2 \\
4.3 \\
8.0\end{array}$ & $\begin{array}{r}1.8 \\
22.5 \\
5.7 \\
5.4 \\
11.2 \\
4.2 \\
5.4 \\
11.9 \\
21.0\end{array}$ & $\begin{array}{r}5.1 \\
35.9 \\
11.9 \\
16.4 \\
20.4 \\
14.8 \\
17.0 \\
26.0 \\
40.9\end{array}$ \\
\hline
\end{tabular}


Table $\mathrm{Cl}$ (Continued)

\begin{tabular}{|c|c|c|c|c|c|c|c|}
\hline \multirow[b]{2}{*}{$\begin{array}{c}\text { Course } \\
\text { No. }\end{array}$} & \multirow[b]{2}{*}{$\begin{array}{l}\text { Test } \\
\text { No. }\end{array}$} & \multirow[b]{2}{*}{$\begin{array}{c}\text { Roughness } \\
\text { rms }\end{array}$} & \multirow[b]{2}{*}{$\begin{array}{c}\text { Speed } \\
\mathrm{mph}\end{array}$} & \multicolumn{3}{|c|}{$\begin{array}{c}\text { Average Absorbed } \\
\text { Power, watts }\end{array}$} & \multirow{2}{*}{$\begin{array}{c}\text { Total } \\
\text { Average } \\
\text { Absorbed } \\
\text { Power } \\
\text { watts } \\
\end{array}$} \\
\hline & & & & Vertical & $\begin{array}{l}\text { Side- } \\
\text { to-Side }\end{array}$ & $\begin{array}{l}\text { Fore- } \\
\text { to-Aft }\end{array}$ & \\
\hline \multicolumn{8}{|c|}{ M520-XM198 (Continued) } \\
\hline $\mathrm{T}-3$ & $\begin{array}{l}0380 \\
0381 \\
0382 \\
0383 \\
0384 \\
0385 \\
0386\end{array}$ & 1.01 & $\begin{array}{r}15.6 \\
11.1 \\
12.3 \\
9.9 \\
13.8 \\
6.5 \\
6.9\end{array}$ & $\begin{array}{r}38.2 \\
4.9 \\
9.7 \\
4.9 \\
54.3 \\
0.6 \\
1.7\end{array}$ & $\begin{array}{r}18.5 \\
6.6 \\
7.7 \\
6.6 \\
34.5 \\
4.6 \\
4.7\end{array}$ & $\begin{array}{r}12.1 \\
7.4 \\
23.3 \\
3.3 \\
21.7 \\
0.9 \\
1.8\end{array}$ & $\begin{array}{r}68.8 \\
18.9 \\
40.7 \\
14.8 \\
110.5 \\
6.1 \\
8.2\end{array}$ \\
\hline $\mathrm{T}-4$ & $\begin{array}{l}0354 \\
0355 \\
0356 \\
0357 \\
0358 \\
0359 \\
0360 \\
0361 \\
0362\end{array}$ & 1.44 & $\begin{array}{r}6.1 \\
6.8 \\
7.6 \\
7.4 \\
9.2 \\
4.3 \\
5.1 \\
7.2 \\
11.0\end{array}$ & $\begin{array}{c}4.7 \\
4.3 \\
5.0 \\
6.0 \\
11.8 \\
0.2 \\
0.9 \\
5.6 \\
11.4\end{array}$ & $\begin{array}{r}5.7 \\
6.7 \\
6.0 \\
8.9 \\
8.1 \\
5.4 \\
5.9 \\
10.4 \\
9.9\end{array}$ & $\begin{array}{r}6.0 \\
6.7 \\
7.2 \\
7.3 \\
13.0 \\
0.5 \\
1.3 \\
7.5 \\
15.5\end{array}$ & $\begin{array}{r}16.4 \\
17.7 \\
18.2 \\
22.2 \\
32.9 \\
6.1 \\
8.1 \\
23.5 \\
36.8\end{array}$ \\
\hline$S R-1$ & $\begin{array}{l}0334 \\
0335 \\
0336 \\
0337 \\
0338\end{array}$ & 0.09 & $\begin{array}{l}32.5 \\
19.8 \\
11.3 \\
22.73 \\
25.98\end{array}$ & $\begin{array}{l}0.5 \\
0.4 \\
0.04 \\
0.7 \\
0.5\end{array}$ & $\begin{array}{l}0.2 \\
0.1 \\
0.0 \\
0.2 \\
0.1\end{array}$ & $\begin{array}{l}0.4 \\
0.2 \\
0.0 \\
2.6 \\
0.3\end{array}$ & $\begin{array}{l}1.08 \\
0.73 \\
0.04 \\
3.46 \\
0.88\end{array}$ \\
\hline $\mathrm{CC}-2$ & ** & \multicolumn{5}{|c|}{$M 561-M 102$} & \\
\hline $\mathrm{CC}-3$ & $\begin{array}{l}0408 \\
0409 \\
0410 \\
0411 \\
0412 \\
0413 \\
0414\end{array}$ & 0.92 & $\begin{array}{r}7.4 \\
11.3 \\
15.0 \\
16.6 \\
17.1 \\
10.5 \\
8.3\end{array}$ & $\begin{array}{l}0.9 \\
3.5 \\
4.1 \\
8.1 \\
6.0 \\
3.3 \\
1.7\end{array}$ & $\begin{array}{l}0.3 \\
1.5 \\
1.4 \\
1.4 \\
2.4 \\
2.8 \\
2.3\end{array}$ & $\begin{array}{l}2.6 \\
6.5 \\
1.9 \\
3.5 \\
3.0 \\
4.8 \\
3.8\end{array}$ & $\begin{array}{r}3.8 \\
11.5 \\
7.4 \\
13.0 \\
11.4 \\
10.9 \\
7.8\end{array}$ \\
\hline $\mathrm{CC}-4$ & $\star *$ & & & & & & \\
\hline
\end{tabular}


Table C1 (Continued)

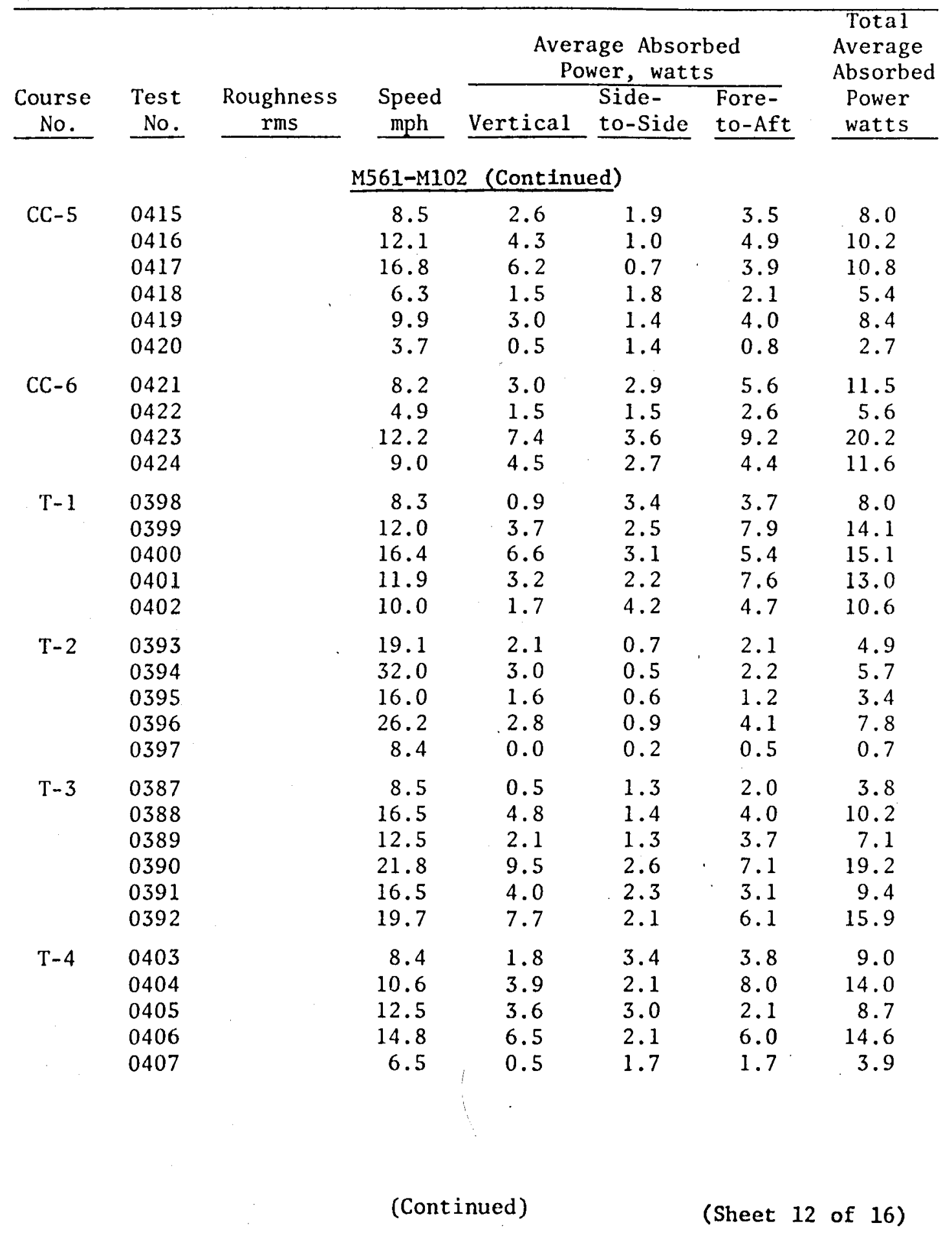


Table C1 (Continued)

\begin{tabular}{|c|c|c|c|c|c|c|c|}
\hline \multirow[b]{2}{*}{$\begin{array}{c}\text { Course } \\
\text { No. } \\
\end{array}$} & \multirow[b]{2}{*}{$\begin{array}{r}\text { Test } \\
\text { No. }\end{array}$} & \multirow[b]{2}{*}{$\begin{array}{c}\text { Roughness } \\
\text { rms } \\
\end{array}$} & \multirow[b]{2}{*}{$\begin{array}{c}\text { Speed } \\
\text { mph }\end{array}$} & \multicolumn{3}{|c|}{$\begin{array}{c}\text { Average Absorbed } \\
\text { Power, watts }\end{array}$} & \multirow{2}{*}{$\begin{array}{l}\text { Total } \\
\text { Average } \\
\text { Absorbed } \\
\text { Power, } \\
\text { watts }\end{array}$} \\
\hline & & & & Vertical & $\begin{array}{l}\text { Side- } \\
\text { to-Side }\end{array}$ & $\begin{array}{l}\text { Fore- } \\
\text { to-Aft }\end{array}$ & \\
\hline \multicolumn{8}{|c|}{ M109A1 } \\
\hline $\mathrm{CC}-2$ & $\begin{array}{l}0486 \\
0487 \\
0488 \\
0489 \\
0490 \\
0491\end{array}$ & 1.74 & $\begin{array}{r}14.1 \\
15.0 \\
18.8 \\
15.2 \\
7.6 \\
10.4\end{array}$ & $\begin{array}{l}4.2 \\
4.2 \\
5.9 \\
3.7 \\
2.4 \\
2.9\end{array}$ & $\begin{array}{l}1.3 \\
1.8 \\
2.3 \\
1.9 \\
0.9 \\
1.3\end{array}$ & $\begin{array}{l}1.9 \\
2.8 \\
0.8 \\
0.6 \\
2.0 \\
0.4\end{array}$ & $\begin{array}{l}7.4 \\
8.8 \\
9.0 \\
6.2 \\
5.3 \\
4.6\end{array}$ \\
\hline $\mathrm{CC}-3$ & $\begin{array}{l}0466 \\
0467 \\
0468 \\
0469 \\
0470 \\
0471\end{array}$ & 0.92 & $\begin{array}{r}10.6 \\
14.0 \\
21.1 \\
18.6 \\
22.7 \\
6.6\end{array}$ & $\begin{array}{l}1.0 \\
2.5 \\
2.7 \\
3.5 \\
2.6 \\
0.6\end{array}$ & $\begin{array}{l}0.3 \\
1.3 \\
0.2 \\
0.8 \\
1.1 \\
0.5\end{array}$ & $\begin{array}{l}0.2 \\
0.7 \\
0.6 \\
0.4 \\
0.2 \\
0.3\end{array}$ & $\begin{array}{l}1.5 \\
4.5 \\
3.5 \\
4.7 \\
3.9 \\
1.4\end{array}$ \\
\hline $\mathrm{CC}-4$ & $\begin{array}{l}0430 \\
0431 \\
0432 \\
0433 \\
0434 \\
0435 \\
0436 \\
0437\end{array}$ & 0.69 & $\begin{array}{r}6.2 \\
11.2 \\
18.4 \\
21.5 \\
20.4 \\
22.2 \\
16.7 \\
22.5\end{array}$ & $\begin{array}{l}0.7 \\
1.4 \\
2.0 \\
3.3 \\
1.6 \\
2.5 \\
1.5 \\
2.2\end{array}$ & $\begin{array}{l}0.1 \\
0.2 \\
0.3 \\
0.9 \\
0.1 \\
0.3 \\
0.2 \\
0.7\end{array}$ & $\begin{array}{l}0.3 \\
0.1 \\
0.4 \\
0.2 \\
0.0 \\
0.0 \\
0.2 \\
0.1\end{array}$ & $\begin{array}{l}1.1 \\
1.7 \\
2.7 \\
4.4 \\
1.7 \\
2.8 \\
1.9 \\
3.0\end{array}$ \\
\hline $\mathrm{CC}-5$ & $\begin{array}{l}0472 \\
0473 \\
0474 \\
0475 \\
0476 \\
0477 \\
0478 \\
0479\end{array}$ & 0.87 & $\begin{array}{r}6.0 \\
9.0 \\
15.9 \\
5.4 \\
21.3 \\
12.0 \\
15.4 \\
6.4\end{array}$ & $\begin{array}{l}3.3 \\
3.6 \\
3.7 \\
2.2 \\
3.6 \\
3.4 \\
2.9 \\
2.5\end{array}$ & $\begin{array}{l}0.8 \\
0.6 \\
1.1 \\
0.6 \\
1.6 \\
0.8 \\
0.9 \\
0.6\end{array}$ & $\begin{array}{l}1.9 \\
0.3 \\
0.9 \\
1.8 \\
0.8 \\
0.5 \\
0.6 \\
1.6\end{array}$ & $\begin{array}{l}6.0 \\
4.5 \\
5.7 \\
4.6 \\
6.0 \\
4.7 \\
4.4 \\
4.7\end{array}$ \\
\hline $\mathrm{CC}-6$ & $\begin{array}{l}0480 \\
0481 \\
0482 \\
0483 \\
0484 \\
0485\end{array}$ & 1.29 & $\begin{array}{r}14.0 \\
12.8 \\
18.6 \\
10.0 \\
18.3 \\
6.1\end{array}$ & $\begin{array}{l}3.6 \\
4.2 \\
4.5 \\
4.7 \\
4.7 \\
2.9\end{array}$ & $\begin{array}{l}1.5 \\
1.7 \\
2.1 \\
1.9 \\
1.3 \\
0.9\end{array}$ & $\begin{array}{l}1.2 \\
1.2 \\
1.0 \\
0.5 \\
0.2 \\
3.3\end{array}$ & $\begin{array}{l}6.3 \\
7.1 \\
7.6 \\
7.1 \\
6.2 \\
7.1\end{array}$ \\
\hline
\end{tabular}

(Continued) 
Table C1 (Continued)

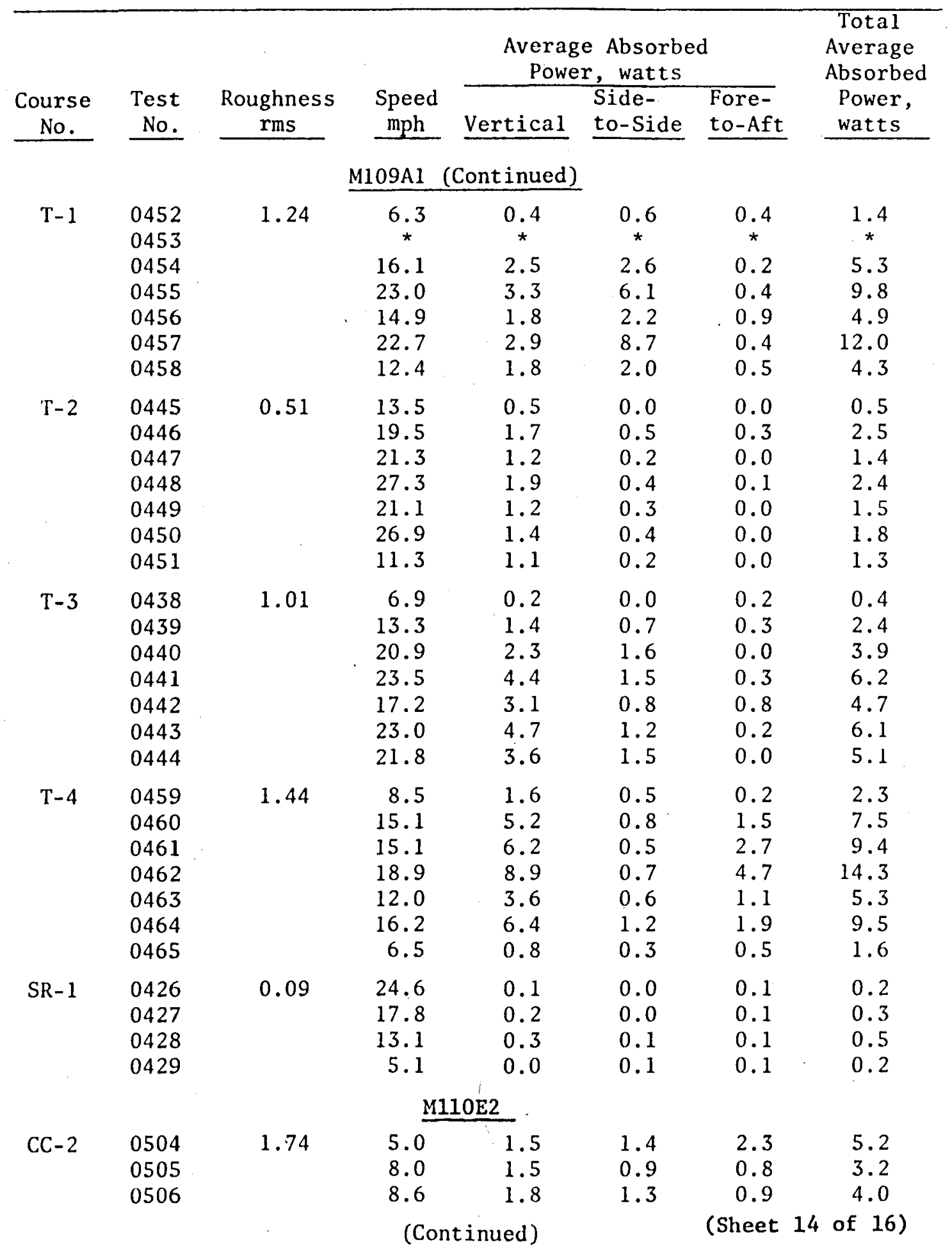


Table Cl (ContInued)

\begin{tabular}{|c|c|c|c|c|c|c|c|}
\hline \multirow[b]{2}{*}{$\begin{array}{c}\text { Course } \\
\text { No. } \\
\end{array}$} & \multirow[b]{2}{*}{$\begin{array}{l}\text { Test } \\
\text { No. }\end{array}$} & \multirow[b]{2}{*}{$\begin{array}{c}\text { Roughness } \\
\text { rms } \\
\end{array}$} & \multirow[b]{2}{*}{$\begin{array}{l}\text { Speed } \\
\text { mph }\end{array}$} & \multicolumn{3}{|c|}{$\begin{array}{c}\text { Average Absorbed } \\
\text { Power, watts }\end{array}$} & \multirow{2}{*}{$\begin{array}{l}\text { Total } \\
\text { Average } \\
\text { Absorbed } \\
\text { Power, } \\
\text { watts }\end{array}$} \\
\hline & & & & Vertical & $\begin{array}{l}\text { Side- } \\
\text { to-Side }\end{array}$ & $\begin{array}{l}\text { Fore- } \\
\text { to-Aft }\end{array}$ & \\
\hline & & & M110E2 & (Continued) & & & \\
\hline $\mathrm{CC}-2$ & $\begin{array}{l}0528 \\
0529 \\
0530 \\
0531 \\
0532 \\
0533\end{array}$ & 1.74 & $\begin{array}{r}5.5 \\
9.2 \\
18.7 \\
14.6 \\
16.2 \\
16.2\end{array}$ & $\begin{array}{l}1.5 \\
3.0 \\
9.9 \\
3.7 \\
4.0 \\
5.1\end{array}$ & $\begin{array}{l}1.5 \\
1.6 \\
3.8 \\
2.2 \\
2.6 \\
2.1\end{array}$ & $\begin{array}{l}1.8 \\
2.0 \\
1.9 \\
1.8 \\
2.3 \\
0.7\end{array}$ & $\begin{array}{r}4.8 \\
6.6 \\
15.6 \\
7.7 \\
8.9 \\
7.9\end{array}$ \\
\hline $\mathrm{CC}-3$ & $\begin{array}{l}0492 \\
0493 \\
0494 \\
0495 \\
0496 \\
0497 \\
0498\end{array}$ & 0.92 & $\begin{array}{r}5.4 \\
8.2 \\
14.3 \\
14.8 \\
16.5 \\
11.8 \\
17.0\end{array}$ & $\begin{array}{l}0.1 \\
0.2 \\
0.7 \\
0.9 \\
0.7 \\
0.2 \\
0.8\end{array}$ & $\begin{array}{l}0.3 \\
0.2 \\
0.6 \\
0.5 \\
0.4 \\
0.1 \\
0.5\end{array}$ & $\begin{array}{l}0.2 \\
0.2 \\
0.3 \\
0.0 \\
0.2 \\
0.0 \\
0.3\end{array}$ & $\begin{array}{l}0.6 \\
0.6 \\
1.6 \\
1.4 \\
1.3 \\
0.3 \\
1.6\end{array}$ \\
\hline $\mathrm{CC}-4$ & $\begin{array}{l}\text { No } \\
\text { data }\end{array}$ & 0.69 & & & & & \\
\hline $\mathrm{CC}-5+$ & $\begin{array}{l}0492 \\
0493 \\
0494 \\
0495 \\
0496\end{array}$ & 0.87 & $\begin{array}{r}9.2 \\
5.3 \\
15.3 \\
5.4 \\
20.1\end{array}$ & $\begin{array}{l}1.4 \\
1.3 \\
1.6 \\
0.8 \\
1.9\end{array}$ & $\begin{array}{l}0.5 \\
1.0 \\
1.0 \\
0.7 \\
0.9\end{array}$ & $\begin{array}{l}0.4 \\
1.6 \\
0.7 \\
1.0 \\
0.1\end{array}$ & $\begin{array}{l}2.3 \\
3.9 \\
3.3 \\
2.5 \\
2.9\end{array}$ \\
\hline $\mathrm{CC}-6$ & $\begin{array}{l}0499 \\
0500 \\
0501 \\
0502 \\
0503 \\
0534\end{array}$ & no test) & $\begin{array}{r}5.5 \\
9.0 \\
15.3 \\
12.0 \\
17.5\end{array}$ & $\begin{array}{l}1.2 \\
1.5 \\
1.8 \\
1.9 \\
2.2 .\end{array}$ & $\begin{array}{l}1.7 \\
1.1 \\
1.1 \\
1.5 \\
2.1\end{array}$ & $\begin{array}{l}1.7 \\
1.0 \\
0.7 \\
0.6 \\
0.9\end{array}$ & $\begin{array}{l}4.6 \\
3.6 \\
3.6 \\
4.0 \\
5.2\end{array}$ \\
\hline $\mathrm{T}-1$ & $\begin{array}{l}0518 \\
0519 \\
0520 \\
0521 \\
0522\end{array}$ & 1.24 & $\begin{array}{r}5.8 \\
15.7 \\
9.4 \\
18.9 \\
22.5\end{array}$ & $\begin{array}{l}0.3 \\
2.1 \\
1.1 \\
4.5 \\
4.4\end{array}$ & $\begin{array}{l}0.9 \\
3.4 \\
2.7 \\
3.7 \\
5.1\end{array}$ & $\begin{array}{l}0.5 \\
1.1 \\
0.9 \\
1.0 \\
1.0\end{array}$ & $\begin{array}{r}1.7 \\
6.6 \\
4.7 \\
9.2 \\
10.5\end{array}$ \\
\hline
\end{tabular}

(Continued) 
Table C1 (Concluded)

\begin{tabular}{|c|c|c|c|c|c|c|c|}
\hline $\begin{array}{c}\text { Course } \\
\text { No. } \\
\end{array}$ & $\begin{array}{l}\text { Test } \\
\text { No. }\end{array}$ & $\begin{array}{l}\text { Roughness } \\
\text { rms } \\
\end{array}$ & $\begin{array}{c}\text { Speed } \\
\text { mph }\end{array}$ & \multicolumn{3}{|c|}{$\begin{array}{l}\text { Average Absorbed } \\
\text { Power, watts }\end{array}$} & $\begin{array}{c}\text { Total } \\
\text { Average } \\
\text { Absorbed } \\
\text { Power, } \\
\text { watts } \\
\end{array}$ \\
\hline & & & $110 \mathrm{E} 2$ & Continued) & & & \\
\hline $\mathrm{T}-2$ & $\begin{array}{l}0514 \\
0515 \\
0516 \\
0517\end{array}$ & 0.51 & $\begin{array}{r}6.0 \\
25.6 \\
15.6 \\
20.9\end{array}$ & $\begin{array}{l}0.1 \\
1.2 \\
0.8 \\
1.1\end{array}$ & $\begin{array}{l}0.0 \\
1.0 \\
0.3 \\
0.3\end{array}$ & $\begin{array}{l}0.0 \\
0.1 \\
0.3 \\
0.1\end{array}$ & $\begin{array}{l}0.1 \\
2.3 \\
1.4 \\
1.5\end{array}$ \\
\hline $\mathrm{T}-3$ & $\begin{array}{l}0508 \\
0509 \\
0510 \\
0511 \\
0512 \\
0513\end{array}$ & & $\begin{array}{r}4.7 \\
7.9 \\
15.2 \\
19.5 \\
18.9 \\
24.1\end{array}$ & $\begin{array}{l}0.2 \\
0.3 \\
2.0 \\
2.9 \\
2.8 \\
5.6\end{array}$ & $\begin{array}{l}0.0 \\
0.3 \\
0.9 \\
1.1 \\
0.9 \\
1.9\end{array}$ & $\begin{array}{l}0.1 \\
0.5 \\
0.9 \\
0.7 \\
0.5 \\
1.3\end{array}$ & $\begin{array}{l}0.3 \\
1.1 \\
3.8 \\
4.7 \\
4.2 \\
8.8\end{array}$ \\
\hline$T-4$ & $\begin{array}{l}0523 \\
0524 \\
0525 \\
0526 \\
0527\end{array}$ & & $\begin{array}{r}6.0 \\
9.5 \\
15.2 \\
17.8 \\
21.6\end{array}$ & $\begin{array}{r}1.2 \\
1.8 \\
4.5 \\
5.1 \\
11.3\end{array}$ & $\begin{array}{l}1.0 \\
1.1 \\
0.8 \\
2.7 \\
1.1\end{array}$ & $\begin{array}{l}1.0 \\
1.4 \\
1.9 \\
0.9 \\
1.9\end{array}$ & $\begin{array}{r}4.0 \\
4.3 \\
7.2 \\
8.7 \\
14.3\end{array}$ \\
\hline
\end{tabular}


Table C2

Obstacle Height-Speed Data

\begin{tabular}{|c|c|c|c|c|c|c|c|c|c|}
\hline \multirow{2}{*}{$\begin{array}{l}\text { Test } \\
\text { No. } \\
\end{array}$} & \multirow{2}{*}{$\begin{array}{l}\text { Obstacle } \\
\text { Height, in. }\end{array}$} & \multirow{2}{*}{$\begin{array}{l}\text { Time } \\
\text { sec }\end{array}$} & \multirow{2}{*}{$\begin{array}{c}\text { Speed } \\
\mathrm{mph}\end{array}$} & \multicolumn{6}{|c|}{$\begin{array}{l}\text { No. of Occurrences } \\
\text { Peak Acceleration Levels, g }\end{array}$} \\
\hline & & & & $\geq 1 \leq 1: 5$ & $>1.5 \leq 2.0$ & $>2.0 \leq 2.5$ & $>2.5 \leq 3$ & $\geq 3 \leq 4$ & $\geq 4$ \\
\hline \multicolumn{10}{|c|}{ M35A2-XM198 } \\
\hline 121 & 6 & 14.9 & & 1 & 0 & 0 & 0 & 0 & 0 \\
\hline 122 & 6 & 14.2 & & 1 & 0 & 0 & 0 & 0 & 0 \\
\hline 123 & 6 & 8.2 & & 2 & 0 & 0 & 0 & 0 & 0 \\
\hline 124 & 6 & 6.2 & & 2 & 1 & 0 & 0 & 0 & 0 \\
\hline 125 & 8 & $14: 3$ & & 0 & 1 & 0 & 0 & 0 & 0 \\
\hline 126 & 8 & 11.2 & & 2 & 2 & 0 & 0 & 0 & 0 \\
\hline 127 & 8 & 8.0 & & 2 & 2 & 1 & 0 & 0 & 0 \\
\hline 128 & 8 & 6.1 & & 3 & 2 & 0 & 0 & 0 & 0 \\
\hline 129 & 8 & 5.4 & & 2 & 0 & 1 & 0 & 0 & 0 \\
\hline 130 & 12 & 14.3 & & 3 & 0 & 1 & 0 & 0 & 0 \\
\hline 131 & 12 & 11.2 & & 6 & 0 & 1 & 0 & 0 & 0 \\
\hline 132 & 12 & 9.1 & & 2 & 3 & 1 & 0 & 0 & 0 \\
\hline \multicolumn{10}{|c|}{ M35A2-M102 } \\
\hline 156 & 6 & 14.4 & & 1 & 0 & 0 & 0 & 0 & 0 \\
\hline \multirow{2}{*}{\multicolumn{10}{|c|}{$\begin{array}{l}\text { (bent rims on M102) } \\
\text { M813-XM198 }\end{array}$}} \\
\hline & & & & & & & & & \\
\hline 224 & 12 & 19.4 & .3 .5 & 3 & 0 & 0 & 0 & 0 & 0 \\
\hline 225 & 12 & 11.8 & 5.8 & 1 & 5 & 2 & 2 & 0 & 0 \\
\hline 226 & 8 & 11.8 & 5.8 & 6 & 0 & 1 & 0 & 0 & 0 \\
\hline 227 & 8 & 9.6 & 7.1 & 5 & 1 & 2 & 0 & 0 & 0 \\
\hline 228 & 8 & 7.5 & 9.1 & 3 & 5 & 0 & 0 & 0 & 0 \\
\hline 229 & 8 & 5.5 & 12.4 & 5 & 3 & 1 & 1 & 0 & 0 \\
\hline 230 & 8 & 4.7 & 14.5 & 3 & 4 & 0 & 1 & 1 & 0 \\
\hline \multicolumn{10}{|c|}{ M813 } \\
\hline 303 & 12 & 13.0 & 5.2 & 7 & 0 & 2 & 0 & 0 & 0 \\
\hline 304 & 8 & 6.3 & 10.8 & 3 & 0 & 2 & 0 & 0 & 0 \\
\hline 305 & 8 & 4.9 & 13.9 & 3 & 2 & 0 & 2 & 0 & 0 \\
\hline 306 & 8 & 6.1 & 11.2 & 1 & 4 & 0 & 1 & 0 & 0 \\
\hline 307 & 6 & 2.3 & 29.7 & 4 & 0 & 0 & 1 & 0 & 0 \\
\hline 308 & 6 & 2.2 & 31.0 & 4 & 0 & 1 & 1 & 0 & 0 \\
\hline 309 & 6 & 2.5 & 27.3 & 2 & 1 & 2 & 1 & 0 & 0 \\
\hline 310 & 6 & 2.7 & 25.3 & 3 & 0 & 1 & 1 & 0 & 0 \\
\hline 311 & 6 & 2.8 & 24.4 & 1 & 0 & 1 & 0 & 0 & 0 \\
\hline \multicolumn{7}{|c|}{ (Continued) } & \multicolumn{3}{|c|}{ (Sheet 1 of 2 ) } \\
\hline
\end{tabular}


Table C2 (Concluded)

\begin{tabular}{|c|c|c|c|c|c|c|c|c|c|}
\hline Test & Obstacle & Time & Speed & & $\begin{array}{l}\text { No. of } \\
\text { Peak Acce }\end{array}$ & $\begin{array}{l}\text { f Occurren } \\
\text { leration }\end{array}$ & $\begin{array}{l}\text { cees } \\
\text { evels, g }\end{array}$ & & \\
\hline No. & Height, in. & $\underline{\sec }$ & $\mathrm{mph}$ & $\geq 1 \leq 1.5$ & $\geq 1.552 .0$ & $\geq 2.0<2.5$ & $\geq 2.5 \leq 3$ & $\geq 3 \leq 4$ & $\geq 4$ \\
\hline \multicolumn{10}{|c|}{ M109AI } \\
\hline 492 & 6 & 10.0 & 6.8 & 0 & 0 & 0 & 0 & 0 & 0 \\
\hline 493 & 6 & 5.1 & 13.4 & 0 & 0 & 0 & 0 & 0 & 0 \\
\hline 494 & 6 & 2.9 & 23.5 & 2 & 0 & 0 & 0 & 0 & 0 \\
\hline 495 & 6 & 2.7 & 25.2 & 0 & 0 & 0 & 0 & 0 & 0 \\
\hline 496 & 8 & 9.6 & 7.1 & 0 & 0 & 0 & 0 & 0 & 0 \\
\hline 497 & 8 & 5.3 & 12.9 & 0 & 0 & 0 & 0 & 0 & 0 \\
\hline 498 & 8 & 3.5 & 19.5 & 0 & 0 & 0 & 0 & 0 & 0 \\
\hline 499 & 8 & 2.7 & 25.2 & 0 & 0 & 0 & 0 & 0 & 0 \\
\hline 500 & 8 & 2.7 & 25.2 & 2 & 0 & 0 & 0 & 0 & 0 \\
\hline 501 & 12 & 10.0 & 6.8 & 0 & 0 & 0 & 0 & 0 & 0 \\
\hline 502 & 12 & 5.5 & 12.4 & 1 & 0 & 1 & 0 & 0 & 0 \\
\hline 503 & 12 & 5.4 & 12.6 & 3 & 0 & 0 & 0 & 0 & 0 \\
\hline \multicolumn{10}{|c|}{ M110E2 } \\
\hline 534 & 6 & 7.3 & 9.3 & 0 & 0 & 0 & 0 & 0 & 0 \\
\hline 535 & 6 & 3.7 & 18.4 & 0 & 0 & 0 & 0 & 1 & 0 \\
\hline 536 & 6 & 4.2 & 16.2 & 0 & 0 & 0 & 0 & 0 & 0 \\
\hline 537 & 8 & 11.9 & 5.7 & 0 & 0 & 0 & 0 & 0 & 0 \\
\hline 538 & 8 & 7.4 & $9: 2$ & 0 & 0 & 0 & 0 & 0 & 0 \\
\hline 539 & 8 & 4.7 & 14.5 & 0 & 0 & 0 & 1 & 0 & 0 \\
\hline 540 & 12 & 8.5 & 8.0 & 1 & 0 & 0 & 0 & 0 & 0 \\
\hline 541 & 12 & 7.5 & 9.1 & 1 & 0 & 0 & 0 & 0 & 0 \\
\hline 542 & 12 & 4.8 & 14.2 & 1 & 0 & 0 & 0 & 0 & 0 \\
\hline
\end{tabular}


1. Generalized terrain data were available for nine countries, referred to as countries A, B, C, D, E, F, G, H, and I. These data were prepared from limited available information and maps with scales ranging between $1: 325,000$ and 1:3,200,000. Principally because of the sma11 scales of the maps, only type of surface material, soil strength (CI), slope, obstacle height, and vegetation diameter and spacing data were used to describe these nine study areas.

2. The terrain factors and classes used are given in Table D1. Terrain maps of these, study areas are available at the U. S. Army Engineer Waterways Experiment Station. Legends describing the terrain units, terrain factor class numbers, and percentages of area occupied by the terrain units are given in Table D2 through D10. 
Table D1

Terrain Factors and Classes Used in Describing Terrain or Map Units

1. Surface Composition

\begin{tabular}{|c|c|c|c|}
\hline Soil & $\begin{array}{l}\text { S Snow Strength, } C \\
\text { Surface Materia1* }\end{array}$ & Class Range, CI & $\begin{array}{l}\text { Value Used in } \\
\text { Prediction, CI }\end{array}$ \\
\hline 1 & Fine-grained soil & $>150$ & 150 \\
\hline 2 & Fine-grained soil & $75-150$ & 112 \\
\hline 3 & Fine-grained soil & $45-75$ & 60 \\
\hline 4 & Fine-grained soil & $>45$ & 22 \\
\hline 5 & $\begin{array}{l}\text { Coarse-grained } \\
\text { soil }\end{array}$ & $<150$ & 150 \\
\hline
\end{tabular}

2. Slope

\begin{tabular}{ccc} 
Unit & Class Range, $\%$ & \multicolumn{2}{c}{$\begin{array}{c}\text { Value Used in } \\
\text { Prediction, } \%\end{array}$} \\
$\frac{1}{1}$ & $0-10$ & 5 \\
2 & $10-30$ & 20 \\
3 & $>30$ & 30
\end{tabular}

3. Surface Geometry

Height of Vertical Obstacle, in.

\begin{tabular}{lccc} 
Unit & Class Range, in. & Value Used in Prediction, in. \\
\cline { 2 - 3 } 1 & $<3.9$ & 7 \\
2 & $3.9-9.8$ & 24 \\
3 & $9.8-39.4$ & 39 \\
4 & $>39.4$ &
\end{tabular}

* For country D, fine-grained and coarse-grained soil-vehicle relations were used. 
Table D1 (Continued)

\section{Vegetation}

Tropical Climate

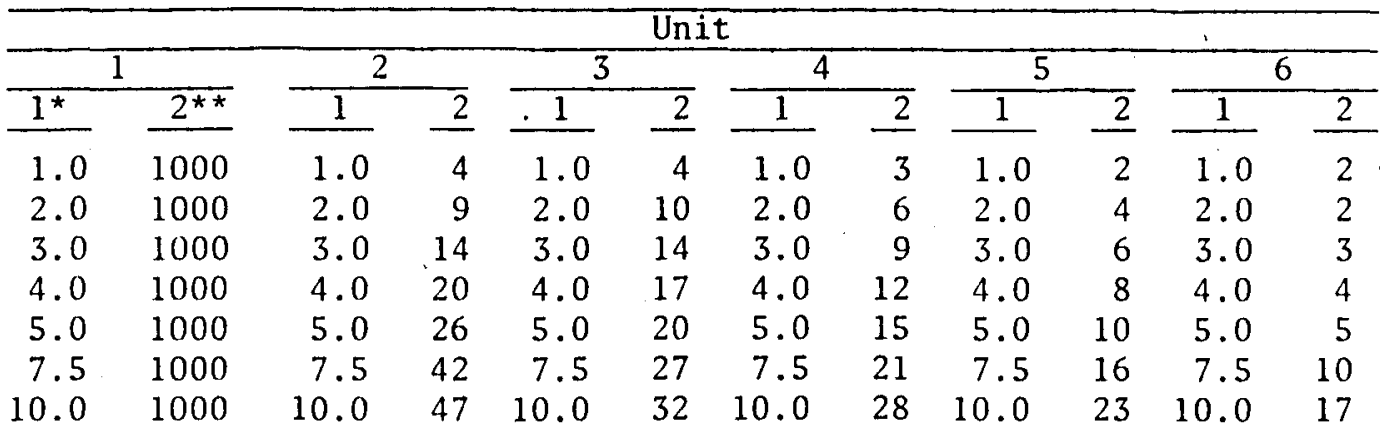

Temperate C1imate

\begin{tabular}{|c|c|c|c|c|c|c|c|c|c|c|c|}
\hline \multicolumn{12}{|c|}{ Unit } \\
\hline \multicolumn{2}{|c|}{1} & \multicolumn{2}{|c|}{2} & \multicolumn{2}{|c|}{3} & \multicolumn{2}{|c|}{$\overline{4}$} & \multicolumn{2}{|c|}{$\overline{5}$} & \multicolumn{2}{|c|}{6} \\
\hline 1 & 2 & 1 & $\overline{2}$ & 1 & 2 & 1 & 2 & 1 & 2 & 1 & 2 \\
\hline 1.0 & 1000 & 1.0 & 11 & 1.0 & 10 & 1.0 & 8 & 1.0 & 6 & 1.0 & 4 \\
\hline 2.0 & 1000 & 2.0 & 15 & 2.0 & 11 & 2.0 & 9 & 2.0 & 7 & 2.0 & 4 \\
\hline 3.0 & 1000 & 3.0 & 20 & 3.0 & 13 & 3.0 & 10 & 3.0 & 7 & 3.0 & 5 \\
\hline 4.0 & 1000 & 4.0 & 26 & 4.0 & 15 & 4.0 & 12 & 4.0 & 8 & 4.0 & 6 \\
\hline 5.0 & 1000 & 5.0 & 29 & 5.0 & 16 & 5.0 & 13 & 5.0 & 10 & 5.0 & 6 \\
\hline 7.5 & 1000 & 7.5 & 31 & 7.5 & 22 & 7.5 & 19 & 7.5 & 15 & 7.5 & 10 \\
\hline 10.0 & 1000 & 10.0 & 33 & 10.0 & 30 & 10.0 & 26 & 10.0 & 22 & 10.0 & 19 \\
\hline
\end{tabular}

Arid Climate

\begin{tabular}{|c|c|c|c|c|c|c|c|c|c|c|c|}
\hline \multicolumn{12}{|c|}{ Unit } \\
\hline \multicolumn{2}{|c|}{1} & \multicolumn{2}{|c|}{2} & \multicolumn{2}{|c|}{3} & \multicolumn{2}{|c|}{4} & \multicolumn{2}{|c|}{5} & \multicolumn{2}{|c|}{6} \\
\hline 1 & 2 & 1 & 2 & 1 & 2 & 1 & 2 & 1 & 2 & 1 & 2 \\
\hline 1.0 & 1000 & 1.0 & 13 & 1.0 & 7 & 1.0 & 6 & 1.0 & 5 & 1.0 & 4 \\
\hline 2.0 & 1000 & 2.0 & 19 & 2.0 & 11 & 2.0 & 7 & 2.0 & 5 & 2.0 & 5 \\
\hline 3.0 & 1000 & 3.0 & 24 & 3.0 & 15 & 3.0 & 8 & 3.0 & 6 & 3.0 & 5 \\
\hline 4.0 & 1000 & 4.0 & 29 & 4.0 & 19 & 4.0 & 11 & 4.0 & 7 & 4.0 & 6 \\
\hline 5.0 & 1000 & 5.0 & 34 & 5.0 & 22 & 5.0 & 14 & 5.0 & 10 & 5.0 & 7 \\
\hline 7.5 & 1000 & 7.5 & 40 & 7.5 & 29 & 7.5 & 25 & 7.5 & 18 & 7.5 & 12 \\
\hline 10.0 & 1000 & 10.0 & 43 & 10.0 & 36 & 10.0 & 33 & 10.0 & 27 & 10.0 & 20 \\
\hline & & & & (Col & int & & & & & & \\
\hline
\end{tabular}

* Stem diameter $\geq$, in.

* Stem spacing $\geq$, ft. 
Table D1 (Concluded)

Example:

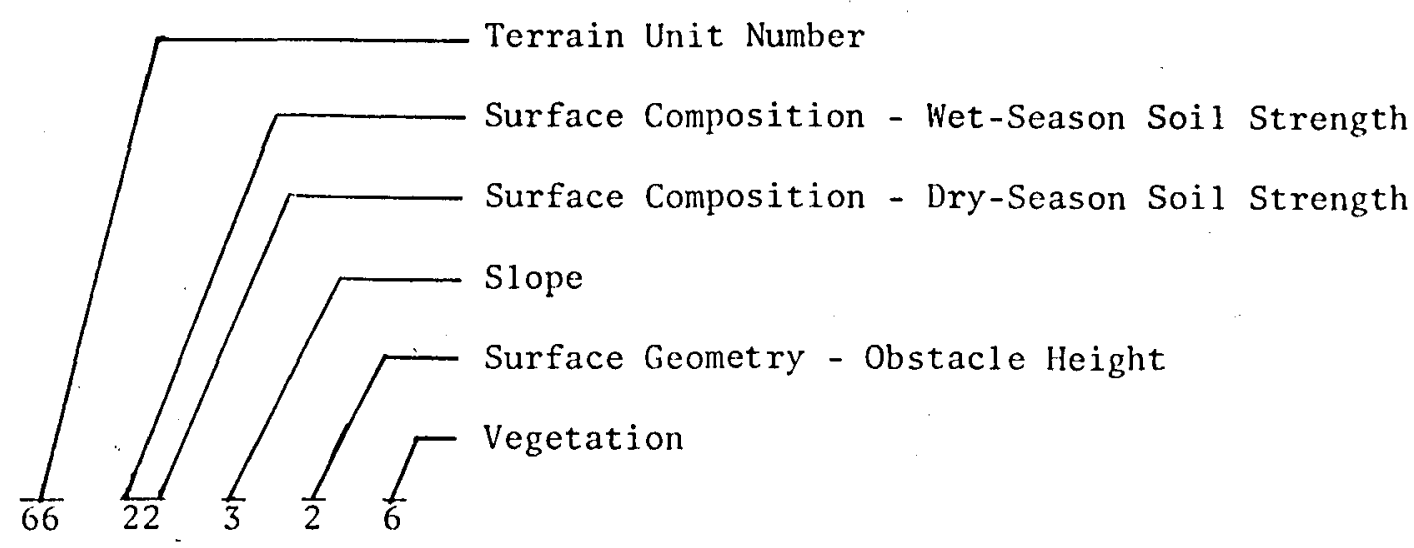


Table D2

Terrain Description and Percentage of Area Occupied by Each Terrain Unit in Country A

C.NINTEY A

\begin{tabular}{|c|c|c|c|c|c|c|}
\hline \multirow[b]{2}{*}{$\begin{array}{l}\text { TERRAIN } \\
\text { IINI I }\end{array}$} & \multicolumn{5}{|c|}{ TI RRAIN FAFIOR I:LASS } & \multirow[b]{2}{*}{$\begin{array}{l}\text { AlFA } \\
\text { PEPSTINAT }\end{array}$} \\
\hline & $\begin{array}{l}\text { COINF } \\
\text { HFT T }\end{array}$ & $\begin{array}{c}\text { INIIFX } \\
\text { DPY }\end{array}$ & SLAPE & $\begin{array}{l}\text { MBSTARIF } \\
\text { HFIG:TT }\end{array}$ & 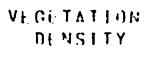 & \\
\hline 3 & 1 & 1 & 1 & 1 & .3 & 7.03 \\
\hline 5 & 1 & 1 & 1 & 1 & 4 & 11.03 \\
\hline 6 & 1 & 1 & 1 & 1 & 5 & 11.05 \\
\hline 14 & 1 & 1 & 1 & 1 & $?$ & ก. \\
\hline 16 & 1 & 1 & 1 & 1 & 3 & $n, 0.3$ \\
\hline 17 & 1 & 1 & 1 & 1 & 4 & $1.7 ?$ \\
\hline is & 1 & i & 1 & 1 & 5 & $n \cdot 07$ \\
\hline $2 ?$ & $i$ & 1 & 1 & $?$ & 3 & $n .1 .3$ \\
\hline 24 & 1 & 1 & 1 & $?$ & 3 & 3.42 \\
\hline 25 & $i$ & 1 & 1 & $?$ & 5 & 1.18 \\
\hline 51 & 1 & 1 & $?$ & 1 & ? & $n .>1$ \\
\hline 52 & 1 & 1 & $?$ & $i$ & 4 & 3.95 \\
\hline 53 & 1 & 1 & 2 & $i$ & 5 & 0.35 \\
\hline 58 & 1 & 1 & ? & 2 & 4 & 4.56 \\
\hline 59 & 1 & 1 & $?$ & ? & 5 & 5.06 \\
\hline 81 & 1 & 1 & 3 & 1 & 4 & 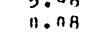 \\
\hline 82 & 1 & 1 & 3 & 1 & 5 & $11 . \cap 4$ \\
\hline 86 & 1 & 1 & 3 & ? & 4 & 11.04 \\
\hline 87 & 1 & 1 & 3 & ? & 5 & 11.71 \\
\hline 102 & 2 & ; & 1 & 1 & 3 & $n \cdot \cap 8$ \\
\hline 103 & 2 & 1 & 1 & 1 & 4 & $n \cdot n 2$ \\
\hline ing & 2 & 1 & 1 & 1 & $?$ & $n .64$ \\
\hline 111 & $?$ & 1 & 1 & 1 & $A$ & 4.47 \\
\hline 112 & ? & $i$ & 1 & $i$ & 5 & $n \cdot n_{3}$ \\
\hline 113 & 2 & 1 & 1 & 2 & $?$ & 11.26 \\
\hline 116 & 2 & $i$ & 1 & $?$ & 4 & 7.144 \\
\hline 117 & 2 & 1 & 1 & 2 & 5 & 1.71 \\
\hline 138 & 2 & 1 & $?$ & 1 & $?$ & $11 .>n$ \\
\hline 140 & 2 & 1 & $?$ & 1 & 4 & 3.61 \\
\hline 141 & ? & 1 & $?$ & 1 & 5 & ก.ก8 \\
\hline 144 & 2 & 1 & 2 & 2 & $?$ & 11.05 \\
\hline 149 & ? & 1 & $?$ & $?$ & 4 & 6.79 \\
\hline 150 & 2 & 1 & $?$ & $?$ & 5 & 2.78 \\
\hline 164 & $?$ & 1 & 3 & 1 & 4 & U.ก? \\
\hline 169 & 2 & 1 & 3 & $?$ & 4 & 11.08 \\
\hline 179 & 3 & 1 & 1 & 1 & $?$ & $n, 14$ \\
\hline 181 & 3 & 1 & 1 & 1 & 1 & 1.10 \\
\hline 182 & 3 & 1 & 1 & 1 & 5 & 11.06 \\
\hline 184 & 3 & 1 & 1 & 2 & 4 & 1.24 \\
\hline $\begin{array}{l}189 \\
185\end{array}$ & 3 & 1 & 1 & 2 & 5 & $\begin{array}{l}0.14 \\
n\end{array}$ \\
\hline 196 & 3 & 1 & $?$ & 1 & $?$ & $\begin{array}{l}11.11 \text { ? } \\
10\end{array}$ \\
\hline $\begin{array}{l}197 \\
\end{array}$ & 3 & 1 & $?$ & 1 & 1 & $\begin{array}{lll}0.02 \\
1.00\end{array}$ \\
\hline 198 & 3 & 1 & $?$ & ? & ? & n.na \\
\hline $\begin{array}{l}19 n \\
? \cap 0\end{array}$ & 3 & 1 & $?$ & $?$ & $\frac{?}{4}$ & $\begin{array}{l}0.06 \\
? .44\end{array}$ \\
\hline $2 n 1$ & 3 & $i$ & $?$ & $?$ & 5 & $1.8 \pi$ \\
\hline 208 & 3 & 1 & 3 & ? & 1 & 11.15 \\
\hline 2.13 & 4 & $i$ & 1 & 1 & 3 & $1.3 n$ \\
\hline 214 & 4 & 1 & 1 & 1 & 4 & $n . .33$ \\
\hline 215 & 4 & 1 & 1 & 1 & 3 & $n . n 3$ \\
\hline 216 & 4 & 1 & 1 & 1 & 2 & 1.18 \\
\hline 217 & 4 & 1 & 1 & 1 & 4 & $7 . \Delta 1$ \\
\hline
\end{tabular}

\begin{tabular}{|c|c|c|c|c|c|c|}
\hline \multirow[b]{2}{*}{ 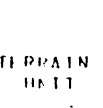 } & \multicolumn{5}{|c|}{ 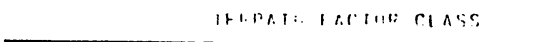 } & \multirow[b]{2}{*}{ 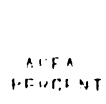 } \\
\hline & 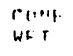 & $\begin{array}{l}\text { IPIir.x } \\
\text { HIPY }\end{array}$ & 5 SיF & 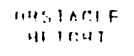 & 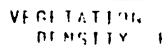 & \\
\hline ?18 & 4 & 1 & 1 & 1 & is & ॥. ०9 \\
\hline $\begin{array}{l}221 \\
2 ? 2\end{array}$ & 4 & 1 & 1 & $?$ & 4 & $\because 75$ \\
\hline $\begin{array}{l}3,20 \\
? 30\end{array}$ & 4 & 1 & 1 & $?$ & 5 & $n \cdot 1 n$ \\
\hline $\begin{array}{l}2.30 \\
2.31\end{array}$ & 4 & 1 & $?$ & 1 & 1 & 10.3 .3 \\
\hline $\begin{array}{l}2,31 \\
233\end{array}$ & $\hat{A}$ & 1 & 2 & $?$ & ? & $n, n 3$ \\
\hline 334 & $\begin{array}{l}4 \\
1\end{array}$ & 1 & $?$ & ? & 1 & $1 \cdots n$ \\
\hline 2.38 & $\hat{s}$ & 1 & ? & $?$ & $n$ & $" .71$ \\
\hline 3.39 & $\begin{array}{l}5 \\
5\end{array}$ & $\begin{array}{l}1 \\
1\end{array}$ & 1 & 1 & $?$ & $1.0 n$ \\
\hline 24.3 & 3 & 1 & 1 & $\begin{array}{l}1 \\
?\end{array}$ & 4 & 1.76 \\
\hline 347 & 5 & 1 & $?$ & 1 & $\begin{array}{l}4 \\
4\end{array}$ & $1 ., n 3$ \\
\hline $24 \mathrm{~B}$ & 5 & 1 & $?$ & $?$ & $\begin{array}{l}4 \\
3\end{array}$ & 11.15 \\
\hline 249 & 5 & 1 & ? & $?$ & $\frac{3}{4}$ & $n \cdot n 1$ \\
\hline 250 & 5 & 1 & $?$ & $?$ & $\begin{array}{l}1 \\
5\end{array}$ & 1.41 \\
\hline 255 & b & 1 & 3 & $i$ & $\begin{array}{l}5 \\
4\end{array}$ & $1 . n 8$ \\
\hline 256 & 5 & 1 & $\hat{3}$ & 1 & $\begin{array}{l}4 \\
4\end{array}$ & 10.116 \\
\hline 257 & 5 & 1 & 3 & 1 & $\begin{array}{l}4 \\
5\end{array}$ & $n \cdot n 3$ \\
\hline $2 n 0$ & 5 & 1 & 3 & $?$ & 5 & $n \cdot 07$ \\
\hline 261 & 5 & 1 & 3 & $?$ & 4 & n.र9. \\
\hline $3 \cap 7$ & $?$ & ? & $?$ & 3 & 5 & $1 . \therefore 3$ \\
\hline 339 & 3 & ? & 1 & 1 & 4 & $\ln \cap 8$ \\
\hline $34 n$ & 3 & $?$ & 1 & 1 & 3 & $n . n ?$ \\
\hline 34.3 & 3 & $?$ & 1 & 1 & 1 & $n .1 n$ \\
\hline 344 & 3 & $?$ & 1 & 1 & $?$ & 1.46 \\
\hline 345 & 3 & $?$ & 1 & 1 & 4 & $3.3 ?$ \\
\hline 346 & 3 & $?$ & 1 & 1 & 5 & 0.115 \\
\hline 347 & 3 & 3 & 1 & $?$ & 3 & 0.14 \\
\hline 348 & 3 & $?$ & 1 & $?$ & 4 & 3.155 \\
\hline 357 & 3 & $?$ & $?$ & $?$ & 5 & $n .8 n$ \\
\hline 358 & 3 & $?$ & $?$ & 1 & $?$ & 0.17 \\
\hline 359 & $\ddot{3}$ & $?$ & $?$ & 1 & 4 & 2.77 \\
\hline 361 & 3 & $?$ & $?$ & $?$ & 5 & $11.1 n$ \\
\hline $36 ?$ & 3 & $?$ & $?$ & $?$ & 1 & $1.0 R$ \\
\hline 365 & 3 & $?$ & 3 & & 5 & 1.114 \\
\hline 366 & 3 & $?$ & 3 & $\begin{array}{l}1 \\
?\end{array}$ & 4 & 110.1 .3 \\
\hline 368 & 4 & $?$ & 1 & $?$ & 5 & $B \cdot \cap A$ \\
\hline 369 & 4 & $?$ & 1 & $\begin{array}{l}1 \\
1\end{array}$ & 3 & $\because . n 6$ \\
\hline $37 n$ & 4 & $?$ & 1 & $?$ & 4 & 10.14 \\
\hline 371 & 4 & $?$ & $?$ & 1 & 4 & 1.06 \\
\hline 372 & 4 & ? & $?$ & 1 & 4 & $\begin{array}{l}n \cdot n ? \\
n=04\end{array}$ \\
\hline 373 & 4 & ? & $?$ & ? & 1 & $\begin{array}{l}110.14 \\
11.18\end{array}$ \\
\hline 411 & 4 & 3 & 1 & 1 & $?$ & \\
\hline 412 & 1 & 3 & $i$ & 1 & 3 & ".*n \\
\hline 113 & 4 & 3 & 1 & 1 & 4 & $n \cdot n$ \\
\hline 414 & 4 & 3 & $i$ & 1 & $\hat{2}$ & 0.03 \\
\hline 415 & 4 & 3 & 1 & 1 & $?$ & 1.75 \\
\hline 416 & 4 & 3 & $i$ & $?$ & 4 & $\because .17$ \\
\hline $4 ? 4$ & 4 & 1 & $i$ & 1 & 3 & $1 . .27$ \\
\hline 428 & 4 & 4 & 1 & $?$ & 4 & $1 .<0$ \\
\hline 439 & 4 & 1 & $?$ & 1 & 3 & $" 0.3$ \\
\hline
\end{tabular}


Table D3

Terrain Description and Percentage of Area Occupied by Each Terrain Unit in Country B

C:OUNIDY H

\begin{tabular}{|c|c|c|c|c|c|c|c|c|c|c|c|c|c|}
\hline \multirow[b]{3}{*}{$\begin{array}{l}\text { TERRAIN } \\
\text { INIT }\end{array}$} & \multicolumn{6}{|c|}{ rinUNIDY } & \multirow[b]{3}{*}{ 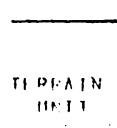 } & \multirow{2}{*}{\multicolumn{5}{|c|}{ 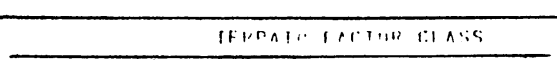 }} & \\
\hline & \multicolumn{5}{|c|}{ II DRAIN TACITB rIASS } & \multirow[b]{2}{*}{$\begin{array}{l}\text { ANFA } \\
\text { PF:CFAT }\end{array}$} & & & & & & & \multirow[b]{2}{*}{ 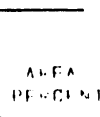 } \\
\hline & $\begin{array}{l}\text { CONF } \\
\text { HFT }\end{array}$ & $\begin{array}{l}\text { INUFXX } \\
\text { IIPYY }\end{array}$ & SI.nPF & $\begin{array}{c}\text { nosina:tF } \\
\text { Hillid1 }\end{array}$ & $\begin{array}{l}\text { VHFi IATIONA } \\
\text { DENSTIYY }\end{array}$ & & & $\begin{array}{l}\text { ri... } \\
\text { Wh r }\end{array}$ & 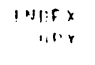 & SI I F F & 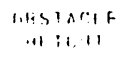 & 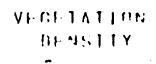 & \\
\hline 20 & 1. & 1 & 1 & ? & $?$ & 11.11 & 246 & 5 & 1 & 1 & 3 & 2 & 11.013 \\
\hline $2 ?$ & $i$ & 1 & 1 & ? & 3 & 1.49 & 248 & 5 & 1 & ? & ? & 3 & n.4h \\
\hline 28 & 1 & 1 & 1 & $?$ & 3 & $11 \cdot 11.3$ & 25,1 & s & 1 & $?$ & $?$ & $?$ & 11.16 \\
\hline 31 & 1 & 1 & 1 & $?$ & $s^{3}$ & $1 " \cdot n 1$ & 2क? & 3 & 1 & $?$ & $?$ & 3 & 1.01 \\
\hline 36 & 1 & 1 & 1 & 3 & $?$ & 11.14 & 253 & ; & 1 & ? & $?$ & 5 & 11.05 \\
\hline $3 \AA$ & 1 & 1 & 1 & 3 & 3 & 11.48 & 234 & s & 1 & $?$ & 3 & $?$ & $n . n 1$ \\
\hline 55 & 1 & 1 & $?$ & ? & $?$ & 1.115 & 258 & 5 & 1 & 3 & $?$ & ? & $n .09$ \\
\hline 57 & 1 & 1 & 2 & $?$ & 3 & 6.24 & 250 & b & 1 & 3 & ; & 3 & 1.74 \\
\hline 59 & 1 & 1 & 2 & $?$ & 5 & 11.05 & 261 & 5 & & 3 & $?$ & 5 & $n \cdot n H$ \\
\hline o? & 1 & 1 & 2 & $?$ & ? & $n .07$ & $26 ?$ & 5 & 1 & 3 & $?$ & $?$ & $n \cdot n H$ \\
\hline 65 & 1 & 1 & $?$ & $?$ & 3 & $? .10$ & 20.3 & s & 1 & 3 & $?$ & ; & $4+.51$ \\
\hline 70 & 1 & 1 & $?$ & 3 & 3 & $n .05$ & 364 & s & 1 & 3 & $?$ & ; & 14.75 \\
\hline 72 & 1 & 1 & $?$ & 3 & 3 & $n \cdot n \geqslant$ & 265 & 2 & 1 & 3 & 3 & 3 & $11 . n 4$ \\
\hline 85 & 1 & 1 & 3 & 2 & 3 & 1.05 & 374 & 5 & $?$ & 1 & $?$ & $?$ & $n \cdot{ }^{3 n}$ \\
\hline 87 & 1 & 1 & 3 & $?$ & 5 & $n .05$ & 375 & 5 & $?$ & 1 & $?$ & $?$ & 11.75 \\
\hline $9 n$ & 1 & 1 & 3 & ? & $?$ & n."R & 376 & 5 & $?$ & 1 & $?$ & 3 & ". . .3 \\
\hline 9.3 & 1 & 1 & 3 & ? & 3 & 1.107 & 377 & "' & $?$ & 1 & 3 & $?$ & $11 \cdot 57$ \\
\hline 96 & 1 & 1 & 3 & $?$ & 5 & $\| .4 ?$ & 378 & b & ? & $?$ & $?$ & 3 & "." \\
\hline 99 & 1 & 1 & 3 & 3 & 3 & 11.04 & 379 & 5 & $?$ & $?$ & $?$ & $?$ & 2.46 \\
\hline 113 & 2 & 1 & 1 & $?$ & $?$ & 0.31 & $38 \pi$ & 5 & $?$ & $?$ & $?$ & 3 & $11 \cdot 0.3$ \\
\hline 115 & 2 & $i$ & 1 & ? & 3 & n.n? & 381 & 5 & $?$ & 3 & $?$ & 2 & $n \cdot n_{1}$ \\
\hline 120 & $?$ & $i$ & 1 & 2 & ? & 11.76 & 382 & 5 & ? & 3 & $?$ & 3 & 10.14 \\
\hline 122 & 2 & 1 & 1 & $?$ & 3 & $n, 1 ?$ & 383 & 5 & $?$ & 3 & $?$ & $\equiv$ & 0.16 \\
\hline 126 & 2 & 1 & $i$ & 3 & ? & 1.45 & 384 & 5 & $?$ & 3 & $?$ & 3 & $n .1 .3$ \\
\hline 128 & 2 & 1 & 1 & 3 & 3 & $1 ., n 4$ & 385 & 5 & $?$ & 3 & $?$ & 5 & $11 \cdot 0.5$ \\
\hline 153 & $?$ & 1 & $?$ & $?$ & $?$ & 1.10 & 417 & 5 & 3 & $?$ & $?$ & 3 & 1.41 \\
\hline 158 & 2 & 1 & ? & 3 & 3 & $n, n 5$ & 418 & 5 & 3 & $?$ & $?$ & $?$ & 1.03 \\
\hline 165 & 2 & 1 & 3 & ? & 2 & ". n1 & 419 & b & 3 & $?$ & $?$ & 3 & 10.05 \\
\hline 167 & 2 & 1 & 3 & $?$ & 3 & ". n. & $42 \pi$ & 5 & 3 & $?$ & 3 & ? & $n .05$ \\
\hline $\begin{array}{l}171 \\
171\end{array}$ & 2 & 1 & 3 & $?$ & $?$ & $0 . n 0$ & 471 & 5 & 3 & 3 & $?$ & 3 & 1.05 \\
\hline 173 & ? & 1 & 3 & $?$ & 3 & 11.77 & 443 & 5 & 4 & 1 & ? & $?$ & 10.05 \\
\hline 199 & 3 & 1 & 2 & $?$ & 3 & ".5n & 114 & 5 & 1 & 1 & 3 & ? & 11.17 \\
\hline 203 & 3 & 1 & $?$ & $?$ & ? & $11, n k$ & 145 & 5 & 4 & ? & $?$ & , & it."? \\
\hline 204 & 3 & 1 & $?$ & 3 & $?$ & $\pi .13$ & 446 & 5 & A & $?$ & $?$ & 3 & 11.113 \\
\hline 206 & 3 & 1 & 2 & 3 & 3 & n.114 & 447 & 5 & 4 & 3 & $?$ & $?$ & $n . n 1$ \\
\hline 207 & 3 & 1 & 3 & $?$ & 3 & 0.12 & 148 & $b$ & 1 & 3 & 2 & 3 & 11.05 \\
\hline$? 19$ & 4 & 1 & 1 & $?$ & $?$ & $n .16$ & 449 & 5 & 1 & 3 & 3 & ? & nor? \\
\hline 220 & 4 & 1 & 1 & $?$ & 3 & $n . n 3$ & & & & & & & \\
\hline 223 & 4 & 1 & 1 & $?$ & $?$ & $n . n 1$ & & & & & & & \\
\hline $2 ? 4$ & 4 & 1 & 1 & $?$ & ? & $11 .>0$ & & & & & & & \\
\hline$? 25$ & 4 & 1 & 1 & 3 & ? & $? \cdot n g$ & & & & & & & \\
\hline 227 & 4 & 1 & 1 & 3 & 3 & 11.11 & & & & & & & \\
\hline 231 & 4 & 1 & $?$ & $?$ & $?$ & n. n? & & & & & & & \\
\hline ?32 & 4 & 1 & $?$ & $?$ & 3 & r."n & & & & & & & \\
\hline 235 & 4 & $i$ & $?$ & 3 & $?$ & $n \cdot n z$ & & & & & & & \\
\hline 236 & 4 & 1 & 3 & 3 & $?$ & 1.14 & & & & & & & \\
\hline 740 & 5 & 1 & 1 & $?$ & 1 & 11.07 & & & & & & & \\
\hline 241 & 5 & 1 & 1 & $?$ & $?$ & n. 14 & & & & & & & \\
\hline 242 & 5 & 1 & 1 & $?$ & 3 & 11.18 & & & & & & & \\
\hline 244 & 5 & 1 & 1 & $?$ & $?$ & n. no & & & & & & & \\
\hline 245 & 5 & 1 & 1 & $?$ & 3 & 11.25 & & & & & & & \\
\hline
\end{tabular}


Table D4

Terrain Description and Percentage of Area Occupied for Each Terrain Unit in Country C

\begin{tabular}{|c|c|c|c|c|c|c|}
\hline \multirow[b]{3}{*}{$\begin{array}{c}\text { TERHAJN } \\
\text { IINII }\end{array}$} & \multicolumn{6}{|c|}{ COIINIRY } \\
\hline & & & $|R P A| N$ & FACIOE $\because 1$ & & \\
\hline & $\begin{array}{l}\text { C.IINF } \\
\text { WFT }\end{array}$ & $\begin{array}{r}\text { INIFX } \\
\text { MPY }\end{array}$ & SI. NHF & $\begin{array}{l}\text { ORSTARIF } \\
\text { HF IGHT }\end{array}$ & $\begin{array}{c}\text { VH GHTAIIIN } \\
\text { II-NSIIY }\end{array}$ & $\begin{array}{c}A 1 \cdot F A \\
\text { H.FIIIH NI }\end{array}$ \\
\hline $1 n$ & 1 & 1 & 1 & 1 & 3 & $1 . \triangle 8$ \\
\hline 22 & 1 & 1 & 1. & $?$ & 3 & 11.69 \\
\hline 78 & 1 & 1 & 1 & 2 & $?$ & 11.6 .6 \\
\hline 36 & 1 & 1 & 1 & 3 & $?$ & 1.54 \\
\hline 47 & 1 & 1 & $?$ & 1 & $?$ & 11.114 \\
\hline 49 & 1 & 1 & $?$ & 1 & 3 & 211.3 .4 \\
\hline 5,7 & 1 & 1 & $?$ & $?$ & 3 & $11 . M 0$ \\
\hline $6 ?$ & 1 & 1 & $?$ & $?$ & $?$ & $1 \mathrm{~A} \cdot \mathrm{x}$ \\
\hline $7 n$ & 1 & 1 & $?$ & 3 & $?$ & ก.11? \\
\hline 75 & 1 & 1 & $?$ & 3 & 3 & $n .1 n$ \\
\hline 79 & 1 & 1 & 3 & 1 & 3 & 1.27 \\
\hline 45 & 1 & 1 & 3 & $?$ & 3 & 1.25 \\
\hline $1 \cap 7$ & $?$ & 1 & 1 & 1 & 3 & $1.1 n$ \\
\hline 115 & $?$ & 1 & 1 & $?$ & 3 & 11.97 \\
\hline $1 ? n$ & 2 & 1 & 1 & $?$ & $?$ & 11.114 \\
\hline 126 & $?$ & 1 & 1 & 3 & $?$ & 11.15 \\
\hline 1.35 & $?$ & 1 & $?$ & 1. & 3 & 11.0 .1 \\
\hline 146 & $?$ & 1 & $?$ & $?$ & 3 & $\|. x\|$ \\
\hline 15.3 & 2 & 1 & $?$ & $?$ & $?$ & 11.10 \\
\hline 167 & $?$ & 1 & 3 & $?$ & 3 & $n . n$ \\
\hline $19 n$ & .3 & 1 & 1 & .3 & $?$ & 1). 84 \\
\hline 2.37 & $b$ & 1 & 1 & 1 & 3 & $11.0 ?$ \\
\hline 271 & $?$ & $?$ & 1 & 1 & .3 & S. 4 \\
\hline $28 n$ & $?$ & $?$ & 1 & $?$ & 3 & 11.110 \\
\hline PP, 5 & $?$ & $?$ & 1 & $?$ & $?$ & $1 .>1$ \\
\hline 202 & $?$ & $?$ & 1 & 3 & $?$ & מ.? \\
\hline $3 \cap 4$ & $?$ & $?$ & $?$ & 1 & 3 & $1.1 n$ \\
\hline 311 & $?$ & $?$ & $?$ & $?$ & 3 & 11.0 .3 \\
\hline 3.14 & $?$ & $?$ & $?$ & $?$ & $?$ & 11.27 \\
\hline 327 & $?$ & $?$ & 3 & 1 & 3 & 11.18 \\
\hline 3.37 & $?$ & $?$ & 3 & $?$ & .3 & 1111 \\
\hline 342 & 3 & $?$ & 1 & 1 & 3 & 11.15 \\
\hline 346 & 3 & $?$ & 1 & $?$ & .3 & 1116 \\
\hline 351 & 3 & $?$ & 1 & 3 & $?$ & .1 .17 \\
\hline 397 & 1 & 3 & 1 & 1 & 3 & $\|.1\|$ \\
\hline 390 & 3 & 3 & 1 & 3 & $?$ & 11.017 \\
\hline
\end{tabular}


Table D5

Terrain Description and Percentage of Area Occupied for Each Terrain Unit in Country D

\begin{tabular}{|c|c|c|c|c|c|c|c|c|c|c|c|c|c|}
\hline \multirow[b]{3}{*}{$\begin{array}{l}\text { TERRAIN } \\
\text { UNIT }\end{array}$} & \multicolumn{6}{|c|}{ cnUsteY } & \multicolumn{7}{|c|}{ inuminer D } \\
\hline & \multicolumn{6}{|c|}{ HRRAIN EACTHE RLASS } & \multicolumn{6}{|c|}{ IFRRAI. BATTHR CIASS } & \multirow[b]{2}{*}{ 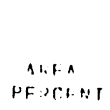 } \\
\hline & $\begin{array}{l}\text { ronit } \\
\text { Wit T }\end{array}$ & $\begin{array}{l}\text { INifex } \\
\text { ney }\end{array}$ & SI NPF & $\begin{array}{l}\text { 1:12STA:LAF } \\
\text { HI } 16.41\end{array}$ & 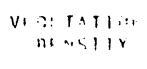 & $\left\{\begin{array}{l}n+1+n \\
\because \cdots+1\end{array}\right.$ & 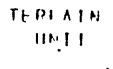 & $\begin{array}{l}r \cap \cdots 1 \\
H+r\end{array}$ & $\begin{array}{l}\text { INUFX } \\
\text { HPY }\end{array}$ & SI HFF & $\begin{array}{l}\text { MRSTRE:IF } \\
\text { "Hitridt }\end{array}$ & $\begin{array}{l}\text { VH R. IATIONN } \\
\text { III NSITY }\end{array}$ & \\
\hline 9 & 1 & , & 1 & 1 & ? & .24 & 331 & $?$ & 2 & 3 & $?$ & $?$ & $v^{r} \cdot$ \\
\hline 26 & 1 & 1 & 1 & $?$ & n & $\because c_{j}$ & 334 & $?$ & $?$ & 3 & ? & a & $1 \ldots$ \\
\hline 29 & 1 & 1 & 1 & $?$ & 3 & $" \cdot 17$ & 364 & 3 & ? & 3 & 1 & $?$ & I: . " f \\
\hline 35 & 1 & 1 & 1 & $?$ & 6 & $\because * 4$ & $39 n$ & 3 & 3 & 1 & 1 & $?$ & $11.7 \mathrm{R}$ \\
\hline 37 & 1 & 1 & 1 & 3 & $?$ & 11.43 & 391 & 3 & 3 & $i$ & 1 & 3 & $11 \cdot 0.3$ \\
\hline 41 & 1 & 1 & 1 & 3 & 6 & $11 \cdot 03$ & 30.3 & & $x^{2}$ & 1 & 1 & 5 & $n, n 3$ \\
\hline 18 & 1 & 1 & $?$ & 1 & $?$ & 0.06 & 31,5 &. & $x^{2}$ & 1 & $?$ & $?$ & $n \cdot n$ \\
\hline 56 & 1 & 1 & ? & $?$ & $?$ & $n \cdot n k$ & Afli & 3 & 3 & 1 & 3 & $?$ & $1 \% \cdots$ \\
\hline $6, n$ & 1 & 1 & $?$ & $?$ & 6 & 11.13 & $4 n 1$ & s & z & 1 & 3 & 4 & $\because n$ \\
\hline 6.3 & 1 & 1 & $?$ & ? & $?$ & 3.1 .3 & 40.3 & $s$ & ' & $i$ & 3 & $?$ & $\therefore . . ?$ \\
\hline 64 & 1 & 1 & $?$ & $?$ & 3 & $1.1 \mu$ & 404 & $\pi$ & ; & $i$ & 3 & 4 & 1.018 \\
\hline 69 & 1 & 1 & $?$ & $?$ & 6 & 0.14 & 405 & 3 & 3 & 1 & 3 & 6 & $n \cdot 14$ \\
\hline 71 & 1 & 1 & $?$ & 3 & 2 & $11 \cdot 11$ & 408 & 3 & 3 & $?$ & 1 & 2 & 11.74 \\
\hline 84 & 1 & 1 & 3 & $?$ & $?$ & n. n? & $4 n 9$ & 3 & 3 & $?$ & 3 & $?$ & $n .75$ \\
\hline 91 & 1 & 1 & 3 & 2 & $?$ & 3.69 & 410 & 3 & 3 & $?$ & 3 & 6 & 0.05 \\
\hline $9 ?$ & 1 & 1 & 3 & 2 & 3 & $n .12$ & 427 & 4 & 4 & 1 & 1 & 3 & 11.00 \\
\hline 97 & 1 & 1 & 3 & $?$ & 6 & 6.16 & & & & & & & \\
\hline 13.3 & 2 & 1 & 2 & 1 & 2 & 2.35 & & & & & & & \\
\hline 151 & 2 & 1 & $?$ & $?$ & 6 & 0.46 & & & & & & & \\
\hline 154 & 2 & 1 & $?$ & 2 & 2 & 0.04 & & & & & & & \\
\hline 162 & 2 & 1 & 3 & 1 & $?$ & 2.87 & & & & & & & \\
\hline 166 & 2 & 1 & 3 & $?$ & $?$ & 1.09 & & & & & & & . \\
\hline 170 & 2 & 1 & 3 & $?$ & 6 & $n \cdot 10$ & & & & & & & \\
\hline 172. & ? & 1 & 3 & $?$ & $?$ & $n \cdot n 8$ & & & & & & & \\
\hline 269 & 2 & $?$ & 1 & 1 & 2 & 10.40 & & & & & & & \\
\hline , 270 & 2 & $?$ & 1 & 1 & 3 & $n \cdot n 7$ & & & & & & & \\
\hline$\cdot 274$ & $?$ & $?$ & 1 & 1 & 6 & 0.33 & & & & & & & \\
\hline $\begin{array}{r}276 \\
270\end{array}$ & $?$ & $?$ & 1 & 1 & 6 & 0.12 & & & & & & & \\
\hline 279 & $?$ & $?$ & 1 & $?$ & $?$ & 0.ก8 & & & & & & & \\
\hline 283 & $?$ & $?$ & 1 & 2 & 6 & 5.53 & & & & & & & \\
\hline 286 & $?$ & $?$ & 1 & $?$ & 2 & 0.15 & & & & & & & \\
\hline $28 \mathrm{~A}$ & $?$ & $?$ & 1 & $?$ & 3 & $n \cdot n 4$ & & & & & & & \\
\hline 293 & $?$ & $?$ & 1 & 3 & $?$ & 3.17 & & & & & & & \\
\hline 294 & 2 & 2 & 1 & 3 & 3 & $0 . n 3$ & & & & & & & \\
\hline 297 & 2 & $?$ & 1 & 3 & 6 & $n \cdot 11$ & & & & & & & \\
\hline 298 & $?$ & $?$ & 1 & 3 & $?$ & $0 . n 6$ & & & & & & & \\
\hline $3 \cap 2$ & $?$ & $?$ & $?$ & 1 & 2 & $1 . n 1$ & & & & & & & \\
\hline 303 & 2 & $?$ & $?$ & 1 & 3 & 1.41 & $\cdots$ & & & & & & \\
\hline 306 & 2 & $?$ & 2 & 1 & 6 & 0.32 & & & & & & & \\
\hline 308 & $?$ & $?$ & ? & 1 & 6 & 0.12 & & & & & & & \\
\hline 309 & $?$ & $?$ & $?$ & $?$ & $?$ & 11.96 & & & & & & & \\
\hline 310 & 2 & ? & $?$ & $?$ & 3 & $n \cdot \wedge \pi$ & & & & & & & \\
\hline 313 & 2 & ? & $?$ & ? & 6 & 20.07 & & & & & & & \\
\hline 320 & ? & $?$ & $?$ & ? & 6 & 11.05 & & & & & & & \\
\hline 321 & 2 & ? & 2 & 3 & $?$ & 2.74 & & & & & & & \\
\hline 323 & ? & $?$ & ? & 3 & 6 & n.1? & & & & & & & \\
\hline 324 & $?$ & $?$ & $?$ & 4 & $?$ & $n \cdot 05$ & & & & & & & \\
\hline 32.5 & $?$ & $?$ & 3 & 1 & $?$ & 2.40 & & & & & & & \\
\hline 326 & 2 & ? & 3 & 1 & 3 & 0.37 & & & & & & & \\
\hline 329 & $?$ & $?$ & 3 & 1 & 6 & 11.59 & & & & & & & \\
\hline $33 n$ & $?$ & $?$ & 3 & 1 & 6 & 11.115 & & & & & & & \\
\hline
\end{tabular}


Table D6

Terrain Description and Percentage of Area Occupied for Each Terrain Unit in Country E

\begin{tabular}{|c|c|c|c|c|c|c|c|c|c|c|c|c|c|c|}
\hline \multirow[b]{3}{*}{$\begin{array}{l}\text { TERRA IN } \\
\text { IINIT }\end{array}$} & \multicolumn{6}{|c|}{ C.niminert } & & & \multicolumn{6}{|c|}{ E:MHIPY E } \\
\hline & \multicolumn{5}{|c|}{ 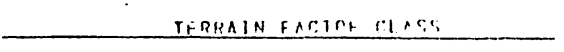 } & \multirow[b]{2}{*}{$\begin{array}{l}\text { AUFA } \\
\text { HFICFANT }\end{array}$} & & \multirow[b]{2}{*}{$\begin{array}{l}\text { THPKNIN } \\
\text { WNII }\end{array}$} & \multicolumn{5}{|c|}{ IFHEAM IARTHR rIASE } & \multirow[b]{2}{*}{$\begin{array}{l}\text { AHFA } \\
\text { PEHC:CNA }\end{array}$} \\
\hline & $\begin{array}{l}\text { CONF } \\
\text { HET }\end{array}$ & $\begin{array}{c}\text { IMNFX } \\
\text { DPY }\end{array}$ & SLAPE & $\begin{array}{l}\text { MIASTASIE } \\
\text { HEIGHT }\end{array}$ & $\begin{array}{l}\text { WFFH TATIOMA } \\
\text { OFNGIIY }\end{array}$ & & & & $\begin{array}{l}\operatorname{rininf} \\
\text { wi } \mathrm{r}\end{array}$ & $\begin{array}{c}\text { Imirex } \\
\text { iner }\end{array}$ & SL.UPF & $\begin{array}{c}\text { mosishe:1F } \\
\text { Hi } !(6: 11\end{array}$ & $\begin{array}{c}\text { verfit ia TinN } \\
\text { nitisitr }\end{array}$ & \\
\hline 2 & 1 & 1 & 1 & 1 & ? & $n .01$ & & 148 & $?$ & 1 & $?$ & ? & 4 & $11.2 ?$ \\
\hline 7 & 1 & 1 & 1 & 1 & 6 & n.on & & 149 & $?$ & 1 & $?$ & $?$ & 5 & $1 . .3 n$ \\
\hline 9 & 1 & 1 & $i$ & 1 & $?$ & $n \cdot n 6$ & & 131 & ? & 1 & $?$ & $?$ & n & n."? \\
\hline 11 & 1 & 1 & 1 & 1 & 4 & 2.54 & & 156 & $?$ & 1 & $?$ & $?$ & 5 & 11.19 \\
\hline 12 & 1 & 1 & 1 & 1 & 5 & 11.05 & & 157 & $?$ & 1 & $?$ & 3 & & 11.04 \\
\hline 13 & 1 & 1 & 1 & 1 & 6 & $n \cdot n ?$ & & 159 & $?$ & 1 & $?$ & 3 & 5 & 11.05 \\
\hline 15 & 1 & 1 & 1 & $i$ & $?$ & 0.16 & & 169 & $?$ & 1 & 3 & 2 & 5 & $1 . ? 3$ \\
\hline 17 & 1 & $i$ & 1 & 1 & 5 & $\|, n$ ? & & $\cdot 176$ & 3 & 1 & 1 & 1 & $?$ & $11.2 ?$ \\
\hline 19 & 1 & 1 & $i$ & ? & 1 & 0.79 & & $\begin{array}{l}178 \\
170\end{array}$ & 3 & 3 & 1 & 1 & 4 & n.ng \\
\hline 21 & 1 & $i$ & 1 & $?$ & $?$ & 0.11 & & $1 \mathrm{HO}$ & 3 & 1 & 1 & 1 & $?$ & $n .01$ \\
\hline 23 & 1 & $i$ & 1 & $?$ & 4 & $\| \cdot n 1$ & & 186 & 3 & 1 & 1 & $?$ & 6 & $n .04$ \\
\hline 24 & 1 & 1. & 1 & $?$ & 5 & 7.46 & & 191 & 3 & 1 & 1 & 3 & 2 & R.A.3 \\
\hline 26 & 1 & $i$ & 1 & 2 & 6 & 5.34 & & 193 & 3 & 1 & 1 & 4 & $?$ & 0.05 \\
\hline 29 & 1 & 1 & 1 & ? & ? & ". n? & & 21? & 1 & 1 & 1 & 1 & $?$ & 11.10 \\
\hline 33 & 1 & 1 & 1 & $?$ & 4 & n..32 & & 226 & 4 & 1 & 1 & 3 & $?$ & $4 . n n$ \\
\hline 34 & 1 & 1 & 1 & $?$ & 5 & 2.23 & & $? 28$ & 4 & 1 & 1 & 3 & 5 & n. 1 \\
\hline 35 & $i$ & 1 & $i$ & $?$ & 6 & 1.64 & & 267 & $?$ & $?$ & 1 & 1 & $?$ & $\because .11$ \\
\hline 37 & 1 & 1 & $i$ & 3 & 2 & 10.13 & . & 368 & $?$ & $?$ & 1 & 1 & 5 & n. n 1 \\
\hline 39 & 1 & $i$ & 1 & 3 & 4 & $n \cdot n_{1}$ & & 273 & $?$ & $?$ & 1 & 1 & 4 & 11.113 \\
\hline 40 & $i$ & 1 & j & 3 & 5 & $n .11$ & & 274 & $?$ & $?$ & 1 & 1 & 6 & $n \cdot n 1$ \\
\hline 42 & 1 & $i$ & 1 & 4 & 5 & $n .04$ & & 275 & 2 & $?$ & 1 & 1 & ? & $n . n 1$ \\
\hline 44 & 1 & 1 & 2 & 1 & 5 & $n \cdot n 1$ & & 276 & 2 & $?$ & 1 & 1 & 6 & $n \cdot n 1$ \\
\hline 50 & 1 & 1 & $?$ & 1 & 4 & 0.00 & & $\begin{array}{l}277 \\
279\end{array}$ & $?$ & $?$ & 1 & $?$ & 1 & $0.1 \mathrm{~A}$ \\
\hline 52 & 1 & 1 & 2 & 1 & 5 & $n \cdot n 1$ & & $\begin{array}{l}279 \\
287\end{array}$ & $?$ & $?$ & 1 & $?$ & $?$ & 11.67 \\
\hline 54 & 1 & $i$ & 2 & 2 & 1 & 11.11 & : & $\begin{array}{l}28 ? \\
283\end{array}$ & $?$ & 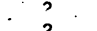 & 1 & $?$ & 5 & 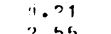 \\
\hline 58 & 1. & 1 & $i$ & ? & 5 & 0.83 & & $\begin{array}{l}283 \\
003\end{array}$ & $?$ & $?$ & 1 & $?$ & 5 & 30,46 \\
\hline 60 & 1 & 1 & -2 & $?$ & 6 & ח. & . & 296 & $?$ & $?$ & $\frac{1}{1}$ & 3 & $\begin{array}{l}3 \\
5\end{array}$ & $\begin{array}{l}? .46 \\
\text { n..nin }\end{array}$ \\
\hline $\begin{array}{l}67 \\
68\end{array}$ & 1 & 1 & $\begin{array}{l}2 \\
2\end{array}$ & $?$ & $\begin{array}{l}4 . \\
5\end{array}$ & $\begin{array}{l}1.80 \\
5.06\end{array}$ & & 312 & $?$ & $?$ & ? & $?$ & 5 & $n, 18$ \\
\hline 69 & 1 & 1 & 2 & $?$ & 6 & 5.89 & & 313 & 2 & $?$ & $?$ & $?$ & 6 & $? .19$ \\
\hline 71 & 3 & 1 & 2 & 3 & , & $n \cdot n 1$ & & 315 & 2 & $?$ & $?$ & $?$ & $?$ & 11. ก? \\
\hline 86 & 1 & 1 & 3 & $?$ & 5 & 10.5 & & 319 & $?$ & ? & $?$ & ? & $i$ & .13 \\
\hline 94 & 1 & 1 & & ? & 4 & "*As & & $\begin{array}{l}321 \\
322\end{array}$ & 3 & $?$ & $?$ & 3 & 3 & $\because \cdots$ \\
\hline 95 & 1 & 1 & 3 & $?$ & 5 & $7.7 \mathrm{H}$ & & 334 & $?$ & $?$ & $\overrightarrow{3}$ & $?$ & $\begin{array}{l}1 \\
i\end{array}$ & $\because \cdots$ \\
\hline $\begin{array}{l}97 \\
98\end{array}$ & 1 & 1 & 3 & $\begin{array}{l}? \\
3\end{array}$ & $\begin{array}{l}6 \\
2\end{array}$ & $\begin{array}{r}10.55 \\
0.04\end{array}$ & & 336 & $?$ & ? & 3 & $?$ & $\begin{array}{l}n \\
h\end{array}$ & ". $11 ?$ \\
\hline $\begin{array}{l}101 \\
101\end{array}$ & 2 & j & 1 & 1 & $?$ & $n . n 1$ & & 337 & 2 & ? & 3 & 3 & $?$ & 11.07 \\
\hline 106 & $?$ & 1 & 1 & $i$ & $?$ & 11.115 & & 338 & $?$ & $?$ & 3 & 3 & 5 & "."1 \\
\hline $\begin{array}{l}100 \\
108\end{array}$ & $?$ & 1 & 1 & 1 & 4. & 1.44 & & 387 & 3 & 3 & 1 & 1 & $?$ & ก.?7 \\
\hline 110 & $?$ & 1 & 1 & 1 & ? & $\therefore$.n & & 388 & 3 & 3 & 1 & 1 & 1 & $n .0 .3$ \\
\hline 111 & $?$ & 1 & 1. & 1 & 5 & 1.119 & & 389 & 3 & 3 & 1 & 1 & 6 & .0 .05 \\
\hline 116 & $?$ & 1 & $i$ & $?$ & 5 & $\ldots .17$ & & 397 & 3 & 3 & 1 & $?$ & i. & $n, n ?$ \\
\hline 118 & $?$ & 1 & 1 & ? & 6 & $n \cdot n \beta$ & & $4 \pi n$ & 3 & 3 & 1 & 3 & $?$ & $n . n 1$ \\
\hline 124 & $?$ & 1 & $i$ & $?$ & 5 & 8.18 & - & $40 ?$ & 3 & 3 & 1 & 3 & $n$ & 0.05 \\
\hline 125 & 2 & 1 & 1 & 3 & 1 & $r .1 n$ & & $4 n 6$ & 3 & 3 & 1 & 4 & $?$ & $n \cdot n 6$ \\
\hline 127 & $?$ & 1 & j & 3 & $?$ & 1.53 & & 407 & 3 & 3 & 1 & 1 & 6 & 2.43 \\
\hline 129 & $?$ & 1 & 1 & 3 & 5 & $n \cdot n a$ & & $\begin{array}{l}473 \\
43 ?\end{array}$ & $\begin{array}{l}4 \\
4\end{array}$ & 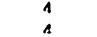 & i & 1 & $?$ & $\begin{array}{l}1.08 \\
\text { i1,?0n }\end{array}$ \\
\hline 131 & $?$ & 1 & 1 & 4 & 5 & $n \cdot 14$ & & $\begin{array}{l}43 ? \\
434\end{array}$ & 4 & 4 & 1 & 4 & $?$ & $\begin{array}{l}10.2 n \\
0.115\end{array}$ \\
\hline 137 & 2 & 1 & $?$ & 1 & 4 & 0.27 & & $\begin{array}{l}4.34 \\
437\end{array}$ & 4 & 4 & 1 & 4 & $?$ & 0.115 \\
\hline 139 & $?$ & 1 & ? & 1 & 3 & $n \cdot n 1$ & & $\begin{array}{l}437 \\
44 ?\end{array}$ & 4 & 4 & 3 & ? & $\begin{array}{l}\hat{b} \\
h\end{array}$ & $\begin{array}{l}n .05 \\
\because .25\end{array}$ \\
\hline $14 ?$ & $?$ & $i$ & $?$ & 1 & $6^{\prime}$ & 1.01 & & $44 ?$ & & & & & & 11.35 \\
\hline
\end{tabular}


Table D7

Terrain Description and Percentage of Area Occupied for Each Terrain Unit in Country $F$

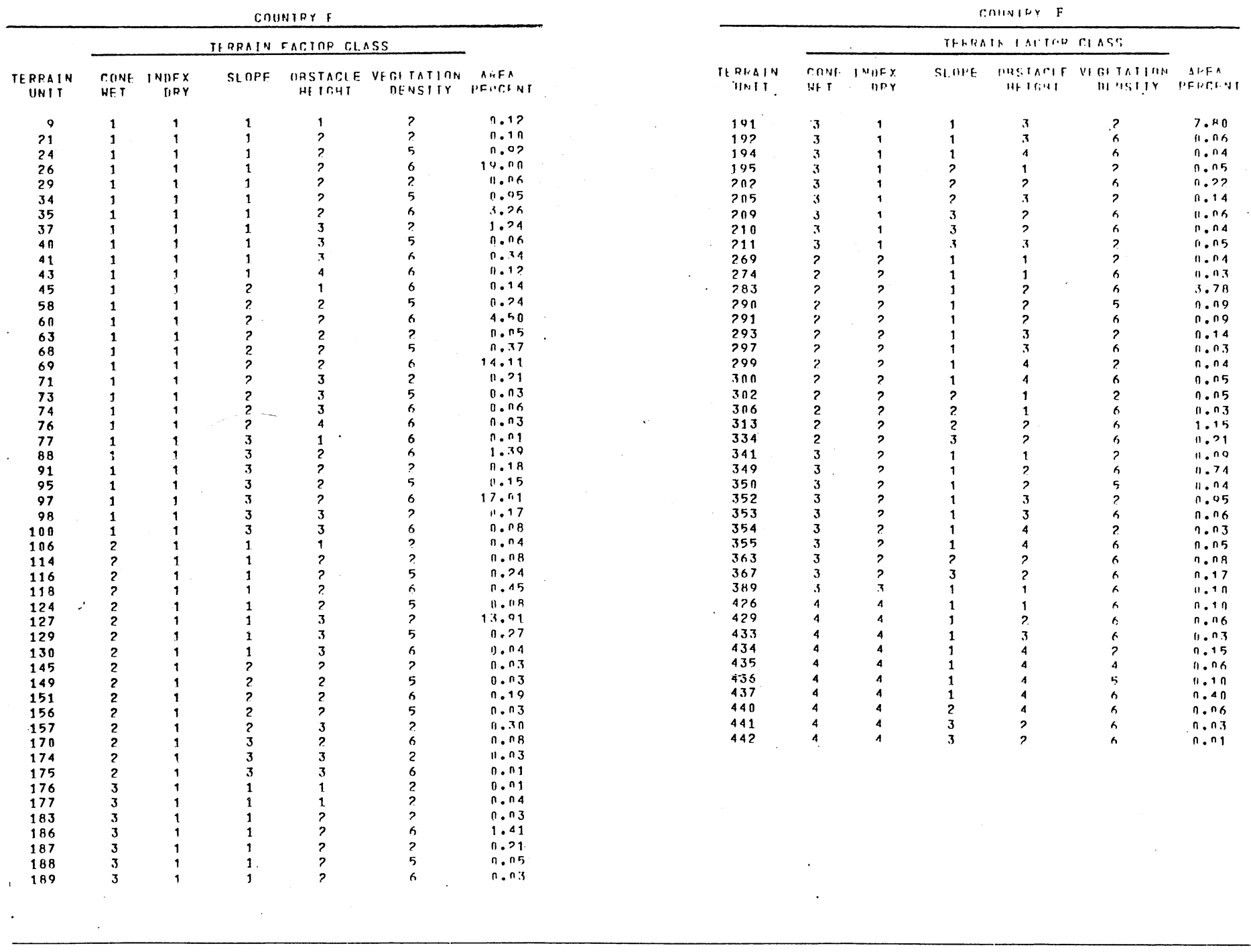


Table D8

Terrain Description and Percentage of Area Occupied for Each Terrain Unit in Country G

CONUNTRY

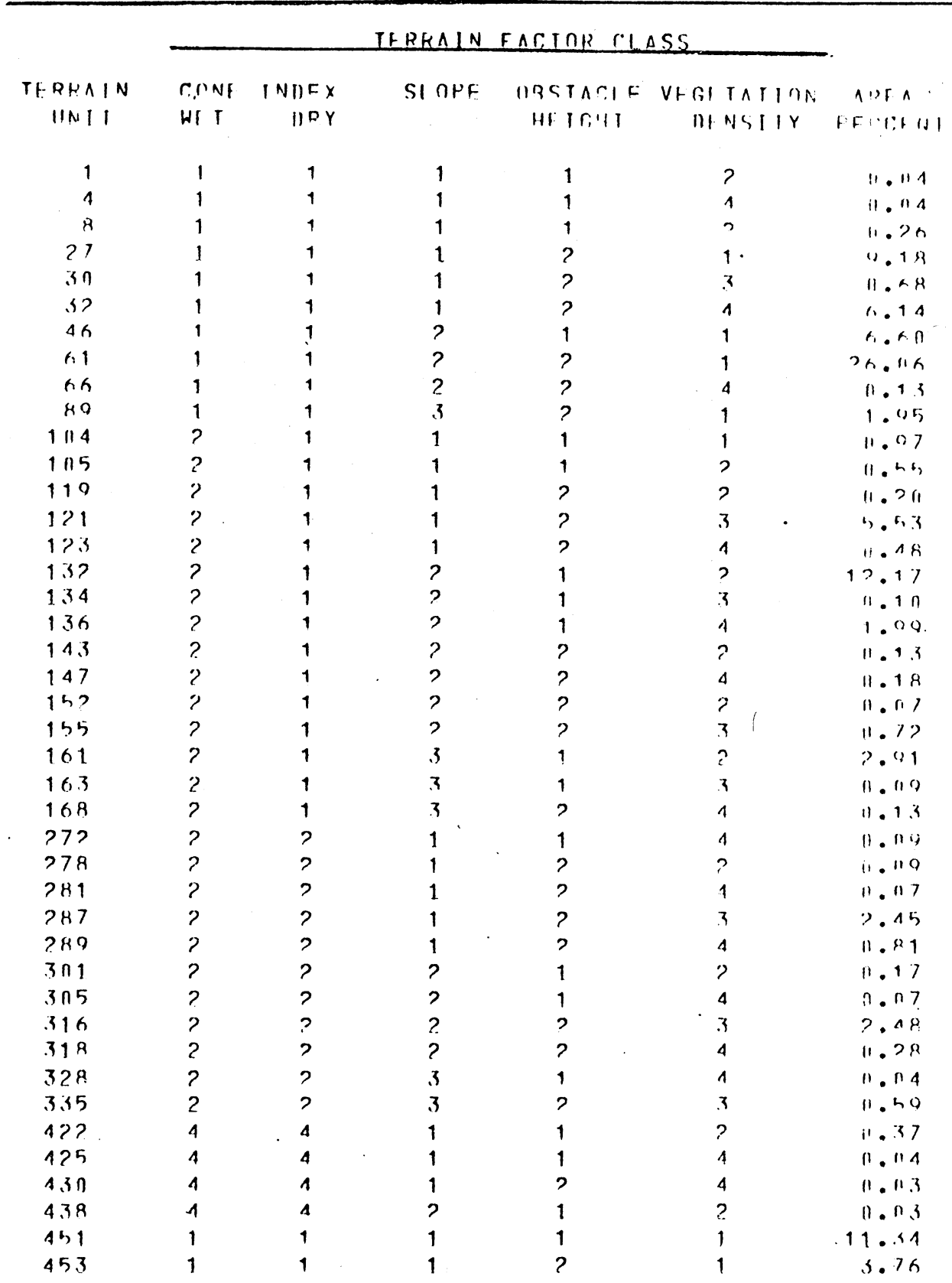


Table D9

Terrain Description and Percentage of Area Occupied for Each Terrain Unit in Country $H$ (; RUN IRY H

\section{TFRRAIN FAITTR CII SSS}

\begin{tabular}{|c|c|c|c|c|c|c|}
\hline $\begin{array}{l}\text { I RIAIN } \\
\text { IINII }\end{array}$ & $\begin{array}{l}\text { RONF } \\
\text { WIT }\end{array}$ & $\begin{array}{r}\text { INDEX } \\
\text { DRY }\end{array}$ & SLNPE & $\begin{array}{l}\text { IRSTACIF } \\
\text { HF IFHT }\end{array}$ & $\begin{array}{c}\text { VEGFTATIOM } \\
\text { DINSIIY }\end{array}$ & $\begin{array}{c}\text { ARFA } \\
\text { PFHCINT }\end{array}$ \\
\hline$? 7$ & 1 & 1 & 1 & $?$ & 1 & $1: .41$ \\
\hline 01 & 1 & 1 & $?$ & $?$ & 1 & 7.153 \\
\hline 83 & 1 & 1 & 3 & $?$ & 1 & 4.36 \\
\hline 119 & 1 & 1 & 3 & $?$ & 1 & 5.5 .48 \\
\hline 386 & 3 & 3 & 1 & 1 & $?$ & $n .01$ \\
\hline 394 & 3 & 3 & 1 & $?$ & 1 & $11 .<5$ \\
\hline 396 & 3 & 3 & 1 & $?$ & $?$ & 11.10 \\
\hline $45 n$ & 1 & 1 & 1 & 1 & $?$ & 11.18 \\
\hline $45 ?$ & 1 & 1 & 1 & $?$ & 1 & 2.4 .20 \\
\hline 454 & 1 & 1 & 3 & $?$ & 1 & ?. 10 \\
\hline
\end{tabular}


Table D10

Terrain Description and Percentage of Area Occupied for Each Terrain Unit in Country I

COHNTRY I

\begin{tabular}{|c|c|c|c|c|c|c|}
\hline \multirow[b]{2}{*}{$\begin{array}{l}\text { TFRRAIN } \\
\text { IINIT }\end{array}$} & \multirow{2}{*}{\multicolumn{2}{|c|}{$\begin{array}{lr} \\
\text { CONHI INIIFX } \\
\text { WFT NOY }\end{array}$}} & \multicolumn{3}{|c|}{ IFRRAIN FACTOR CIASS } & \multirow[b]{2}{*}{$\begin{array}{c}\text { ARFA } \\
\text { PFRRENT }\end{array}$} \\
\hline & & & SLOPE & $\begin{array}{l}\text { ORSTACLLE } \\
\text { HF IGHT }\end{array}$ & $\begin{array}{c}\text { VI GETATION } \\
\text { OINSITY }\end{array}$ & \\
\hline 1 & 1 & 1 & 1 & 1 & 1 & $n \cdot n, 3$ \\
\hline 2 & 1 & 1 & 1 & 1 & ? & 1.29 \\
\hline 3 & 1 & 1 & 1 & 1 & 4 & $n . n 1$ \\
\hline 4 & 1 & 1 & 1 & $?$ & 1 & 16.70 \\
\hline 5 & 1 & 1 & 1 & $?$ & $?$ & 0.114 \\
\hline 6 & 1 & 1 & 1 & 2 & 4 & $n .2 n$ \\
\hline 7 & 1 & 1 & 1 & $?$ & 6 & n. ก? \\
\hline 8 & 1. & 1 & 1 & 3 & 1 & $11 . n 6$ \\
\hline 9 & 1 & 1 & 1 & 4 & $\Delta$ & $n . n 1$ \\
\hline $1 n$ & 1 & 1 & $?$ & 1. & 1 & 0.117 \\
\hline 11 & 1 & 1 & $?$ & 1 & $?$ & 11.30 \\
\hline $1 ?$ & 1 & 1 & $?$ & 1 & 4 & $n . n 3$ \\
\hline 13 & 1 & 1 & $?$ & $?$ & 1 & 10.47 \\
\hline 14 & 1 & 1 & $?$ & $?$ & $?$ & 0.15 \\
\hline 15 & 1 & 1 & $?$ & $?$ & 1 & 0.15 \\
\hline 16 & 1 & 1 & $?$ & $?$ & 6 & $0 . ? 7$ \\
\hline 17 & 1 & 1 & $?$ & 3 & 1 & 11.113 \\
\hline 18 & 1 & 1 & $?$ & 4 & 1 & $n \cdot n, 3$ \\
\hline 19 & 1 & 1 & 3 & 1 & $?$ & 0.18 \\
\hline$? n$ & 1 & 1 & 3 & 1 & 4 & 0.07 \\
\hline 71 & 1 & 1 & 3 & 1 & 6 & n. $n 1$ \\
\hline $2 ?$ & 1 & 1 & 3 & $?$ & 1 & 3.34 \\
\hline 2.3 & 1 & 1 & 3 & $?$ & $?$ & 0.14 \\
\hline 24 & 1 & 1 & 3 & $?$ & 4 & $n .2 n$ \\
\hline 25 & 1 & 1 & .3 & $?$ & 6 & 11.86 \\
\hline 26 & 1 & 1 & 3 & 3 & 2 & $n . n 1$ \\
\hline 27 & 1 & 1 & 3 & 1 & 1 & $n \cdot n ?$ \\
\hline 28 & 1 & 1 & 3 & 4 & 6 & n. n? \\
\hline$? 9$ & $?$ & 1 & 1 & 1 & 1 & n. n? \\
\hline $3 n$ & $?$ & 1 & 1 & 1 & $?$ & $n .77$ \\
\hline 31 & $?$ & 1 & 1 & $?$ & 1 & $11.0 n$ \\
\hline 32 & $?$ & 1 & 1 & $?$ & 4 & 11.14 \\
\hline 33 & $?$ & 1 & 1 & 3 & $?$ & $n \cdot n 1$ \\
\hline 34 & $?$ & 1 & $?$ & 1 & 1 & 0.111 \\
\hline .35 & $?$ & 1 & $?$ & 1 & $?$ & 11.11 \\
\hline 36 & $?$ & 1 & $?$ & 1 & 4 & $0 . .43$ \\
\hline 37 & $?$ & 1 & $?$ & 1 & 6 & $n .11$ \\
\hline 38 & $?$ & 1 & $?$ & $?$ & 1 & $? .17$ \\
\hline 30 & 2 & 1 & $?$ & ? & 4 & 11.70 \\
\hline $4 n$ & $?$ & 1 & $?$ & $?$ & 6 & 0.07 \\
\hline 41 & $?$ & 1 & $?$ & 4 & 4 & 0.155 \\
\hline $4 ?$ & $?$ & 1 & 3 & 1 & 4 & $11 . n 3$ \\
\hline 4.3 & $?$ & 1 & 3 & 2 & 1 & 0.14 \\
\hline 44 & 3 & 1 & 1 & 1 & $?$ & 1.05 \\
\hline 45 & 3 & 1 & 1 & ? & 1 & 1.89 \\
\hline 46 & 3 & 1 & 1 & $?$ & $?$ & $n .01$ \\
\hline 47 & 3 & $?$ & 1 & 1 & 2 & $n \cdot n 3$ \\
\hline 48 & 5 & 5 & 1 & $i$ & 1 & 8.39 \\
\hline 49 & 5 & 5 & $?$ & 1 & 1 & 11.65 \\
\hline $5 n$ & 5 & 5 & 3 & 1 & 1 & $0.3 n$ \\
\hline 51 & 1 & 1 & 1 & 4 & 1 & 18.49 \\
\hline
\end{tabular}




\section{APPENDIX E: BASIC PERFORMANCE PREDICTIONS FOR HIMO WEST GERMANY AND MID-EAST STUDY AREAS}

1. Appendix $\mathrm{E}$ contains most of the basic performance data (speed profiles and diagnostic statistics) for the selected study vehicles in the HIMO West Germany and Mid-East study areas and for the same vehicles over the selected artillery missions. Also included in Appendix $E$ are the speed profile data for the selected groups of vehicles traveling together in the HIMO study areas.

2. The speed profile data (see paragraphs 27 and 28 main text) for the selected study vehicles over primary roads, secondary roads, trails, and off-road terrain for two conditions are given in Tables El-E43 as follows :

\begin{tabular}{ll} 
Table & Speed Profiles for Study Vehicles \\
\hline E1 & M561-M102 \\
E2 & M561-M204 \\
E3 & M561-XM198 \\
E4 & M35A2-M102 \\
E5 & M35A2-XM204 \\
E6 & M35A2-XM198 \\
E7 & M813-XM204 \\
E8 & M813-M114A1 \\
E9 & M813-XM198 \\
E10 & M813-FH70 \\
E11 & M813-XM(130-mm) \\
E12 & M656-XM204 \\
E13 & M656-XM198 \\
E14 & TDW901-XM204 \\
E15 & TDW901-M114A1 \\
E16 & TDW901-XM198 \\
E17 & TDW901-FH70 \\
E18 & TDW901-XM(130-mm) \\
E19 & M520E1-XM204 \\
&
\end{tabular}




\begin{tabular}{ll} 
Tab1e & Speed Prof1les for Study Vehicles \\
\hline E20 & M520E1-XM198 \\
E21 & M520E1-FH70 \\
E22 & M125E1-XM198 \\
E23 & M548E1-XM204 \\
E24 & M548E1-XM198 \\
E25 & M548E1-FH70 \\
E26 & UET-XM198 \\
E27 & UET-FH70 \\
E28 & ASV-XM204 \\
E29 & ASV-XM198 \\
E30 & ASV-FH70 \\
E31 & M109A1 \\
E32 & M107 \\
E33 & M110E2 \\
E34 & M113A1 \\
E35 & M60A2 \\
E36 & M548E1 \\
E37 & M577A1 \\
E38 & XM723 \\
E39 & XM1 \\
E40 & ASV \\
E41 & M561 \\
E42 & M813 \\
E43 & UET \\
&
\end{tabular}

3. The diagnostic statistics (see paragraph 31, main text) for the selected study vehicles over primary roads, secondary roads, and trails for two conditions in the HIMO West Germany and Mid-East study areas are given in Tables E44-E87 as follows:

Table

E44

E45
Diagnostic Statistics for Study Vehicles M561-M102

M561-XM204 
Table

E46

E47

E48

E49

E50

E51

E52

E53

E54

E55

E56

E57

E58

E59

E60

E61

E62

E63

E64

E65

E66

E67

E68

E69

E70

E71

E72

E73

E74

E75

E76

E77

E78
Diagnostic Statistics for Study Vehicles M561-XM198

M35A2-M102

M35A2-XM204

M35A2-XM198

M813-XM204

M813-M114A1

M813-XM198

M813-FH70

M813-XM(130-mm)

M656-XM204

M656-XM198

TDW901-XM204

TDW901-M114A1

TDW901-XM198

TDW901-FH70

TDW901-XM(130-mm)

M520E1-XM204

M520E1-XM198

M520E1-FH70

M125E1-XM198

M548E1-XM204

M548E.1-XM198

M548E1-FH70

UET-XM198

UET-FH70

ASV-XM204

ASV-XM198

ASV-FH70

M109A1

M107

M110E2

M113A1

M60A2 
Table

E79

E80

E81

E82

E83

E84

E85

E86
Diagnostic Statistics for Study Vehicles

M548E1

M577A1

XM723

XM1

ASV

M561

M813

UET

4. The diagnostic statistics for the selected vehicles in offroad terrain of the HIMO West Germany and Mid-East study areas for two conditions are given in Table E87. The speed profile data for the selected groups of vehicles (see paragraph 36 and 37 , main text) traveling together over primary roads, secondary roads, trails, and off-road terrain for two conditions are given in Tables E88-E91 as follows :

Table

$\mathrm{E} 88$

E89

E90

E91 $\underline{\text { Vehicle Group }}$

1

2

3

4

5. The time to complete the selected artillery missions in the West Germany study area for two conditions are given in Table E92 for the study prime mover-towed artillery combinations, and in Table E93 for the self-propelled artillery and reference vehicles. 


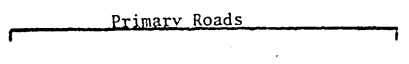

DERIE.it TULAL HISIAHCE

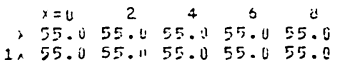

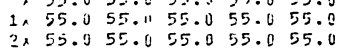
$\frac{2}{3} \times 55.055 .055 .055 .054 .9$

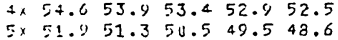

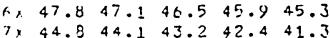
$\begin{array}{llllll}9 \times & 44.9 & 4.1 & 43.2 & 42.4 & 41.3 \\ 3 \times & 39.9 & 37.8 & 36.6 & 35.3 \\ 102 & 34.9 & 32.9 & 31.9 & 30.9 & 29.6\end{array}$ $10 \times 28.2$

PERI:FN TETAL DISTANCE

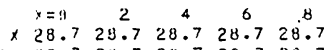

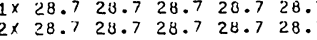

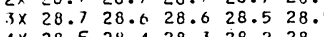

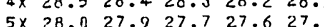
$\begin{array}{llllll}6 \times & 27.4 & 77.3 & 27.2 & 27.1 & 27.0\end{array}$ $\begin{array}{llllll}7 \times & 25.4 & 26.9 & 26.8 & 26.6 & 26.4\end{array}$ $\begin{array}{llllll}8 \times & 26.1 & 25.7 & 25.4 & 25.0 & 24.5 \\ 9 \times & 24.0 & 23.6 & 23.1 & 22.7 & 22.0\end{array}$ $\begin{aligned} 9 \times & 24.0 \\ 10 \times & 21.3\end{aligned}$$$
\text { - }
$$

PERTEMt TIITAL DISIANTE.

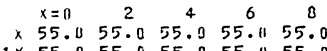

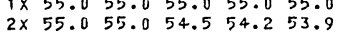
$\begin{array}{llllll}3 \times & 53.6 & 53.4 & 53.2 & 53.1 & 52.9 \\ 4 \times & 52.8 & 52.5 & 52.1 & 51.7 & 51.3\end{array}$

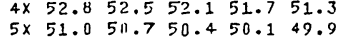
$\begin{array}{llllll}5 x & 51.0 & 511.7 & 50.4 & 50.1 & 49.9 \\ 6 \times & 49.7 & 49.5 & 40.3 & 49.1 & 48.9\end{array}$ $\begin{array}{llllll}7 \times & 48.7 & 48.5 & 43.2 & 47.7 & 47.3 \\ 3 \times & 46.4 & 45.5 & 45.7 & 45.1 & 44.3\end{array}$ $\begin{array}{llllll}3 \times & 46.4 & 45.3 & 45.7 & 45.1 & 44.3 \\ 3 \times & 43.0 & 41.1 & 38.3 & 35.8 & 33.7\end{array}$ $9 \times 43.0$
$10 \times 32.0$

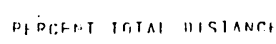

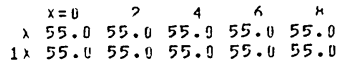
$\begin{array}{llllll}1 \times & 55.0 & 55.0 & 55.0 & 55.0 & 55.0 \\ 2 \times & 55.0 & 55.0 & 54.5 & 54.0 & 55.0 \\ 3 \times & 53.6 & 53.0 & 53.0\end{array}$

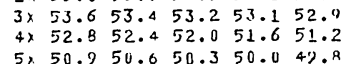
$\begin{array}{llllll}5 \times & 50.9 & 50.6 & 50.3 & 50.0 & 49.8 \\ 6 \times & 49.6 & 49.4 & 49.2 & 49.0 & 48.8\end{array}$ $\begin{array}{rrrrrrr}8 \times & 46.5 & 45.4 & 45.3 & 44.6 & 43.8 \\ 32 & 42.4 & 40.5 & 37.6 & 35.1 & 33.0 \\ 10 \times & 31.2\end{array}$

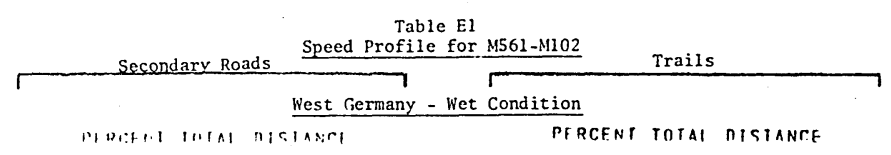

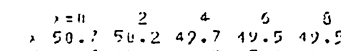

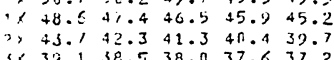

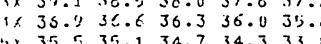

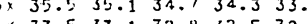

$\begin{array}{llllll}4 \times & 33.5 & 33.1 & 32.8 & 32.5 & 32.2 \\ 1 \times & 31.0 & 31.4 & 31.0110 .1 & 30.1\end{array}$

$\begin{array}{llllll}2 x & 29.6 & 29.1 & 28.5 & 27.9 & 27.2 \\ 3 \times & 26.6 & 25.9 & 25.3 & 21.6 & 23.7\end{array}$

\section{West Sermany - Snow Condition}

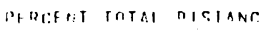

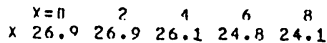

$\begin{array}{llllll}1 \times & 23.8 & 26.5 & 26.1 & 24.8 & 24.8 \\ 2 x & 22.8 & 23.8 & 22.5\end{array}$

$3 \times 20.620 .3 \quad 20.1 \quad 19.8 \quad 19.3$

$\begin{array}{llllll}4 \times & 18.6 & 17.9 & 17.4 & 16.9 & 16.5\end{array}$

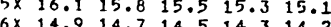

$7 \times 14.013 .7 \quad 13.3 \quad 12.7 \quad 12.2$

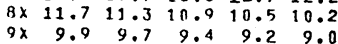

PERCFNT TOTAI. DISTANCE

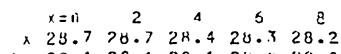

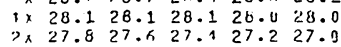

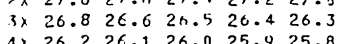

$5 \times \quad 25.6 \quad 25.5 \quad 25.4 \quad 25.3 \quad 25.2$

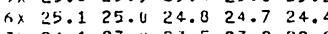

$\begin{array}{lllllll}7 x & 24.1 & 23.0 & 23.5 & 23.2 & 22.4 \\ 3 x & 22.6 & 22.2 & 21.8 & 21.3 & 220\end{array}$

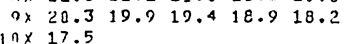

Mid-East - Wet Condition

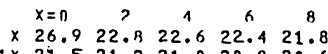

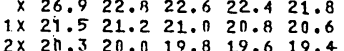

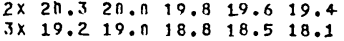

$4 \times \quad 17.517 .0 \quad 16.516 .1 \quad 15.8$

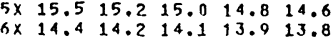

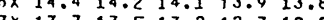

$8 \times 11.711 .3 \quad 10.910 .510 .2$

inx

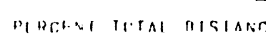

$\begin{array}{rrrrrr} & x=0 & 2 & 4 & 6 & 8 \\ \{ & 50.7 & 50.7 & 50.2 & 49.9 & 40.7\end{array}$

$2 \times \quad 49.4 \quad 48.8 \quad 48.0 \quad 46.4 \quad 45.0$

$\begin{array}{llllll}3 \times & 43.8 & 42.9 & 42.1 & 41.4 & 40.8\end{array}$

$\begin{array}{llllll}4 \times & 40.2 & 39.8 & 39.4 & 39.0 & 38.6 \\ 5 \times 38.3 & 38.0 & 37.8 & 37.5 & 37.0\end{array}$

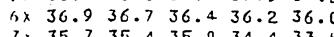

$\begin{array}{llllll}7 \times & 35.7 & 35.4 & 35.0 & 34.4 & 33.8 \\ 4 \times & 33.11 & 31.8 & 30.7 & 20.7 & 23.9\end{array}$

$\begin{array}{llllll}4 \times & 33.0 & 31.8 & 30.7 & 29.7 & 23.3 \\ 9 \times & 28.1 & 27.3 & 26.7 & 26.1 & 25.5\end{array}$

$9 \times$
$\ln \times 24.8$

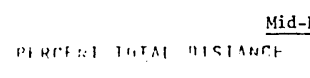

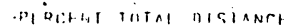

$\begin{array}{rrrrrr} & x=0 & 2 & 4 & 6 & 8 \\ x & 39.3 & 39.3 & 49.3 & 39.3 & 39.3 \\ x & 39.3 & 39.3 & 39.3 & 39.3 & 39.3\end{array}$

$\begin{array}{lllllll}2 \times & 31.3 & 38.9 & 38.2 & 37.7 & 37.2 \\ 3 \times & 36.9 & 36.5 & 36.3 & 36.0 & 35.9\end{array}$

$4 \times \quad 35.6 \quad 35.4 \quad 35.3 \quad 35.1 \quad 35.0$

$5 \times 34.834 .7 \quad 34.534 .434 .2$

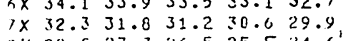

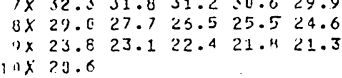

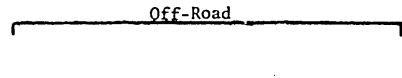

DI PCLAt Intal mistunet

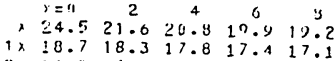

2x $16.916 .7 \quad 16.5 \quad 16.316$.

$\begin{array}{lllllll}4 & 25.8 & 15.5 & 15.3 & 15.2 & 15.0 \\ 1 \times & 44.9 & 14.7 & 14.6 & 14.4 & 14 .\end{array}$

$\begin{array}{lllllll}\text { i) } & 14.1 & 14.0 & 13.8 & 13.7 & 13.5 \\ \text { hX } & 13.4 & 13.2 & 13.1 & 13.9 & 13\end{array}$

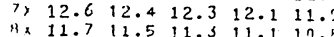

$\begin{array}{rrrrrr}12 \times & 5.4 & 2.5 & 11.5 & 11.1 & 10.9 \\ 10 \times & 0.9 & & & & \end{array}$

riRl:H. THIAL MISIANCH

$\begin{array}{llcccc} & x=0 & 2 & 4 & 5 & 8 \\ \times x & 21.8 & 20.2 & 19.8 & 19.3 & 18.8 \\ x & 18.4 & 18.2 & 17.8 & 17.5 & 17.8\end{array}$ $\begin{array}{llllll}2 \times & 16.9 & 16.8 & 16.6 & 16.4 & 16.2\end{array}$

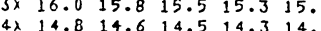
$\begin{array}{lllllll}5 \times & 14.0 & 13.8 & 13.7 & 13.5 & 13.4\end{array}$ $\begin{array}{llllll}6 x & 13.2 & 13.1 & 12.9 & 12.8 & 12.6 \\ 7 x & 12.5 & 12.3 & 12.2 & 12.0 & 11.8 \\ 9 x & 12.7 & 5.5 & 12.3 & 12 & 11.9\end{array}$ $8 \times 11.7 \quad 11.511 .3 \quad 11.110 .9$ $9 \times 10.7$
$10 \times 1.0$

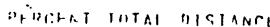

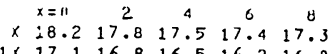
$2 \times 15.7 \quad 15.5 \quad 15.216 .216 .0$ $\begin{array}{lllllll}3 \times & 14.7 & 14.6 & 14.4 & 14.3 & 14.1\end{array}$ $\begin{array}{llllll}1 \times & 14.0 & 13.9 & 13.8 & 13.6 & 13.5 \\ 5 \times & 13.4 & 13.3 & 13.2 & 13.1 & 13.0\end{array}$ $\begin{array}{lllllll}6 \times & 12.8 & 12.7 & 12.5 & 12.3 & 12.1 \\ 7 \times & 11.8 & 11.7 & 11.5 & 11.3 & 11.1\end{array}$ $3 \times 10.510 .4 \quad 5.5 \quad 2.5 \quad 1.6$ $\begin{array}{ccc}97 \times & 1.2 \\ 1 \cap \times & 0.6\end{array}$

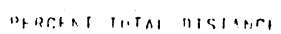

$\begin{array}{lccccc}x=11 & 2 & 4 & 6 & 8 \\ x & 17.3 & 16.8 & 16.8 & 16.5 & 16^{8}\end{array}$ $\begin{array}{lllllll}1 \times & 15.7 & 15.4 & 14.9 & 14.0 & 14.2 \\ 2 x & 13.7 & 13.3 & 12.9 & 12.6 & 12.3 \\ 3 x & 12.0 & 11.5 & 120.4 & 10.4 & 1.0\end{array}$ $\begin{array}{lllllll}4 \times & 0.7 & 0.5 & 0.4 & 0.4 & 0.3\end{array}$ $\begin{array}{cccccc}5 x & 0.3 & 1.3 & 0.3 & 0.3 & 0.3 \\ 30 \times & 0.2 & 0.3 & 0.2 & 0.3 & 0.2\end{array}$ $: \begin{array}{llllll}6 x & 0.2 & 0.2 & 0.2 & 0.2 & 0.2 \\ 7 x & 0.2 & 0.2 & 0.2 & 0.2 & 0.2\end{array}$ $\begin{array}{llllll}3 x & 0.2 & 0.2 & 0.2 & 0.2 & 0.2 \\ 0 x & 0.2 & 0.2 & 0.2 & 0.2 & 0.2\end{array}$ 


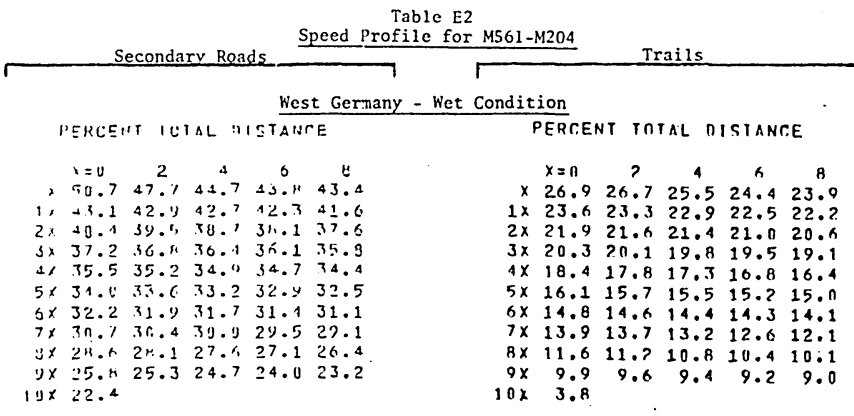

West Germany - Snow Condition

percenit total oistante

P'ERTEHT TITAL DISTANTE

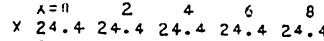

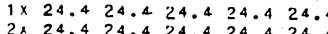
$3 \times 24.424 .424 .424 .024$.

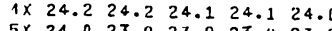

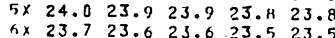
$\begin{array}{llllll}7 \times & 23.4 & 23.4 & 23.3 & 23.2 & 23.1 \\ 9 \times & 22.9 & 22.7 & 22.5 & 22.3 & 21.9 \\ 9 \times & 21.6 & 21.3 & 21.0 & 20.6 & 20.1\end{array}$ $\begin{aligned} 9 \times & 21.6 \\ 111 \times & 19.6\end{aligned}$

PERCENT TIIIAL DISTANCE

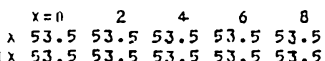

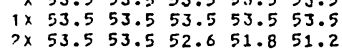

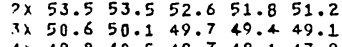

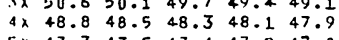
$\begin{array}{llllll}5 x & 47.7 & 47.5 & 47.4 & 47.2 & 47.0 \\ 9 x & 46.9 & 46.8 & 46.6 & 46.5 & 46.2 \\ 7 x & 46.0 & 45.8 & 45.5 & 45.2 & 44.0\end{array}$ $\begin{array}{llllll}7 \times & 46.0 & 45.8 & 45.5 & 45.2 & 44.8 \\ 8 \times & 44.4 & 44.0 & 43.5 & 42.9 & 42.2\end{array}$ $9 \times$
$1 n \times 31.1$
10

Dencent rutal uisiance

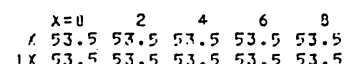

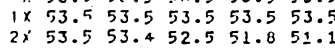

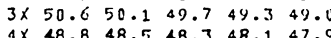
$\begin{array}{llllll}4 \times & 48.8 & 48.5 & 48.3 & 48.1 & 47.9 \\ 5 X & 47.7 & 47.5 & 47.3 & 47.2 & 47.0\end{array}$ $\begin{array}{lllllll}4 x & 46.9 & 46.8 & 46.6 & 46.4 & 46.2\end{array}$

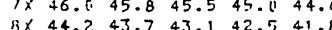
$\begin{array}{llllll}8 \times & 44.2 & 43.7 & 43.1 & 42.5 & 41.8 \\ 9 \times & 40.6 & 38.4 & 36.2 & 33.9 & 32.0\end{array}$

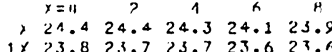
$\begin{array}{llllll}3 x & 23.3 & 23.2 & 23.2 & 23.2 & 23.2\end{array}$ $\begin{array}{llllll}4 x & 23.1 & 23.0 & 23.0 & 23.9 & 23 .\end{array}$

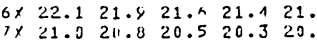
$\begin{array}{llllll}9 x & 19.9 & 1 \% .6 & 19.3 & 18.9 & 19.5 \\ 3 x & 18.2 & 17.8 & 17.4 & 16.0 & 16.4\end{array}$ $\begin{array}{rl}30 & 18.2 \\ 10 \times & 10.1\end{array}$$$
\text { Mid-E }
$$

Mid-E

$\begin{array}{rrrrr}x=0 & 2 & 4 & 6 & 8 \\ \times 23.1 & 21.1 & 21.1 & 20.4 & 80\end{array}$

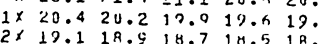
$3 \times 17.9 \quad 17.5 \quad 17.216 .016 .4$ $\begin{array}{llllll}1 \times & 16.1 & 15.7 & 15.1 & 15.1 & 14.8\end{array}$ $6 x \quad 13.7 \quad 14.4$ 14.2 14.013 .0 $\begin{array}{llllll}7 \times & 12.8 & 12.7 & 12.2 & 11.8 & 11.3\end{array}$ $\begin{array}{cccccc}8 \times & 10.9 & 10.5 & 10.2 & 9.9 & 9.7 \\ 9 \times & 9.4 & 9.2 & 9.0 & 8.8 & 8.6\end{array}$ $\begin{array}{rr}9 \times & 9.4 \\ 10 \times & 6.2\end{array}$

$\begin{array}{rrrrrr}r=0 & 2 & 4 & 6 & 8 \\ \times & 50.7 & 50.7 & 47.7 & 45.7 & 44.7 \\ 1 \times & 44.2 & 43.8 & 43.6 & 43.4 & 43.2\end{array}$ $2 x \quad 43.142 .842 .3311 .340 .5$ $4 \times \quad 37.5 \quad 37.2 \quad 36.9 \quad 36.7 \quad 36.5$ $5 \times \quad 36.3 \quad 36.1 \quad 35.935 .7 \quad 35.5$ $6 \times 35.3 \quad 35.134 .734 .4 \quad 34.0$ $\begin{array}{llllll}3 \times & 31.4 & 30.3 & 20.3 & 28.5 & 27.7 \\ 8 \times & 27.0 & 26.3 & 25.7 & 25.2 & 24.7\end{array}$

$$
\text { Mid-East }
$$

Protent TItAl DISIA.MeE $\begin{array}{lllccc}x=0 & 2 & 4 & 6 & 8 \\ x & 39.3 & 39.3 & 38.3 & 37.6 & 37.3 \\ x & 37.1 & 36.9 & 36.4 & 36.8 & 36.7\end{array}$

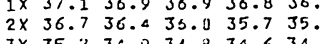
$3 x \quad 35.234 .934 .035 .634 .4$ $5 \times 33.6 \quad 33.7 \quad 33.6 \quad 33.4 \quad 33.0$ 6x $32.7 \quad 32.4 \quad 32.031 .7 \quad 31$. $\begin{array}{llllll}7 \times & 31.6 & 30.5 & 3.9 .1 & 29.5 & 28 . \\ 8 & 25.0 & 26.8 & 25.7 & 24.8 & 24.0\end{array}$ $18 \times 20.2$
PeDrent tutal nISIANCE

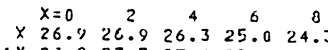
$\begin{array}{llllll}1 \times & 23.9 & 23.7 & 23.4 & 23.1 & 22.8\end{array}$

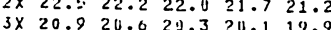

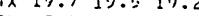
$\begin{array}{llllll}5 x & 17.8 & 17.3 & 16.3 & 16.0 & 16.3 \\ 6 y & 16.0 & 15.7 & 15.5 & 15.5 & 15.1\end{array}$ $7 \times 14.214 .114 .6 \quad 14.414 .3$ $\begin{array}{llllll}7 \times & 14.2 & 14.1 & 14.6 & 14.4 & 14.3 \\ 8 . x & 14.0 & 13.3 & 12.9 & 12.2 & 11 . .1\end{array}$

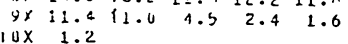
ast - Sand Condition

TERLENI IOTAL UISTARCE

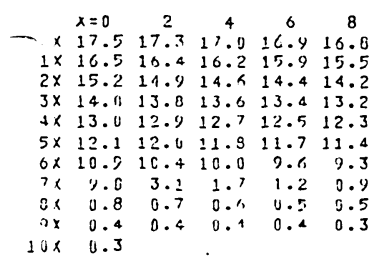
$\begin{array}{llllll}1 \times & 23.8 & 24.3 .7 & 23.7 & 23.6 & 23.4 \\ 2 \times & 23.5 & 23.4 & 23.4 & 23.3 & 23.3\end{array}$

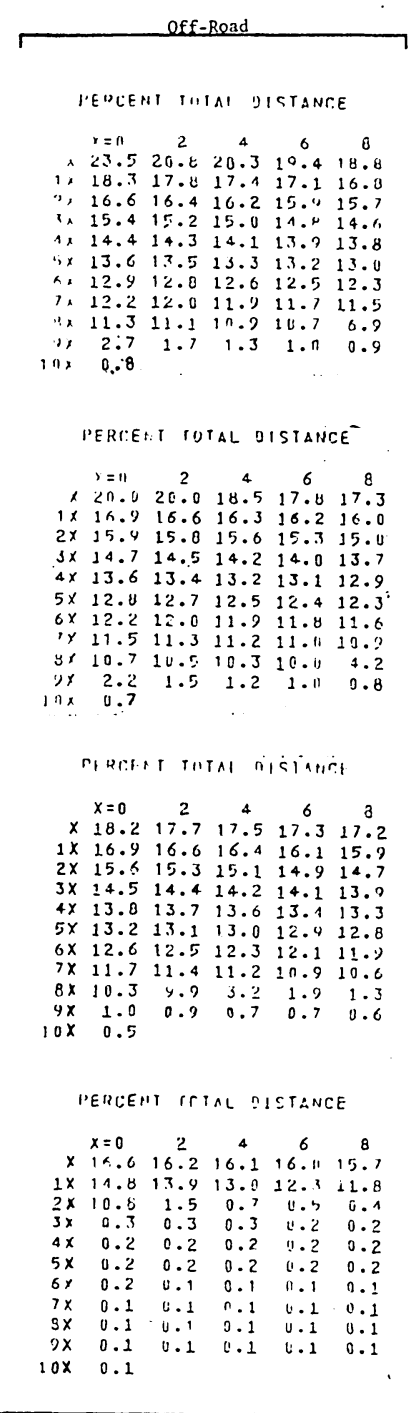




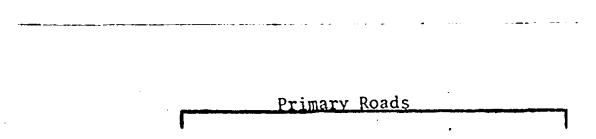

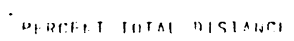

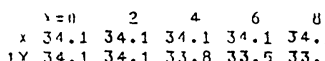
$\begin{array}{llllll}2 \times & 33.0 & 32.0 & 33.8 & 33.5 & 33.2 \\ 3 \times & 32.5 & 32.4 & 32.3 & 32.6 & 32.5 \\ 4 & 32.3 & 32.4 & 32.2\end{array}$ $\begin{array}{llllll}3 \times & 32.5 & 32.4 & 32.3 & 32.3 & 32.2 \\ 4 \times & 32.2 & 32.1 & 31.9 & 31.7 & 31.5\end{array}$ $4 \times 31.431 .2 \quad 30.930 .7 \quad 30.4$

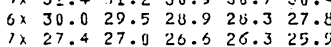

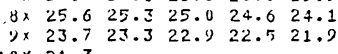
$11 \times 21.3$

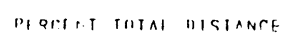
$\begin{array}{llllll}r=11 & 2 & 4 & 6 & 0 \\ x & 16.9 & 16.9 & 16.9 & 16.3 & 15.0\end{array}$ $2 x 15.1 \quad 15.415 .0015 .215$. $\begin{array}{llllll}3 \times & 14.9 & 14.9 & 14.9 & 14.9 & 14.9\end{array}$ 4x $14.8 \quad 14.814 .814 .8 \quad 14.8$ $5 x$ 14.8 14.014 .714 .714$. $7 x \quad 14.314 .2 \quad 14.114 .114 .0$ $\begin{array}{rrrrrrr}8 \times & 13.8 & 4.7 & 2.2 & 1.5 & 1.1 \\ 9 x & 0.9 & 0.8 & 0.7 & 0.6 & 0.6\end{array}$ $\begin{array}{rr}9 \times & 0.9 \\ .10 \times & 0.5\end{array}$

PFREFH TMTAL DISIANTE

$\begin{array}{llllll}x=n & 2 & 4 & 6 & & \\ x & 34.1 & 34.1 & 34.1 & 34.1 & 34.1\end{array}$ $\begin{array}{llllll}1 \times & 34.1 & 34.1 & 34.1 & 34.1 & 34.1 \\ 2 x & 34.1 & 34.1 & 33.9 & 33.5 & 33.1\end{array}$ $\begin{array}{llllll}3 x & 32.7 & 32.4 & 32.1 & 31.9 & 31.7 \\ 4 \times & 31.5 & 31.3 & 31.2 & 31.0 & 30.9\end{array}$ $\begin{array}{llllll}5 \times 30.8 & 30.7 & 30.6 & 30.4 & 30.3\end{array}$ $\begin{array}{llllll}6 x & 30.2 & 30.1 & 30.1 & 30.0 & 29.9\end{array}$ $\begin{array}{rrrrrr}7 \times & 29.8 & 29.7 & 29.5 & 29.4 & 29.3 \\ 9 \times & 29.2 & 29.1 & 28.9 & 28.7 & 28.5 \\ 9 \times & 28.2 & 27.6 & 26.5 & 25.4 & 24.5 \\ 10 \times & 23.7 & & & & \end{array}$ $\begin{array}{ll}10 \times & 23.7\end{array}$

PFRSFNT TITAI MISTANCF $\begin{array}{cccccc}x=0 & 2 & 4 & 6 & 8 \\ x & 34.1 & 34.1 & 34.1 & 34.1 & 34.1\end{array}$

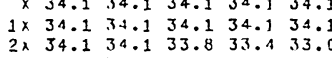
$3 \times 32.6 \quad 32.3 \quad 32.0 \quad 31.831$. $\begin{array}{lllllll}4 \times & 31.4 & 31.3 & 31.1 & 31.0 & 30.0 \\ 5 \times & 30.7 & 30.6 & 30.5 & 30.4 & 30.0\end{array}$ $\begin{array}{llllll}6 \times & 30.2 & 30.1 & 30.0 & 29.9 & 20.9\end{array}$ $7 \times 29.82 \% .6 \quad 29.5 \quad 29.4 \quad 29.4$ $\begin{array}{llllll}8 \times & 29.1 & 29.0 & 28.8 & 28.6 & 28.4 \\ 1 \times & 28.0 & 27.4 & 26.2 & 25.1 & 24.2\end{array}$

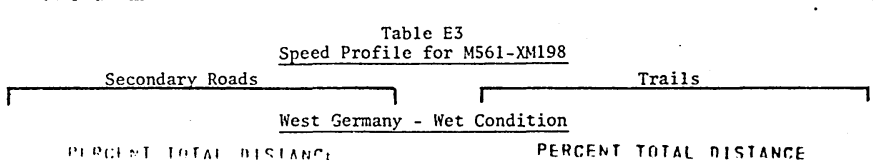

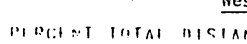
Percent total nistanfe.

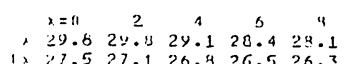
$2,26.126 .1125 .925 .425 .7$

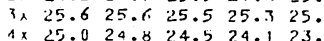
Sर $23.4 \quad 33.122 .0322 .5522 .2$ $\begin{array}{lll} & \end{array}$ $8 \times<0.01 \% .719 .514 .219 .0$ $11 \times 17.1$

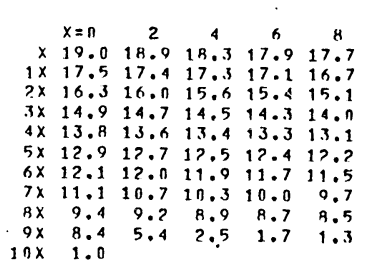

\section{West Germany - Snow Condition}

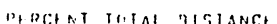 \\ PERCENT TITAL DISIANGE}

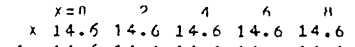

$\begin{array}{llllll}1 \times & 14.6 & 14.6 & 14.6 & 14.6 & 14.6 \\ 2 x & 14.0 & 14.6 & 14.5 & 14.6 & 14.6 \\ 3 & 14.4 & 14.3\end{array}$

$\begin{array}{lllllll}3 x & 14.2 & 14.6 & 14.0 & 13.9 & 13.8 \\ 1 \times & 13.8 & 13.7 & 13.6 & 13.5 & 13.5\end{array}$

$5 \times 13.4 \quad 13.4 \quad 13.3 \quad 13.2 \quad 13.0$

$\begin{array}{lllllll}5 \times & 12.9 & 12.7 & 12.6 & 12.2 & 13.0 \\ 7 \times & 2.6 & 1.5 & 12.6 & 12.3 & 10.1\end{array}$

$\begin{array}{llllll}8 \times & 0.6 & 0.6 & 0.5 & 0.5 & n .4 \\ 9 x & 0.4 & 0.4 & 0.4 & 0.3 & n .5\end{array}$

$\begin{array}{rr}9 \times & 0.4 \\ 1 n \times & 0.3\end{array}$

\section{Mid-East - Wet Condition}

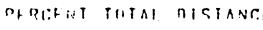

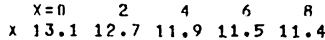

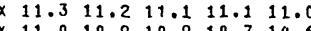
$\begin{array}{cccccc}4 \times & 9.3 & 8.8 & 8.5 & 8.2 & 7.9\end{array}$ $\begin{array}{llllll}4 x & 1 & 0.9 & 0.3 & 4.5 & 1.9\end{array}$ $\begin{array}{lllllll}7 x & 0.5 & 0.4 & 0.4 & 0.4 & 0.5\end{array}$ $\begin{array}{lllll}0.3 & 0.3 & n .3 & 0.3 & 0.3\end{array}$ $\begin{array}{lll}2 & 0.3 \\ x & 0.2 & 0\end{array}$

$x=11 \quad 2 \quad 4 \quad 6 \quad 0$

$\begin{array}{rrrrrr}\times & 29.8 & 29.8 & 29.8 & 29.8 & 29.8 \\ 1 \times & 29.0 & 28.2 & 27.7 & 27.3 & 27.0\end{array}$

$\begin{array}{llllll}2 x & 26.8 & 26.6 & 26.4 & 26.3 & 26.1 \\ 3 \times & 26.011 & 26.0 & 25.9 & 25.8 & 25.7\end{array}$

$4 \times \quad 25.7 \quad 25.6 \quad 25.6 \quad 25.6 \quad 25.5$

$\begin{array}{llllll}5 \times & 25.5 & 25.5 & 25.4 & 25.4 & 25.4 \\ 6 \times & 25.3 & 25.3 & 25.2 & 25.1 & 25.0\end{array}$

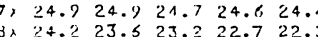

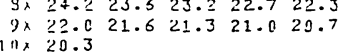

\section{Mid-East - Sand Condition}

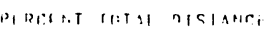

$\begin{array}{cccccc} & x=11 & 2 & 4 & 6 & n \\ \times & 29.8 & 29.8 & 29.8 & 29.8 & 29.5 \\ 1 \times & 28.4 & 27.8 & 27.5 & 27.0 & 26.7\end{array}$

$3 \times 26.526 .3 \quad 26.226 .025 .9$

$\times 25.925 .825 .725 .725 .6$

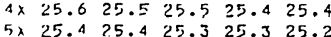

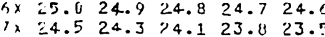

$\begin{array}{llllll}1 x & 24.5 & 24.3 & 14.1 & 23.8 & 23.5 \\ n_{x} & 23.1 & 22.3 & 21.7 & 21.1 & 23.5\end{array}$

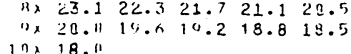

PHECFET TITAL जISTAMCF

$\begin{array}{rrrrrr}x=11 & 2 & 4 & 6 & y \\ \times & 19.0 & 10.4 & 18.7 & 18.6 & 18.5 \\ 1 \times & 18.2 & 18.0 & 17.8 & 17.6 & 17.5\end{array}$ $\begin{array}{llllll}1 \times & 18.2 & 18.0 & 17.8 & 17.6 & 17.5 \\ 2 \times & 17.2 & 17.0 & 16.8 & 16.6 & 15.5\end{array}$ $\begin{array}{llllll}4 \times & 15.9 & 14.8 & 11.6 & 14.3 & 14 . \\ 5 \times & 13.4 & 13.8 & 13.6 & 13.5 & 13 .\end{array}$ $\begin{array}{llllll}5 x & 13.0 & 13.8 & 13.6 & 13.5 & 13.4 \\ 7 x & 12.4 & 12.0 & 12.8 & 12.7 & 12.5\end{array}$

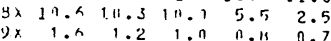

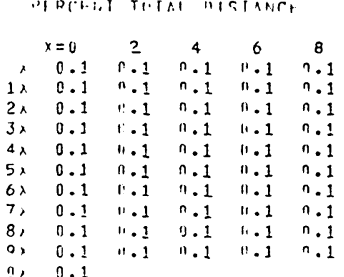

$r$

Ph RIFHT TIITAL DISIANCI

$x=1124 \quad 6 \quad 6$

1) $15.5 \quad 14.7 \quad 14.213 .7 \quad 13.3$ 2x $13.012 .612 .4 \quad 12.1 \quad 11.9$ $1 x$ 10.6 111.5110 .211 .010 $\begin{array}{llllll}3 x & 9.8 & 9.6 & 9.5 & 9.4 & 3 . ? \\ h x & 9.1 & 8.9 & 8.8 & 8.6 & 8.5 \\ 7 x & 1.3 & 0.1 & 4.0 & 2.6 & 1.3\end{array}$ $\begin{array}{llllll}7 x & 2.3 & 8.1 & 4.0 & 2.0 & 1.5\end{array}$ $\begin{array}{llllll}4 x & 1.0 & 0.8 & 0.7 & 0.6 & 0.6 \\ 9 x & 0.5 & 0.5 & 0.4 & 0.4 & 0.4 \\ 10 x & 0.0 & & & & \end{array}$

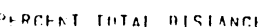

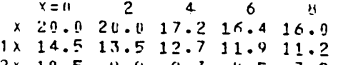
$2 \times \quad 10.5 \quad 4.9 \quad 9.3 \quad 4.5 \quad 7.2$ $\begin{array}{llllll}3 \times & 4.7 & 3.4 & 2.4 & 1.1 & 0.7 \\ 1 \times & 0.5 & 0.4 & 0.4 & 1.3 & 0.3\end{array}$ $\begin{array}{llllll}5 x & 0.3 & 11.3 & 0.3 & 11.2 & 0.2 \\ x \times & 0.2 & 0.2 & 0.2 & 11.2 & 0.2\end{array}$ $\begin{array}{llllll}7 x & 0.2 & 1.2 & 0.2 & 10.2 & 0.2 \\ 3 x & 0.2 & 0.2 & 0.2 & 0.2 & 0.2 \\ 3 x & 0.2 & 0.2 & 0.2 & 1.2 & 0.2\end{array}$ $\begin{array}{llllll}3 x & 0.2 & 0.2 & 0.2 & 0.2 & 0.2 \\ 9 x & 0.2 & 0.2 & 0.2 & 0.2 & 0.2 \\ 1 . x & 0.1 & & & & \end{array}$ $\begin{array}{rr}9 \lambda & 0.2 \\ \ln x & 0.1\end{array}$

PI REFET THTA MISTANG

$\begin{array}{cccccc} & x=11 & 2 & 1 & 0 & 8 \\ \times & 15.7 & 15.3 & 15.2 & 14.2 & 3.3 .5\end{array}$ $\begin{array}{llllll}1 \times & 13.1 & 12.8 & 12.2 & 11.2 & 12.5 \\ 2 x & 12.0 & 11.8 & 11.5 & 12.5 & 12.2 \\ 3 x & 11.5 & 11.3\end{array}$

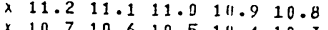
$\begin{array}{lllllll}5 \times & 10.2 & 10.1 & 10.0 & 9.9 & 9.8\end{array}$ $\begin{array}{llllll}7 x & 9.7 & 9.6 & 9.5 & 9.4 & 3.2 \\ 7 x & 9.0 & 8.8 & 4.0 & 2.011 & 1.3\end{array}$ $\begin{array}{llllll}3 x & 1.0 & 0.8 & 0.7 & 11.6 & 0.6 \\ 9 x & 0.5 & 0.5 & 0.4 & 0.4 & 0.4 \\ 9 x & 0 & & & & \end{array}$ 1.9.

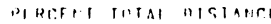

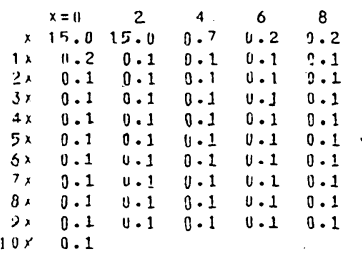




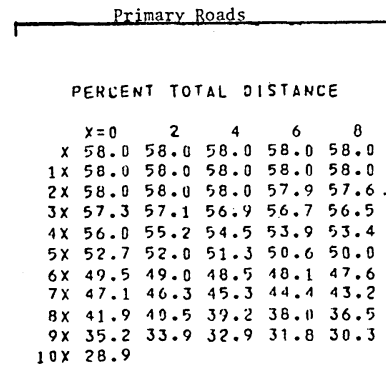

PERGEMT TOTAL PISTANGE

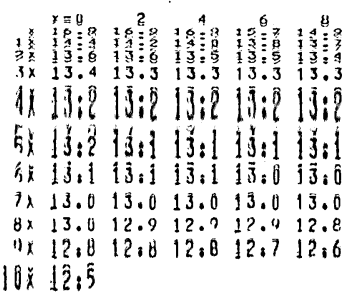

perrent fotal doftance

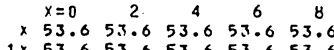
$3 \times 53.653 .6 \quad 53.0 \quad 53.653 .6$ $3 \times 52.752 .652 .552 .452 .3$ $4 \times 52.251 .951 .551 .150 .7$ $\begin{array}{lllll}5 \times 50.4 & 50.1 & 19.8 & 49.5 & 49.3\end{array}$ $\begin{array}{llllll}7 \times & 48.0 & 47.8 & 47.5 & 47.1 & 46.0 \\ 8 \times & 46.1 & 45.5 & 45.11 & 44.4 & 43 \\ 9 \times & 42.3 & 40.5 & 37.0 & 35.4 & 33\end{array}$ $10 \times 31.0$

MERTENT IQTAL DISTANGE

$\begin{array}{lcccc}x=0 & 2 & 4 & 4 & 0 \\ 53.6 & 53^{\circ} .6 & 53.6 & 53.6 & 53.6\end{array}$ $1153,6 \quad 53: 6 \quad 53: 6 \quad 53: 6 \quad 53: 6$

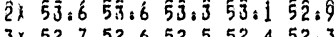
$3 \times 52.752 .6 \quad 52.5 \quad 52.452 .3$ $4 \times 52.251 .851 .451 .050 .7$

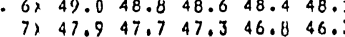

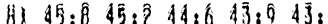

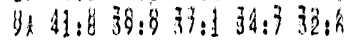

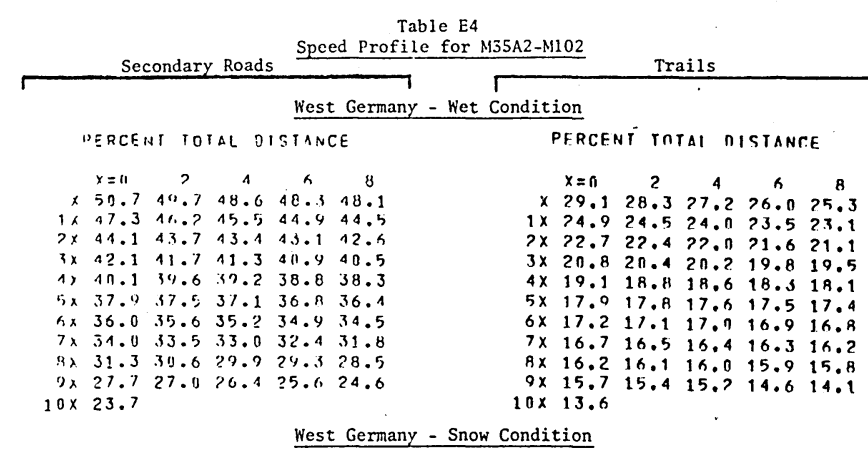

PERCENT TOTAL DISTANCE

PFRCENT IOTAI MISTANGE

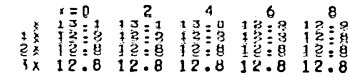
1) $12 i 812 i$ 15il $12 ; 12 i$

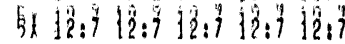

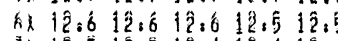
कर 12.512 .512 .412 .412$. द) 12.412 .312 .312 .212 .3 iii) $1: 5$

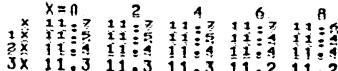
4h lli2 llil llil llil lial

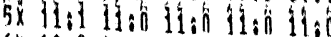

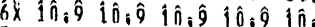

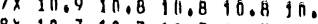
ax $10,6 \quad 10,510,310,0$ a. lñx $3 \hat{\imath}$

\section{Mid-East - Met Conditio}

PERIENT FUTAL DISTARCE

l'ERGENA fOTAL DISTAHEE

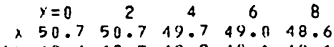
$2 \times 48.0 \quad 47.6 \quad 47.0 \quad 40.5 \quad 16.1$ $3 \times 45.7 \quad 45.4 \quad 45.2 \quad 45.1144 .8$ $\begin{array}{lllllll}4 x & 44.6 & 44.4 & 44.2 & 44.0 & 43 .\end{array}$ $5 \times 43.3 \quad 43.0 \quad 42.7 \quad 42.4 \quad 42.0$

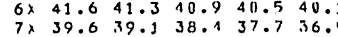

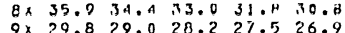
$10 \times 26.0$

\section{Mid-East - Sartd Condition} PEHHEMT rorAL GISTAMEE I'EREEN IOTAL DISIANGE

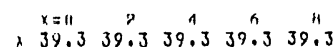

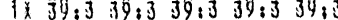

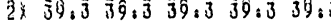
$3 \times 39.3 \quad 39.3 \quad 39.3 \quad 39.3 \quad 39.3$ $4 \times 39.3 \quad 39.239 .038 .838$.

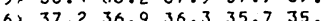
7. $34.6 \quad 34.0 \quad 33.3 \quad 32.531 .6$ B) $30: 629: 427: 8 \quad 20: 6 \quad 23: 6$

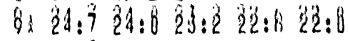
1.1. 21.2 $\begin{array}{cccccc} & x=0 & 2 & 4 & 6 & 8 \\ \times & 28.5 & 28.4 & 27.8 & 60.5 & 25.7 \\ 1 \times & 25.2 & 24.0 & 27.8\end{array}$ $2423.923 .7 \quad 23.422 .424 .2$ $3 \times 21.821 .121 .120 .420 .5$

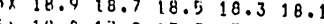

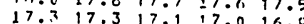

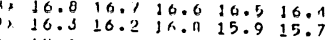

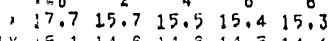

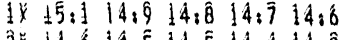
$2 \times 14.6 \quad 14.55 \quad 14.5 \quad 14.4 \quad 14.2$ $\begin{array}{llllll}\therefore & 14.1 & 14.0 & 13.9 & 13.4 & 12.1\end{array}$ $\begin{array}{rrrrrr}4 \times & 11.0 & 10.2 & 9.6 & 9.1 & 8.7 \\ -\rightarrow 32 & 8.3 & 2.8 & 1.4 & 1.0 & 0.7\end{array}$ $\begin{array}{llllll}0 . & 0.6 & 0.5 & 0.5 & 0.4 & 0.4\end{array}$ $\begin{array}{llllll}11 & 0.4 & 0.3 & 0.3 & 0.3 & 0.3 \\ 3 & 1.3 & 0.3 & 0.3 & 0.3 & 0.3\end{array}$ (3) $11: 2$ b:2 $8: 2$ 8:2 $0: 2$ $10,11.2$ 4) $20.320 .619 .1 \quad 19.410 .1$

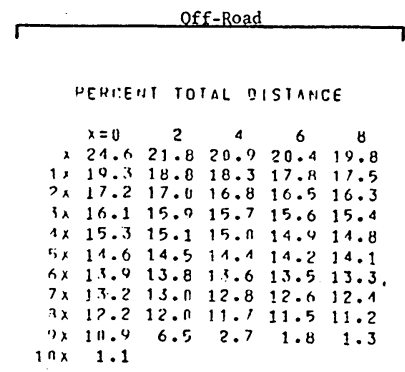

PFRCENT TUTAL DISIANCE

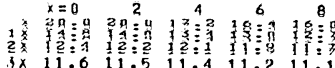
1) $11 i 0111010 i d 10 i$ 10i6

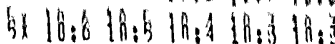

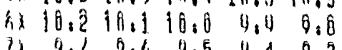
$\begin{array}{llllll}31 & 9.1 & 5.6 & 9.5 & 19.4 & 0.2 \\ 4 \times & 9.1 & 8.9 & 7.4 & 2.7 & 1.2\end{array}$ $\begin{array}{llllll}4 x & 9.1 & 8.0 & 7.0 & 2.7 & 1.7\end{array}$ in $0: 6$

bergent total distance

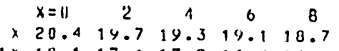

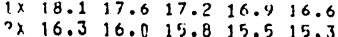
id $15.1 \quad 14.9 \quad 14.7 \quad 14.5 \quad 14.4$ $\begin{array}{llllll}\text { is } 11.3 & 14.1 & 14.0 & 13.9 & 13.7\end{array}$ $\begin{array}{lllllll}5 x & 13.6 & 13.4 & 13.2 & 13.1 & 12.9\end{array}$

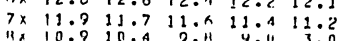

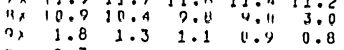
11100.7

MEPGENF IIITAL GISTANGE

$x=110^{2} \quad 5^{4}, 15^{\circ}, 15^{4}$ 1) $14,714.313,11301011.7$ $\hat{2} \hat{k} \quad 1.5 \quad 11 . \overline{7} \quad 0.5 \quad 11.4 \quad 0.3$ $\begin{array}{llllll}3 x & n .3 & 11.2 & n .2 & 11.2 & 0.2\end{array}$ $\begin{array}{llllll}4 \times & n .2 & 11.2 & n .2 & 1.2 & 0.2 \\ 5 x & 11.2 & 11.2 & n .2 & 11.2 & 0.2\end{array}$ $\begin{array}{llllll}6 x & n .1 & 1.2 & 0.1 & 1.1 & n\end{array}$

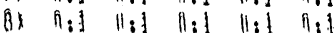

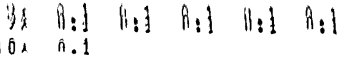




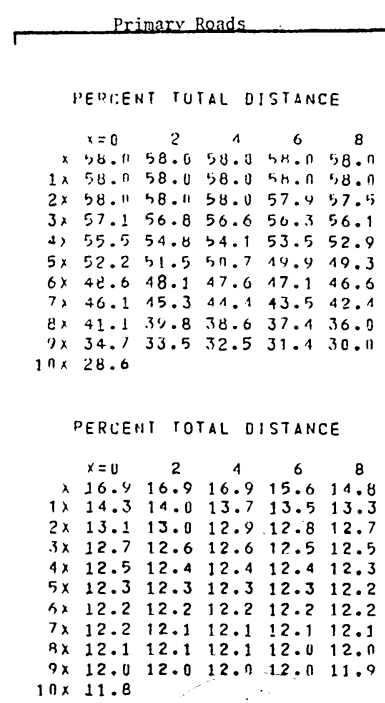

PEDCENT total Distance

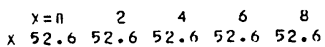

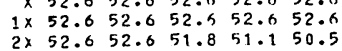

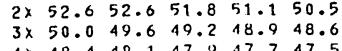

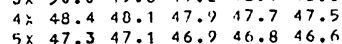

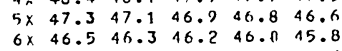

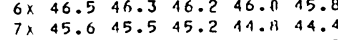

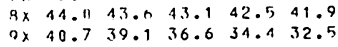
$\begin{array}{rr}2 \times & 40.7 \\ 10 \times & 30.8\end{array}$

\section{Dercent total Distance}

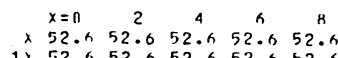

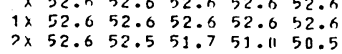

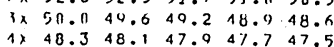
$5 x \quad 47.3 \quad 47.146 .926 .746 .5$

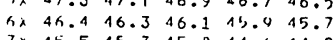
$7 \times 45.545 .3 \quad 15.0444 .644 .2$ $\begin{array}{llllll}4 \times & 43.7 & 13.2 & 42.7 & 12.1 & 41.1 \\ 2 x & 40.2 & 38.5 & 35.0 & 33.7 & 31.7 \\ n \times & 30.1 & & & & \end{array}$

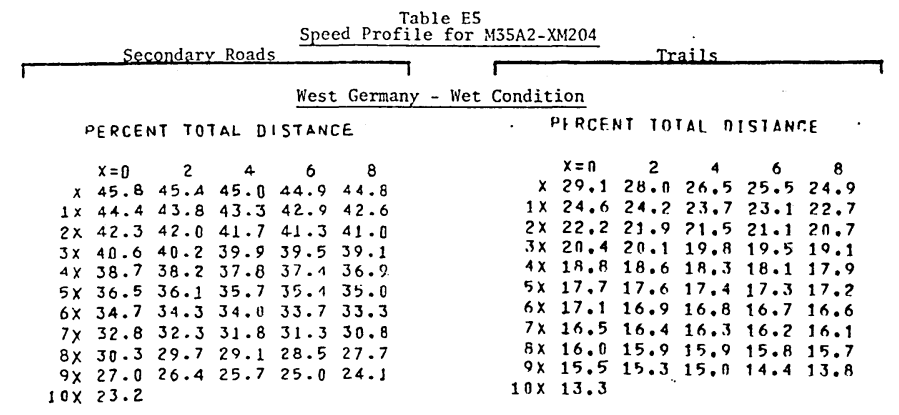

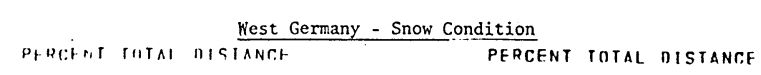

$\begin{array}{rrrrrr}x=11 & 2 & 4 & 6 & 8 \\ x & 13.1 & 12.9 & 12.4 & 12.2 & 12.1 \\ 1 x & 12.1 & 12.9 & 12.0 & 12.0 & 12.11\end{array}$

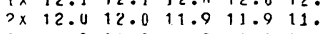

$\begin{array}{llllll}3 \times & 11.9 & 11.9 & 11.9 & 11.9 & 11.9 \\ 1 \times & 11.8 & 11.8 & 11.8 & 11.8 & 11.8\end{array}$

$\begin{array}{llllll}4 x & 11.8 & 11.8 & 11.8 & 11.8 & 11.8 \\ 5 x & 11.8 & 11.8 & 11.8 & 11.8 & 11.8\end{array}$

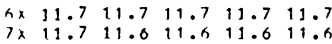

$\begin{array}{rrrrrrr}8 \times & 11.6 & 11.5 & 11.5 & 11.4 & 11.4 \\ 9 x & 11.4 & 11.3 & 11.5 & 11.0 & 5.4 \\ 10 \times & 2.6 & & \end{array}$

Mid-East - Wet Condition

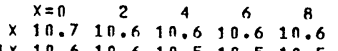

$3 x \quad 10.3 \quad 10.3 \quad 10.3 \quad 10.3 \quad 10.3$

$\begin{array}{llllll}4 \times & 10.3 & 10.2 & 10.2 & 10.2 & 10.2\end{array}$

$6 x 10.210 .2$ 1n.2 10.2 1n.2

$\begin{array}{lllllll}7 \times & 10.1 & 10.1 & 10.1 & 10.1 & 10.1 \\ 8 \times & 10.0 & 10.0 & 10.0 & 10.0 & 0.0\end{array}$

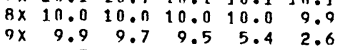

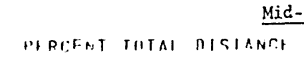

percent rotal disiance

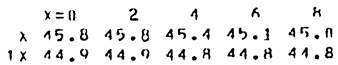

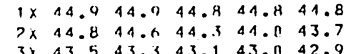

$\begin{array}{ll}1 \times 42.7 \quad 42.6 & 42.5 \quad 12.4 \quad 42 .\end{array}$

$\begin{array}{cccccc}5 x & 11.9 & 41.6 & 41.4 & 41.1 & 40.7\end{array}$

$8 \times 35.133 .1 \quad 32.436 .836$ 30.

$\begin{aligned} 9 \times & 29.4 \\ 111 \times & 25.7\end{aligned}$

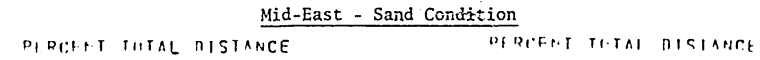

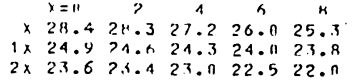

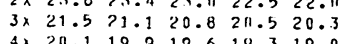
4x $20.110 .9 \quad 10.5 \quad 19.319 .0$ bx $18.7 \quad 11 \% .4 \quad 18.3 \quad 16.218$. $\begin{array}{llllll}6 \times & 17.8 & 17.7 & 77.6 & 17.5 & 17.4 \\ 7 \times & 17.2 & 17.1 & 17.0 & 10.9 & 15.8 \\ 7 \times & 16.7 & 10.0 & 16.5 & 10.4 & 16.3\end{array}$

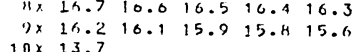

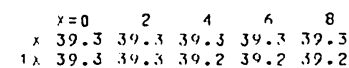

$\begin{array}{llllll}1 \times & 39.3 & 39.4 & 39.2 & 39.2 & 39.2 \\ 2 \times & 39.2 & 30.2 & 39.2 & 34.7 & 39.2 \\ 3 x & 39.2 & 30.2 & 39.2 & 34.2 & 30.2\end{array}$

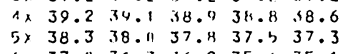

$\begin{array}{llllll}6 \times & 37.0 & 36.7 & 36.2 & 35.6 & 35 . \\ 7 \times & 34.5 & 33.9 & 37.2 & 32.4 & 35.5\end{array}$

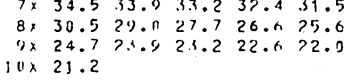

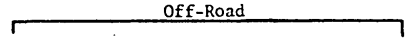

pIRI:HN TITAL DISTANCH.

$\begin{array}{cccccc} & x=0 & 2 & 4 & 6 & B \\ x & 24.1 & 21.2 & 20.6 & 20.0 & 19.4 \\ x & 18.9 & 18.4 & 17.9 & 17.5 & 17.2\end{array}$

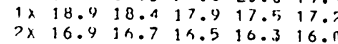

$\begin{array}{lllll}3 x & 15.8 & 15.0 & 15.5 \\ 4 \times & 15.0 & 14.0 & 14.5\end{array}$

$\begin{array}{lllllll}5 \times & 14.4 & 14.7 & 14.1 & 14.01 & 13.4\end{array}$

$\begin{array}{llllll}5 x & 3.7 & 13.5 & 13.4 & 13.2 & 13.3 \\ 7 \times & 12.4 & 12.8 & 12.6 & 12.4 & 12 .\end{array}$

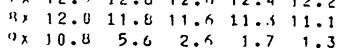

$\begin{array}{ccc}7 \times & 10.8 \\ 10 \times \quad 1.0\end{array}$

BIPCHNT TIITAL DISTANCH

$\begin{array}{rlrrrr}x & x=0 & 2 & 4 & 6 & 8 \\ x \quad 20.0 & 211.0 & 17.2 & 10.4 & 16.0\end{array}$

$2 \times 11.8 \quad 11.6 \quad 11.4 \quad 11.3 \quad 11.1$

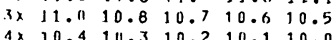

$5 \times \quad 9.9$ c..

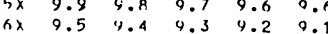

$\begin{array}{llllll}7 \times & 9.1 & 4.11 & 8.8 & 8.7 & 8.5 \\ 8 \times & 8.4 & 7.3 & 2.7 & 1.7 & 1.2\end{array}$

$\begin{array}{llllll}A x & 8.4 & 7.3 & 2.7 & 1.7 & 1.4 \\ 9 x & 1.0 & 1.0 & 0.7 & 0.6 & n .6 \\ 10 x & 0.5 & & & & \end{array}$

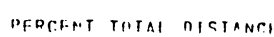

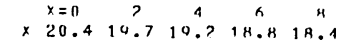

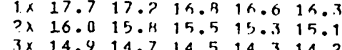

$\begin{array}{llllll}4 \times & 14.0 & 13.0 & 13.8 & 13.7 & 13.5\end{array}$

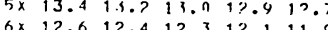

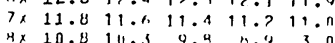

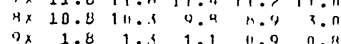

$\ln x \quad 0.7$

DI RIENT TITAL DISIARECE

$\begin{array}{llll}x=0 & 2 & 1 & 6\end{array}$ $\begin{array}{rrrrrrr}1 \times & 14.3 & 1 \% .8 & 10.4 & 1.3 & 0.6 \\ 2 x & 0.4 & 0.3 & 0.3 & 0.2 & 0.2 \\ 4 x & 0.2 & 0.2 & 0.2 & 0.2 & 0.2\end{array}$ $\begin{array}{llllll}2 x & 0.4 & 0.3 & 0.3 & 0.2 & 0.2 \\ 3 x & 0.2 & 0.2 & 0.2 & 0.2 & 0.2 \\ 4 x & 0.2 & 0.2 & 0.2 & 0.1 & 0.2\end{array}$ $\begin{array}{llllll}4 x & 0.2 & 0.2 & 0.2 & 0.1 & 0.1 \\ 5 x & 0.1 & 6.1 & 0.1 & 0.1 & 0.1 \\ 4 x & 0.1 & 0.1 & 0.1 & 0.1 & 0.1\end{array}$ $\begin{array}{llllll}3 x & 0.1 & 0.1 & 0.1 & 0.1 & 0.1 \\ 7 x & 0.1 & 0.1 & 0.1 & 0.1 & 0.1 \\ 3 x & 0.1 & 0.1 & 0.1 & 1.1 & 0.1\end{array}$ $\begin{array}{llllll}3 x & 0.1 & 0.1 & 0.1 & 11 & 0.1 \\ 2 x & 0.1 & 0.1 & 0.1 & 11 & 0.1 \\ 10 x & 0.1 & 0.1 & 0.1 & 11.1 & 0.1\end{array}$ 


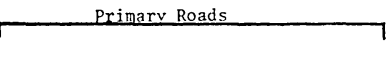

percent total distance

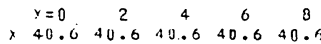
1) $40.0 \quad 40.6 \quad 40.6 \quad 40.6 \quad 40.6$ $\begin{array}{llllll}2 \times & 40.6 & 10.6 & 40.6 & 40.6 & 40.5 \\ 3 \times & 40.4 & 40.4 & 40.3 & 30.2 & 0.5\end{array}$ $4 \times \quad 40.0 \quad 39.8 \quad 39.5 \quad 34.1 \quad 39.7$ $5 \times 38.4 \quad 38.0 \quad 37.5 \quad 37.1036 .5$ $\begin{array}{llllll}6 \times & 36.0 & 35.6 & 35.2 & 34.8 & 31.5\end{array}$ $8 \times 32.1131 .4 \quad 30.7 \quad 30.139 .6$ $8 \times$
$9 \times 28.5$
$10 \times 24.7$

\section{Derient tutal distance}

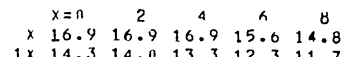
$? \times 11.2 \quad 10.8 \quad 10.6 \quad 10.3 \quad 10$. $\begin{array}{rrrrrr}3 \times & 10.0 & 9.8 & 9.7 & 9.6 & 9.5 \\ 4 \times & 9.4 & 9.4 & 9.3 & 9.2 & 9.2 \\ 5 \times & 9.1 & 9.1 & 9.0 & 9.0 & 9.2\end{array}$ $\begin{array}{llllll}4 x & .4 & 9.4 & 9.3 & 9.6 & 9.5 \\ 5 x & 9.1 & 9.1 & 9.0 & 9.0 & 9.0 \\ 7 x & 8.9 & 8.9 & 8.9 & 8.9 & 8.0\end{array}$ $\begin{array}{llllll}7 \times & 8.7 & 8.7 & 4.6 & 8.6 & 8.0 \\ 8 \times & 2.5 & 1.0 & 1.2 & 0.9 & 0.8\end{array}$ $\begin{array}{llllll}8 x & 2.5 & 1.6 & 1.2 & 0.9 & 0.8 \\ 9 x & 0.7 & 0.6 & 0.5 & 0.5 & 0.5 \\ 10 x & 0.4 & & & & \end{array}$

\section{PERCENT TOTAL DISTANCE}

$\begin{array}{rrrrrr} & x=0 \\ \times & 39.1 & 39.1 & 49.1 & 6 & 8 \\ 1 \times & 39.1 & 30.1\end{array}$ $2 \times 39.139 .139 .139 .139 .1$ $\begin{array}{cccccc}3 \times & 38.7 & 38.6 & 38.6 & 38.5 & 38.5 \\ 4 \times & 38.5 & 38.4 & 38.2 & 38.1 & 38.0\end{array}$

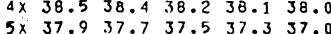
$\begin{array}{llllll}6 \times & 36.8 & 36.6 & 36.5 & 36.3 & 36.1 \\ 7 \times & 35.9 & 35.7 & 35.5 & 35.2 & 35.1\end{array}$ $\begin{array}{lllllll}7 \times & 35.9 & 35.7 & 35.5 & 35.2 & 35.0 \\ 8 \times & 34.8 & 34.5 & 34.3 & 33.9 & 33.6\end{array}$ $\begin{array}{lllllll}8 \times & 34.8 & 34.5 & 34.3 & 33.9 & 33.6 \\ 9 \times & 33.0 & 32.0 & 30.4 & 24.11 & 27.7\end{array}$ $\begin{aligned} 9 \times & 33.0 \\ 10 \times & 26.6\end{aligned}$

PHREEN TIITAL DICIAMTE

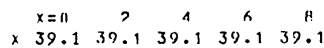

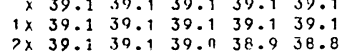
$3 \times 38.7 \quad 36.6 \quad 38.6 \quad 38.5 \quad 38.5$ $4 \times 33.538 .438 .238 .138$. 6) $36.736 .0 \quad 36.436 .236$.

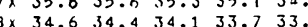

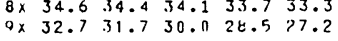
$10 \times 26.1$
Table E6
Speed Profile for M35A2-XM198

\begin{tabular}{|c|c|}
\hline Secondary Road & Trails \\
\hline \multicolumn{2}{|c|}{ West Germany - Wet Condition } \\
\hline DFRIEET TOIAI DISIANCE & PERCENT TOTAL DISTANTEE \\
\hline 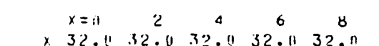 & $?=2^{4}$ \\
\hline 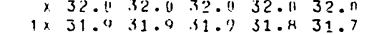 & $\begin{array}{ll}6 \\
.6 & 21.1 \\
2 & 18\end{array}$ \\
\hline $\begin{array}{llllllll}3 \times & 31.5 & 31.3 & 51.1 & 310 & 30.7\end{array}$ & 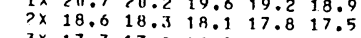 \\
\hline $3 \times 30.5 \quad 30.129 .8 \quad 29.529 .2$ & 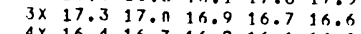 \\
\hline 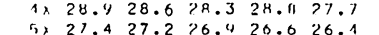 & $\begin{array}{lllllll}4 \times & 16.4 & 16.3 & 16.2 & 16.1 & 16.0\end{array}$ \\
\hline 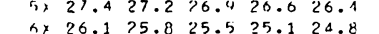 & $\begin{array}{llllll}5 x & 15.9 .15 .8 & 15.6 & 15.5 & 15.4\end{array}$ \\
\hline 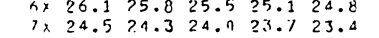 & 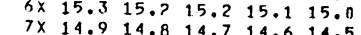 \\
\hline 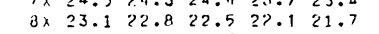 & 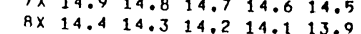 \\
\hline $2 \times 21.4 \quad 21.1 \quad 20.7 \quad 211.210 .7$ & $9 \times 13.713 .312 .9 \quad 8.4 \quad 3.1$ \\
\hline $1.0 \times 19.1$ & $10 \times 1.9$ \\
\hline
\end{tabular}

West Germany - Snow Condition

DEREFT TIITAK DISIANRE

PERCENT TOTAL MISTANCF

$\begin{array}{cccccc}x=11 & 2 & 1 & 0 & 0 \\ x & 13.1 & 12.5 & 11.0 & 10.0 & 10.1 \\ 10 & 9.4 & 9.6 & 0.4 & 4.2 & 0.1\end{array}$

$\begin{array}{rrrrrr}1 \times & 9.4 & 9.6 & 0.4 & 4.2 & 9.1 \\ 2 x & 9.11 & 8.9 & 8.8 & 8.8 & 8.7\end{array}$

$\begin{array}{llllll}3 \times & 8.7 & 8.7 & 8.6 & 8.0 & 8 . \\ 4 x & 0.5 & 0.4 & 0.4 & 0.3 & 8.5\end{array}$

$\begin{array}{llllll}4 x & 8.5 & 8.4 & 8.4 & 8.3 & 8.3 \\ 5 x & 8.2 & 8.2 & 8.1 & 8.1 & 8.0 \\ 7 x & 8.0 & 2.8 & 1.5 & 1.1 & 0.8\end{array}$

$\begin{array}{llllll}5 x & 8.0 & 2.8 & 1.5 & 1.1 & 0.8 \\ 7 \times & 1.7 & 0.6 & 0.5 & 0.5 & 0.4 \\ 8 x & 0.4 & 0.4 & 0.3 & 0.3 & 0.3\end{array}$

$\begin{array}{llllll}8 x & 0.4 & 0.4 & 0.3 & 0.3 & 0.3 \\ 2 x & 0.3 & 0.3 & 0.3 & 0.3 & 0.3\end{array}$

$\ln x=2$

Mid-East - Wet Condition

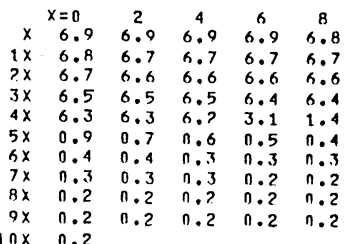

"EPCENT TOTAL DISTANCE

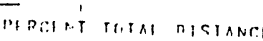

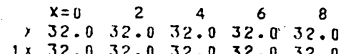
$2 \times 32.0 .31 .9 \quad 31.9831 .931 .9$

$\begin{array}{llllll}3 \times & 31.9 & 31.9 & 31.9 & 31.8 & 31.8 \\ 4 \times & 31.7 & 31.6 & 31.5 & 31.4 & 31.3\end{array}$

$\begin{array}{llllll}5 \times & 31.2 & 31.2 & 31.1 & 31.11 & 30 .\end{array}$

$7 \times 29.079 .8 \quad 29.5 \quad 29.2 \quad 28$.

$\begin{array}{lllllll}4 \times & 28.4 & 77.6 & 24.8 & 26.1 & 25.5 \\ 0 \times & 25.0 & 21.5 & 24.0 & 23.5 & 23.1\end{array}$

$11 \times 25$
$17 \times 22.0$

- Sand Condition

\begin{tabular}{|c|c|c|c|c|c|c|}
\hline \multirow{2}{*}{\multicolumn{7}{|c|}{ 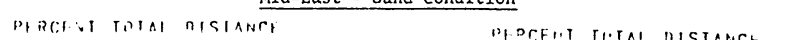 }} \\
\hline & & Dipef & I זיו & AI & & \\
\hline $\begin{array}{l}x= \\
31\end{array}$ & & & $0^{2}$ & & & \\
\hline 31.231 .231 & & $\begin{aligned} 12.4 \\
0.1\end{aligned}$ & 0.1 & 0.1 & 0.1 & \\
\hline 31.231 .231 .231 .231 .2 & - & $\begin{array}{lll}1 \times & 0.1 \\
2 \times & 0.1\end{array}$ & 0.1 & $\begin{array}{l}0.1 \\
0.1\end{array}$ & $\begin{array}{l}n=1 \\
n=1\end{array}$ & \\
\hline $\begin{array}{lllll}31.2 & 31.2 & 31.2 & 31.1 & 31.0 \\
30.9 & 30.8 & 30.8 & 30.7 & 30.6\end{array}$ & & $3 \times \quad 0.1$ & 0.1 & 0.1 & 0.1 & \\
\hline $30.6 \quad 30.530 .430 .229 .9$ & & $\begin{array}{lll}1 x & 0.1 \\
5 x & 0.1\end{array}$ & $\begin{array}{l}0.1 \\
0.1\end{array}$ & $\begin{array}{l}0.1 \\
0.1\end{array}$ & $\begin{array}{l}0.1 \\
0.1\end{array}$ & \\
\hline $29.7 \quad 20.5 \quad 29.3 \quad 29.11 \quad 20.8$ & & n 0.1 & 0.1 & 0.1 & 0.1 & \\
\hline 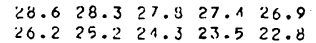 & & $\begin{array}{ll}7 x & 0.1 \\
8 x & 0.1\end{array}$ & 0.1 & 0.1 & 0.1 & \\
\hline $\begin{array}{lllll}26.2 & 25.2 & 21.5 & 23.5 & 22.8 \\
22.1 & 21.5 & 21.4 & 20.5 & 20.1\end{array}$ & & $9 \times \quad 0.1$ & 0.1 & 0.1 & 0.1 & \\
\hline .4 & & $10 \times 0.1$ & & & & \\
\hline
\end{tabular}

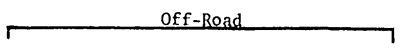

MDIFE TIIAL DISTANCF

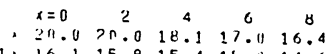

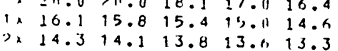

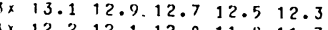
$\begin{array}{llllll}5 \times & 11.6 & 11.5 & 11.4 & 11.3 & 11.2 \\ 6 x & 11.1 & 11.0 & 11.8 & 10.7 & 10.5\end{array}$

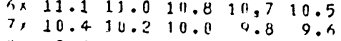
$\begin{array}{cccccc}. \times & 2.4 & 1.1 & 8.7 & 8.1 & 3.1 \\ .1 .1 & 1.4 & 0.8\end{array}$

PFOCFN TIITA OISIANCE

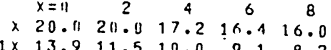
$\begin{array}{cccccc}2 \times & 13.9 & 11.5 & 10.0 & 9.1 & 8.0 \\ 2 \times & 7.3 & 1.2 & 0.6 & 0.4 & 0.2 \\ 3 & 0.5 & 0.3 & 0.5 & 0.2 & 0.2\end{array}$ $\begin{array}{llllll}3 x & 0.3 & 0.3 & 0.2 & 0.2 & 0.2 \\ 4 x & 0.2 & 0.2 & 0.2 & 0.2 & 0.2\end{array}$ $\begin{array}{llllll}5 x & 0.2 & 0.2 & 0.2 & 0.2 & 0.2 \\ 0 \times & 0.2 & 0.1 & 0.2 & 0.2 & 0.2\end{array}$ $\begin{array}{llllll}5 x & 0.2 & 0.2 & 0.2 & 0.2 & 0.2 \\ 6 x & 0.2 & 0.1 & 0.1 & n .1 & 0.1 \\ 7 x & 0.1 & 0.1 & 0.1 & 0.1 & 0.1\end{array}$ $\begin{array}{lllllll}8 \times & 0.1 & 0.1 & 0.1 & 0.1 & 0.1 \\ 9 x & 0.1 & 0.1 & 0.1 & 0.1 & 0.1\end{array}$ $\begin{array}{ccc}9 x & 0.1 \\ 10 x & 0.1\end{array}$

perrent total distance

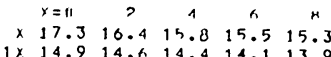
$\begin{array}{lllllll}1 \times & 14.9 & 14.6 & 14.4 & 14.1 & 13.9 \\ 3 \times & 13.7 & 13.4 & 13.3 & 13.1 & 12.9 \\ 3 \times & 13.0 & 12.6 & 13.5 & 2.3 & 12.2\end{array}$ $4 \times 12.0 \quad 11.9 \quad 11.8$ $\begin{array}{llllll}5 \times & 11.4 & 11.3 & 11.2 & 11.0 & 10.9 \\ 6 x & 10.8 & 10.7 & 10.6 & 10.4 & 10.2\end{array}$ $\begin{array}{llllll}7 \times & 10.0 & 9.7 & 9.3 & 8.9 & 8.4\end{array}$ $\begin{array}{rrrrrr}8 \times & 7.9 & 7.4 & 1.5 & 8.9 & 8.4 \\ 2 \times & 1.2 & 0.9 & 0.8 & 1.9 & 1.5 \\ 10 \times & 0.6 & & & 1.7 & 0.6\end{array}$

PERIELT THTAL DISTANCE

$\begin{array}{lllll}x=0 & 2 & 4 & 6 & 8\end{array}$ $\begin{array}{lllllll}1 \times & 0.2 & 0.1 & 0.2 & 11.3 & 0.2 \\ 2 x & 0.1 & 0.1 & 0.1 & 11.1 & 0.1\end{array}$ $\begin{array}{llllll}3 x & 0.1 & 0.1 & 0.1 & 11.1 & 0.1 \\ 1 \times & 0.1 & 0.1 & 0.1 & 11 & 0.1\end{array}$ $\begin{array}{llllll}5 x & 0.1 & 0.1 & 0.1 & 11.1 & 0.1 \\ 5 x & 0.1 & 0.1 & 0.1 & 1.1 & 0.1\end{array}$ $\begin{array}{llllll}2 x & 0.1 & 0.1 & 0.1 & 11.1 & 0.1\end{array}$ $\begin{array}{cccccc}8 \times & 0.1 & 0.1 & 0.1 & 0.1 & 0.1 \\ 9 x & 0.1 & 0.1 & 0.1 & 1.1 & 0.1 \\ 10 x & 0.1 & & & & \end{array}$ 


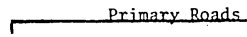

Dercent total miciante

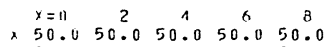

$1 \times 50.050 .050 .0050 .050 .0$

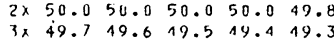

$\begin{array}{llllll}4 . & 49.1 & 48.4 & 47.8 & 47.2 & 46.7\end{array}$

5) $45.345 .7 \quad 45.244 .644 .1$

$7 x+41.041 .1 \quad 40.5 \quad 34.8 \quad 38.9$

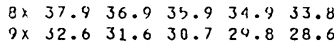

percent tutal distance

$\begin{array}{rrrrrr} & \lambda=0 & 2 & 4 & 6 & 8 \\ x & 16.9 & 16.9 & 16.9 & 15.6 & 14.8 \\ 1 \times & 14.3 & 14.0 & 13.7 & 13.4 & 13.2\end{array}$ $\begin{array}{llllll}2 \times & 13.0 & 12.9 & 12.7 & 12.6 & 12.6\end{array}$ $\begin{array}{lllllll}3 x & 12.5 & 12.4 & 12.4 & 12.3 & 12 .\end{array}$ $\begin{array}{llllll}5 \times & 12.1 & 12.1 & 12.0 & 12.4 & 12.0\end{array}$ $\begin{array}{llllll}6 \times & 12.0 & 12.0 & 11.9 & 11.9 & 12.0 \\ 7 \times & 11.8 & 11.7 & 11.7 & 11.7 & 11.8\end{array}$

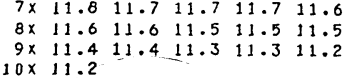

PERCENT TUTAL DISIANCE

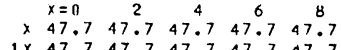
$2 \times \quad 47.7 \quad 47.7 \quad 47.5 \quad 47.2 \quad 47.0$

$\begin{array}{llllll}3 x & 46.9 & 16.7 & 46.6 & 46.5 & 46.4\end{array}$

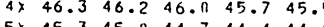

$\begin{array}{llllll}5 \times & 45.3 & 45.0 & 44.7 & 44.4 & 44.1 \\ 6 \times & 43.9 & 43.7 & 43.4 & 43.2 & 43.1 \\ 7 \times & 42.9 & 42.7 & 42.5 & 42.3 & 41.9\end{array}$

$\begin{array}{rlllll}8 \times & 41.6 & 41.2 & 40.8 & 40.4 & 39.8 \\ 9 \times & 38.9 & 37.4 & 35.1 & 33.1 & 31.3 \\ 10 \times & 29.8 & & \end{array}$

Percent total oisiance

$\begin{array}{rlrrrr} & x=0 & 2 & 4 & 6 & 8 \\ x & 47.7 & 47.7 & 47.7 & 47.7 & 47^{8} .7 \\ 1 \times & 47.7 & 47.7 & 47.7 & 17.7 & 47.7\end{array}$

$2 \times 47.787$

$\begin{array}{cccccc}3 \times & 46.9 & 46.7 & 46.6 & 46.5 & 46.4 \\ 4 \times & 46.3 & 46.2 & 45.9 & 45.7 & 45.4\end{array}$

$\begin{array}{llllll}5 x & 45.2 & 44.9 & 44.6 & 44.3 & 44.1 \\ 6 \times & 43.8 & 43.6 & 43.4 & 43.2 & 43.0\end{array}$

$\begin{array}{lllllll}7 \times & 42.8 & 42.7 & 42.4 & 42.2 & 41.8 \\ 8 x & 41.4 & 41.0 & 40.5 & 40.1 & 39\end{array}$

$\begin{array}{llllll}8 \times & 41.4 & 41.0 & 40.5 & 40.1 & 39.5 \\ 9 \times & 38.4 & 36.9 & 34.6 & 32.5 & 30.7\end{array}$
Table E7
Speed Profile for M813-XM204

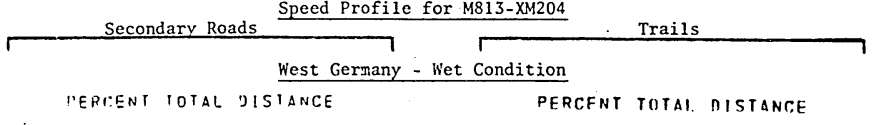

$x=0$
$\times \quad 35$

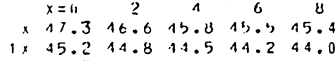

$\begin{array}{llllll}2 \times & 43.3 & 42.7 & 42.2 & 41.8 & 41.0 \\ 3 \times & 40.9 & 40.5 & 40.2 & 39.8 & 39\end{array}$

$4 \times 38.4 \quad 38.5 \quad 38.1 \quad 37.7 \quad 37$.

$\begin{array}{llllll}5 \times & 34.1 & 33.6 & 33.2 & 32.7 & 32.3 \\ 7 \times & 31.9 & 31.5 & 31.0 & 30.5 & 29.9\end{array}$

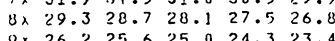

$9 \times 26.2$
$10 \times 22.6$

West Ger

PERCENT TOTAL DISTANCE

$\begin{array}{llllll}1 \times & 27.5 & 26.4 & 25.3 & 24.6 & 24.0 \\ 2 x & 23.6 & 23.1 & 22.7 & 22.3 & 22.0\end{array}$

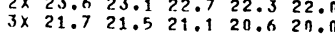

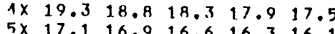

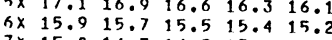

$\begin{array}{llllll}8 \times & 12.5 & 12.1 & 11.7 & 11.3 & 11.0 \\ 9 \times & 10.7 & 10.5 & 10.2 & 10.0 & 9.8\end{array}$

$\begin{array}{rrrrrr}9 \times & 10.7 & 10.5 & 10.2 & 10.0 & 9.8 \\ 10 \times & 3.9 & & & & \end{array}$

PERCFN TOTAL DISTANGE

$\begin{array}{rlrcrc} & x=0 & 2 & 4 & 0 & 8 \\ x & 13.1 & 12.9 & 12.2 & 12.0 & 11.8 \\ 1 \times & 11.7 & 11.7 & 11.6 & 11.6 & 11.6\end{array}$

$\begin{array}{llllll}2 x & 11.6 & 11.5 & 11.5 & 11.5 & 11.5 \\ 3 x & 11.5 & 11.4 & 11.4 & 11.5 & 11.5\end{array}$

$4 \times 11.311 .411 .411 .411 .5$

$\begin{array}{llllll}5 x & 11.1 & 11.1 & 11.1 & 11.1 & 11.1 \\ 6 x & 11.0 & 11.0 & 11.0 & 11.0 & 10.9\end{array}$

$\begin{array}{llllll}7 x & 10.9 & 10.9 & 11.9 & 11.8 & 10.9 \\ 8 \times & 0.8 & 10.8 & 10.8\end{array}$

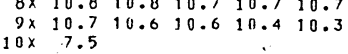

Mid-East - Het Condition

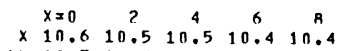

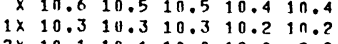

$\begin{array}{cccccc}2 \times & 10.1 & 10.1 & 10.0 & 10.0 & 0.9 \\ 3 \times & 9.9 & 9.9 & 9.8 & 9.8 & 9.8\end{array}$

$\begin{array}{llllll}3 x & 9.9 & 9.9 & 9.8 & 9.8 & 9.8 \\ 4 x & 9.7 & 9.7 & 9.7 & 9.6 & 9.6\end{array}$

$\begin{array}{llllll}5 x & 9.5 & 9.5 & 9.4 & 9.4 & 9.4 \\ 6 \times & 9.3 & 9.3 & 9.2 & 9.2 & 9.2\end{array}$

$\begin{array}{cccccc}7 \times & 9.1 & 9.1 & 8.9 & 8.7 & 8.5 \\ 9 \times & .4 & 9.7 & 0.6 & 0.5 & 9.9\end{array}$

$\begin{array}{llllll}8 \times & 8.4 & 8.3 & 8.1 & 8.0 & 7.9 \\ 9 \times & 7.8 & 7.7 & 7.6 & 7.5 & 7.5\end{array}$

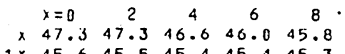

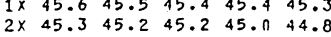

$\begin{array}{llllll}3 \times & 44.7 & 44.5 & 44.4 & 44.3 & 44.2 \\ 4 \times & 44.1 & 44.0 & 43.8 & 43.7 & 43.3\end{array}$

$5 \times 42.9 \quad 42.6 \quad 42.2 \quad 41.9 \quad 41.5$

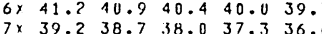

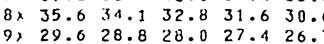

$1: 1 \times 25.9$

Mid-E

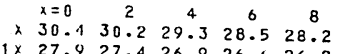

$2 \times 25.524 .924 .926 .626 .2$

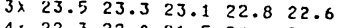

$5 \times 22.522 .021 .520 .820 .2$

$\begin{array}{llllll}5 x & 19.6 & 19.1 & 18.7 & 18.3 & 18.0 \\ 6 x & 17.7 & 17.4 & 17.1 & 16.9 & 16.7 \\ 7 x & 16.5 & 16.3 & 16.1 & 15.9 & 15.8\end{array}$

$\begin{array}{lllllll}7 \times & 16.5 & 16.3 & 16.1 & 15.9 & 15.8\end{array}$

$\begin{array}{lllllll}8 \times & 15.6 & 15.4 & 15.1 & 14.4 & 13.8 \\ 9 \times & 13.3 & 12.9 & 12.4 & 12.1 & 11.7\end{array}$

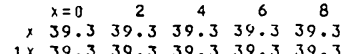
percent rotal Distance

$\begin{array}{llllll}1 \times & 3 \times .3 & 39.3 & 39.3 & 39.3 & 39.3\end{array}$

$\begin{array}{llllll}2 \times & 39.3 & 39.3 & 39.3 & 39.3 & 39.3 \\ 3 \times & 39.3 & 39.3 & 39.3 & 39.3 & 39.3 \\ 1 \times & 39.3 & 39.2 & 38.9 & 38.6 & 38.3\end{array}$

$\begin{array}{llllll}5 \times & 38.0 & 37.7 & 37.5 & 37.2 & 37.0 \\ 6 x & 36.7 & 36.4 & 35.9 & 35.3 & 34.8\end{array}$

$\begin{array}{llllll}7 \times & 34.3 & 33.7 & 32.9 & 32.2 & 31.3 \\ 8 \times & 30.3 & 28.8 & 27.6 & 26.5 & 25.5 \\ 9 \times & 24.6 & 73.8 & 23.1 & 22.5 & 21.9\end{array}$

$10 \times 24.1$ $\begin{array}{lccccc}x=0 & 2 & 4 & 6 & 8 \\ x & 17 . .1 & 13.5 & 13.3 & 13.3 & 13.2 \\ 1 x \times & 13.1 & 13.0 & 13.0 & 12.0 & 12.7\end{array}$

$\begin{array}{lllllll}1 \times & 13.1 & 13.0 & 13.3 & 13.3 & 12.9 & 12.2 \\ 2 \times & 12.6 & 12.5 & 12.0 & 12.9 & 12.7\end{array}$

$\begin{array}{rrrrrrr}3 x & 12.0 & 11.9 & 11.7 & 11.6 & 11.5 \\ 1 \times & 11.4 & 10.8 & 10.3 & 4.8 & 9.5\end{array}$

$\begin{array}{lllllll}5 \times & 0.1 & 2.9 & 1.4 & 1.0 & 0.7 \\ 6 x & 0.6 & 0.5 & 0.5 & 1.0 & 0.4\end{array}$

$\begin{array}{llllll}7 \times & 0.4 & 0.3 & 0.3 & 11.4 & 0.4 \\ 8 x & 0.3 & 0.3 & 0.2 & 0.2 & 0.3 \\ 8 x & 0.2 & 0.2 & 0.2 & 1.2 & 0.2\end{array}$ $\begin{array}{llllll}8 x & 0.3 & 0.3 & 0.2 & 0.2 & 0.2 \\ 9 x & 0.2 & 0.2 & 0.2 & 0.2 & 0.2\end{array}$

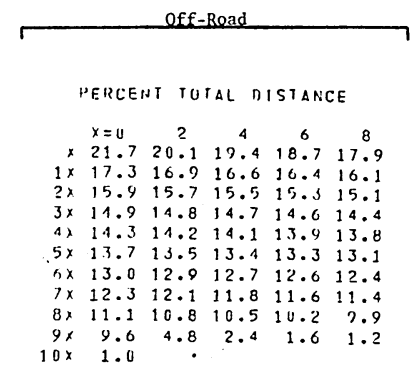

PIRIEET TOTAL DISIANCE

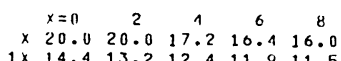
$\begin{array}{llllll}7 \times & 14.4 & 13.2 & 12.4 & 11.9 & 11.5 \\ 2 x & 11.2 & 10.9 & 10.7 & 10.5 & 110.4 \\ 3 \times & 10.2 & 10.5 & 10.7 & 90.5 & 9.9\end{array}$ $\begin{array}{rrrrrr}3 \times & 10.2 & 10.1 & 10.0 & 9.9 & 9.8 \\ 4 \times & 9.6 & 9.5 & 9.5 & 9.4 & 9.3\end{array}$ $\begin{array}{llllll}5 \times & 9.2 & 9.2 & 9.1 & 9.4 & 9.0 \\ 6 x & 8.9 & 8.8 & 8.8 & 8.7 & 8.0 \\ 7 x & 9.5 & 0.4 & 8.3 & 8.2 & 8.0\end{array}$ $\begin{array}{llllll}7 \times & 8.5 & 8.4 & 8.3 & 8.2 & 8.6 \\ \times & 7.9 & 7.7 & 7.5 & 7.2 & 8.0\end{array}$ $\begin{array}{llllll}9 \times & 2.8 & 1.7 & 7.3 & 7.3 & 0.9 \\ 9 \times & 0.8 & & 1.3 & 1.0 & 0.9\end{array}$

PERCENT TOTAL DISTANCE

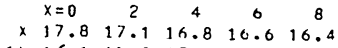

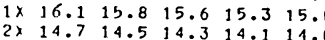
$\begin{array}{lllllll}3 x & 13.8 & 13.7 & 13.5 & 13.4 & 13.0\end{array}$ $\begin{array}{llllll}4 x & 13.0 & 12.8 & 12.6 & 12.5 & 12.3 \\ 5 x & 12.1 & 11.9 & 1.6 & 12.5 & 12.3\end{array}$ $6 \times 11.0 \quad 10.9 \quad 10.7 \quad 10.510 .0$ $\begin{array}{cccccc}7 \times & 10.0 & 9.7 & 9.5 & 9.3 & 9.3 \\ 8 \times & 8.7 & 8.3 & 7.9 & 7.4 & 7 . \\ 90 & 3.9 & 2.1 & 1.5 & 1.2 & 1.0\end{array}$ $\begin{array}{rr}9 \times & 3.9 \\ 10 \times & 0.8\end{array}$

PIRCHET TITAT MISTANCE

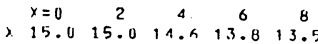
$\begin{array}{rrrrrrr}1 \times & 12.0 & 11.1 & 8.3 & 6.6 & 5.4 \\ 2 \times & 1.1 & 11.0 & 0.4 & 1.0 & 5.3\end{array}$ $\begin{array}{llllll}2 x & 1.1 & 1.6 & 0.4 & 10.3 & n .3 \\ 3 \times & 0.3 & 1.2 & 0.2 & n .2 & n .2 \\ 5 x & 0.2 & 11.2 & 0.2 & 0.2 & 0.2\end{array}$ $\begin{array}{llllll}4 x & 0.2 & 1.2 & n .2 & 11.2 & n .2 \\ 5 x & 0.2 & 0.2 & n .2 & 11.1 & n .1 \\ 6 x & 0.2 & 1.2 & n .1 & 0.1 & n .1\end{array}$ $\begin{array}{llllll}\sigma_{x} & 0.1 & 0.1 & n .1 & 1.1 & n .1 \\ n_{2} & 0.1 & 0.1 & 0.1 & 0.1 & n\end{array}$

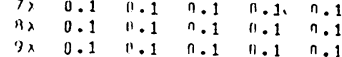
$\begin{array}{llllll}3 \times & 0.1 & n .1 & n .1 & n \cdot 1 & n\end{array}$ 
Table E8

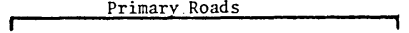

perient total olstance

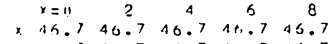
$\begin{array}{lllllll}2 x & 45.7 & 46.7 & 46.7 & 10.7 & 46 .\end{array}$ $3 \times 46.146 .2 \quad 46.1 \quad 15.445 .9$ $4 x$ is.6 45.1 4h. $\begin{array}{llllll}4 \times & 39.1 & 42.6 & 41.9 & 41.03 & 40.4\end{array}$ $7 \times 36.135 .5 \quad 35.131 .533 .9$ $\begin{array}{llllll}3 \times & 33.1 & 32.4 & 31.7 & 31.0 & 30.1 \\ 9 \times & 29.3 & 28.5 & 27.9 & 27.2 & 26.2\end{array}$ $10 \times 25.2$

PERCEHT TOTAL DISTANCE

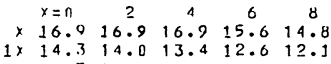
$\begin{array}{rrrrrr}3 \times & 11.0 & 11.5 & 11.3 & 10.9 & 10.1 \\ 4 \times & 10.0 & 10.0 & 9.9 & 9.8 & 9.8\end{array}$ $\begin{array}{llllll}5 \times & 9.7 & 9.7 & 9.6 & 9.6 & 9.5 \\ 6 \times & 9.5 & 9.5 & 0.4 & 9.4 & 9.4\end{array}$ $\begin{array}{llllll}8 \times & 9.2 & 9.2 & 9.2 & 9.1 & 9.1 \\ 9 \times & 9.1 & 0.0 & 0.11 & 9.11 & 9.0\end{array}$

PERCENT TUTAL DISIANC

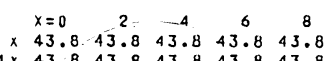

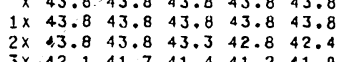
$4 x \quad 40.7 \quad 40.6 \quad 40.4 \quad 40.2 \quad 10.1$ $\begin{array}{llllll}5 \times & 40.0 & 34.8 & 39.6 & 39.4 & 39.3 \\ 6 \times & 39.1 & 39.0 & 38.8 & 38.7 & 38.6\end{array}$ $\begin{array}{llllll}6 \times & 39.1 & 39.0 & 38.8 & 38.7 & 38.6\end{array}$ $\begin{array}{lllllll}7 \times & 38.4 & 38.2 & 38.0 & 37.8 & 37.6 \\ 8 \times & 37.3 & 37.1 & 36.8 & 30.4 & 36.0 \\ 9 \times & 35.3 & 34.2 & 32.3 & 30.6 & 29.2\end{array}$ $10 \times .27 .0$

PERCENT tOTAL Distance

$\begin{array}{cccccc}x=11 & 2 & 4 & 6 & 8 \\ \times & 43.8 & 43.8 & 43.8 & 43.8 & 43.8\end{array}$ $\begin{array}{lllllll}2 \times & 43.8 & 43.8 & 43.3 & 42.8 & 42.4\end{array}$ $\begin{array}{llllllll}3 \times & 42.1 & 41.7 & 4.3 & 41.2 & 40.9 \\ 4 x & 40.7 & 40.6 & 40.4 & 40.2 & 40.9\end{array}$ $2 x+40.740 .640 .440 .240 .1$

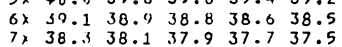
$\begin{array}{llllll}8 \times & 37.2 & 36.9 & 33.6 & 36.2 & 35.8 \\ 9 \times & 35.0 & 33.8 & 36.8 & 36.2 & 35.8\end{array}$

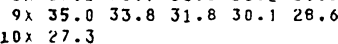

Speed Profile for M813-M114A1 West Germany - Wet Condition Trails PERCENT TOTAL DISTANCE

"ERIEEIT TIITAI BISIAHCE

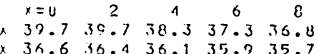
$\begin{array}{rrrrrr}3 \times & 5.6 & 35.5 & 35.4 & 35.3 & 35.2 \\ 3 \times & 34.9 & 31.7 & 34.5 & 31.2 & 33.9 \\ 4 \times & 33.5 & 32.8 & 32.2 & 31.6 & 31.1\end{array}$ $6 \times 28.978 .7 \quad 28.4 \quad 28.1 \quad 27.9$

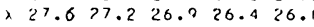
$\begin{array}{llllll}\text { B) } & 25.0 & 25.1 & 24.7 & 24.2 & 23.8 \\ \text { 9x } 23.3 & 22.9 & 22.4 & 21.0 & 21.2\end{array}$

\section{West Ge} PERIEENT TOIAL OISIANCE $\begin{array}{rrrrrr}x & =11 & 2 & 1 & 6 & 8 \\ \times & 13.1 & 12.5 & 11.0 & 10.0 & 10.4\end{array}$ $\begin{array}{rrrrrr}1 \times & 0.1 & 9.9 & 9.8 & 9.7 & 9.6 \\ 2 \times & 9.5 & 9.5 & 9.4 & 4.4 & 0.3\end{array}$ $\begin{array}{llllll}3 \times & 9.3 & 9.3 & 9.2 & 9.2 & 9.1\end{array}$ $\begin{array}{llllll}4 \times & 9.1 & 9.0 & 9.0 & 9.0 & 8.9\end{array}$ $\begin{array}{lllllll}5 \times & 8.9 & 8.9 & 8.9 & 8.8 & 8.0\end{array}$

$\begin{array}{llllll}6 \times & 8.8 & 8.8 & 8.8 & 8.7 & 8.7 \\ 7 \times & 8.7 & 8.7 & 8.7 & 8.6 & 8.6 \\ 8 \times & 8.6 & 8.0 & 0.4 & 8.0 & 0.5\end{array}$

$\begin{array}{llllll}8 \times & 8.6 & 8.6 & 8.5 & 8.6 & 8.5 \\ 9 x & 8.5 & 8.5 & 8.4 & 4.1 & 2.3\end{array}$ $10 \times \quad 1.6$

PERGENT TOTAL DISIANCE

$\begin{array}{rrrrrr} & x=0 & 2 & 4 & 6 & 8 \\ \times & 39.7 & 30.7 & 39.7 & 39.7 & 39.4 \\ 1 \times & 38.6 & 38.0 & 37.6 & 37.3 & 37.1\end{array}$

$\begin{array}{lllllll}2 \times & 37.0 & 36.8 & 36.7 & 36.6 & 36.4\end{array}$

$\begin{array}{llllll}3 \times & 36.3 & 36.2 & 36.1 & 36.0 & 35.9 \\ 4 \times & 35.8 & 35.8 & 35.7 & 35.7 & 35.9\end{array}$

$\begin{array}{llllll}5 \times & 35.5 & 35.5 & 35.4 & 35.2 & 35.0 \\ 6 \times & 34.7 & 34.5 & 34.3 & 34.1 & 33.9\end{array}$

$\begin{array}{llllll}8 \times & 31.4 & 30.3 & 29.3 & 28.5 & 27.7 \\ 9 \times & 27.0 & 26.3 & 25.8 & 25.2 & 24 .\end{array}$

$10 \times 24.0$

Mid-East - Sand Condition

percent total Disiance percent rutal misiance

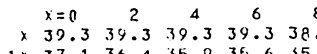

$\begin{array}{llllll}2 \times & 35.1 & 34.9 & 34.8 & 34.7 & 34.6 \\ 3 \times & 34.5 & 34.4 & 34.3 & 34.3 & 34.2\end{array}$

$4 \times 34.2 \quad 34.1 \quad 34.134 .034 .0$

$\begin{array}{llllll}3 \times & 32.9 & 32.6 & 32.3 & 31.9 & 31.6 \\ 7 \times & 31.2 & 30.8 & 30.2 & 24.6,29.0\end{array}$

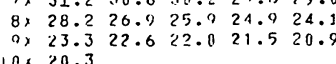

$\begin{array}{cccccc}x=0 & 2 & 4 & 6 & 8 \\ x & 25.7 & 25.7 & 24.9 & 04.3 & 23.9\end{array}$

$2 \times 21.322 .720 .421 .921 .5$

$3 \times 19.819 .519 .7 \quad 18.818 .3$

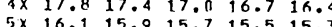

$914.8 \quad 14.7 \quad 14.5$

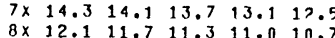

$9 \times 10.410 .710 .89 .890 .6$

dition

$\begin{array}{cccccc} & x=0 & 2 & 1 & 6 & 8 \\ x & 7.8 & 7.8 & 7.8 & 7.8 & 7.8 \\ 1 \times & 7.8 & 7.7 & 7.7 & 7.7 & 7.6 \\ & 7.6\end{array}$

$\begin{array}{llllll}2 x & 7.6 & 7.6 & 7.8 & 7.8 & 7.8 \\ 3 x & 7.6 & 7.6 & 7.6 & 7.6 & 7.6 \\ 4 x & 7.5 & 7.5 & 7.5 & 7.5 & 7.5\end{array}$

$\begin{array}{llllll}4 \times & 7.5 & 7.5 & 7.5 & 7.5 & 7.5 \\ 5 \times & 7.5 & 7.5 & 7.5 & 7.5 & 7.5 \\ 6 x & 7.4 & 7.5 & 7.5 & 7.5 & .5\end{array}$

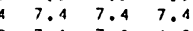

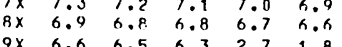

$\begin{array}{rrrrrr}9 \times & 6.6 & 6.5 & 6.3 & 2.7 & 1.8 \\ 10 \times & 1.3 & & 0.8 & \end{array}$

PERCEN TUIAL DISIANCE
Pation

$\begin{array}{rrrrrr}x & x=0 & 2 & 4 & 6 & 8 \\ x & 25.7 & 25.7 & 25.6 & 25, .6 & 25.1 \\ 1 \times & 24.6 & 24.11 & 23.4 & 23.011 & 22.5 \\ 3 x & 22.1 & 21.7 & 21.4 & 21.0 & 20.9\end{array}$

$\begin{array}{llllll}2 \times & 22.1 & 21.7 & 21.4 & 21.0 & 20.9 \\ 3 \times & 20.7 & 211.5 & 20.3 & 211 . & 19.9 \\ 5 x & 19.6 & 10.9 & 10.9 & 10.3 & 19.9\end{array}$

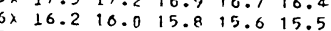

$8 \times 14.6 \quad 14.414 .214 .914 .8$

$\begin{array}{llll} & \end{array}$

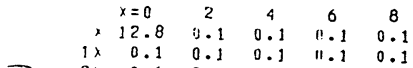
$\begin{array}{llllll}1 x & 0.1 & 0.1 & 0.1 & n .1 & 0.1 \\ 2 x & 0.1 & 0.1 & 0.1 & n .1 & 0.1\end{array}$ $\begin{array}{llllll}3 \times & 0.1 & 0.1 & 0.1 & 10.1 & 0.1 \\ 4 \times & n .1 & 0.1 & 0.1 & 1.1 & 0.1 \\ 5 \times & .1 & 0.1 & 0.1 & 0.1 & 0.1\end{array}$

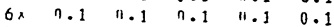
$\begin{array}{llllll}7 x & n .1 & 0.1 & n .1 & 1.1 & 0.1 \\ 8) & n .1 & 0.1 & n .1 & n .1 & 0.1\end{array}$

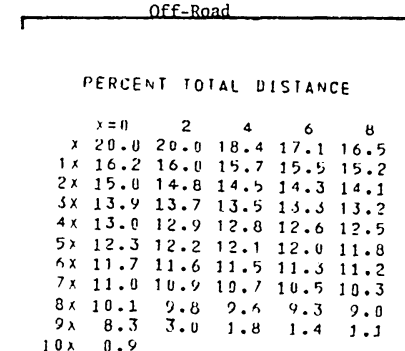

PERlitNi TOTAL DISTANCE

$\begin{array}{lllll}x=11 & 2 & 4 & 6\end{array}$

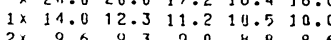

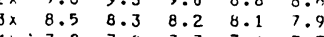
$\begin{array}{llllll}5 x & 7.5 & 7.8 & 7.7 & 7.6 & 7.5 \\ 6 x & 7.2 & 7.4 & 7.3 & 7.3 & 7.2 \\ 0 x & 6.8 & 0.7 & 7.5 & 7.0 & 5.9\end{array}$ $\begin{array}{llllll}7 \times & 5.8 & 0.7 & 0.5 & 0.1 & 2.0\end{array}$

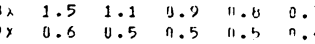

PERCENT TUTAL UISIANCE

$\begin{array}{cccccc} & x=0 & 2 & 4 & 6 & 8 \\ x & 17.8 & 16.2 & 15.6 & 15.2 & 14.9 \\ 1 & 14.8 & 14.6 & 14.4 & 14.1 & 13.9\end{array}$ $\begin{array}{lllllll}2 \times & 13.6 & 13.5 & 13.3 & 13.2 & 13.0\end{array}$ $\begin{array}{cccccc}3 \times & 12.9 & 12.8 & 12.6 & 12.5 & 12.0 \\ 4 \times & 12.2 & 12.1 & 12.0 & 12.5 & 12.5\end{array}$ $\begin{array}{llllll}5 x & 11.4 & 11.2 & 11.9 & 10.8 & 10.5\end{array}$ $\begin{array}{rrrrrr}6 x & 10.5 & 10.4 & 10.2 & 11.0 & 9.8 \\ 7 \times & 9.6 & 9.4 & 0.1 & 8.9 & 8.7\end{array}$

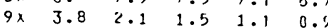
$10 x \quad n .8$

Percent total Distance

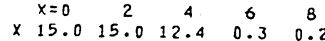
$\begin{array}{llllll}1 x & 0.2 & 0.2 & 0.1 & 0.1 & 0.2 \\ 2 x & 0.1 & 0.1 & 0.1 & 0.1 & 0.1\end{array}$ $\begin{array}{lllllll}3 x & 0.1 & 0.1 & 0.1 & 0.1 & 0.1 \\ 4 x & 0.1 & 0.1 & 0.1 & 0.1 & 0.1\end{array}$ $\begin{array}{llllll}5 x & 0.1 & 0.1 & 0.1 & 0.1 & 0.1 \\ 6 x & 0.1 & 0.1 & 0.1 & 0.1 & 0.1\end{array}$ $\begin{array}{lllllll}7 x & 0.1 & 0.1 & 0.1 & 0.1 & 0.1 \\ 8 x & 0.1 & 0.1 & 0.1 & 0.1 & 0.1\end{array}$ $\begin{array}{llllll}8 x & 0.1 & 0.1 & 0.1 & 0.1 & 0.1 \\ 9 x & 0.1 & 0.1 & 0.1 & 0.1 & 0.1\end{array}$ 


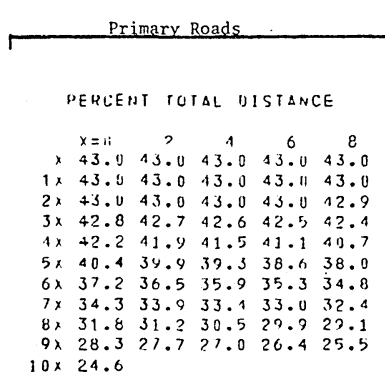

percent total distance

$\begin{array}{cccccc}x=11 & 2 & 1 & 0 & 8 \\ \times & 16.9 & 16.9 & 16.9 & 15.0 & 14.8\end{array}$ $\begin{array}{rrrrrr}\times & 16.9 & 16.9 & 16.9 & 15.6 & 14.8 \\ 2 \times & 14.3 & 14.0 & 1.3 .3 & 12.2 & 11.4 \\ 3 \times & 10.8 & 10.4 & 10.1 & 0.8 & 9.6\end{array}$ $\begin{array}{rrrrrr}2 \times & 10.8 & 10.4 & 10.1 & 9.8 & 9.6 \\ 3 \times & 9.4 & 9.2 & 9.1 & 9.0 & 8.9\end{array}$ $\begin{array}{llllll}4 x & 8.8 & 8.7 & 8.6 & 8.6 & 8.5 \\ 6 x & 8.4 & 0.4 & 8.3 & 8.2 & 8.2\end{array}$ $\begin{array}{llllll}5 x & 8.4 & 8.4 & 8.3 & 8.2 & 8.2 \\ 6 x & 0.1 & 8.1 & 8.1 & 8.0 & 8 .\end{array}$ $\begin{array}{llllll}7 \times & 7.9 & 1.8 & 7.1 & 8.0 & 8.0 \\ 8 \times & 7.7 & 7.8 & 7.7 \\ 9 \times & 7.5 & 7.5 & 7.5 & 7.6 & 7.4\end{array}$ $\begin{array}{rrrrrr}8 \times & 7.7 & 1.6 & 7.6 & 7.6 & 7.5 \\ 9 \times & 7.5 & 7.5 & 7.5 & -7.4 & 7.4 \\ 10 x & 7.4 & & & & \end{array}$

Percent total oIstance

$\begin{array}{rrrrrr}x=0 & 2 & 4 & 6 & 8 \\ x & 41.11 & 41.0 & 41.0 & 41.0 & 41.0\end{array}$ $\begin{array}{llllll}2 x & 41.0 & 41.0 & 40.7 & 40.4 & 40.2\end{array}$ $\begin{array}{llllll}3 \times & 39.9 & 39.7 & 39.5 & 39.3 & 39.1\end{array}$ $5 \times 38.4 \quad 38.3 \quad 38.1 \quad 37.9 \quad 37.7$ $6 \times 37.6 \quad 37.4 \quad 37.3 \quad 37.2 \quad 37.1$

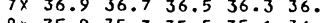
$\begin{array}{lllllll}8 \times & 35.9 & 35.7 & 35.5 & 35.1 & 34 .\end{array}$ $10 \times 27.2$

PERCENT TUTAL DISTANCE

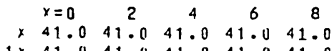

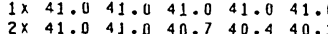
$3 \times 39.9 \quad 39.7 \quad 39.5 \quad 39.3 \quad 39.1$ $\begin{array}{llllll}4 \times & 39.0 & 38.8 & 38.7 & 38.6 & 38.5\end{array}$ $5 \times 38.4 \quad 38.238 .0 \quad 37.837 .7$ $7 \times \quad 36.8 \quad 36.6 \quad 36.5 \quad 36.3 \quad 36.0$ $\begin{array}{rrrrrr}8 \times & 35.8 & 35.6 & 35.3 & 34.9 & 34.5 \\ 9 \times & 33.8 & 32.7 & 30.9 & 29.3 & 27.9 \\ 10 \times & 26.7 & & & & \end{array}$
Table E9
Speed Profile for M813-XM198

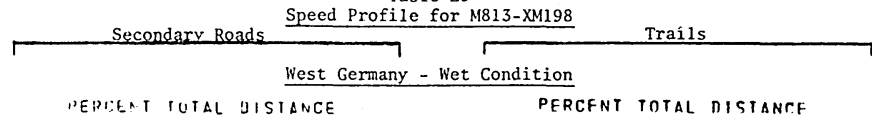
PERCFNt total Distane.

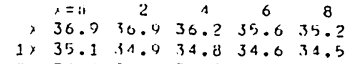
$\begin{array}{llllll}2 \times & 51.4 & 34.4 & 34.3 & 34.1 & 34.0\end{array}$ $\begin{array}{llllll}3 x & 33.8 & 3.3 .5 & 33.3 & 35.0 & 32.6 \\ 4 \times & 32.2 & 31.7 & 31.1 & 30.6 & 50.1\end{array}$ $\begin{array}{llllll}5 \times & 29.7 & 29.3 & 29.0 & 28.7 & 28.4 \\ 62 & 28.1 & 27.8 & 27.6 & 27.3 & 27.4\end{array}$ $7 \times 26.826 .5 \% 0.125 .725$.

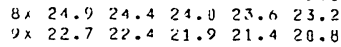
$10 \times 20.2$

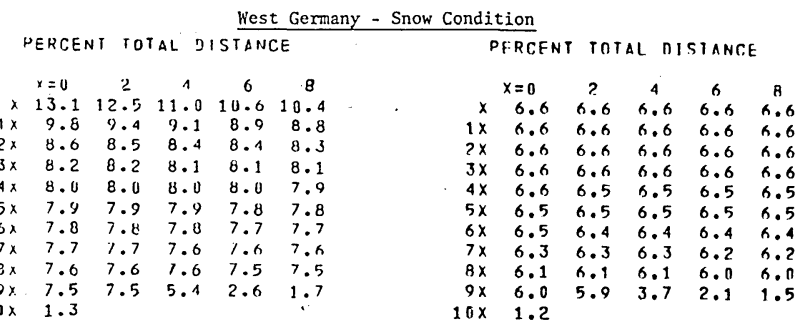
Mid-East - Wet Condition

Percent total Distance Percent total Distance

$\begin{array}{cccccc} & x=0 & 2 & 4 & 6 & 8 \\ \times & 36.9 & 36.4 & 36.9 & 36.9 & 36.7 \\ 1 \times & 36.2 & 35.9 & 35.9 & 35.5 & 35.3\end{array}$ $2 \times 35.235 .135 .1135 .0034 .9$ $3 \times 34.8 \quad 34.8 \quad 34.7 \quad 34.6 \quad 34.6$ $4 \times 34.534 .534 .534 .434 .4$ $6 \times 33.7 \quad 33.4 \quad 33.2 \quad 32.9 \quad 32.7$ $7 \times 32.5 \quad 32.3 \quad 31.931 .5 \quad 31.1$

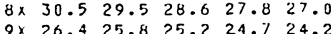
$10 \times 23.6$

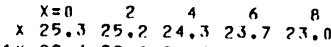

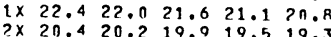
$19.018 .8 \quad 18.518 .1$ 19.

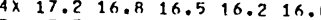
$6 x+14,15.515 .3 \quad 15.115 .0$ $7 \times 14.013 .813 .412 .812 .0$ .99 .790 .5

\section{PERCENT TOTAL DISTANCE}

$\begin{array}{cccccc}x=0 & 2 & 4 & 6 & 8 \\ \times & 36.9 & 36.9 & 36.9 & 36.9 & 36.1\end{array}$

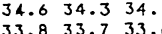
$3 \times \quad 33.5 \quad 33.5 \quad 33.4 \quad 33.4 \quad 33.4$ $\begin{array}{llllll}4 \times & 33.3 & 33.3 & 33.3 & 33.2 & 33.4 \\ 5 \times & 33.2 & 32.9 & 32.2 & 32.5 & 3.2\end{array}$ $\begin{array}{llllll}5 \times & 33.1 & 332.9 & 32.7 & 32.5 & 32.3 \\ 6 \times & 32.0 & 31.7 & 31.4 & 32.1 & 30.0\end{array}$ $\begin{array}{llllll}7 \times & 30.4 & 30.1 & 29.5 & 29.0 & 28.4\end{array}$ $\begin{array}{llllll}8 \times & 27.6 & 26.4 & 25.4 & 24.5 & 23.7 \\ 9 \times & 23.0 & 22.3 & 21.7 & 21.2 & 20.7\end{array}$

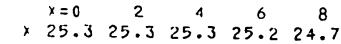

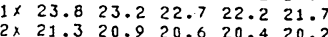
$\begin{array}{llllll}3 \times & 19.9 & 19.6 & 19.3 & 19.1 & 18.9\end{array}$ $6 \times 15.7 \quad 15.6 \quad 15.4 \quad 15.1215 .9$ $9 \times 12.2 \quad 11.8 \quad 11.5 \quad 11.2 \quad 10.9$ dition

\section{PECENT TOTAL DISTANCE}

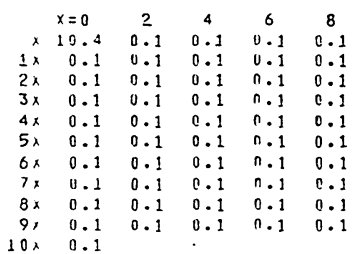

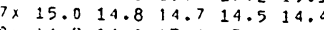

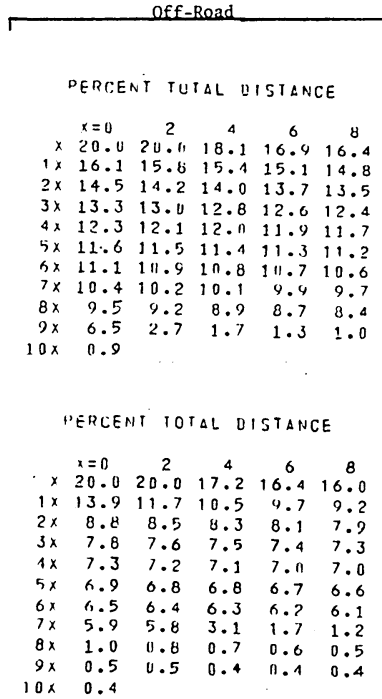

Perlent total vistance

$\begin{array}{ccccc}x=0 & 2 & 4 & 6 & 8 \\ \times 17.4 & 10.0 & 15.5 & 15.1 & 14.9\end{array}$ $\begin{array}{llllll}3 \times & 12.6 & 12.4 & 12.3 & 12.1 & 12.0\end{array}$

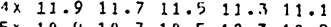
$6 \times 10.098 .80 .7 \quad 9.5 \quad 9.4$ $\begin{array}{llllll}7 \times & 9.1 & 8.9 & 8.7 & 8.5 & 8.2 \\ 8 \times & 7.9 & 7.5 & 7.2 & 6.0 & 2.8\end{array}$ $\begin{array}{llllll}3 x & 7.9 & 7.5 & 7.2 & 6.6 & 2.8 \\ 9 x & 1.8 & 1.3 & 1.0 & 0.9 & 0.7\end{array}$

PERCENT TOTAL DistanCE

$\begin{array}{lllll}x=0 & 2 & 4 & 6 & 8\end{array}$ $\begin{array}{rrrrrr}15.0 & 15.0 & 1.2 & 0.3 & 0.2 \\ 1 \times & 0.2 & 0.1 & 0.1 & 0.1 & 0.1\end{array}$ $\begin{array}{llllll}2 x & 0.1 & 0.1 & 0.1 & 11.1 & 0.1 \\ 3 x & 0.1 & 0.1 & 0.1 & 10.1 & 0.1\end{array}$ $\begin{array}{llllll}4 x & 0.1 & 0.1 & 0.1 & n .1 & 0.1 \\ 5 x & 0.1 & 0.1 & 0.1 & 0.1 & 0.1\end{array}$ $\begin{array}{llllll}6 x & 0.1 & 0.1 & 0.1 & n .1 & 0.1 \\ 7 x & 0.1 & 0.1 & 0.1 & 0.1 & 0.1\end{array}$ $\begin{array}{llllll}8 \times & 0.1 & 0.1 & 0.1 & 0.1 & 0.1 \\ 3 \times & 0.1 & 0.1 & 0.1 & 0.1 & 0.1 \\ 10 \times & 0.1 & & & & \end{array}$ 


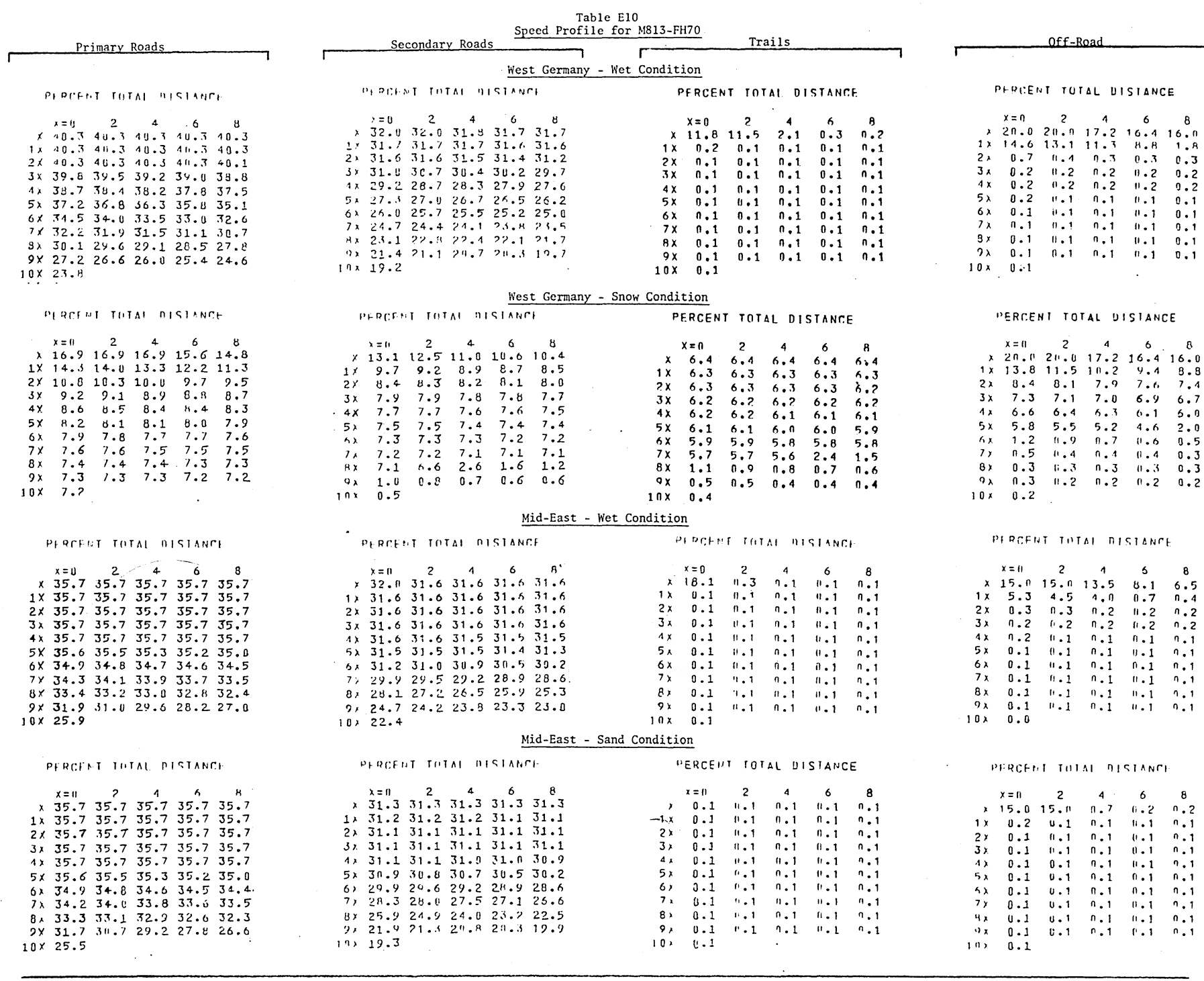




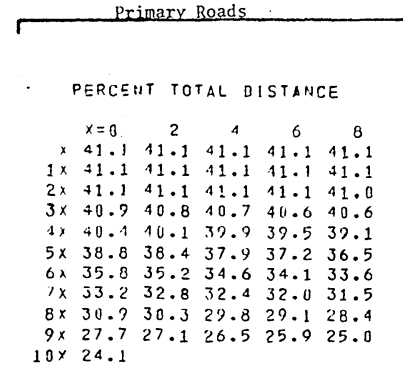

Percent total Distance

$\begin{array}{rccccc}x=0 & 2 & 4 & 6 & 8 \\ x & 16.9 & 16.9 & 16.9 & 15.6 & 14.8 \\ 1 \times & 14.3 & 14.0 & 13.3 & 12.2 & 11.4 \\ 3 x & 14.8 & 1 . .4 & 0.1 & 0.0 & 0.6\end{array}$ $\begin{array}{rrrrrr}2 \times & 10.8 & 10.4 & 10.1 & 9.8 & 9.6 \\ 3 \times & 9.4 & 9.2 & 9.1 & 8.9 & 8.8 \\ 5 \times & 8.9 & 8.0 & 0.6 & 0.5 & 8.4\end{array}$ $\begin{array}{llllll}4 \times & 8.7 & 8.6 & 8.6 & 8.5 & 8.4 \\ 5 \times & .3 & .3 & 0.6 & 8.2 & 8.1\end{array}$ $\begin{array}{llllll}5 x & 8.3 & 8.3 & 8.2 & 8.2 & 8.1 \\ 6 x & 8.0 & 8.0 & 8.0 & 7.9 & 7.8\end{array}$ $\begin{array}{llllll}7 \times & 7.8 & 7.7 & 7.7 & 7.7 & 7.6 \\ 8 \times & 7.6 & 7.6 & 7.5 & 7.5 & 7.5 \\ 9 x & 7.4 & 7.4 & 7.5 & -7.4 & 7.5\end{array}$ $10 \times \quad 7.3$

PERCENT TOTAL DISTANCE

$\begin{array}{rrrrrr}x=0 & 2 & 4 & 6 & 8 \\ x & 39.3 & 39.3 & 39.3 & 39.3 & 39.3 \\ 1 x & 39.3 & 39.3 & 39.3 & 39.3 & 39.3\end{array}$ $\begin{array}{llllll}1 \times & 39.3 & 39.3 & 39.3 & 39.3 & 39.3 \\ 2 \times & 39.3 & 39.3 & 39.1 & 38.9 & 38.8\end{array}$ $\begin{array}{lllllll}2 \times & 38.6 & 38.4 & 38.3 & 38.1 & 38.0 & \end{array}$ $\begin{array}{llllll}4 \times & 37.9 & 37.8 & 37.7 & 37.6 & 37.6 \\ 5 \times & 37.5 & 37.4 & 37.2 & 37.0 & 36.8\end{array}$ $\begin{array}{llllll}5 x & 37.5 & 37.4 & 37.2 & 37.0 & 36.8 \\ 6 x & 36.7 & 36.5 & 36.4 & 36.3 & 36.1\end{array}$ $\begin{array}{llllll}6 \times & 36.7 & 36.5 & 36.4 & 36.0 & 36.8 \\ 7 \times & 35.9 & 35.7 & 35.6 & 35.4 & 35.2\end{array}$ $\begin{array}{llllll}8 \times & 35.0 & 34.8 & 34.6 & 34.3 & 34.0 \\ 9 \times & 33.4 & 32.4 & 30.7 & 29.2 & 27.9\end{array}$ $9 \times 33.4$
$10 \times 26.8$

percent total distance

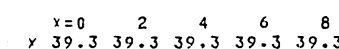

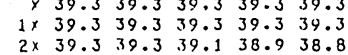
$\begin{array}{llllll}2 \times & 39.3 & 39.3 & 39.1 & 38.9 & 38.8 \\ 3 \times & 38.6 & 38.4 & 38.3 & 38.1 & 38.0\end{array}$ $4 \times \quad 37.9 \quad 37.8 \quad 37.7 \quad 37.6 \quad 37.6$ $\begin{array}{llllll}5 \times & 37.5 & 37.3 & 37.1 & 36.9 & 36.8\end{array}$ $6 \times 36.636 .5 \quad 36.3 \quad 36.236$. $7 \times 35.935 .7 \quad 35.5 \quad 35.3 \quad 35$.

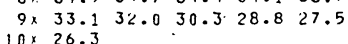

Table E11
Speed Profile for M813-XM(130 mm)

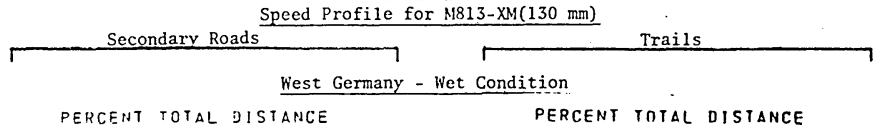

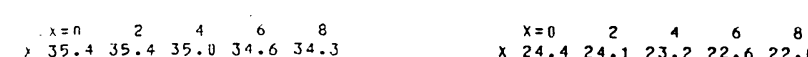

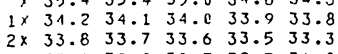

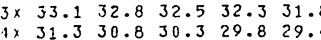

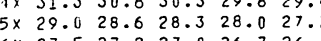

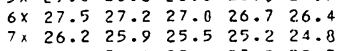
$8 \times 24.424 .0 \quad 23.6 \quad 23.2 \quad 22.7$

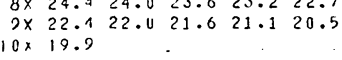
West Germany - Snow Condition

PERCENT TOTAL DISTANCE

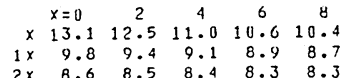

$\begin{array}{llllll}1 \times & 9.8 & 9.4 & 9.1 & 8.9 & 8.7 \\ 2 \times & 8.6 & 8.5 & 8.4 & 8.3 & 8.3 \\ 3 \times & 8.2 & 8.2 & 8.1 & 8.1 & 8.0 \\ 4 x & 8.0 & 8.0 & 7.9 & 7.9 & 7.9\end{array}$

$\begin{array}{llllll}3 \times & 8.2 & 8.2 & 8.1 & 8.1 & 8.0 \\ 4 \times & 8.0 & 8.0 & 7.9 & 7.9 & 7.9\end{array}$

$\begin{array}{llllll}5 x & 7.8 & 7.8 & 7.8 & 7.8 & 7.7 \\ 6 x & 7.7 & 7.7 & 7.6 & 7.6 & 7.6 \\ 7 x & 7.6 & 7.5 & 7.5 & 7.5 & 7.5\end{array}$ $\begin{array}{llllll}7 \times & 7.6 & 7.5 & 7.5 & 7.5 & 7.5 \\ 8 x & 7.5 & 7.4 & 7.4 & 7.4 & 7.3\end{array}$ $\begin{array}{rr}9 \times & 7.1 \\ 10 \times & 1.3\end{array}$ Mid-East - Wet Condition

\section{percent total Distance} $\begin{array}{rlcccc} & x=0 & 2 & 4 & 6 & 8 \\ x & 35.4 & 35.4 & 35.4 & 35.4 & 35.3 \\ 1 x & 34.9 & 34.7 & 34.5 & 34.4 & 34.3 \\ 2 x & 44.3 & 34.2 & 34.1 & 34.1 & 34.0 \\ 3 x & 3.0 & 33.9 & 33.9 & 3.9 & 33.0\end{array}$

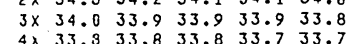
$\begin{array}{llllll}4 x & 33.8 & 33.8 & 33.8 & 33.7 & 33.7 \\ 5 x & 33.7 & 33.6 & 33.6 & 33.5 & 33.3\end{array}$ $7 \times 33.732 .9431 .6 \quad 32.5352 .03$ $\begin{array}{llllll}8 \times & 29.7 & 28.8 & 27.9 & 27.2 & 26.5 \\ 9 \times & 25.9 & 25.3 & 24.8 & 24.3 & 23.9\end{array}$ $9 \times 25.9$
$10 \times 23.2$

Mid-E

Dereent cotal distance

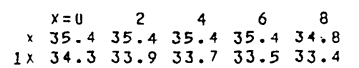
$\begin{array}{lllllll}1 \times & 34.3 & 33.9 & 33.7 & 33.5 & 33.4 \\ 2 \times & 33.3 & 33.2 & 33.1 & 33.0 & 33.0\end{array}$ $\begin{array}{lll}32.7 & 32.7\end{array}$ $\begin{array}{llllll}4 \times & 32.8 & 32.8 & 32.7 & 32.7 & 32.7 \\ 5 \times & 32.6 & 32.4 & 32.3 & 32.0 & 31.6\end{array}$ $\begin{array}{llllll}6 \times & 31.3 & 30.9 & 30.7 & 30.4 & 30.1 \\ 7 \times & 29.7 & 30.4 & 20.9 & 20.4 & 27.8\end{array}$ $\begin{array}{llllll}7 \times & 29.7 & 29.4 & 28.9 & 28.4 & 27.8 \\ 8 \times & 27.1 & 25.9 & 25.0 & 24.1 & 23.3\end{array}$

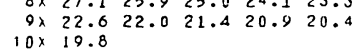

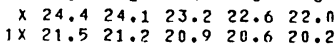
$\begin{array}{lllllll}2 \times & 19.9 & 19.7 & 19.4 & 19.1 & 18.8\end{array}$

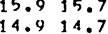
$\begin{array}{llllll}6 x & 14.6 & 14.4 & 14.2 & 14.1 & 13.9\end{array}$ $8 \times 11.7 \quad 11.311 .0010 .7 \quad 10.4$

PERCENT TOTAL DISTANCE

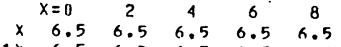
$\begin{array}{llllll}x & 6.5 & 6.5 & 6.5 & 6.5 & 6.5 \\ 1 \times & 6.5 & 6.5 & 6.5 & 6.5 & 6.5 \\ 2 \times & 6.5 & 0.5 & 6.5 & 0.5 & 6.5\end{array}$ $\begin{array}{llllll}2 x & 6.5 & 6.5 & 6.5 & 6.5 & 6.5 \\ 3 x & 6.5 & 6.5 & 6.5 & 6.4 & 6.4\end{array}$ $\begin{array}{llllll}4 x & 6.5 & 6.5 & 6.5 & 6.4 & 6.4 \\ 5 x & 6.4 & 6.4 & 6.4 & 6.4 & 6.4\end{array}$ $\begin{array}{llllll}5 x & 6.4 & 6.4 & 5.3 & 6.3 & 6.3 \\ 6 x & 6.3 & 6.3 & 6.3 & 6.3 & 6.2\end{array}$ $\begin{array}{llllll}7 \times & 6.2 & 6.2 & 6.1 & 6.1 & 5.1 \\ 8 \times & 6.0 & 6.0 & 6.0 & 5.9 & 5.9\end{array}$

Percent total distance

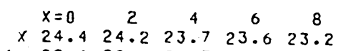
$\begin{array}{llllll}1 \times & 22.6 & 22.1 & 21.7 & 21.3 & 20.9 \\ 2 x & 20.6 & 20.3 & 20.1 & 19.9 & 19.7\end{array}$ $\begin{array}{llllll}3 \times & 19.4 & 19.1 & 18.9 & 18.7 & 18.5\end{array}$ $\begin{array}{lllllll}4 \times & 18.3 & 18.0 & 17.6 & 17.2 & 16.9 \\ 5 \times & 16.6 & 16.3 & 16 . & 15.9 & 15.9\end{array}$ $\begin{array}{llllll}5 \times & 16.6 & 16.3 & 16.1 & 15.9 & 15.9\end{array}$ $\begin{array}{llllll}7 x & 14.8 & 14.4 & 14.25 & 15.1 & 14.9\end{array}$ $\begin{array}{lllllll}8 \times & 14.0 & 13.8 & 13.4 & 12.9 & 12.4 \\ 9 \times & 12.0 & 11.7 & 11.3 & 11.1 & 10.8\end{array}$ $10 \times 0.4$ PERLEn: total distance

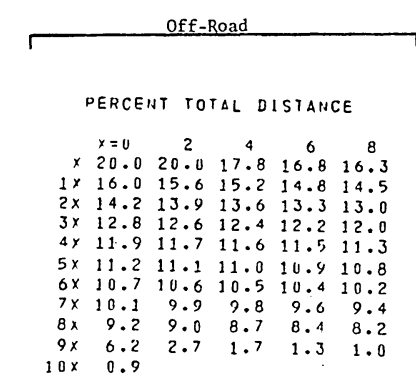

PERCENT TOTAL DISTANCE

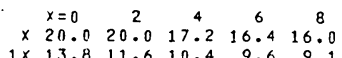
$\begin{array}{lllllll}2 \times & 8.7 & 8.4 & 8.2 & 8.0 & 7.8 \\ 3 \times & 7.0 & 7.5 & 7.4 & 0.3 & 0.0\end{array}$ $\begin{array}{llllll}3 \times & 7.6 & 7.5 & 7.4 & 7.3 & 7.2 \\ 4 \times & 7.1 & 7.0 & 6.9 & 6.8 & 6.7\end{array}$ $\begin{array}{llllll}5 \times & 7.1 & 7.0 & 6.9 & 0.8 & 0.7 \\ 5 \times & 6.0 & 6.5 & 6.4 & 6.2 & 6.1 \\ 6 x & 6.0 & 5.8 & 5.6 & 5.3 & 4.7\end{array}$ $\begin{array}{lllllll}6 x & 6.0 & 5.8 & 5.6 & 5.3 & 4.7 \\ 7 x & 2.7 & 1.6 & 1.1 & 0.9 & 0.7\end{array}$ $\begin{array}{llllll}8 \times & 0.6 & 0.6 & 0.5 & 0.5 & 0.4 \\ 9 \times & 0.4 & 0.4 & 0.4 & 0.3 & 0.3\end{array}$ $\begin{array}{ccc}10 \times & 0.0\end{array}$

PERCENT TOTAL DISTANCE

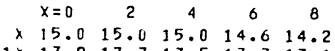
$\begin{array}{llllll}12 x & 13.9 & 13.7 & 13.5 & 13.6 & 13.1 \\ 3 \times & 12.9 & 12.8 & 12.7 & 12.5 & 12.4\end{array}$ $\begin{array}{cccccc}3 \times & 12.2 & 12.0 & 11.8 & 11.7 & 11.5 \\ 4 x & 11.4 & 11.2 & 11.0 & 10.9 & 10.7\end{array}$

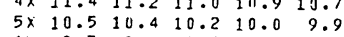
$\begin{array}{lllllll}6 \times & 9.7 & 9.6 & 9.5 & 9.3 & 9.9 \\ 7 \times & 8.9 & 8.7 & 8.5 & 9.7 & 9.1 \\ 0 \times & 7.0 & 7.4 & 7.1 & 0.7 & 8.1\end{array}$ $\begin{array}{llllll}8 \times & 7.8 & 7.4 & 7.1 & 0.7 & 0.3 \\ 9 \times & 3.4 & 2.0 & 1.4 & 1.1 & 0.3\end{array}$ $\begin{array}{rr}9 \times & 3.4 \\ 10 x & 0.8\end{array}$

PERCENT TOTAL DISTANCE $\begin{array}{rrrrrr}x=1 & 2 & 4 & 6 & 8 \\ x & 10.4 & 0.1 & 0.1 & 0.1 & 0.1 \\ 12 & 0.1 & 0.1 & 0.1 & 0.1 & 0.1 \\ 3 & 0.1 & 0.1 & 0.1 & 0.1 & 0.1\end{array}$ $\begin{array}{llllll}12 & 0.1 & 0.1 & 0.1 & 0.1 & 0.1 \\ 2 x & 0.1 & 0.1 & 0.1 & 0.1 & 0.1\end{array}$ $\begin{array}{llllll}3 x & 0.1 & 0.1 & 0.1 & 0.1 & 0.1 \\ 4 x & 0.1 & 0.1 & 0.1 & 0.1 & 0.1 \\ 5 y & 0.1 & 0.1 & 0.1 & 0.1 & 0.1\end{array}$ $\begin{array}{llllll}5 x & 0.1 & 0.1 & 0.1 & 0.1 & 0.1 \\ 6 x & 0.1 & 0.1 & 0.1 & 0.1 & 0.1 \\ 8 x & 0.1 & 0.1 & 0.1 & 0.1 & 0.1\end{array}$ $\begin{array}{lllllll}7 \times & 0.1 & 0.1 & 0.1 & 0.1 & 0.1\end{array}$ $\begin{array}{llllll}8 . & 0.1 & 0.1 & 0.1 & 0.1 & 0.1 \\ 9, & 0.1 & 0.1 & 0.1 & 0.1 & 0.1\end{array}$ $\begin{array}{cccccc}1 \times & 0.2 & 0.1 & 0.2 & 0.3 & 0.2 \\ 2 x & 0.1 & 0.1 & 0.1 & 0.1 & 0.1 \\ 3 x & 0.1 & 0.1 & 0.1 & 0.1 & 0.1\end{array}$ $\begin{array}{llllll}4 \times & 0.1 & 0.1 & 0.1 & 0.1 & 0.1 \\ 4 \times & 0.1 & 0.1 & 0.1 & 0.1\end{array}$ $\begin{array}{llllll}5 x & 0.1 & 0.1 & 0.1 & 0.1 & 0.1 \\ 6 x & 0.1 & 0.1 & 0.1 & 0.1 & 0.1 \\ 0 x & 0.1 & 0.1 & 0.1 & 0.1 & 0.1\end{array}$ $\begin{array}{llllll}7 \times & 0.1 & 0.1 & 0.1 & 0.1 & 0.1 \\ 8 \times & 0.1 & 0.1 & 0.1 & 0.1 & 0.1\end{array}$ $\begin{array}{rrrrrr}9 \times & 0.1 & 0.1 & 0.1 & 0.1 & 0.1 \\ 10 x & 0.1 & 0.1 & 0.1 & 0.1 & 0.1\end{array}$ $\begin{array}{lllll}x=0 & 2 & 4 & 6 & 8\end{array}$ 


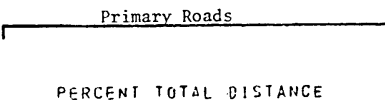

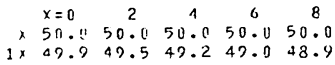
$2 \times 48.748 .6 \quad 48.548 .448$. $4 \times \quad 47.8 \quad 47.4 \quad 46.9 \quad 46.5 \quad 46$. $5 \times 45.6 \quad 45.1 \quad 44.6 \quad 43.8 \quad 43$.

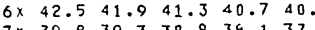
$\begin{array}{llllll}7 \times & 39.8 & 39.3 & 38.8 & 38.7 & 37.3 \\ 8 \times & 36.4 & 35.5 & 34.6 & 33.7 & 32.7\end{array}$

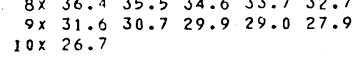

PERCENT TOTAL DISIANCE

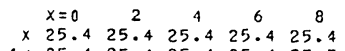
$\begin{array}{llllll}1 \times & 25.4 & 25.4 & 25.4 & 25.4 & 25.3 \\ 2 x & 25.3 & 25.3 & 25.3 & 25.3 & 25.3 \\ 3 \times & 25.2 & 25.3 & 25.3 & 25.3 & 25.2\end{array}$

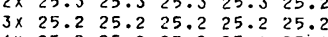
$\begin{array}{llllll}4 \times & 25.2 & 25.25 & 25.2 & 25.1 & 25.1 \\ 5 \times & 25.0 & 25.0 & 25.9 & 24.8 & 24.6\end{array}$ $\begin{array}{llllll}6 \times & 24.5 & 24.3 & 24.2 & 24.1 & 23.9 \\ 7 x & 23.8 & 23.7 & 23.6 & 23.5 & 23.4\end{array}$ $\begin{array}{llllll}8 \times & 23.2 & 23.0 & 22.7 & 22.5 & 23.4 \\ 9 \times & 21.7 & 21.4 & 21.1 & 20.5 & 20.2\end{array}$ $\begin{aligned} 9 \times & 21.7 \\ 10 \times & 19.7\end{aligned}$

Percent iotal oIsIANCE

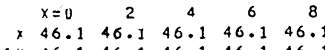
$\begin{array}{llllll}1 \times & 46.1 & 46.1 & 46.1 & 46.1 & 46.1 \\ 2 \times & 46.1 & 46.1 & 45.8 & 45.4 & 45.1 \\ 3 \times & 44.9 & 44.7 & 44.5 & 44.3 & 44.1\end{array}$ $\begin{array}{llllll}4 x & 44.0 & 43.9 & 43.7 & 43.6 & 43.6 \\ 5 x & 43.5 & 43.3 & 43.1 & 42.9 & 42.8\end{array}$ $\begin{array}{lllllll}6 x & 42.6 & 42.4 & 42.3 & 42.1 & 41.9 \\ 7 x & 41.7 & 41.6 & 41.4 & 41.2 & 40.9\end{array}$ $\begin{array}{llllll}8 \times & 40.6 & 40.3 & 40.0 & 39.5 & 39.0 \\ 9 \times & 38.1 & 36.7 & 34.5 & 32.6 & 30.9\end{array}$ $9 \times 38.1$
$10 \times \quad 29.4$

PERcent total Distance

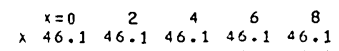
$\begin{array}{llllll}1 \times & 46.1 & 46.1 & 46.1 & 46.1 & 46.1 \\ 2 \times & 46.1 & 46.1 & 45.7 & 45.4 & 45.1\end{array}$ $3 \times 44.9 \quad 44.6 \quad 44.5 \quad 44.3 \quad 44.1$ $\begin{array}{llllll}4 x & 44.0 & 43.9 & 43.7 & 43.6 & 43.5 \\ 5 x & 43.4 & 43.3 & 43.1 & 42.9 & 42.8\end{array}$ $6 \times \quad 42.6 \quad 42.4 \quad 42.2 \quad 42.1 \quad 41.9$ $\begin{array}{llllll}8 \times & 40.5 & 40.1 & 39.7 & 39.2 & 38.7 \\ 9 \times & 37.7 & 36.2 & 34.0 & 32.0 & 30.3\end{array}$ $\begin{aligned} 9 \times & 37.7 \\ 15 \times & 28.8\end{aligned}$
Table E12
ofile for $M 6$

Table E12
Secondary Roads
Peest Germany - Wet Condition
PERCENT TUTAL DISTANCE

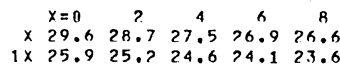

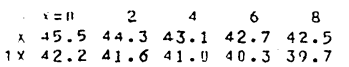

$\begin{array}{llllll}2 \times & 39.2 & 38.7 & 38.3 & 38.0 & 37.7 \\ 3 \times & 37.4 & 37.7 & 30.36 & 38.2 & 35\end{array}$

$\begin{array}{lllllll}4 \times & 35.4 & 35.0 & 34.7 & 34.3 & 33.9\end{array}$

$\begin{array}{lllllll}5 \times & 33.5 & 33.1 & 32.7 & 32.3 & 32.0 \\ 6 \times & 31.7 & 31.4 & 31.0 & 33.0 & 32.0\end{array}$

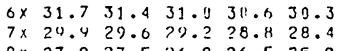

$\begin{array}{llllll}8 \times & 27.9 & 27.5 & 26.9 & 26.5 & 25.9 \\ 3 \times & 25.3 & 24.7 & 24.2 & 23.6 & 22.8\end{array}$

West Ger

$\begin{array}{llllll}1 \times & 25.9 & 25.2 & 24.6 & 24.1 & 23.6 \\ 2 \times & 23.2 & 23.0 & 22.7 & 22.4 & 23.0 \\ 3 x & 21.7 & 21.5 & 2.7 & 20.4 & 20.3\end{array}$

$\begin{array}{lllllll}4 \times & 19.7 & 19.1 & 18.5 & 18.1 & 17.7\end{array}$

$\begin{array}{lllllll}5 \times & 17.3 & 17.0 & 16.8 & 16.5 & 16.3\end{array}$

$10 \times 2 \times 2.0$
19 $14.7 \quad 14.514 .3$ $\begin{array}{rrrrrrr}8 \times & 14.1 & 13.9 & 13.8 & 13.6 & 13.5 \\ 9 \times & 13.3 & 13.2 & 13.1 & 17.8 & 17.4\end{array}$

PERCENT TOTAL DISTANCE

condition

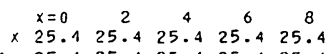

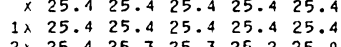

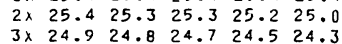

$\begin{array}{llllll}4 \times & 24.2 & 23.9 & 23.8 & 23.6 & 23.4 \\ 5 \times 23.3 & 23.1 & 23.0 & 22.8 & 23.4\end{array}$

$\begin{array}{lllll}6 \times 22.6 & 22.5 & 22.3 & 22.2 & 22.7\end{array}$

$\begin{array}{llllll}7 \times & 21.8 & 21.6 & 21.3 & 21.1 & 20.9 \\ 8 \times & 20.6 & 20.3 & 20.0 & 19.6 & 19.2\end{array}$

$\begin{array}{llllll}8 \times & 20.6 & 20.3 & 20.0 & 19.6 & 19.2 \\ 9 x & 18.8 & 18.5 & 18.1 & 17.7 & 17.1\end{array}$

$10 \times 16.5$

\section{Mid-East - Wet Condition}

PERCENT TOTAL DISIANTE

PERCENT IUTAL DISTANCE

$\begin{array}{ccccc}x=0 & 2 & 4 & 6 & 8 \\ x \quad 24.2 & 24.0 & 23.3 & 22.4 & 21.9\end{array}$

$1 \times 21.6 \quad 21.321 . ? \geqslant 0.920 .6$

$\begin{array}{llllll}2 x & 2 n .3 & 20.1 & 19.9 & 19.7 & 10.6 \\ 3 x & 10.3 & 10.1 & 19.9 & 10.6 & 18.5\end{array}$

$\begin{array}{llllll}3 \times & 19.3 & 19.1 & 18.9 & 18.6 & 18.2\end{array}$

$\begin{array}{llllll}5 \times & 16.1 & 15.9 & 15.7 & 15.5 & 15.3\end{array}$

$\begin{array}{llllll}6 x & 15.1 & 15.0 & 14.8 & 14.7 & 14.6 \\ 7 \times & 14.4 & 14.6 & 14.8 & 13.0 & 13.6\end{array}$

$\begin{array}{llllll}7 x & 14.4 & 14.2 & 14.0 & 13.8 & 13.6 \\ 8 \times & 13.5 & 13.3 & 13.7 & 13.0 & 12.9\end{array}$

$9 \times 12.812 .7 \quad 12.612 .311 .9$

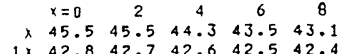

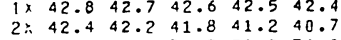

$\begin{array}{llllll}3 \times & 40.2 & 39.8 & 39.5 & 39.2 & 38.9 \\ 4 \times & 38.7 & 38.5 & 38.3 & 38.1 & 37.9\end{array}$

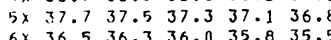

$7 \times \quad 35.3 \quad 35.034 .5 \quad 34.033 .5$

$\begin{array}{llllll}8 \times & 32.7 & 31.5 & 30.4 & 29.5 & 28.6 \\ 0 \times & 27.6 & 77.1 & 26.5 & 25.9 & 25.4\end{array}$

$10 \times 24.6$

\section{Mid-East - Sand Conditio}

PERCENT TOTAL DISTANCE

\section{Pertent intal distance}

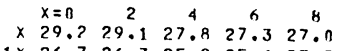

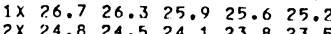

$\begin{array}{llllll}3 \times & 23.2 & 23.0 & 22.9 & 22.6 & 22.4\end{array}$

$\begin{array}{llllll}4 \times & 22.1 & 21.9 & 21.4 & 20.9 & 20.4\end{array}$

$6 \times 19.819 .3 \quad 18.9 \quad 18.518 .1$

$\begin{array}{ll}0.5 & 0\end{array}$

$\begin{array}{llllll}8 \times & 15.8 & 15.6 & 15.4 & 15.1 & 14.9 \\ 9 x & 14.7 & 14.5 & 14.3 & 14.2 & 13.9\end{array}$

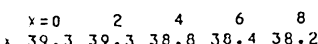

$\begin{array}{llllll}1 \times & 38.1 & 38.1 & 38.0 & 38.0 & 37.9 \\ 2 \times & 37.9 & 37.7 & 37.5 & 37.2 & 37.0\end{array}$

$4 \times 36.3 \quad 36.2 \quad 36.1 \quad 36.0 \quad 35.0$

$5 \times 35.6 \quad 35.4 \quad 35.3 \quad 35.1 \quad 34.8$

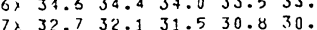

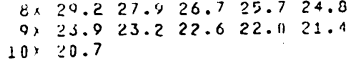

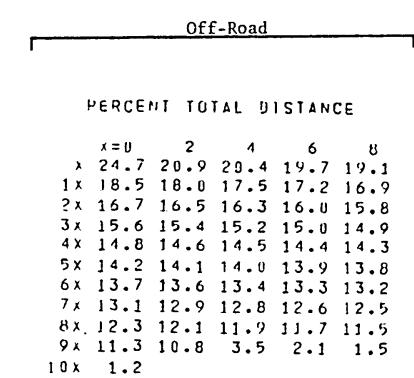

PERCENT TUTAL DISTANCE

$\begin{array}{cccccc}x=0 & 2 & 4 & 6 & 8 \\ x & 20.5 & 20.0 & 19.4 & 68.7 & 18.2 \\ x & 17.8 & 17.5 & 17.2 & 18.7 & 18.2\end{array}$ $2 \times \quad 16.5 \quad 16.3 \quad 16.2 \quad 16.0 \quad 15.8$ $\begin{array}{llllll}3 \times & 15.6 & 15.5 & 15.3 & 15.1 & 15.8 \\ 4 \times & 14.8 & 14.7 & 14.5 & 14.4 & 14.8\end{array}$ $\begin{array}{lllllll}4 \times & 14.8 & 14.7 & 14.5 & 14.4 & 14.2 \\ 5 \times & 14.1 & 14.0 & 3.9 & 13.8 & 13.0 \\ 7 \times & 12.5 & 13.4 & 3.9 & 13.8 & 13.6\end{array}$ $\begin{array}{llllll}6 x & 13.5 & 13.4 & 13.3 & 13.1 & 13.0 \\ 7 x & 12.9 & 12.8 & 12.6 & 12.5 & 12.3\end{array}$ $\begin{array}{rrrrrr}8 \times & 12.1 & 11.9 & 11.7 & 11.6 & 11.4 \\ 9 \times & 11.2 & 10.8 & 3.6 & 2.1 & 1.5\end{array}$

$10 \times \quad 1.2$

PERCEN TOTAL DISIANCE

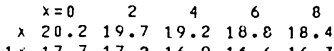
$\begin{array}{llllll}1 \times & 17.7 & 17.2 & 16.9 & 16.6 & 16.3 \\ 2 x & 16.1 & 15.8 & 15.5 & 15.3 & 15.1 \\ 3 x & 14.9 & 15.8 & 14.5 & 14.3 & 14.2\end{array}$

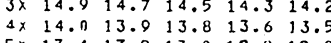
$\begin{array}{llllll}5 \times & 13.4 & 13.2 & 13.0 & 13.6 & 12.5 \\ 6 x & 12.7 & 12.5 & 12.0 & 12.9 & 12.0\end{array}$ $\begin{array}{llllll}6 \times & 12.7 & 12.5 & 12.4 & 12.3 & 12 . \\ 7 x & 12.0 & 11.9 & 1.4 & \end{array}$ $\begin{array}{rrrrrr}8 \times & 11.1 & 10.8 & 10.4 & 10.1 & 5.4 \\ 9 x & 2.5 & 1.6 & 1.2 & 1.11 & 0.8\end{array}$

PERCENT TOTAL DISTANCE

$\begin{array}{lccccc}x=0 & 2 & 4 & 6 & 8 \\ , & 15.0 & 15.0 & 14.8 & 14.5 & 14.4\end{array}$ $\begin{array}{lllllll}1 \times & 14.0 & 13.6 & 13.3 & 13.1 & 12.9\end{array}$ $\begin{array}{llllll}12 x & 12.7 & 12.5 & 12.4 & 12.2 & 12.9 \\ 3 x & 11.8 & 11.6 & 11.4 & 11.3 & 12.0 \\ 4 x & 11.0 & 10.8 & 10.6 & 0.3 & 9.9\end{array}$ $\begin{array}{rrrrrrr}4 x & 11.0 & 10.8 & 10.6 & 10.3 & 9.9 \\ 5 x & 9.4 & 2.9 & 1.4 & 1.0 & 0.7\end{array}$ $\begin{array}{llllll}6 \times & 0.6 & 0.5 & 0.5 & 0.4 & 0.4 \\ 7 \times & 0.4 & 0.3 & 0.3 & 0.3 & 0.3\end{array}$ $\begin{array}{llllll}87 & 0.3 & 0.3 & 0.2 & 0.7 & 0.2\end{array}$ $\begin{array}{cc}9 \times & 0.2 \\ 10, & 0.2\end{array}$ 
Table E13

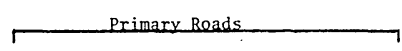

PERCENT tOTAL DISTANCE

$\begin{array}{rrrrrr}x=11 & 2 & 4 & 6 & 8 \\ \times \quad 4 & 1.4 & 41.4 & 11.4 & 41.4 & 41.4\end{array}$

$2 \times 41.4 \quad 41.4 \quad 41.4 \quad 41.4 \quad 41.3$

$3 \times 41.241 .141 .0 \quad 10.940 .0$

$5 \times 38.8 \quad 38.3 \quad 37.8 \quad 37.2 \quad 36.0$

$6 \times 36.0 \quad 35.4 \quad 34.9 \quad 34.4 \quad 33.9$

$\begin{array}{llllll}7 \times & 33.5 & 33.2 & 32.8 & 32.3 & 31.9 \\ 8 \times & 31.2 & 30.7 & 30.1 & 29.5 & 28.7\end{array}$

$\begin{array}{lll}9 \times & 28.0 \\ 10 \times & 24.3 & \end{array}$

PERCENT TUTAL DISIANCE

$\begin{array}{llllll}x=0 & & 2 & 4 & 6 & 8 \\ x & 17.7 & 17^{2} .7 & 17.7 & 17.7 & 17^{8} .7\end{array}$

$\begin{array}{lllllll}1 x & 17.7 & 17.7 & 17.7 & 17.6 & 17.6 \\ 2 x & 17.5 & 17.5 & 17.4 & 17.6 & 17.6 \\ 3 x & 17.3 & 17.02 & 17.4 & 17.4 & 17.4\end{array}$

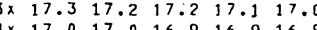

$\begin{array}{llllll}4 \times & 17.0 & 17.0 & 16.9 & 16.9 & 16.8 \\ 5 \times & 16.8 & 16.8 & 16.8 & 16.7 & 16.7 \\ 7 \times & 16.5 & 10.5 & 16.4 & 10.0 & 16.6\end{array}$

$6 \times \quad 16.716 .7 \quad 16.6 \quad 10.6 \quad 16.6$

$\begin{array}{lllllll}8 \times & 16.3 & 16.3 & 16.2 & 16.1 & 16.0 \\ 9 x & 16.0 & 15.9 & 15.8 & 15.7 & 15.4\end{array}$

$10 \times 15$

PERCENT TOTAL DISTANCE

$\begin{array}{rrrrrr} & x=0 & 2 & 4 & 6 & 8 \\ x \quad 39.1 & 39.1 & 39.1 & 39.1 & 39.1 \\ 1 \times & 39.1 & 39.1 & 39.1 & 39.1 & 39.1\end{array}$

$2 \times 39.1 \quad 39.1 \quad 38.9 \quad 38.7 \quad 38$.

$\begin{array}{cccccc}3 \times & 38.4 & 38.2 & 38.1 & 38.0 & 37.9 \\ 4 \times & 37.9 & 37.8 & 37.7 & 37.6 & 37.4 \\ 5 \times & 37.3 & 37.2 & 37.0 & 36.0 & 36.6\end{array}$

$5 \times 37.3 \quad 37.237 .036 .836 .4$

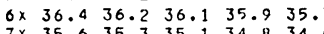

$7 \times 35.6 \quad 35.3 \quad 35.1 \quad 34.834 .6$

$\begin{array}{lllllll}8 \times & 34.4 & 34.2 & 34.0 & 33.7 & 33 . \\ 9 \times & 32.8 & 31.8 & 30.2 & 28.8 & 27.5\end{array}$

$10 \times 26.4$

PERCENT TOTAL DISTANCE

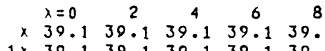
$2 \times 39.139 .1 \quad 38.9 \quad 38.7 \quad 38.5$ $\begin{array}{llllll}3 \times & 38.4 & 38.2 & 38.1 & 38.0 & 37.9 \\ 4 \times & 37.9 & 37.8 & 37.7 & 37.5 & 37.4\end{array}$

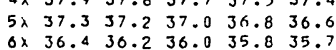
$7 \times 35.5 \quad 35.2 \quad 35.0 \quad 34.8 \quad 34.5$ $\begin{array}{lllllll}8 \times & 34.3 & 34.1 & 33.9 & 33.5 & 33.1 \\ 9 \times & 32.5 & 31.5 & 29.9 & 28.4 & 27.1\end{array}$
Speed Profile for M656-XM198

West Germany - Wet Condition

DERCENT TOTAL DISTANCE PERCENT TOTAL Distance

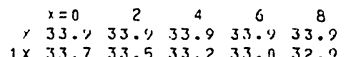

$2 \lambda \quad 32.7 \quad 32.5 \quad 32.3 \quad 32.1 \quad 31.9$

$\begin{array}{llllll}2 \lambda & 31.6 & 31.2 & 30.8 & 30.4 & 30.9 \\ 4 \lambda & 29.7 & 29.3 & 28.9 & 28.5 & 28.2\end{array}$

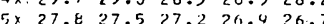

$6 \times \quad 26.4 \quad 26.2 \quad 26.0 \quad 25.7 \quad 25.4$

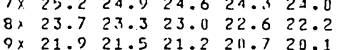

$10 \times 19.6$

$$
\text { West }
$$

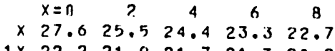

$2 \times 20.620 .42 n, ? .19 .8$

$\begin{array}{llllll}3 \times & 19.3 & 19.1 & 18.8 & 18.4 & 17.9\end{array}$

$4 \times 17.517 .116 .716 .416 .2$

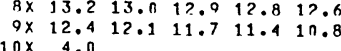

West Germany - Snow Condi

percent IUTAL Distance

PFRCENT TOTAL DISTANCE

$\begin{array}{rrrrrr} & x=0 & 2 & 4 & 6 & 8 \\ x & 17.7 & 11.7 & 17.7 & 17.7 & 17.7 \\ 1 \times & 17.7 & 11.7 & 17.7 & 17.7 & 17.7 \\ 2 x & 17.0 & 17.6 & 17.5 & 17.5 & 17.4\end{array}$

$2 \times 17.6 \quad 17.6 \quad 17.5 \quad 17.5 \quad 17.4$

$\begin{array}{llllll}3 \times & 17.4 & 17.3 & 17.3 & 17.2 & 17.2 \\ 4 \times & 17.1 & 17.0 & 17.0 & 16.9 & 16.8\end{array}$

$5 \times 16.8 \quad 16.7 \quad 16.6 \quad 16.5 \quad 16.4$

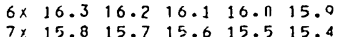

$\begin{array}{lllllll}8 x & 15.3 & 15.2 & 15.0 & 14.8 & 14.7 \\ 9 x & 14.5 & 14.3 & 14.1 & 13.7 & 13.4\end{array}$

Mid-East - Wet Condition

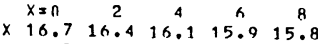

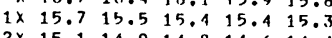

$3 x+14.214 .914 .094 .6514 .4$

$\begin{array}{lllllll}4 \times & 13.5 & 13.4 & 13.3 & 13.2 & 13.1\end{array}$

$\begin{array}{llllll}5 \times & 13.0 & 12.9 & 12.8 & 12.7 & 12.5\end{array}$

$7 x \quad 11.9211 .812 .2512 .112 .0$

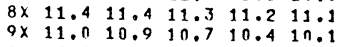

PERCENT TUTAL DISTANCE

PERCENT TOTAL DISTANCE

$\begin{array}{rrcccc} & x=0 & 2 & 4 & 6 & 8 \\ x & 33.9 & 33.9 & 43.9 & 33.9 & 33.9 \\ 1 \times & 33.9 & 33.9 & 33.9 & 33.9 & 33.9 \\ 2 x & 33.9 & 33.8 & 33.7 & 33.06 & 33.6\end{array}$

$\begin{array}{llllll}2 \times & 33.8 & 33.8 & 33.7 & 33.6 & 33.6 \\ 3 \times & 33.5 & 33.4 & 33.5 & 33.2 & 33.1\end{array}$

$5 \times 32.632 .5232 .932 .032 .7$

$6 \times 32.0 \quad 31.8 \quad 31.7 \quad 31.431 .1$

$7 \times 30.930 .6 \quad 30.3 \quad 30.029 .7$

$\begin{array}{llllll}8 \times & 29.1 & 28.2 & 27.4 & 26.7 & 26.1 \\ 9 \times & 25.5 & 24.9 & 21.4 & 24.11 & 23.5\end{array}$

Mid-East - Sand Condition

PERCENT TUTAL UISTANCE

$\begin{array}{llcccc}x=0 & 2 & 4 & 6 & 8 \\ \times & 25.4 & 25.3 & 25.2 & 25.1 & 24.6\end{array}$

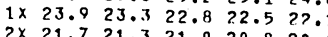

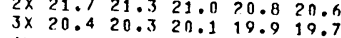

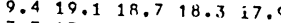

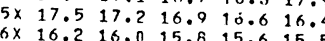

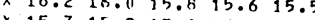
$8 \times 14.414 .214 .014 .814 .6$ $\begin{array}{cccccc}9 \times & 13.3 & 12.8 & 4.7 & 2.4 & 1.6\end{array}$

PERGENT TOTAI DISTANCE

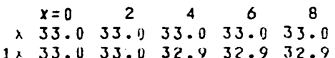

$2 \times 32.9 \quad 32.9 \quad 32.7 \quad 32.9 \quad 32.8$

$\begin{array}{lllllll}3 \times & 32.7 & 32.6 & 32.6 & 32.5 & 32.5\end{array}$

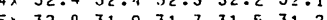

$\begin{array}{llllll}6 \times & 30.9 & 30.6 & 30.3 & 30.1 & 29.8 \\ 7 \times & 29.5 & 29.2 & 28.7 & 28.2 & 27.6\end{array}$

$\begin{array}{llllll}8 \times & 26.9 & 25.8 & 21.4 & 24.0 & 23.2 \\ 97 & 22.5 & 21.9 & 21.4 & 20.8 & 20.4\end{array}$

$\begin{array}{rl}9 & 22.5 \\ 10 \times 19.7 & \end{array}$

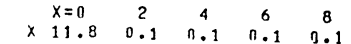
$\begin{array}{llllll}1 \times & n .1 & 0.1 & 0.1 & n .1 & 0.1\end{array}$ $\begin{array}{llllll}2 \times & 0.1 & 0.1 & 0.1 & 0.1 & 0.1 \\ 3 x & 0.1 & 0.1 & 0.1 & 0.1 & 0.1\end{array}$ $6 \times \begin{array}{llllll}6 \times & 0.1 & 0.1 & 0.1 & 0.1 & n .1 \\ 8 \times & 0.1 & 0.1 & 0.1 & 0.1 & 0.1\end{array}$ $\begin{array}{llllll}7 x & 0.1 & 0.1 & 0.1 & 0.1 & 0.1 \\ 8 x & 0.1 & 0.1 & 0.1 & 0.1 & 0.1\end{array}$ $\begin{array}{llllll}8 \times & 0.1 & 0.1 & 0.1 & 0.1 & 0.1 \\ 0 \times & 0.1 & n .1 & 0.1 & 0.1 & 0.1\end{array}$ $\begin{array}{llllll}5 x & 0.1 & 0.1 & 0.1 & n .1 & 0.1\end{array}$
$\Gamma$

PERCENT TOTAL DISIANCE

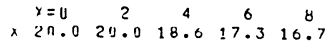

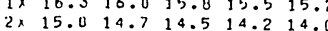
$\begin{array}{llllll}3 \times & 13.8 & 13.6 & 13.4 & 13.2 & 13.0\end{array}$ $5 \times 12.1 \quad 12.011 .911 .811 .7$

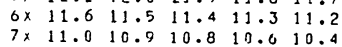

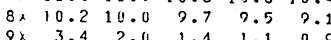
$10 \wedge \quad 0.0$

FERCENT TUTAL DISIANCE

$\begin{array}{rrrrrr}x=0 & 2 & 4 & 6 & 8 \\ \times & 20.0 & 20.0 & 17.3 & 60.5 & 16.1\end{array}$ $\begin{array}{lllllll}1 \times & 15.8 & 15.3 & 14.9 & 14.6 & 14.2\end{array}$ $\begin{array}{llllll}2 x & 12.8 & 12.6 & 13.4 & 13.2 & 13.0 \\ 4 x & 12.1 & 12.7 & 12.5 & 12.3 & 12.2\end{array}$ $\begin{array}{lllllll}4 \times & 12.1 & 11.9 & 11.8 & 11.7 & 11.5 \\ 5 \times & 11.4 & 11.3 & 11.2 & 11.1 & 11.5\end{array}$ $\begin{array}{llllll}5 \times & 11.4 & 11.3 & 11.2 & 11.1 & 11.0 \\ 6 \times & 10.9 & 10.8 & 10.7 & 10.6 & 10.5 \\ 7 x & 10.3 & 10.2 & 10.0 & 0.9 & 0.5\end{array}$ $\begin{array}{llllll}8 \times & 9.6 & 7.3 & 8.9 & 0.3 & 2.8 \\ 0 x & 1.7 & 1.3 & 1.0 & 0.9 & 0.7\end{array}$ $\begin{array}{ccc}80 x & 0.6 \\ 10 \times & 0.7\end{array}$

fEREent TOTAL DISTAHCE

$\begin{array}{rrrrrr} & x=0 & 2 & 4 & 6 & 8 \\ \times & 18.3 & 17.0 & 17.1 & 16.3 & 16.0\end{array}$ $\begin{array}{llllll}1 \times & 15.8 & 15.5 & 15.1 & 14.8 & 14.5 \\ 2 \times & 14.3 & 14.0 & 13.8 & 13.6 & 13.4\end{array}$ $\begin{array}{cccccc}3 \times & 13.2 & 13.1 & 12.9 & 12.7 & 12.4 \\ 4 \times & 12.4 & 12.3 & 12.2 & 12.1 & 12.0\end{array}$ $\begin{array}{lllllll}5 x & 11.9 & 11.8 & 11.7 & 11.6 & 11.5 \\ 6 x & 11.4 & 11.3 & 11.2 & 11.6 & 10.5\end{array}$ $\begin{array}{llllll}6 \times & 11.4 & 11.3 & 11.2 & 11.1 & 10.9 \\ 7, & 10.7 & 10.4 & 10.2 & 14.0 & 9\end{array}$ 8) $9.50 .5 \quad 8.9 \quad 4.1 \quad 2.2$ $\begin{array}{lll}0 \times & 0 . \\ 10 \times & 0.5\end{array}$

"ERCEnT tOTAL DISTANCE

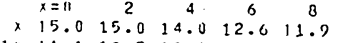
$\begin{array}{llllll}2 \times & 0.4 & 0.3 & 0.3 & 0.2 & 0.2 \\ 3 \times & 0.2 & 0.2 & 0.2 & 0.2 & 0.2\end{array}$ $\begin{array}{lllllll}4 \lambda & 0.2 & 0.2 & 0.2 & 0.1 & 0.1 \\ 5 x & 0.1 & 0.1 & 0.1 & 0.1 & 0.1 & 0.1\end{array}$ $\begin{array}{lllllll}5 x & 0.1 & 0.1 & 0.1 & 0.1 & 0.1 \\ 7 x & 0.1 & 0.1 & 0.1 & 0.1 & 0.1\end{array}$ $\begin{array}{cccccc}8 \times & 0.1 & 0.1 & 0.1 & 0.1 & 0.1 \\ 0 \times & 0.1 & 0.1 & 0.1 & 0.1 & 0.1\end{array}$ 


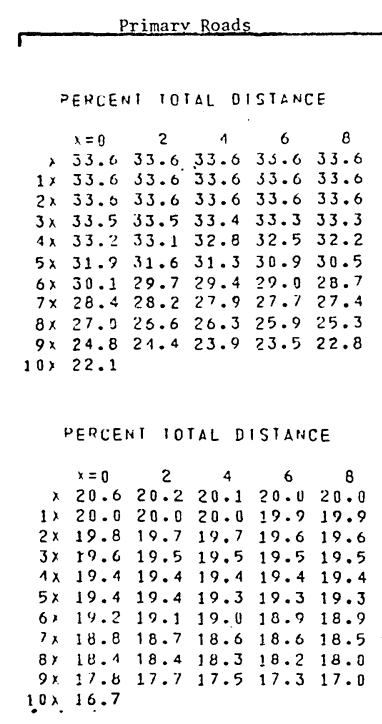

PERLENT TOTAL DISIANCE

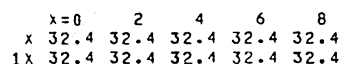

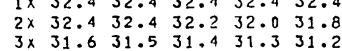
$\begin{array}{llllll}3 \times & 31.6 & 31.5 & 31.4 & 31.3 & 31.2 \\ 4 \times & 31.1 & 31.0 & 30.9 & 30.8 & 30.7\end{array}$ $\begin{array}{llllll}5 \times & 30.7 & 30.6 & 30.5 & 30.3 & 30.1 \\ 6 \times & 30.0 & 29.8 & 29.7 & 29.5 & 29.4 \\ 7 \times & 29.5 & 29.1 & 28.9 & 20.8 & 30.7\end{array}$ $\begin{array}{llllll}6 \times & 30.0 & 29.8 & 29.7 & 29.5 & 29.4 \\ 7 \times & 29.3 & 29.1 & 28.9 & 28.8 & 28.7\end{array}$ $\begin{array}{llllll}7 \times & 29.3 & 29.1 & 28.9 & 28.8 & 28.7 \\ 8 \times & 28.5 & 28.4 & 28.2 & 28.0 & 27.8\end{array}$ $\begin{aligned} & 9 \times \times 27.5 \\ & 10 \times 23.2\end{aligned}$

percent tutal distance

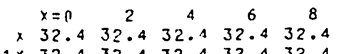
$2 \times 32.4 \quad 32.4 \quad 32.2 \quad 32.0 \quad 31.8$ $\begin{array}{llllll}3 \times & 31.6 & 31.5 & 31.4 & 31.3 & 31.2\end{array}$

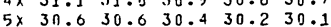
$\begin{array}{llllll}6 \times & 29.9 & 29.8 & 29.6 & 29.5 & 29.4 \\ 7 \times & 29.2 & 29.1 & 28.9 & 28.8 & 28.6\end{array}$

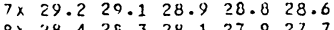

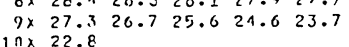

Table E14
Sneed Profile for TDN901-XM204

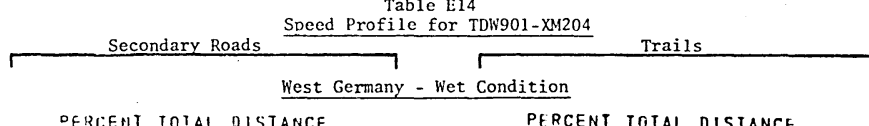

Dercent total DISTANCE

PERCENT TOTAL DISTANCE

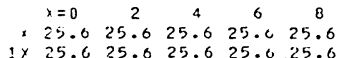

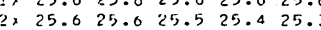

$\begin{array}{llllll}3 \times & 25.1 & 25.0 & 24.8 & 24.6 & 24.4\end{array}$

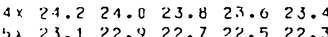

$6 \times 22.122 .1121 .821 .621 .4$

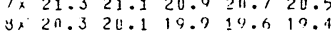

$9 \times 19.1$
$10 \times 17.5$

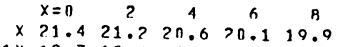

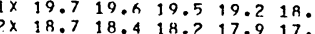

$\begin{array}{llllll}2 x & 18.7 & 18.4 & 18.7 & 17.9 & 17.7 \\ 3 x & 17.5 & 17.4 & 17 . ? & 17.1 & 17.0\end{array}$

$\begin{array}{llllll}4 \times & 16.9 & 16.8 & 16.7 & 16.6 & 16.5 \\ 5 \times & 16.3 & 16.8 & 16.7 & 16.0 & \end{array}$

$6 \times 15.8 \quad 15.7 \quad 15.6 \quad 15.5 \quad 15.4$

$\begin{array}{cccccc}7 \times & 15.3 & 15.7 & 15.1 & 15.0 & 14.9\end{array}$

$9 \times 14.2 \quad 14.1 \quad 13.8 \quad 14.6 \quad 14.4$

West Germany - Snow Condition

perrenit total mistance

PFRCENT TOTAL DISTANC:

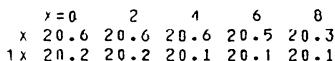

$\begin{array}{llllll}1 \times & 2 n .2 & 20.2 & 20.1 & 20.1 & 20.1 \\ 2 \times & 20.1 & 20.0 & 20.0 & 20.0 & 19.9\end{array}$

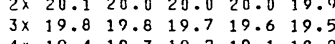

$\begin{array}{cccccc}4 \times & 19.4 & 19.3 & 19.2 & 19.1 & 18 . \\ 5 \times & 18.8 & 18.1 & 18.6 & 18.4 & 18 .\end{array}$

$\begin{array}{llllll}5 \times & 18.8 & 18.7 & 18.6 & 18.4 & 18.3 \\ 6 \times & 18.2 & 18.1 & 18.0 & 17.9 & 17.7 \\ 7 \times & 17.6 & 17.5 & 17.4 & 17.9 & 17.7\end{array}$

$\begin{array}{lllllll}8 \times & 16.9 & 16.7 & 16.5 & 10: 3 & 16.1 \\ 2 x & 15.8 & 15.6 & 15.4 & 15.1 & 14.7\end{array}$

Mid-East - Wet Condition

- percent total Distance

$\begin{array}{rccccc} & x=0 & 2 & 4 & 6 & 8 \\ \times & 25.6 & 25.0 & 25.6 & 25.0 & 25.6 \\ 1 \times & 25.0 & 25.06 & 25.6 & 25.0 & 25.6\end{array}$

$\begin{array}{llllll}1 \times & 25.6 & 25.6 & 25.6 & 25.6 & 25.6 \\ 2 \times & 25.6 & 25.6 & 25.6 & 25.6 & 25.6\end{array}$

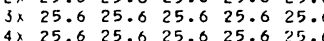

$\begin{array}{llllll}5 \times & 25.6 & 25.5 & 25.5 & 25.5 & 25.4 \\ 6 \times & 25.4 & 25.3 & 25.2 & 25.1 & 25.0\end{array}$

64.023 .523 .022 .622 .2
$\times 210$

$9 \times 21.8$
$10 \times 20.2$ Mid-E

$\begin{array}{cccccc}x=0 & 2 & 4 & 6 & 8 \\ x & 19.5 & 19.5 & 19.1 & 18.7 & 18.5\end{array}$ $\begin{array}{lllllll}1 \times & 18.4 & 18.3 & 18.2 & 18.0 & 17.5 \\ 2 x & 17.6 & 17.4 & 17.0 & \end{array}$ $3 \times 16.7 \quad 170.617 .2 \quad 17.016 .9$ $\begin{array}{llllll}3 \times & 16.2 & 16.1 & 16.0 & 15.9 & 15.8\end{array}$ $\begin{array}{llllll}5 \times & 15.7 & 15.6 & 15.5 & 15.4 & 15.3\end{array}$ $7 \times \quad 14.8 \quad 14.7 \quad 14.6 \quad 14.0 \quad 14.9$ $\begin{array}{llllll}8 \times & 14.4 & 14.3 & 14.2 & 14.1 & 14.0 \\ 9 \times & 13.9 & 13.7 & 13.6 & 13.2 & 13\end{array}$

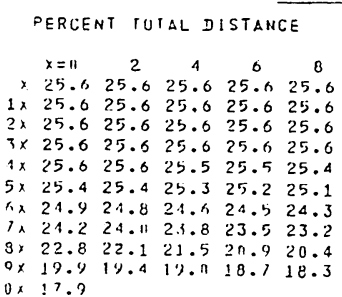

PERCFA TITAL DISTANCE

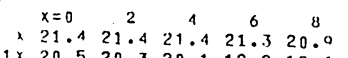
$\begin{array}{llllll}1 \times & 20.5 & 20.3 & 20.1 & 19.9 & 10.6 \\ 2 x & 19.4 & 19.1 & 10.0 & 18.8 & 18.7 \\ 3 x & 9 . .6 & 10.4 & 10.3 & 18.8 & 18.7\end{array}$

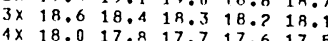

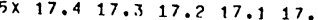
Sx $16.9 \begin{array}{llll}76.8 & 16.7 & 16.6 & 16 .\end{array}$

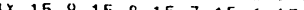
$7 x 15.215 .014 .8 \quad 14.6 \quad 14.4$ $\ln \times 13$.

PERCENT TOTLL DISIAHCE

$\begin{array}{cccccc}x=0 & 2 & 4 & 6 & 8 \\ -\quad x & 12.4 & 10.5 & 10.4 & 11.3 & 10.3 \\ 1 x & 10.3 & 10.3 & 10.3 & 10.2 & 100.3\end{array}$ $\begin{array}{llllll}2 \times & 10.2 & 10.2 & 10.2 & 11.2 & 10.2 \\ 3 x & 10.2 & 10.2 & 10.2 & 10.2 & 10.2\end{array}$ $\begin{array}{lllllll}3 \times & 10.2 & 10.2 & 10.2 & 10.2 & 10.2 \\ 4 x & 10.2 & 10.2 & 10.1 & 10.1 & 10.1\end{array}$ $5 x \quad 1 n .110 .1 \quad 1 n, 010.010 .0$ $\begin{array}{llllll}5 x & 9.9 & 9.9 & 9.9 & 9.8 & 9.8 \\ 7 \times & 9.8 & 9.7 & 9.6 & 9.6 & 9.5 \\ 9 x & 9.4 & 9.1 & 0.0 & 3.4 & 0.7\end{array}$ $\begin{array}{llllll}8 \times & 9.3 & 9.1 & 8.8 & 3.4 & 9.0 \\ 9 x & 1.4 & 1.1 & 0.9 & 0.8 & n .7\end{array}$

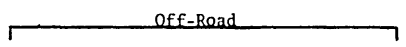

pertient total vistance

$\begin{array}{rrrrrr} & x=0 & 2 & 4 & 6 & 8 \\ \times & 21.0 & 20.0 & 18.1 & 17.0 & 16.4 \\ 1 \times & 16.1 & 15.9 & 15.6 & 15.3 & 15.1\end{array}$ 2) $14.914 .7 \quad 14.5 \quad 14.3 \quad 14.2$ $\begin{array}{llllll}3 x & 14.1 & 13.9 & 13.8 & 13.6 & 13.5 \\ 4 x & 13.3 & 13.2 & 13.1 & 12.9 & 12.8\end{array}$ $\begin{array}{lllllll}5 x & 12.7 & 12.6 & 12.5 & 12.4 & 12.3 \\ 3 \times & 12.2 & 12.1 & 12.1 & 12.0 & 11\end{array}$ $7 \times 11.8 \quad 11.7 \quad 11.611 .511 .4$

$\begin{array}{rrrrrr}8 \times & 11.3 & 11.2 & 11.0 & 10.9 & 10.7 \\ 9 x & 10.6 & 10.4 & 10.2 & 4.7 & 2.4 \\ 10 x & 1.6 & & & & \end{array}$

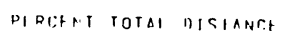

$\begin{array}{rlcccc}x=11 & 2 & 4 & 6 & 6 \\ \times & 210.0 & 20.0 & 18.4 & 17.2 & 16.0\end{array}$ $\begin{array}{cccccc}1 \times & 16.3 & 16.11 & 15.4 & 15.6 & 15.4 \\ 2 x & 15.3 & 15.1 & 15.0 & 14.4 & 14.8 \\ 3 x & 15.0 & 14.5 & 14.4 & 14.3 & 14.1\end{array}$ $\begin{array}{lllllll}3 \times & 14.6 & 14.5 & 14.4 & 14.3 & 14 . \\ 4 \times & 14.0 & 13.9 & 13.9 & 13.0 & 13\end{array}$ $\begin{array}{llllll}5 \times & 13.4 & 13.2 & 13.1 & 13.0 & 12.9\end{array}$

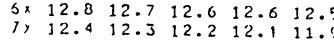
$\begin{array}{llllll}8 \times & 11.8 & 11.7 & 11.5 & 11.4 & 11.3 \\ 9 \times & 11.1 & 10.9 & 10.7 & 5.2 & 0.5\end{array}$

percent TOTAL DISIANCE

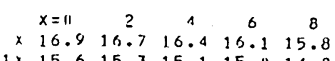
$\begin{array}{cccccc}1 \times & 15.6 & 15.3 & 15.4 & 15.0 & 15.8 \\ 2 \times & 14.0 & 14.4 & 15.2 & 15.0 & 1.4 .8\end{array}$ $\begin{array}{llllll}2 x & 14.6 & 14.4 & 14.2 & 14.1 & 13.9 \\ 3 x & 13.8 & 13.7 & 13.5 & 13.4 & 13.3\end{array}$

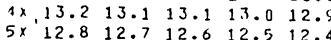
$\begin{array}{lllllll}5 \times & 12.3 & 12.2 & 12.1 & 12.11 & 11.9\end{array}$

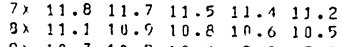
$\begin{array}{rr}9 \times & 10.3 \\ 10 \times & 3.1\end{array}$

PIREFT TOTAL DIGIAHA

$\begin{array}{lllll}x & x=0 \\ x & 2 & 4 & 6 & 8\end{array}$ $\begin{array}{llllll}x & 11.0 & 11.0 & 14.1 & 12.7 & 12.1 \\ 2 x & 11.0 & 11.2 & 10.9 & 111.7 & 10.5\end{array}$ $\begin{array}{cccccc}2 x & 10.3 & 11.2 & 11.1 & 10.11 & 0.9 \\ 3 x & 9.8 & 9.8 & 9.7 & 9.6 & 0.5 \\ 4 x & 0.5 & 9.4 & 9.3 & 9.3 & 0.2\end{array}$

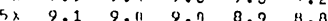
$\begin{array}{llllll}6 \times & 8.7 & 8.6 & 8.4 & 8.2 & 7.9\end{array}$ $\begin{array}{llllll}6 \times & 7.7 & 1.0 & 1.0 & 1.3 & 1.0\end{array}$ $\begin{array}{llllll}3 x & 11.8 & 1 . .7 & 0.6 & 1.5 & 0.5 \\ 9 x & 0.5 & 1 . .4 & 0.4 & 0.1 & 0.4\end{array}$ 


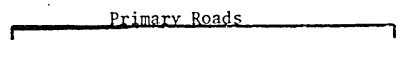

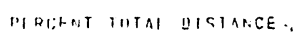

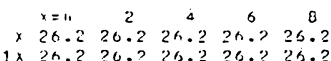
$\begin{array}{llllll}1 \times 2 h .2 & 20.2 & 215.2 & 20 . ? & 25.2 \\ 2 \times & 26.2 & 20.2 & 26.2 & 26.2 & 26.2 \\ 3 \times & 20.2 & 20.2 & 20.2 & 20.2 & 26.2\end{array}$ $1 \times 25.2206 .2$ $\begin{array}{llllll}5 \times & 25.7 & 25.5 & 25.4 & 25.2 & 25.0\end{array}$

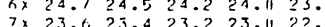
3) $22.722 .5 \quad 22.322 .121 .7$ $\begin{array}{ll}9 \times 2 & 21.4 \\ 10 \times & 19.5\end{array}$

PRPCHT TETAL MISIANT $\begin{array}{rrrrrr} & x=11 & 2 & 4 & 6 & 8 \\ x & 17.6 & 17.0 & 17.4 & 17.3 & 17^{8} \cdot 2\end{array}$ $\begin{array}{lllllll}2 \times & 17.1 & 17.1 & 17.1 & 17.1 & 17\end{array}$ $\begin{array}{cccccc}3 \times & 17.1 & 17.1 & 17.1 & 17.0 & 17.0\end{array}$

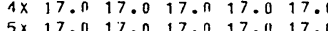
Sx $16.9 \quad 16.9 \quad 16.9$

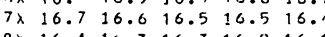
$\begin{array}{llllll}8 \times & 16.4 & 16.3 & 16.3 & 16.2 & 16.1 \\ \text { ax } & 16.0 & 15.9 & 15.8-15.7 & 15.4\end{array}$ and
$17 \times 15 . ?$

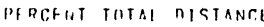

$\begin{array}{cccccc}x=0 & 2 & 1 & 6 & 8 \\ x & 26.1 & 26.1 & 26.1 & 20.1 & 26\end{array}$

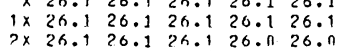
$3 \times 26.126 .026 .025 .925$. $\begin{array}{llllll}4 \times & 25.9 & 25.9 & 25.9 & 25.8 & 25.0 \\ 5 \times & 25.7 & 25.7 & 25.9 & 25.8 & 25.4\end{array}$

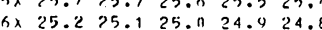
$\begin{array}{llllll}6 \lambda & 25.25 & 25.1 & 25.0 & 24.9 & 24.8 \\ 7 \lambda & 24.7 & 24.0 & 24.5 & 24.4 & 24.8\end{array}$

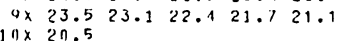

PERCFNT TIITAL DISIANSI

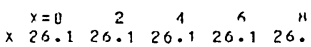
$\begin{array}{llllll}1 \times & 26.1 & 26.1 & 26.1 & 26.1 & 26.1 \\ 2 \times & 26.1 & 26.1 & 26.1 & 26.1\end{array}$

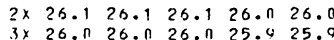

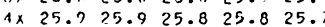
$\begin{array}{llllll}5 \times 25.7 & 25.7 & 25.6 & 25.5 & 25.3\end{array}$ $\begin{array}{llllll}\text { Sx } & 25.2 & 25.1 & 25.0 & 24.4 & 24.8\end{array}$

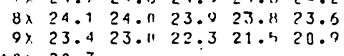
$\begin{array}{r}9 \times 23.4 \\ 10 \times 2 \pi .3 \\ \hline\end{array}$

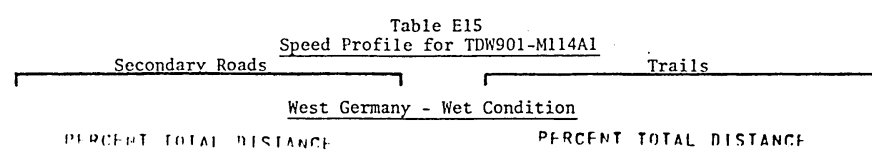

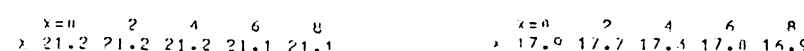

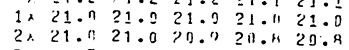

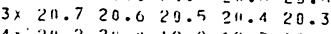

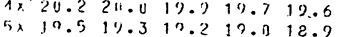

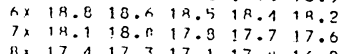
$\begin{array}{llllll}8 \times & 17.4 & 17.3 & 17.1 & 17.11 & 11.0 \\ 9 \times & 16.7 & 16.5 & 16.3 & 16.11 & 15.8\end{array}$ West Germany - Snow Condition

DHRGH THIAI DIGTANC, PERCENT IOTAL DISTANCE

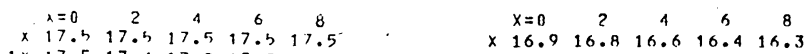

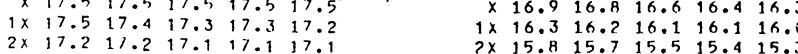

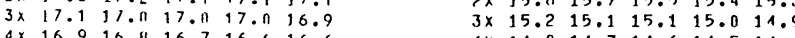
$\begin{array}{llllll}4 \times & 16.9 & 16.8 & 16.7 & 16.6 & 16.6 \\ 5 \times & 16.5 & 16.4 & 16.3 & 16.2 & 16.6\end{array}$ Sx $16.015 .915 .915 .8 \quad 15.7 \quad 15.6$ $7 \times 15.5 \quad 15.4 \quad 15.3 \quad 15.715 .1$ $\begin{array}{llllll}8 \times & 15.0 & 14.9 & 14.7 & 14.5 & 11.4 \\ O x & 14.2 & 14.1 & 13.9 & 13.6 & 13.3\end{array}$

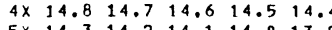

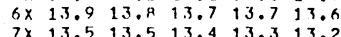
$7 \times 13.513 .513 .4 \quad 13.3 \quad 13.6$ $\begin{array}{rrrrrr}8 \times & 13.2 & 13.1 & 13.0 & 12.9 & 13.8 \\ 9 \times & 12.7 & 12.5 & 12.4 & 12.0 & 11.7\end{array}$ $10 \times 11.4$

Mid-East - Wet Condition

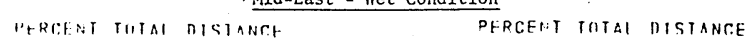

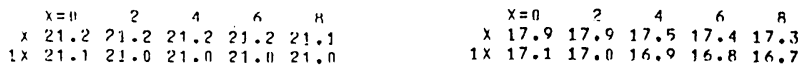

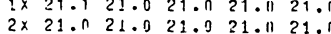

$3 x \quad 21.021 .0211 .020 .9210 .9$

$1 \times 211.9$
$5 \times$ 20.920 .9211 .920 .9

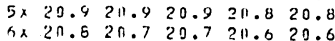

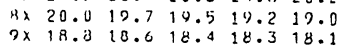

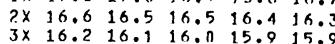

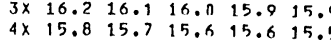

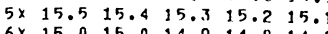
$10 \times 17.8$

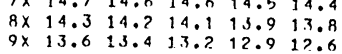
Mid-East - Sand-Gondition

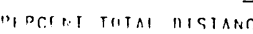
Percent total nistance

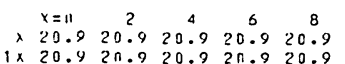

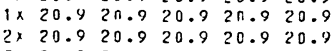
$\begin{array}{cccccc}1 \times & 20.9 & 20.9 & 20.9 & 20.9 & 20.8 \\ 5 \times 2 & 20.8 & 20.7 & 20.7 & 20.6 & 20.6\end{array}$ xx $20.6 \quad 20.5 \quad 20.5 \quad 20.4 \quad 20.3$ $\begin{array}{lllllll}8 \times & 10.4 & 10.0 & 18.0 & 18.2 & 17.8 \\ 0 \times & 17.5 & 17.2 & 17.0 & 10.1 & 15.5\end{array}$ 10.16 .1

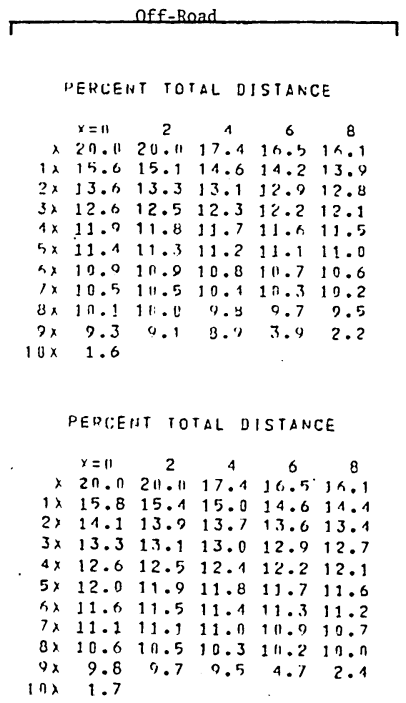

PIRIENT TOTOL DISIANCE

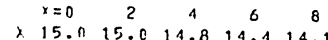

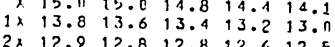

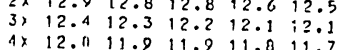
5x $11.6 \quad 11.5 \quad 11.4 \quad 11.3 \quad 11.3$

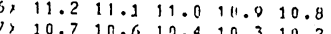
$\begin{array}{llllll}4 \times & 10.0 & 9.9 & 9.1 & 4.6 & 0.4 \\ 3 \times & 9.3 & 4.1 & 0.0 & 0.8 & 9.3\end{array}$ $10 \times \quad 2.8$

PFRCEHT TOTAL DISILNCE

$\begin{array}{lllll}x=11 & 2 & 4 & 6 \\ \times & 15.11 & 15.11 & 8\end{array}$

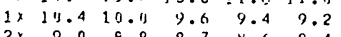
$\begin{array}{llllll}2 x & 0.0 & A .3 & 8.7 & 8.6 & 8.4 \\ 3 x & 8.3 & 0.2 & 8.0 & 7.7 & 6.4\end{array}$ $\begin{array}{llllll}4 x & 1.8 & 1.0 & 0.7 & 0.6 & 0.5 \\ 5 x & 0.4 & 11.4 & 0.3 & 0.3 & 0.5\end{array}$ $\begin{array}{llllll}3 x & 0.4 & 1.0 & 0.7 & 0.6 & 0.5 \\ 3 x & 0.3 & 0.3 & 0.2 & 0.2 & 0.3 \\ 0.2 & 0.2 & 0.2 & 0.2 & 0.2 & 0.2\end{array}$ $\begin{array}{llllll}7 \times & 0.2 & 11.2 & 0.2 & 0.2 & 0.2 \\ 8 x & 0.2 & 11.2 & 0.2 & 0.2 & 0.2\end{array}$ $\begin{array}{rrrrrr}9 \times & 0.2 & 1.2 & 0.2 & 0.2 & 0 . ? \\ 1 n \times & 0.2 & & & & \end{array}$ 


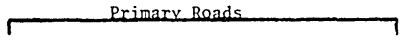

percent total distance

$\begin{array}{rrrrrr}x & =0 & 2 & 4 & 6 & 8 \\ x & 25.0 & 25.0 & 25.0 & 25.0 & 25.0 \\ 1 x & 25.0 & 25.0 & 24.0 & 24.08 & 24 .\end{array}$

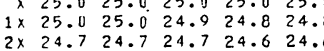
$3 \times 24.624 .624 .6 \quad 24.524 .5$

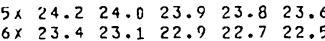
$\times 22.322 .222 .021 .921 .7$ $9 \times 20.520 .320 .1 \quad 19.8 .19 .4$ $10 \times 18.9$

PeRcent total distance

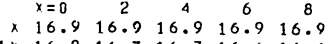

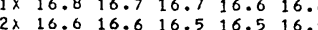
$\begin{array}{lllllll}3 \times & 16.5 & 16.5 & 16.5 & 16.5 & 16.5 \\ 4 \times & 16.5 & 16.5 & 16.5 & 16.4 & 16.5\end{array}$

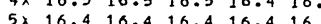
$7 \times 16.1 \quad 16.0 \quad 15.915 .916 .9$ $\begin{array}{llllll}8 \times & 15.8 & 15.7 & 15.7 & 15.6 & 15.5 \\ 9 \times & 15.4 & 15.3 & 15.3 & 15.2 & 15.0\end{array}$ $10 \times 14.7$

PERCENT IOTAL DISTANCE

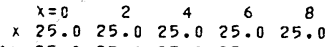

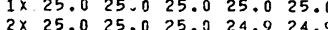
$\begin{array}{llllll}3 \times & 24.9 & 24.8 & 24.8 & 24.8 & 24.9\end{array}$ $\begin{array}{llllll}4 \times & 24.8 & 24.8 & 24.7 & 24.7 & 24.6\end{array}$

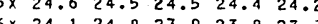

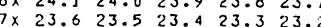
$\begin{array}{llllll}8 \times & 23.1 & 23.0 & 22.9 & 22.8 & 22.7 \\ 9 \times & 22.5 & 22.2 & 21.5 & 20.9 & 20.3\end{array}$ $10 \times 19.8$

Percent total distance

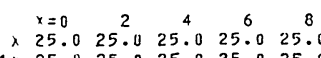
$\begin{array}{cccccc}1 \times & 25.0 & 25.0 & 25.0 & 25.0 & 25.0 \\ 2 \times & 25.0 & 25.0 & 25.0 & 24.9 & 24.0\end{array}$ $\begin{array}{llllll}2 x & 25.0 & 25.0 & 25.0 & 24.9 & 24.9 \\ 3 x & 24.9 & 24.8 & 24.8 & 24.8 & 24.8\end{array}$ $\begin{array}{llllll}4 \times & 24.8 & 24.7 & 24.7 & 24.7 & 24.6\end{array}$

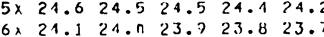

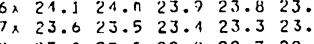
$\begin{array}{llllll}8 \times & 23.0 & 22.9 & 22.8 & 22.7 & 22.6 \\ 9 \times & 22.4 & 22.1 & 21.4 & 20.7 & 20.1\end{array}$ $10 \times 19.6$
Table E16

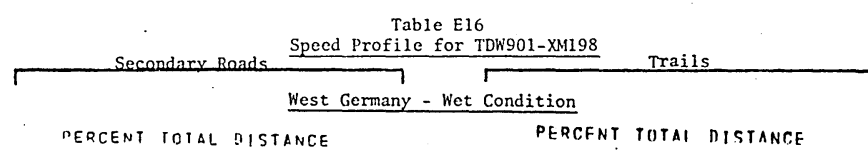

$\begin{array}{llllllll}x & x & 0 & 2 & 4 & 6\end{array}$

$\begin{array}{rrrrrr}\times & 20.5 & 20.5 & 20.5 & 20.4 & 20.3 \\ 12 & 20.2 & 20.2 & 20.2 & 20.2 & 20.1\end{array}$

$\begin{array}{llllll}2 \times & 20.1 & 20.1 & 20.1 & 20.0 & 20.0 \\ 3 \times & 19.9 & 19.8 & 19.7 & 19.6 & 19.6 \\ 5 \times & 19.4 & 10.3 & 10.2 & 19.6 & 19.6\end{array}$

$\begin{array}{cccccc}5 \times & 19.4 & 10.3 & 10.2 & 19.1 & 18.9\end{array}$

$\begin{array}{llllllll}5 \times & 18.8 & 18.7 & 18.5 & 18.4 & 18.3 \\ 6 \times & 18.5 & 18.0 & 17.9 & 18.4 & 18.6\end{array}$

$\begin{array}{llllll}6 \times & 18.1 & 18.0 & 17.9 & 17.8 & 17.6 \\ 7 \times & 17.5 & 17.4 & 17.3 & 17.2 & 17.0 \\ 8 \times & 10.9 & 16.7 & 16.6 & 16.5 & 16.4\end{array}$

$9 \times 16.2 \quad 16.0 \quad 15.8 \quad 15.6 .15 .3$

West

$\begin{array}{rlcccc} & x=0 & 2 & 4 & 6 & 8 \\ x & 16.7 & 16.6 & 16.3 & 16.2 & 16.1 \\ 1 \times & 16.0 & 16.0 & 15.0 & 15.8 & 15.7\end{array}$

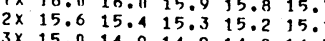

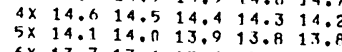

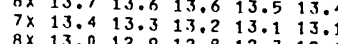

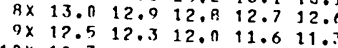

PERCENT TOTAL DISTANCE

PERCENT TOTAL DISTANCE

$\begin{array}{cccccc}x=0 & 2 & 4 & 6 & 8 \\ \times & 16.8 & 16.8 & 16.8 & 16.7 & 16.7 \\ 10 & 16.7 & 16.6 & 16.6 & 16.5 & 16.5\end{array}$

$\begin{array}{llllll}1 \times & 16.7 & 16.6 & 16.6 & 16.5 & 16.5 \\ 2 \times & 16.5 & 16.4 & 16.4 & 16.4 & 16.5 \\ 3 \times & 16.4 & 16.4 & 16.3 & 16.3 & 16.9\end{array}$

$\begin{array}{lllllll}3 \times & 16.4 & 16.3 & 16.3 & 16.3 & 16.3 \\ 4 \times & 16.2 & 16.1 & 16.1 & 16.0 & 15.9\end{array}$

$\begin{array}{llllllll}5 \times & 15.8 & 15.7 & 15.7 & 15.6 & 15.5\end{array}$

$6 \times 15.4 \quad 15.3 \quad 15.2 \quad 15.1 \quad 15.0$

$\begin{array}{llllll}7 \times & 14.9 & 14.8 & 14.8 & 14.7 & 14.6 \\ 8 \times & 14.4 & 14.3 & 14.1 & 14.0 & 13.9\end{array}$

$\begin{array}{rr}9 \times & 13.7 \\ 10 \times & 12.6\end{array}$$$
\text { Mid-E }
$$

\begin{tabular}{|c|}
\hline 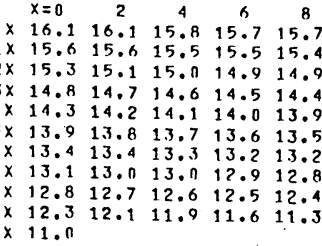 \\
\hline
\end{tabular}

PERCENT TOTAL DISTANCE

\section{Percent TOTAL Distance}

$\begin{array}{rccccc} & x=0 & 2 & 4 & 6 & 8 \\ x & 20.5 & 20.5 & 20.5 & 20.5 & 20.4 \\ 1 \times & 20.3 & 20.3 & 20.2 & 20.2 & 20.2\end{array}$

$\begin{array}{llllll}1 \times & 20.3 & 20.3 & 20.2 & 20.2 & 20.4 \\ 2 \times & 20.2 & 20.2 & 20.1 & 20.1 & 20.1\end{array}$

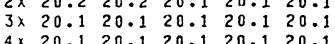

$\begin{array}{llllll}4 \times & 20.1 & 20.1 & 20.1 & 20.1 & 20.1 \\ 5 \times & 20.1 & 20.1 & 20.0 & 20.0 & 20.0\end{array}$

$\begin{array}{lllllll}7 \times & 19.8 & 19.7 & 19.6 & 19.5 & 19.4 \\ 8 \times & 19.3 & 19.1 & 18.8 & 18.6 & 18.4\end{array}$ $2 \times 18.2$

Mid-East

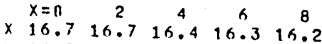

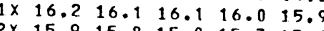
$3 \times 15.5 \quad 15.4 \quad 15.3 \quad 15.315 .6$ $4 \times 15.115 .115 .015 .014 .9$

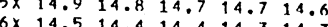
$7 x \quad 14.214 . ? \quad 14.1 \quad 14.013 .0$ $\begin{array}{llllll}8 X & 13.8 & 13.7 & 13.6 & 13.5 & 13.03 \\ 9 x & 13.1 & 12.0 & 17.6 & 12.3 & 10.0\end{array}$ $10 \times 10.3$

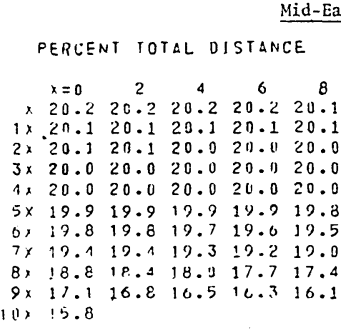

PERCENT TOTAL DISTANCE

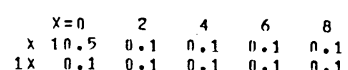

$\begin{array}{llllll}1 \times & 0.1 & 0.1 & 0.1 & 0.1 & n .1 \\ 2 \times & 0.1 & 0.1 & n .1 & 0.1 & n .1 \\ 3 x & 0.1 & 0.1 & 0.1 & 0.1 & n\end{array}$

$\begin{array}{llllll}3 \times & 0.1 & 0.1 & n .1 & n .1 & n .1 \\ 4 \times & 0.1 & 0.1 & n .1 & 0.1 & 0.1\end{array}$

$\begin{array}{llllll}5 x & 0.1 & 0.1 & n .1 & n .1 & n .1 \\ 6 x & 0.1 & n .1 & n .1 & 0.1 & n .1\end{array}$

$\begin{array}{llllll}6 x & n .1 & n .1 & n .1 & 0.1 & n .1 \\ 7 x & n .1 & n .1 & 0.1 & 0.1 & 0.1\end{array}$

$\begin{array}{llllll}8 x & n .1 & 0.1 & n .1 & 0.1 & n .1 \\ 0 \times & n=1 & n .1 & n .1 & 0.1 & n .1\end{array}$

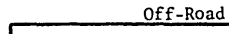

PERLENT TOTAL DISIANCE

$\begin{array}{cccccc}x=0 & 2 & 4 & 6 & 8 \\ \times & 20.0 & 20.0 & 17.3 & 10.4 & 16 .\end{array}$

$\begin{array}{lllllll}1 \times & 15.5 & 14.8 & 14.3 & 13.8 & 16.4 \\ 2 \times & 13.1 & 12.9 & 12.7 & 12.8 & 13.4 \\ 3 \times & 13.1 & 12.0 & 1 . .6 & 12.5 & 12\end{array}$

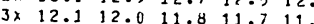

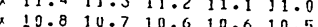

$\begin{array}{cccccc}6 x & 10.4 & 10.7 & 10.6 & 10.6 & 10.9 \\ 7 \times & 10.0 & 9.9 & 0.2 & 10.2 & 10.1\end{array}$

$\begin{array}{llllll}8 \times & 2.5 & 9.3 & 9.2 & 9.0 & 8.9\end{array}$

$\begin{array}{llllll}9 \times & 8.7 & 8.5 & 8.0 & 3.1 & 1.9 \\ 0 . x & 1.4 & & & & \end{array}$

PERCENT tUIAL Distance

$\begin{array}{cccccc}x=0 & 2 & 4 & 6 & 8 \\ x & 20.0 & 20.0 & 17.2 & 16.4 & 16.0 \\ 2 x & 15.0 & 15.2 & 19.2 & 16.3 & 14.1\end{array}$

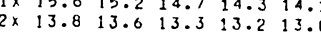

$\begin{array}{lllllll}3 x & 12.8 & 12.7 & 12.5 & 12.4 & 12.3 \\ 4 x & 12.1 & 12.0 & 11.0 & 11.4 & 12.7\end{array}$

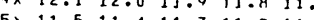

6x $11.0 \quad 10.9 \quad 10.910 .8 \quad 10.7$

$\begin{array}{rrrrrr}7 \times & 10.6 & 10.5 & 10.4 & 10.3 & 10.1 \\ 8 \times & 10.0 & 9.8 & 9.7 & 9.5 & 9.3\end{array}$

$\begin{array}{rr}9 \times & 9.1 \\ 10 \times & 1.6\end{array}$

percent tutal disiance

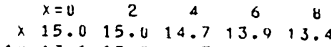
$\begin{array}{lllllll}1 \times & 13.1 & 12.9 & 12.7 & 12.0 & 12.4 \\ 2 x & 12.4 & 12.3 & 12.2 & 12.0 & 12.5 \\ 3 x & 11.9 & 11.0 & 11.2 & 11.7 & 12.6\end{array}$

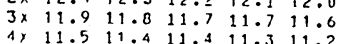
$\begin{array}{llllll}4 x & 11.5 & 11.4 & 11.4 & 11.3 & 11.2 \\ 5 x & 11.1 & 11.0 & 10.9 & 10.8 & 10.2\end{array}$

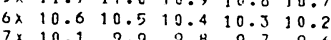
$\begin{array}{llllll}8 \times & 9.4 & 9.3 & 9.1 & 9.0 & 8.8 \\ 9 \times & 8.6 & 8.5 & 8.1 & 8.2 & 5.9\end{array}$

PERCEII tutal Distance

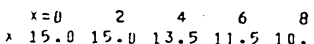
$\begin{array}{lllllll}1 \times & 10.0 & 9.4 & 8.9 & 4.9 & 0.7\end{array}$ $\begin{array}{llllll}2 x & 1.5 & 0.3 & 10.3 & 0.3 & 0.2 \\ 3 x & 0.2 & 0.2 & 0.2 & 0.2 & 0.2\end{array}$ $\begin{array}{llllll}4 x & 0.2 & 0.2 & 0.2 & 0.2 & 0.2 \\ 5 x & 0.1 & 0.1 & 0.2 & 0.1 & 0.1\end{array}$ $\begin{array}{llllll}5 x & 11.1 & 0.1 & 0.1 & 0.1 & 0.1 \\ 6 x & 0.1 & 0.1 & 0.1 & 0.1 & 0.1\end{array}$ $\begin{array}{llllll}7 \times & 0.1 & 0.1 & 0.1 & 0.1 & 0 . \\ 8 \times & 0.1 & 0.1 & 0.1 & 0.1 & 0.1\end{array}$ $\begin{array}{cccccc}9 x & 0.1 & 0.1 & 0.1 & 0.1 & 0.1 \\ 10 x & 0.1 & & & 0.1 & 0.1\end{array}$ 


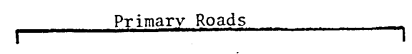

PERCENT TOTAL DISTANCE

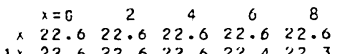
$2 \times 22.222 .122 .0222 .021 .9$ $\begin{array}{llllll}3 \times & 21.9 & 21.8 & 21.8 & 21.8 & 21.7 \\ 4 \times & 21.7 & 21.7 & 21.7 & 21.7 & 21.6\end{array}$ $5 \times 21.621 .521 .421 .3 \quad 21.2$ $\begin{array}{llllll}6 \times & 21.0 & 20.9 & 20.8 & 20.6 & 20.5 \\ 7 \times & 20.3 & 20.2 & 20.1 & 19.9 & 19.8\end{array}$ $\begin{array}{llllll}8 \times & 19.7 & 19.6 & 19.5 & 19.3 & 19.1 \\ 9 x & 18.9 & 18.8 & 19.6 & 18.4 & 18.0\end{array}$ $10 \times 17.7$

\section{PERCENT TOTAL DISTANCE}

$\begin{array}{rrrrrr}x=0 & 2 & 4 & 6 & 8 \\ x & 16.9 & 16.9 & 16.9 & 16.6 & 16.3 \\ 1 x & 16.1 & 16.0 & 15.9 & 15.8 & 15.7\end{array}$

$\begin{array}{llllll}1 \times & 16.1 & 16.0 & 15.9 & 15.8 & 15.7 \\ 2 \times & 15.7 & 15.7 & 15.6 & 15.6 & 15.6 \\ 3 \times & 15.0 & 15.5 & 55.5 & 15.5 & 15.5\end{array}$

$3 \times \quad 15.6 \quad 15.5 \quad 15.5 \quad 15.5 \quad 15.6$

$\begin{array}{llllll}4 \times & 15.5 & 15.5 & 15.5 & 15.5 & 15.5 \\ 5 \times & 15.5 & 15.4 & 15.4 & 15.4 & 15.4\end{array}$

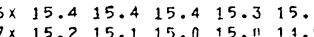

$\begin{array}{rrrrrrr}8 \times & 14.9 & 14.8 & 14.8 & 14.7 & 14.7 \\ 9 \times & 11.6 & 14.6 & 14.5 & 14.4 & 14.2 \\ 10 \times & 14.0 & & & & & \end{array}$

PERCENT tOTAL DISTANCE

$\begin{array}{rccccc} & x=0 & 2 & 4 & 6 & 8 \\ x & 22.6 & 22.6 & 22.6 & 22.6 & 22.5 \\ 1 \times 2 & 22.6 & 22.6 & 22.6 & 22.0 & 22.6\end{array}$

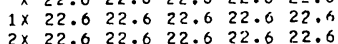

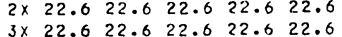

$\begin{array}{llllll}3 \times & 22.6 & 22.6 & 22.6 & 22.6 & 22.6 \\ 4 x & 22.6 & 22.6 & 22.6 & 22.6 & 22.5 \\ 5 x & 22.5 & 22.5 & 22.4 & 22.4 & 22.5\end{array}$

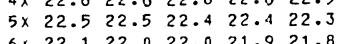

$\begin{array}{llllll}6 \times & 22.1 & 22.0 & 22.0 & 21.9 & 21.8 \\ 7 \times & 21.7 & 21.0 & 21.6 & 21.5 & 21.4\end{array}$

$\begin{array}{llllll}8 \times & 21.3 & 21.2 & 21.1 & 21.0 & 20.0 \\ 9 \times & 20.7 & 20.5 & 20.0 & 19.5 & 19.0\end{array}$

$10 \times 18.6$

-PERCENT TOTAL DISTANCE

$\begin{array}{rrrrrr} & x=0 & 2 & 4 & 6 & 8 \\ \times & 22.6 & 22.6 & 22.6 & 22.6 & 22.6 \\ 1 \times & 22.6 & 22.6 & 22.6 & 22.0 & 22.6\end{array}$

$\begin{array}{llllll}1 \times & 22.6 & 22.6 & 22.6 & 22.6 & 22.6 \\ 2 x & 22.6 & 22.6 & 22.6 & 22.6 & 22.6\end{array}$

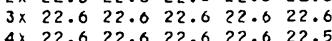

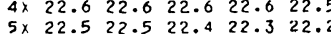

$\begin{array}{llllll}6 \times & 22.1 & 22.0 & 21.9 & 21.8 & 21.8 \\ 7 \times & 21.7 & 21.6 & 21.5 & 21.4 & 21.3\end{array}$

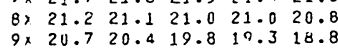

$\begin{array}{r}9 \times 20.7 \\ 10 \times 18.4 \\ \hline\end{array}$

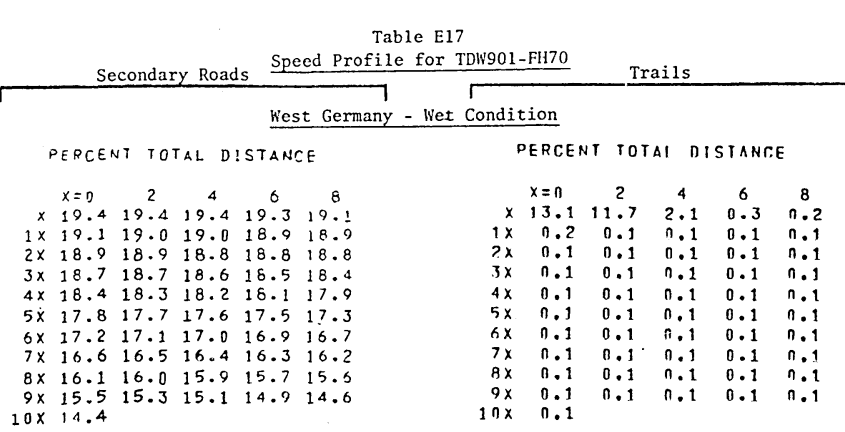

\section{West Germany - Snow Condition}

PERCENT TOTAL DISTANCE PERCENT total Distance

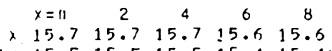

$2 \times 15.415 .4 \quad 15.415 .4$

$\begin{array}{llllll}3 \times & 15.4 & 15.4 & 15.3 & 15.3 & 15.3\end{array}$

$4 \times 15.315 .215 .115 .15: 5.0$

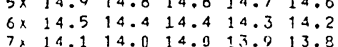

$\begin{array}{llllll}8 \times & 13.7 & 13.5 & 13.4 & 13.3 & 13.2 \\ 9 \times & 13.1 & 12.9 & 12.7 & 12.5 & 12.2\end{array}$

Mid-East - Wet Condition

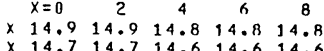

$2 \times 14.4 \quad 14.3 \quad 14.3 \quad 14.2 \quad 14.6$

$\begin{array}{llllll}3 \times & 14.0 & 13.9 & 13.8 & 13.8 & 13.7 \\ 1 \times & 13.6 & 13.5 & 13.4 & 13.3 & 13.7\end{array}$

$\begin{array}{llllll}5 \times & 13.2 & 13.1 & 13.0 & 12.9 & 13.2\end{array}$

$\begin{array}{llllll}5 x & 12.7 & 12.7 & 12.6 & 12.5 & 12.5\end{array}$

$\begin{array}{llllll}7 \times & 12.4 & 17.4 & 2.6 & 12.5 & 12.5 \\ 8 X & 12.1 & 17.0 & 17.0 & 12.2 & 12.2 \\ 8 \times & 1.7 & 11.9 & 11.8\end{array}$

$\begin{array}{ll}9 x & 12.1 \\ 10 \times & 10.3\end{array}$

PERCENT TOTAL DISTANCE

PERCENT IDTAL DISTANCE

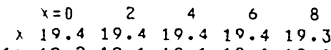

$\begin{array}{llllll}1 \times & 19.2 & 19.4 & 19.4 & 10.4 & 19.3 \\ 2 \times & 19.0 & 18.9 & 18.9 & 19.0 & 19.0\end{array}$

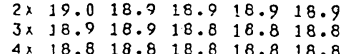

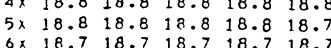

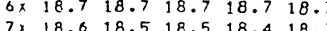

$\begin{array}{lllllll}8 \times & 18.2 & 18.0 & 17.9 & 17.7 & 17.5 \\ 9 \times & 17.4 & 17.3 & 17.1 & 17.0 & 16.9\end{array}$

Mid-E

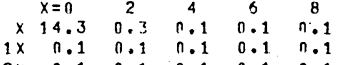

$\begin{array}{llllll}1 \times & n .1 & 0.1 & n .1 & 0.1 & n .1 \\ 2 x & n .1 & 0.1 & n .1 & 0.1 & n .1\end{array}$

$\begin{array}{llllll}3 x & 0.1 & 0.1 & n .1 & 0.1 & n .1 \\ 4 x & 0.1 & 0.1 & n .1 & 0.1 & n .1\end{array}$

$\begin{array}{llllll}5 x & 0.1 & n .1 & n .1 & 0.1 & n .1 \\ 6 x & 0.1 & n .1 & n .1 & 0.1 & n .1\end{array}$

$\begin{array}{llllll}7 x & 0.1 & n .1 & n .1 & 0.1 & n .1\end{array}$

$\begin{array}{llllll}8 \times & n .1 & n .1 & n .1 & n .1 & n \cdot 1 \\ 9 x & 0.1 & 0.1 & n .1 & 0.1 & n .1\end{array}$

id-East - Sand Condition

PERCENT TOTAL DISTANCE

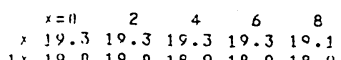

$\begin{array}{lllllll}1 \times & 19.0 & 19.0 & 18.9 & 18.9 & 18.9 \\ 2 \times & 18.9 & 18.9 & 18.8 & 18.8 & 18.8\end{array}$

$\begin{array}{llllll}3 \times & 18.8 & 18.8 & 18.8 & 18.8 & 18.0 \\ 5 & 18.8 & 18.8 & 18.8 & 18.8 & 18.0\end{array}$

5) $18.7 \quad 18.7 \quad 18.7 \quad 18.7 \quad 18.7$

$6 \times 18.7 \quad 18.5 \quad 18.6 \quad 18.5 \quad 18.5$

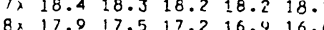

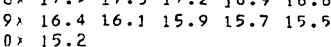

PHRCENT TOTAL DISTANCE

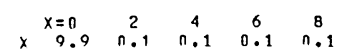

$\begin{array}{llllll}1 \times & 0.1 & 0.1 & n .1 & 0.1 & n .1 \\ 2 x & 0.1 & 0.1 & n=1 & n=1 & n .1\end{array}$

$\begin{array}{llllll}3 x & n .1 & 0.1 & n .1 & 0.1 & n .1 \\ 4 x & 0.1 & n .1 & n .1 & n .1 & n .1\end{array}$

$\begin{array}{llllll}4 x & 0.1 & n .1 & n .1 & 0.1 & n .1 \\ 5 x & n .1 & n .1 & n .1 & 0.1 & n .1\end{array}$

$\begin{array}{llllll}6 \times & 0.1 & 0.1 & n .1 & n .1 & n .1\end{array}$

$\begin{array}{llllll}7 x & n .1 & n .1 & n .1 & 0.1 & n .1 \\ 8 x & 0.1 & 0.1 & 0.1 & 0.1 & n .1\end{array}$

$\begin{array}{rrrrrr}9 x & 0.1 & 0.1 & 0.1 & 0.1 & n .1 \\ 10 x & 0.1 & & 0 & & \end{array}$

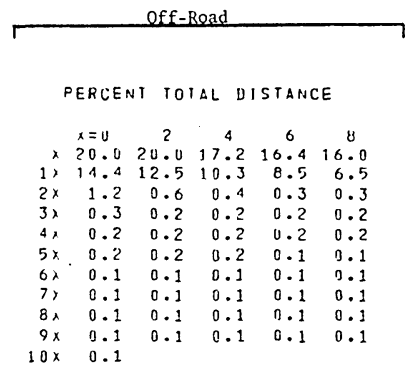

PERCENT TOTAL DISTANCE

$\begin{array}{ccccc}x=n & 2 & 4 & 6 & 8 \\ \times 20.0 & 20.0 & 17.2 & 16.4 & 16.0\end{array}$

$\begin{array}{lllllll}2 \times & 13.2 & 13.0 & 12.8 & 12.6 & 12.4\end{array}$

$3 \times 12.312 .112 .011 .911 .7$

$\begin{array}{llllll}4 \times & 11.6 & 11.5 & 11.4 & 11.2 & 11.1\end{array}$

$\begin{array}{lllllll}5 x & 11.0 & 10.9 & 10.8 & 10.7 & 10.6 \\ 6 x & 10.6 & 10.5 & 10.4 & 10.3 & 10.2\end{array}$

$\begin{array}{rrrrrr}7 \times & 10.1 & 10.0 & 9.9 & .8 & 0.7 \\ 8 \times & 0.6 & 9.4 & 9.3 & 0.1 & 8.8 \\ 9 \times & 0.6 & 0.4 & 9.0 & 9.4 & 2.3\end{array}$

$\begin{array}{rr}9 \times & 8.6 \\ 10 \times & 1.6\end{array}$

PERCENT IOTAL DISTANCE

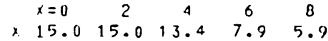

$\begin{array}{lllllll}1 \times & 5.0 & 4.4 & 4.0 & 2.9 & 0.8 \\ 2 \times & 0.5 & 0.3 & 0.3 & 0.3 & 0.2 \\ 3 \times & 0.2 & 0.2 & 0.2 & 0.2 & 0.2\end{array}$

$\begin{array}{llllll}3 \times & 0.2 & 0.2 & 0.2 & 0.2 & 0.2 \\ 3 \times & 0.2 & 0.2\end{array}$

$\begin{array}{llllll}4 x & 0.2 & 0.2 & 0.2 & 0.2 & 0.1 \\ 5 x & 0.1 & 0.1 & 0.1 & 0.1 & 0.1\end{array}$

$\begin{array}{llllll}5 \times & 0.1 & .1 & 0.1 & 0.1 & 0.1 \\ 7 x & 0.1 & 0.1 & 0.1 & 0.1 & 0.1\end{array}$

$\begin{array}{llllll}8 \times & 0.1 & 0.1 & 0.1 & 0.1 & 0.1 \\ 0 \times & 0.1 & 0.1 & 0.1 & 0.1 & 0.1\end{array}$

$10 \times 0.1$

PERCENT TOTAL DISTANCE

$\begin{array}{lllll},=0 & 2 & 1 & 6 & 8\end{array}$

$\begin{array}{lllllll}1 \times & 0.2 & 0.1 & 0.1 & 0.1 & 0.1\end{array}$

$\begin{array}{llllll}2 \times & 0.1 & 0.1 & 0.1 & 0.1 & 0.1 \\ 3 \times & 0.1 & 0.1 & 0.1 & 0.1 & 0.1\end{array}$

$\begin{array}{llllll}4 x & 0.1 & 0.1 & 0.1 & 0.1 & 0.1 \\ 5 x & 0.1 & 0.1 & 0.1 & 0.1 & 0.1\end{array}$

$\begin{array}{llllll}6 \lambda & 0.1 & 0.1 & 0.1 & 0.1 & 0.1 \\ 7 \lambda & 0.1 & 0.1 & 0.1 & 0.1 & 0.1\end{array}$

$\begin{array}{llllll}7 \lambda & 0.1 & 0.1 & 0.1 & 0.1 & 0.11 \\ 8 \gamma & 0.1 & 0.1 & 0.1 & 0.1 & 0.1\end{array}$

$\begin{array}{cccccc}9 \times & 0.1 & 0.1 & 0.1 & 0.1 & 0.1 \\ 10 \times & 0.1 & & & & \end{array}$ 


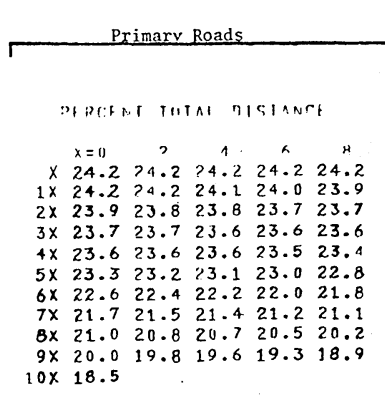

Dhocent ratal misianci.

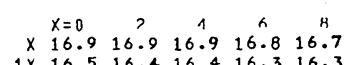

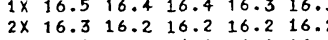
$\begin{array}{lllllll}3 \times & 16.2 & 16.2 & 16.2 & 16.1 & 16.1\end{array}$ $\begin{array}{llllll}4 \times & 16.1 & 16.1 & 16.1 & 16.1 & 16.1 \\ 5 \times & 16.1 & 16.1 & 16.1 & 16.1 & 16.0\end{array}$ $\begin{array}{llllll}6 \times & 16.0 & 16.0 & 16.0 & 15.9 & 15.8 \\ 7 \times & 15.8 & 15.7 & 15.6 & 15.6 & 15.5 \\ 6 \times & & \end{array}$ $\begin{array}{llllll}7 \times & 15.8 & 15.7 & 15.6 & 15.6 & 15.5 \\ 8 X & 15.4 & 15.4 & 15.3 & 15.3 & 15.2\end{array}$ $\begin{aligned} 9 \times & 15.1 \\ 10 \times & 14.5\end{aligned}$

PPRCHA TATAL DICIANC

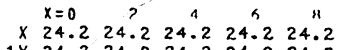

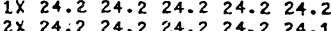
$\begin{array}{lllllll}3 \times & 24.1 & 24.1 & 24.1 & 24.1 & 24.1\end{array}$ $\begin{array}{llllll}4 \times & 24.1 & 24.1 & 24.0 & 24.0 & 24.0 \\ 5 \times & 23.9 & 23.9 & 24.0 & 24.7 & 24.0\end{array}$ $6 \times 23.523 .423 .8323 .723 .6$ $\begin{array}{llllll}7 \times & 23.0 & 22.9 & 22.8 & 22.7 & 23.6\end{array}$

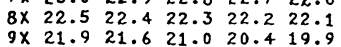
$9 \times 21.9$
$10 \times 19.4$

PRPCNI TITAL DISIANTE

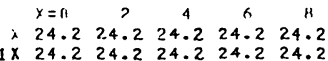

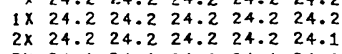
$\begin{array}{llllll}2 x & 24.2 & 24.2 & 24.2 & 24.2 & 24.1 \\ 3 \times & 24.1 & 24.1 & 24.1 & 24.1 & 24.1\end{array}$ $\begin{array}{llllll}4 \times & 24.1 & 24.0 & 24.0 & 24.0 & 23.9 \\ 5 \times & 23.5 & 23.9 & 23 . & 23 & 23.6\end{array}$ $\begin{array}{llllll}5 \times & 23.9 & 23.9 & 23.8 & 23.7 & 23.6\end{array}$

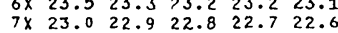
$\begin{array}{llllll}8 \times & 22.5 & 22.4 & 22.3 & 22.2 & 22.0 \\ 9 x & 21.8 & 21.5 & 20.9 & 20.3 & 19.7\end{array}$ $10 \times 19.2$
Table E18

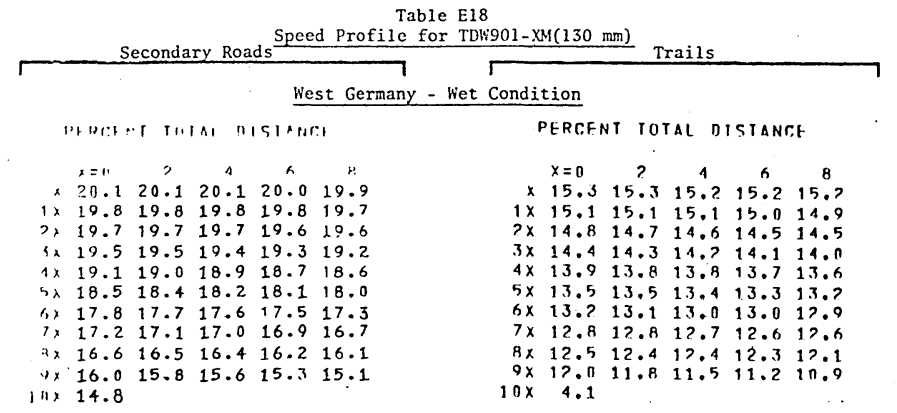

\section{West Germany - Snow Condition}

DIRCHNT TIITA DIGIANFI PERCFNT TOTAL DISTANCE

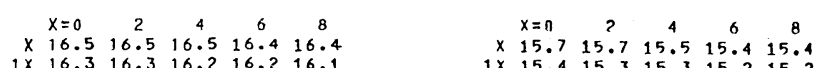

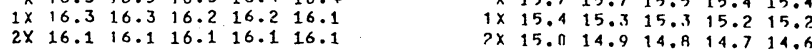

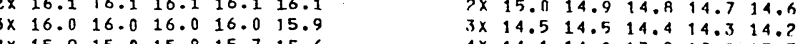

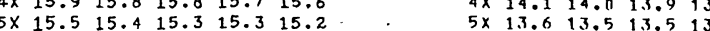

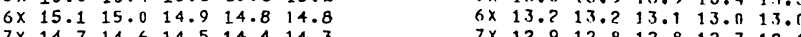

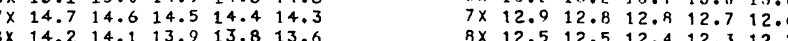

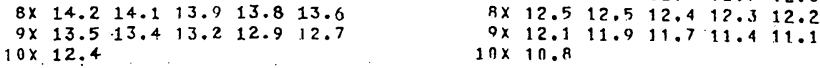

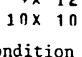

Mid-East - Wet Condition

PHDL:H THAL MIGTANCI PERCENT TOTAL MISIANTE

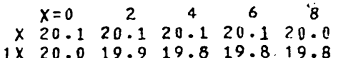
$2 \times 19.8 \quad 19.8 \quad 19.719 .7 \quad 19.7$ $3 \times 19.719 .7 \quad 19.7 \quad 19.719 .7$

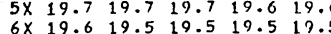
$7 \times 19.419 .319 .319 .2 \quad 10.1$

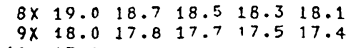
$10 \times 17.1$

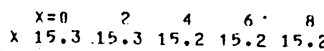

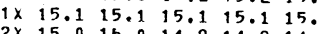
$3 \times 14,7 \quad 14,7 \quad 14,6 \quad 14,5,14,0$ $4 \times 14,4 \quad 24.414 .4414 .314 .0$

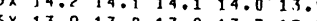

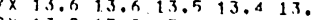

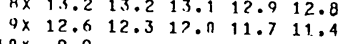

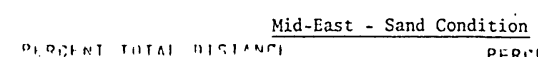
$\begin{array}{rlcccc} & x=0 & 2 & 4 & 6 & 8 \\ x & 19.9 & 19.9 & 19.9 & 19.9 & 19.8 \\ 1 \times & 19.8 & 19.7 & 19.7 & 19.7 & 19.7 \\ 2 \times & 19.7 & 19.7 & 19.7 & 19.7 & 19.7 \\ 3 & 19.7 & 19.7 & 19.7 & 19.7 & 19.6\end{array}$

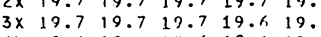
$\begin{array}{lllllll}4 \times & 19.6 & 19.6 & 19.6 & 19.6 & 19.6\end{array}$ $\begin{array}{llllll}5 \times & 19.6 & 19.5 & 19.5 & 19.5 & 19.5 \\ 6 \times & 19.4 & 19.4 & 19.4 & 19.3 & 19.3\end{array}$ $\begin{array}{llllll}7 \times & 1 . .1 & 19.0 & 18.9 & 18.9 & 19 . \\ 8 x & 18.5 & 18.1 & 17.8 & 17.4 & 17 .\end{array}$ $\begin{array}{lllllll}8 \times & 18.5 & 18.1 & 17.8 & 17.4 & 17.1 \\ 9 \times & 16.8 & 16.6 & 16.3 & 16.1 & 15.9\end{array}$ $10 \times 15.6$

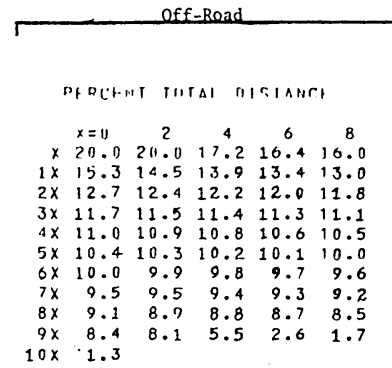

DFPCHN TITAL DISTANCI

$\begin{array}{cccccc}x=0 & 2 & 4 & 6 & 8 \\ x & 20.0 & 20.0 & 17.2 & 16.4 & 16.0 \\ 1 \times & 15.0 & 15.0 & 14.0 & 14.2 & 13.9\end{array}$

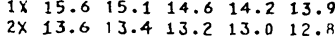
$\begin{array}{llllll}3 \times & 12.7 & 12.5 & 12.4 & 12.2 & 12.9 \\ 4 \times & 11.9 & 11.0 & 11.7 & 12.6 & 11.5\end{array}$ $5 \times 11.4 \quad 11.2 \quad 11.111 .0 \quad 11.0$ $\begin{array}{llllllll}6 \times & 10.9 & 10.8 & 10.7 & 10.6 & 10.5\end{array}$ $\begin{array}{llllll}8 \times & 9.8 & 9.6 & 9.5 & 9.3 & 9.1\end{array}$ $\begin{array}{rl}9 \times & 8.9 \\ 10 \mathrm{x} & 1.6\end{array}$

pirifhi thetal DIGIAHe

$\begin{array}{rrrrrr}x=n & 0 & 1 & 5 & \\ x & 15.0 & 15.0 & 14.6 & 13.6 & 13.1 \\ 1 \times & 12.8 & 12.6 & 12.4 & 12.2 & 12.0\end{array}$ $2 x \quad 11.911 .811 .711 .611 .5$ $\begin{array}{llllll}3 \times & 11.4 & 11.4 & 11.3 & 11.2 & 11.5 \\ 1 \times & 11.3 & 11.0 & 110.9 & 11.8 & 10.7\end{array}$

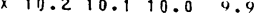
$\begin{array}{clllll}3 \times & 0.7 & 9.5 & 0.1 & 9.3 & 7.2 \\ 4 \times & 9.1 & 8.9 & 8.3 & 11.0 & 8.5\end{array}$ $\begin{array}{llllll}3 \times & 9.1 & 8.9 & 8.8 & 11.6 & 8.5 \\ 4 \times & 8.3 & 8.2 & 8.1 & 1.4 & 5.8 \\ 10 x & 8.7 & & & & \end{array}$ $\ln x \quad 2.7$

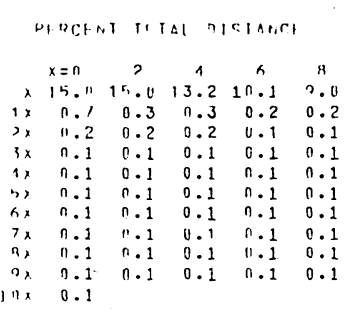




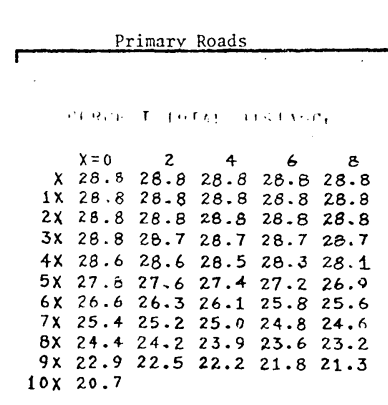

DPREFA THTAL MISIANCH

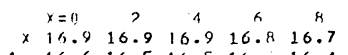
$\begin{array}{llllll}1 x & 16.6 & 16.5 & 16.9 & 16.4 & 16.4 \\ 3 x & 16.4 & 6.4 & 16.4 & 16.4 & 16.4\end{array}$ $\begin{array}{lllllll}3 x & 16.3 & 16.3 & 16.3 & 16.3 & 16.3\end{array}$ $\begin{array}{llllll}1 \times & 16.3 & 16.3 & 16.3 & 16.2 & 16.2 \\ 5 x & 16.2 & 16.2 & 16.2 & 16.2 & 16.2\end{array}$

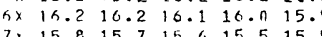
$\begin{array}{llllll}4 x & 15.4 & 15.3 & 15.2 & 15.2 & 15.1 \\ \Rightarrow ; & 15.0 & 15.0 & 14.9 & 14.8 & 14.6\end{array}$ in 14.4

PFPCFH TUTAL DISTAMEF

$\begin{array}{cccccc}x=0 & 2 & 4 & 6 & 8 \\ x & 28.2 & 28.2 & 28.2 & 28.2 & 28.2 \\ x & 28.2 & 28.2 & 28.2 & 28.2 & 28.2\end{array}$ $\begin{array}{llllll}1 X & 28.2 & 28.2 & 28.2 & 28.2 & 28.2 \\ 2 X & 28.2 & 28.2 & 28.2 & 28.2 & 28.0\end{array}$ $\begin{array}{llllll}2 x & 28.2 & 28.2 & 28.2 & 28.2 & 28.0 \\ 3 X & 27.8 & 27.7 & 27.5 & 27.4 & 27.0\end{array}$

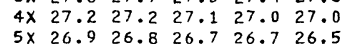
$\begin{array}{llllll}5 x & 26.9 & 26.8 & 26.7 & 26.7 & 26.5 \\ 6 x & 26.4 & 26.3 & 26.2 & 26.1 & 26.0\end{array}$ $\begin{array}{cccccc}6 \times & 26.4 & 26.3 & 26.2 & 26.1 & 26.0 \\ 7 \times & 25.9 & 25.8 & 25.7 & 25.6 & 25.5\end{array}$ $\begin{array}{llllll}8 \times & 25.4 & 25.3 & 25.2 & 25.1 & 25.1 \\ 9 \times & 24.9 & 24.4 & 23.6 & 22.8 & 22.1\end{array}$ $\begin{aligned} 9 \times & 24.9 \\ 10 \times & 21.5\end{aligned}$

PHREFW THIAL DISIANRI

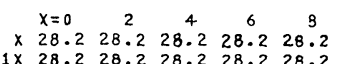
$\begin{array}{llllll}1 \times & 28.2 & 28.2 & 28.2 & 28.2 & 28.2 \\ 2 X & 28.2 & 28.2 & 28.2 & 28.1 & 27.9\end{array}$

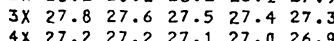

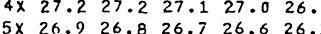
$\begin{array}{lllllll}6 \times & 26.4 & 26.3 & 26.1 & 26.0 & 26.5\end{array}$ $\begin{array}{llllll}7 \times & 25.9 & 25.8 & 25.7 & 25.6 & 25.5 \\ 8 \times & 25.4 & 25.3 & 25.2 & 25.1 & 25.0\end{array}$ $\begin{array}{llllll}8 \times & 25.4 & 25.3 & 25.2 & 25.1 & 25.0 \\ 9 \times & 24.8 & 24.3 & 23.4 & 22.6 & 21.9\end{array}$

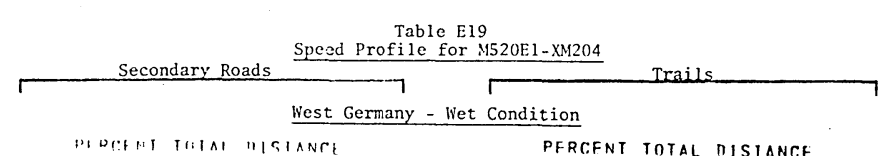

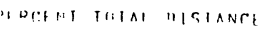

PFRGFNT TOTAL MISIANCF $\begin{array}{rccccc} & y=0 & 2 & 4 & 6 & 8 \\ \times & 26.8 & 26.5 & 26.0 & 25.8 & 25.7 \\ 1 \times & 25.6 & 25.4 & 25.0 & 24.7 & 24.4 \\ 2 x & 24.6 & 23.8 & 23.0 & 23.4\end{array}$

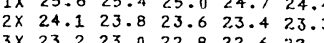
$\begin{array}{llllll}3 \times & 23.2 & 23.0 & 22.8 & 22.6 & 22.3 \\ 4 \times & 22 & 22.4\end{array}$ $\begin{array}{llllll}5 \times & 21.3 & 21.1 & 20.9 & 20.7 & 21.5 \\ 7 \times & 20.5 & 0.5\end{array}$

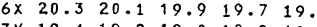
$\begin{array}{lllllll}7 \times & 19.4 & 19.2 & 19.0 & 18.8 & 18.6 \\ 8 \times & 18.4 & 18.2 & 18.0 & 17.8 & 17.7\end{array}$

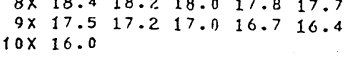

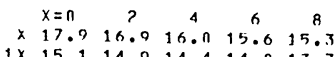
$2 \times 13.513 .313 .4$ 13.0 13.

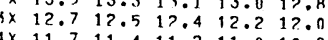
$\begin{array}{lllllll}5 \times & 10.6 & 10.4 & 10.3 & 10.2 & 10.1\end{array}$ $\begin{array}{cccccc}6 \times & 10.0 & 9.9 & 9.8 & 9.7 & 9.6\end{array}$

$\begin{array}{llllll}8 \times & 8.9 & 8.4 & 9.2 & 9.1 & 9.0 \\ 9 \times & 8.9 & 8.8 & 8.6 & 8.5\end{array}$

phochlol intal nISTANCh PERCEN TOTAL DISTANCE

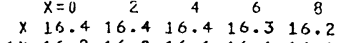
$\begin{array}{llllll}3 \times & 16.0 & 16.0 & 16.0 & 16.0 & 15.9\end{array}$ $\begin{array}{lllllll}4 \times & 15.9 & 15.8 & 15.6 & 15.5 & 15.4\end{array}$ $\begin{array}{llllll}6 \times & 14.7 & 14.6 & 14.5 & 14.9 & 14.8 \\ 7 \times & 4.2 & 14.6 & 14.0 & 13.0 & 13.7\end{array}$ $\begin{array}{lllllll}7 \times & 14.2 & 14.1 & 14.0 & 13.9 & 13.7 \\ 8 X & 13.6 & 13.5 & 13.4 & 13.3 & 13.1\end{array}$ $\begin{array}{llllll}8 X & 13.6 & 13.5 & 13.4 & 13.3 & 13.1 \\ 9 \times & 13.0 & 12.9 & 12.7 & 12.6 & 12.3\end{array}$

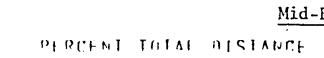

\section{PERCEN TOTAL DISTANCE}

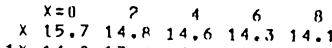

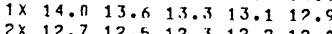
$3 \times 11.811 .7 \quad 11.5 \quad 11.411 .2$ $\begin{array}{cccccc}4 \times & 10.9 & 10.7 & 10.5 & 10.4 & 10.2 \\ 5 x & 10.1 & 10.0 & 9.9 & 9.7 & 9.4\end{array}$

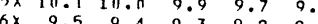
$\begin{array}{llllll}7 \times & 9.0 & 8.9 & 8.8 & 8.7 & 8.6 \\ 8 \times & 8.5 & 8.4 & 8.4 & .9 & 0.6\end{array}$ $\begin{array}{lllllll}8 \times & 8.5 & 8.4 & 8.4 & 8.3 & R .7 \\ 9 \times & 8.1 & 8.1 & 8.0 & 7.9 & 7.8 \\ 10 X & . .7 & & & & \end{array}$ Condition

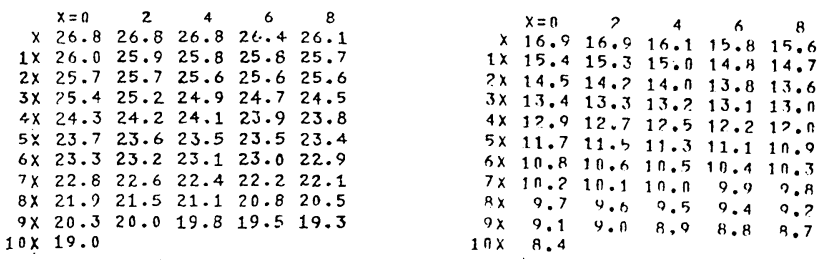

Mid-East - Sand Condition

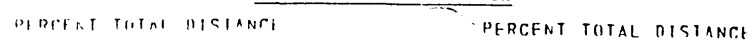

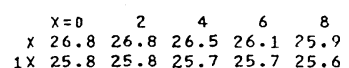

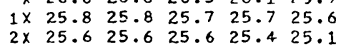

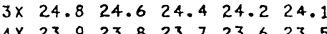
$\begin{array}{lllllll}5 \times & 23.4 & 23.3 & 23.2 & 23.1 & 23.0\end{array}$ $\begin{array}{llllll}6 \times & 22.9 & 22.8 & 22.7 & 22.5 & 22.3\end{array}$

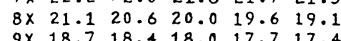

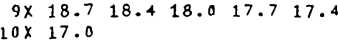

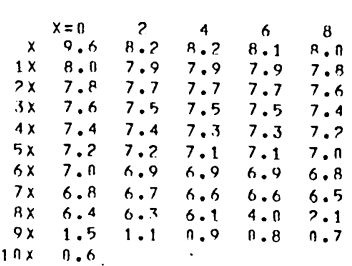

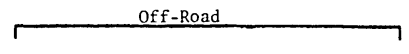

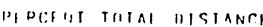

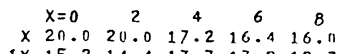

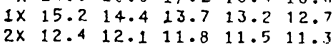
$3 \times 11.110 .910 .8 \quad 10.6 \quad 10.5$ $\begin{array}{llllll}5 \times & 9.7 & 9.6 & 9.4 & 9.3 & 0.2 \\ 6 \times & 0.0 & 8.9 & 8.8 & 8.7 & 0.5\end{array}$ $\begin{array}{llllll}6 \times & 9.0 & 8.9 & 8.8 & 8.7 & 8.5 \\ 7 \times & 8.4 & 8.2 & 8.1 & 7.9 & 7.8\end{array}$ $\begin{array}{llllll}8 \times & 7.6 & 7.4 & 7.2 & 7.0 & 6.8 \\ 9 x & 6.6 & 6.3 & 6.0 & 5.7 & 2.8\end{array}$ $10 \times 1.8$

DI RCFET TOTAL DICTANCI

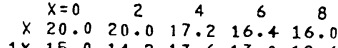
$2 \times 12.2 \quad 11.911 .6 \quad 611.311 .1$ $\begin{array}{llllll}3 \times & 10.9 & 10.8 & 10.6 & 10.5 & 10.4 \\ 4 X & 10.2 & 10.1 & 10.0 & 9.9 & 9.7\end{array}$ $\begin{array}{rrrrrr}4 \times & 10.2 & 10.1 & 10.0 & 9.9 & 9.7 \\ 5 \times & 9.6 & 9.5 & 9.3 & 9.2 & 9.1\end{array}$ $\begin{array}{lllllll}6 \times & 8.9 & 8.8 & 8.7 & 8.5 & 8.4 \\ 7 X & 8.3 & 8.1 & 8.0 & 7.8 & 7.7\end{array}$ $\begin{array}{llllll}8 \times & 7.5 & 7.3 & 7.2 & 7.0 & 6.8 \\ 9 \mathrm{X} & 6.6 & 6.4 & 6.1 & 5.8 & 3.5\end{array}$ $\begin{array}{rr}9 \times & 6.6 \\ 10 \times & 2.1\end{array}$

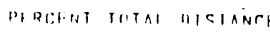

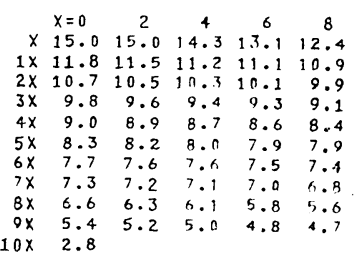

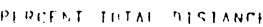

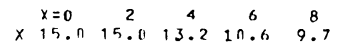
$\begin{array}{lllllll}1 \times & 9.1 & 8.4 & 8.3 & 8.0 & 7.8 \\ 2 x & 7.6 & 7.5 & 7.4 & 7.3 & 7.1\end{array}$ $\begin{array}{llllll}3 x & 7.0 & 6.9 & 6.8 & 6.7 & 6.6 \\ 4 \times & 6.5 & 6.4 & 6.4 & 6.3 & 6.2\end{array}$ $\begin{array}{llllll}5 x & 6.1 & 0.0 & 5.9 & 5.8 & 5.6 \\ 6 x & 5.5 & 5.3 & 5.1 & 4.9 & 4.7\end{array}$

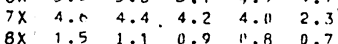
$\begin{array}{lllllll}9 x & 0.6 & 0.5 & 0.5 & 0.4 & 0.4 \\ 10 x & 0.4 & & & & \end{array}$ 


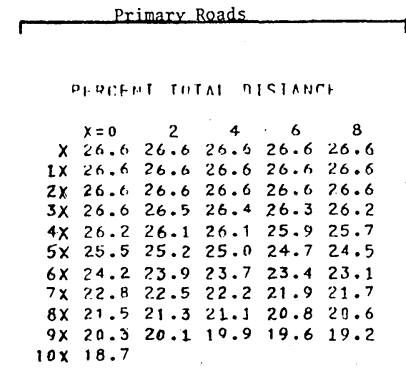

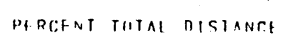

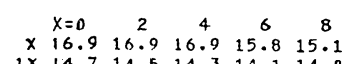
$\begin{array}{lllllll}1 x & 14.7 & 14.5 & 14.3 & 14.1 & 14.0 \\ 2 x & 13.9 & 13.8 & 13.7 & 13.6 & 13.6 \\ 3 x & 13.5 & 13.4 & 13.4 & 33.6 & 13.3\end{array}$ $\begin{array}{lllllll}2 x & 13.5 & 13.4 & 13.4 & 13.3 & 13.3\end{array}$ $\begin{array}{llllll}4 \times & 13.2 & 13.2 & 13.2 & 13.1 & 13.1\end{array}$ $\begin{array}{cccccc}6 \mathrm{X} & 13.0 & 13.0 & 12.9 & 12.9 & 12.9 \\ 7 \mathrm{X} & 12.8 & 12.8 & 12.8 & 12.7 & 12.6\end{array}$

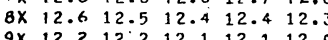
$\begin{array}{rr}9 \times & 12.2 \\ 10 x & 11.9\end{array}$

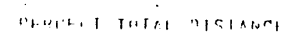

$\begin{array}{cccccc}x=0 & 2 & 2 & 4 & 6 & 8 \\ x & 25.0 & 25.0 & 25.0 & 25.0 & 25.0\end{array}$ $\begin{array}{lllllll}1 \times & 25.0 & 25.0 & 25.0 & 25.0 & 25.0 \\ 2 \times & 25.0 & 25.0 & 25.0 & 25.0 & 25.0\end{array}$ $\begin{array}{llllll}3 \times & 24.7 & 24.5 & 24.4 & 24.4 & 24.3\end{array}$ $\begin{array}{lllllll}4 \times & 24.2 & 24.1 & 24.1 & 24.4 & 24.3 \\ 5 \times & 23.0 & 24.0\end{array}$

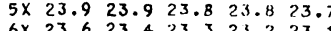

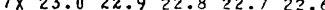
$\begin{array}{llllll}8 \times & 22.5 & 22.4 & 22.3 & 22.3 & 22.2 \\ 9 \times & 22.1 & 21.8 & 21.2 & 21.6 & 20.0\end{array}$ $9 \times 22.1$
$10 \times 19.6$

PERCFAT TIITAL MISTANCF

$\begin{array}{cccccc}x=0 & 2 & 4 & 6 & 8 \\ \times & 25.0 & 25.0 & 25.0 & 25.0 & 25.0\end{array}$

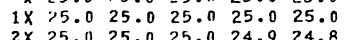

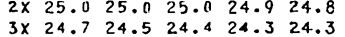
$\begin{array}{lllllll}4 \times & 24.2 & 24.1 & 24.1 & 24.0 & 24.0\end{array}$ $\begin{array}{llllll}5 \times & 23.9 & 23.9 & 23.8 & 23.8 & 23.7 \\ 6 x & 23.5 & 23.4 & 23.3 & 23.1 & 23.0\end{array}$

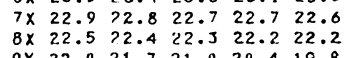

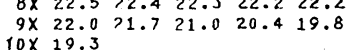

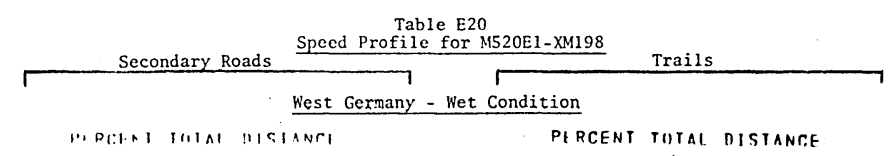

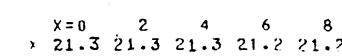

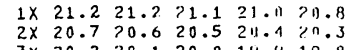
$\begin{array}{llllll}4 X & 19.6 & 19.5 & 19.3 & 14.2 & 1 \% \\ 5 X & 18.8 & 18.6 & 18.3 & 18.1 & 17 \\ 6 X & 17.7 & 17.5 & 17.4 & 17.2 & 17\end{array}$

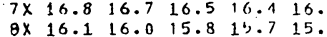
$\begin{array}{llllll}9 \times & 15.4 & 15.2 & 14.9 & 14.7 & 14 .\end{array}$

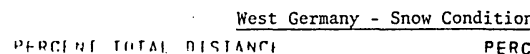
$x=0 \quad 4 \quad 6 \quad 8$
$\times 15$ $\begin{array}{llllll}1 \times & 13.8 & 13.4 & 13.1 & 12.9 & 11.0 \\ 3 \times & 12.5 & 13.3 & 13.1 & 12.9 & 110\end{array}$ $3 x \quad 11.511 .311 .2211 .0010 .7$ $\begin{array}{llllll}4 x & 10.6 & 10.4 & 1 n .2 & 10.1 & 1 n .0\end{array}$ $\begin{array}{llllll}5 \times & 9.8 & 9.7 & 9.6 & 9.5 & 9.4 \\ 6 \times & 9.3 & 9.2 & 9.1 & 9.0 & 8.9\end{array}$ $\begin{array}{lllllll}7 \times & 8.8 & 8.7 & 8.6 & 8.5 & 8.9 \\ 8 X & 8.3 & 8.2 & 8.2 & 6.1 & 0.4\end{array}$

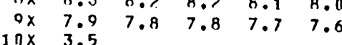

$\begin{array}{rrrrrr}x=0 & 2 & 4 & 6 & 8 \\ x & 13.3 & 13.3 & 13.3 & 13.3 & 13.3 \\ x & 13.3 & 13.3 & 13.3 & 13.3 & 13.3\end{array}$ $2 \times 13.3 \quad 13.3 \quad 13.2 \quad 13.2 \quad 13.1$ $\begin{array}{lllllll}3 \times & 13.1 & 13.0 & 12.9 & 12.9 & 12.8 \\ 4 \times & 12.8 & 12.7 & 12.6 & 12.6 & 12.5\end{array}$ $\begin{array}{llllll}5 X & 12.4 & 12.3 & 12.2 & 12.2 & 12.1\end{array}$

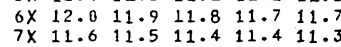

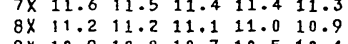
$\begin{array}{rrrrrr}8 \times & 11.2 & 11.2 & 11.1 & 11.0 & 10.9 \\ 9 \times & 10.9 & 10.8 & 10.7 & 10.5 & 10.4\end{array}$

Mid-East - Wet Condition

PERCENT TOTAL DISTANTE

I ancr.

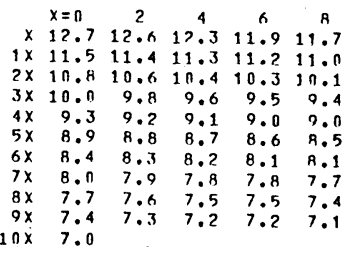

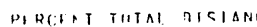
PERCENT TOTAL DISTANTE

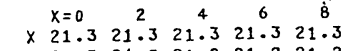

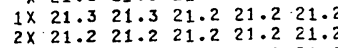
$3 \times 21.221 .2 \quad 21.2 \quad 21.121$. $\begin{array}{llllll}4 \times & 20.9 & 20.9 & 20.8 & 20.8 & 21 . \\ 5 \times & 20.7 & 20.6 & 20.6 & 20.6 & 20\end{array}$

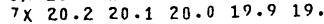
$\begin{array}{llllll}8 \times & 19.6 & 19.4 & 19.1 & 18.9 & 18.7\end{array}$ $\begin{aligned} 9 \times & 18.5 \\ 10 \times & 17.6\end{aligned}$ Mid-East - Sand Condition

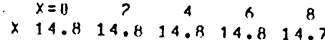

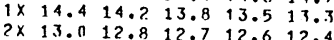

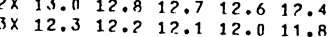
$\begin{array}{llllll}4 \times & 11.7 & 11.5 & 11.3 & 11.1 & 10.9 \\ 5 x & 10.7 & 10.5 & 10.4 & 10.3 & 10.2\end{array}$ $\begin{array}{cccccc}7 \times & 9.6 & 9.5 & 9.8 & 9.8 & 9.7 \\ 9 \times & 9.4 & 9.3 & 9.4 & 9.7 & 9.6\end{array}$ $\begin{array}{rrrrrr}8.0 .0 & 9.0 \\ 0 \times & 8.5 & 8.9 & 8.8 & 8.7 & 8.6 \\ 10 \times & 7.5 & 8.4 & 8.3 & 8.2 & 8.0\end{array}$

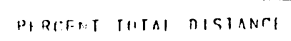
PFRCENT InTAL TISTANCE.

$\begin{array}{cccccc}x=0 & 2 & 4 & 6 & 8 \\ x & 21.3 & 21.3 & 21.3 & 21.3 & 21.3 \\ 1 x & 21.3 & 21.2 & 21.2 & 21.2 & 21.2\end{array}$ $\begin{array}{llllll}1 x & 21.3 & 21.2 & 21.2 & 21.2 & 21.2 \\ 2 x & 21.2 & 21.2 & 21.2 & 21.2 & 21.2\end{array}$ $\begin{array}{llllll}3 \times & 21.1 & 21.1 & 21.0 & 20.9 & 20.9\end{array}$ $\begin{array}{lllllll}4 x & 20.8 & 20.7 & 20.7 & 20.6 & 20.6 \\ 5 \times & 20.6 & 711.5 & 20.5 & 20.4 & 20.4\end{array}$

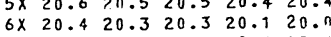
$8 \times 19.2 \quad 18.8 \quad 18.4 \quad 18.017 .7$ $\begin{array}{rrrrrrr}8 \times & 19.2 & 18.8 & 18.4 & 18.0 & 17.7 \\ 9 \times & 17.3 & 17.1 & 16.8 & 16.6 & 15.3 \\ 10 \times & 16.0 & & & & & \end{array}$

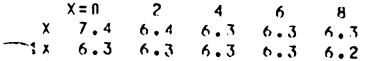
$\begin{array}{llllll}3 x & 6.3 & 0.3 & h .3 & 0.3 & 6.2 \\ 3 x & 5.2 & 6.2 & 6.2 & 6.2 & 6.2 \\ 3 x & 6.2 & 6.1 & 6.1 & 0.1 & 0.2\end{array}$
$\begin{array}{llllll}4 x & 6.1 & 0.1 & 6.1 & 6.1 & 6.0\end{array}$
$\begin{array}{llllll}5 x & 6.011 & 6.0 & 6.0 & 5.9 & 5.9 \\ 6 x & 5.9 & 5.9 & 5.9 & 5.8 & 5.0\end{array}$
$\begin{array}{lllllll}7 \times & 5.8 & 5.8 & 5.97 & 5.7 & 5.0 \\ 2 \times & 5.4 & 4.7 & 4.7 & 5.4 & 5.0\end{array}$
$\begin{array}{llllll}8 x & 5.4 & 4.7 & 4.1 & 2.4 & 1.6 \\ 7 x & 1.2 & 1.0 & 0.8 & 0.7 & 0.6 \\ 10 x & 0.6 & & & & \end{array}$

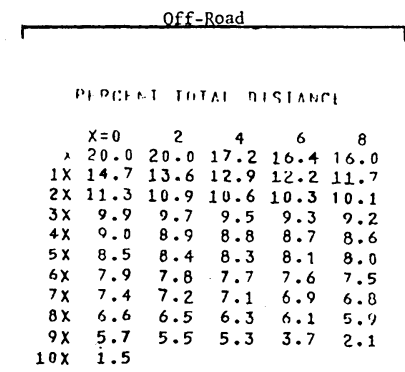

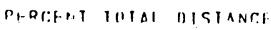

$\begin{array}{cccccc}x=0 & 2 & 4 & 6 & 8 \\ \times & 20.0 & 20.0 & 17.2 & 16.4 & 16.0\end{array}$ $\begin{array}{rrrrrr}2 x & 10.9 & 10.5 & 10.2 & 9.9 & 9.7 \\ 3 \times & 9.5 & 9.3 & 9.2 & 9.1 & 8.9\end{array}$ $\begin{array}{llllll}3 x & 9.5 & 9.3 & 9.2 & 9.1 & 8.9 \\ 4 x & 8.8 & 8.7 & 8.6 & 8.5 & 8.4 \\ 5 x & 8.3 & 0.1 & 8.0 & 7.9 & 7.8\end{array}$ $\begin{array}{llllll}4 \times & 8.3 & 8.7 & 8.6 & 8.5 & 8.4 \\ 5 \times & . .2 & 7.6 & 8.0 & 7.9 & 7.8\end{array}$ $\begin{array}{llllll}6 x & 7.7 & 7.6 & 7.5 & 7.4 & 7.3 \\ 7 \mathrm{x} & 7.2 & 7.0 & 6.9 & 6.8 & 6.6 \\ 6 \times & 6.5 & 6.4 & 6.2 & 6.1 & 5.9\end{array}$ $\begin{array}{llllll}8 \mathrm{X} & 6.5 & 6.4 & 6.2 & 6.1 & 5.9 \\ 9 \mathrm{x} & 5.7 & 5.5 & 5.3 & 5.1 & 3.2\end{array}$

PERCENT TOTAL DISTANCE

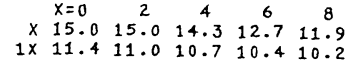
$\begin{array}{cccccc}2 \times & 10.0 & 9.8 & 9.6 & 9.4 & 9.2 \\ 3 \times & 9.1 & 8.9 & 8.8 & 9.4 & 9.2 \\ 4 \times & 8.3 & 8.9 & 8.0 & 8.6 & 8.5\end{array}$ $\begin{array}{llllll}4 \times & 8.3 & 8.2 & 8.0 & 7.9 & 7.8 \\ 5 x & .7 & 7.6 & 7.5 & 7.5 & 7.4\end{array}$ $\begin{array}{llllll}5 x & 7.7 & 7.6 & 7.5 & 7.5 & 7.4 \\ 6 x & 7.3 & 7.2 & 7.1 & 6.9 & 6.8 \\ 7 x & 6.8 & 6.7 & 6.6 & 6.4 & 6.8\end{array}$ $\begin{array}{llllll}7 \mathrm{X} & 6.8 & 6.7 & 6.6 & 6.4 & 6.8 \\ 8 \mathrm{X} & 6.0 & 5.7 & 5.5 & 5.3 & 5.1 \\ 9 \mathrm{x} & 4.9 & 4.8 & 4.6 & 4.5 & 4.3\end{array}$ $9 \times \quad 4.9 \quad 5.8 \quad 5.5 \quad 5.350 .1$ $10 \times \quad 2.6$

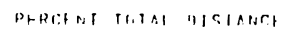

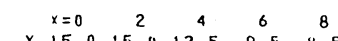
$\begin{array}{rrrrrr}1 \times & 7.8 & 7.3 & 6.8 & 0.5 & 6.5 \\ 2 \times & 6.0 & 5.2 & 1.7 & 0.8 & 0.5 \\ 3 \times & .4 & 0.3 & 0.3 & 0.3 & 0.5\end{array}$ $\begin{array}{llllll}3 x & 0.4 & 0.3 & 0.3 & 11.3 & 0.2 \\ 4 x & 0.2 & 0.2 & 0.2 & 0.2 & 0.2\end{array}$ $\begin{array}{llllll}5 x & 0.2 & 0.2 & 0.2 & 0.2 & 0.2 \\ 6 x & 0.2 & 0.2 & 0.2 & 11.2 & 0.1\end{array}$ $\begin{array}{llllll}7 x & 0.1 & 0.1 & 0.1 & 0.1 & 0.1 \\ 8 x & 0.1 & 0.1 & 0.1 & 0.1 & 0.1\end{array}$ $\begin{array}{rrrrrr}9 \times & 0.1 & 0.1 & 0.1 & 0.1 & 0.1 \\ 10 x & 0.1 & & 0.1 & 0.1 & 0.1\end{array}$ 


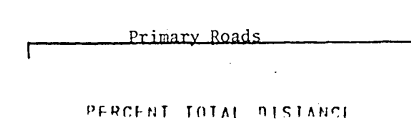

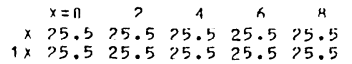

$\begin{array}{llllll}2 \times & 25.5 & 25.5 & 25.5 & 25.5 & 25 . \\ 3 \times & 25.5 & 25.4 & 25.3 & 25.3 & 25.3\end{array}$

4) 25.525 .425 .325 .325 .2

$\begin{array}{llllll}5 x & 24.5 & 24.3 & 24.1 & 23.9 & 23.6 \\ 6 x & 23.4 & 23.1 & 22.9 & 22.6 & 22.6\end{array}$

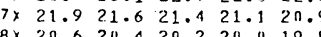

$\begin{array}{llllll}2 \times & 20.6 & 211.4 & 20.2 & 20.11 & 19.8 \\ 0 \times & 19.6 & 19.4 & 19.2 & 18.9 & 18.5\end{array}$

$1 \mathrm{nx} 18.1$

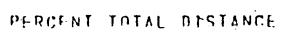

$\begin{array}{rrrrrr}x=0 & 2 & 4 & 6 & 8 \\ x & 16.9 & 10.9 & 16.9 & 15.6 & 14.8 \\ 10 & 14.3 & 14.0 & 13.8 & 13.0 & 13.5\end{array}$

$\begin{array}{lllllll}2 x & 13.4 & 1.3 .3 & 3.3 & 13.2 & 13.2 \\ 3 x & 13.1 & 13.1 & 13.3 & 13.2 & 13.2\end{array}$

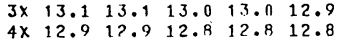

$\begin{array}{llllll}4 X & 12.9 & 12.9 & 12.8 & 12.8 & 12.8 \\ 5 X & 12.8 & 12.8 & 12.7 & 12.7 & 12.7 \\ 6 x & 12.7 & 12.5 & 12.6 & 12.6 & 12.5\end{array}$

$\begin{array}{llllll}6 \times & 12.7 & 12.7 & 12.6 & 12.6 & 12 . \\ 7 \times & 12.5 & 12.5 & 12.4 & 12.4 & 12 .\end{array}$

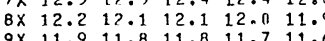

$10 \times 11.5$

PERCENT TCTAL DISTANCE

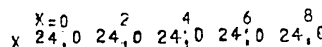

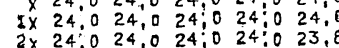

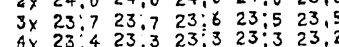

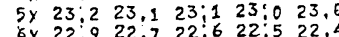

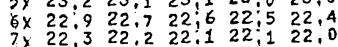

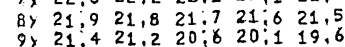
$10 \times 19: 1$

PERCENT TCTAL DISTANCE

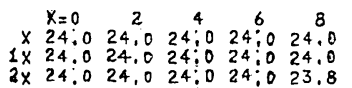

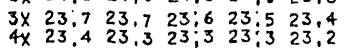
$\begin{array}{llllllll}5 x & 23.2 & 23,1 & 23 & 1 & 23 & 0 & 22.9 \\ 6 x & 22.8 & 22,7 & 22,6 & 22 & 5 & 22.4\end{array}$ $2 \times 22,3 \quad 22,2 \quad 22 ; 1 \quad 22: 022$;

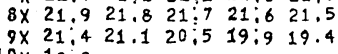

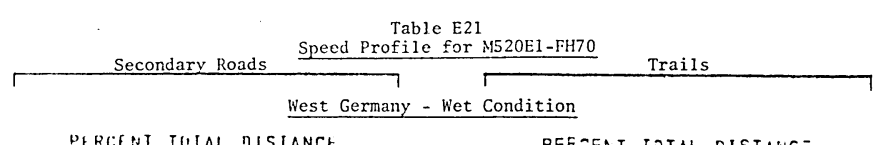

PFREFHT THIAL DISTANR

PEFIENT TOTAL DISTANCE

$\begin{array}{cccccc}x=n & 2 & 4 & 6 & 8 \\ x & 20.4 & 20.4 & 20.4 & 20.4 & 20.4 \\ 1 x & 20.4 & 20.3 & 20.3 & 20.2 & 20.1\end{array}$

$\begin{array}{llllll}1 \times & 20.4 & 213.3 & 20.3 & 20.2 & 20.1 \\ 2 \times & 20.0 & 19.9 & 19.8 & 19.7 & 19.6\end{array}$

$\begin{array}{llllll}3 \times & 19.5 & 19.5 & 19.4 & 19.2 & 19.1 \\ 4 \times & 19 & 19.4 & 19.4 & \end{array}$

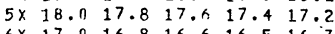

$\begin{array}{llllll}6 \times & 17.0 & 16.8 & 16.5 & 16.5 & 16.3 \\ 7 \times & 16.2 & 16.0 & 15.9 & 15.7 & 15.6\end{array}$

$\begin{array}{llllll}8 \times & 15.4 & 15.3 & 15.2 & 15.0 & 14.9 \\ 9 \times & 14.7 & 14.5 & 14.3 & 14.1 & 13.9\end{array}$

$9 \times 14.7$
$1 \mathrm{n} \times 13.6$

$$
\frac{\text { West Germ }}{\text { ANrt }}
$$

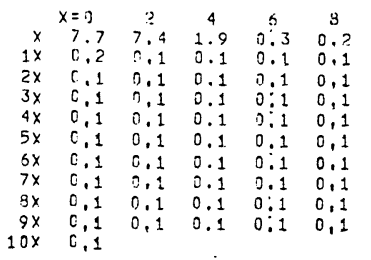

PLREFWT TIITA DistanCE PERC

$\begin{array}{rrrrrr} & x=0 & 2 & 4 & 6 & 8 \\ x & 13.1 & 13.0 & 12.8 & 12.7 & 12.7 \\ 1 & 13.7 & 12.7 & 12.7 & 12.7 & 12.6\end{array}$

$\begin{array}{llllll}2 \times & 12.6 & 12.6 & 12.6 & 12.5 & 12.4\end{array}$

$\begin{array}{llllll}2 x & 12.4 & 12.6 & 12.6 & 12.5 & 12.4 \\ 4 x & 12.4 & 12.3 & 12.2 & 12.2 & 12.1\end{array}$

$\begin{array}{llllll}5 x & 11.8 & 11.7 & 11.6 & 11.5 & 11.4\end{array}$

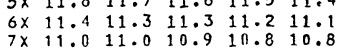

$\begin{array}{cccccc}8 \times & 10.7 & 10.6 & 10.6 & 10.5 & 10.4 \\ 9 \times & 10.4 & 10.3 & 10.2 & 10.1 & 10.0\end{array}$

Mid

PEPCHNT TIITAL DISTANTH

$\begin{array}{rlrrrr} & x=11 & 2 & 4 & 6 & 8 \\ x & 12.1 & 11.9 & 11.3 & 10.9 & 0.8 \\ 1 x & 10.6 & 10.5 & 10.4 & 10.4 & 10.2 \\ 3 x & 10.1 & 9.9 & 0.8 & 9.6 & 9.5\end{array}$

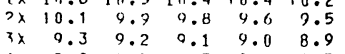

$\begin{array}{llllll}4 \times & 8.8 & 0.7 & 0.7 & 8.6 & 8.9 \\ 5 x & 0.4 & 0.4 & 0.3 & 8.2 & 8.5\end{array}$

$\begin{array}{lllllll}5 x & 0.0 & 0.7 & 0.7 & 8.0 & 0.5 \\ 6 x & 8.11 & 1.9 & 7.9 & 7.2 & 8.1 \\ 3 x & 7.0 & 7.5 & 7.5 & 7.8 & 7.7\end{array}$

$\begin{array}{llllll}7 x & 7.7 & 7.6 & 7.5 & 7.5 & 7.4 \\ 8 \times & 7.4 & 7.3 & 7.3 & 7.2 & 7.2\end{array}$

$\begin{array}{rrrrrr}8 \times & 7.4 & 7.3 & 7.3 & 7.2 & 7.9 \\ 9 x & 7.0 & 7.0 & 7.0 & 6.9 & 4.8\end{array}$

Mid-East - Wet Condition

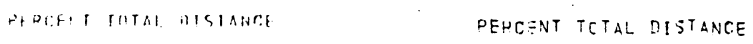

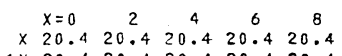

$\begin{array}{llllll}1 \times & 20.4 & 20.4 & 20.4 & 20.4 & 20.4 \\ 2 \times & 20.4 & 20.4 & 20.4 & 20.4 & 20.4\end{array}$

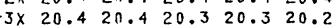

$\begin{array}{llllll}4 \times & 20.2 & 20.1 & 20.1 & 20.0 & 20.0\end{array}$

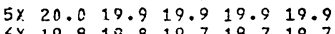

7x $19.619 .4+19.2 \quad 19.018 .8$

$\begin{array}{llllll}8 \times & 18.7 & 18.5 & 18.3 & 18.1 & 17.9 \\ 9 x & 17.7 & 17.6 & 17.4 & 17.3 & 17.2\end{array}$

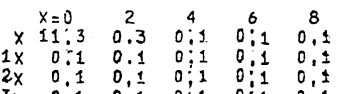

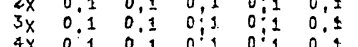

$4 x \quad 0.1$

$\begin{array}{llll}x & 0.1 & 0.1 & 0.1\end{array}$

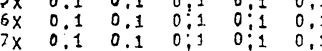

$\begin{array}{lllllll}8 \times & 0.1 & 0.1 & 0: 1 & 0 & 0 & 0.1 \\ 9 \times & 0.1 & 0.1 & 0.1 & 0 & 0.1 & 0.1\end{array}$

PIRI:FNT TOTAL DISTANST

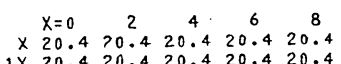

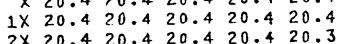

$\begin{array}{llllll}2 \times & 20.4 & 20.4 & 20.4 & 20.4 & 20.3 \\ 3 \times & 20.3 & 20.3 & 20.2 & 20.2 & 20.1 \\ 4 \times & 20.1 & 20.0 & 20.0 & 20.0 & 19.9\end{array}$

$\begin{array}{llllll}5 \times & 19.9 & 19.9 & 19.8 & 19.8 & 19.7 \\ 6 \times & 19.7 & 19.7 & 19.6 & 19.5 & 19.4\end{array}$

$\begin{array}{lllllll}6 x & 19.7 & 19.7 & 19.8 & 19.5 & 19.7 \\ 8 \times & 19.3 & 19.1 & 18.9 & 18.7 & 18.5 \\ 8 \times & 18.3 & 17.9 & 17.6 & 17.3 & 17.0\end{array}$

$\begin{array}{llllll}8 \times & 18.3 & 17.9 & 17.6 & 17.3 & 17.0 \\ 9 \times & 16.7 & 16.5 & 16.2 & 16.0 & 15.8\end{array}$ $10 \times 15.5$
Condition

\begin{tabular}{|c|c|c|c|c|c|}
\hline & & & & & \\
\hline $1 x$ & 0.1 & 0,1 & $0: 1$ & $0: 1$ & 0.1 \\
\hline & 0.1 & 0.1 & $0: 1$ & $0: 1$ & 0,1 \\
\hline $\begin{array}{l}3 x \\
4 x \\
4 x\end{array}$ & 0.11 & 0.1 & $\begin{array}{l}0 ; 1 \\
0 ; 1\end{array}$ & $\begin{array}{l:l}0 \\
0 \\
0\end{array}$ & $\begin{array}{l}0: 1 \\
0: 1\end{array}$ \\
\hline & & 0, & $0: 1$ & $0: 1$ & 0.1 \\
\hline$e x$ & 0. & 0.1 & 0.2 & 0.1 & 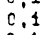 \\
\hline & 0. & 01 & $0 ; 1$ & $0: 1$ & .1 \\
\hline & $0_{0}^{0.1}$ & & & & \\
\hline
\end{tabular}

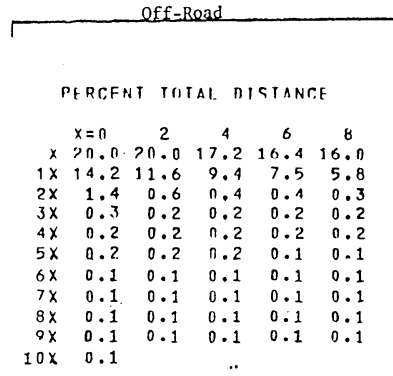

PEPCENT TOTAI DISTANTH

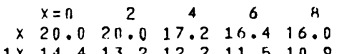

$\begin{array}{lllllll}2 \times & 10.5 & 10.1 & 9.8 & 9.5 & 9.3\end{array}$

$\begin{array}{llllll}3 \times & 9.1 & 8.9 & 8.8 & 8.6 & 8.5 \\ 4 \times & 8.4 & 0.3 & 0.2 & 0.1 & 0.0\end{array}$

$\begin{array}{lllllll}5 \times & 7.9 & 7.8 & 7.7 & 7.6 & 7.5\end{array}$

$\begin{array}{llllll}6 \times & 7.4 & 7.3 & 7.2 & 7.1 & 7.0 \\ 7 \times & 5.9 & 6.7 & 6.6 & 6.5 & 6.4\end{array}$

$\begin{array}{llllll}8 \times & 6.2 & 6.1 & 5.9 & 5.8 & 5.6 \\ 9 x & 5.5 & 5.3 & 5.1 & 4.9 & 3.8\end{array}$

$10 \times \quad 2.2$

PERCENT TETAL DISTANCE

$\begin{array}{rrrrrr}x & =0 & 2 & 4 & 6 & 8 \\ x & 15.0 & 15,0 & 12: 2 & 6: 9 & 5.5 \\ 1 \times & 4: 7 & 4,3 & 3: 9 & 3: 3 & 0.8\end{array}$

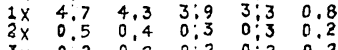

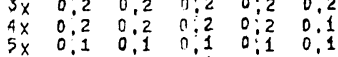

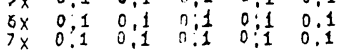

$\begin{array}{llllll}8 \times & 0.1 & 0.1 & 0.1 & 0: 1 & 0.1 \\ \times & 0.1 & 0.1 & 0.1 & 0: 1 & 0.1\end{array}$

$\begin{aligned} 9 \times & 0.1 \\ 10 \times & 0.1\end{aligned}$

PERCFNT TCTAL. DISTTACEE

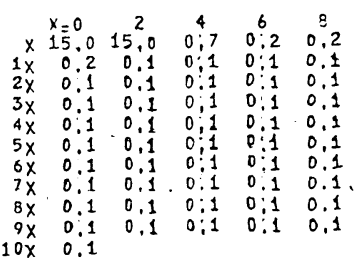

$10 \times 0$ 


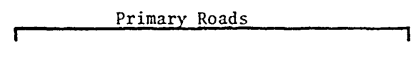

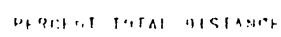

$\begin{array}{rrrrrr} & x=0 & 2 & 4 & 6 & 8 \\ \times & 29.8 & 29.8 & 29.8 & 24.8 & 29.8 \\ 1 \times & 29.8 & 29.8 & 29.6 & 29.3 & 20.1\end{array}$

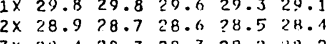
$\begin{array}{llllll}3 \times & 28.4 & 78.3 & 28.3 & 28.2 & >8.2 \\ 4 \times & 28.1 & 28.1 & 28.0 & 27.4 & 27.7\end{array}$ $5 x .27 .6 \quad 27.5 \quad 27.4 \quad 27.2 \quad 27.0$ $\begin{array}{llllll}6 \times & 26.8 & 26.6 & 26.4 & 26.2 & 26.11 \\ 7 \times & 25.8 & 25.6 & 25.5 & 25.0 & 25.1\end{array}$

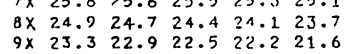
$9 \times 23.3$
$10 \times 21.0$

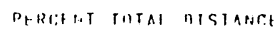

$\begin{array}{rrrrrr}x=0 & 2 & 4 & 6 & 8 \\ x & 16.9 & 16.9 & 16.9 & 15.6 & 11.8 \\ 1 & 14.3 & 14.0 & 13.4 & 12.7 & 12.3\end{array}$ $2 \times 11.9 \quad 11.6 \quad 11.4$ 11.2 11.1 $\begin{array}{llllll}3 \times & 10.9 & 10.8 & 10.7 & 10.6 & 10.6 \\ 4 \times & 10.5 & 10.4 & 10.4 & 10.6 & 10.3\end{array}$

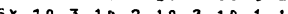

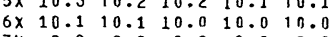
$\begin{array}{llllll}7 x & 9.9 & 9.9 & 9.8 & 9.8 & 9.8 \\ 8 \times & 9.7 & 9.7 & 9.7 & 9.7 & 9.6\end{array}$ $\begin{array}{rrrrrr}8 \times & 9.7 & 9.7 & 9.7 & 9.7 & 9.6 \\ 9 \times & 9.6 & 9.6 & 9.6 & 9.5 & 9.5\end{array}$

Drochent THTAI DISTANCE

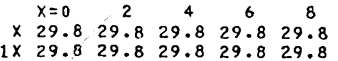
$\begin{array}{llllll}1 \times & 29.8 & 29.8 & 29.8 & 29.8 & 29.8 \\ 2 \times & 29.8 & 29.8 & 29.7 & 29.6 & 29.5\end{array}$

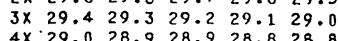

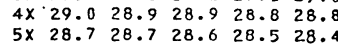
$\begin{array}{llllll}5 x & 28.7 & 28.7 & 28.6 & 28.5 & 28.4 \\ 6 x & 28.2 & 28.1 & 28.0 & 27.9 & 27.8\end{array}$

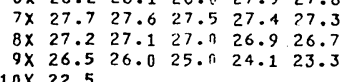

ph.pliant ratal MISIANTH

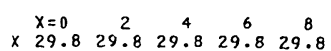

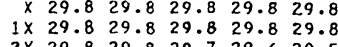

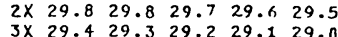

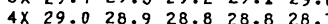
$\begin{array}{llllll}5 \times & 28.7 & 28.7 & 28.6 & 28.5 & 28.3\end{array}$ $\begin{array}{llllll}6 \times & 28.2 & 28.1 & 28.0 & 28.5 & 28.7 \\ 7 \times & 27.6 & 27.5 & 27.4 & 27.8 & 27.7\end{array}$ $\begin{array}{llllll}7 \times & 27.6 & 27.5 & 27.4 & 27.3 & 27 . \\ 8 \times & 27 & 1 & 27.0 & 27.9 & \end{array}$

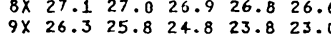
\begin{aligned} & $9 \times 26.3 \\ & 10 \times 22.2 \\ &$\hline\end{aligned}

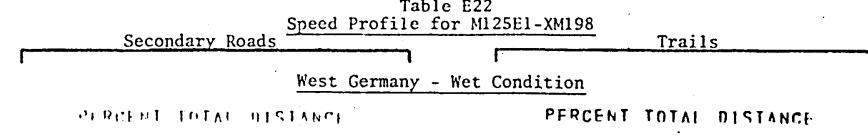

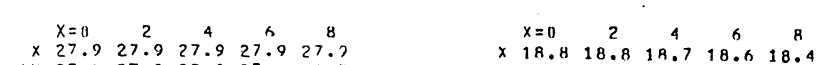

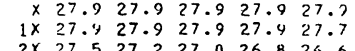
$\begin{array}{lllll}2 x & 27.5 & 27.2 & 27.0 & 26.8 \\ 3 \times & 26.5 & 26.3 & 26.2 & 26.0 \\ 4 x & 25.7 & 25.5 & 25.3 & 25.0\end{array}$

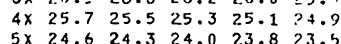

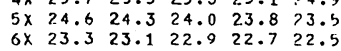
$\begin{array}{llllll}7 \times & 22.3 & 22.1 & 22.0 & 21.7 & 21.5 \\ 8 \times & 21.3 & 21.0 & 20.7 & 20.4\end{array}$

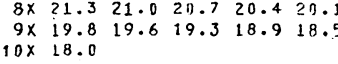

$$
\text { West Ger }
$$

$\begin{array}{rlcccc} & x=0 & 2 & 4 & 6 & 8 \\ x & 18.4 & 18.8 & 18.7 & 18.6 & 18.4 \\ 1 \times & 18.3 & 18.3 & 18.2 & 18.0 & 18.7 \\ 2 x & 17.5 & 17.2 & 17.0 & 16.0 & 17.5\end{array}$

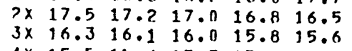

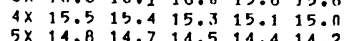

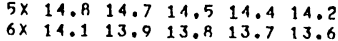

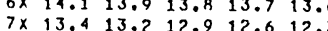

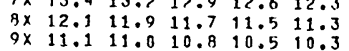

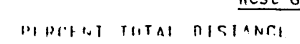
Condition $\begin{array}{rrrrrr} & x=0 & 2 & 4 & 6 & 8 \\ x & 13.1 & 12.5 & 11.0 & 10.6 & 10.4 \\ 1 x & 10.2 & 10.0 & 9.9 & 9.8 & 9.8 \\ 2 x & 9.7 & 9.7 & 9.7 & 9.6 & 9.6 \\ 3 x & 9.5 & 9.6 & 9.5 & 9.5 & 9.5 \\ 4 x & 9.5 & 9.4 & 9.4 & 9.4 & 9.4 \\ 5 x & 9.4 & 9.4 & 9.4 & 9.3 & 9.3 \\ 6 x & 9.3 & 9.3 & 9.3 & 9.3 & 9.2 \\ 7 \times & 9.2 & 9.2 & 9.2 & 9.2 & 9.2 \\ 8 \times & 9.1 & 9.1 & 9.1 & 9.1 & 9.1 \\ 9 x & 9.1 & 9.0 & 8.9 & 8.9 & 8.7 \\ 10 x & 4.0 & & & & \end{array}$

Mid-E

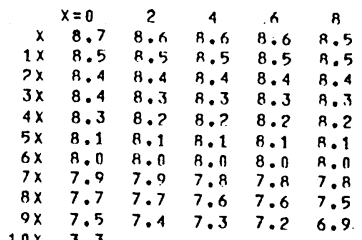

$\begin{array}{cccccc}x=0 & 2 & 4 & 6 & 8 \\ \times & 27.9 & 27.9 & 27.9 & 27.9 & 27.9\end{array}$ $\begin{array}{cccccc}1 \times & 27.9 & 27.9 & 27.9 & 27.9 & 27.9 \\ 2 \times & 27.9 & 27.9 & 27.9 & 27.9 & 27.9 \\ 3 \times & 27.9 & 27.9 & 27.9 & 27.9 & 27.9\end{array}$

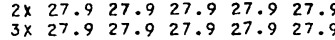
$\begin{array}{llllll}4 \times & 27.8 & 27.8 & 27.7 & 27.6 & 27.6\end{array}$ $\begin{array}{llllll}5 \times & 27.5 & 27.4 & 27.3 & 27.2 & 27.1 \\ 6 x & 27.1 & 27.0 & 26.9 & 26.7 & 26.6\end{array}$ $\begin{array}{llllll}7 x & 26.5 & 26.4 & 26.2 & 26.1 & 25.9 \\ 8 \times & 25.6 & 24.9 & 24.4 & 23.9 & 23.4\end{array}$

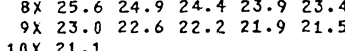

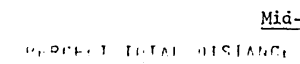
percent total mistance -East - Sand Condition

$\begin{array}{rrrrrr} & x=0 & 7 & 4 & 6 & 8 \\ x & 18.8 & 18.6 & 18.4 & 18.3 & 18.2 \\ 1 \times & 18.2 & 18.1 & 18.1 & 18.0 & 17.9\end{array}$

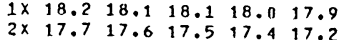
$\begin{array}{llllll}3 \times & 17.1 & 17.6 & 16.5 & 16.4 & 17.2 \\ 4 \times & 16.3 & 16.2 & 16.0 & 16.9 & 16.5 \\ 5 X & 5.9 & 15.7\end{array}$

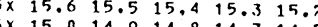

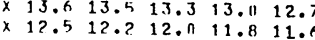

$\begin{array}{llcccc}x=0 & 2 & 4 & 6 & 8 \\ x & 27.6 & 27.6 & 27.6 & 27.6 & 27.6 \\ x & 27.6 & 27.6 & 277.6 & 27.6 & 27.6\end{array}$

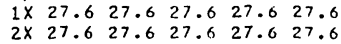

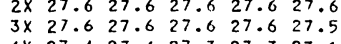

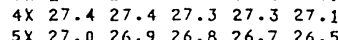
$\begin{array}{lllllll}5 x & 27.0 & 26.9 & 26.8 & 26.7 & 26.5 \\ 6 x & 26.4 & 26.3 & 26.1 & 26.0 & 25.9 \\ 7 x & 25.7 & 25.5 & 25.1 & 24.9 & 24.6\end{array}$ $\begin{array}{llllll}8 \times & 24.1 & 23.2 & 22.5 & 21.8 & 21.2 \\ 9 \times & 20.7 & 20.2 & 19.8 & 19.4 & 19.0\end{array}$ $10 \times 18.5$
PERCFNT TIIAL DISTANT.

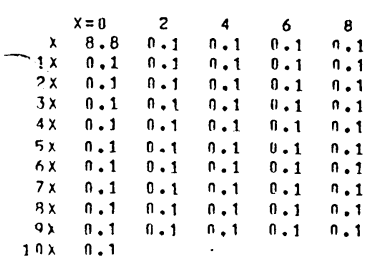

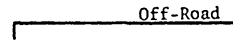

bipilan tittai mistanti.

$\begin{array}{lllll}x=0 & 2 & 2 & 6 & 8\end{array}$ $\begin{array}{llllll}1 \times & 15.8 & 15.3 & 14.8 & 14.5 & 14.1 \\ 2 x & 13.8 & 13.5 & 13.2 & 12.9 & 12.7 \\ 3 x & 12.5 & 12.3 & 12.1 & 12.9 & 12.7\end{array}$

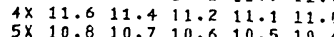

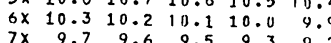
$\begin{array}{llllll}7 \times & 9.7 & 9.6 & 9.5 & 9.3 & 9 . \\ 8 \times & 9.0 & 8.8 & 8.7 & 8.5 & 9.3\end{array}$ $\begin{array}{rrrrrr}9 x & 8.1 & 5.2 & 2.5 & 8.5 & 8.3 \\ 10 x & 1.0 & . & & & 1.3\end{array}$

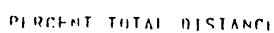

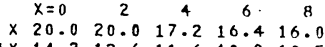
$\begin{array}{llllll}2 \times & 10.1 & 9.8 & 9.6 & 9.4 & 9.2\end{array}$ $\begin{array}{llllll}3 \mathrm{X} & 9.1 & 9.0 & 8.8 & 8.7 & 8.6 \\ .4 \mathrm{X} & 8.5 & 8.4 & 8.4 & 8.3 & 8.2\end{array}$ $\begin{array}{llllll}5 \times & 8.2 & 8.1 & 8.0 & 8.0 & 7.9 \\ 6 x & 7.8 & 7.7 & 7.7 & 7.6 & 7.5 \\ 7 \times & 7.4 & 7.3 & 7.2 & 7.1 & 7.0\end{array}$ $\begin{array}{llllll}7 \times & 7.4 & 7.3 & 7.2 & 7.6 & 7.5 \\ 8 \times & 0.4 & 0.7 & 0.5 & 3.5 & 2.0\end{array}$ $\begin{array}{llllll}8 \mathrm{x} & 6.9 & 6.7 & 6.3 & 3.5 & 2.0 \\ 9 \mathrm{x} & 1.4 & 1.1 & 0.9 & 0.8 & 0.7\end{array}$ $10 \times 0.0$

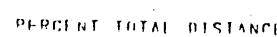

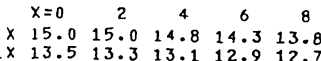
$\begin{array}{rrrrrr}1 \times & 13.5 & 13.3 & 13.1 & 12.9 & 12.8 \\ 2 x & 12.6 & 12.4 & 12.2 & 12.1 & 12.0\end{array}$ $\begin{array}{llllll}2 x & 12.6 & 12.4 & 12.2 & 12.1 & 12.0 \\ 3 x & 11.9 & 11.7 & 11.6 & 11.4 & 11.3\end{array}$ $\begin{array}{llllll}4 \times & 11.1 & 11.0 & 110.8 & 10.7 & 10.5 \\ 5 \times & 10.4 & 10.3 & 10.1 & 10.0 & 9.5\end{array}$ $\begin{array}{rrrrrr}5 \times & 10.4 & 10.3 & 10.1 & 10.0 & 9.8 \\ 6 \times & 9.7 & 9.5 & 9.4 & 9.3 & 9.1\end{array}$ $\begin{array}{llllll}7 \times & 9.0 & 8.9 & 8.7 & 8.6 & 8.4 \\ 8 \times & 8.2 & 8.0 & 7.8 & 7.5 & 7.4 \\ 9 \times & 5.8 & 2.6 & 1.7 & 1.3 & 1.0\end{array}$ $\begin{array}{rrrrrrr}9 \times & 5.8 & 2.6 & 1.7 & 1.3 & 1.0 \\ 10 \times & 0.9 & & & & \end{array}$

PIRTHAT TIITA RICIAME

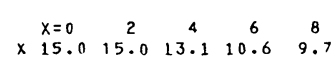
$\begin{array}{rrrrrr}1 x & 1.2 & 0.4 & 0.3 & 0.2 & 0.2 \\ 2 x & 0.2 & 4.2 & 0.2 & 0.2 & 0.1\end{array}$ $\begin{array}{llllll}2 x & 0.2 & 0.2 & 0.2 & 0.2 & 0.2 \\ 3 \times & 0.1 & 0.1 & 0.1 & 0.1 & 0.1 \\ 5 x & 0.1 & 0.1 & 0.1 & 0.1 & 0.1\end{array}$ $\begin{array}{llllll}4 \times & 0.1 & 0.1 & 0.1 & 0.1 & 0.1 \\ 5 \times & 0.1 & 0.1 & 0.1 & 0.1 & 0.1 \\ 6 x & 0.1 & 0.1 & 0.1 & 0.1 & 0.1\end{array}$ $\begin{array}{llllll}6 x & 0.1 & 0.1 & 0.1 & 0.1 & 0.1 \\ 7 x & 0.1 & 0.1 & 0.1 & 0.1 & 0.1\end{array}$ $\begin{array}{llllll}9 x & 0.1 & 0.1 & 0.1 & 0.1 & 0.1\end{array}$ 


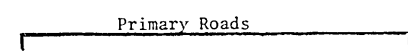

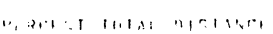

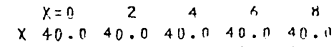
3X 411.040 .040 .040 .040 .040$.

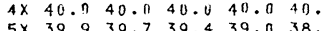

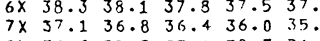
$8 \times 34.6 \quad 33.8 \quad 33.1 \quad 32.3 \quad 31$. $9 \times 30.4$
$10 \times 25.9$

(5.

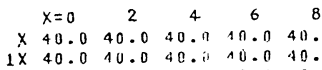
$\begin{array}{llllll}2 x & 40.0 & 10.0 & 40.0 & 40.040 .0 \\ 3 x & 40.0 & 40.0 & 40.0 & 40.0 & 40.0\end{array}$ $4 \times 40.040 .0 \quad 40.0 \quad 40.040 .0$ $5 \times \quad 40.0 \quad 40.0 \quad 39.7 \quad 39.5 \quad 39.2$

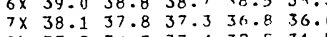

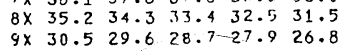

PFRCENT TOTAL DISTANCE $\begin{array}{rrrrrr}X=0 & 2 & 4 & 6 & 8 \\ x & 40.0 & 40.0 & 40.0 & 40.0 & 40.0 \\ 1 \times & 40.0 & 40.0 & 40.0 & 40.0 & 40.0\end{array}$ $2 \times 40.0 \quad 40.0 \quad 40.0 \quad 40.04 \pi .0$.

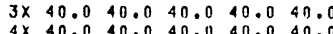
$5 \times 40.0400040 .040 .0440$ $\begin{array}{llllll}6 \times & 40.0 & 40.0 & 40.0 & 40.0 & 39.9\end{array}$

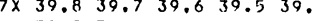
$\begin{array}{llllll}8 \times & 39.2 & 39.0 & 38.7 & 38.5 & 38.0 \\ 9 \times & 37.1 & 35.8 & 33.8 & 31.9 & 3 n .3\end{array}$

\section{PERCENT TOTAL DISTANCE}

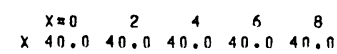

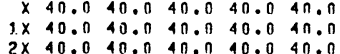

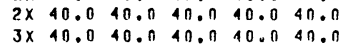
$\begin{array}{cccccc}3 x & 40.0 & 40.0 & 40.0 & 40.0 & 4 n .0 \\ 4 x & 40.0 & 40.0 & 40.0 & 40.0 & 40.0\end{array}$ $5 \times \quad 40.0 \quad 40.0 \quad 40.0 \quad 40.0 \quad 40.0$ $6 \times \quad 40.0 \quad 40.0 \quad 40.0 \quad 40.0 \quad 39.9$ $\begin{array}{llllll}7 \times & 39.8 & 39.7 & 39.6 & 39.4 & 39 . \\ 8 \times & 39.1 & 38.8 & 38.5 & 38.1 & 37.6\end{array}$ $\begin{array}{llllll}8 \times & 39.1 & 38.8 & 38.5 & 38.1 & 37.6 \\ 9 \times & 36.7 & 35.3 & 33.2 & 31.3 & 29.7\end{array}$ aable E23

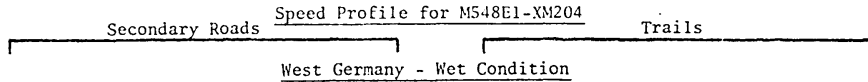

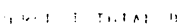

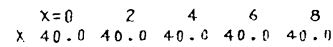
$\begin{array}{llllll}1 X & 41.0 & 40.0 & 40.0 & 39.7 & 39.3 \\ 2 X & 38.3 & 37.4 & 36.7 & 36.1 & 35.6 \\ 3 X & 35.2 & 34.9 & 34.6 & 34.3 & 34.1\end{array}$ $\begin{array}{lllllll}3 x & 35.2 & 34.9 & 34.6 & 34.3 & 34.1 \\ 4 X & 33.8 & 33.5 & 33.2 & 32.9 & 32.6\end{array}$ $\begin{array}{llllll}5 X & 32.3 & 32.1 & 31.9 & 31.6 & 31.3 \\ 6 X & 31.1 & 30.8 & 30.4 & 30.1 & 29.7\end{array}$ $\begin{array}{llllll}7 \times & 29.2 & 28.8 & 28.4 & 28.0 & 27.6 \\ 8 \times & 27.1 & 26.7 & 26.2 & 25.7 & 25.1\end{array}$ $\begin{array}{llllll}8 \times & 27.1 & 26.7 & 26.2 & 25.7 & 25.1 \\ 9 \times & 24.6 & 24.1 & 23.6 & 23.0 & 22.3\end{array}$ $9 \times 24.6$
$10 \times 21.5$ West

,

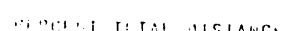

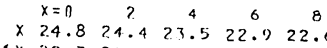
$\begin{array}{llllll}1 \times & 24.8 & 24.4 & 23.5 & 22.9 & 22.6 \\ 2 \times & 20.0 & 21.8 & 21.3 & 20.9 & 211.4 \\ 3 x & 20.5 & 10.7 & 19.5 & 10.2 & 10.9\end{array}$ $\begin{array}{lllll}3 \times & 18.5 & 18.2 & 19.0 & 17.6\end{array}$ $\begin{array}{llllll}4 \times & 16.8 & 16.4 & 16.1 & 15.6 & 15.5 \\ 5 \times & 15.3 & 15.4 & 14.0 & 14.7 & 14.5\end{array}$

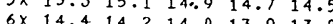
$\begin{array}{llllll}7 \times & 13.6 & 13.4 & 14.0 & 13.9 & 13.6\end{array}$

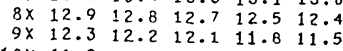
$10 \times 11.2$

$\begin{array}{rrrrrr}x=0 & 2 & 4 & 6 & 8 \\ x & 39.3 & 39.3 & 39.3 & 39.3 & 39.3\end{array}$

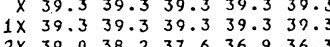
$\begin{array}{lllllll}2 x & 39.0 & 38.2 & 37.6 & 36.9 & 36 . \\ 3 x & 35.9 & 35.5 & 35.1 & 34.8 & 34 .\end{array}$ $\begin{array}{lllllll}4 \times & 34.3 & 34.1 & 33.9 & 33.7 & 33.5 \\ 5 \times & 33.4 & 33.2 & 33.0 & 32.8 & 33.5\end{array}$ $\begin{array}{llllll}5 \times & 33.4 & 33.2 & 33.0 & 32.8 & 32.6 \\ 6 \times & 32.3 & 32.0 & 31.7 & 31.3 & 30.0\end{array}$

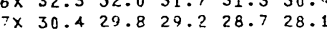
$\begin{array}{llllll}7 \times & 30.4 & 29.8 & 29.2 & 28.7 & 28.1 \\ 8 \times & 27.5 & 26.8 & 26.0 & 25.3 & 24.5 \\ 9 \times & 23.7 & 23.0 & 22.3 & 21.6 & 20.6\end{array}$ $10 \times 19.7$$$
\text { Mid-E }
$$

PERCENT TOTAL DISTANCE

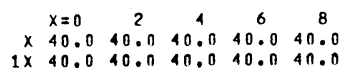
$2 x \quad 40.0 \quad 40.0 \quad 40.0 \quad 39.1 \quad 38.3$

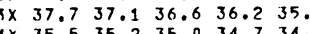
$\begin{array}{lllllll}5 \times & 34.3 & 34.2 & 34.0 & 33.9 & 33.7\end{array}$ $\begin{array}{llllll}6 \times & 33.6 & 33.4 & 33.2 & 33.0 & 33.8 \\ 7 \times & 32.7 & 32.5 & 337.2 & 31.8 & 31.3\end{array}$ $8 \times \quad 30.729 .7 \quad 28.8$ 27.9 27.2 $\begin{array}{rr}9 \times & 26.5 \\ 10 \times & 23.7\end{array}$

$$
25.925 .424 .824 .4
$$

$$
\text { Mid-E }
$$

PERCENT TOTAL DISTANCE id-East - Sand Comlition

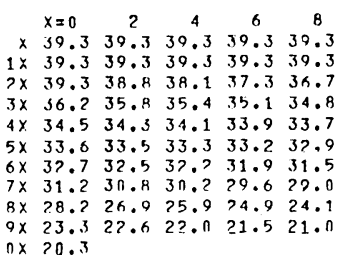

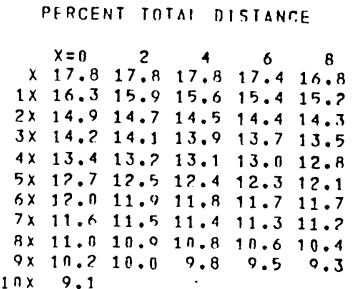

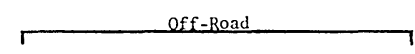

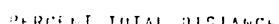

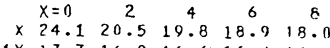

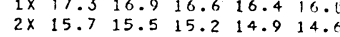
$\begin{array}{lllllll}3 \times & 14.4 & 14.2 & 14.1 & 13.9 & 13.9 \\ 4 \times & 13.6 & 13.4 & 13.3 & 13.1 & 13.0\end{array}$ $\begin{array}{lllllll}5 \times & 12.8 & 12.7 & 12.6 & 12.4 & 12 .\end{array}$ $\begin{array}{llllll}7 \times & 11.5 & 11.3 & 11.2 & 11.0 & 10.9\end{array}$ $\begin{array}{cccccc}8 \times & 10.7 & 10.5 & 10.3 & 10.1 & 9.9 \\ 9 \times & 3.5 & 2.0 & 1.4 & 1.1 & 0.9\end{array}$

$\begin{array}{rr}9 \times & 3.5 \\ 10 \times & 0.8\end{array}$

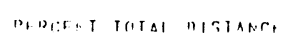

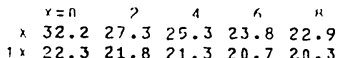
$\begin{array}{llllll}1 \times & 22.3 & 21.8 & 21.3 & 20.7 & 20.3 \\ 3 \times & 19.9 & 19.4 & 19.0 & 18.6 & 18.3\end{array}$ $\therefore \begin{array}{rlllll}\therefore & 15.9 & 15.7 & 15.4 & 15.2 & 15.0\end{array}$ $7 \times 13.7 \quad 13.5 \quad 13.3 \quad 13.112 .9$ $\begin{array}{rrrrrr}4 \times & 12.7 & 12.5 & 12.3 & 12.1 & 12.9 \\ r \times & 11.6 & 4.1 & 2.2 & 1.5 & 1.9 \\ 1.2 & 1.2\end{array}$

PERCENT TOTAL DISIANCE

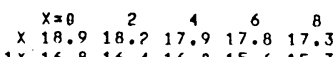

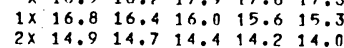
$\begin{array}{lllllll}2 x & 14.9 & 14.7 & 14.4 & 14.2 & 14.0 \\ 3 x & 13: 8 & 13.7 & 13.5 & 13.4 & 13 .\end{array}$ $\begin{array}{lllllll}5 \times & 12.3 & 12.2 & 12.1 & 11.9 & 11.8\end{array}$ $\begin{array}{cccccc}6 x & 11.7 & 11.6 & 11.5 & 11.3 & 11 . \\ 7 x & 10.9 & 10.7 & 11.5 & 110.4 & 11\end{array}$ $\begin{array}{cccccc}7 \times & 10.9 & 10.7 & 10.6 & 10.4 & 10.2 \\ 8 \times & 10.1 & 9.9 & 9.7 & 0.5 & \end{array}$ $\begin{array}{rrrrrr}9 \times & 2.6 & 1.7 & 1.3 & 1.0 & 0.9 \\ 10 \times & 0.8 & & & & \end{array}$

PERCENT TITAL DISTANCE

$\begin{array}{rlrrrr} & x=0 & 2 & 4 & 6 & 8 \\ x & 17.1 & 16.3 & 15.6 & 15.2 & 14.9 \\ 1 x & 14.7 & 14.6 & 14.5 & 14.4 & 14 . ?\end{array}$ $\begin{array}{llllll}2 x & 14.0 & 13.7 & 13.5 & 13.2 & 13 .\end{array}$ $\begin{array}{cccccc}3 \times & 12.8 & -12.5 & 12.3 & 12.1 & 11.9 \\ 4 \times & 11.7 & 11.6 & 11.4 & 11.3 & 11.9\end{array}$

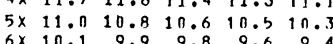
$\begin{array}{llllll}7 \times & 9.3 & 9.1 & 8.9 & 8.8 & 9.6 \\ 8 \times & 8.4 & 8.2 & 8.0 & 7.8 & 9.6 \\ 9 x & 3.1 & 1.9 & 1.4 & 1.8 & 0.9\end{array}$ $\begin{array}{rrrrrr}9 \times & 3.1 & 1.9 & 1.4 & 1.1 & 0.9 \\ 10 \times & 0.8 & & & & \end{array}$ 


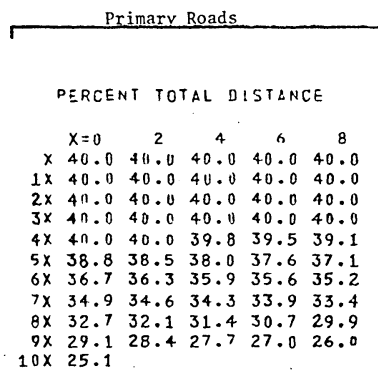

PERCENT TOTAL DISTANCE

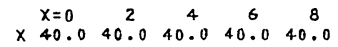
$\begin{array}{cccccc}1 \times & 40.0 & 10.0 & 40.0 & 40.0 & 40.0 \\ 2 \times & 40.0 & 40.0 & 40.0 & 40.11 & 40.0\end{array}$

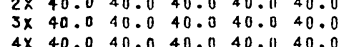
$\begin{array}{llllll}4 \times & 40.0 & 40.0 & 40.0 & 40.0 & 40.0 \\ 5 \times & 39.8 & 39.5 & 39.2 & 38.8 & 38.4\end{array}$ $6 \times 38.0$ $\begin{array}{lllllll}7 \times & 36.6 & 36.3 & 35.8 & 35.4 & 34.7\end{array}$ $\begin{array}{llllll}8 \times & 33.9 & 33.1 & 32.3 & 31.5 & 30.6 \\ 9 \times & 29.6 & 28.8 & 28.0 & 27.3 & 26.2\end{array}$

PERCENT TOTAL DISTANC:E

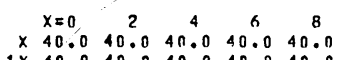

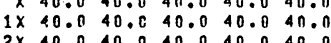

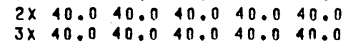

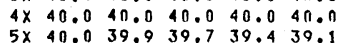
$\begin{array}{llllll}6 \times & 38.9 & 38.6 & 38.4 & 38.2 & 38.1 \\ 7 \times & 37.8 & 37.6 & 37.4 & 37.2 & 37.0 \\ 8 \times & 36.8 & 36.6 & 36.4 & 36.1 & 35.7\end{array}$ $\begin{array}{rrrrrrr}8 \times & 36.8 & 36.6 & 36.4 & 36.1 & 35.7 \\ 9 \times & 35.0 & 33.9 & 32.0 & 30.4 & 20.0\end{array}$ $10 \times 27.7$

PERCENT IOTAL DISTANCE

$\begin{array}{rrrrrr}x=0 & 2 & 4 & 6 & B \\ x & 40.0 & 40.0 & 4 n^{4} .0 & 40.0 & 4 n^{3} .0 \\ x & 40.0 & 40.0 & 40.0 & 40.0 & 40.0\end{array}$

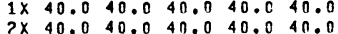
$3 \times \quad 40.0 \quad 40.0 \quad 40.0 \quad 40.0 \quad 40.0$ $\begin{array}{llllll}4 \times & 40.0 & 40.0 & 40.0 & 40.0 & 40.0\end{array}$ $\begin{array}{lllllll}5 \times & 40.0 & 39.8 & 39.5 & 39.3 & 39.0 & 0\end{array}$ $7 \times 37.7737 .5 \quad 37.3 \quad 37.136 .9$ $\begin{array}{rrrrrr}8 \times & 36.7 & 36.4 & 36.2 & 35.8 & 35.4 \\ 9 \times & 34.6 & 33.5 & 31.6 & 79.9 & 28.4 \\ 10 \times & 27.2 & & & & \end{array}$ $10 \times 27.2$

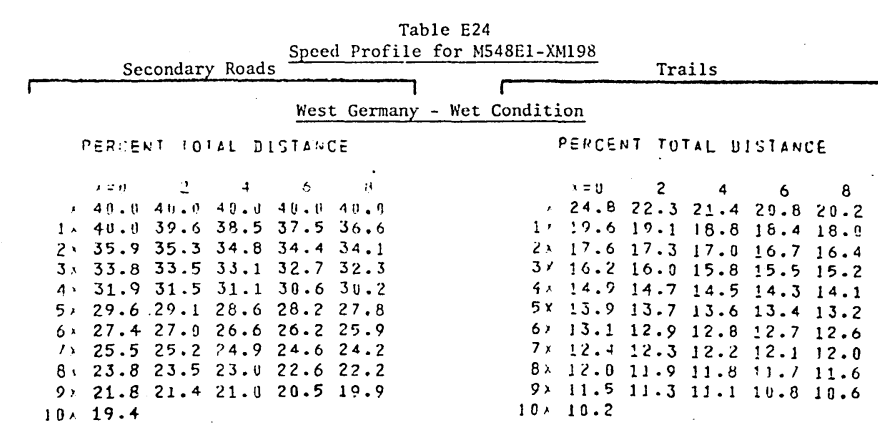

\section{PERCENT TOTAL DISTANCE \\ PERCENT TOTAL DISTANCE}

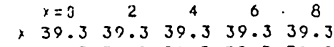

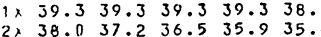

3. $35.0 \quad 34.7 \quad 34.4 \quad 34.2 \quad 33.0$

$\begin{array}{llllll}4 \times & 33.7 & 33.4 & 33.1 & 32.8 & 32.4\end{array}$

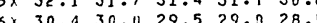

7 ) 28.427 .527 .026 .525 .9

$\begin{array}{llllll}61 & 25.3 & 24.6 & 24.0 & 23.4 & 22.7 \\ 9 x & 22.1 & 21.5 & 20.9 & 20.3 & 19.4\end{array}$ $9 \times 22.1$

\section{Mid-East - Wet Conditio}

Percent total Distance

$\begin{array}{ccccc}x=0 & 2 & 4 & 0 & 8 \\ 24.8 & 24.8 & 24.7 & 24.0 & 23.4\end{array}$

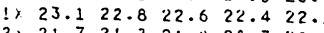
$3 \times 20.1 \quad 19.9 \quad 19.819 .410 .9$

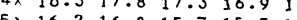
$\begin{array}{llllll}5, & 16.2 & 16.9 & 15.7 & 15.5 & 15.5 \\ 6.15 .1 & 15.9 & 14.9 & 14.8 & 14.0 & 14.5\end{array}$ $\begin{array}{lllllll}1 . & 14.3 & 14.1 & 15.9 & 13.7 & 13.5\end{array}$

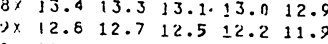
PERCFNT TOTAL Distanf.F

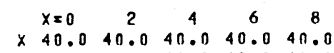
$\begin{array}{cccccc}1 \times & 40.0 & 40.0 & 40.0 & 40.0 & 40.0 \\ 2 \times & 40.0 & 40.0 & 40.0 & 39.0 & 30.0 \\ 3 x & 30.0 & 30.0 & 30.0\end{array}$ $\begin{array}{llllll}2 x & 40.0 & 40.0 & 40.0 & 39.1 & 38.0 \\ 3 x & 37.7 & 37.1 & 36.6 & 36.2 & 35.8\end{array}$ $\begin{array}{llllll}4 \times & 35.5 & 35.2 & 35.0 & 34.7 & 34 . \\ 5 \times & 34.3 & 34.2 & 34.0 & 33.9 & 33 .\end{array}$ $\begin{array}{llllll}6 x & 33.6 & 33.3 & 33.1 & 32.8 & 33.5 \\ 7 \times & 32.2 & 32.0 & 31.7 & 31.3 & 3 n .9\end{array}$ $\begin{array}{llllll}8 \times & 10.3 & 29.3 & 28.4 & 27.6 & 26.9 \\ 9 \times & 26.2 & 25.7 & 25.1 & 24.6 & 24.1\end{array}$ $9 \times 26.2$
$10 \times 23.5$

$$
\text { Mid-E }
$$

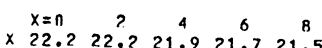

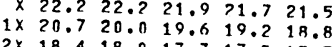
$\begin{array}{llllll}2 \times & 18.4 & 18.0 & 17.7 & 17.5 & 17.3\end{array}$

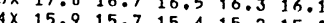
$5 \times 14.8 \quad 14.6 \quad 14.4 \quad 14.3 \quad 14.1$ $\begin{array}{llllll}6 x & 13.9 & 13.8 & 13.7 & 13.5 & 13.4\end{array}$ $\begin{array}{llllll}7 x & 13.3 & 13.1 & 13.0 & 17.9 & 17.8 \\ 8 \times & 12.6 & 12.5 & 17.4 & 12.3 & 12.8\end{array}$ ax $12.011 .311 .7 \quad 11.511 .3$

Percent total Distanfie

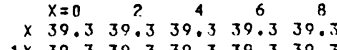
$\begin{array}{lllllll}1 \times & 39.3 & 39.3 & 39.3 & 39.3 & 39.5 \\ 3 \times & 39.3 & 38.8 & 38.1 & 37.3 & 36.7\end{array}$ $\begin{array}{llllll}3 \times & 36.2 & 35 . & 35.4 & 35.1 & 34.7\end{array}$ $\begin{array}{lllllll}4 \times & 34.5 & 34.5 & 34.1 & 33.9 & 33.7 \\ 5 \times & 33 & 33.6 & 33.5 & 33.5 & 33.0 & 32.7\end{array}$ $\begin{array}{llllll}5 \times & 33.6 & 33.5 & 34.1 & 33.9 & 33.7 \\ 6 \times & 32.3 & 32.0 & 31.7 & 33.0 & 39\end{array}$ $\begin{array}{llllll}7 \times & 30.7 & 30.3 & 29.8 & 99.2 & 28.6\end{array}$ $\begin{array}{llllll}8 \times & 27.8 & 26.6 & 25.6 & 24.7 & 23.6\end{array}$

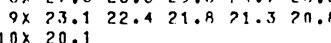

PERCENT TOTAL DISTANCE

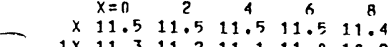
$? x 10.810 .8$ 1n $10.7 \quad 11.0010 .9$ $\begin{array}{llllll}3 \times & 10.3 & 10.7 & 10.1 & 9.9 & 9.8\end{array}$ $\begin{array}{cccccc}4 \times & 9.8 & 9.7 & 9.6 & 9.5 & 9.4 \\ 5 x & 9.4 & 9.5 & 0.2 & 9.1 & 0.1\end{array}$ $\begin{array}{llllll}8 \times & 8.3 & 8.6 & 8.6 & 8.5 & 8.4 \\ 9 \times & 7.6 & 7.4 & 8.1 & 8.0 & 7.9\end{array}$ $\begin{array}{llllll}6 x & 9.4 & 9.3 & 9.2 & 9.1 & 0.1\end{array}$ $\begin{array}{llllll}9 x & 7.6 & 7.4 & 7.1 & 8.0 & 7.9 \\ 10 \times & 3.0 & & & & \end{array}$

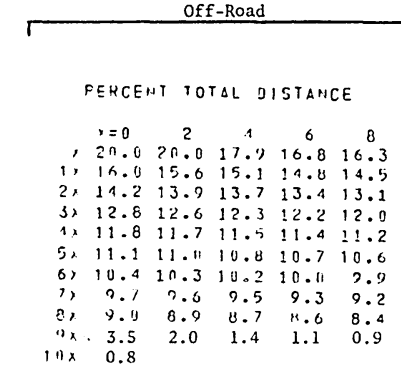

PERLE.T TOIAL DISTANCE

$\begin{array}{lllll}x=0 & 2 & 1 & 6 & 8\end{array}$ $2 \times 18.4 \quad 18.419 .919 .4 \quad 18.4$

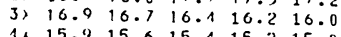
$\begin{array}{lllllll}14 & 15.9 & 15.6 & 15.4 & 15.2 & 15.0 \\ 5 . & 11.8 & 14.6 & 14.3 & 14.1 & 13.9\end{array}$

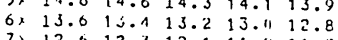
$\begin{array}{rrrrrrr}8 \times & 11.5 & 11.3 & 11.1 & 11.8 & 10.6 \\ 3 \times & 10.4 & 3.9 & 2.2 & 1.5 & 1.2\end{array}$ $10 \times 1,0$

PERCENT TOTAL. DISTANCE

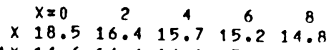
$\begin{array}{rrrrrr}1 \times & 14.6 & 14.4 & 14.1 & 13.2 & 14.8 \\ 2 x & 13.5 & 13.4 & 13.2 & 13.0 & 13.7 \\ 3 x & 12.5 & 13.6 & 13.4 & 13.3 & 12.9\end{array}$

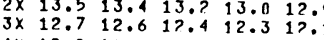
$\begin{array}{llllll}4 x & 12.0 & 11.9 & 11.7 & 11.6 & 11.5 \\ 5 x & 11.3 & 11.2 & 11.1 & 11.0 & 110.0\end{array}$ $\begin{array}{cccccc}6 \times & 10.7 & 10.6 & 10.4 & 10.3 & 1 \mathrm{n} .1\end{array}$ $\begin{array}{llllll}7 \times & 9.9 & 9.7 & 0.6 & 9.4 & 9 .\end{array}$ $\begin{array}{rrrrrr}8 \times & 9.1 & 8.9 & 8.7 & 8.6 & 5.8 \\ 9 x & 2.6 & 1.7 & 1.3 & 1.0 & 0.9 \\ 10 x & 0.7 & & & & \end{array}$

PERCENT TOTAL DISTANCE

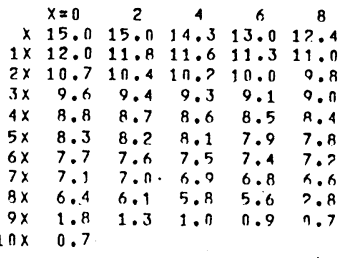




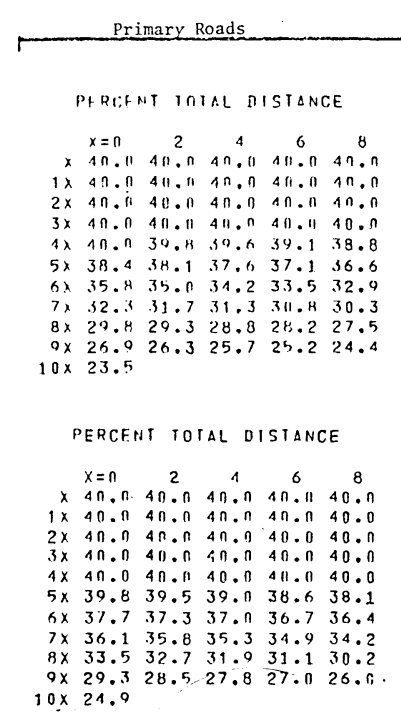

PERCENT TOTAL DISTANCE

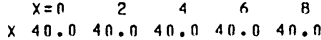
$\begin{array}{cccccc}1 \times & 40.0 & 40.0 & 4 n .0 & 10.0 & 4 n .0 \\ 2 x & 40.0 & 40.0 & 40.0 & 0.0 & 40\end{array}$ $3 x \quad 40.040 .0 \quad 4 n, 0 \quad 40.0 \quad 4 n$. $\begin{array}{llllll}4 \times & 40.0 & 40.0 & 39.9 & 39.7 & 39.9\end{array}$

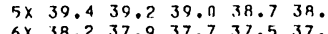
$7 \times 37.136 .836 .6 \quad 36.4 \quad 36.2$ $8 \times 36.035 .8 \quad 35.6 \quad 35.2 \quad 34$.

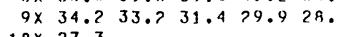

\section{PERCENT TOTAL DISTANC.}

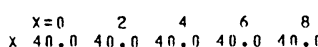

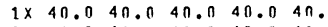
$3 x 4 n .04 n .04 n \cdot 040.04 n$.

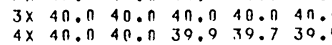
$5 \times \quad 39.4 \quad 39.2 \quad 38.9 \quad 38.6 \quad 38$. $\begin{array}{llllll}6 \times & 38.1 & 37.8 & 37.6 & 37.4 & 37.2\end{array}$

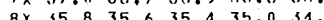
$\begin{array}{llllll}8 \times & 35.8 & 35.6 & 35.4 & 35.0 & 31 . \\ 9 \times & 33.9 & 32.8 & 31.0 & 29.4 & 28 .\end{array}$ $10 \times 26.8$

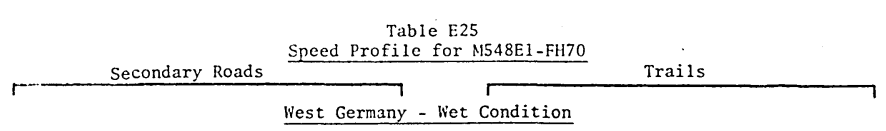

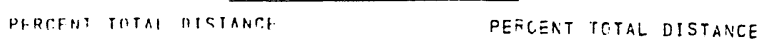

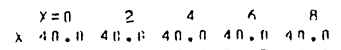

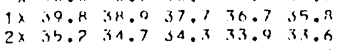

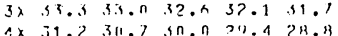

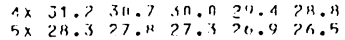

$\begin{array}{cccccc}6 \times & 26.1 & 25.7 & 75.4 & 21,011 & 24.7 \\ 7 \times & 24.4 & 74.7 & 73.8 & 23.5 & 23.2\end{array}$

$8 \times 22.872 .422 .1121 .671 .6$

$10 \times 18.6$

West Germany - Snow Condition

PerTent THTAL Distant.E

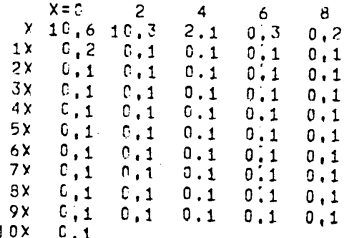

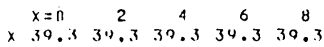

$\begin{array}{llllll}1 \times & 30.3 & 30.3 & 30.3 & 39.3 & 38.8 \\ 7 \times & 37.8 & 36.9 & 36.3 & 35.7 & 35.3\end{array}$

$\begin{array}{llllll}2 x & 37.8 & 36.9 & 36.3 & 35.7 & 35.7 \\ 3 x & 34.9 & 34.0 & 34.3 & 34.0 & 33.7\end{array}$

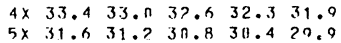

$\begin{array}{llllll}5 \times & 31.6 & 31.2 & 30.8 & 30.4 & 20.9 \\ 6 x & 29.4 & 00.11 & 78.5 & 28.0 & 77.6\end{array}$

$\begin{array}{lllllll}7 \times & 27.1 & 26.6 & 26.1 & 25.6 & 25.1 \\ 8 \times & 24.5 & 23.9 & 23.3 & 22.7 & 22.1\end{array}$

ox $71.5 \quad 2 n .020 .119 .719$.

$1 \mathrm{n} \times 18$.

\section{Mid-East - Wet Condition}

PFRCFNT TIINL DISTANGE PERCFIT TSTAL DIGTANCE

$\begin{array}{cccccc}x=0 & 2 & 4 & 6 & 8 \\ \times & 40.0 & 411.0 & 4 n \cdot 0 & 40 \cdot n & 4 n^{2} \cdot n\end{array}$

$2 x+40,0 \quad 40,040,039.1 \quad 38.3$

$3 \times 37.7 \quad 37.136 .6 \quad 36.235 .4$

$4 \times 35.5 \quad 35 . ? 35.634 .7 \quad 34.5$

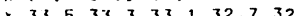

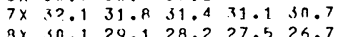

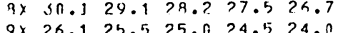

$1 \mathrm{n} \times 23.4$

Ph:RIEnt TGtal DISTANG,

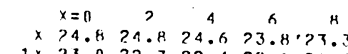

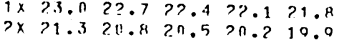

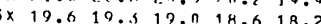

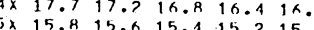

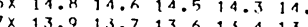

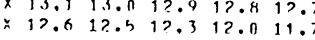

$$
\text { prroent totTal mistante }
$$$$
\text { Mid- }
$$

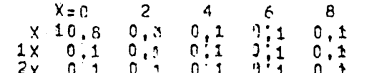

$\begin{array}{ll}2 x & 0 \\ 3 x & 0 \\ 4 x & 0 \\ 5 x & 0 \\ 2 x & 1\end{array}$

$4 x$
$5 x$

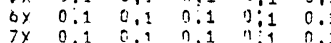

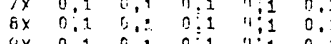
$10 \times \quad 0.1$

$\begin{array}{ccccc}x=0 & 2 & 4 & 6 & 8 \\ x \quad 39.3 & 34.3 & 30.3 & 39.3 & 30.3\end{array}$

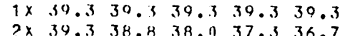

$3 \times 36.235 .435 .4435 .134 .7$

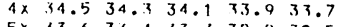

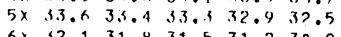

$7 x \quad 30.5311 .1120 .620 .030 .9$

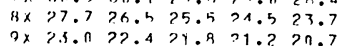

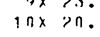

PEacer

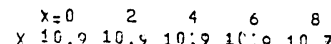

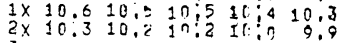

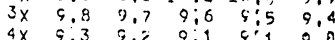

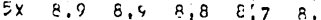

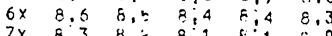

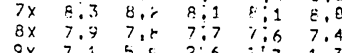

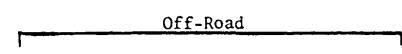

PFPCENT TOIAI DISIANTE

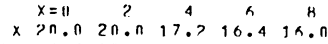

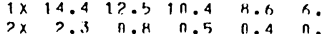
$\begin{array}{llllll}3 x & n \cdot 3 & n \cdot ? & n \cdot ? & 11.2 & n \cdot ? \\ 4 x & n: ? & n . ? & n \cdot ? & 11 . ? & n \cdot ?\end{array}$ $\begin{array}{llllll}5 x & n . ? & 1.2 & n \cdot 2 & n .2 & n \cdot ? \\ 6 x & n .1 & 0.2 & 0.1 & 0.1 & n\end{array}$

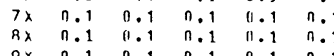
$\begin{array}{rr}9 \times & 0.1 \\ 10 \times & 0.1\end{array}$

PEREFAT TITAL DISIANC.

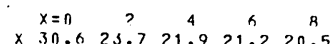

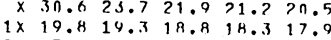
$? \times 37.517 .317 .916 .8$ 16.6 $\begin{array}{ccccccc}3 \times & 16.3 & 16.1 & 15.9 & 15.6 & 15.4\end{array}$ $5 \times 14.4 \quad 14.2 \quad 13.9013 .7$ 11.6 $\begin{array}{llllll}6 x & 13.3 & 13.3 & 13.0 & 13.8 & 17.6\end{array}$

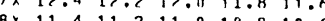
$\begin{array}{rrrrrr}8 \times & 11.4 & 11.2 & 11.0 & 10.8 & 10.6 \\ 9 x & 10.3 & 3.9 & 7.2 & 1.5 & 1.9\end{array}$

PERCENTT TCTAL DISTANCE

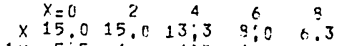

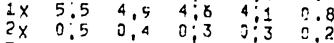
$\begin{array}{llllll}3 x & 0: 2 & 0.2 & 0: 2 & 0 ; 2 & 0.2\end{array}$

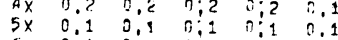

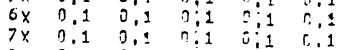

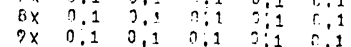
$\begin{array}{cc}9 \times & 0.1 \\ 10 \times & 0.1\end{array}$

PERCENT TGTAL DISTANCE

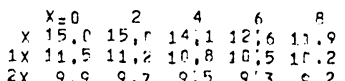

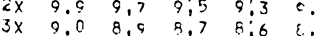

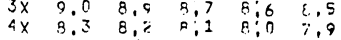

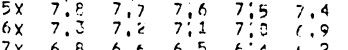

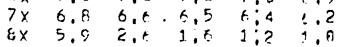
$\begin{array}{ccccc}9 x & 0.8 & 0.7 & 0 & 0 \\ 10 x & 0.5 & 0 & \end{array}$ 


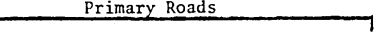

opremi total gistance

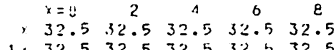

$2 \times 32.532 .5 \quad 32.5 \quad 32.532 .5$

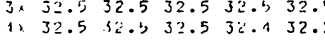

$5 \times 32.131 .931 .731 .5 \quad 31$.

is $29.228 .928 .7 \quad 26.4 \quad 28.2$

$\begin{array}{llllll}8 \times & 27.8 & 27.4 & 27.9 & 26.0 & 26.0 \\ 9 \times & 25.5 & 25.0 & 24.5 & 24.11 & 23.3\end{array}$

$9 \times 25.5$
$10 \times 22.6$

PERCENT TOTAL DISTANCE

$\begin{array}{rrcccc} & x=0 & 2 & 4 & 6 & 8 \\ \times \quad 32.5 & 32.5 & 32.5 & 32.5 & 32.5 \\ 1 \times & 32.5 & 32.5 & 32.5 & 32.5 & 32.5\end{array}$

$\begin{array}{llllll}2 \lambda & 32.5 & 32.5 & 32.5 & 32.5 & 32.5 \\ 3 \times & 32.5 & 32.5 & 32.5 & 32.5 & 32.5\end{array}$

$\begin{array}{llllll}4 \times & 32.5 & 32.5 & 32.5 & 32.5 & 32.5 \\ 5 \times & 32.5 & 32.4 & 32.2 & 32.1 & 31.9\end{array}$

$6 \times 31.8 \quad 31.6 \quad 31.5 \quad 31.3 \quad 31$.

$7 \times 31.131 .0 \quad 30.830 .6 \quad 30$.

$\begin{array}{llllll}8 \times & 29.7 & 29.1 & 28.6 & 28.0 & 27.3 \\ 9 \times & 26.7 & 26.0 & 25.5 & 24.0 & 24\end{array}$

$10 \times 23.2$

PERCENT TOTAL DISIANCE

$\begin{array}{rrrrrr}x=0 & 2 & 4 & 6 & 8 \\ \times & 32.5 & 32.5 & 32.5 & 32.5 & 32.5 \\ x & 32.5 & 32.5 & 32.5 & 32.5 & 32.5\end{array}$

$\begin{array}{llllll}1 \times & 32.5 & 32.5 & 32.5 & 32.5 & 32.5 \\ 2 x & 32.5 & 32.5 & 32.5 & 32.5 & 37.5\end{array}$

$\begin{array}{llllll}2 x & 32.5 & 32.5 & 32.5 & 32.5 & 32.5 \\ 3 x & 32.5 & 32.5 & 32.5 & 32.5 & 32.5\end{array}$

$\begin{array}{lllllll}4 x & 32.5 & 32.5 & 32.5 & 32.5 & 32.5 \\ 5 x & 32.5 & 32.5 & 32.5 & 32.4 & 32.3\end{array}$

$6 \times 32.1 \quad 32.0 \quad 31.931 .8 \quad 31.9$

$\begin{array}{lllllll}7 \times & 31.6 & 31.5 & 31.4 & 31.3 & 31.3\end{array}$

$\begin{array}{llllll}8 \times & 31.2 & 31.1 & 31.0 & 30.9 & 3 n .8 \\ 9 \times & 30.3 & 29.6 & 28.2 & 27.0 & 25.9\end{array}$

PERCENT TOTAL DISTANCE

$\begin{array}{cccccc}x=0 & 2 & 4 & 6 & 8 \\ \times & 32.5 & 32.5 & 32.5 & 32.5 & 32.5\end{array}$

$1 \times 32.5 \quad 32.5 \quad 32.5 \quad 32.5 \quad 32.5$

$3 \times 32.532 .5332 .532 .537 .5$

$\begin{array}{lllllll}3 \times & 32.5 & 32.5 & 32.5 & 32.5 & 32.5 \\ 4 \times & 32.5 & 32.5 & 32.5 & 32.5 & 32.5\end{array}$

$\begin{array}{llllll}5 \times & 32.5 & 32.5 & 32.5 & 32.3 & 32.2\end{array}$

6x $32.1 \quad 31.9 \quad 31.8 \quad 31.7 \quad 31.6$

$\begin{array}{llllll}7 x & 31.5 & 31.4 & 31.4 & 31.3 & 31.2 \\ 8 \times & 31.1 & 31.1 & 31.0 & 30.9 & 31.6\end{array}$

$\begin{array}{llllll}8 \times & 31.1 & 31.1 & 31.0 & 31.9 & 31.6 \\ 9 \times & 30.2 & 29.3 & 28.0 & 76.7 & 25.6\end{array}$
Table E26
Speed Profile for UET-XM198

Secondary Roads
West Germany - Wet Condition

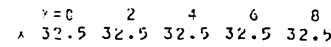

$\begin{array}{rrrrrr}i=0 & 2 & 4 & 0 & 8 \\ 21.3 & 23.9 & 23.2 & 22.0 & 22.0\end{array}$

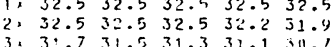

$\begin{array}{lllllll}4 & 31.3 & 29.9 & 29.4 & 29.0 & 28.6 \\ 5 \times & 28.2 & 27.9 & 27.5 & 27.2 & 27.0\end{array}$

$\begin{array}{llllll}3 \times & 25.0 & 25.4 & 25.1 & 24.8 & 24.5 \\ 3 \times & 21.4 & 23.9 & 23.5 & 23.1 & 22.7 \\ 3 \times & 22.3 & 22.0 & 21.5 & 21.1 & 20.5\end{array}$

0.19 .9

$$
\text { West Germany - Snow Condition }
$$

DEFCENT IOTAL 21 STANCE

$\begin{array}{cccccc}x=0 & 2 & 4 & 6 & 8 \\ \times & 32.5 & 32.5 & 32.5 & 32.5 & 32.5 \\ 12 & 32.5 & 32.5 & 32.5 & 3.5 & 32.5\end{array}$

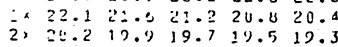
$\begin{array}{lllllll}3 & 19.1 & 19.0 & 18.8 & 13.6 & 18.5\end{array}$

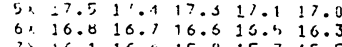
$\begin{array}{lllllll}8 \% & 15.4 & 15.3 & 15.2 & 15.11 & 14.8 \\ \%, & 14.7 & 14.5 & 14.2 & 13.7 & 13.3\end{array}$ 19. 14.

$\begin{array}{llllll}1 \times & 32.5 & 32.5 & 32.5 & 32.5 & 32.5 \\ 2 x & 32.5 & 32.5 & 32.5 & 32.5 & 32.5\end{array}$

$\begin{array}{llllll}2 \times & 32.5 & 32.5 & 32.5 & 32.5 & 32.5 \\ 3 x & 32.5 & 32.5 & 32.5 & 32.3 & 32.0\end{array}$

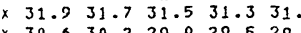

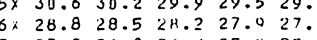

$\begin{array}{llllll}7 \times & 27.2 & 26.8 & 26.5 & 25.9 & 25.5 \\ 8 \times & 25.0 & 24.5 & 23.9 & 23.3 & 22.7\end{array}$

$\begin{aligned} 9 \times & 27.2 \\ 9 \times & 22.1 \\ 10 \times & 18.7\end{aligned}$

Percent rotal distance

PERCENT TOTAL DISTANCE. Mid-East - Het Condition

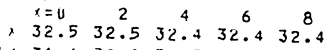
$\begin{array}{llllll}1 * & 31.6 & 30.9 & 30.3 & 29.5 & 28.9\end{array}$ $\begin{array}{llllll}2 \times & 28.5 & 28.1 & 27.6 & 27.1 & 26.5 \\ 3 \times & 26.1 & 25.6 & 25.1 & 24.5 & 24.5\end{array}$

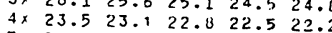
$5 \times 21.921 .721 .521 .321 .0$

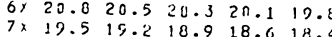
$7 \times 19.519 .218 .928 .618 .4$ $\begin{array}{lll}177 & \end{array}$ $10 \times 14$

PERCENT TOTAL DISTANCE

$\begin{array}{cccccc} & x=0 & 2 & 4 & 6 & 8 \\ \times & 32.5 & 32.5 & 32.5 & 32.5 & 37^{8} .5 \\ 1 \times & 32.5 & 32.5 & 32.5 & 32.5 & 32.5\end{array}$

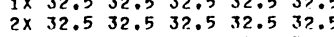
$\begin{array}{llllll}3 \times & 32.5 & 32.5 & 32.5 & 32.5 & 32.5\end{array}$ $\begin{array}{llllll}4 \times & 32.5 & 32.5 & 32.5 & 32.5 & 32.5\end{array}$ $\begin{array}{lllllll}5 x & 32.5 & 32.5 & 32.5 & 32.4 & 32.3 \\ 6 x & 32.2 & 32.1 & 31.9 & 31.6 & 31.3\end{array}$ $\begin{array}{llllll}7 \times & 31.1 & 30.8 & 30.5 & 30.2 & 29.8 \\ 3 \times & 29.3 & 28.4 & 27.6 & 20.8 & 26.8\end{array}$

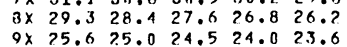
$9 \times 25.6$
$10 \times 23.0$

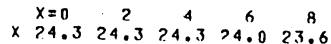
$\begin{array}{llllll}1 x & 23.3 & 23.0 & 22.7 & 22.0 & 23.6 \\ 2 x & 21.2 & 20.9 & 20.6 & 22.1 & 21.6 \\ 3 x & 10.6 & 20.6 & 20.7 & 20.4 & 20.2\end{array}$

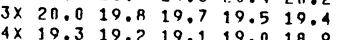
$\begin{array}{ccccccc}5 \times & 18.7 & 18.6 & 18.4 & 18.3 & 18.9\end{array}$

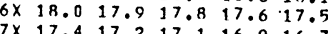
$8 \times 16.516 .4$

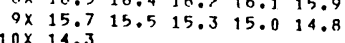

PERCENT TOTAL DISTANCE PERCENT TDTAL DISTANTE

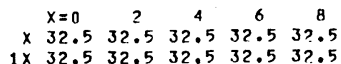

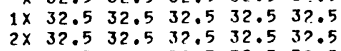

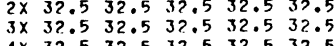

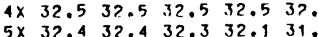
$\begin{array}{llllll}5 \times & 32.4 & 32.4 & 32.3 & 32.1 & 31.5 \\ 6 \times & 31.4 & 31.0 & 3 \mathrm{n} .7 & 30.5 & 3 \mathrm{n}\end{array}$

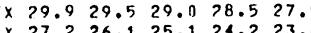

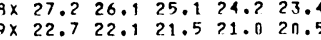
$10 \times 19.9$

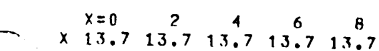
$\begin{array}{llllll}1 x & 13.5 & 13.4 & 13.3 & 13.7 & 13.7 \\ 2 x & 13.13 & 13.1 & 13.3 & 13.2 & 13.2\end{array}$ $\begin{array}{llllll}2 x & 13.1 & 13.1 & 13.1 & 13.1 & 13.0\end{array}$ $2 \times 12.6 \quad 12.612 .5$ 12.8 12.9

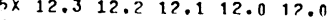

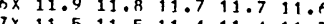
$8 \times 11.7 \quad 11.1 \quad 11.0 \quad 10.911 .7$

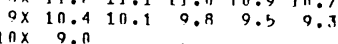

Off-Read

PERCENT tulal oIstance

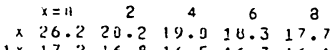
$\begin{array}{llllll}1 \times & 17.2 & 16.8 & 16.5 & 16.3 & 16.1 \\ 2 x & 15.9 & 15.7 & 15.5 & 15.3 & 15.1 \\ 3 x & 14.9 & 1.78 & 14.5 & 15.4 & 15.3\end{array}$

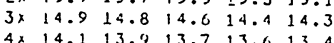
$\begin{array}{lllllll}4 \times & 14.1 & 13.9 & 13.7 & 13.6 & 13.4 \\ 5 \times & 13.3 & 13.2 & 13.1 & 13.0 & 12.9\end{array}$ $\begin{array}{cccccc}6 \times & 12.7 & 12.6 & 12.5 & 12.4 & 12.3 \\ 7 \times & 12.1 & 12.0 & 11.9 & 11.7 & 12.5\end{array}$

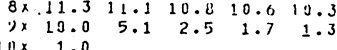

Perient total distal:ce

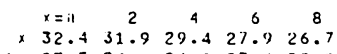

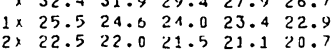
$3 \times 20.219 .9 \quad 19.5 \quad 19.218 .9$ $\begin{array}{llllll}4 \times & 18.7 & 18.5 & 18.3 & 18.1 & 17.9 \\ 57 & 17.7 & 17.5 & 17.3 & 17.1 & 16.9\end{array}$ $\begin{array}{lllllll}6 \times & 16.7 & 16.6 & 16.4 & 16.2 & 16.0 \\ 7 \times & 15.7 & 15.5 & 15.3 & 15.1 & 14.8\end{array}$ ox $14.614 .3 \quad 14.0013 .5 \quad 13.1$ $19 \times \quad 1.1$

PERCENT tOTAL Distance

$\begin{array}{rrrrrr}x=0 & 2 & 4 & 6 & 8 \\ x & 19.0 & 18.4 & 17.9 & 17.5 & 17.2\end{array}$

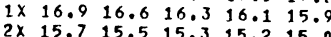
$3 \times 14.914 .814 .7 \quad 15.215 .0$ $\begin{array}{llllll}4 \times & 14.2 & 14.1 & 14.0 & 13.8 & 13.7\end{array}$ $\begin{array}{cccccc}5 \times & 13.5 & 13.4 & 13.3 & 13.1 & 13.0 \\ 7 x & 12.9 & 12.0 & 13.0 & 13.5 & \end{array}$ $8 \times 10.8 \quad 10.4 \quad 9.0 \quad 9.4 \quad 3.2$ $\begin{array}{rrrrrr}9 \times & 1.9 & 1.4 & 1.1 & 0.9 & n .2\end{array}$

PERCENT TOTAL DISTANCE

$\begin{array}{rrrrr}x=0 & 2 & 4 & 6 & 8 \\ \times & 15.0 & 15.0 & 14.8 & 14.4\end{array}$

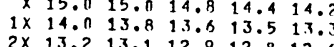
$\begin{array}{llllll}2 x & 13.2 & 13.1 & 17.9 & 12.8 & 17.6 \\ 3 x & 12.5 & 12.4 & 12 & \end{array}$ $4 \times 11.811 .7 \quad 12.512 .112$. 5x $11.2 \quad 11.1 \quad 10.9 \quad 10.8 \quad 10.7$ $\begin{array}{llllll}6 \times & 10.5 & 10.3 & 10.9 & 10.8 & 10.7\end{array}$ $\begin{array}{llllll}7 x & 9.6 & 9.3 & 9.0 & 8.6 & 9.9 \\ 8 \times & 8.1 & 7.0 & 9.0 & 8.6 & 3.4\end{array}$ $\begin{array}{rrrrrr}9 x & 1.9 & 1.4 & 1.6 & 7.3 & 3.3 \\ 10 x & 0.7 & & & 0.9 & 0.8\end{array}$ 


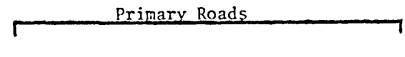

PHPCFNT TIIAL DISTANCA

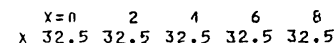
$\begin{array}{llllll}1 \times & 32.5 & 32.5 & 32.5 & 32.5 & 32.5\end{array}$ $\begin{array}{llllll}3 \times & 32.5 & 32.5 & 32.5 & 32.5 & 32.5 \\ 3 \times & 32.5 & 32.5 & 32.5 & 33.5 & 32.5\end{array}$ $4 \times 32.5 \quad 32.5 \quad 32.5 \quad 32.4 \quad 32.2$ $\begin{array}{llllll}5 \times & 32.0 & 31.9 & 31.7 & 31.4 & 31.0\end{array}$ $\begin{array}{rlllll}6 \times & 30.6 & 30.2 & 29.8 & 29.5 & 29.1\end{array}$ $\begin{array}{cccccc}7 \times & 28.4 & 28.6 & 28.3 & 28.1 & 27.8 \\ 8 \times & 27.4 & 27.1 & 26.7 & 26.3 & 25.7\end{array}$

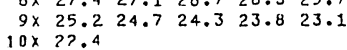

PEPCFNT TOTAL DISIANTE

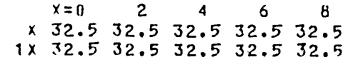
$\begin{array}{llllll}2 \times & 32.5 & 32.5 & 32.5 & 32.5 & 32.5 \\ 3 \times & 32.5 & 32.5 & 32.5 & 32.5 & 32.5\end{array}$ $\begin{array}{llllll}3 \times & 32.5 & 32.5 & 32.5 & 32.5 & 32.5 \\ 4 x & 32.5 & 32.5 & 32.5 & 32.5 & 32.5\end{array}$ $\begin{array}{lllllll}4 \times & 32.5 & 32.5 & 32.5 & 32.5 & 32.5 \\ 5 \times & 32.5 & 32.4 & 32.2 & 32.1 & 31.8\end{array}$ $6 \times 31.4 \quad 31.1 \quad 30.8 \quad 30.530$. $\begin{array}{llllll}7 \times & 30.1 & 29.9 & 29.7 & 29.4 & 29.1 \\ 8 \times & 28.6 & 28.1 & 27.7 & 27.1 & 26.5\end{array}$ $\begin{array}{rrrrrr}8 \times & 28.6 & 28.1 & 27.7 & 27.4 & 26.5 \\ 9 \times & 25.9 & 25.3 & 24.8 & 24.2 & 23.4\end{array}$ $9 \times 25.9$
$10 \times 22.6$

PERCENT TOTAL DISTANCE

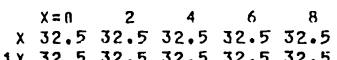
$\begin{array}{rlllll}1 \times & 32.5 & 32.5 & 32.5 & 32.5 & 32.5 \\ 2 \times & 32.5 & 32.5 & 32.5 & 32.5 & 32.5\end{array}$

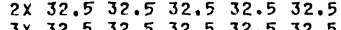
$4 \times 32.532 .532 .532 .532 .5$ $\begin{array}{lllllll}5 \times & 32.5 & 32.5 & 32.4 & 32.3 & 32.2\end{array}$ $6 \times 32.1 \quad 32.0 \quad 31.8 \quad 31.7 \quad 31.6$ $8 \times 31.531 .531 .431 .331 .2$

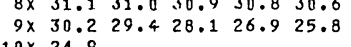
$9 \times 30.2$
$10 \times 24.9$

PFRCENT TOTAL DISTANTCE

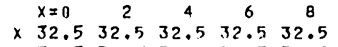
$\begin{array}{llllll}1 \times & 32.5 & 32.5 & 32.5 & 32.5 & 32.5 \\ 2 \times & 32 & 32 & 32.5 & 32.5 & 32.5\end{array}$ $\begin{array}{llllll}3 x & 32.5 & 32.5 & 32.5 & 32.5 & 32.5\end{array}$ $\begin{array}{llllll}4 \times & 32.5 & 32.5 & 32.5 & 32.5 & 32.5 \\ 5 \times & 32.5 & 32.5 & 32.4 & 32.5 & 32.5\end{array}$

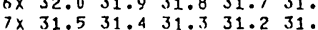
$\begin{array}{llllll}8 \times & 31.0 & 31.0 & 30.8 & 30.7 & 30.5 \\ 9 \times & 30.0 & 29.2 & 27.8 & 26.6 & 25.5\end{array}$

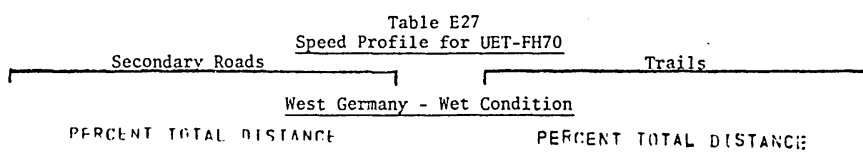

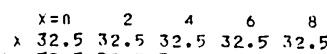

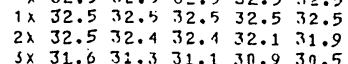

$\begin{array}{llllll}4 \times & 30.0 & 29.5 & 29.0 & 28.6 & 28.2 \\ 5 \times & 27.0 & 27.5 & 27.2 & 26.9 & 26.6\end{array}$

$\begin{array}{llllll}4 x & 26.4 & 26.1 & 25.9 & 25.9 & 26.6 \\ 7 x & 25.7 & 25.5\end{array}$

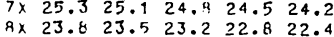

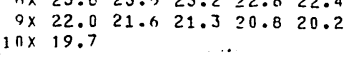

West G

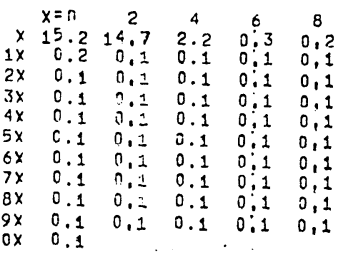

PFREFNT TITAL MIGTANCE

$\begin{array}{rrrrrr} & x=0 & 2 & 4 & 6 & 8 \\ \times & 32.5 & 32.5 & 32.5 & 32.5 & 32.5 \\ 1 \times & 32.5 & 32.5 & 32.5 & 32.5 & 32.5\end{array}$

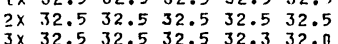

$\begin{array}{llllll}4 \times & 31.9 & 31.7 & 31.3 & 30.9 & 30 .\end{array}$

$\begin{array}{lllllll}5 \times & 30.1 & 29.7 & 29.3 & 29.0 & 28.6 \\ 6 \times & 28.3 & 27.9 & 27.7 & 27.4 & 27.6\end{array}$

$\begin{array}{lllllll}7 \times & 26.7 & 26.3 & 25.9 & 25.4 & 25.0\end{array}$

$\begin{array}{llllll}8 \times & 24.6 & 24.1 & 23.5 & 23.0 & 22.4 \\ 9 \times & 21.7 & 21.0 & 23.6 & 20.0 & 19.4\end{array}$

$\begin{aligned} & 9 \times 21.7 \\ & 10 \times 18.5\end{aligned}$

Mid-East - Wet Condition

$$
\text { PERCENT TOTAL MISIANTEt }
$$

$\begin{array}{ccccc}x=0 & 2 & 4 & 6 & 8 \\ \times \quad 32.5 & 32.5 & 32.5 & 32.5 & 32.5\end{array}$

$\begin{array}{lllllll}1 \times & 32.5 & 32.5 & 32.5 & 32.5 & 32.5 \\ 3 \times & 32.5 & 32.5 & 32.5 & 32.5 & 32.5\end{array}$

$\begin{array}{llllll}2 x & 32.5 & 32.5 & 32.5 & 32.5 & 32.5 \\ 3 \times & 32.5 & 32.5 & 32.5 & 32.5 & 32.5\end{array}$

$\begin{array}{llllll}4 \times & 32.5 & 32.5 & 32.5 & 32.5 & 32.5 \\ 5 \times & 32.5 & 32.5 & 32.4 & 32.3 & 32.3\end{array}$

$\begin{array}{lllllll}5 \times & 32.5 & 32.5 & 32.4 & 32.3 & 32.3 \\ 6 \times & 32.2 & 32.0 & 31.9 & 31.5 & 31.2\end{array}$

$\begin{array}{llllll}6 \times & 32.2 & 32.6 & 31.9 & 31.5 & 31.2 \\ 7 \times & 30.9 & 30.6 & 30.3 & 30.0 & 29.6\end{array}$

$\begin{array}{llllll}8 x & 29.0 & 28.2 & 27.4 & 26.6 & 26.0 \\ 0 \times & 25.4 & 24.9 & 24.4 & 23.9 & 23.5\end{array}$

$9 \times 25.4$
$10 \times 22.9$$$
\text { Mid- }
$$

PFREENT TOTAL DISTANGE

$\begin{array}{rrrrrr} & x=0 & 2 & 4 & 5 & 8 \\ \times & 32.5 & 32.5 & 32.5 & 32.5 & 32.5 \\ 1 \times & 32.5 & 32.5 & 32.5 & 32.5 & 32.5\end{array}$

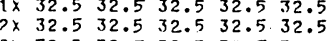

$\begin{array}{llllll}3 \times & 32.5 & 32.5 & 32.5 & 32.5 & 32.5 \\ 4 \times & 32.5 & 32.5 & 32.5 & 32.5 & 32.4\end{array}$

$\begin{array}{llllll}5 \times & 32.4 & 32.4 & 32.3 & 32.0 & 31.6 \\ 6 x & 31.3 & 30.9 & 30.6 & 30.3 & 30.0\end{array}$

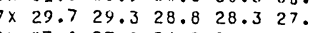

$\begin{array}{llllll}R \times & 27.0 & 25.9 & 24.9 & 24.1 & 23.3 \\ 0 \times & 22.6 & 22.0 & 22.4 & 20.9 & 20.4\end{array}$
Condition

RCFNT TOTAL DISTANCF

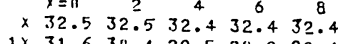

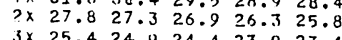

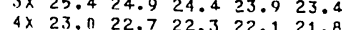
$5 \times 21.621 .3 \quad 21.1 .20 .920 .6$ $\begin{array}{llllll}5 \times & 20.4 & 20.1 & 19.9 & 19.7 & 19.4\end{array}$

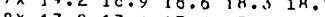
$9 x \quad 16.9 \quad 16.7 \quad 16.4 \quad 15.915 .2$

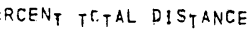

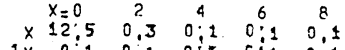

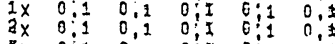

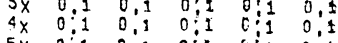

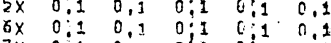

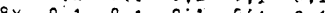
$\begin{array}{rrrrrr}9 x & 0: 1 & 0.1 & 0: 1 & 0: 1 & 0.1 \\ 10 x & 0: 1 & & & & \end{array}$ dition

PFREEHT TIIAL DISTANCI

$\begin{array}{rrrrrr}x=0 & 2 & 4 & 6 & 8 \\ x & 12.7 & 12.6 & 12.5 & 12.5 & 12.5 \\ 1 x & 12.4 & 12.4 & 12.4 & 12.4 & 12.4\end{array}$ $2 \times 12.4 \quad 12.4 \quad 12.4 \quad 12.412$. $4 x \quad 12.312 .3 \quad 12.212 .212 .1$ $5 \times 11.7 \quad 11.7 \quad 11.6 \quad 11.511 .5$ 6x $11.4 \quad 11.411 .3 \quad 11.211$. Ox $11.111 .1 \quad 11.011 .010 .9$

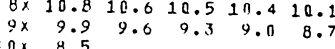

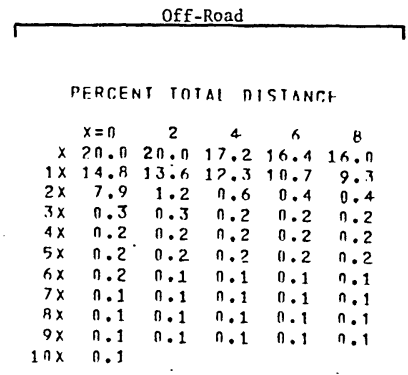

PERCENT TOTAL DISTANC

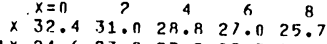

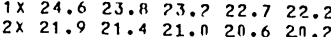

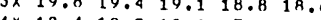
$\begin{array}{llllll}4 \times & 18.4 & 18.2 & 18.0 & 17.8 & 17.6 \\ 5 \times & 17.4 & 17.2 & 18.0 & 16.8\end{array}$ $6 \times 16.516 .316 .016 .816 .6$ $\times 15.415 .215 .014 .8$. $\begin{array}{rrrrrr}8 \times & 14.3 & 14.0 & 13.7 & 13.3 & 12.9 \\ 9 x & 12.5 & 11.0 & 3.6 & 2.1 & 1.5\end{array}$ $10 \times \quad 1.2$

PERCENT TCTAL DISTANCE

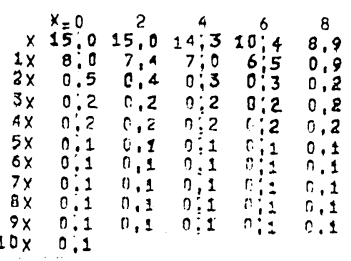

PERCENT TSTAL DISTANCE

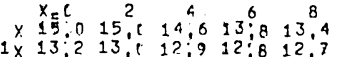

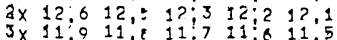
$5 \times 10: 310$, $11,111: 010$, $6 \times 10: 110,10,80940.3$

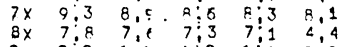
$\begin{array}{rrrrr}9 x & 2: 2 & 1,2 & 1 ; ? \\ 10 x & 0,7 & 1: 0 & 0.8\end{array}$ 


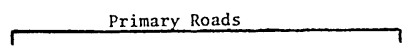

PERCENT TUTLL PISTALE

, $\begin{aligned} & \because=0 \\ & +3.1\end{aligned} 43.745 .745 .143 .7$

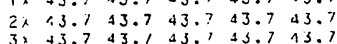

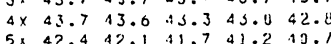
$\begin{array}{llllll}61 & 40.3 & 39.9 & 39.5 & 39.2 & 38.9 \\ 7 . & 38.6 & 38.3 & 37.8 & 37.2 & 36.5\end{array}$ $\begin{array}{llllll}8 \times & 35.7 & 34.8 & 34.01 & 33.1 & 32.1 \\ 9 . & 31.2 & 30.3 & 29.5 & 28.6 & 27.5\end{array}$ $\begin{aligned} & 9 \times 31.2 \\ & 10 \times 26.4\end{aligned}$

PERCENT TOTAL UISTENCE

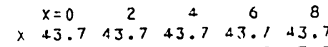
$\begin{array}{llllll}1 \times & 43.7 & 43.7 & 43.7 & 43.7 & 43.7 \\ 2 \times & 43.7 & 43.7 & 43.7 & 43.7 & 43.7\end{array}$ $\begin{array}{lllllll}2 \times & 43.7 & 43.7 & 43.7 & 33.7 & 43.7 \\ 3 \times & 43.7 & 43.7 & 43.7 & 43.7 & 43.7\end{array}$ $\begin{array}{lllllll}1 \times & 43.7 & 43.7 & 43.7 & 43.6 & 43.6\end{array}$ $\begin{array}{llllll}5 x & 43.5 & 43.1 & 42.7 & 42.3 & 42.0 \\ 6 x & 41.7 & 41.4 & 41.1 & 40.9 & 40.6\end{array}$ $7 \times 40.4 \quad 39.9 \quad 30.2 \quad 38.6 \quad 37$. $\begin{array}{lllllll}7 \times & 40.4 & 39.9 & 39.2 & 38.6 & 37.8 \\ 8 \times & 36.8 & 35.7 & 34.8 & 33.8 & 32.6 \\ 9 \times & 31.5 & 30.5 & 29.6 & 28.7 & 27.5\end{array}$ $10 \times 26$.

PFRCENT TOTAL DISTANCE

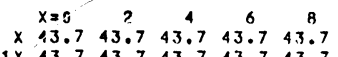
$\begin{array}{llllll}2 x & 43.7 & 43.7 & 43.7 & 43.7 & 43.7 \\ 3 x & 43.7 & 43.7 & 43.7 & 43.7 & 43.7\end{array}$ $\begin{array}{llllll}3 x & 43.7 & 43.7 & 43.7 & 43.7 & 43.7 \\ 4 x & 43.7 & 43.7 & 43.6 & 43.6 & 43.5\end{array}$ $\begin{array}{llllll}4 x & 43.7 & 43.7 & 43.6 & 43.6 & 43.5 \\ 5 x & 43.4 & 43.2 & 43.0 & 42.7 & 47.5\end{array}$ $7 \times \quad 41.4 \quad 11.2 \quad 41.1 \quad 40.940 .6$ $8 \times 40.440 .1 \quad 39.8$ 39.4 38.9 $10 \times 29.4$

PERCENT IOTAL DISTANCE

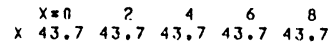
$\begin{array}{lllllll}1 x & 43.7 & 43.7 & 43.7 & 43.7 & 43.7 \\ 2 x & 43.7 & 43.7 & 43.7 & 43.7 & 43.7\end{array}$ $\begin{array}{llllll}3 x & 43.7 & 43.7 & 43.7 & 43.7 & 43.7\end{array}$ $\begin{array}{lllllll}4 x & 43.7 & 43.7 & 43.6 & 43.6 & 43.5\end{array}$

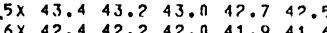

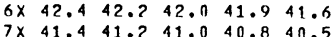
$\begin{array}{rrrrrr}8 \times & 40.3 & 39.9 & 39.5 & 39.1 & 38.6 \\ 9 \times & 37.6 & 36.1 & 33.9 & 31.9 & 3 n .2\end{array}$ $10 \times 28.7$
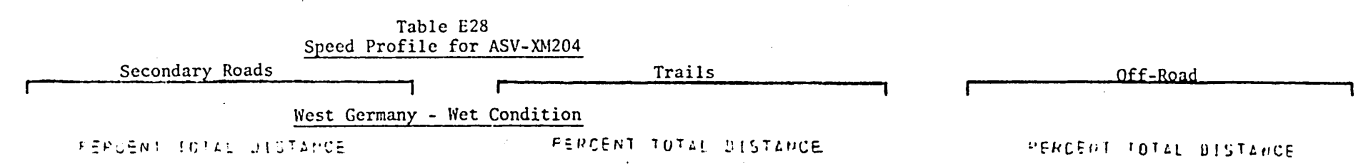

"EHCERT TOTLL OISTANCE

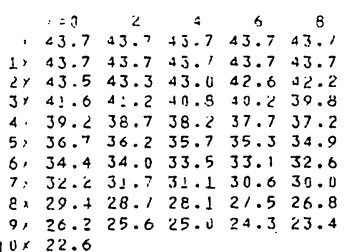

$\begin{array}{ccccc}x=0 & 2 & 4 & 6 & 8 \\ 34.2 & 32.3 & 31.3 & 30.8 & 30.5\end{array}$

2) $28.0 \quad 27.5 \quad 27.1 \quad 26.8 \quad 26$.

$4 \times 26.525 .725 .425 .124 .0$

$5.23 .122 .8 \quad 22.5 \quad 22.3 \quad 22.0$

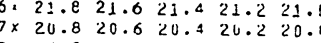

$2 \times 29 .+28.128 .1221 .526 .0$

$10 \times 22.0$

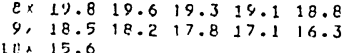

West Germany - Snow Condition

PEPCEN, TOIAL D!STAKCE

PEKCENT TDTAL DiSTANCE

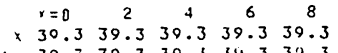

$\begin{array}{llllll}1 \times & 39.3 & 39.3 & 39.3 & 3 \% .3 & 39.3 \\ 21 & 39.3 & 39.3 & 39.3 & 39.3 & 37.3\end{array}$

3) $39.3 \quad 39.3 \quad 39.2 \quad 39.1 \quad 36.8$

$\begin{array}{llllll}2 \times & 38.6 & 38.2 & 31.9 & 37.6 & 37.2 \\ 5 \times & 36.9 & 36.6 & 36.2 & 35.9 & 35.5 \\ 0 \times & 35.1 & 34.8 & 34.3 & 33.8 & 33.2\end{array}$

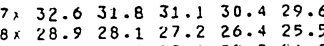

$9 \times 24.7$
10.20 .3

$\begin{array}{lcccc}\quad=0 & 2 & 4 & 6 & e \\ 43.7 & 43.6 & 43.0 & 43.6 & 43.3\end{array}$

$\begin{array}{llllll}1 \times & 41.9 & 40.8 & 30.0 & 39.5 & 38.8 \\ 2 . x & 38.2 & 37.8 & 30.9 & 36.2 & 35.6\end{array}$

$\begin{array}{llllll}2 \times & 38.2 & 37.5 & 30.9 & 36.2 & 35.6 \\ 3 x & 34.8 & 34.1 & 33.4 & 32.9 & 32.4 \\ 5 x & 32 . & 3 . .6 & 31.4 & 30.9 & 30.6\end{array}$

$\begin{array}{llllll}4 \times & 32.0 & 31.6 & 31.3 & 30.9 & 30.6\end{array}$

$\begin{array}{llllll}5 x & 30.3 & 29.9 & 29.5 & 29.2 & 28.9 \\ 6 x & 28.6 & 28.1 & 27.6 & 27.2 & 26.6\end{array}$

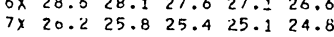

$\begin{array}{llllll}8 \times & 24.5 & 24.2 & 23.4 & 25.6 & 24.6 \\ 8 \times & 22.0 & 23.2\end{array}$

$\begin{array}{llllllll}9 x & 22.7 & 24.5 & 231.8 & 20.7 & 19.5\end{array}$ Mid-East - Wet Condition

PERCENT TOTAL DISTANCE

PERCENT TOIAL DISTANCE

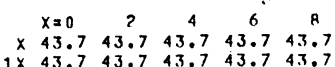
$\begin{array}{llllll}2 x & 43.7 & 43.7 & 43.7 & 43.7 & 43.7 \\ 3 x & 43.7 & 43.7 & 43.7 & 43.7 & 43.7\end{array}$

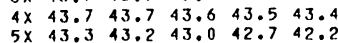
$\begin{array}{llllll}6 \times & 41.8 & 41.5 & 41.0 & 40.6 & 40.2 \\ 7 \times & 39.7 & 39.7 & 38.5 & 37.8 & 37.0\end{array}$ $\begin{array}{llllll}7 \times & 39.7 & 39.7 & 38.5 & 37.8 & 37.0 \\ 8 \times & 35.9 & 34.4 & 33.1 & 31.9 & 30.8\end{array}$ $\begin{array}{llllll}8 \times & 35.9 & 34.4 & 33.1 & 31.9 & 30.9 \\ 9 \times & 29.9 & 29.0 & 28.2 & 77.5 & 26.9\end{array}$ $10 \times 26.0$

Mid-East - Sand Condition

$\begin{array}{cccccc} & x=0 & 2 & 4 & 6 & 8 \\ x & 32.8 & 32.8 & 32.7 & 32.0 & 31.5 \\ 1 x & 31.1 & 30.7 & 30.7 & 79.7 & 29.5\end{array}$ $\begin{array}{llllll}2 \times & 29.0 & 28.6 & 28.4 & 28.1 & 27.9 \\ 3 \times & 27.8 & 27.6 & 27.4 & 77.1 & 26.9 \\ 4 \times & 26.7 & 26.5 & 26.3 & 26.0 & 25.7\end{array}$ $\begin{array}{llll}5 \times & 25.5 & 25.3 & 25.5\end{array}$ $\begin{array}{llllll}6 x & 24.4 & 24.7 & 23.9 & 23.6 & 23.7\end{array}$

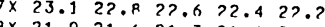

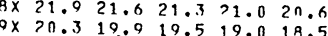
$10 \times 15$

PERCENT IOTAI DISTANCE PERCENT TIITAL DISTANCI

$\begin{array}{rrrrr}x \times 0 & 2 & 2 & 6 & 8 \\ \times \quad 39.3 & 39.3 & 39.3 & 39.3 & 39 . .3\end{array}$ $\begin{array}{llllll}1 \times & 39.3 & 39.3 & 39.3 & 39.3 & 39.3 \\ 2 \times & 39.3 & 39.3 & 39.3 & 39.3 & 30.3\end{array}$ $3 \times 39.3 \quad 39.3 \quad 39.3 \quad 39.3 \quad 39.3$ $\begin{array}{lllllll}4 \times & 39.3 & 39.3 & 39.3 & 39.3 & 30.0 & 0.3\end{array}$ $\begin{array}{lllllll}5 x & 38.9 & 38.5 & 38 . ? & 37.9 & 37.6 \\ 6 x & 37.4 & 37.0 & 36.5 & 35.9 & 35.3\end{array}$ $\begin{array}{llllll}7 \times & 34.8 & 34.1 & 33.4 & 32.6 & 31.7\end{array}$ $8 \times \quad 30.7 \quad 29: 1 \quad 27.8 \quad 96.7 \quad 25.7$ $9 \times 24.8$

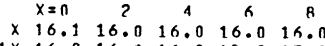
$2 \times \quad 15.8 \quad 15.7 \quad 15.6 \quad 15.4 \quad 15.3$ $\begin{array}{lllllll}3 x & 15.2 & 15.0 & 14.9 & 14.8 & 14.7\end{array}$ $4 \times 14.614 .414 .3 \quad 14.8 \quad 14.1$ $\begin{array}{llllll}5 x & 14.0 & 13.9 & 13.8 & 13.7 & 13.7 \\ 6 x & 13.6 & 13.5 & 13.4 & 13.3 & 13 .\end{array}$ $\begin{array}{llllll}7 x & 13.2 & 13.1 & 13.4 & 13.9 & 13.8\end{array}$ $\begin{array}{llllll}8 \times & 17.7 & 12.6 & 1 ? .5 & 12.4 & 1 ? . \\ 9 x & 11.9 & 11.7 & 11.4 & 11.1 & 10.8\end{array}$ $\begin{array}{ccccc}x=0 & 2 & 4 & 6 & y \\ \times 28.0 & 22.1 & 21.9 & 20.4 & 1 \%\end{array}$

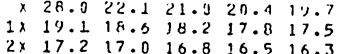
$\begin{array}{llllll}3 x & 10.1 & 15.9 & 15.8 & 16.5 & 16.3 \\ 4 x & 15.0 & 15.5 & 15.3\end{array}$

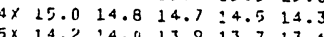
$6 \times 13.413 .313 .2$ $\begin{array}{llllll}7 \times & 12.8 & 12.7 & 12.5 & 12.3 & 12.2\end{array}$ $\begin{array}{cccccc}8 x & 12.0 & 11.9 & 11.7 & 11.5 & 11.4 \\ 9 x & 11.2 & 10.9 & 8.9 & 3.1 & 1.9\end{array}$

PEKIEENT IOIDL DISTANCE

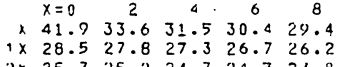
$\begin{array}{llllll}3 x & 23.4 & 23.0 & 22.5 & 22.1 & 21.7 \\ 4 x & 21.3 & 20.9 & 20.5 & 20.2 & 10.7\end{array}$ $\begin{array}{lllllll}5 \times & 19.6 & 19.3 & 19.0 & 18.7 & 18.4 \\ 6 \times & 18.2 & 19.0 & 19.8 & 17.5 & 17.3\end{array}$ $\begin{array}{llllll}7 \times & 17.1 & 16.9 & 16.6 & 16.4 & 16.2\end{array}$

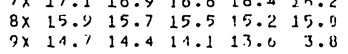
inx 14.2

PERGENT total Distante

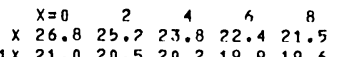
$\begin{array}{lllllll}2 \times & 19.3 & 18.8 & 18.4 & 18.1 & 17.7\end{array}$ $\begin{array}{llllll}3 \times & 17.4 & 17.1 & 16.8 & 16.6 & 16.3\end{array}$ $5 \times 16.1$ 15. 15.6 15.4 15.1 $6 \times 14.1 \quad 14.013 .8$ 13.6 13.5 $\begin{array}{llllll}7 \times & 13.3 & 13.1 & 17.9 & 12.8 & 17.6\end{array}$ $\begin{array}{rrrrrr}8 x & 12.4 & 12.2 & 17.0 & 11.9 & 11.7 \\ 9 x & 11.5 & 11.3 & 11.1 & 10.9 & 6.2\end{array}$

PERcent total Distante

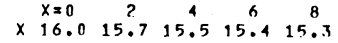

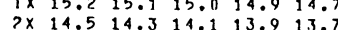
$\begin{array}{llllllll}3 \times & 13.5 & 13.4 & 13.2 & 13.1 & 13.0\end{array}$ $\begin{array}{llllll}4 x & 12.8 & 17.7 & 12.6 & 12.5 & 17.3 \\ 5 x & 12.2 & 12.1 & 11.9 & 12.5 & 11.7\end{array}$ $\begin{array}{llllll}5 \times & 12.2 & 12.1 & 11.9 & 11.8 & 11.7 \\ 6 x & 11.5 & 11.4 & 11.2 & 11.1 & 10.9\end{array}$

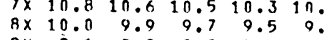

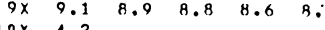




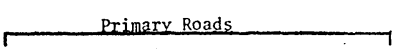

Peptent foral distance

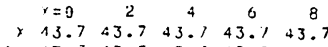

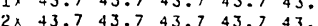
3. $43.7 \quad 43.7 \quad 43.7 \quad 43.7 \quad 43.7$

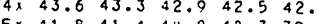

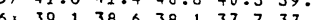
$7 \times 36.9 \quad 36.5 \quad 36.135 .6 \quad 350$ $\begin{array}{lllllll}8 \times & 34.2 & 33.5 & 32.7 & 32.0 & 31.0 \\ 9 \times & 30.1 & 29.3 & 28.6 & 27.8 & 26.0\end{array}$ $10 \times 25.8$

PERLENT TOTLL DISIARICE

$\begin{array}{rrrrrr} & x=0 & 2 & 4 & 6 & 8 \\ \times & 43.7 & 43.7 & 43.7 & 43.7 & 43.7\end{array}$ $\begin{array}{llllll}1 \times & 43.7 & 43.7 & 43.7 & 43.7 & 43.7 \\ 2 x & 43.7 & 43.7 & 43.7 & 43.7 & 43.7 \\ 3 \times & 43.7 & 43.7 & 3.7 & 43.7 & 43.7\end{array}$

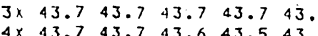

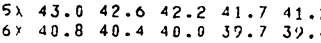

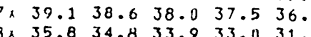
$9 \times 30.8$ $10 \times 25.9$

PERCENT TOTAL DISTANCE

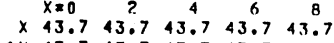

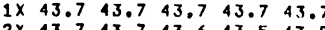
$3 \times 43.443 .4 \quad 43.3 \quad 43.343 . ?$ $\begin{array}{lllllll}4 x & 43.2 & 43.2 & 43.0 & 42.8 & 47.5 \\ 5 x & 42.3 & 42.1 & 42.0 & 41.5 & 40.5\end{array}$ $\begin{array}{llllll}5 \times & 42.3 & 42.1 & 41.8 & 41.5 & 41.3 \\ 6 x & 41.0 & 40.8 & 40.6 & 40.4 & 40.2\end{array}$ $7 \times \quad 40.0 \quad 39.8 \quad 39.5 \quad 39.3 \quad 39.0$ $\begin{array}{lllllll}8 \times & 38.8 & 38.5 & 38.2 & 37.9 & 37.4 \\ 9 \times & 36.6 & 35.3 & 33.3 & 31.5 & 30.0\end{array}$ $9 \times 36.6$
$10 \times 28.6$

PERCENT TOTAL DISTANCE

$\begin{array}{rrrrrr}x=0 & 2 & 4 & 6 & 8 \\ \times & 43.7 & 43.7 & 43^{6} .7 & 43.7 & 43^{8}\end{array}$ $\begin{array}{llllll}1 \times & 43.7 & 43.7 & 43.7 & 43.7 & 43.7\end{array}$

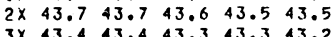

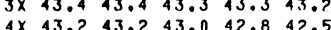
$\begin{array}{llllll}5 \times & 42.3 & 42.1 & 41.8 & 41.5 & 41.2\end{array}$ $6 \times \quad 11.0 \quad 40.8 \quad 40.6 \quad 40.4 \quad 4 n$.? $\begin{array}{lllllll}7 \times & 39.9 & 39.7 & 39.4 & 39.2 & 38.9\end{array}$ $8 \times 36.2 \quad 34.932 .8 \quad 31.0 \quad 37.1$ $10 \times 28.0$

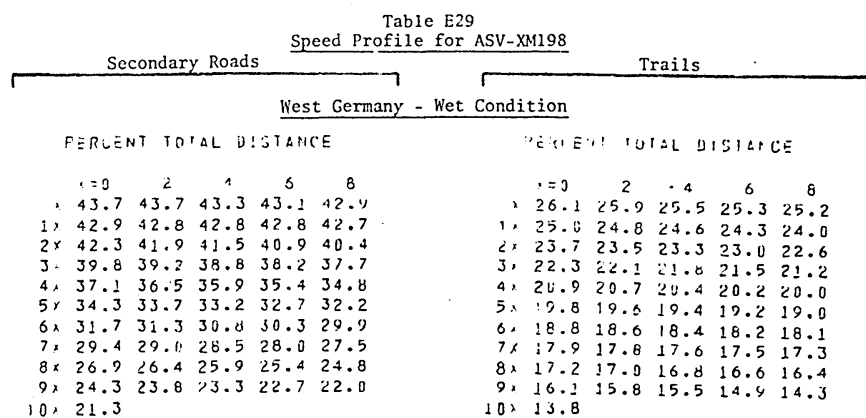

West Germany - Snow Condition

PERCENT TOTAL DISTANCE

$\begin{array}{lcccc}r=0 & 2 & 4 & 6 & 8 \\ x, 39.3 & 39.3 & 39.3 & 39.3 & 39.3\end{array}$

$\begin{array}{llllll}1, & 39.3 & 339.3 & 39.3 & 39.3 & 39.3 \\ 2, & 39.3 & 39.3 & 39.3 & 39.5 & 39.2\end{array}$

$3 \times 39.2 \quad 39.1 \quad 38.9 \quad 38.6 \quad 38.2$

$5 \times 35.735 .3 \quad 34.934 .4 \quad 34.0$

6) $33.533 .0 \quad 32.0 \quad 32.1 \quad 31.5$

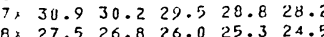

$9 \times 23.7$
$10 \times 19.7$

PERCENT TOTLL DISTANCE

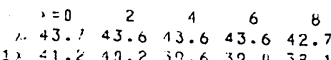

$\begin{array}{llllll}21 & 37.3 & 36.4 & 35.5 & 34.5 & 33.7 \\ 3 \times & 33.1 & 32.5 & 32.0 & 31.6 & 31.2\end{array}$

4: $30.8 \quad 30.5 \quad 30.129 .729 .4$

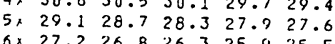

$\begin{array}{llllll}6 \times & 27.2 & 26.8 & 26.3 & 25.9 & 25.6 \\ 7 . & 25.1 & 24.8 & 2.4 .4 & 24 . & 25\end{array}$

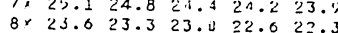

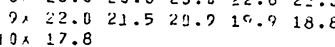
Mid-East - Wet Condition

PERCENT TOTAL DISTANCE

PERCENT total Distance

$\begin{array}{ccccc}x=0 & 2 & 4 & 6 & 8 \\ x \quad 43.7 & 43.7 & 43.7 & 43.7 & 43.5\end{array}$

$\begin{array}{llllll}x & 43.3 & 13.2 & 43.1 & 43.0 & 43.0 \\ 2 x & 43.3 & 43.2 & 43.0 & 42.9 & 42.9\end{array}$

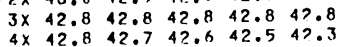

$5 \times \quad 42.1 \quad 42.0 \quad 41.7 \quad 41.3 \quad 4 n \cdot 8$

$7 \times \quad 38.3 \quad 37.9 \quad 37.3 \quad 36.6 \quad 35.9$

$\begin{array}{cccccc}8 \times & 34.9 & 33.5 & 37.2 & 31.1 & 3 n .1 \\ 9 \times & 29.2 & 28.4 & 27.7 & 27.0 & 26.4\end{array}$

$9 \times 29.2$
$10 \times \quad 25.6$

Mid-E

PERCENT IOTAL DistanCEE

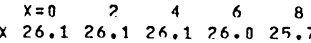

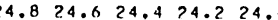

$\begin{array}{llllll}3 \times & 23.8 & 23.6 & 23.4 & 23.3 & 23 . \\ 4 \times & 22.9 & 22.7 & 23.5 & 22.3 & 23 .\end{array}$

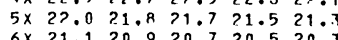

$\begin{array}{llllll}7 \times & 20.2 & 20.0 & 19.8 & 19.6 & 10.4\end{array}$ $\begin{array}{llllll}8 \times & 19.1 & 18.9 & 18.7 & 18.4 & 18.1 \\ 9 x & 17.8 & 17.5 & 17.9 & 16.8 & 16.5\end{array}$

$\begin{array}{rccccc} & x=0 & 2 & 4 & 6 & 8 \\ \times & 39.3 & 39.3 & 39.3 & 39.3 & 39.3 \\ 1 \times & 39.3 & 39.3 & 399.3 & 39.3 & 39.3\end{array}$

$\begin{array}{llllll}2 \times & 39.3 & 39.3 & 30.3 & 39.3 & 30.3\end{array}$

$\begin{array}{llllll}4 \times & 39.3 & 39.2 & 39.1 & 78.9 & 39.7\end{array}$

$\begin{array}{llllll}5 \times & 38.4 & 38.1 & 37.8 & 37.4 & 37.0\end{array}$

$6 \times 36.636 .257 .7937 .4 \quad 31.0$

$\begin{array}{llllll}8 \times & 30.2 & 28.7 & 27.5 & 26.4 & 25.4\end{array}$

$9 \times$
9 $24.5 \quad 23.7 \quad 23.072 .4 \quad 21$.

$\begin{aligned} 9 \times & 24.5 \\ 10 \times & 21.1\end{aligned}$ ition

PHRCFNT TOTAL DISTANCE

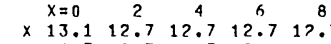
$\begin{array}{llllll}1 \times & 12.7 & 12.7 & 12.7 & 12.7 & 12.7 \\ 2 \times & 12.5 & 12.5 & 12.4 & 12.6 & 12.0\end{array}$

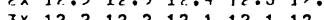
$4 \times 12.011 .9 \quad 11.9 \quad 11.911$. $\begin{array}{lllllll}5 x & 11.8 & 11.7 & 11.7 & 11.6 & 11.6 \\ 6 x & 11.5 & 11.5 & 11.5 & 11.4 & 11.4\end{array}$

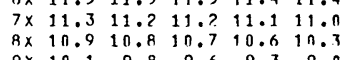
$\begin{array}{rr}9 \times & 1.1 \\ 10 \times & 8.7\end{array}$

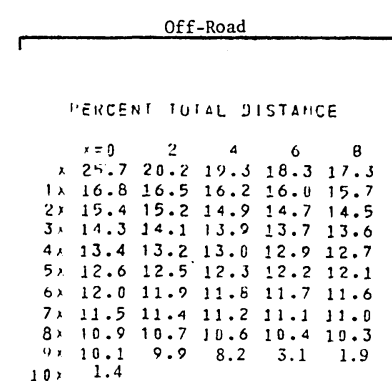

PERTEII TOTAL DESTAHCE

$\begin{array}{rrrrr}\quad y=0 & 2 & 4 & 6 & 8 \\ \times \quad 39.8 & 33.0 & 40.3 & 28.9 & 27.9\end{array}$

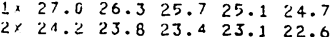

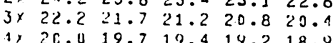
$\begin{array}{llllll}5 \times & 18.6 & 18.3 & 18.1 & 17.8 & 17.6 \\ 0 \times & 17.4 & 17.2 & 17.0 & 16.8 & 16.5\end{array}$ $7 \times 10.3 \quad 16.0 \quad 15.8 \quad 16.515 .5$

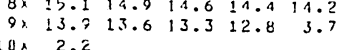

a. 2.2

Percent total distance

$\begin{array}{cccccc}x=0 & 2 & 4 & 6 & 8 \\ \times & 20.4 & 19.5 & 18.8 & 18.2 & 17.0\end{array}$

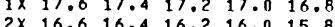
$3 x \quad 15.7 .15 .5 \quad 15.3 \quad 15.1 \quad 14.9$ $\begin{array}{lllllll}4 \times & 14.7 & 14.6 & 14.4 & 14.2 & 14.0\end{array}$ $\begin{array}{lllllll}5 x & 13.8 & 13.6 & 13.4 & 13.2 & 13.1 \\ 6 x & 12.9 & 12.7 & 17.5 & 12.4 & 17.2\end{array}$ $\begin{array}{cccccc}7 \times & 12.1 & 11.9 & 11.8 & 11.6 & 11.4\end{array}$

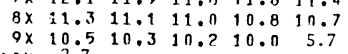

PERCENT TOTAL DISTANCE

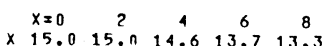
$\begin{array}{llllll}1 \times & 13.0 & 12.8 & 12.0 & 13.4 & 13.3 \\ 2 x & 12.1 & 12.0 & 12.9 & 11.8 & 12.2 \\ 3 x & 11.6 & 11.5 & 11.4 & 11.3 & 11.7\end{array}$ $\begin{array}{llllll}3 \times & 11.6 & 11.5 & 11.4 & 11.3 & 11.2 \\ 4 x & 11.1 & 11.0 & 10.9 & 10 & 110.7\end{array}$ $\begin{array}{cccccc}5 \times & 10.1 & 10.0 & 9.8 & 9.7 & 0.6 \\ 7 \times & 0.4 & 0.0 & 0.8 & 9.0 & 0.9\end{array}$ $\begin{array}{cccccc}7 x & 9.4 & 9.3 & 9.1 & 9.0 & 8.9\end{array}$ $\begin{array}{rrrrrr}8 \times & 8.7 & 8.5 & 8.4 & 8.2 & 8.0 \\ 9 x & 7.9 & 7.7 & 7.5 & 7.3 & 7.1 \\ 17 \times & 3.8 & & & & \end{array}$ 


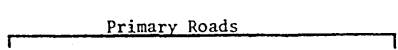

PIRCENT TOTAL DISTANC:

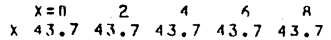
$\begin{array}{llllll}1 x & 43.7 & 43.7 & 43.7 & 43.7 & 43.7 \\ 3 x & 43.7 & 43.7 & 43.7 & 13.7 & 43.7\end{array}$ $\begin{array}{lllllll}3 \times & 43.7 & 43.7 & 43.7 & 13.7 & 43.7\end{array}$ $\begin{array}{llllll}4 \times & 43.6 & 43.3 & 42.9 & 12.4 & 42.0 \\ 5 \times & 41.6 & 41.1 & 40.5 & 39.9 & 39.2\end{array}$

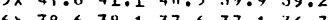
$7 \times 36.3 \quad 35.9 \quad 35.5 \quad 35.034 .4$ $\begin{array}{llllll}8 \times & 33.7 & 33.0 & 32.2 & 31.5 & 30.6 \\ 9 \times & 29.7 & 29.0 & 28.3 & 77.5 & 26.5\end{array}$ $7 \times$
$9 \times$
$9 \times 29.7$
$10 \times 25.5$

PRRENT TUTAL MISTANTF

$\begin{array}{rrrrrr}x=0 & 2 & 4 & 6 & 8 \\ x & 43.7 & 43.7 & 43.7 & 43.7 & 43.7 \\ x & 43.7 & 43.7 & 43.7 & 43.7 & 43.7\end{array}$ $\begin{array}{llllll}1 \times & 43.7 & 43.7 & 43.7 & 43.7 & 43.7 \\ 2 \times & 43.7 & 43.7 & 43.7 & 43.7 & 43.7\end{array}$

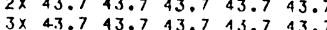
$\begin{array}{llllll}4 \times & 43.7 & 43.7 & 43.5 & 43.7 & 43.7\end{array}$

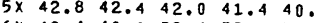
7x $40.450 .039 .6 \quad 39.238 .9$ $8 \times 35.538 .1 \quad 37.6 \quad 37.036 .0$ $\begin{array}{ccccc}9 \times & 30.6 \quad 29.7 \quad 28.8 \quad 28.0 \quad 26.9\end{array}$ $10 \times 25.8$

PFRCENT TITAL MISTANCE

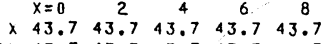

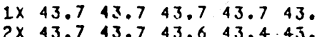
$3 \times \quad 43.2 \quad 43.1 \quad 43.1 \quad 43.042 .9$ $\begin{array}{lllllll}4 \times & 42.9 & 42.8 & 42.6 & 42.4 & 42.1\end{array}$ $5 \times 41.941 .6 \quad 41.441 .14 n$. $7 \times 39.439 .2 \quad 38.938 .7 \quad 38.4$ $8 \times 38.2 \quad 37.9 \quad 37.6 \quad 37.2 \quad 36.8$ $9 \times 36.034 .8 \quad 32.931 .1 \quad 29.6$

PFRCENT TOTAL DISTANGE

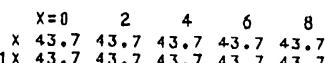
$\begin{array}{llllll}2 x & 43.7 & 43.7 & 43.6 & 43.4 & 43.3\end{array}$

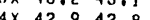

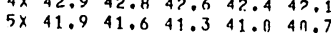
$\begin{array}{llllll}6 \times & 40.4 & 40.2 & 4 n .0 & 39.8 & 39.6 \\ 7 \times & 39.3 & 39.1 & 38.8 & 38.6 & 38.6\end{array}$ $8 \times 38.0 \quad 37.837 .4 \quad 37.036 .0$

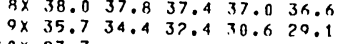

Table E30

Speed Profile for ASV-FH70

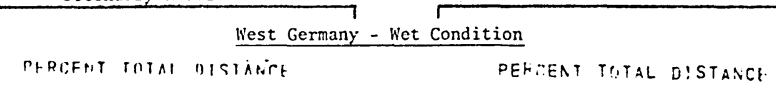

Trails

$\begin{array}{ccccc}x=0 & 2 & 4 & 6 & 8 \\ \times \quad 43.7 & 43.7 & 43.7 & 42.7 & 42.5 .\end{array}$ $2 x \quad 42.3 \quad 42.342 .2 \quad 42.2 \quad 42.1$

$\begin{array}{llllll}3 \times & 39.0 & 38.5 & 38.0 & 37.4 & 36.0\end{array}$

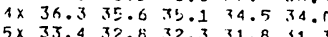

$2 \times 30.8 \quad 311.3 \quad 29.8 \quad 29.4428$.

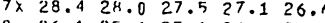

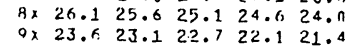

$10 \times 20.8$

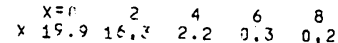

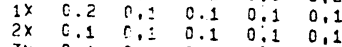
$\begin{array}{llllll}3 x & 0.1 & 0.1 & 0.1 & 0.1 & 0.1 \\ 3 x & 0.1 & 0.3 & 0.1 & 0.1 & 0.1\end{array}$ $\begin{array}{llllll}4 x & 0.1 & 0.3 & 0.1 & 0.1 & 0.1 \\ 5 x & 0.1 & 0.3 & 0.1 & 0.1 & 0.1\end{array}$ $\begin{array}{llllll}5 x & 0.1 & 0.2 & 0.1 & 0.1 & 0.1 \\ 7 x & 0.1 & 0.1 & 0.1 & 0.1 & 0.1\end{array}$

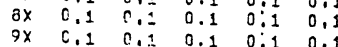

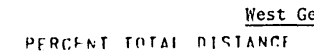
$10 x \quad 0,1$

Nest Germany - Snow Condition

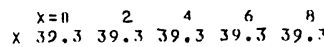

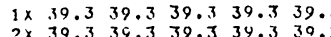

$\begin{array}{llllll}2 \times & 39.3 & 39.3 & 39.3 & 34.3 & 30.2 \\ 3 \times & 39.1 & 34.0 & 38.6 & 38.3 & 37.2\end{array}$

$\begin{array}{llllll}5 \times & 35.2 & 34.8 & 34.3 & 33.8 & 33.4\end{array}$

$\begin{array}{llllll}6 x & 32.9 & 32.4 & 32.0 & 31.5 & 30.9 \\ 7 \mathrm{x} & 3 \mathrm{n} .2 & 29.6 & 28.9 & 28.3 & 27.6\end{array}$

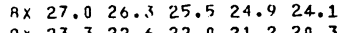

$9 \times 23.322 .6 \quad 22 . n 21.220 .3$

$10 \times 19.5$

$$
\text { Mid-East - Wet Condition }
$$

DIANCET

PERCENT TOTAL MISTANTI

$x=0 \quad ? \quad 4 \quad 6 \quad n$

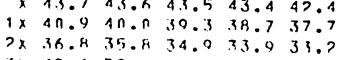
$1 \times 3 n .433 .1130 .531 .23 n .4$ $5 x>78.628 .7 \quad 27.8 \quad 77.4 \quad 27.0$ 6x $26.726 .725 .825 .4 \quad 25.0$

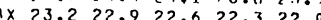
6 ? $1 . ? 20.6 \quad 19.6 \quad 18.5$

$\begin{array}{lllll}x=0 & 2 & 4 & 5\end{array}$ PEREENT TCTAL DISTANCE

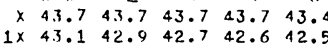

$\begin{array}{llllll}2 x & 42.5 & 42.4 & 42.4 & 42.3 & 42.3\end{array}$

$\begin{array}{llllll}3 x & 42.3 & 42.3 & 42.2 & 42.2 & 42.2 \\ 4 x & 42.2 & 42.2 & 42.0 & 42.0 & 41.7\end{array}$

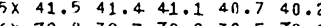

$\begin{array}{llllll}7 \times & 37.7 & 37.3 & 36.6 & 36.0 & 35.3\end{array}$

$\begin{array}{llllll}8 \times & 34.4 & 33.0 & 31.8 & 30.7 & 29.8\end{array}$ $9 \times 28.9$
$10 \times 25.4$

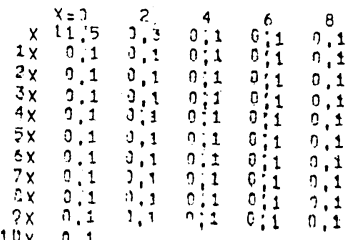

Meast - Sand Condi

PFRCFNT TIIAL MISTANTF

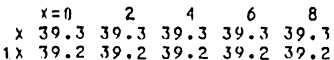

$\begin{array}{llllll}1 \times & 39.2 & 39.2 & 39.2 & 39.2 & 39.2 \\ 2 \times & 39.2 & 39.2 & 39.2 & 39.2 & 39.1\end{array}$

$3 \times \quad 39.1 \quad 39.1 \quad 39.1 \quad 39.1 \quad 39$.

$\begin{array}{llllll}4 \times & 39.1 & 39.1 & 38.8 & 38.7 & 38.4 \\ 5 \times & 38.1 & 37.8 & 37.5 & 37.1 & 36.7\end{array}$

$\begin{array}{llllll}5 \times & 36.1 & 37.8 & 37.5 & 37.2 & 36.7 \\ 6 \times & 36.3 & 35.8 & 35.3 & 34.8 & 34.3\end{array}$

$\begin{array}{rrrrrr}R \times & 29.9 & 28.5 & 27.3 & 26.2 & 25.2 \\ 9 \times & 24.4 & 23.6 & 22.9 & 22.3 & 21.7\end{array}$

$10 \times 21.0$
PIRCERT TIISAL MISTINCE

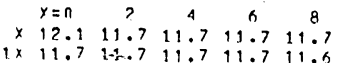
$3 x \quad 11.5 \quad 11.5 \quad 11.5 \quad 11.4 \quad 11.4$

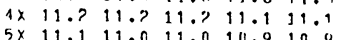
$\begin{array}{cccccc}5 x & 11.9 & 11.0 & 11.0 & 10.9 & 10.0 \\ 6 x & 10.9 & 10.0 & 10.8 & 10.8 & 10.7\end{array}$ $7 x \quad 10.7$ 11.6 10.5 to.5 10.4 $\begin{array}{cccccc}8 \times & 10.3 & 11.2 & 10.1 & 11.0 & 0.7 \\ 0 \times & 0.5 & 9.2 & 8.0 & 8.7 & 9.4\end{array}$ $\ln x$ :

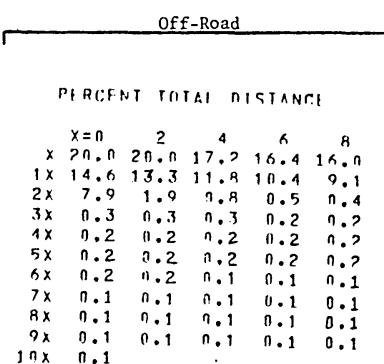

PERCFNT TIITAL MISIANSF

$\begin{array}{rrrrr} & x=0 \\ \times & 39.7 \quad 32.2 & 29.6 & 6 & 6 \\ 1 & 8\end{array}$

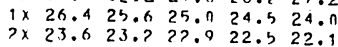

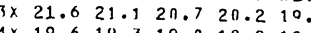
$\begin{array}{lllllll}5 \times & 18.2 & 17.9 & 17.7 & 17.5 & 17.5\end{array}$ $\begin{array}{lllllll}5 x & 17.1 & 16.9 & 16.7 & 16.4 & 16.2\end{array}$ $\begin{array}{cccccc}7 \times & 16.0 & 15.7 & 15.5 & 15.3 & 15.2\end{array}$

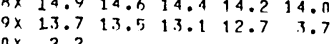

$10 \times \quad 2.2$

DERCENT TETAL DISTANCE

$x=0$
$x+5$
15

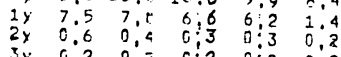

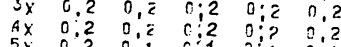

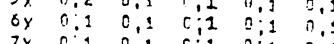

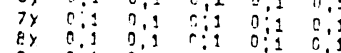

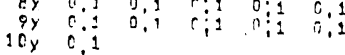

PERCENT TCTAL DISTANCE

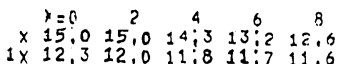

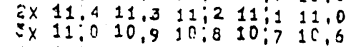

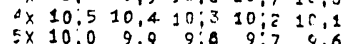

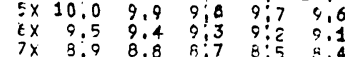

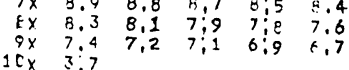




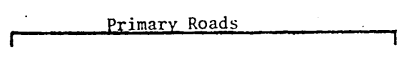

PERCENT TUTAL DISTANCE

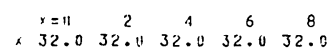

$\begin{array}{llllll}1 \times & 32.0 & 32.0 & 32.0 & 32.0 & 32.0\end{array}$

$\begin{array}{llllll}2 \times & 32.0 & 32.0 & 32.0 & 32.0 & 32.0 \\ 3 \times & 32.0 & 32.0 & 32.0 & 32.0 & 32.0\end{array}$

1) $32.0 \quad 32.0 \quad 32.0 \quad 32.0 \quad 31.9$

$\begin{array}{lllllll}5 \times & 31.9 & 31.8 & 31.6 & 31.4 & 31.1 \\ 6 \times & 39.8 & 30.6 & 30.3 & 30.1 & 29.8\end{array}$

$\begin{array}{lllllll}7 \times & 29.6 & 29.5 & 29.3 & 29.1 & 28.8 \\ 8 \times & 28.4 & 28.0 & 27.6 & 27.1 & 26.5\end{array}$

$\begin{array}{rrrrr}9 \times 26.0 & 25.4 & 25.0 & 24.4 & 23.7 \\ 10 \times 22.9 & & & & \end{array}$

PERCENT TUIAL DISTANCE

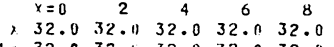

$\begin{array}{lllllll}3 \times & 32.0 & 32.0 & 32.0 & 32.0 & 32.0\end{array}$

$\begin{array}{llllllllll}4 x & 32.0 & 32.0 & 32.0 & 32.0 & 32.0 & 0.0\end{array}$

$\begin{array}{llllll}6 x & 31.3 & 31.1 & 30.9 & 30.8 & 30.6\end{array}$

$\begin{array}{llllll}7 \times & 30.4 & 30.3 & 30.1 & 29.9 & 29.5 \\ 8 \times & 29.1 & 28.6 & 28.1 & 27.5 & 26.9\end{array}$

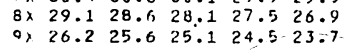

$\begin{array}{rl}10 \times 26.2 & 25 . \\ 10 \times 22.9\end{array}$

PERCENT total Distance

$\begin{array}{cccccc}x=0 & 2 & 4 & 6 & 6 \\ \times & 32.0 & 32.0 & 32.0 & 32.0 & 32^{8}\end{array}$ $\begin{array}{rrrrrr}1 \times & 32.0 & 32.0 & 32.0 & 32.0 & 32.0 \\ 2 \times & 32.0 & 32.0 & 32.0 & 32.0 & 32.0 \\ 32 & 32.0 & 32.0\end{array}$ $\begin{array}{llllll}3 \times & 32.0 & 32.0 & 32.0 & 32.0 & 32.0\end{array}$ $\begin{array}{lllllll}4 \times & 32.0 & 32.0 & 32.0 & 32.0 & 32.0 & 32.0\end{array}$ $\begin{array}{llllll}5 x & 32.0 & 32.0 & 32.0 & 32.0 & 31.9 \\ 6 x & 31.9 & 31.9 & 31.9 & 31.8 & 31.8\end{array}$ $\begin{array}{llllll}7 \times & 31.8 & 31.8 & 31.8 & 31.7 & 31.5\end{array}$

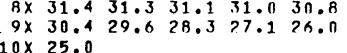

PERCENT TOTAL DISTANCE

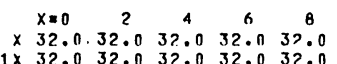
$\begin{array}{lllllll}2 \times & 32.0 & 32.0 & 32.0 & 32.0 & 32.0\end{array}$ $\begin{array}{llllll}3 \times & 32.0 & 32.0 & 32.0 & 32.0 & 32.0 \\ 4 \times & 32.0 & 32.0 & 32.0 & 32.0 & 32.0\end{array}$

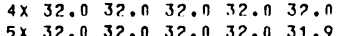
$\begin{array}{llllll}5 \times & 32.0 & 32.0 & 32.0 & 32.0 & 31.9 \\ 6 \times & 31.9 & 31.9 & 31.9 & 31.8 & 31.8\end{array}$ $\begin{array}{llllll}7 x & 31.8 & 31.8 & 31.7 & 31.6 & 31.5 \\ 8 x & 31.3 & 31.7 & 31.1 & 30.9 & 30.7\end{array}$ $\begin{array}{llllll}8 \times & 31.3 & 31.2 & 31.1 & 30.9 & 3 n .7 \\ 9 \times & 30.2 & 29.4 & 28.0 & 26.7 & 25.5\end{array}$ $\begin{array}{r}9 \times \\ 11 \times 24.6 \\ \hline\end{array}$
Table 531

$\sqrt{\text { Secondary Roads }} \frac{\text { Speed Profile for M109A1 }}{\text { WESt Germany - Wet Condition }}$ Trails

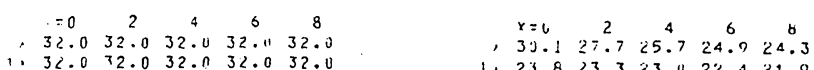

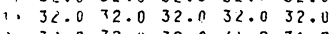

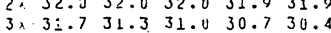

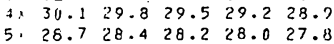

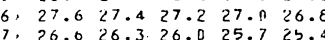

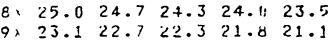

$10 \times 20.5$

$\begin{array}{llllll}1, & 23.8 & 23.3 & 23.0 & 22.4 & 21.9 \\ 2, & 21.5 & 21.1 & 20.8 & 20.4 & 21.9\end{array}$

3. $19.919 .6 \quad 19.4 \quad 19.1 \quad 18$.

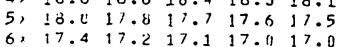

$\begin{array}{llllll}6, & 17.4 & 17.2 & 17.1 & 17.0117 .0 & 17.0\end{array}$

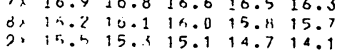
in) 13.0

West Germany - Snow Condition

DERLEHT IOTAL DISTLICEE PERCENT TOTAL DISTLACE

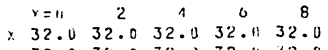

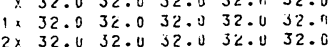

$3 \times 32.0 \quad 32.0 \quad 32.032 .031 .9$

$4 \times 31.931 .8 \quad 31.531 .230 .9$

$\begin{array}{llllll}5 \times & 30.6 & 30.3 & 30.1 & 29.8 & 29.5 \\ 6 \times 29.3 & 29.1 & 28.9 & 28.6 & 28.3\end{array}$

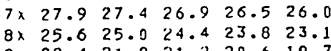

$\begin{array}{rr}9 \times & 22.4 \\ 10 \times & 18.9\end{array}$

$x=0 \quad 2 \quad 4 \quad 6$

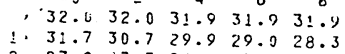

$\begin{array}{llllll}2, & 31.7 & 30.7 & 29.9 & 29.9 & 28.3 \\ 3 \times & 27.8 & 27.3 & 26.6 & 25.7 & 25.1\end{array}$

$\begin{array}{lllllll}3 x & 24.5 & 24.0 & 23.6 & 23.1 & 22.5 \\ 4 x & 22.1 & 21.7 & 21.4 & 21.1 & 20.8\end{array}$

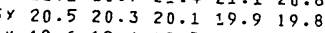

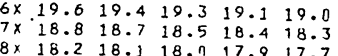

$\begin{array}{llllll}8 \times & 18.2 & 18.1 & 18.7 & 17.9 & 17.7 \\ 9 \times & 17.6 & 17.4 & 17.1 & 16.5 & 15.7\end{array}$

\section{Mid-East - Wet Condition}

PERCENT TOTAL DISTANCE

PERCENT total Distance

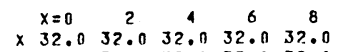

$\begin{array}{rrrrrr}1 \times & 32.0 & 32.0 & 32.0 & 32.0 & 32.0 \\ 2 \times & 32.0 & 32.0 & 32.0 & 32.0 & 32.0\end{array}$

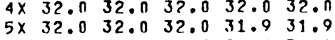

$\begin{array}{llllll}6 \times & 31.8 & 31.7 & 31.5 & 31.4 & 31.9\end{array}$

$8 \times 30.930 .730 .530 .429$.

$9 \times \quad 25.5 \quad 25.0 \quad 24.5 \quad 24.0 \quad 23.6$

$10 \times 23.0$

\section{Mid-}

PERCENT TOTAL Distance

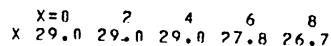

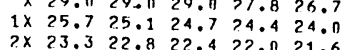

$\begin{array}{llllll}2 x & 23.3 & 22.8 & 22.4 & 22.4 & 24.6 \\ 3 x & 21.3 & 21.0 & 20.7 & 21.6 \\ 4 x & 20.5 & 21.0 & 20.5\end{array}$

$4 \times \quad 20.019 .7 \quad 19.5 \quad 19.3 \quad 10.2$

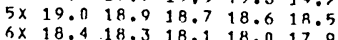

$\begin{array}{llllll}6 \times & 18.4 & 18.3 & 18.1 & 18.0 & 17.5 \\ 7 \times & 17.8 & 17.7 & 17.6 & 17.5 & 17.4\end{array}$

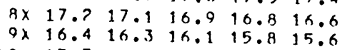

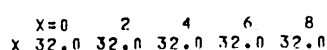

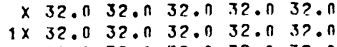

$\begin{array}{llllll}2 x & 32.0 & 32.0 & 32.0 & 32.0 & 32.0 \\ 3 x & 32.0 & 32.0 & 32.0 & 32.0 & 32.0\end{array}$

$\begin{array}{llllll}3 x & 32.0 & 32.0 & 32.0 & 32.0 & 32.0 \\ 4 \times & 32.0 & 32.0 & 32.0 & 32.0 & 33.0\end{array}$

$\begin{array}{llllll}4 \times & 32.0 & 31.9 & 31.9 & 31.0 & 31.5\end{array}$

$\begin{array}{llllll}6 \times & 31.2 & 31.0 & 30.7 & 30.4 & 30.2 \\ 7 \times & 29.9 & 29.5 & 29.0 & 78.5 & 27.9\end{array}$

$8 \times \quad 27.726 .1 \quad 25.1 \quad 24.2 \quad 23.4$

$\begin{aligned} 9 \times & 22.7 \\ 10 \times & 19.9\end{aligned}$
PFRCFNT TOTAL. DIGIANTE

$\begin{array}{ccccc}x=0 & 7 & 4 & 6 & 8 \\ x & 15.2 & 15.2 & 15 . ? & 15.2 \\ 2 x & 15.1 & 15.1\end{array}$

$\begin{array}{llllll}1 x & 15.1 & 15.1 & 15.1 & 15.0 & 14.9 \\ 2 x & 14.8 & 14.7 & 14.6 & 14.5 & 14.4\end{array}$

$\begin{array}{lllllll}3 x & 14.3 & 14.3 & 14.7 & 14.1 & 14.0 \\ 4 \times & 13.9 & 13.8 & 13.8 & 13.7 & 1.3 .6\end{array}$

$\begin{array}{lllllll}5 x & 13.6 & 13.5 & 13.4 & 13.4 & 13.3 \\ 7 x & 13.3 & 13.2 & 13 . & 13.1 & 13.0\end{array}$

$\begin{array}{lllllll}8 \times & 12.6 & 12.5 & 12.4 & 12.3 & 17.1 \\ 9 \times & 11.8 & 11.6 & 11.3 & 11.0 & 10.8\end{array}$ $\begin{array}{cccccc}7 \times & 13.0 & 12.9 & 12.9 & 12.8 & 13.0 \\ 9 x & 12.0 & 12.5 & 12.4 & 12.3 & 12.7\end{array}$

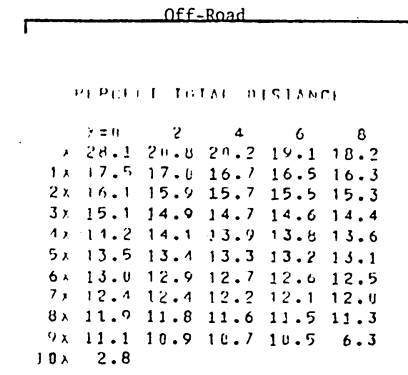

"ERCENT tUtal motance

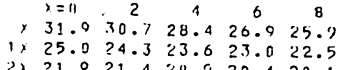
2) 21.921 .420 .920 .420 .1 $\begin{array}{llllll}3 \lambda & 17.8 & 19.5 & 19.2 & 18.9 & 18.7 \\ 4 \lambda & 18.4 & 18.2 & 18.0 & 17.8 & 17.6\end{array}$ $\begin{array}{lllllll}5 \times & 17.4 & 17.3 & 17.1 & 16.9 & 16.7 \\ 6 \lambda & 16.6 & 10.4 & 16.3 & 16.9 & 16.7\end{array}$ $7 \times 15.815 .7 \quad 15.5 \quad 15.415 .2$ $\begin{array}{cccccc}8 \times & 15.0 & 14.8 & 14.7 & 14.5 & 14.3 \\ 3 \times & 14.1 & 13.2 & 13.6 & 13.3 & 12.9\end{array}$

\section{PERCENT total Distance}

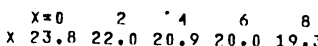
$\begin{array}{llllll}1 \times & 18.6 & 18.1 & 17.7 & 17.3 & 17.0\end{array}$ $\begin{array}{llllll}2 x & 16.7 & 16.5 & 16.3 & 16.1 & 15.0 \\ 3 x & 15.7 & 15.5 & 15.4 & 15.2 & 15.1\end{array}$ $\begin{array}{llllll}4 \times & 14.9 & 14.7 & 14.6 & 14.4 & 14.93\end{array}$ $\begin{array}{llllll}5 x & 14.1 & 14.0 & 13.9 & 13.7 & 13.6 \\ 6 x & 13.4 & 13.2 & 13.1 & 13.0 & 12.8\end{array}$ $8 \times 12.712 .6 \quad 12.512 .317 .2$ $\begin{array}{rrrrrr}8 \times & 12.1 & 12.0 & 11.8 & 11.7 & 11.6 \\ 9 \times & 11.4 & 11.3 & 11.1 & 10.9 & 10.7 \\ 10 \times & 5.8 & & & & \end{array}$

Percent total Distance

$\begin{array}{rrrrrr} & x=0 & 2 & 4 & 6 & 8 \\ \times & 15.2 & 15.9 & 15.1 & 14.9 & 14.8 \\ x & 14.7 & 14.6 & 14.4 & 14.3 & 14.2\end{array}$ $\begin{array}{llllll}1 \times & 14.7 & 14.6 & 14.4 & 14.3 & 14.8 \\ 3 x & 14.1 & 14.0 & 13.9 & 13.8 & 13.7\end{array}$ $\begin{array}{cccccc}3 x & 13.5 & 13.4 & 13.3 & 13.2 & 13.1 \\ 4 x & 13.0 & 12.9 & 12.7 & 12.6 & 13.5\end{array}$ $\begin{array}{lllllll}5 x & 12.4 & 12.3 & 12.2 & 12.1 & 11 . \\ 6 x & 11.0 & 0\end{array}$ $7 \times 11.211 .110 .9 \quad 10.8$ a 11.4 $\begin{array}{rrrrrr}8 \times & 10.5 & 10.3 & 10.1 & 9.9 & 0.7 \\ 9 \times & 9.5 & 9.4 & 9.2 & 9.0 & 8.9\end{array}$ 


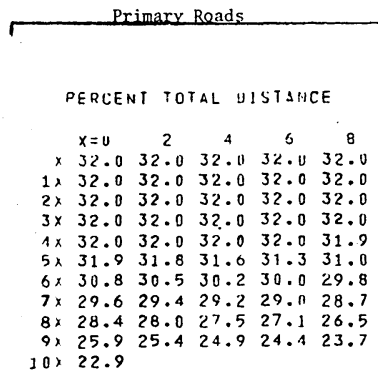

Derlen: tutal mistance

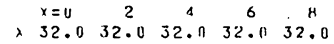

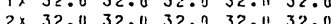
$\begin{array}{lllllll}3 \times & 32.0 & 32.0 & 32.0 & 32.0 & 32.0 & 32.0\end{array}$ $\begin{array}{llllll}4 \times & 32.0 & 32.0 & 52.0 & 32.01 & 32.0 \\ 5 \times & 32.0 & 32.0 & 31.9 & 31.8 & 31.5\end{array}$ $6 \times 31.3 \quad 31.030 .8 \quad 30.7 \quad 30.5$ $\begin{array}{lllll}7 \times & 30.3 & 30.2 & 30.0 & 29.4 \\ 8 \times & 29.0 & 29.4\end{array}$ $\begin{array}{llllll}9 x & 26.1 & 25.5 & 28.0 & 27.4 & 26.8 \\ 9 x & 25.4 & 24.4 & 23.6\end{array}$

\section{PERCEnt total DISTANCE.}

$\begin{array}{cccccc}x=0 & 2 & 2 & 6 & 8 \\ \times 32.0 & 32.0 & 32.0 & 32.0 & 32.0\end{array}$ $\begin{array}{rllllll}1 \times & 32.0 & 32.0 & 32.0 & 32.0 & 32.0 & 32.0\end{array}$ $\begin{array}{llllll}2 \times & 32.0 & 32.0 & 32.0 & 32.0 & 32.0 \\ 3 \times & 32.0 & 32.0 & 32.0 & 32.0 & 32.0\end{array}$ $\begin{array}{llllll}3 \times & 32.0 & 32.0 & 32.0 & 32.0 & 32.0 \\ 4 \times & 32.0 & 32.0 & 32.0 & 32 & 32.0\end{array}$ $5 \times 32.032 .032 .032 .0 \quad 31.9$ $6 \times 31.931 .8 \quad 31.8 \quad 31.8 \quad 31.8$

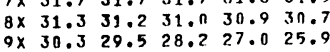
$9 \times 30.3$
$10 \times 24.9$

PERCENT TOTAL DISTANC.

$\begin{array}{rrrrrr}x=0 & 2 & 4 & 6 & 8 \\ \times & 32.0 & 32.0 & 32.0 & 32.0 & 32.0 \\ x & 32.0 & 32.0 & 32.0 & 32.0 & 32.0\end{array}$ $2 \times 32.0 \quad 32.0 \quad 32.0 \quad 32.0 \quad 32.0$ $\begin{array}{lllllll}3 x & 32.0 & 32.0 & 32.0 & 32.0 & 32.0 \\ 4 x & 32.0 & 32.0 & 32.0 & 32.0 & 32.0\end{array}$

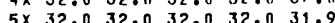
$6 \times 31.931 .8 \quad 31.8 \quad 31.6 \quad 31.8$ $7 \times \quad 31.731 .7 \quad 31.7 \quad 31.6 \quad 31.4$ $\begin{array}{cccccc}8 \times & 31.3 & 31.1 & 31.0 & 30.8 & 30.6 \\ 9 \times & 30.1 & 29.3 & 27.9 & 76.6 & 25.5\end{array}$

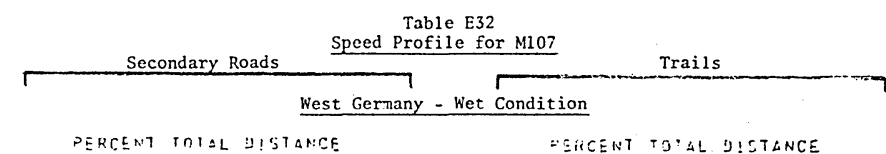

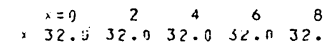

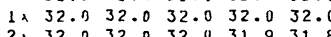
$\begin{array}{llllll}2 \wedge & 32.0 & 32.0 & 32.0 & 31.0 & 31.8 \\ 3 \times & 31.6 & 31.3 & 30.9 & 30.6 & 30.3\end{array}$

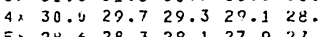
$\begin{array}{llllll}5 \times & 28.6 & 28.3 & 28.1 & 27.9 & 27.7 \\ 6 x & 27.5 & 27.3 & 27.1 & 26.8 & 26.5\end{array}$ $\Rightarrow 26.225 .925 .625 .325 .0$ $10 \times 20.3$

\section{West Germany - Snow Condition}

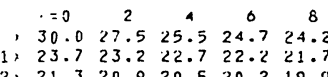

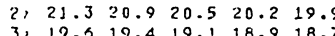

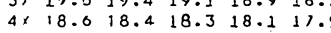

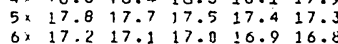
$\begin{array}{llllll}6 \times & 17.2 & 17.1 & 17.0 & 10.9 & 16.8 \\ 7 \times & 10.7 & 16.6 & 10.5 & 10.3 & 16.2\end{array}$ $\begin{array}{llllll}\times x & 10.7 & 16.6 & 10.5 & 16.3 & 16.2 \\ 8 & 10.1 & 15.9 & 15.8 & 15.7 & 15.5\end{array}$ 15.215 .011 .511 .0

DERLENT IGTAL TISTAMCE UERIENT IUIAL UISTAMCE

$\begin{array}{rrrrrr}r=11 & 2 & 4 & 6 & 8 \\ \times & 32.0 & 32.0 & 32.0 & 32.0 & 32.9\end{array}$ $\begin{array}{llllll}1 \times & 32.0 & 32.0 & 32.11 & 32.0 & 32.9 \\ 3 \times & 32.0 & 32.0 & 32.0 & 32.0 & 32.0\end{array}$ $\begin{array}{llllll}3 \times & 32.0 & 32.0 & 32.0 & 32.0 & 31.9 \\ 4 x & 31.8 & 31.6 & 31.4 & 31.1 & 30.8\end{array}$ $5 \times 30.430 .129 .929 .629$.

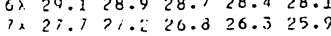
$\begin{array}{llllll}3 x & 25.4 & 24.9 & 21.2 & 23.7 & 23.0 \\ 0 \times & 22.3 & 21.7 & 21.1 & 20.5 & 19.6\end{array}$ $10 \times 18.9$

$$
\text { Mid-Ea }
$$$$
\therefore \begin{array}{lcccc}
32.0 & 2 & 4 & 6 & 8 \\
31.0 & 32.0 & 31.9 & 31.9 & 31.9
\end{array}
$$

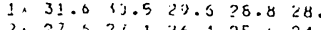
3. 21.423 .923 .5233 .022$. $\begin{array}{ccccc}4 \times & 22.0 & 21.6 & 21.3 & 21.020 .7\end{array}$ $6 \times 21.520 .220 .919 .119 .7$ 7. 18.818 .618 .518 .418 .3

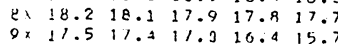
$10 \times 15.14$

PERCENT total Distance Perent total DISTANCE

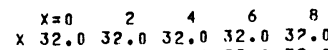
$\begin{array}{llllll}1 \times & 32.0 & 32.0 & 32.0 & 32.0 & 32.0\end{array}$

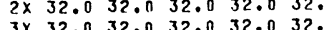
$4 \times 32.032 .032 .032 .032 .0$ $\begin{array}{llllll}5 \times & 32.0 & 32.0 & 32.0 & 31.9 & 31.9\end{array}$ $6 \times 31.831 .731 .6 \quad 31.331$. $9 \times 25.525 .024 .524 .023 .6$ $10 \times 23.0$

$\begin{array}{llll}x & =0 & 2 & 4\end{array}$

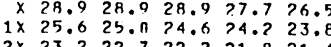

$\begin{array}{lll}-2 & 21.8 & 23.4\end{array}$

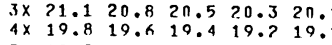

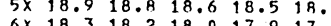
$\begin{array}{lllllll}7 x & 17.7 & 17.6 & 17.5 & 17.4 & 17.9\end{array}$ $8 \times 17.117 .016 .8 \quad 16.7$ 16.5 Qx $16.316 .1 \quad 15.9 \quad 15.6 \quad 15.4$ PERCENT TOTAL DISTANCF dition

$\begin{array}{rrrrr}x=0 \\ \times \quad 32.0 & 32.0 & 32.0 & 32.0 & 30^{8}\end{array}$

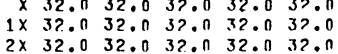
$\begin{array}{llllll}3 x & 32.0 & 32.0 & 32.0 & 32.0 & 32.0 \\ 4 x & 32.0 & 32.0 & 32.0 & 32.0 & 32.0\end{array}$ $5 \times \quad 32.031 .9 \quad 31.9231 .8 \quad 31.5$ $6 \times 31.230 .9 \quad 30.7 \quad 30.430 .1$

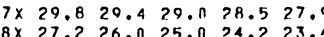

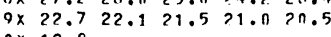

PFRCFNT TOTAL MISTANFE

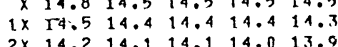
$\begin{array}{llllll}2 x & 14.2 & 14.1 & 14.1 & 14.0 & 13.9 \\ 3 x & 13.9 & 13.8 & 13.8 & 13.7 & 13.6\end{array}$ $\begin{array}{llllll}7 \times & 12.7 & 12.6 & 12.6 & 12.5 & 12.4 \\ 9 x & 12.3 & 12.3 & 12.6 & 12.0 & 11.4\end{array}$ $\begin{array}{ccccc}x=0 & ? & 4 & 6 & 8 \\ \times \quad 14.8 & 14.5 & 14.5 & 14.5 & 14.5\end{array}$

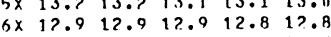

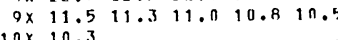

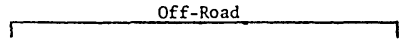

"ERIENT TOTAL DISTANCE

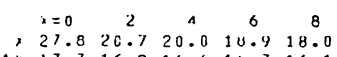

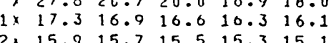
$\begin{array}{llllllll}2 \times & 15.9 & 15.7 & 15.5 & 15.3 & 15.1 \\ 3 \times & 14.9 & 14.7 & 14.5 & 14.4 & 14.2\end{array}$

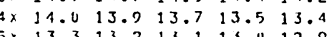
$\begin{array}{lllllll}6 x & 12.8 & 12.1 & 12.6 & 12.5 & 12.4\end{array}$

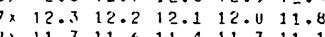

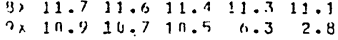
$10 \times 1.8$

"EREENT TOTAL DISIANCE

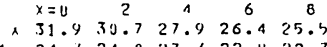
$\therefore 24.124 .023 .522 .4222 .3$ $\begin{array}{llllll}3 \times & 10.6 & 19.3 & 10.0 & 18.4 & 18.5\end{array}$ a

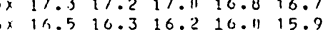
$\begin{array}{lllllll}7 \times & 15.8 & 15.6 & 15.1 & 15.3 & 15.1 \\ 4 x & 14.4 & 14.8 & 14.5 & 14.5 & 14.3\end{array}$

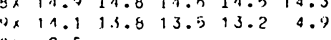

PERCENT TOTAL DISTANCE

$\begin{array}{cccccc} & x=0 & 2 & 4 & 6 & 8 \\ x & 23.9 & 22.1 & 20.9 & 19.9 & 19.1 \\ x & 18 & 19.9 & 17.5 & 17.2 & 16.9\end{array}$ $\begin{array}{rlllll}1 x & 18.4 & 17.9 & 17.5 & 17.2 & 16.9 \\ 2 x & 16.6 & 16.4 & 16.2 & 16.0 & 15.8\end{array}$ $\begin{array}{llllll}2 x & 16.6 & 16.4 & 16.2 & 16.0 & 15.8 \\ 3 \times & 15.6 & 15.4 & 15.2 & 15.1 & 14.9 \\ 4 x & 14.7 & 14.0 & 14.4 & 14.3 & 14.1\end{array}$ $\begin{array}{lllllll}5 \times & 14.0 & 13.8 & 13.7 & 13.5 & 13.4\end{array}$ $\begin{array}{llllll}5 x & 14.0 & 3.0 & 34.4 & 14.3 & 14.1 \\ 7 x & 13.2 & 13.9 & 12.9 & 12.8 & 17.4\end{array}$

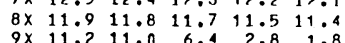
$10 \times \quad 1.3$

PERCENT TOTAL DISTANTE

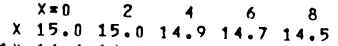
$\begin{array}{llllll}1 \times & 14.4 & 14.2 & 14.1 & 14.7 & 14.5 \\ 3 \times & 13.8 & 13.7 & 13.6 & 13.0 & 13.9\end{array}$ $\begin{array}{llllll}3 x & 13.2 & 13.1 & 13.0 & 12.9 & 17.8\end{array}$ $\begin{array}{llllll}1 \times & 12.7 & 12.6 & 12.5 & 12.4 & 12.2 \\ 5 x & 12.12 & 12.0 & 11.9 & 11.8 & 11.7\end{array}$ $\begin{array}{llllll}5 x & 12.1 & 12.0 & 11.9 & 11.8 & 11.7 \\ 6 x & 11.6 & 11.5 & 11.4 & 11.2 & 1.7\end{array}$ $7 \times \quad 11.010 .910 .7 \quad 10.510 .4$

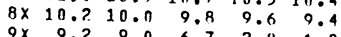
$\begin{array}{llllll}9 x & 9.2 & 9.0 & 6.7 & 2.8 & 1.8\end{array}$ 


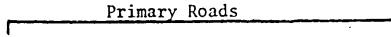

DERLEHT TOTAL UISTAMCE

$\begin{array}{rrrrrr} & i=0 & 2 & 4 & 6 & 8 \\ \times & 32.0 & 32.0 & 32.0 & 32.0 & 32.0\end{array}$ $\begin{array}{llllll}1 \times & 32.0 & 32.0 & 32.9 & 32.0 & 32.0 \\ 2 \times & 32.0 & 32.0 & 32.0 & 32.0 & 32.0\end{array}$ $\begin{array}{lllllll}3 \times & 32.0 & 32.0 & 32.0 & 32.0 & 32.0 \\ 4 \times & 32.0 & 32.0 & 32.0 & 32.0 & 31.0\end{array}$ $\begin{array}{llllll}5 \times & 31.9 & 31.8 & 31.6 & 31.3 & 31.0 \\ 6 \times & 30.8 & 30.5 & 30.2 & 30.0 & 20.8\end{array}$ $\begin{array}{llllll}8 \times & 28.4 & 28.0 & 27.5 & 27.1 & 26.5 \\ \text { 7) } & 25.9 & 25.4 & 24.9 & 24.4 & 23.7\end{array}$ $10 \times 22.9$

PERCENT TUTAL DISTANCE

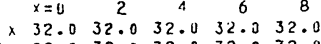

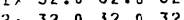

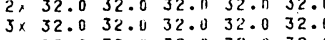
$4 \times 32.032 .0 \quad 32.032 .032$. $\begin{array}{lllllll}5 \times & 32.0 & 32.0 & 31.9 & 31.8 & 31.5 \\ 6 \times & 31.3 & 31.0 & 30.8 & 30.7 & 310.5\end{array}$ $7 \times 30.3 \quad 30.230 .029 .829 .4$ $\begin{array}{llllll}8 \times & 29.0 & 28.5 & 28.0 & 27.4 & 26.8 \\ 9 \times & 26.1 & 25.6 & 25.0 & 24.4 & 23.6\end{array}$ $14 \times 22.8$

PERCENT TOTAL DISTANCE

$\begin{array}{rrrrrr}x=0 & =0 \\ \times 32.0 & 32.0 & 32.0 & 32.0 & 32 & 8\end{array}$ $\begin{array}{llllll}1 \times & 32.0 & 32.0 & 32.0 & 32.0 & 32.0\end{array}$ $\begin{array}{lllllll}2 x & 32.0 & 32.0 & 32.0 & 32.0 & 32.0 \\ 3 x & 32.0 & 32.0 & 32.0 & 32.0 & 32.0\end{array}$ $4 \times 32.032 .032 .032 .032 .0$ $\begin{array}{llllll}5 \times & 32.0 & 32.0 & 32.0 & 32.0 & 31.9 \\ 6 \times & 31.9 & 31.8 & 31.8 & 31.8 & 31.8\end{array}$ $\begin{array}{llllll}7 \times & 31.9 & 31.7 & 31.7 & 31.6 & 31.5 \\ 8 \times & 31.7 & 31.2 & 31.0 & 30.9 & 30.7\end{array}$

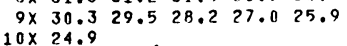

PFRCFNT TITAL DISTANCE

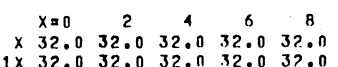
$\begin{array}{llllll}1 \times & 32.0 & 32.0 & 32.0 & 32.0 & 32.0 \\ 32 & 32.0 & 32.0 & 32.0 & 32.0 & 32.0\end{array}$ $\begin{array}{lllllll}3 \times & 32.0 & 32.0 & 32.0 & 32.0 & 32.0 \\ 4 \times & 32.0 & 32.0 & 32.0 & 32.0 & 32.0\end{array}$

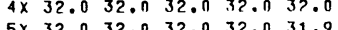
$\begin{array}{llllll}5 \times & 32.0 & 32.0 & 32.0 & 32.0 & 31.9 \\ 6 \times & 31.9 & 31.8 & 31.8 & 31.8 & 31.8 \\ 7 \times & 31.7 & 31.7 & 31.7 & 31.6 & 31.4\end{array}$ $\begin{array}{llllll}7 \times & 31.7 & 31.7 & 31.7 & 31.6 & 31.4 \\ 8 \times & 31.3 & 31.1 & 31.0 & 30.8 & 30.6\end{array}$

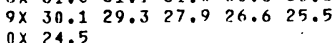

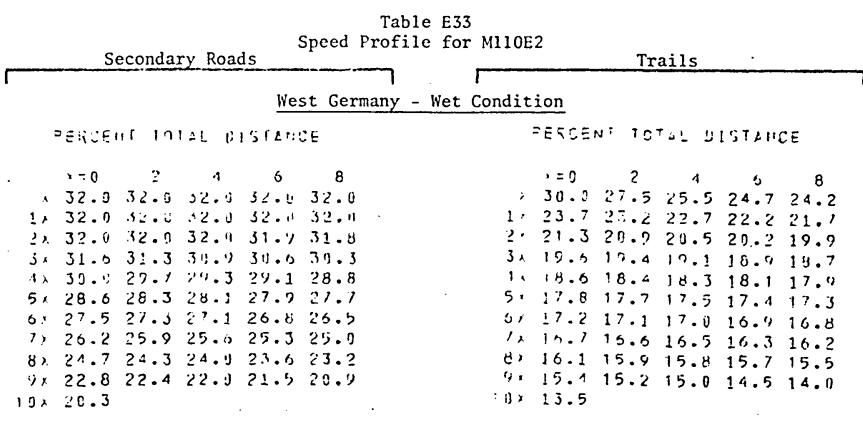

West Germany - Snow Condition

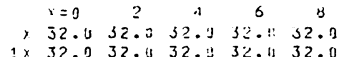

$\begin{array}{llllll}2 * & 32.0 & 32.0 & 32.0 & 32.11 & 32.0 \\ 3 ; & 32.5 & 32.0 & 32.0 & 32.11 & 31.0\end{array}$

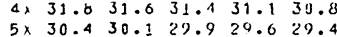

$\begin{array}{llllll}5 x & 29.1 & 28.9 & 28.7 & 28.4 & 28.4\end{array}$

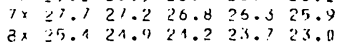

2. 22.321 .721 .126 .519 .0

Mid-E

PERCENT TOTAL DISTANTE

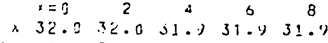

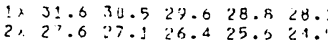

$3 \times 24.423 .723 .523 .022$.

$+x 22.1123 .621 .321 .029$.

$6.19 .519 .4 \quad 19.219 .118$.

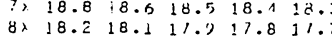

$4 \times 17.5 \quad 27.417 .0$ 10.4 15.7

$\begin{array}{rrrrrr}x=0 & 2 & 4 & 6 & 8 \\ \times & 32.0 & 32.0 & 32.0 & 32.0 & 32.0 \\ 12 & 32.0 & 32.0 & 32.0 & 32.0 & 32.0\end{array}$

$\begin{array}{llllll}1 \times & 32.0 & 32.0 & 32.0 & 32.0 & 32.0 \\ 2 \times & 32.0 & 32.0 & 32.0 & 32.0 & 32.0\end{array}$

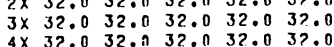

$5 \times 32.032 .037 .0 \quad 31.931 .9$

$\begin{array}{llllll}6 \times & 31.8 & 31.7 & 31.6 & 31.3 & 31.1 \\ 7 \times & 30.9 & 30.7 & 30.4 & 30.1 & 20.7\end{array}$

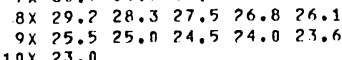

Mid-

PERCENT TRTAL DISTANTE

PERCENT TOTAL DISIANGE

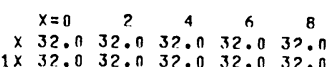

$\begin{array}{llllll}2 \times & 32.0 & 32.0 & 32.0 & 32.0 & 32 \\ 3 \times & 32.0 & 32.0 & 32.0\end{array}$

$\begin{array}{llllll}3 \times & 32.0 & 32.0 & 32.0 & 32.0 & 32.0 \\ 4 \times & 32.0 & 32.0 & 32.0 & 32.0 & 32.0\end{array}$

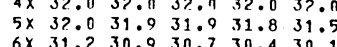

$\begin{array}{llllll}6 \times & 31.2 & 30.9 & 30.7 & 30.4 & 30.1 \\ 7 \times & 29.8 & 29.4 & 29.0 & 78.5 & 27.9\end{array}$

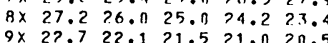

$\begin{aligned} 9 \times & 19.8\end{aligned}$ $\begin{array}{ccccccc}x=0 & 2 & 4 & 6 & 8 \\ \times & 78.9 & 28.9 & 28.9 & 77^{6} .7 & 286^{-5}\end{array}$

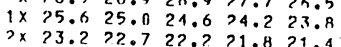

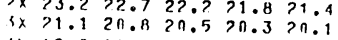

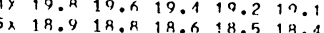
$\begin{array}{llllll}6 \times & 18.3 & 18.4 & 18.6 & 18.5 & 18.4 \\ 7 x & 18.0 & 17.9 & 17.8\end{array}$

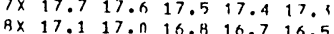
$9 \times 16.316 .115 .9 \quad 15.6 \quad 15.4$

PERCENT TUTAL DISTANC.

$\begin{array}{rrrrrr} & x=0 & 2 & 4 & 6 & 8 \\ x & 14.8 & 14.5 & 14.5 & 14.5 & 14.5 \\ 1 x & 14.5 & 14.4 & 14.4 & 14.4 & 14.3\end{array}$ $\begin{array}{lllllll}4 x & 13.5 & 13.5 & 13.4 & 13.4 & 13.3 \\ 5 x & 13 . & 13.2 & 13.1 & 13.5 & 13.0\end{array}$

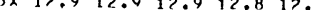
$\begin{array}{llllll}7 x & 12.7 & 12.6 & 17.6 & 17.5 & 1 ? .4 \\ 9 x & 12.5 & 12.8 & 12.4 & 12.0 & 11.8\end{array}$ $\begin{array}{lllllll}8 x & 12.3 & 12.2 & 12.1 & 12.0 & 11.8 \\ 9 x & 11.5 & 11.3 & 11.0 & 10.8 & 10.5\end{array}$ $\begin{array}{llllll}2 \times & 14.2 & 14.1 & 14.1 & 14.0 & 13.0\end{array}$ $\begin{array}{llllll}3 \times & 13.9 & 13.8 & 13.8 & 13.7 & 13.6\end{array}$

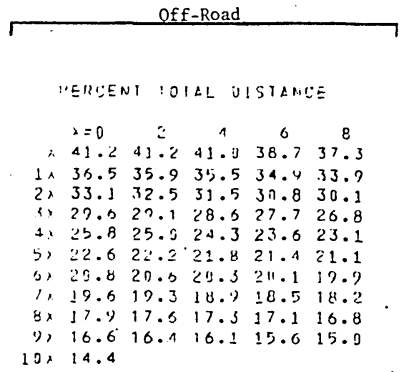

PERCENT total DISTANCE

$\begin{array}{rlcccc} & x=0 & 2 & & \\ x & 31.9 & 30.7 & 27.9 & 6 & 8 \\ 1 \times & 24.7 & 24.0 & 23.3 & 26.4 & 25.5 \\ 2 x & 21.7 & 21.2 & 20.7 & 22.8 & 22.3\end{array}$

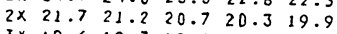
$\begin{array}{llllll}3 \times & 19.6 & 19.3 & 19.0 & 18.8 & 18.5 \\ 4 \times & 18.3 & 18.1 & 17.9 & 17.7 & 17.5 \\ 5 x & 17.3 & 17.1 & 17.0 & 16.8 & 16.6\end{array}$ $\times 17.317 .117 .016 .8 \quad 16.6$ $\begin{array}{lllllll}5 \times & 16.5 & 16.3 & 16.2 & 16.0 & 15.0 \\ 7 \times & 15.7 & 15.6 & 15.4 & 15.3 & 15.9\end{array}$ $\begin{array}{llllll}8 \times & 14.9 & 14.7 & 14.6 & 14.4 & 14.2 \\ 9 \times & 14.0 & 13.8 & 13.5 & 13.2 & 12.8\end{array}$ $10 \times 4.1$

PERCENT tOTAL Distance

$\begin{array}{rrrrr}x=0 & 2 & 4 & 6 & 8 \\ \times \quad 23.9 & 22.1 & 20.9 & 10^{8} .9 & 10^{\circ}\end{array}$

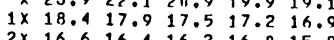

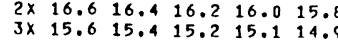
$\begin{array}{lllllll}4 \times & 14.7 & 14.6 & 14.4 & 14.3 & 14.9\end{array}$ $\begin{array}{ccccccc}5 \times & 14.0 & 13.8 & 13.7 & 13.5 & 13.4\end{array}$

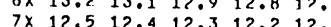

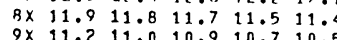

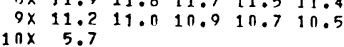

Percent total DistanteE

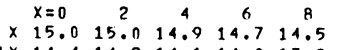

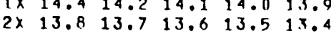
$\begin{array}{lllllll}3 x & 13.2 & 13.1 & 13.0 & 12.9 & 12.8\end{array}$ $\begin{array}{llllll}5 \times & 12.7 & 13.6 & 13.5 & 12.4 & 12.8\end{array}$

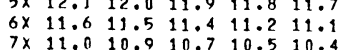
$\begin{array}{rrrrrr}8 \times & 10.2 & 10.0 & 9.8 & 9.6 & 9.4 \\ 9 \times & 9.3 & 9.1 & 8.9 & 8.8 & 8.6\end{array}$ 


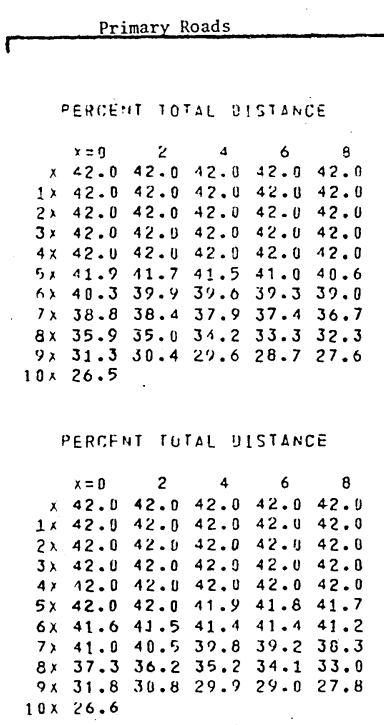

PERCENT TOTAL DISTAHTE -

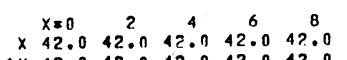

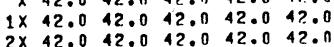
$\begin{array}{cccccc}2 x & 42.0 & 42.0 & 42.0 & 42.0 & 42.0 \\ 3 \times & 42.0 & 42.0 & 42.0 & 42.0 & 42.0\end{array}$ $\begin{array}{llllll}4 \times & 42.0 & 42.042 .0 & 42.0 & 12.0 \\ 5 \times & 42.0 & 42.0 & 42.0 & 42.0 & 42.0\end{array}$ $6 x \quad 42.0 \quad 42.042 .042 .041$. $7 \times \quad 41.941 .941 .841 .641 .4$ $\begin{array}{llllll}8 \times & 41.2 & 40.9 & 40.6 & 40.2 & 39.7 \\ 9 \times & 38.7 & 37.3 & 35.0 & 33.0 & 31.3\end{array}$ $10 \times 29.8$

PERCENT TOTAL DISTANGE

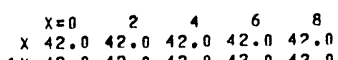

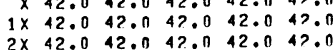
$\begin{array}{cccccc}2 x & 42.0 & 42.0 & 42.0 & 42.0 & 4 ? .0 \\ 3 x & 42.0 & 42.0 & 42.0 & 42.0 & 4 ? .0\end{array}$

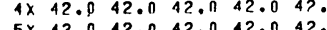

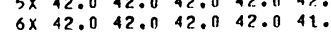
$\begin{array}{cccccc}7 x & 41.9 & 41.9 & 41.8 & 41.5 & 41.3 \\ 8 x & 41.0 & 40.7 & 40.3 & 39.9 & 30.3\end{array}$ $\begin{array}{llllll}8 \times & 41.0 & 40.7 & 40.3 & 39.9 & 30.3 \\ 9 \times & 38.2 & 36.7 & 34.4 & 32.4 & 3 n .6\end{array}$
Table E34
Profile for M113A1

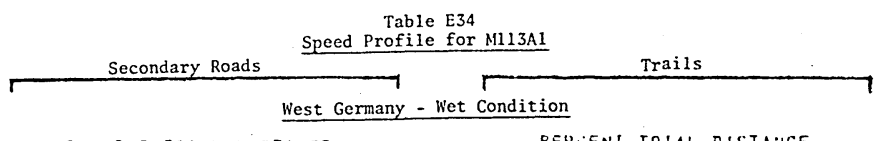

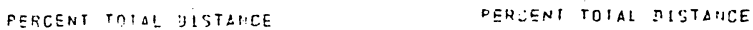

$\begin{array}{ccccc}x=0 & 2 & 4 & 6 & 8 \\ , 42.0 & 42.0 & 42.0 & 42.0 & 42.0\end{array}$

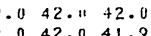

$3 \times 41.8 \quad 41.7 \quad 41.7 \quad 41.4 \quad 41.1$

$\begin{array}{llllll}57 & 38.4 & 38.0 & 39.6 & 39.1 & 39.8 \\ 57 & 37.5 & 37.5 & 31.2\end{array}$

$7 \times 35.134 .6 \quad 34.0 \quad 33.5 \quad 32.9$

$\begin{array}{llllll}81 & 32.4 & 31.7 & 30.9 & 30.2 & 2 \% .4\end{array}$

$9 \times 28.5$

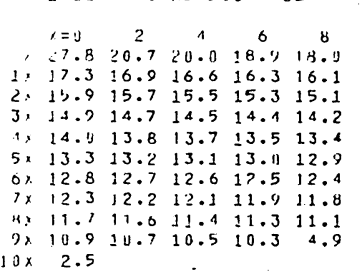

West Germany - Snow Condition

Dercent total distance

PERCENT IJIAL DISTAACE

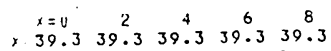

$\begin{array}{llllll}1 \times & 39.3 & 39.3 & 39.3 & 39.3 & 39.5 \\ 22 & 39.3 & 39.3 & 39.3 & 39.3 & 39.3\end{array}$

$\begin{array}{llllll}2 \times & 39.3 & 39.3 & 39.3 & 39.3 & 39.3 \\ 3 \times & 39.3 & 39.3 & 39.3 & 39.3 & 39.3\end{array}$

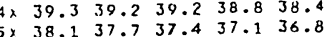

$\begin{array}{lllllll}6 \times & 36.6 & 36.4 & 36.0 & 35.5 & 34.9\end{array}$

$\begin{array}{lllllll}7 \times & 34.3 & 33.7 & 32.9 & 32.1 & 31.3 \\ 8 \times & 30.5 & 29.6 & 28.6 & 27.7 & 26.7 \\ 3 \times & 25.7 & 24.9 & 24.11 & 23.1 & 22.0\end{array}$

$0 \times 21.0$

$$
\text { Mid-East - Wet Condition }
$$

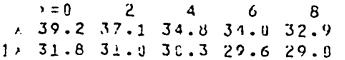

$\begin{array}{llllll}27 & 21.8 & 31.0 & 36.3 & 23.6 & 29.0 \\ 32 & 26.5 & 28.0 & 27.6 & 27.3 & 20.0\end{array}$

$4 x .23 .422 .8 \quad 22.2221 .821 .4$

6. 10.719 .519 .319 .118$.

$8 \times 17.916 .816 .6 \quad 16.4 \quad 16.2$ $30 \times 13.9$

PFRCENT TOTAL DISTANTE PFRCFNT TOTAL DISTANC.

$\begin{array}{ccccc}x=0 & 2 & 4 & 6 & 8 \\ x \quad 42.0 & 42^{2} .0 & 42.0 & 42.0 & 42^{2} .0\end{array}$

$1 \times 42.0 \quad 42.0 \quad 42.0 \quad 42.0 \quad 42.0$

$2 x \quad 42.042 .0 \quad 42.042 .042 .0$

$4 \times 42.042 .042 .042 .047 .0$

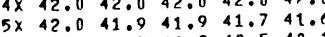

$\begin{array}{llllll}6 x & 41.4 & 41.3 & 4 n .9 & 40.5 & 4 n .1 \\ 7 \times & 39.6 & 39.1 & 38.5 & 37.7 & 37.0\end{array}$

$8 \times 35.934 .4 \quad 33.0 \quad 31.930 .8$

$9 \times 29.9$
$10 \times 26.0$

$$
\text { Mid-E }
$$

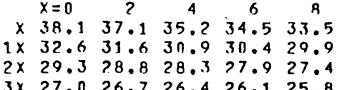
$\begin{array}{llllll}3 x & 27.0 & 26.7 & 26.4 & 36.1 & 25.8 \\ 4 x & 25.5 & 25.0 & 24.3 & 33.7 & 23.1\end{array}$

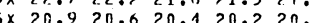

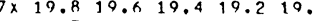
$8 x \quad 18.7 \quad 18.418 .1 \quad 1 \% .8 \quad 17$.

PERCENT TDTAI DISTANCE PE-East - Sand Condition

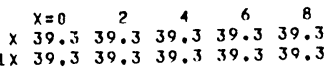

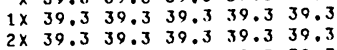

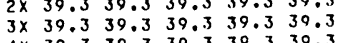
$\begin{array}{llllll}3 \times & 39.3 & 39.3 & 39.3 & 39.3 & 30.3\end{array}$ $5 \times 39.2 \quad 39.1 \quad 38.9 \quad 38.7 \quad 38.4$ $\begin{array}{llllll}6 \times & 38.1 & 37.8 & 37.3 & 36.6 & 36.0 \\ 7 \times & 35.4 & 34.7 & 34.0 & 33.1 & 32.2\end{array}$ $8 \times 31.1 \quad 29.5 \quad 28.727 .0 \quad 26.0$

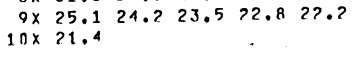

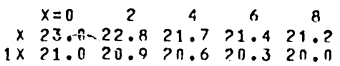
$\begin{array}{llllll}4 \times & 18.0 & 17.8 & 17.7 & 17.5 & 17.4\end{array}$ $\begin{array}{lllllll}5 x & 17.2 & 17.1 & 16.9 & 16.7 & 16.6\end{array}$ $\begin{array}{cccccc}6 x & 16.5 & 16.3 & 16.2 & 16.1 & 15.9 \\ 7 X & 15.7 & 15.6 & 15.4 & 15.2 & 15.0\end{array}$ $\begin{array}{llllll}8 \times & 14.9 & 14.7 & 14.6 & 14.4 & 14.1 \\ 9 \times & 13.7 & 13.4 & 13.0 & 12.6 & 10.2\end{array}$

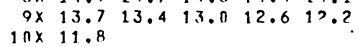
$\begin{array}{llllll}2 x & 19.8 & 19.5 & 19.3 & 19.1 & 18.9 \\ 3 x & 18.7 & 18.6 & 18.4 & 18.3 & 18.2\end{array}$

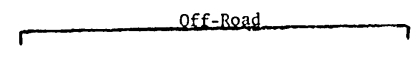

PERIENT TOIAL DISTIANCE

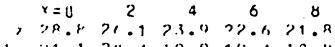

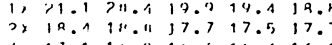

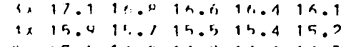

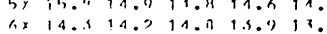

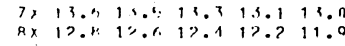
$\ln x \quad 1.0$

DERCEn fotal jistance

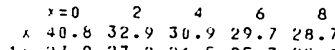

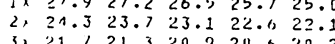
$\begin{array}{lllll}1 \times 19 . \% & 19.0 & 19.3 & 19.1 & 18.8\end{array}$ $\begin{array}{llllll}5 \times & 18.6 & 18.4 & 18.1 & 17.9 & 17.7 \\ 6 x & 17.5 & 17.4 & 17.2 & 77.0 & 176.0\end{array}$ $\begin{array}{llllll}7 \times & 16.6 & 16.4 & 16.2 & 16.0 & 15.7 \\ 8 \times & 15.5 & 15.3 & 15.1 & 14.9 & 14.7\end{array}$ $\begin{array}{llllll}7 \times & 14.4 & 13.9 & 6.9 & 2.9 & 1 .\end{array}$

PERCENT IMTAL DISTANGE $\begin{array}{cccccc}x=0 & 2 & 4 & 6 & 8 \\ x & 31.9 & 30.1 & 28.1 & 25.2 & 23.6 \\ 1 \times 2 & 22.3 & 21.5 & 20.9 & 20.0 & 10.3\end{array}$ $2 \times 18.7 \quad 18.3 \quad 18.0 \quad 17.6 \quad 17$. $\begin{array}{llllll}3 \times & 16.9 & 16.6 & 16.3 & 16.1 & 15.8 \\ 4 \times & 15.6 & 15.4 & 15.3 & 15.1 & 14.9\end{array}$

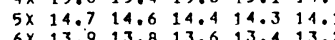
$7 \times 13.012 .8 \quad 12.6 \quad 12.4130 .2$ $\begin{array}{rrrrrr}7 x & 13.0 & 12.8 & 13.4 & 12.4 & .2 \\ 8 \times & 12.0 & 11.7 & 11.3 & 10.7 & 3.2 \\ 9 \times & 1.9 & 1.4 & 1.1 & 0.9 & 0.8\end{array}$ $\begin{array}{rr}9 \times & 1.9 \\ 10 x & 0.7\end{array}$

PERCENT total Distanct

$\begin{array}{rrrrrr}x=0 & 2 & 4 & 6 & 8 \\ x & 21.4 & 21.3 & 21.3 & 21.3 & 21.2\end{array}$ $\begin{array}{llllll}1 \times & 21.0 & 20.5 & 19.9 & 19.5 & 19.2 \\ 2 X & 18.9 & 18.6 & 18.4 & 18.1 & 17.2\end{array}$ $\begin{array}{llllll}2 \times & 18.9 & 18.6 & 18.4 & 18.1 & 17.8 \\ 3 \times & 17.6 & 17.3 & 17.0 & 16.8 & 16.6\end{array}$ $\begin{array}{llllll}4 \times & 16.4 & 16.1 & 15.9 & 15.7 & 15.5\end{array}$ $\begin{array}{cccccc}5 \times & 15.3 & 15.1 & 14.9 & 14.8 & 14.6 \\ 6 x & 14.4 & 14.2 & 14.0 & 13.8 & 13.5\end{array}$

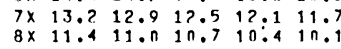

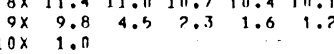




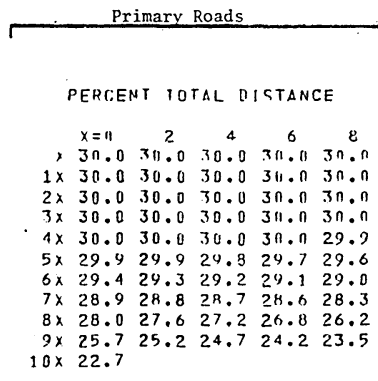

PERCENT TOtal nistance

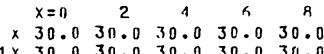
$3 \times 30.0 \quad 30.0 \quad 30.0 \quad 30.030$.

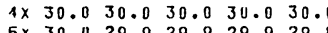
$6 \times 29.7 \quad 29.6 \quad 29.6 \quad 29.5$ $\begin{array}{lllll}7 \times 29.4 & 29.3 & 29.2 & 29.0 & 28.7\end{array}$

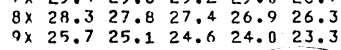
$9 \times 25.7$
$10 \times 22.5$

\section{PERCENT TOTAL OISTANCE}

$\begin{array}{cccccc}x=0 & 2 & 4 & 6 & 8 \\ x & 30.0 & 30.0 & 30.0 & 30.0 & 30\end{array}$ $\begin{array}{llllll}1 \times & 30.0 & 30.0 & 30.0 & 30.0 & 30.0 \\ ? \times & 30.0 & 30.0 & 30.0 & 30.0 & 30.0\end{array}$ $\begin{array}{llllll}3 \times & 30.0 & 30.0 & 30.0 & 30.0 & 30.0\end{array}$ $\begin{array}{cccccc}4 \times & 30.0 & 30.0 & 30.0 & 30.0 & 29.9 \\ 5 \times & 29.9 & 29.9 & 29.9 & 29.9 & 29.9\end{array}$

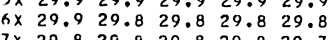

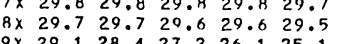
$9 \times 29.1$
$10 \times 24.2$

PERCENT TOTAL DISTANCE

$\begin{array}{rrrrrr} & x=0 & 2 & 4 & 6 & 8 \\ \times & 30.0 & 30.0 & 30.0 & 30.0 & 30.0 \\ x & 30.0 & 30.0 & 30.0 & 30.0 & 30.0\end{array}$ $2 \times 30.0 \quad 30.0 \quad 30.0 \quad 30.0 \quad 30.0$ $3 \times \quad 30.0 \quad 30.0 \quad 30.0 \quad 30.0 \quad 30.0$ $\begin{array}{ccccccc}4 \times & 30.0 & 30.0 & 30.0 & 30.0 & 29.9 \\ 5 \times & 29.9 & 29.9 & 20.0 & 29.9 & 29.9\end{array}$ $6 \times 29.8 \quad 29.829 .8 \quad 29.829 .8$

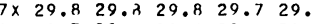

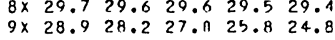
$9 \times 28.9$
$10 \times \quad 23.8$

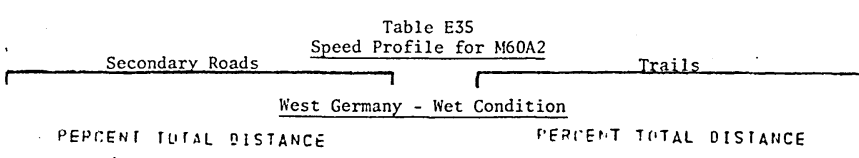

$\begin{array}{cccccc} & x=0 & 2 & 4 & 6 & 8\end{array} \times$ $\begin{array}{llllll}1 \times & 29.9 & 29.9 & 29.0 & 29.9 & 29.9 \\ 2 \times & 29.9 & 29.9 & 29.9 & 29.8 & 29.9\end{array}$ $\begin{array}{llllll}2 x & 29.9 & 24.9 & 29.9 & 29.8 & 29.8 \\ 3) & 29.7 & 29.7 & 29.6 & 29.5 & 29.4\end{array}$

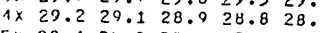
$\begin{array}{llllll}5 \times & 28.4 & 24.2 & 28.0 & 27.8 & 27.6 \\ 6 x & 27.4 & 27.2 & 27.0 & 26.8 & 26.6 \\ 6 \times & 26.4 & 28.2 & 25.9 & 25.7 & 25.4\end{array}$ $\begin{array}{llllll}7 x & 26.4 & 2 \pi .2 & 25.9 & 25.7 & 25.4 \\ 8 x & 25.1 & 24.8 & 24.5 & 24.1 & 23.7\end{array}$

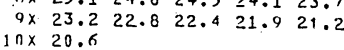

\section{West Germany - Snow Condition}

PERCENT TOTAL DISTANCE $\begin{array}{llll}x=11 & 2 & 4 & 6\end{array}$

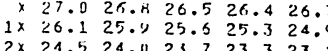
$\begin{array}{llllll}3 \times & 21.5 & 24.011 & 25.3 .7 & 23.3 & 23.9\end{array}$ $\begin{array}{llllll}4 \times & 21.8 & 21.5 & 21.5 & 21.3 & 21.1 \\ 5 x & 21.0 & 211.4 & 20.6 & 20.4 & 20 .\end{array}$ $6 \times 20.010 .020 .720 .4250$ $7 \times 19.018 .818 .618 .518 .0$

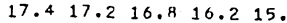

pertent IIITAL UISTANCE

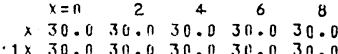
$\begin{array}{lllllll}2 \times & 30.0 & 36.0 & 30.0 & 30.11 & 30.0 \\ 3 \times & 30.0 & 30.0 & 29.9 & 29.9 & 29.9\end{array}$ $\begin{array}{llllll}4 \times & 29.9 & 29.8 & 29.7 & 29.6 & 29.5 \\ 5 \times & 29.4 & 29.2 & 29.7 & 29.0 & 20.4\end{array}$ 6x 28.628 .6 $\begin{array}{llllll}7 \times & 27.5 & 27.1 & 26.7 & 26.3 & 25.9 \\ 8 \times & 25.4 & 24.9 & 24.3 & 23.7 & 23.1\end{array}$

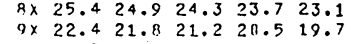
$17 \times 18.9$

$$
\text { Mid-E }
$$

$\begin{array}{ccccc}x=11 & 2 & 4 & 6 & 8 \\ \times .30 .0 & 311.0 & 29.9 & 29.9 & 29.9\end{array}$ $\begin{array}{llllll}1 \times & 29.9 & 29.9 & 29.9 & 29.8 & 29.9 \\ 2 \times & 29.5 & 29.2 & 28.9 & 28.7 & 28.7\end{array}$ $\begin{array}{llllll}3 \times & 28.1 & 27.8 & 27.3 & 26.1 & 26.3\end{array}$

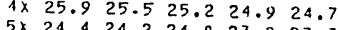
$\begin{array}{llllll}5 x & 23.5 & 23.3 & 23.1 & 23.9 & 23.7\end{array}$ $\begin{array}{lllllll}7 \times & 22.1 & 21.8 & 21.4 & 21.1 & 20.9\end{array}$ $\begin{array}{llllll}8 \times & 20.6 & 20.3 & 20.1 & 19.9 & 19.7 \\ 9 \times & 19.5 & 19.3 & 18.9 & 18.1 & 17.2\end{array}$ percent total distance tion

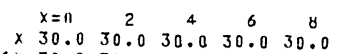
$\begin{array}{llllll}1 \times & 30.0 & 30.0 & 30.0 & 30.0 & 29.9 \\ 2 \times & 29.9 & 29.9 & 29.9 & 29.9 & 29.9\end{array}$ $\begin{array}{llllll}2 x & 29.9 & 29.9 & 29.9 & 29.9 & 29.9 \\ 3 \times & 29.9 & 29.9 & 29.9 & 29.9 & 29.9\end{array}$ $\begin{array}{lllll}4 \times 29.9 & 29.9 & 29.9 & 29.9 & 29.9\end{array}$ $\begin{array}{llllll}5 x & 29.9 & 29.9 & 29.9 & 29.9 & 29.9 \\ 6 x & 29.9 & 29.8 & 20.7 & 29.6 & 29.5 \\ 7 x & 20.5 & 29.4 & 29.5 & 29.6 & 29.5\end{array}$

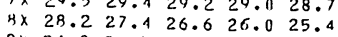

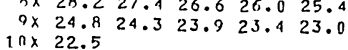
Mid-East - Sand Condition -

PERTENT TOTAL DISTANCE Pepcifnt total oistante

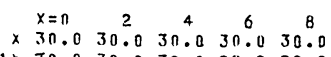
2x 29.920 .929 .929 .929 .9

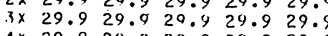
$\begin{array}{llllll}4 \times & 29.9 & 29.9 & 29.9 & 29.9 & 29.9 \\ 5 \times & 29.9 & 29.9 & 29.9 & 29.8 & 29.7\end{array}$

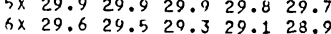
$\begin{array}{llllll}7 \times & 28.7 & 24.4 & 28.0 & 27.5 & 27.0\end{array}$ $\begin{array}{rrrrrr}8 \times & 26.3 & 25.3 & 24.4 & 23.6 & 22.8 \\ 9 \times & 22.2 & 21.6 & 21.1 & 20.6 & 20.1\end{array}$ $\begin{array}{lllll}x=0 & 2 & 4 & 6 \\ x & 17.0 & 8 & 0\end{array}$ $1 \times 16.816 .8$ 16.9 $16 . \mathrm{H} \quad 16.9$ $\begin{array}{llllll}3 x & 16.4 & 16.7 & 16.6 & 16.5 & 16.9 \\ 4 x & 16.0 & 15.2 & 16.2 & 16.2 & 16 .\end{array}$ $\begin{array}{llllll}4 \times & 16.0 & 15.9 & 15.9 & 15.4 & 15.9 \\ 5 x & 15.6 & 15.5 & 15.4 & 15.3 & 15\end{array}$ $\begin{array}{lllllll}6 x & 15.2 & 15.1 & 15.0 & 14.9 & 14.0 \\ 7 x & 14.8 & 14.7 & 14.6 & 14.5 & 14.4\end{array}$

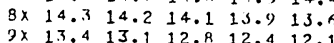
$9 \times 13.4 \quad 13.214 .1215 .412 .1$

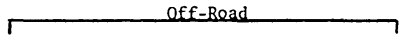

PERTENT TOTAL OISTANTE

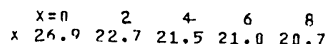
$\begin{array}{llllll}1 \times & 20.2 & 19.7 & 19.3 & 19.0 & 18.7 \\ 2 x & 18.3 & 17.9 & 17.6 & 17.4 & 17.2\end{array}$ $\begin{array}{llllll}3 x & 17.0 & 16.8 & 16.5 & 16.3 & 16.0 \\ 4 \times & 15.8 & 15.6 & 15.4 & 15.1 & 16.0\end{array}$

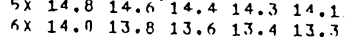

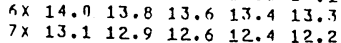

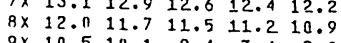

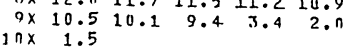

PERCENT total UISTANCE

$\begin{array}{rlcccc} & x=11 & 2 & 4 & 6 & \\ \times & 29.9 & 29.6 & 29.0 & 28.7 & 28.3\end{array}$ $\begin{array}{llllll}1 \times & 26.0 & 25.5 & 25.25 & 24.7 & 24.4\end{array}$ $\begin{array}{llllll}3 \times & 23.9 & 23.5 & 23.0 & 22.5 & 24.4 \\ 5 \times & 21.9 & 21.5 & 0\end{array}$ $\begin{array}{llllll}4 x & 21.7 & 21.3 & 20.9 & 20.5 & 20.2 \\ 5 \times & 19.9 & 19.5 & 19.5 & 10.9 & 19.6\end{array}$ $6 x \quad 18.3 \quad 18.019 .717 .913 .6$

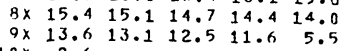

PERCENT TOTAL DISTHNGE

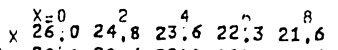

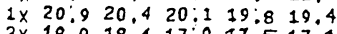

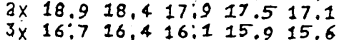

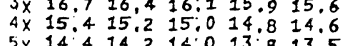
$5 \times 14: 4 \quad 14,2 \quad 14: 0 \quad 13: 8 \quad 13.5$

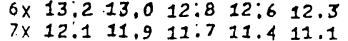

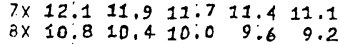
$9 \times \quad 8.7 \quad 4.0 \quad 2 ; 2 \quad 1: 5 \quad 1.2$

PFRCENT TCTAL DISTANCE $\begin{array}{rlcccc} & x=0 & 2 & 4 & 6 & 8 \\ x & 17: 0 & 16,9 & 16: 7 & 16: 7 & 8 \\ 1 \times & 16,3 & 16,2 & 16: 0 & 15: 9 & 16.6\end{array}$

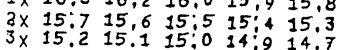

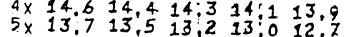

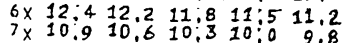
$\begin{array}{rrrrrr}8 \times & 9.5 & 9.3 & 9: 0 & 8: 8 & 8.5 \\ 9 \times & 8: 3 & 8.0 & 7 ; 7 & 7 \vdots 3 & 7.5 \\ 10 \times & 5.1 & & \end{array}$ 


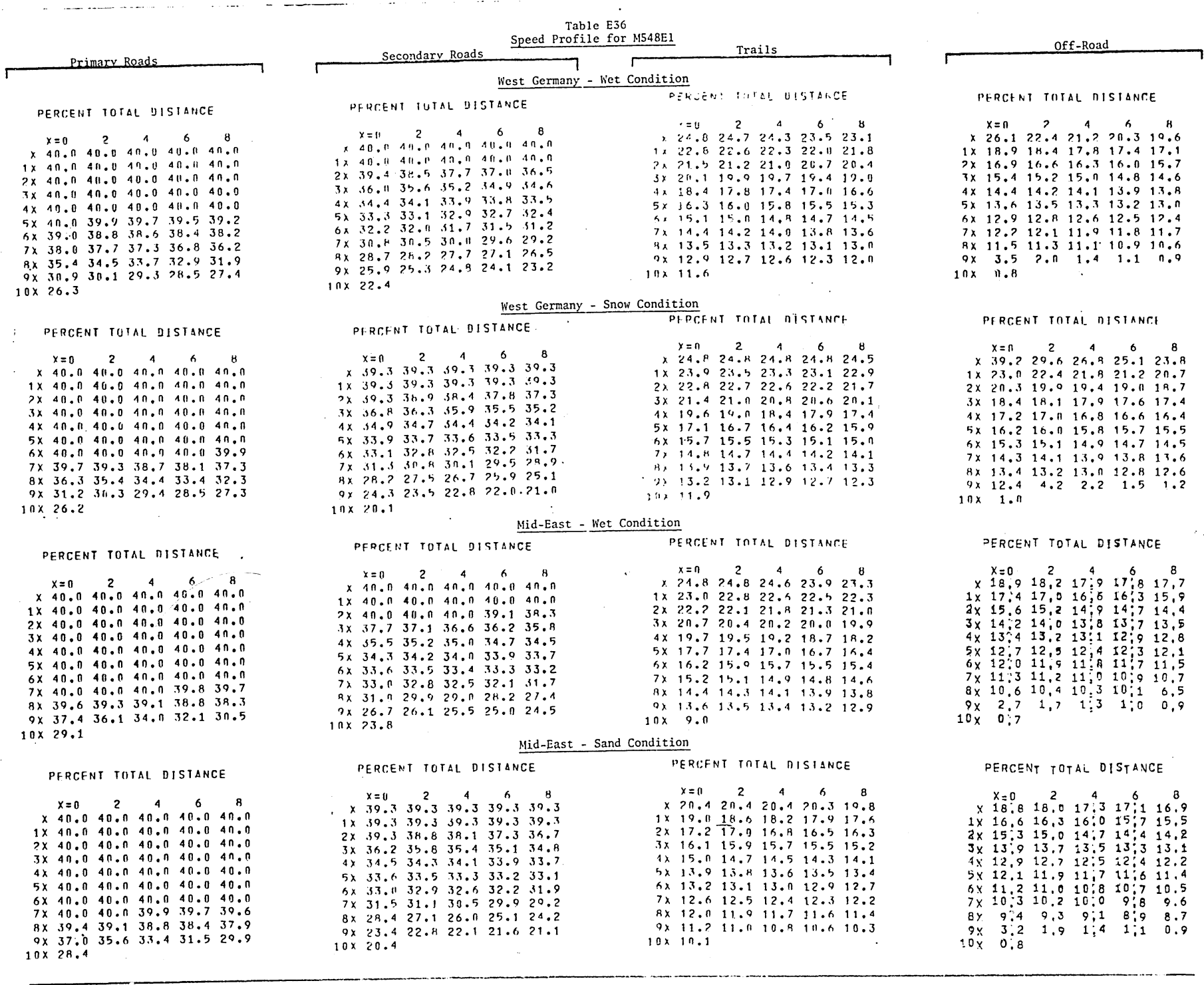




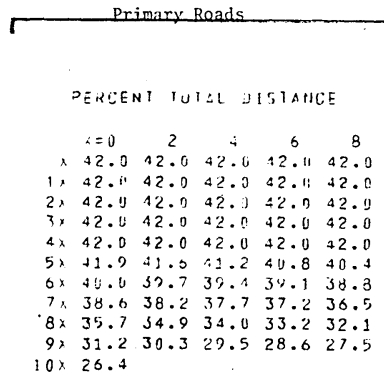

PERCENT TOTAL DISTANCE

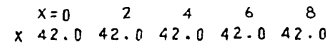

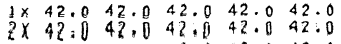

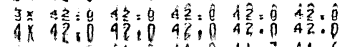

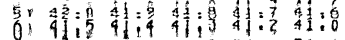

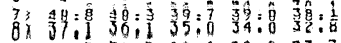

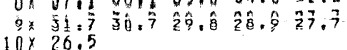

PEETCENT TOTAL FIETANFE

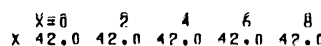

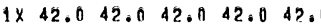
$\begin{array}{llllll}2 x & 42.0 & 42.0 & 42.0 & 42.0 & 42.0 \\ 3 x & 42.0 & 42.0 & 42.0 & 42.0 & 40.0\end{array}$ $\begin{array}{llllll}4 \times & 42.0 & 12.0 & 42.0 & 42.0 & 42.0 \\ 5 \times & 42.0 & 42.0 & 42.0 & 42.0 & 42.0\end{array}$

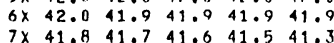
$8 \times \quad 41.140 .840 .5 \quad 40.130 .6$ $9 \times$
$9 \times 38.6$
$10 \times \quad 29.7$

PERCENT TOTAL DISTANTE

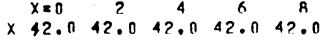
$\begin{array}{llllll}1 \times & 42.0 & 42.0 & 42.0 & 42.0 & 42.0 \\ 2 x & 42.0 & 42.0 & 42.0 & 42.0 & 42\end{array}$ $\begin{array}{llllll}3 x & 42.0 & 42.0 & 42.0 & 42.0 & 47.0\end{array}$ $\begin{array}{lllllll}4 \times & 42.0 & 42.0 & 42.0 & 42.0 & 42.0\end{array}$ $\begin{array}{llllll}5 \times & 42.0 & 42.0 & 42.0 & 42.0 & 42.0 \\ 6 & 42.0 & 41.0 & 41.0 & 41.0 & 41\end{array}$ $8 \times 40.9 \quad 40.6 \quad 40.2 \quad 39.8 \quad 39$.

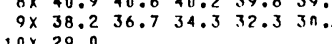

Table E37
Speed Profile for M577A1

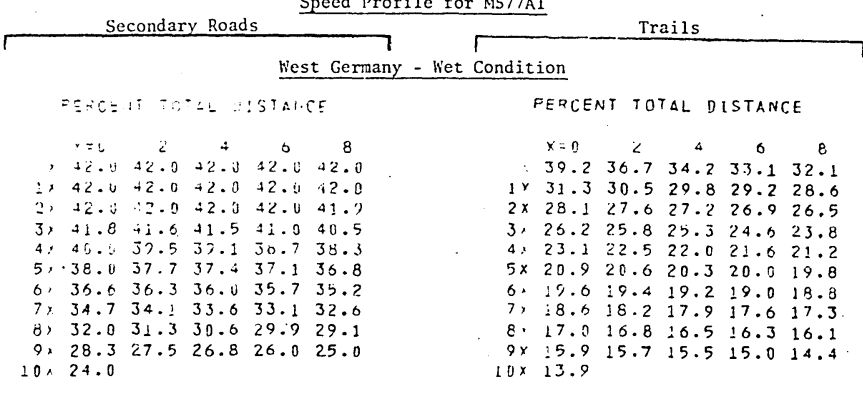

\section{Nest Germany - Snow Conditio}

PERCENT TOTA! BISTANCE PERCENT TOTAL DISTANCE

$\begin{array}{llllll}x=0 & 2 & 4 & 6 & 8 \\ 30.3 & 39.3 & 39.3 & 39.3 & 39.3\end{array}$

$\begin{array}{llllll}1 & 39.3 & 39.3 & 39.3 & 39.3 & 39.5 \\ 2 \times & 39.3 & 39.3 & 39.3 & 39.3 & 39.5\end{array}$

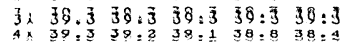

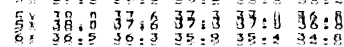

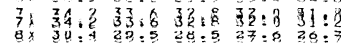

8) $35: 334: 8$ 24:0 $33: 1$ 21:0

Mid=East = Het Conditition

$\begin{array}{ccccc}x=1 & 2 & 4 & 5 & 8 \\ 41.2 & 41.2 & 41.0 & 38.7 & 37.2\end{array}$ 1) 36.435 .8 35.3 34.733 .8 3: 20:5 20:0 $28: 5$ : $27: 726: 0$

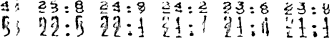

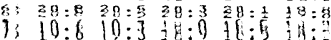

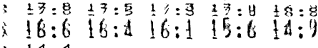
PEREFN TOTAL DISTANGE

PERGENT TUTAL DISTANCE

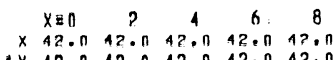

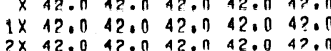

$\begin{array}{llllll}2 x & 42.0 & 42.0 & 42.0 & 42.0 & 47.0 \\ 3 x & 42.0 & 42.0 & 42,0 & 42.0 & 12.0\end{array}$

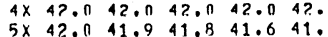

$6 \times 41.040 .740 .3 \quad 39.939 .5$

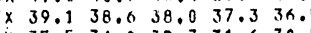

$9 \times 29.6 \quad 28.828 .0 \quad 27.3 \quad 26.7$

$10 \times 25.9$ Mid-East - Sand Condition -

PERCENT TOTAL DISTANCE

\author{
$x=0$
$x$
}

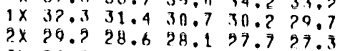

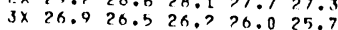

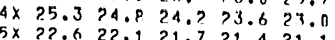

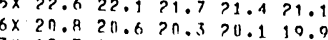

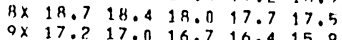
$\begin{array}{llllll}9 x & 17.2 & 17.0 & 16.7 & 16.4 & 15.9\end{array}$ PERCENT TIITAL DISTANC,F

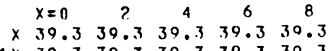
$\begin{array}{lllllll}2 \times & 39.3 & 39.3 & 39.3 & 39.3 & 39.3\end{array}$ $\begin{array}{llllll}3 \times & 39.3 & 39.3 & 39.3 & 39.3 & 30.3\end{array}$ $\begin{array}{llllll}4 \times & 39.3 & 39.3 & 39.3 & 39.3 & 39.3 \\ 5 \times & 39.1 & 38.7 & 38.5 & 38.2 & 37.9\end{array}$

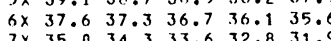
$\begin{array}{llllll}7 \times & 35.0 & 34.3 & 33.6 & 32.8 & 31.9 \\ 8 \times & 30.8 & 20.3 & 28.0 & 26.8 & 25.8\end{array}$ $\begin{array}{lllllll}8 \times & 30.8 & 29.3 & 2 \mathrm{R} .0 & 26.8 & 25.8 \\ 9 \times & 24.9 & 24.9 & 23.4 & 72.7 & 20.1\end{array}$ $10 \times 21.3$

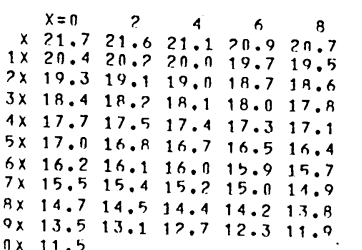

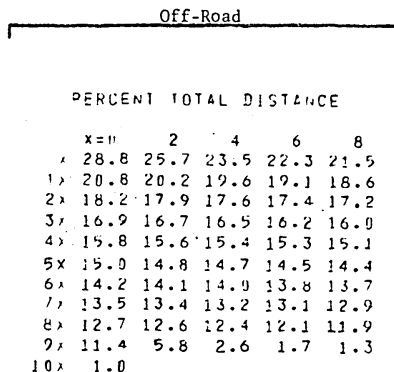

- perlent tidLl nistance

$\begin{array}{cccccc} & x=0 & 2 & 4 & 6 & 8 \\ 1, & 40.8 & 32.3 & 30.5 & 29.4 & 28.4 \\ 12 & 27.6 & 27.0 & 20.3 & 25.5 & 24.8\end{array}$ $\begin{array}{llllll}1 \times & 27.6 & 27.0 & 20.3 & 25.5 & 24.8 \\ 2 & 24.2 & 23.6 & 23.0 & 22.5 & 22.0 \\ 3 & 21.0 & 23.6 & 23.0 & 22.0\end{array}$

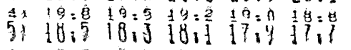

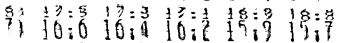

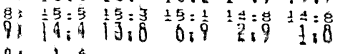

PERGENT TOTAL DISTANGE

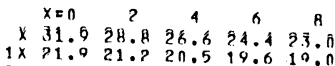
$2 \times 18.418 .0 \quad 17.7 \quad 17.417$. $4 \times \quad 15.5 \quad 15.315 .2$ 15.0 140.

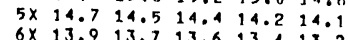
$\begin{array}{llllll}6 \times & 13.9 & 13.7 & 13.6 & 13.4 & 13.2 \\ 7 \times & 13.0 & 12.8 & 12.6 & 12.4 & 13.2\end{array}$

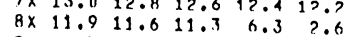
$\begin{array}{rrrrrr}8 x & 11.9 & 11.6 & 11.3 & 6.3 & 2.6 \\ 9 x & 1.7 & 1.2 & 1.0 & 0.8 & 0.7 \\ 10 x & 0.7 & & & & \end{array}$

PERCENT TOTAL DISTANCE $\begin{array}{rrrrrr} & x=0 & 2 & 4 & 6 & 8 \\ \times 20.6 & 20.5 & 20.4 & 20.4 & 20.3 \\ 1 \times 20.0 & 20.0 & 19.6 & 10.2 & 18.9 & 10.6\end{array}$ $2 \times 18,318.619 .218 .918$. $\begin{array}{lllllll}3 \times & 17.2 & 16.9 & 16.7 & 16.4 & 16.2\end{array}$ $\begin{array}{cccccc}4 \times & 16.0 & 15.8 & 15.5 & 15.4 & 15.2\end{array}$ $\begin{array}{llllll}5 \times & 15.1 & 14.9 & 14.7 & 14.5 & 14.3 \\ 6 x & 14.1 & 14.0 & 143.7 & 13.5 & 14.3\end{array}$

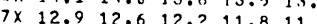
$\begin{array}{rrrrrr}8 x & 11.1 & 10.7 & 10.4 & 0.8 & 0.4 \\ 9 x & 7.1 & 2.8 & 1.8 & 1.3 & 1.7 \\ 10 x & 0.9 & & & & \end{array}$ 


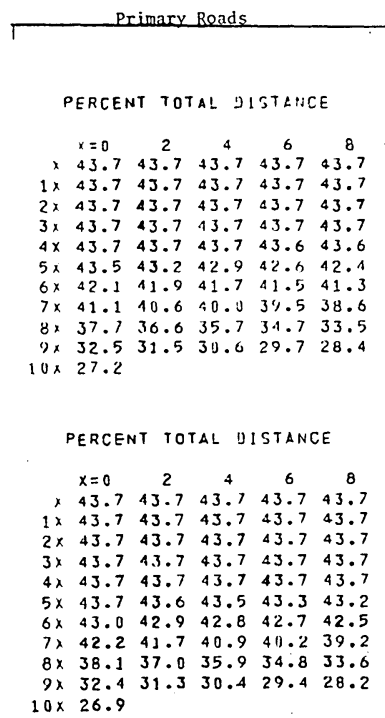

PERCENT TOTAL DISTANCE

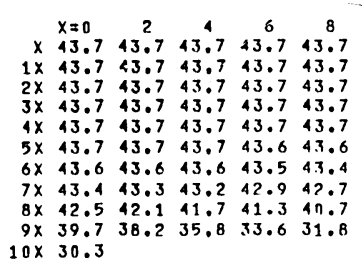

PERCENT total Distance

$\begin{array}{cccccc}x=0 & 2 & 4 & 6 & 8 \\ x & 43.7 & 43.7 & 43.7 & 43.7 & 43.7\end{array}$

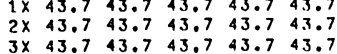
$\begin{array}{llllll}3 x & 43.7 & 43.7 & 43.7 & 43.7 & 43.7 \\ 4 x & 43.7 & 43.7 & 43.7 & 43.7 & 43.7\end{array}$

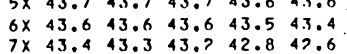
$\begin{array}{llllll}7 \times & 43.6 & 43.6 & 43.6 & 43.5 & 43.6 \\ 8 \times & 42.4 & 43.3 & 43.7 & 42.8 & 47.6 \\ 8 \times & 42.9 & 41.4 & 41.0 & 4 n .3\end{array}$ $9 \times 39.2 \quad 37.6 \quad 35.2 \quad 33.0 \quad 31.2$

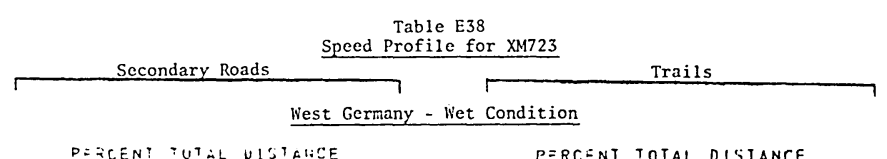

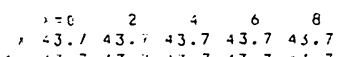 \\ $\begin{array}{llllll}1 \times & 43.7 & 43.7 & 43.7 & 43.7 & 43.7 \\ 2, & 43.7 & 43.7 & 13.7 & 43.6 & 43.5\end{array}$ \\ 3. 43.343 .143 .942 .742 .3

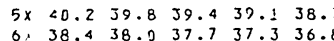 \\ $\begin{array}{lllllll}7 . & 36.3 & 35.7 & 35.1 & 34.5 & 33.8 \\ \text { 8. } & 33.2 & 32.4 & 31.6 & 30.8 & 30.0\end{array}$ \\ 8. 33.2
$9: 29.1$
10.24 .6}

\section{Hest Ger}

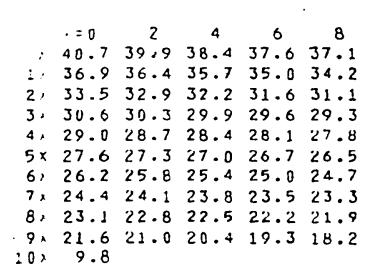

PERCENT TOTAL UISTAMCE

DERCEN TUTAL DISTARCE

$\begin{array}{lcccc}x=0 & 2 & 4 & 6 & 8 \\ 39.3 & 39.3 & 39.3 & 39.3 & 39.3\end{array}$

$\begin{array}{llllll}1 \times & 39.3 & 39.3 & 39.3 & 39.3 & 39.3 \\ 2) & 39.3 & 39.3 & 39.3 & 39.3 & 39.3\end{array}$

$\begin{array}{llllll}27 & 39.3 & 39.3 & 39.3 & 39.3 & 39.3 \\ 3 \times & 39.3 & 39.3 & 39.3 & 39.3 & 39.3\end{array}$

$\begin{array}{llllll}47 & 39.3 & 39.2 & 39.1 & 38.9 & 38.7 \\ 52 & 38.5 & 38.3 & 38.1 & 37.9 & 37.6\end{array}$

$\begin{array}{llllll}6 \times & 37.4 & 37.1 & 36.7 & 30.2 & 35.0 \\ 7 \times & 35.0 & 34.3 & 33.5 & 32.7 & 31.8\end{array}$

$\begin{array}{llllll}8 \times & 31.0 & 30.0 & 29.0 & 28.1 & 27.1 \\ 9 \times & 26.0 & 25.1 & 24.3 & 23.3 & 22.2\end{array}$

\section{Mid-}

PERCENT TOTAL DISTANCE

$\begin{array}{rccccc} & x=0 & 2 & 4 & 6 & 8 \\ x & 43.7 & 43.7 & 43.7 & 43.7 & 43.7 \\ 1 \times & 43.7 & 43.7 & 43.7 & 43.7 & 43.7\end{array}$

$2 x \quad 43.7 \quad 43.7 \quad 43.7 \quad 43.7 \quad 43.7$

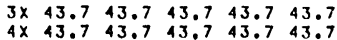

$\begin{array}{llllll}5 \times & 43.6 & 43.5 & 43.4 & 43.2 & 43.0 \\ 6 \times & 42.8 & 42.6 & 42.1 & 41.6 & 41.2 \\ 7 \times & 40.7 & 40.1 & 39.4 & 38.6 & 37.8\end{array}$

$\begin{array}{llllll}8 \times & 36.7 & 35.1 & 33.6 & 32.4 & 31.3 \\ 9 \times & 30.3 & 29.4 & 28.6 & 77.9 & 27.2\end{array}$

$10 \times 26.3$

Mid-East

PERCENT TMTAL DISTANCE

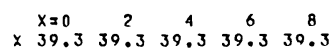

$\begin{array}{llllll}1 \times & 39.3 & 39.3 & 39.3 & 39.3 & 39.3 \\ 2 \times & 39.3 & 39.3 & 39.3 & 39.3 & 39.3\end{array}$

$\begin{array}{llllll}2 \times & 39.3 & 39.3 & 39.3 & 39.3 & 39.3 \\ 3 \times & 39.3 & 39.3 & 39.3 & 39.3 & 39.3\end{array}$

$\begin{array}{llllll}3 \times & 39.3 & 39.3 & 39.3 & 39.3 & 39.3 \\ 4 \times & 39.3 & 39.3 & 39.3 & 39.3 & 39.3\end{array}$

$6 \times 38.0 \quad 37.8 \quad 37.2 \quad 36.5 \quad 36.0$

$7 \times 35.434 .733 .9 \quad 33.0 \quad 37.2$

$\begin{array}{llllll}8 \times & 31.1 & 29.5 & 28.2 & 27.0 & 25.0 \\ 9 \times & 25.0 & 24.2 & 23.5 & 22.8 & 22.2\end{array}$

$10 \times 21.4$

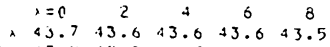

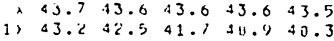

2) $39.8 \quad 39.4 \quad 39.0 \quad 38.0 \quad 38.2$

$4,33.8 \quad 33.3 \quad 32.9 \quad 32.5 \quad 32.1$

$\begin{array}{llllll}5 . & 31.8 & 31.5 & 31.2 & 31.0 & 30.7 \\ 31 & 30.4 & 30.0 & 29.5 & 20.0 & 28.4\end{array}$

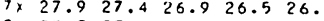

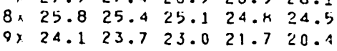

$\begin{array}{llllll} & x=0 \\ x & 40.6 & 40.6 & 40.5 & 39.6 & 38 \\ x & 30.6\end{array}$

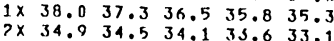

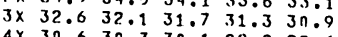

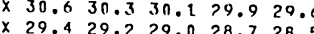

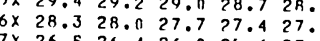

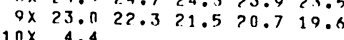
condition

PERCENT TMTAL DISTANCE

$\begin{array}{rrrrrr} & x=0 & 2 & 4 & 6 & 8 \\ \times & 22.6 & 22.6 & 22.6 & 22.6 & 22.5 \\ 1 \times & 22.4 & 22.04 & 72.3 & 72.3 & 22.3\end{array}$

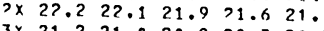

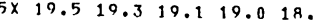
$\begin{array}{llllll}6 x & 18.7 & 18.6 & 18.4 & 18.3 & 18.0 \\ 7 \times & 17.9 & 17.8 & 17.6 & 17.4 & 17.2 \\ 9 \times & 7 . & 17.9 & 6.9 & 564 & 16.2\end{array}$

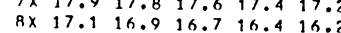

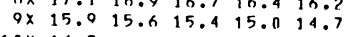

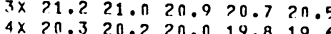

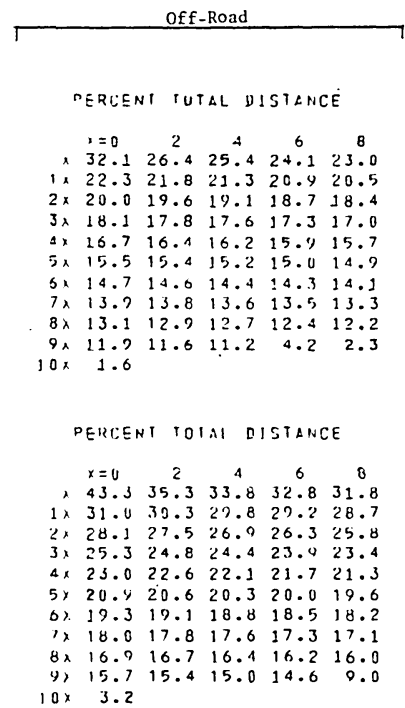

PERGent total Distance

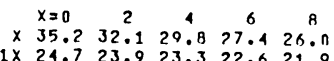
$\begin{array}{llllll}1 \times & 24.7 & 23.9 & 23.3 & 72.6 & 21.9 \\ 2 \times & 21.4 & 21.0 & 20.6 & 20.0 & 10.6 \\ 3 \times & 19.1 & 18.5 & 10.1 & 17.7 & 10.4\end{array}$

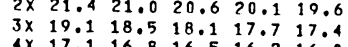
$\begin{array}{llllll}5 \times & 15.8 & 15.5 & 15.3 & 15.1 & 14.9\end{array}$ $\begin{array}{llllll}6 \times & 14.7 & 14.5 & 14.3 & 14.1 & 13.9 \\ 7 \times & 13.7 & 13.5 & 13.4 & 13.2 & 13\end{array}$

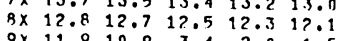
$9 \times 11.9 \quad 10.9 \quad 3.4 \quad 2.0 \quad 1.5$ $10 \times 1.1$

PFREENT TOTAL DISTANC.F

$\begin{array}{cccccc}x=0 & 2 & 2 & 4 & 6 & 8 \\ x & 22.6 & 22.5 & 22.3 & 22.3 & 21.9\end{array}$

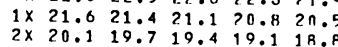
$\begin{array}{lllllll}3 \times & 18.5 & 18.2 & 17.9 & 17.7 & 17.5\end{array}$ $5 \times 16.015 .8 \quad 15.6 \quad 15.315 .0$ 6x $14.9 \quad 14.7 \quad 14.5 \quad 14.3 \quad 14.9$

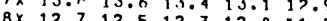

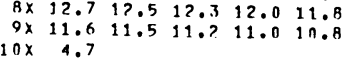




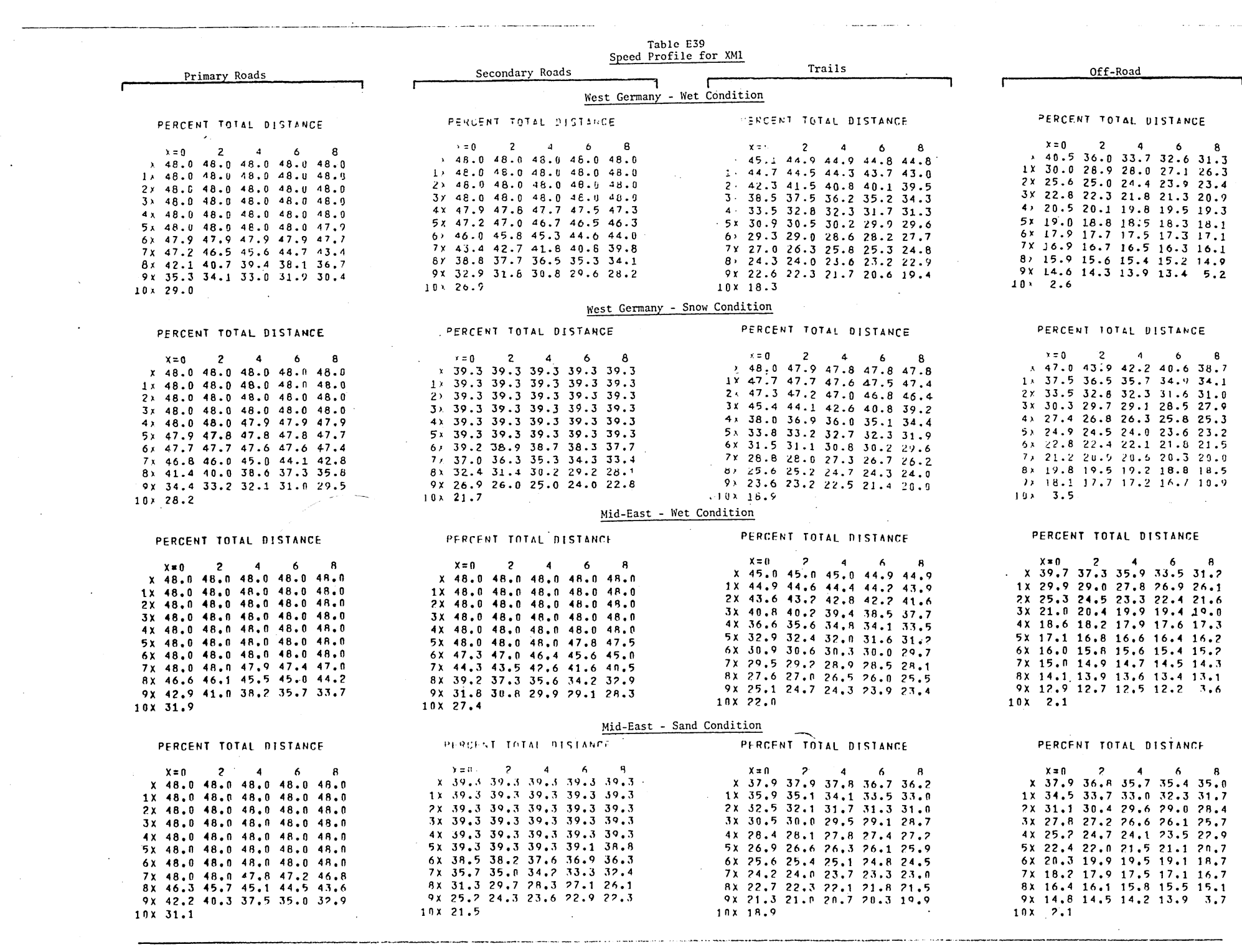


Table E40
Speed Profile for ASV

$\sqrt[\text { Secondary Roads }]{\frac{\text { Speed Profile for ASV }}{\text { West Germany - Wet Condition }} \text { Trails }}$

PERTERT TITAL DISTANCE

$\begin{array}{rrrrrr} & x=11 & 2 & 4 & 6 & 8 \\ x & 43.7 & 43.7 & 43.7 & 43.7 & 43.7 \\ x & 43.7 & 43.7 & 43.7 & 43.7 & 43.7\end{array}$

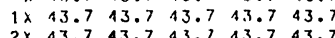

$\begin{array}{llllll}2 x & 43.7 & 43.7 & 43.7 & 43.7 & 43.7 \\ 3 x & 43.7 & 43.7 & 43.7 & 43.7 & 43.7\end{array}$

$5 \times \quad 42.9 \quad 42.5 \quad 42.2 \quad 41.7 \quad 41$.

$\begin{array}{llllll}7 \times & 39.4 & 39.1 & 38.5 & 38.0 & 37.2 \\ 8 \times & 36.4 & 35.5 & 34.6 & 33.7 & 32\end{array}$

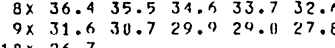

PERCENT total Distance

$\begin{array}{llllcc} & x=0 & 2 & 4 & 6 & 8 \\ x & 43.7 & 43.7 & 43.7 & 43.7 & 43.7\end{array}$

$\begin{array}{llllll}1 x & 43.7 & 43.7 & 43.7 & 43.7 & 43.7 \\ 2 x & 43.7 & 43.7 & 43.7 & 43.7 & 43.7 \\ 3 x & 43.7 & 43.7 & 43.7 & 43.7 & 43.7\end{array}$

3.7 43.7

$\begin{array}{llllll}5 \times & 43.6 & 43.3 & 43.0 & 42.7 & 42.4\end{array}$

$\begin{array}{llllll}5 \times & 42.1 & 41.9 & 41.6 & 11.4 & 41.2\end{array}$

$\begin{array}{llllll}7 \times & 40.9 & 40.4 & 39.8 & 39.1 & 38.2 \\ 8 \times & 37.2 & 36.1 & 35.1 & 34.1 & 32.9\end{array}$

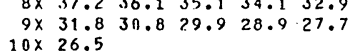

PERCENT TOTAL DISTANCE

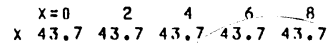

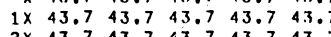

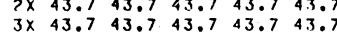

$\begin{array}{llllllll}4 \times & 43.7 & 43.7 & 43.7 & 43.6 & 43.6\end{array}$

$\begin{array}{lllllll}5 x & 43.6 & 43.4 & 43.3 & 43.2 & 43.0 \\ 6 x & 42.9 & 42.8 & 42.7 & 42.2 & 42.0\end{array}$

$\begin{array}{lllllll}7 \times & 12.2 & 42.0 & 41.9 & 11.7 & 41.5 \\ 8 \times & 41.3 & 41.0 & 40.6 & 40.2 & 39.7 \\ 9 x & 38.7 & 37.3 & 35.0 & 33.0 & 31.2\end{array}$ $9 \times 38.7$
$10 \times 29.8$

PERCENT TOTAL DISTANCE

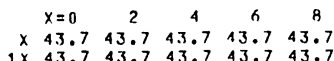

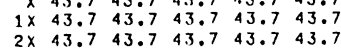

$\begin{array}{llllll}3 \times & 43.7 & 43.7 & 43.7 & 43.7 & 43.7\end{array}$

$\begin{array}{llll}4 \times 43.743 .7 & 43.7 & 43.6 & 43.6\end{array}$

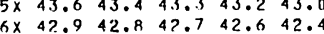

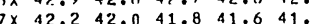

$\begin{array}{lllllll}8 \times & 41.1 & 40.8 & 40.3 & 39.9 & 39.3 \\ 9 \times & 38 & 30.3 & 36.8 & 34.4 & 32.4 & 30.6\end{array}$ $\begin{array}{r}9 \times \\ 10 \times \quad 38.3 \\ \hline\end{array}$
Diont total nicisant

$\begin{array}{rrrrrr} & x=0 & 2 & 4 & 5 & 8 \\ x & 13.7 & 43.7 & 13.7 & 4.6 .7 & 13.7 \\ 1 \times & 43.7 & 43.7 & 43.7 & 4.3 .1 & 43.7\end{array}$

$\begin{array}{llllll}1 x & 43.7 & 43.7 & 43.7 & 4.4 .7 & 43.7 \\ 2 x & 43.61 & 43.5 & 43.3 & 43.01 & 42.8 \\ 3 x & 22.3 & 41.0 & 41.5 & 41.1 & 40.6\end{array}$

$\begin{array}{llllll}3 x & 42.3 & 41.041 .5 & 41.7 & 0.0 \\ 4 x & 40,1 & 31.7 & 39.2 & 38.7 & 38.0 \\ 5 x & 37.4 & 37.3 & 36.9 & 36.5 & 36.0\end{array}$

$\begin{array}{llllll}5 x & 37.4 & 37.3 & 36.9 & 36.3 & 36.0 \\ 6 x & 35.6 & 35.2 & 34.8 & 34.4 & 33.9\end{array}$

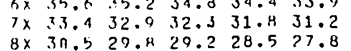

$\begin{array}{llllll}8 \times & 3 \pi .5 & 29.8 & 29.2 & 21.4 & \\ 9 \times & 27.1 & 26.4 & 25.8 & 25.8 & 21.4\end{array}$

$111 \times 23.2$

West Germ

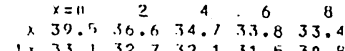

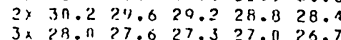

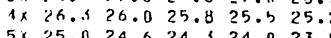

$6 \times 23.523 .2 \quad 23.0$

7) 22.3 $72.1121 .821 .7 \quad 21$.

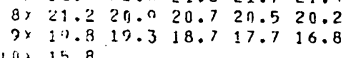

PERTENT TOTAL DISTINAE

$\begin{array}{rrrrrr} & x=0 & 2 & 4 & 6 & 8 \\ x & 39.3 & 39.3 & 34.3 & 39.3 & 39.3 \\ 1 \times & 39.3 & 39.3 & 39.3 & 39.3 & 39.3 \\ 2 x & 39.3 & 39.3 & 30.3 & 34.3 & 39.3\end{array}$

$\begin{array}{llllll}2 \times & 39.3 & 39.3 & 30.3 & 34.3 & 39.3 \\ 3 x & 39.3 & 39.3 & 39.3 & 30.2 & 39.0\end{array}$

$4 \times 38.8 \quad 38.5 \quad 38.3 \quad 37.9 \quad 37.6$

$\begin{array}{cccccc}5 \times & 37.3 & 37.1 & 36.8 & 36.5 & 36.2\end{array}$

$\begin{array}{llllll}6 x & 35.8 & 35.5 & 35.0 & 34.5 & 33.9 \\ 7 \times & 33.3 & 32.8 & 31.8 & 31.1 & 30.3\end{array}$

$8 \times 29.628 .727 .8 \quad 27.1126 .05$

$9 \times$
$9 \times 25.1$
$10 \times 20.6$

(4x 15.8

Mnr.t

PERCENT TIITA DISIANTE

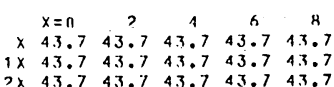

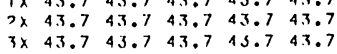

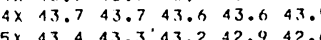

$\begin{array}{llllll}7 x & 4 n .1 & 31.0 & 38.9 & 38.1 & 37.3 \\ 8 x & 36.2 & 34.7 & 3.3 .3 & 37.1 & 31.0\end{array}$

Mid-East - Sand Condition

PHRCFN T TIITAL MISIANCE

$\begin{array}{rrrrrr} & x=0 & 2 & & & \\ x & 39.3 & 39.3 & 30.3 & 39.3 & 8 \\ 1 \times & 39.3 & 39.3 & 39.3 & 39.3 & 39.3 \\ 2 x & 39.3 & 39.3\end{array}$

$\begin{array}{lllllll}2 \times & 39.3 & 35.0 & 39.3 & 39.3 & 39.3 \\ 3 \times & 39.3 & 39 . & 39.3 & 39.3 & 39.3\end{array}$

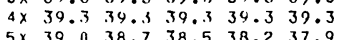

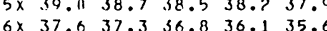

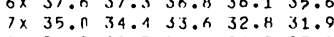

$\begin{array}{llllll}8 \times & 3 n .8 & 29.3 & 74.0 & 26.8 & 25.8 \\ 9 x & 24.0 & 24.1 & 23.4 & 22.7 & 22.1\end{array}$ $111 \times 21.3$

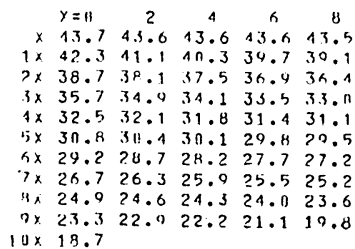

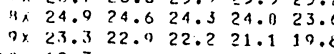

PIREFN TATAL DICIANCE

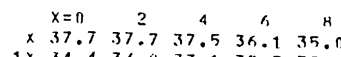

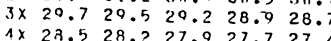
$5 \times 27.220 .227 .827 .727 .4$ $\begin{array}{llllll}6 x & 26.1 & 25.8 & 25.5 & 25.2 & 21.9\end{array}$ $6 x$
$2 x$ 23.2522 .2523 .923 .723 .4

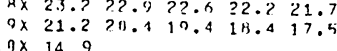

PFRCEN TOTAL DISTANCE $2 \times 17.5 \quad 17.4 \quad 17.3 \quad 17.116$.

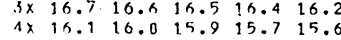
$5 \times 15.415 .3 \quad 15.215 .014 .0$ $6 \times 14.814 .7 \quad 14.614 .514$. $\begin{array}{llllllll}7 \times & 14.3 & 14.2 & 14.2 & 14.0 & 13.9 \\ 8 \times & 13.8 & 13.7 & 13.6 & 13.4 & 13.9 \\ 9 x & 13.9 & 12.7 & 12.4 & 12.4 & 11.9\end{array}$ $\begin{array}{llllll}8 x & 13.8 & 13.7 & 13.6 & 13.4 & 13.2 \\ 2 x & 13.0 & 12.7 & 12.4 & 12.1 & 11.9\end{array}$ $10 \times 11.3$ $\begin{array}{llllll}1 \times & 31.4 & 3.4 .8 & 33.1 & 32.5 & 32.0 \\ 2 x & 31.6 & 31.2 & 30.9 & 30.5 & 30\end{array}$

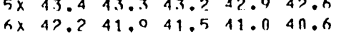

$9 \times 130.029 .228 .477 .727 .0$

$\begin{array}{rlrrrrr} & x=0 & 2 & 4 & 6 & 8 \\ x & 17.7 & 17.7 & 17.7 & 17.7 & 17.7 \\ 1 \times & 17.7 & 17.7 & 17.77 & 17.6 & 17.5\end{array}$

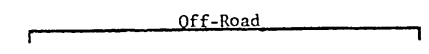

PERCENT TOTAL DISTANCE

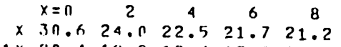
$2 x \quad 18.2 \quad 17.8 \quad 17.6 \quad 17.3 \quad 17.1$ $\begin{array}{llllll}3 \times & 16.9 & 16.6 & 16.0 & 16.1 & 15.9 \\ 4 \lambda & 15.6 & 15.4 & 15.2 & 15.1 & 14.9\end{array}$ $\begin{array}{llllll}7 x & 13.3 & 13.1 & 33.7 & 13.5 & 13.4 \\ 8 x & 12.5 & 12.3 & 13.0 & 12.9 & 12.7\end{array}$ $\begin{array}{rrrrrrr}8 \times & 12.5 & 12.3 & 12.1 & 11.9 & 11.7 \\ 9 x & 11.4 & 11.1 & 8.7 & 3.1 & 1.9\end{array}$ $10 \times 1.4$

PERcent total distance

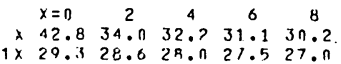
$\begin{array}{llllll}1 \times & 29.3 & 28.6 & 28.0 & 27.5 & 27.0 \\ 2 \times & 26.5 & 25.9 & 25.4 & 24.9 & 24.5\end{array}$

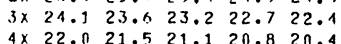

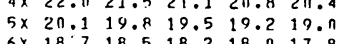

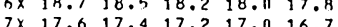

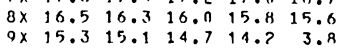
$\ln 2.2$

PERCENT TOTAL DISTANCE

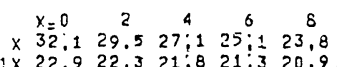

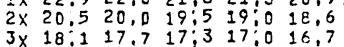

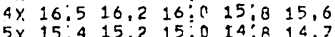
$6 \times 14.514,3 \quad 14: 1 \quad 13: 913,7$

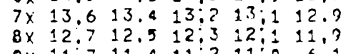
$\begin{array}{rr}9 \times & 11: 7 \\ 10 \times & 2 ; 8\end{array}$

PERCENT TOTAL DISTANCE

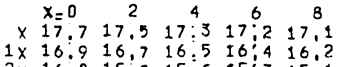

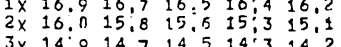
$3 \times 14: 9 \quad 14,7 \quad 14,514: 3 \quad 14,2$

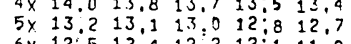

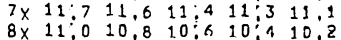
$\begin{array}{rr:rrrrr}8 \times & 11: 0 & 10,8 & 10: 6 & 10: 4 & 10,2 \\ 9 \times & 10: 0 & 9,8 & 9: 6 & 9: 5 & 9.3\end{array}$ $10 \times 4: 4$ 


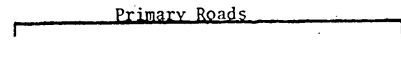

PERLENT total histance

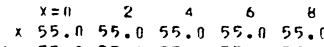

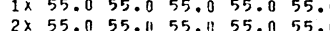
$3 \times 55.055 .0 \quad 55.055 .055$.

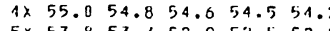
$5 \times 53.853 .352 .05 \% .552$.

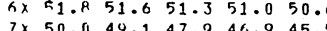
$8 \times 44.0 \quad 42.4 \quad 41.0 \quad 3 \% .3 \quad 38.0$

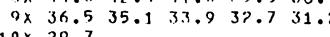

\section{DERIEEN TUTAL DISTANCE}

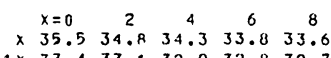
$\begin{array}{llllll}1 \times & 33.4 & 33.1 & 32.9 & 32.8 & 32.6 \\ 2 \times & 32.6 & 32.5 & 32.4 & 32.3 & 32 .\end{array}$ $\begin{array}{llllll}3 \times & 32.6 & 32.5 & 32.4 & 32.3 & 32.3 \\ 3 \times & 32.2 & 32.2 & 32.1 & 32.1 & 32.0\end{array}$ $\begin{array}{llllll}4 \times & 32.0 & 32.0 & 32.0 & 32.1 & 32.0 \\ 5 x & 31.0 & 32.0 & 32.0 & 32.0 & 32 .\end{array}$ $\begin{array}{llllll}5 \times & 31.9 & 31.8 & 31.6 & 31.5 & 31.3 \\ 6 x & 31.1 & 31.0 & 30.9 & 30.8 & 30.6\end{array}$ $\begin{array}{llllll}6 \times & 31.1 & 31.0 & 3 n .9 & 3 n .8 & 3 n \\ 7 \times & 30.5 & 30.4 & 30.2 & 30.0 & 29.6\end{array}$

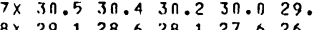
$\begin{array}{llllll}8 \times & 29.1 & 28.6 & 28.1 & 27.6 & 26.9 \\ 9 \times & 26.3 & 25.7 & 25.1 & 24.5 & 23.7 \\ 0 \times & 22.9 & & & & \end{array}$

\section{PERGENT InTAL DISTANCE}

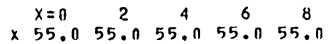

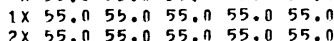
$3 \times 55.055 .055 .055 .055 .0$

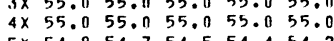

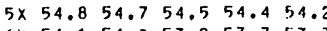
$\begin{array}{llllll}6 \times & 54.1 & 54.0 & 53.9 & 53.7 & 53.3 \\ 7 \times & 53.0 & 52.7 & 52.3 & 51.6 & 51.0\end{array}$

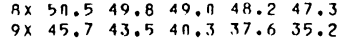
$10 \times 33.3$

pirefet toral nistanth

$\begin{array}{llll}x & =0 & 2 & 4\end{array}$

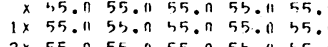
$2 \times 55.055 .055 .055 .055$. $4 \times 55.055055050 .055$. $5 \times 54.854 .7 \quad 54.5 \quad 54.4 \quad 51.2$ $6 \times 54.154 .053 .9 \quad 53.6 \quad 5.3$.

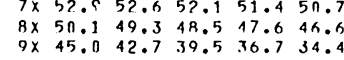
$\begin{aligned} 9 \times & 45.049 \\ 10 \times & 37.4\end{aligned}$

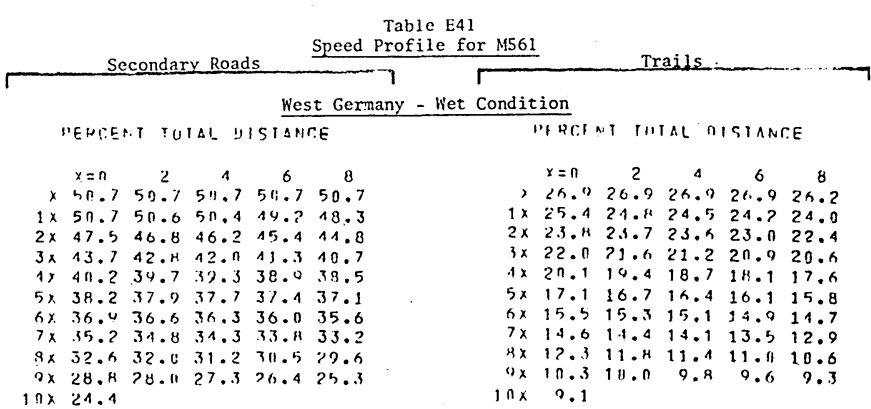

West Gernany - Snow Condition

DERGFNT TUTAL DISIANCE

$\begin{array}{rrrrrr} & x=11 & 2 & 4 & 6 & 8 . \\ x & 33.6 & 3.3 .6 & 33.3 & 33.0 & 32.7 \\ 1 \times & 32.5 & 32.4 & 32.3 & 32.2 & 32.7\end{array}$

$\begin{array}{llllll}1 \times & 32.5 & 32.4 & 32.3 & 32.2 & 32.2 \\ 2 x & 32.1 & 32.1 & 32.0 & 32.11 & 32.0\end{array}$

$\begin{array}{lllllll}3 \times & 31.9 & 31.8 & 31.7 & 31.1 & 31.1 & 31\end{array}$

$\begin{array}{llllll}5 x & 29.7 & 29.5 & 29.3 & 29.2 & 29.0 \\ 6 x & 28.0 & 74.7 & 28.6 & 28.4 & 28.2\end{array}$

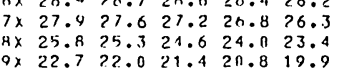

$\begin{array}{ll}9 \times & 22.7 \\ \ln x & 19.1\end{array}$

$$
\text { Mid-East }
$$

PFREEMT TOTAL MISTANFE

d-East - Wet Condition

$\begin{array}{rrrrrr}x=0 & ? & 4 & 5 & 8 \\ x & 50.7 & 501.7 & 50.7 & 50.7 & 50.7 \\ x & 50.7 & 511.7 & 50.7 & 50.7 & 50.7\end{array}$

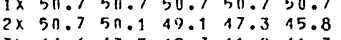

$\begin{array}{llllll}3 x & 44.6 & 43.5 & 42.7 & 41.9 & 41.3 \\ 4 x & 40.7 & 40.2 & 39.8 & 30.4 & 33.4\end{array}$

$5 \times \quad 38.7 \quad 38.459 .1 \quad 37.439 . n$

$\begin{array}{llllll}5 \times & 38.7 & 38.4 & 38.1 & 37.9 & 37.6 \\ 6 \times & 37.4 & 37.2 & 37.0 & 36.7 & 36.5\end{array}$

$\begin{array}{llllll}7 \times & 36.2 & 35.9 & 35.4 & 31.0 & 34.3 \\ 3 \times & 33.4 & 3 \% .2 & 31.0 & 311.0 & 29.1\end{array}$

$\begin{array}{llllll}8 \times & 3.3 .4 & 32.2 & 31.0 & 31.0 & 29.1 \\ 9 \times & 28.3 & 27.6 & 26.9 & 26.3 & 75.7\end{array}$

$9 \times 28.3$
$1 n \times 25.0$

Mid-East - Sand Condition

PERCFNT TOTAL DISTANTE

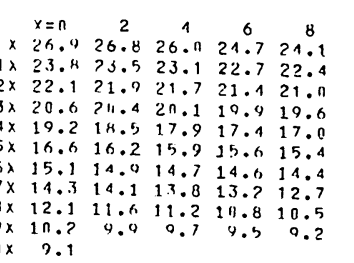

PFreent tGIAL DISTANGE

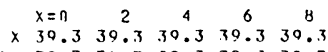

$\begin{array}{llllll}1 \times & 39.3 & 39.3 & 39.3 & 39.3 & 39.3 \\ 2 \times & 39.3 & 38.9 & 38.2 & 37.7 & 37.3\end{array}$

$3 \times 36.9 \quad 36.6 \quad 36.3 \quad 36.0 \quad 35.8$

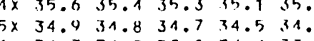

$6 \times 34.3 .34 .233 .835 .433$.

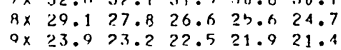

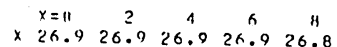

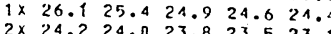
$\begin{array}{llllll}1 \times & 24.1 & 25.4 & 24.9 & 24.6 & 24.4 \\ 3 x & 22.5 & 24.0 & 23.8 & 23.5 & 23 . \\ 4 x & 20.8 & 20.1 & 21.7 & 21.4 & 21 .\end{array}$ $\begin{array}{lllllll}5 \times & 19.7 & 19.2 & 18.7 & 18.2 & 17.7 \\ 6 \times & 17.4 & 17.0 & 16.7 & 16.4 & 16.1\end{array}$ $\begin{array}{cccccc}7 x & 15.9 & 15.7 & 15.5 & 15.3 & 15.1\end{array}$ $\begin{array}{llllll}8 x & 15.0 & 14.9 & 14.6 & 15.9 & 13.3 \\ 9 x & 12.7 & 12.2 & 11.8 & 11.4 & 11.1\end{array}$ ח 1 X 1 ती.

TERCENT TOTAL DISTANCE

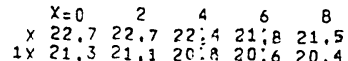

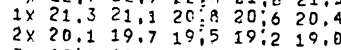

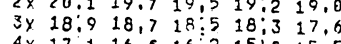

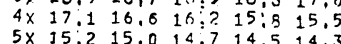

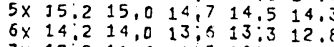
$7 \times 12: 211.611 .210: 710.4$ $\begin{array}{rrrrrr}8 \times & 7.0 & 2.6 & 1: 6 & 1: 2 & 1.0 \\ 9 x & 0: 8 & 0.7 & 0.6 & 0: 6 & 0.5 \\ 10 x & 0: 5 & & & & \end{array}$

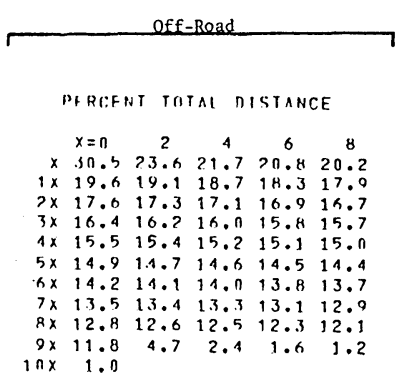

PFREENT TOTAL MISTANCF

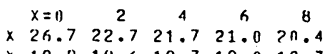

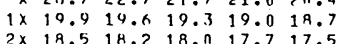
$\begin{array}{lllllll}2 \times & 18.5 & 1 \mathrm{H} .2 & 18.0 & 17.7 & 17.5 \\ 3 \times & 17.3 & 17.1 & 17.0 & 16.8 & 16.6\end{array}$ $\begin{array}{llllll}3 x & 17.3 & 17.1 & 17.0 & 16.8 & 16.0 \\ 5 x & 16.4 & 16.2 & 16.0 & 15.9 & 15.9\end{array}$ 6 14.914 .814 .614 .514 .8

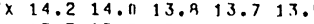
$\begin{array}{rrrrrr}8 \times & 13.3 & 13.2 & 13.0 & 12.8 & 12.6 \\ 9 x & 12.4 & 4.7 & 2.4 & 1.6 & 1.2\end{array}$

nx 1.0

PERCENT TOTAL DISTANCE

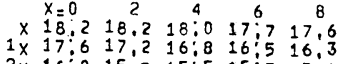
$\begin{array}{llllll}3 x & 16: 0 & 15,7 & 15: 5 & 15: 3 & 15,7 \\ 3 x & 15: 0 & 14: 8 & 14: 7 & 14: 6 & 14,5\end{array}$

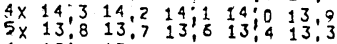

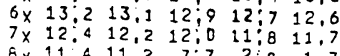

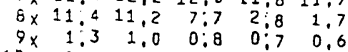

PERCENT TOTAL DISTANCE

$\begin{array}{rrrrrr}x=0 & \bar{c} & 4 & 6 & 8 \\ \times & 18.0 & 17.7 & 17: 6 & 17 \% 5 & 17.4\end{array}$

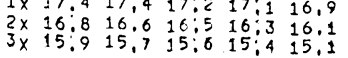
$\begin{array}{lllllll}4 \times & 14.9 & 14.7 & 14: 4 & 14: 2 & 14.0 \\ 5 \times & 13 & 7 & 13.3 & 13: 9 & 12: 4 & 5\end{array}$

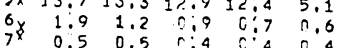

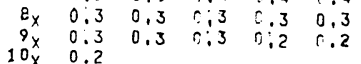




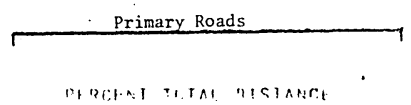

$\begin{array}{cccccc}x=11 & 2 & 4 & 6 & 8 \\ \times & 50.0 & 50.0 & 50.0 & 50.0 & 50.11\end{array}$ $1 \times 50.050 .050 .050 .050 .0$

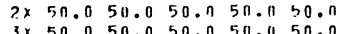

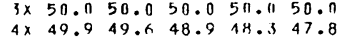

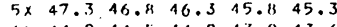
$3 \times 44.9$ 14.5 $44.2 \quad 43.9 \quad 13.6$

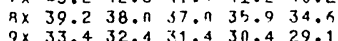
$17 \times 27.9$

PF RCENT TITAL DICTANTI

$\begin{array}{cccccc}x=0 & 2 & 4 & 6 & 8 \\ x & 16.9 & 16.9 & 16.9 & 15.7 & 14.9\end{array}$ $2 \times \quad \begin{array}{llll}x & 13.6 & 13.5 & 13.4\end{array}$ $\begin{array}{llllll}3 \times & 13.2 & 13.1 & 13.0 & 13.0 & 12.9\end{array}$ $\begin{array}{llllll}4 \times & 12.9 & 12.8 & 12.8 & 12.7 & 12.7\end{array}$ $\begin{array}{llllll}5 \times & 12.7 & 12.6 & 12.6 & 12.5 & 12.5 \\ 6 x & 12.5 & 12.5 & 12.4 & 12.4 & 12.5\end{array}$ $\begin{array}{llllll}7 \times & 12.4 & 12.3 & 12.3 & 12.3 & 12.3 \\ 8 \times & 12.3 & 12.3 & 12.3 & 12.2 & 12.2 \\ 9 x & 12.2 & 12.2 & 12.2 & 12.2 & 12.1\end{array}$ $\begin{array}{rr}9 \times & 12.2 \\ 10 \times & 12.0\end{array}$

PERCENT total Distante

$\begin{array}{rrrrr}x=0 & 2 & 4 & 6 & 8 \\ \times & 50.0 & 50^{2} & 50.0 & 50.0 .50 .0\end{array}$ $1 \times 50.050 .050 .050 .050 .0$ $2 \times 50.050 .0 \quad 49.749 .4 \quad 49.2$ $\begin{array}{cccccc}3 \times & 49.0 & 48.8 & 48.6 & 48.5 & 48.4 \\ 4 \times & 48.3 & 48.2 & 48.0 & 47.9 & 47.8\end{array}$ $\begin{array}{cccccc}5 \times & 47.7 & 47.4 & 46.9 & 46.5 & 46.2\end{array}$ $6 \times \quad 45.9 \quad 45.5 \quad 45.3 \quad 45.044 .7$

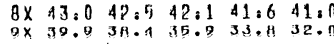
$10 \times 30 \div 4$

PERGENT TOTAL MISTANTE

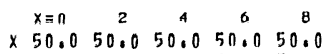
$\times 50.050 .050 .050 .050 .0$
$1 \times 50.050 .050 .050 .050 .0$
$3 \times 50.050 .0$ $2 \times 50.050 .0 \quad 49.6 \quad 19.4 \quad 49.1$ $\begin{array}{cccccc}3 \times & 18.9 & 48.8 & 48.6 & 18.5 & 18.4 \\ 4 \times & 48.3 & 48.1 & 48.0 & 47.9 & 47.8\end{array}$

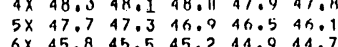
$7 \times 44.544 .244 .043 .643 . ?$ $8 \times \quad 42.742 .344 .841 .244 .6$

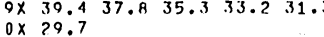

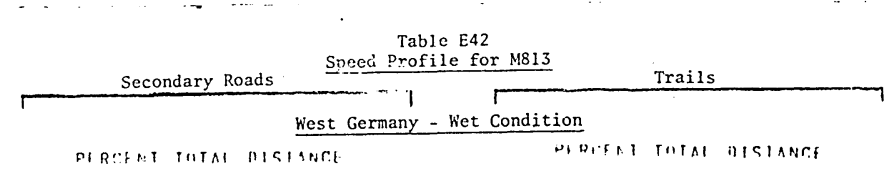

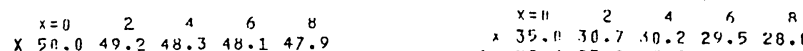

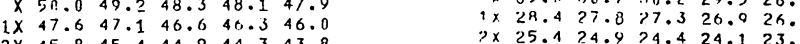

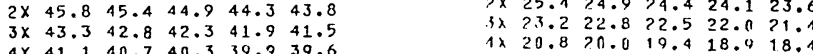

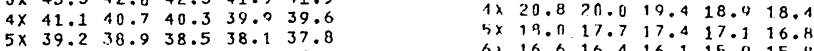

$\begin{array}{llllll}6 \times & 37.4 & 36.9 & 36.3 & 35.7 & 35.1\end{array}$

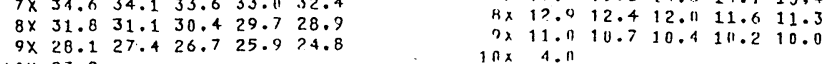

$10 \times 23.9$

West Germany - Snow Condition

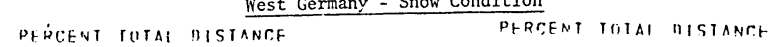

$\begin{array}{cccccc}x=0 & 2 & 4 & 6 & 8 \\ x & 13.1 & 13.1 & 13.0 & 13.0 & 13.0 \\ 1 x & 13.0 & 13.0 & 132.9 & 12.9 & 12.9\end{array}$

$\begin{array}{lllllll}2 \times & 12.9 & 12.8 & 12.8 & 12.7 & 12.7\end{array}$

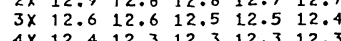

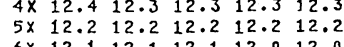

$\begin{array}{llllll}6 \times & 12.1 & 12.1 & 12.1 & 12.0 & 12.0 \\ 7 \times & 12.0 & 11.9 & 11.9 & 11.9 & 11.8\end{array}$

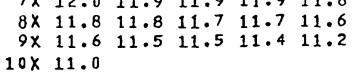

\section{Mid-East - Wet Condition}

DERIENT TITAL MISINNTE

$\begin{array}{rlrrrr} & x=11 & 7 & 4 & 8 & 4 \\ x & 12.0 & 12.0 & 12.0 & 12.0 & 11.9 \\ 1 \times & 11.9 & 11.8 & 11.7 & 11.6 & 11.5\end{array}$ $3 \times 11.511 .4 \quad 11.311 .311 .3$

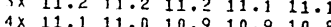

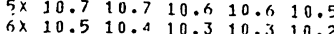
$\begin{array}{cccccc}6 \times & 10.5 & 10.4 & 10.3 & 10.3 & 10.5 \\ 7 \times & 10.1 & 10.1 & 10.0 & 4.7 & 9.5\end{array}$ $\begin{array}{llllll}9 \times & 9.3 & 9.1 & 8.9 & 4.7 & 8.6\end{array}$ $\begin{array}{lll}0 \times & 8.5 \\ 10 x \quad 7.0\end{array}$

Perefent TIIALI DISTAMCE.

$\begin{array}{cccccc}x=0 & 2 & 4 & 6 & 8 \\ x & 50.0 & 50.0 & 49.2 & 48.6 & 48.3 \\ 1 & 48.2 & 46.0 & 48.0 & 47.9 & 47.9\end{array}$

$\begin{array}{rrrrrr}x \times & 48.2 & 46.1 & 48.0 & 47.9 & 47.9 \\ 3 x & 47.8 & 47.6 & 47.3 & 47.1 & 46 .\end{array}$

$\begin{array}{cccccc}3 x & 46.6 & 46.5 & 46.3 & 46.2 & 46.1 \\ 4 x & 45.9 & 45.8 & 45.6 & 45.5 & 45.3\end{array}$

$5 \times 45.1 \quad 44.9 \quad 44.6 \quad 44.2 \quad 43.8$

$7 \times \quad 41.1 \quad 40.5 \quad 39.8 \quad 39.0 \quad 38.1$

$\begin{array}{llllll}8 \times & 37.0 & 35.3 & 33.9 & 32.6 & 31.5\end{array}$

$5 \times$
$10 \times 26.5$
102

Mid=-

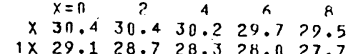

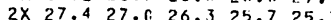
$\begin{array}{llllll}3 \times & 24.9 & 24.5 & 24.2 & 24.0 & 23.7\end{array}$

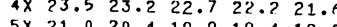
$6 x x^{21.0} \quad 18.4 \quad 19.9 \quad 19.419$. $7 \times 17.2517 .010$ $\begin{array}{llllll}8 \times & 16.2 & 16.0 & 15.7 & 15.81 & 14.3 \\ 0 \times \times & 13.4 & 13.3 & 12.8 & 12.4 & 12.0\end{array}$ PIBTEN IIIAL MISIANCE

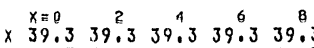
$\begin{array}{llllll}1 \times & 39.3 & 39.39 & 39.3 & 39.3 & 39.3 \\ 2 \times & 39.3 & 39.3 & 39.3 & 39.3 & 39.3\end{array}$ $3 \times \quad 39.3 \quad 39.3 \quad 39.3 \quad 39.339 .3$

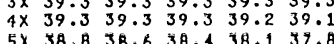
$\begin{array}{llllll}5 \times & 38.8 & 38.6 & 38.4 & 38.1 & 37.8 \\ 6 \times & 37.6 & 37.3 & 36.7 & 36.1 & 35.5\end{array}$ $\begin{array}{llllll}7 \times & 35.0 & 34.3 & 33.6 & 32.7 & 31.9 \\ 8 \times & 30.8 & 29.3 & 27.9 & 26.8 & 25.8\end{array}$ $\begin{array}{rrrrrr}9 \times & 24.9 & 24.1 & 23.3 & 22.7 & 22.1 \\ 10 x & 21.3 & & & & \end{array}$

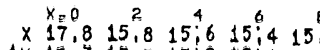
$2 x \quad 14: 8 \quad 14,7 \quad 14: 514: 4$ : 14. $5 \times 11,311.010: 4 \pm 0: 410.1$

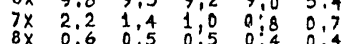

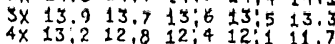
$\begin{array}{llllll}9 x & 0.6 & 0.5 & 0.5 & 0: 4 & 0.4 \\ 9 x & 0.4 & 0.4 & 0.3 & 0: 3 & 0.3\end{array}$

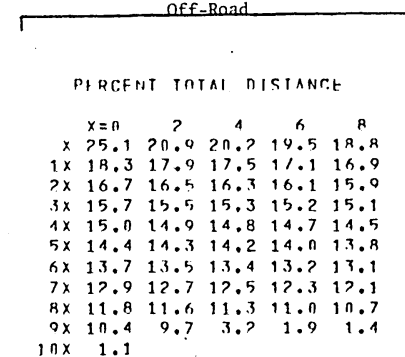

PERCFNT TRTAI. DISTANCE

$\begin{array}{lllll}x=0 & 0 & 4 & 4 & 6\end{array}$ $1 \times 14.0 \quad 14.7 \quad 13.6 \quad 13.1 \quad 19.7$ $\begin{array}{llllll}3 x & 17.3 & 12.0 & 11.8 & 11.6 & 11.4\end{array}$

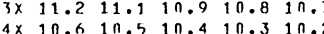
$5 \times 10.1 \quad 10.010 .0 \quad 9.9 \quad 9.8$ $\begin{array}{llllll}5 \times & 9.7 & 9.7 & 0.6 & 9.5 & 9.4 \\ 7 \times & 9.3 & 9.2 & 9.2 & 9.0 & 8.9\end{array}$ $\begin{array}{llllll}8 x & 8.8 & 3.7 & 8.6 & 8.5 & 8.3 \\ 0 x & 8.1 & 7.7 & 3.2 & 1.9 & 1.4 \\ 10 x & 1.1 & & & & \end{array}$

PERCENT TCTAL DISTANCE

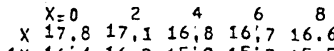

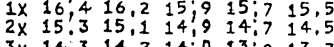

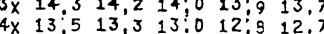
12 : $11: 711.5$

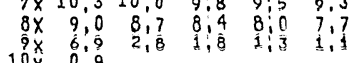

FEHETH TRTAL DIBTENGE

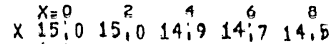

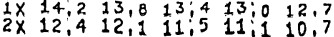
$\begin{array}{cccccc}3 \times & 10: 3 & 9: 9 & 9: 3 & 8: 3 & 2 \\ 4 \times & 1,2 & 0: 9 & 0: 6 & 0,5 & 0: 4\end{array}$

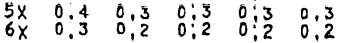
$\begin{array}{rrllll}7 x & 0: 2 & 0.2 & 0: 2 & 0: 2 & 0.2 \\ 8 k & 0: 2 & 0,2 & 0: 2 & 0: 2 & 0.2 \\ 9 x & 0 ; 2 & 0,2 & 0: 2 & 0: 2 & 0.2 \\ 10 x & 0.2 & & & & \end{array}$ $10 x$ 


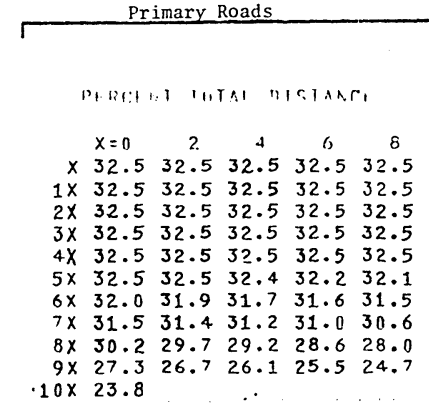

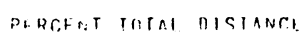

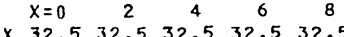
$\begin{array}{llllll}1 \times & 32.5 & 32.5 & 32.5 & 32.5 & 32.5 \\ 2 x & 32.5 & 32.5 & 32.5 & 32.5 & 32.5 \\ 32 & 32.5 & 32.5 & 32.5 & 32.5 & 32.5\end{array}$ $\begin{array}{lllllll}3 \times & 32.5 & 32.5 & 32.5 & 32.5 & 32.5\end{array}$ $\begin{array}{lllll}4 \times 32.5 & 32.5 & 32.5 & 32.5 & 32.4\end{array}$ $\begin{array}{lllllll}6 \times & 32.4 & 32.4 & 32.4 & 32.3 & 32.3\end{array}$ $\begin{array}{llllll}7 \times & 32.3 & 32.2 & 31.9 & 31.7 & 31.2\end{array}$ $\begin{array}{llllll}8 \times & 30.7 & 30.1 & 29.5 & 28.9 & 28.1\end{array}$ $9 \times 27.4$
$10 \times 23.7$

\section{PERCENT totAL Distance}

$\begin{array}{rrrrrr}x=0 & 2 & 4 & 6 & 8 \\ \times 32.5 & 32.5 & 32.5 & 32.5 & 32.5\end{array}$

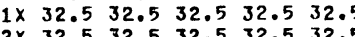
$3 \times 32.5 \quad 32.532 .5 \quad 32.532 .5$ $\begin{array}{llllll}4 \times & 32.5 & 32.5 & 32.5 & 32.5 & 32.5\end{array}$ $\begin{array}{llllll}5 x & 32.5 & 32.5 & 32.5 & 32.5 & 32.5 \\ 6 x & 32.5 & 32.5 & 32.5 & 32.5 & 32.5\end{array}$ $7 \times 32.532 .532 .532 .532 .5$ $9 \times 31.630 .729 .3 \quad 28.026 .8$ $10 \times 25.8$

PERCENT TOTAL DISTANCE

$\begin{array}{ccccc}x=0 & 2 & 4 & 6 & 8 \\ x & 32.5 & 32 & 5 & 32 \\ x & 32 & 32.5 & 32.5\end{array}$ $1 \times 32.5 \quad 32.5 \quad 32.532 .532 .5$ $\begin{array}{llllll}2 \times & 32.5 & 32.5 & 32.5 & 32.5 & 32.5\end{array}$ $\begin{array}{lllll}3 \times & 32.532 .5 & 32.5 & 32.5 & 32.5\end{array}$ $4 \times$
$5 \times 32.5$ $\begin{array}{llllll}5 \times & 32.5 & 32.5 & 32.5 & 32.5 & 32.5 \\ 6 \times & 32.5 & 32.5 & 32.5 & 32.5 & 32.5\end{array}$ $\begin{array}{llllll}7 \times & 32.5 & 32.5 & 32.5 & 32.5 & 32.5\end{array}$ $8 \times 32.5 \quad 32.4 \quad 32.3 \quad 32.2 \quad 32$. 10x 31.430 .529 .0 $10 \times 25.3$

Speed Profile for UET

$\sqrt{\text { Secondary Roads }} \frac{\text { Speed Profile for UET }}{\text { West Germany - Wet Condition }}$ Trails

$\begin{array}{ccccc}x=0 & 2 & 4 & 6 & 8 \\ x \quad 32.5 & 32.5 & 32.5 & 32.5 & 32.5\end{array}$ $\begin{array}{lllllll}1 \times & 32.5 & 32.5 & 32.5 & 32.5 & 32.5 \\ 2 x & 32.5 & 32.5 & 32.5 & 32.5 & 32.5\end{array}$ $\begin{array}{lllllll}2 \times & 32.5 & 32.5 & 32.5 & 32.5 & 32.5 \\ 3 \times & 32.5 & 32.5 & 32.5 & 32.4 & 32.2\end{array}$ $\begin{array}{lllllllll}4 \times & 32.0 & 31.8 & 31.7 & 31.5 & 31.3\end{array}$ $5 \times 31.2 \quad 30.9 \quad 30.6 \quad 30.3 \quad 30.0$ $\begin{array}{llllll}6 x & 29.7 & 29.4 & 29.1 & 28.8 & 28.6 \\ 7 \times & 28.3 & 28.0 & 27.8 & 27.5 & 27.2\end{array}$ 426.025 .525 .0

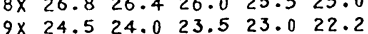
$\begin{aligned} & 9 \times 24.5 \\ & 10 \times 21.5\end{aligned}$

West Germany - Snow Condition

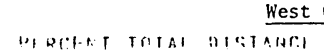

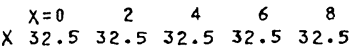

$\begin{array}{lllllll}1 x & 32.5 & 32.5 & 32.5 & 32.5 & 32.5\end{array}$

$\begin{array}{lllllll}2 x & 32.5 & 32.5 & 32.5 & 32.5 & 32.5 \\ 3 \times & 32.5 & 32.5 & 32.5 & 32.5 & 32.5\end{array}$

$\begin{array}{lllllll}4 \times & 32.5 & 32.5 & 32.4 & 32.4 & 32.3\end{array}$

$\begin{array}{lllllll}5 \times & 32.1 & 32.0 & 31.8 & 31.7 & 31.6 \\ 6 \times & 31.4 & 31.3 & 30.9 & 30.6 & 30.6\end{array}$

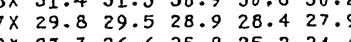

$\begin{array}{llllll}8 \times & 27.3 & 26.6 & 25.9 & 25.2 & 24.4 \\ 9 \times & 23.7 & 23.0 & 22.3 & 21.5 & 20.6\end{array}$

$10 \times 19.7$

Mid

PERCENT TOIAL DISTANCE

$\begin{array}{cccccc}x=0 & 2 & 4 & 6 & 8 \\ x & 32.5 & 32.5 & 32.5 & 32.5 & 32.5 \\ 1 \times & 32.5 & 32.5 & 32.5 & 32.5 & 32.5\end{array}$

$\begin{array}{llllll}1 \times & 32.5 & 32.5 & 32.5 & 32.5 & 32.5 \\ 2 x & 32.5 & 32.5 & 32.5 & 32.5 & 32.5\end{array}$

$\begin{array}{llllll}3 \times & 32.5 & 32.5 & 32.5 & 32.5 & 32.5\end{array}$

$\begin{array}{lllllll}4 \times & 32.5 & 32.5 & 32.5 & 32.5 & 32.5 \\ 5 \times & 32.5 & 32.5 & 32.5 & 32.5 & 32.4\end{array}$

$6 \times 32.3 \quad 32.2 \quad 30.1 \quad 32.0 \quad 31.9$

$\begin{array}{llllll}6 x & 32.3 & 32.7 & 32.1 & 32.0 & 31.9 \\ 7 x & 31.8 & 31.6 & 31.3 & 31.0 & 30.6\end{array}$

$\begin{array}{llllll}8 \times & 30.0 & 29.0 & 28.2 & 27.4 & 26.7 \\ 9 \times & 26.1 & 25.5 & 24.9 & 24.5 & 24.0\end{array}$

$\begin{array}{rr}9 \times & 26.1 \\ 10 \times & 23.4\end{array}$

\section{Mid-E}

\section{PFRCENT TOTAL DISTANCE}

$\begin{array}{rllll}x=n & 2 & 4 & 6 & 8 \\ x \quad 32.5 & 32.5 & 32.5 & 32.5 & 32.5\end{array}$

$1 \times 32.5 \quad 32.5 \quad 32.5 \quad 32.5 \quad 32.5$

$2 \times 32.5 \quad 32.5 \quad 32.5 \quad 32.5 \quad 32.5$

$\begin{array}{llllll}3 x & 32.5 & 32.5 & 32.5 & 32.5 & 32.5\end{array}$

$5 \times 32.532 .532 .532 .5 \quad 32.5$

$\begin{array}{llllll}6 \times & 32.0 & 31.9 & 31.6 & 31.3 & 31.0 \\ 7 \times & 30.7 & 30.3 & 29.8 & 29.7 & 28.6\end{array}$

$\begin{array}{lllllll}8 \times & 27.8 & 26.6 & 75.6 & 24.6 & 23.8\end{array}$

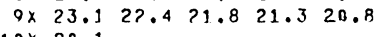

$10 \times 2 n .1$ $\begin{array}{cccccc}\quad=10 & 2 & 4 & 6 & 8 \\ \times & 30.4 & 30.4 & 20.3 & 28.5 & 28.0\end{array}$

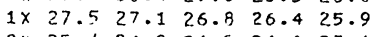

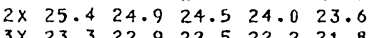

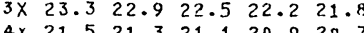

$\begin{array}{llllll}4 \times & 21.5 & 21.3 & 21.1 & 20.9 & 20.7 \\ 5 x & 20.5 & 20.3 & 20.1 & 19.8 & 19.6\end{array}$

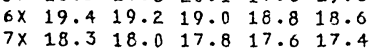

$8 \times 17.2 \quad 17.0 \quad 16.8 \quad 16.7 .16 .5$

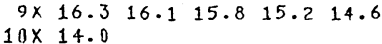

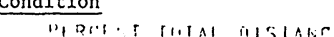

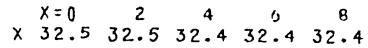
$\begin{array}{lllllll}1 \times & 32.4 & 32.2 & 32.0 & 31.5 & 31.4 \\ 2 \times & 30.8 & 30.4 & 2 . .8 & 23.4 & 29.0\end{array}$ $\begin{array}{llllll}2 x & 30.7 & 30.4 & 29.8 & 29.4 & 29.0 \\ 3 x & 28.7 & 28.3 & 27.8 & 27.0 & 26.3\end{array}$ $\begin{array}{llllll}4 \times & 25.6 & 25.0 & 24.5 & 24.1 & 23.7\end{array}$ $\begin{array}{llllll}5 \times & 23.4 & 23.1 & 22.8 & 22.5 & 22.3\end{array}$ $\begin{array}{llllll}6 \times & 22.1 & 21.9 & 21.7 & 21.5 & 21.2\end{array}$ $\begin{array}{llllll}7 \times & 20.9 & 20.6 & 20.2 & 19.9 & 19.5\end{array}$

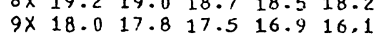
$10 \times 15.4$

PERCENT TOTAL DISTANCE

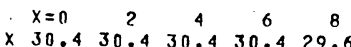
$\begin{array}{rrrrrr}\times & 30.4 & 30.4 & 30.4 & 30.4 & 29.6 \\ 1 \times & 29.0 & 28.5 & 28.2 & 27.9 & 27.5\end{array}$ $\begin{array}{llllll}2 \times & 27.3 & 27.0 & 26.7 & 26.4 & 26.0 \\ 3 \times & 25.9 & 25.6 & 25.7 & 26.4 & 26.0\end{array}$ $\begin{array}{cccccc}3 x & 25.9 & 25.6 & 25.2 & 24.8 & 24.4 \\ 4 \times & 24.0 & 23.7 & 23.3 & 22.9 & 23.0\end{array}$ $\begin{array}{llllll}4 x & 24.0 & 23.7 & 23.3 & 22.9 & 27.6 \\ 5 x & 22.3 & 22.1 & 21.9 & 21.7 & 21.5\end{array}$ $\begin{array}{llllll}5 x & 22.3 & 22.1 & 21.9 & 21.7 & 21.5 \\ 6 \times & 21.3 & 21.1 & 21.0 & 20.8 & 20.6\end{array}$

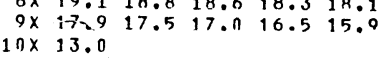
Condition

\section{PERcent total Distance -}

$\begin{array}{cccccc}x=0 & ? & 4 & 6 & 8 \\ \times & 18.7 & 18.7 & 18.5 & 18.4 & 18.3\end{array}$ $\begin{array}{ccccccc}1 \times & 18.3 & 18.2 & 18.1 & 18.1 & 18.0 \\ 2 \times & 17.9 & 17.9 & 17.8 & 17.6 & 17.5\end{array}$ $\begin{array}{llllll}2 \times & 17.9 & 17.9 & 17.8 & 17.6 & 17.5 \\ 3 \times & 17.3 & 17.2 & 17.1 & 17.0 & 16.9\end{array}$ $4 \times 17.317 .2 \quad 17.1 \quad 17.016 .9$ $5 \times 16.316 . ? \quad 16.1 \quad 16.0 \quad 15.4$ $\begin{array}{lllllll}6 \times & 15.7 & 15.6 & 15.5 & 15.4 & 15 . ?\end{array}$ $7 \times 15.1 \quad 15.014 .9 \quad 14.8 \quad 14.7$ $\begin{array}{llllll}8 \times & 14.6 & 14.4 & 14.3 & 14.1 & 14.0 \\ 9 x & 13.7 & 13.5 & 13.2 & 12.9 & 10.7\end{array}$ $10 \times 17.3$

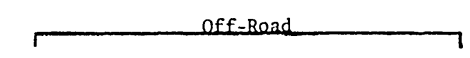

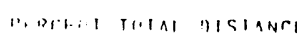

$\begin{array}{rrrrr}x=0 & 2 & 4 & 6 & 8\end{array}$

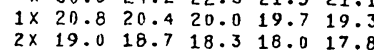
$\begin{array}{llllll}2 \times & 19.0 & 18.7 & 18.3 & 18.0 & 17.8 \\ 3 \times & 17.6 & 17.4 & 17.1 & 16.9 & 16.7\end{array}$ $\begin{array}{llllll}3 \times & 17.6 & 17.4 & 17.1 & 16.9 & 16.7 \\ 4 \times & 16.5 & 16.3 & 16.1 & 15.9 & 15.8\end{array}$ $5 \times 15.6 \quad 15.515 .315 .215 .1$

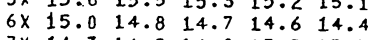
$\begin{array}{lllllll}7 \times & 14.3 & 14.2 & 14.0 & 13.9 & 13.7 \\ 8 \times & 13.6 & 13.4 & 13.2 & 13.9 & 13.7\end{array}$ $\begin{array}{rrrrrr}8 X & 13.6 & 13.4 & 13.2 & 13.0 & 12.8 \\ 9 X & 12.5 & 12.1 & 6.6 & 2.8 & 1.8\end{array}$ $10 \times \quad 1.3$

MDER HITA MICIANRE

$\begin{array}{cccccc}x=0 & 2 & 4 & 6 & 8 \\ x & 32.4 & 32.3 & 32.0 & 31.5 & 30^{8.7}\end{array}$ $\begin{array}{cccccc}1 \times & 30.1 & 29.6 & 29.1 & 28.6 & 28.1 \\ 2 \times & 27.7 & 27.2 & 26.8 & 26.3 & 25.8\end{array}$ $\begin{array}{llllll}3 \times & 25.3 & 24.8 & 24.4 & 23.9 & 23.5\end{array}$

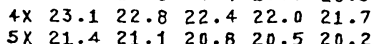
$\begin{array}{llllll}5 \times & 21.4 & 21.1 & 20.8 & 20.5 & 20.2 \\ 6 \times & 20.0 & 19.7 & 19.5 & 19.5 & \end{array}$ $\begin{array}{llllll}6 \times & 20.0 & 19.7 & 19.5 & 19.3 & 19.1\end{array}$ $8 \times 17.9 \quad 17.7 \quad 17.418 .218$. $\begin{array}{rrrrrr}8 \times & 17.9 & 17.7 & 17.4 & 17.2 & 16.9 \\ 9 \times & 16.7 & 16.4 & 16.0 & 5.9 & 2.7\end{array}$ $10 \times 1.8$

PI DEFET THFA DISTANCE

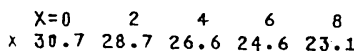
$\begin{array}{rrrrrr}x & 30.7 & 28.7 & 26.6 & 24.6 & 23.1 \\ 1 \times & 22.0 & 21.1 & 200.3 & 19.6 & 19.0 \\ 2 x & 10.5 & 10.1 & 17.6 & 17.5 & 19.0\end{array}$

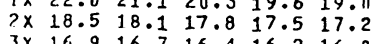
$\begin{array}{llllll}3 \times & 16.9 & 16.7 & 16.4 & 16.2 & 16.0 \\ 4 \times & 15.8 & 15.7 & 15.5 & 15.4 & 15.2\end{array}$ $\begin{array}{llllll}4 \times & 15.8 & 15.7 & 15.5 & 15.4 & 15.2 \\ 5 \times & 15.0 & 14.9 & 14.7 & 14.5 & 14.4\end{array}$ $\begin{array}{llllll}5 x & 15.0 & 14.9 & 14.7 & 14.5 & 14.4 \\ 6 x & 14.3 & 14.1 & 14.0 & 13.9 & 13.8\end{array}$ $7 \times 13.613 .514 .013 .9$. $\begin{array}{llllll}8 X & 12.6 & 13.5 & 13.4 & 13.2 & 13.1 \\ 9 x & 11.5 & 3.7 & 12.5 & 12.3 & 12.1\end{array}$ $\begin{array}{rrrrrr}9 \times & 11.5 & 3.7 & 2.5 & 1.5 & 1.2 \\ 10 \times & 1.0 & & & & \end{array}$

\section{PERCENT TOTAL. DISTANTE}

$\begin{array}{cccccc} & x=0 & 2 & 4 & 6 & 8 \\ \times 18.6 & 18.6 & 18.6 & 18.6 & 18.5\end{array}$ $\begin{array}{ccccccc}1 \times & 18.4 & 18.3 & 18.1 & 17.9 & 17.6\end{array}$ $\begin{array}{llllll}2 x & 17.3 & 17.0 & 16.8 & 16.6 & 16.0\end{array}$ $\begin{array}{lllllll}3 \times & 16.3 & 16.1 & 16.0 & 15.8 & 15.7 \\ 4 x & 15.5 & 15.4 & 15.0 & 15.8 & 14.9\end{array}$ $\begin{array}{llllll}5 \times & 14.5 & 15.4 & 15.2 & 15.1 & 14.9\end{array}$ $\begin{array}{llllll}5 \times & 14.7 & 14.6 & 14.5 & 14.3 & 14.2 \\ 6 \times & 14.0 & 13.9 & 13.7 & 13.6 & 13.4\end{array}$ $\begin{array}{lllllll}7 \times & 13.3 & 13.2 & 13.0 & 13.8 & 13.4\end{array}$ $8 \times 12.4 \quad 12.1 \quad 11.9 \quad 11.6 \quad 11.4$

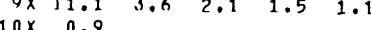


Table E44

Diagnostics Statistics for M561-M102

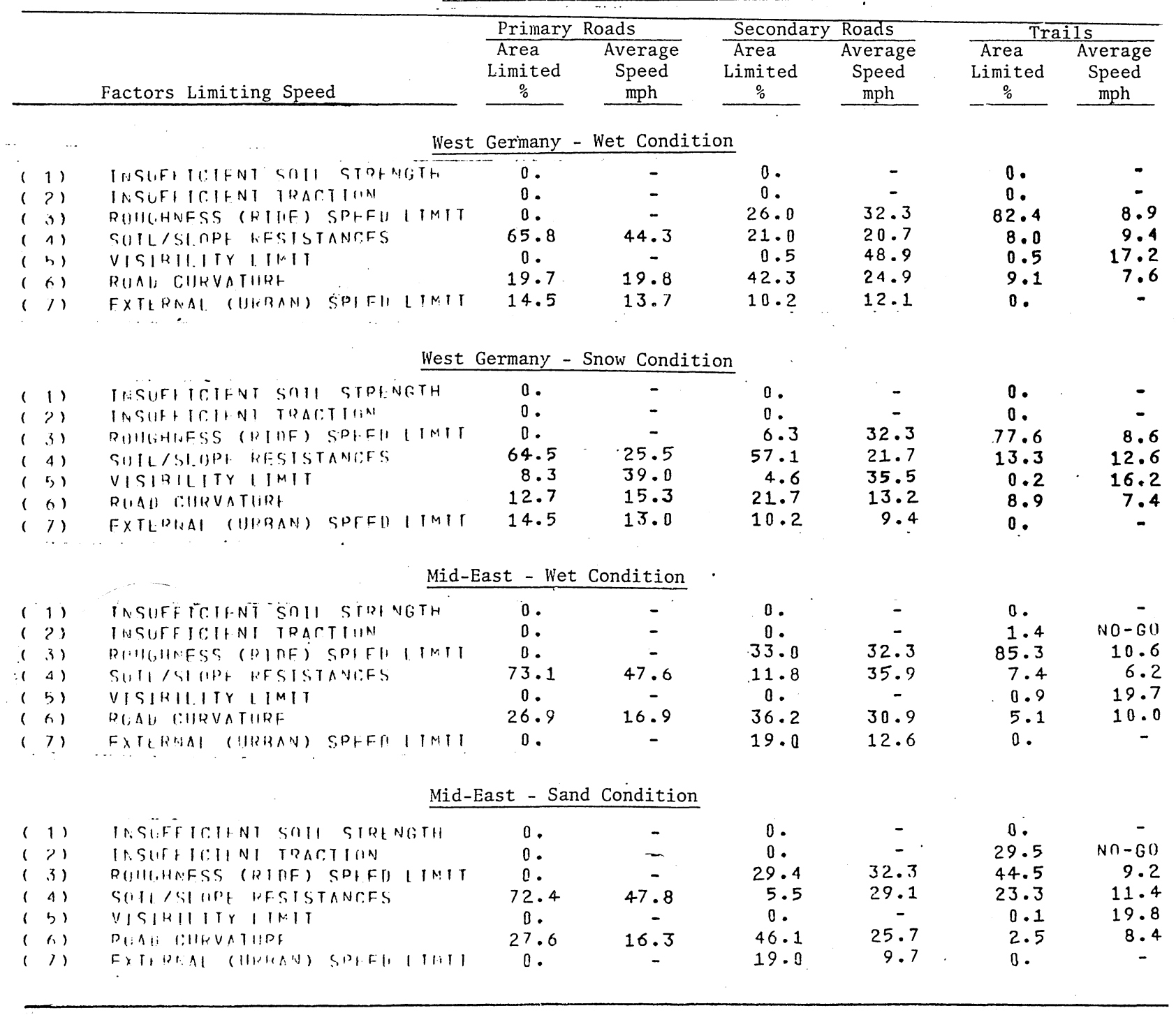


Table E45

Diagnostics Statistics for M561-XM204

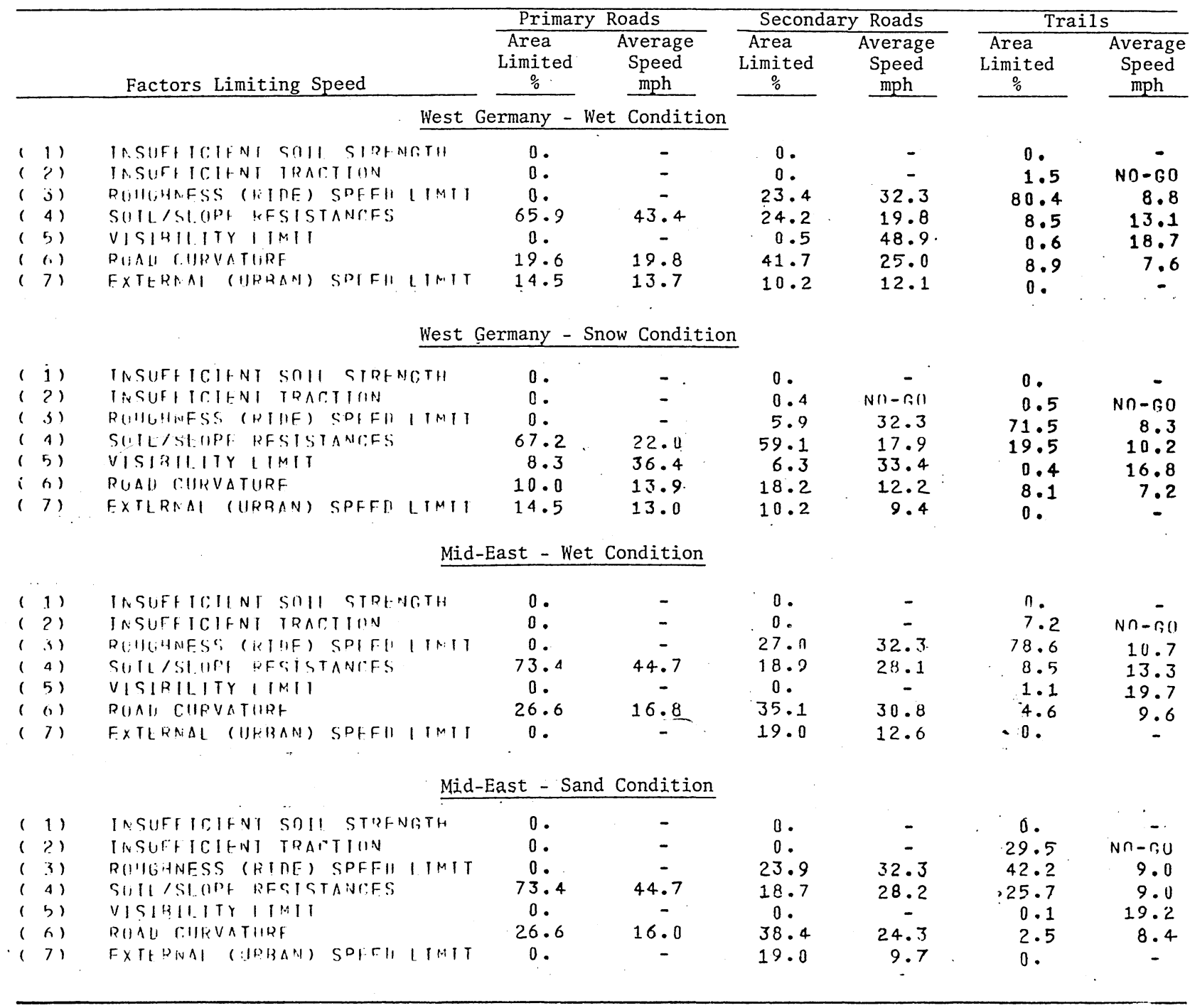


Table E46.

Diagnostics Statistics for M561-XM198

\begin{tabular}{|c|c|c|c|c|c|c|c|c|}
\hline \multirow{2}{*}{\multicolumn{3}{|c|}{ Factors Limiting Speed }} & \multicolumn{2}{|c|}{ Primary Roads } & \multicolumn{2}{|c|}{ Secondary Roads } & \multicolumn{2}{|c|}{ Trails } \\
\hline & & & $\begin{array}{c}\text { Area } \\
\text { Limited } \\
\% \\
\end{array}$ & $\begin{array}{c}\text { Average } \\
\text { Speed } \\
\text { mph } \\
\end{array}$ & $\begin{array}{c}\text { Area } \\
\text { Limited } \\
\% \\
\end{array}$ & $\begin{array}{c}\text { Average } \\
\text { Speed } \\
\text { mph }\end{array}$ & $\begin{array}{c}\text { Area } \\
\text { Limited } \\
\% \\
\end{array}$ & $\begin{array}{c}\text { Average } \\
\text { Speed } \\
\text { mph }\end{array}$ \\
\hline \multicolumn{9}{|c|}{ West Germany - Wet Condition } \\
\hline 1 & 1) & INSUEFIEIFNT SOII GIRFNGTII & B. & - & 0 . & - & 1.5 & $n O=-60$ \\
\hline & $\begin{array}{l}2\} \\
3\} \\
4 \\
51\end{array}$ & 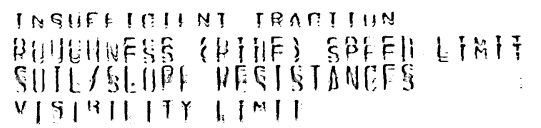 & $\begin{array}{c}0= \\
\hat{0}=0 \\
0 \\
0\end{array}$ & $\begin{array}{c}= \\
25:-2\end{array}$ & $\begin{aligned} 0 & \\
8 & =4 \\
57 & =0 \\
0 & =5\end{aligned}$ & $\begin{array}{l}= \\
33=\overline{3} \\
18: \frac{3}{30} \\
48,9\end{array}$ & $\begin{array}{r}7 \cdot 1 \\
58=0 \\
27=0 \\
1: 7\end{array}$ & 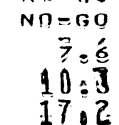 \\
\hline( & (3) & PUAH RUIRVATHEE & $14 . \hat{5}$ & $1 \%=\hat{G}$ & 23.0 & $20 \cdot 0$ & 6.3 & 6.5 \\
\hline & 71 & 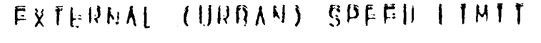 & $14 \cdot 5$ & 13.7 & 10.2 & 12.1 & $\hat{0}$. & - \\
\hline \multicolumn{9}{|c|}{ West Germany - Snow Condition } \\
\hline 1 & 1) & INSUHFICIHNI SOII SIPINETH & 0. & $=$ & 0 . & - & $n$. & - \\
\hline & ?) & IMSUFHICIFNT TRACTIGA & $19 \cdot 1$ & $n \cap-6)$ & $32 \cdot 1$ & $\left.N \cap-R_{0}\right)$ & 44.5 & NO $=60$ \\
\hline ( & .4) & RUII,HNFSS (RINF) SPFFH LIMIT & o. & - & 0. & - & 28.6 & 6.0 \\
\hline( & 1) & SUIL/SI WPH KFSISTANEFS & 62.3 & 13.9 & 48.4 & 12.6 & 23.4 & 9.4 \\
\hline( & i) & VIGIRIIITY I.IMII & $1 \cdot 3$ & 25.8 & 2.8 & 25.9 & 0.7 & 20.0 \\
\hline( & $6)$ & RUAU CUIRVATURF & 2.8 & 10.3 & $6 \cdot 5$ & 10.1 & 2.8 & 6.2 \\
\hline i & 7) & FXTKRNAI (URRAN) SPFFI I IMII & 14.5 & 13.0 & $10 \cdot 2$ & $9 \cdot 4$ & 0 . & - \\
\hline \multicolumn{9}{|c|}{ Mid-East - Wet Condition } \\
\hline 1 & 1) & INSUTFICHNT SOII STPFMGTH & 0 . & - & 0 . & - & $n \cdot$ & - \\
\hline( & 2) & IASUFFICIFNT TRACTII!N & 0 . & - & 0. & - & 14.7 & $N \cap-G)$ \\
\hline( & 3) & RUHGHNFSS (RIIIF) SPFEN LIMIT & 0 . & - & 5.4 & 32.3 & 53.9 & 9.2 \\
\hline( & 4) & SUII/SIURF RFSISTANIES & 79.6 & 28.2 & 64.6 & 23.9 & 27.2 & 11.1 \\
\hline 1 & 51 & VISIRILITY LIMIT & 0 . & - & 0 . & - & 1.3 & 19.4 \\
\hline( & 6) & RUAU CURVATHRF & 20.4 & 14.5 & $11 \cdot 0$ & 20.7 & $2 \cdot 9$ & 8.2 \\
\hline( & 7) & FXTERNAI (URRAN) SPIFU I INIT & 0 . & - & $19 \cdot 0$ & 12.6 & 0 & - \\
\hline \multicolumn{9}{|c|}{ Mid-East - Sand Condition } \\
\hline & 1) & INSUFFICIIFNT SOII STPINGTH & 0 & - & 0 . & - & 100.0 & $n \cap-60$ \\
\hline 1 & ?) & INSUFFICIIINT TRACTIUN & 0 . & - & 0 . & - & 0 . & - \\
\hline 1 & 3) & RQHGHNESS (RIUE) SPFF\| I TMIT & o. & - & 4.4 & 32.3 & 0 . & - \\
\hline ( & 1) & SOILISLUFF KFSISTANCFS & 79.5 & 28.2 & 61.4 & 23.7 & 0 . & - \\
\hline 1 & 5) & VISIRILITY IIMIT & $0 \cdot$ & - & 0 & - & 0 . & - \\
\hline 1 & 6) & RUAI COIRVATHRF & $20 \cdot 5$ & 13.9 & $15 \cdot 2$ & $17 \cdot 5$ & 0 . & - \\
\hline 1 & 7) & FXIEFNAI (URRAN) SDIFH LIMII & 0 . & - & $19 \cdot 0$ & 9.7 & 0 . & - \\
\hline
\end{tabular}


Table E47

Diagnostics Statistics for M35A2-M102

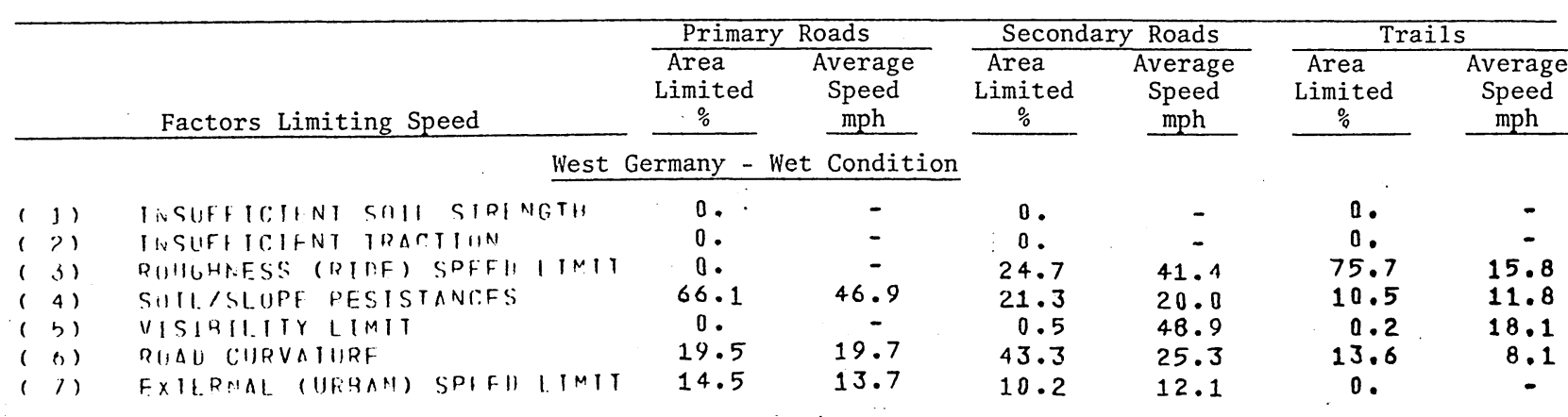

West Germany - Snow Condition

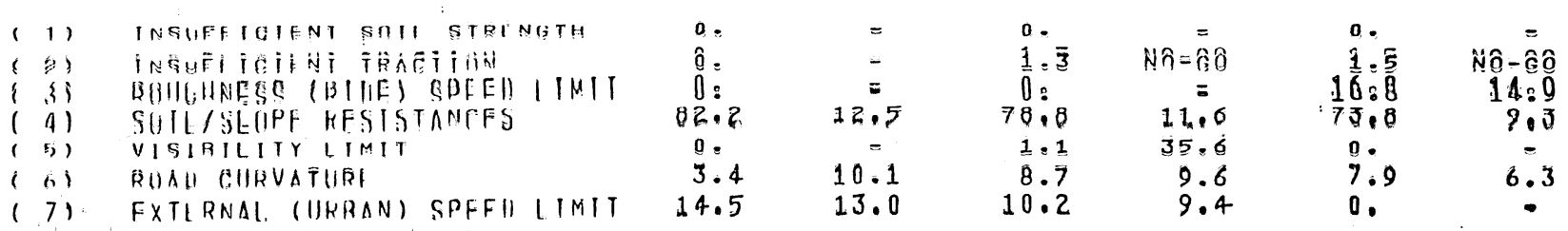

Mid-East - Wet Condition

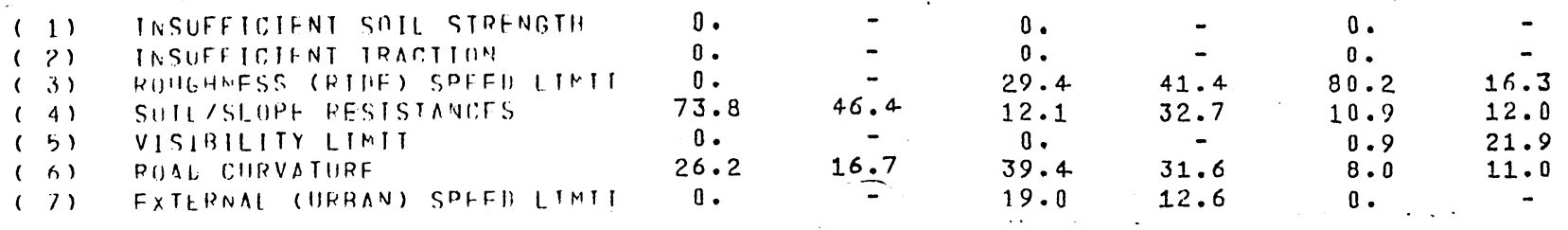

Mid-East - Sand Condition

\begin{tabular}{|c|c|c|c|c|c|c|c|c|c|}
\hline$(1)$ & INSUFFICIENT SOIL & STREN & GTH & D. & - & 0. & - & 0 . & - \\
\hline$(2)$ & IR.SUFI ICIHNT TRAC: & : $: 1111 \mathrm{~N}$ & & 0 . & - & 0 . & - & 49.3 & $n \cap-60$ \\
\hline$(3)$ & R(11)(;HNASSS (KINE) & SPFFN & LIMIT & 0 . & - & 0 . & - & 20.3 & 14.6 \\
\hline$(4)$ & SUIL/SIOUPF KFSIST & TANCFS & & 63.9 & 45.0 & 5.6 & 26.6 & 28.0 & 6.1 \\
\hline (5) & VISIHIIITY IJMIT & & & 9.9 & 57.1 & 0 . & - & 0.1 & 20.7 \\
\hline$(n)$ & RUAW CHIRVATI'RF & & & 26.2 & 15.9 & 75.4 & 29.7 & 2.4 & 8.7 \\
\hline$(1)$ & $F \times T T_{L} \Delta \wedge 1$ (1JPMAN) & SP+FU & IIMIT & 0 . & - & 19.0 & 9.7 & 0 . & - \\
\hline
\end{tabular}


Table E48

Diagnostics Statistics for M35A2-XM204

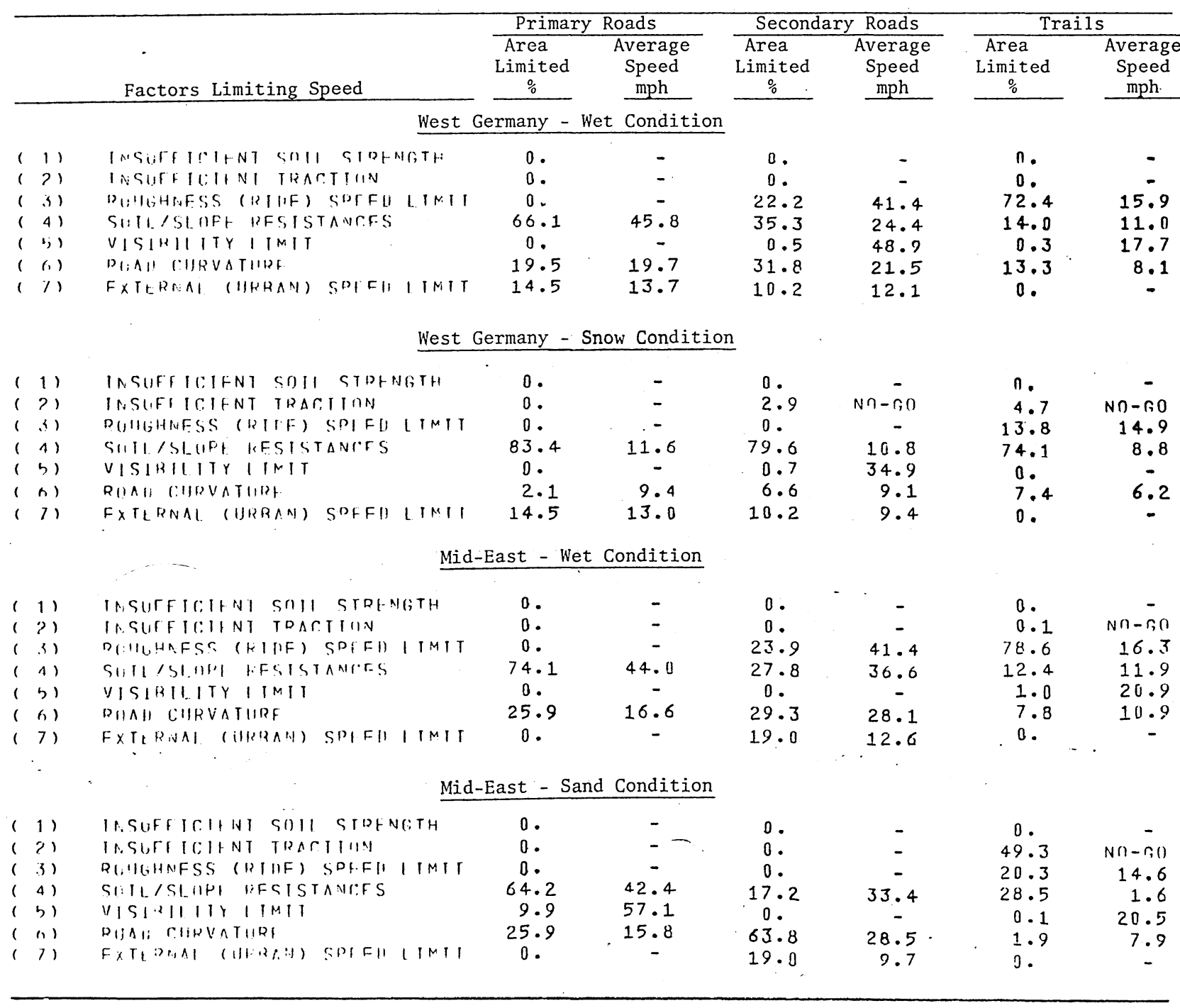


Table E49

Diagnostics Statistics for M35A2-XM198

\begin{tabular}{|c|c|c|c|c|c|c|c|}
\hline & & Primar & Roads & Secons & $y$ Roads & & \\
\hline & Factors Limiting Speed & $\begin{array}{c}\text { Area } \\
\text { Limited } \\
\% \\
\end{array}$ & $\begin{array}{c}\text { Average } \\
\text { Speed } \\
\text { mph }\end{array}$ & $\begin{array}{c}\text { Area } \\
\text { Limi.ted } \\
\% \\
\end{array}$ & $\begin{array}{c}\text { Average } \\
\text { Speed } \\
\text { mph }\end{array}$ & $\begin{array}{c}\text { Area } \\
\text { Limited } \\
\% \\
\end{array}$ & $\begin{array}{c}\text { Average } \\
\text { Speed } \\
\text { mph }\end{array}$ \\
\hline & West & ermany - & Condit & & & & \\
\hline ( 1 ) & 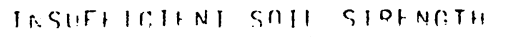 & 0. & - & 0. & - & 1.5 & $N \cap-G 0$ \\
\hline$(7)$ & INSUEFISIINI TRARTIUN & 0 . & - & 0 . & - & 2.9 & NOI-GO. \\
\hline ( i) & DIIULHNESS (RIFF) SDFFI LIMII & 0 . & - & 10.5 & 41.4 & 58.8 & 15.7 \\
\hline$(1)$ & SOI11/SIOHF RFSTSTANCFS & 68.8 & 33.0 & 54.0 & 18.8 & 24.6 & 10.4 \\
\hline$(5)$ & VISIRIIITY ILIMIT & 0 . & - & 0.5 & 48.9 & 0.8 & 18.3 \\
\hline$(n)$ & RUAB I:UYQVATIRH & $16 \cdot 8$ & $18 \cdot 4$ & 24.8 & 20.0 & 11.5 & 7.8 \\
\hline$(1)$ & FXIE HNAL (WREAU) SPFFH ITMIT & 14.5 & 13.7 & 10.2 & 12.1 & n. & - \\
\hline
\end{tabular}

West Germany - Snow Condition

\begin{tabular}{|c|c|}
\hline 1) & IASIIFFICIFNT SOII SIRFNOTH \\
\hline ?) & IR.FIFFICIFNI TRACTHIIN \\
\hline$(5)$ & DI:SGHNFSS (III:F) SPFFI I INII \\
\hline$\left(\begin{array}{l}1\end{array}\right)$ & SULISIOHY HFSISTANIFS \\
\hline$(b)$ & VISIRILITY LIMIT \\
\hline (1) & PUAL CURVATIIDE \\
\hline
\end{tabular}

\begin{tabular}{|c|c|c|c|}
\hline $\begin{array}{c}0 . \\
22.3\end{array}$ & $N \cap \overline{-}-$ & 0. & - \\
\hline 0. & - & $\begin{array}{c}39.4 \\
0 .\end{array}$ & - \\
\hline 53.2 & 7.9 & 49.3 & 7.7 \\
\hline 0. & - & 0. & - \\
\hline $\begin{array}{c}0 . \\
14.5\end{array}$ & 13.0 & 1.1 & 6.6 \\
\hline
\end{tabular}

$\begin{array}{cc}0 . & - \\ 54.8 & N \cap-T 0 \\ 0.0 & - \\ 43.6 & 6.2 \\ 0.0 & - \\ 1.6 & 5.1 \\ 0 . & -\end{array}$

Mid-East - Wet Condition

\begin{tabular}{|c|c|c|c|c|c|c|c|c|c|c|}
\hline 1 & 1) & INSLFFICIFNI SUII & SIRLA & NGTH & 0 . & - & 0 . & - & 0 . & - \\
\hline ( & ?) & INSUFFICIINT IRAS & $\because I I ! N$ & & 0 . & - & 0 . & - & 8.2 & $n n-6,0$ \\
\hline ( & (1) & 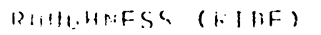 & SDFFII & LIMII & 0 . & - & 10.0 & 41.4 & 60.7 & 15.9 \\
\hline ( & 4) & SUlL/SI UPF PFSISI & IANIFS & & 77.0 & 33.7 & 56.1 & 27.9 & 23.4 & 11.1 \\
\hline ( & 5) & VISIRILITY I IMIT & & & 0 . & - & 0 . & - & 1.5 & 22.4 \\
\hline ( & 6) & RUA口U CUURVATIIRI & & & 23.0 & 15.5 & 14.9 & 22.4 & 6.2 & 10.3 \\
\hline( & 7) & $F \times I E R N A I \quad(11 R R A 4)$ & SPI.FR & IIMII & 0 . & - & $19 \cdot 2$ & 12.6 & 0 & - \\
\hline \multicolumn{11}{|c|}{ Mid-East - Sand Condition } \\
\hline( & 1) & In:SUrFIrItNI SOII & SIDts & NSTH & 0 . & - & 0 . & - & 98.7 & $n(1-B)$ \\
\hline 1 & ?) & II:SUFHICIINI IRAS & CTION & & 0 . & - & 0 . & - & 0.7 & $N \cap-\cap, 10$ \\
\hline 1 & 5) & R(II1,+N:FSS (FIHF) & SPIFI & I. IMIT & 0. & - & 0 . & - & 0.1 & 17.0 \\
\hline ( & 4) & SAIL/SLUPH DFSIST & TANSES & & 67.2 & 31.7 & 45.0 & 26.6 & 0.3 & 1.8 \\
\hline ( & ๖) & $V|S|: 2 \| I .1|Y| I M \mid I$ & & & 9.9 & 57.1 & 0 . & - & 0 . & - \\
\hline ( & 6) & DiAI: COHEVATHRF & & & $23 \cdot 0$ & 14.8 & 36.0 & 24.2 & 0 . & - \\
\hline 1 & 八) & $F \times I R D=\Delta 1 \quad(11+12 A \cdot 1)$ & $S D F ! 1$ & I I I I I & 0 . & - & $19 \cdot 0$ & 9.7 & 0 . & - \\
\hline
\end{tabular}


Table E50

Diagnostics Statistics for M813-XM204

\begin{tabular}{|c|c|c|c|c|c|c|c|c|}
\hline & & & Primary & Roads & Second & $y$ Roads & Tra & \\
\hline & & Factors Limiting Speed & $\begin{array}{c}\text { Area } \\
\text { Limited } \\
\% \\
\end{array}$ & $\begin{array}{c}\text { Average } \\
\text { Speed } \\
\text { mph } \\
\end{array}$ & $\begin{array}{c}\text { Area } \\
\text { Limited } \\
\% \\
\end{array}$ & $\begin{array}{c}\text { Average } \\
\text { Speed } \\
\text { mph } \\
\end{array}$ & $\begin{array}{c}\text { Area } \\
\text { Limited } \\
\% \\
\%\end{array}$ & $\begin{array}{c}\text { Average } \\
\text { Speed } \\
\text { mph } \\
\end{array}$ \\
\hline & & West G & ermany - $H$ & Conditi & & & & \\
\hline ( & 11 & INSLIFFISIFNI SOII SIRENGTH & 0 . & - & 0 . & - & 1.5 & NO-GO. \\
\hline ( & ク & HSSUFFICIFNT TRACTIIIN & 0 . & - & a. & - & 0. & - \\
\hline ( & 3) & RUILHAFSS (RIIEF) SPIFU LIMIT & 0. & - & 22.2 & 44.4 & 71.8 & 9.7 \\
\hline 1 & 4) & SUIL/SIIIPR HFSISTANIES & 66.2 & 41.1 & 45.0 & 25.0 & $15 \cdot 4$ & $11 \cdot 2$ \\
\hline ( & 5) & VISIHILITY IINTI & 0 . & - & 0.4 & 48.7 & 0.3 & 25.0 \\
\hline 1 & (1) & ROAU COURVATIjRA & 19.3 & 19.6 & 22.2 & 17.4 & 11.0 & 8.6 \\
\hline (. & $7)$ & FXTKRNAL (URRAN) SPEFD ITMIT & 14.5 & 13.7 & 10.2 & 12.1 & D. & - \\
\hline & & West G & rmany - S & ow Condit & & & & \\
\hline 1 & 11 & IH SUFHIBIFNI SAII SIRENGTH & 0. & - & 0 & - & 0. & - \\
\hline 1 & 21 & II SUTH IC:IFNT TRACTIWN & 0 . & - & 0.4 & $\left.n \cap-R_{0}\right)$ & 0.5 & NO-GO \\
\hline 1 & 3 & DI:IGINNFSS (RIIIE) SPFFII LIMIT & $0 \cdot$ & - & 0 & - & 31.0 & 6.0 \\
\hline ( & 4) & SUII/SIIUPF RFSISTANR:FS & 83.3 & 10.9 & 81.8 & 10.3 & 63.1 & 8.4 \\
\hline 1 & 5) & VISIRILITY LIMIT & 0. & - & 1.3 & 35.3 & 0.1 & 18.8 \\
\hline 1 & 6) & RUAD CURVATURF. & 2.2 & 9.7 & 6.4 & 9.4 & 5.3 & 6.6 \\
\hline 1 & 1 & $F \times I E R A \wedge I$ (IIHAAN) SPFFH IINIT & $14 \cdot 5$ & $13 \cdot 8$ & $10 \cdot 2$ & 9.4 & $n \cdot$ & - \\
\hline & & Mid-1 & East - Wet & Eondition & & & & \\
\hline( & 1) & INSUFFICIENT SOII STRENGTH & 0. & - & 0 . & - & 0 . & - \\
\hline( & () & INSUFFICIENI TRACTION & 0 . & - & 0 . & - & 0 . & - \\
\hline( & 3) & RI!H,HNFSS (RIDF) SPFFH ITMTT & 0 . & - & 23.9 & 44.4 & 75.9 & 11.2 \\
\hline 1 & 4) & SUIL/SL_UPF RFSISTANIFS & 74.2 & 41.3 & 34.0 & 38.0 & 14.7 & 11.4 \\
\hline 1 & 5) & VISIRILITY I IMIT & 0. & - & 0 . & - & 1.5 & 26.5 \\
\hline( & 6) & ROIAU CIIRVATURF & 25.8 & 16.6 & $23 \cdot 1$ & $25 \cdot 2$ & 7.9 & 12.4 \\
\hline 1 & $7)$ & FXTERNAL (IJRRAN) SPFFI) LIMIT & 0 . & - & 19.0 & 12.6 & 0 . & - \\
\hline & & Mid- & ast - San & Conditior & & & & \\
\hline ( & 1) & INSUFFICIFNT STII STRENGTH & 0 . & - & 0 . & - & 0. & $-\cdots$ \\
\hline( & 2) & INSUFF ICIFNT TRACTIIIN & 0 . & $-\ldots$ & 0 . & - & 49.3 & $N \cap-r, n$ \\
\hline( & 3) & PUIIGHNESS (RIDE) SPIFII LIMIT & 0 . & - & 0 . & - & 18.7 & $7 \cdot 3$ \\
\hline( & 1) & SUIL/SLOPF RESISTANCFS & 74.2 & 41.3 & $17 \cdot 2$ & 32.5 & 30.3 & $10 \cdot 5$ \\
\hline( & 5) & VISIBILITY IIMII & 0 . & - & 0 . & - & 0.1 & $21 \cdot 4$ \\
\hline 1 & (6) & DIIAL CIIIVVATURF & 25.8 & 15.8 & 63.8 & 28.5 & 1.7 & $8 \cdot 3$ \\
\hline i & 1) & FXTHPANI (IIHRAN) SPFEF I.IMII & 0 & - & $19 \cdot 0$ & 9.7 & 0 . & - \\
\hline
\end{tabular}


Table E51

Diagnostics Statistics for M813-M114A1

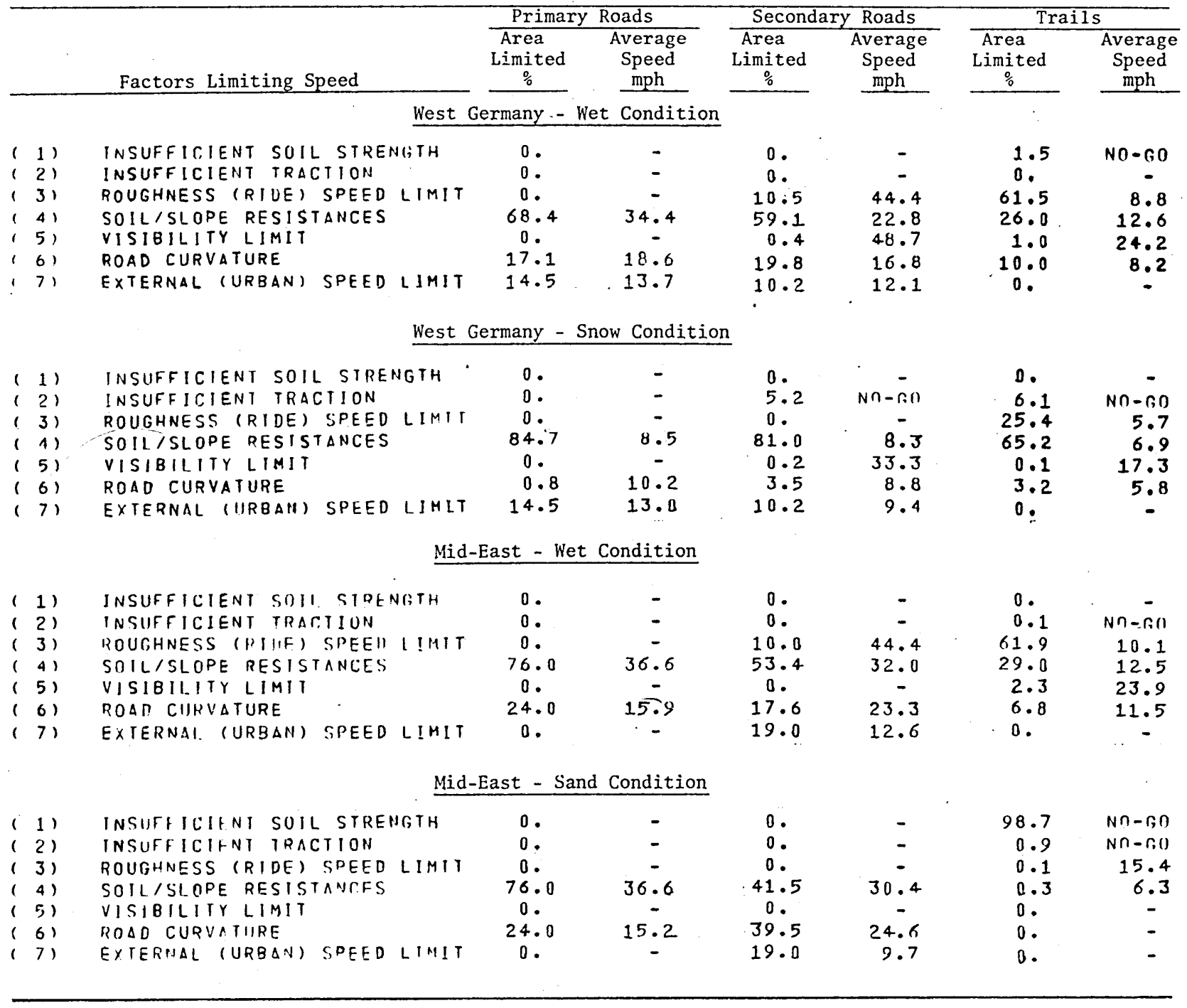


Table E52

Diagnostics Statistics for M813-XM198

\begin{tabular}{|c|c|c|c|c|c|c|c|c|}
\hline & & & Primar & Roads & Second & y Roads & & \\
\hline & & Factors Limiting Speed & $\begin{array}{c}\text { Area } \\
\text { Limited } \\
\% \\
\end{array}$ & $\begin{array}{c}\text { Average } \\
\text { Speed } \\
\text { mph }\end{array}$ & $\begin{array}{c}\text { Area } \\
\text { Limited } \\
\% \\
\end{array}$ & $\begin{array}{c}\text { Average } \\
\text { Speed } \\
\text { mph }\end{array}$ & $\begin{array}{c}\text { Area } \\
\text { Limited } \\
\% \\
\end{array}$ & $\begin{array}{c}\text { Average } \\
\text { Speed } \\
\text { mph } \\
\end{array}$ \\
\hline & & West G & ermany - & t Conditi & & & & \\
\hline 1 & 11 & INSUTFICIINI SOII SIRFHBTH & 0 . & - & 0 . & - & 1.5 & $N \cap-G O$ \\
\hline 1 & 2) & IN.SUFFICIHNI IRACITIUN & 0. & - & 0 . & - & 0. & - \\
\hline( & s) & RUILHNESS (KIIF) SPFFO LIMIT & 0 . & - & 10.5 & 44.4 & 59.6 & 8.7 \\
\hline 1 & 4) & SUIL/SLOPL HFCISTANIFS & 68.7 & 32.7 & 59.4 & 22.0 & 28.2 & 12.2 \\
\hline ( & 5) & VISIRII.ITY LIMII & 0 . & - & 0.4 & 48.7 & 1.2 & 21.9 \\
\hline 1 & (6) & RUAU COUPVATURF & 16.9 & 18.5 & 19.5 & 16.8 & 9.5 & 8.0 \\
\hline 1 & 7) & FXTERAAI (HHBAN) SPFEU ITMTT & $14 \cdot 5$ & 13.7 & 10.2 & 12.1 & 0 . & - \\
\hline & & West G & ermany - & low Condit & & & & \\
\hline ( & 11 & INSUFF ICIENT SOII SIRENGTH & 0 . & - & 0 . & - & 0. & - \\
\hline ( & ?) & INSUFFICIENI TRARTIIIN & 0 . & - & 6.1 & $N \cap-B O$ & 6.5 & NO-GO \\
\hline 1 & 3) & ROUGHNFSS (RINF) SPFFI IIMII & 0 . & - & B. & - & 22.9 & 5.4 \\
\hline ( & 41 & SUII /SLAPF RFSISTANCFS & 85.1 & 6.9 & $81 \cdot 5$ & 0 . & 68.5 & Q. \\
\hline( & b) & VISIRILITY LIMIT & o. & - & 0 . & - & 0.1 & 16.6 \\
\hline ( & 6) & RUAII GURVATURF & 0.4 & 9.0 & 2.2 & 9.5 & 1.9 & 6.2 \\
\hline ( & 71 & FXIERNAL (WRRAN) SPEFH LIMIT & 14.5 & 13.0 & $10 \cdot 2$ & 9.4 & 0. & - \\
\hline & & $\underline{\text { Mid }}$ & ast $-W$ & Conditic & & & & \\
\hline 1 & 11 & INSUFFICIENT SOII STPENGTH & 0 . & - & 0 . & - & 0 . & - \\
\hline 1 & 2) & INSUFFICIENT TRACIIIIN & 0 . & - & 0 . & - & 0.1 & $n \cap-R n$ \\
\hline( & 31 & P(IIlGHNFSS (HINF) SPIFH LIMII & 0 . & - & $10 \cdot 0$ & 44.4 & 59.9 & 10.0 \\
\hline( & 4) & SUIL/SLUPE KFSISTANCFS & 76.7 & 35.2 & 53.4 & 30.6 & 31.3 & 10.2 \\
\hline 1 & b) & VISIRIIIIYYIIMII & 0 . & - & D. & - & 2.4 & 22.5 \\
\hline 1 & (6)) & RUAII C.IIRVATURF & 23.3 & 15.6 & 17.6 & 23.3 & 6.4 & 11.3 \\
\hline 1 & 7) & $F \times I \mid D H A L$ (IIKBAN) SPIF\| IIMIT & 0 . & - & 19.0 & 12.6 & 0 . & - \\
\hline & & $\underline{\mathrm{Mi}}$ & East - & Conditic & & & & \\
\hline 1 & 1) & INSUFF ICIHNT SOII STPENGTH & 0 . & - & 0 . & - & .98 .7 & $N \cap-S O$ \\
\hline( & 2) & INSUFFICIFNI TRACITIIN & 0 . & - & 0 . & - & 0.9 & $N \cap-G O$ \\
\hline 1 & 3) & PUIHUHNFSS (KINF) SPFFI ITMII & 0 . & - & 0 . & - & 0.1 & 11.8 \\
\hline 1 & 4) & SUIL /SIOOPF HESISTANCFS & 76.7 & 35.1 & 41.5 & 29.2 & 0.3 & 5.7 \\
\hline 1 & 51 & VISIBII.ITY IIMIT & B. & - & o. & - & 0 . & - \\
\hline 1 & (f) & PUAB CIHKVATHRF & 23.3 & 14.9 & 39.5 & 24.6 & 0 . & - \\
\hline ( & 71 & $F \times T_{E} P P_{N} A L$ (IIH'HAN) SOFFH IIMIT & 0. & - & 19.9 & 9.7 & 0 . & - \\
\hline
\end{tabular}


Table E53

Diagnostics Statistics for M813-FH70

\begin{tabular}{|c|c|c|c|c|c|c|c|c|}
\hline & \multirow{2}{*}{\multicolumn{2}{|c|}{ Factors Limiting Speed }} & \multirow{2}{*}{\multicolumn{2}{|c|}{$\begin{array}{cc}\text { Primary } & \text { Roads } \\
\text { Area } & \text { Average } \\
\text { Limited } & \text { Speed } \\
\% & \text { mph } \\
\end{array}$}} & \multicolumn{2}{|c|}{ Secondary Roads } & \multicolumn{2}{|c|}{ Trails } \\
\hline & & & & & $\begin{array}{c}\text { Area } \\
\text { Limited } \\
\% \\
\end{array}$ & $\begin{array}{c}\text { Average } \\
\text { Speed } \\
\text { mph } \\
\end{array}$ & $\begin{array}{c}\text { Area } \\
\text { Limited } \\
\% \\
\end{array}$ & $\begin{array}{c}\text { Average } \\
\text { Speed } \\
\text { mph }\end{array}$ \\
\hline \multicolumn{9}{|c|}{ West Germany - Wet Condition } \\
\hline 1 & 1) & INSUFHISIFNT SOII SIDFNITH & 0. & - & 0 . & - & 96.2 & NO- $R O$ \\
\hline 1 & 2) & INSIFFICIFNT TIDC.IIIIN & 0 . & - & 0 . & - & 0 . & - \\
\hline 1 & .5) & RUUI,HNFSS (HIIF) SPHFH IINII & 0 . & - & $8 \cdot 0$ & 44.4 & 2.6 & 9.1 \\
\hline 1 & 4) & SUIL/SLIIPI WFSISTANCFS & 69.1 & 30.5 & 63.2 & 20.6 & 1.0 & 10.8 \\
\hline 1 & (1) & VISIHILITY IIN:IT & 0 . & - & 0.4 & 48.7 & 0. & 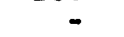 \\
\hline 1 & 6) & RA,AL rHIRVATHRT. & 16.4 & 18.4 & 18.2 & 16.3 & 0.3 & 5.7 \\
\hline ( & 71 & FXTHRNAI (INBAN) SPIFH IIMII & 14.5 & 13.7 & $10 \cdot 2$ & 12.1 & 0. & - \\
\hline \multicolumn{9}{|c|}{ West Germany - Snow Condition } \\
\hline 1 & 1) & IASUFI ICIINT SHIL SIPENGTH & 0 . & - & 0 . & - & o. & - \\
\hline 1 & ?) & INSUFH IPIFNT TRACIIIN & 0 - & - & 18.1 & $N n-\infty O$ & 25.9 & NO-GO \\
\hline( & $\because$ & 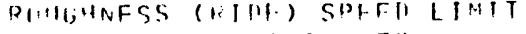 & 0. & - & 0. & - & 15.5 & 5.0 \\
\hline 1 & 4) & SUII SLLUPL KFSISTANCFS & 85.2 & 6.7 & 70.9 & 6.8 & 57.4 & 5.8 \\
\hline 1 & \$) & VISIRIIIIIY IIMII & 0 . & - & 0 . & - & 0 . & - \\
\hline ( & h) & RUAU liHRVATHRH. & 0.3 & 8.5 & 0.8 & 7.7 & 1.2 & $5.8^{\circ}$ \\
\hline 1 & 71. & FXIIDNAL (IJWRAN) SPIFH LIMII & 14.5 & $13 \cdot 0$ & $10 \cdot 2$ & 9.4 & 0 & - \\
\hline \multicolumn{9}{|c|}{ Mid-East - Wet Condition } \\
\hline ( & 11 & INSUFTICI+VT SOHI SIDFVRTH & 0 . & - & 0 . & - & 98.7 & $N n-n, 0$ \\
\hline 1 & 2) & INSUFFICIFNT TRACITIIN & 0 . & - & 0 . & - & 0 . & - \\
\hline 1 & s) & DUII(-WHFSS (IIUF) SDHFH IIMIT & 0 . & - & 4.4 & 44.4 & 0.7 & 17.5 \\
\hline ( & 1) & SUIL SULER DESISTANCFS & 77.0 & 32.4 . & 61.7 & 28.2 & 0.5 & 7.4 \\
\hline 1 & म) & VISIHILIIY IIMII & 0 & - & 0 . & - & 0 . & - \\
\hline 1 & 6) & 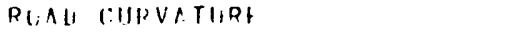 & 23.0 & 15.5 & 14.8 & 22.3 & 0.1 & 9.4 \\
\hline 1 & 7) & FXIFPN:AL (UKGAN) SPEFU.IIMIT & $0 \cdot$ & -2 & 19.0 & 12.6 & 0 & - \\
\hline \multicolumn{9}{|c|}{ Mid-East - Sand Condition } \\
\hline 1 & 11 & INSUFIICIINT SOII SIRENGTH & 0 . & - & 0 . & - & 100.0 & $h n-60$ \\
\hline ( & ?) & IN.SUFFICIHNI IRACIIIINI & $0 .$. & - & 0 . & - & 0 . & - \\
\hline( & 31 & PUIG,HNESS (KIDF) SPFFH I.IMIT & 0 . & - & 0 . & - & D. & - \\
\hline ( & 4) & SWIL/SIUHE HFSISTANR:FS & 77.0 & 32.4 & 45.0 & 26.0 & 0 . & - \\
\hline r & ら) & VISIMILITY IIMII & 0 . & - & 0 . & - & 0 . & - \\
\hline 1 & 6) & $D_{11} \wedge 1 \cdot\{: 11 D \vee A T 1,12 F$ & $23 \cdot 0$ & 14.8 & 36.0 & $24 \cdot 2$ & 0 . & - \\
\hline 1 & 7) & 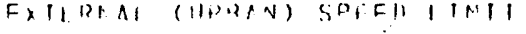 & 0 . & - & 19.0 & 9.7 & 0 . & - \\
\hline
\end{tabular}


Table E54

Diagnostics Statistics for M813-XM(130 mm)

\begin{tabular}{|c|c|c|c|c|c|c|c|c|}
\hline \multirow{2}{*}{\multicolumn{3}{|c|}{ Factors Limiting Speed }} & \multirow{2}{*}{\multicolumn{2}{|c|}{$\begin{array}{cc}\text { Primary } & \text { Roads } \\
\text { Area } & \text { Average } \\
\text { Limited } & \text { Speed } \\
\% & \text { mph } \\
\end{array}$}} & \multicolumn{2}{|c|}{ Secondary Roads } & \multicolumn{2}{|c|}{ Trails } \\
\hline & & & & & $\begin{array}{c}\text { Area } \\
\text { Limited } \\
\% \\
\end{array}$ & $\begin{array}{c}\text { Average } \\
\text { Speed } \\
\text { mph }\end{array}$ & $\begin{array}{c}\text { Area } \\
\text { Limited } \\
\% \\
\end{array}$ & $\begin{array}{c}\text { Average } \\
\text { Speed } \\
\text { mph } \\
\end{array}$ \\
\hline \multicolumn{9}{|c|}{ West Germany - Wet Condition } \\
\hline ( & 11 & WHSUFFICHINT SOHI STRFNGTH & 0 . & - & 0 . & - & 1.5 & NO-GO \\
\hline ( & ?) & INSUFIICIINT IRACIIION & 0 . & - & 0 . & - & 0 . & - \\
\hline ( & .1) & PIIIGHAFSS (IINF) SPFFO LIMII & 0. & - & 10.5 & 44. 4: & 59.2 & 8.6 \\
\hline ( & 4) & SHIL/SL.HPF RESISTANCFS & 68.7 & 31.5 & 59.4 & 21.4 & 28.7 & 11.7 \\
\hline 1 & 5) & VISIRIILITYIIMIT & 0 . & - & 0.4 & 48.7 & 1.1 & 23.0 \\
\hline ( & 6) & DUAL, PHHVATURF & $16 \cdot 8$ & 18.6 & 19.5 & 16.8 & 9.5 & 8.0 \\
\hline ( & 71 & FXTIRMAI (URRAN) SPFFH IIMIT & 14.5 & 13.7 & 10.2 & 12.1 & B. & - \\
\hline \multicolumn{9}{|c|}{ West Germany - Snow Condition } \\
\hline ( & 1) & INSUFFICIHNI SIII STRINGTH & 0 . & - & 0 . & - & n. & - \\
\hline i & ?) & INSILFITCIRNI TRACTIUN & 0 . & - & 6.5 & $N \cap-C, 1$ & 7.0 & $N \cap-G O$ \\
\hline 1 & .3) & 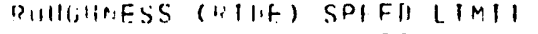 & 0 . & - & 0 . & - & 20.4 & 5.4 \\
\hline( & 4) & SHII/SLUFE KESISTANEFS & 85.2 & 6.8 & $81 \cdot 4$ & 6.6 & 70.7 & 5.5 \\
\hline c & 5) & VISIIILITY I.IMIT & 0 & - & $0 \cdot$ & - & 0.1 & 16.3 \\
\hline ( & (o) & RUAU CIIRVATURF & 0.3 & 8.5 & 1.9 & 9.0 & 1.8 & 6.0 \\
\hline 1 & 71 & FXITRNAL (IBHHAN) SPFFU L.IMIT & $14 \cdot 5$ & 13.0 & $10 \cdot 2$ & 9.4 & 0 . & - \\
\hline \multicolumn{9}{|c|}{ Mid-East - Wet Condition } \\
\hline ( & 1) & INSUFFICIFNI SOII STRENGTH & 0 . & - & 0 . & - & 0 . & - \\
\hline ( & 2) & IN.SIJFHICIENT IRACTIIIN & 0 . & - & 0 . & - & 0.1 & NO- $R_{0}$ \\
\hline ( & 31 & RUIHGiNNFSS (KIDF) SPFFII I IMII & 0 . & - & $10 \cdot 0$ & 44.4 & 58.4 & 9.8 \\
\hline 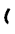 & 4) & SUILSLLOPI RFSISITANCFS & 76.7 & 34.1 & 53.4 & 29.5 & 33.0 & 9.7 \\
\hline( & 5) & VISIPILITY LIMIT & 0 & - & 0. & - & 2.3 & 22.9 \\
\hline 1 & 6) & RUAU COURVATIIRF & $23 \cdot 3$ & 15.6 & 17.6 & 23.3 & 6.2 & 11.2 \\
\hline ( & 71 & FXIFRNAL (UPAAN) SPFFB IIMIT & 0 & - & $19 \cdot 0$ & 12.6 & 0 . & - \\
\hline \multicolumn{9}{|c|}{ Mid-East - Sand Condition } \\
\hline( & 11 & II. SUFFICIFNT SOII STRFNGTH & 0 . & $-\ldots$ & 0 . & - & 98.7 & $N \cap-n \cap$ \\
\hline ( & 2) & INSUFIICIIFNT IRAC:TION & 0 . & - & 0 . & - & 0.9 & $N \cap-60$ \\
\hline ( & 3) & DUIIGUNFSS (HIDE) SPFFU IIMIT & B. & - & 0 . & - & 0.1 & 11.8 . \\
\hline ( & 4) & SUIL/SLUPH PFSISTANCFS & 76.7 & 34.1 & 41.6 & 28.0 & 0.3 & 8.9 \\
\hline & () & VISIMIIITY IIMIT & 0 . & - & 0 . & - & 0 . & - \\
\hline & 61 & SUAH CIIPVAFHDI & $23 \cdot 3$ & 14.9 & 39.4 & 24.6 & 0 . & - \\
\hline & 71 & $F \times T E R P A L \quad(U P A A N)$ SPFFH IIMII & 0 . & - & 19.0 & 9.7 & 0 . & - \\
\hline
\end{tabular}


Table E55

Diagnostics Statistics for M656-XM204

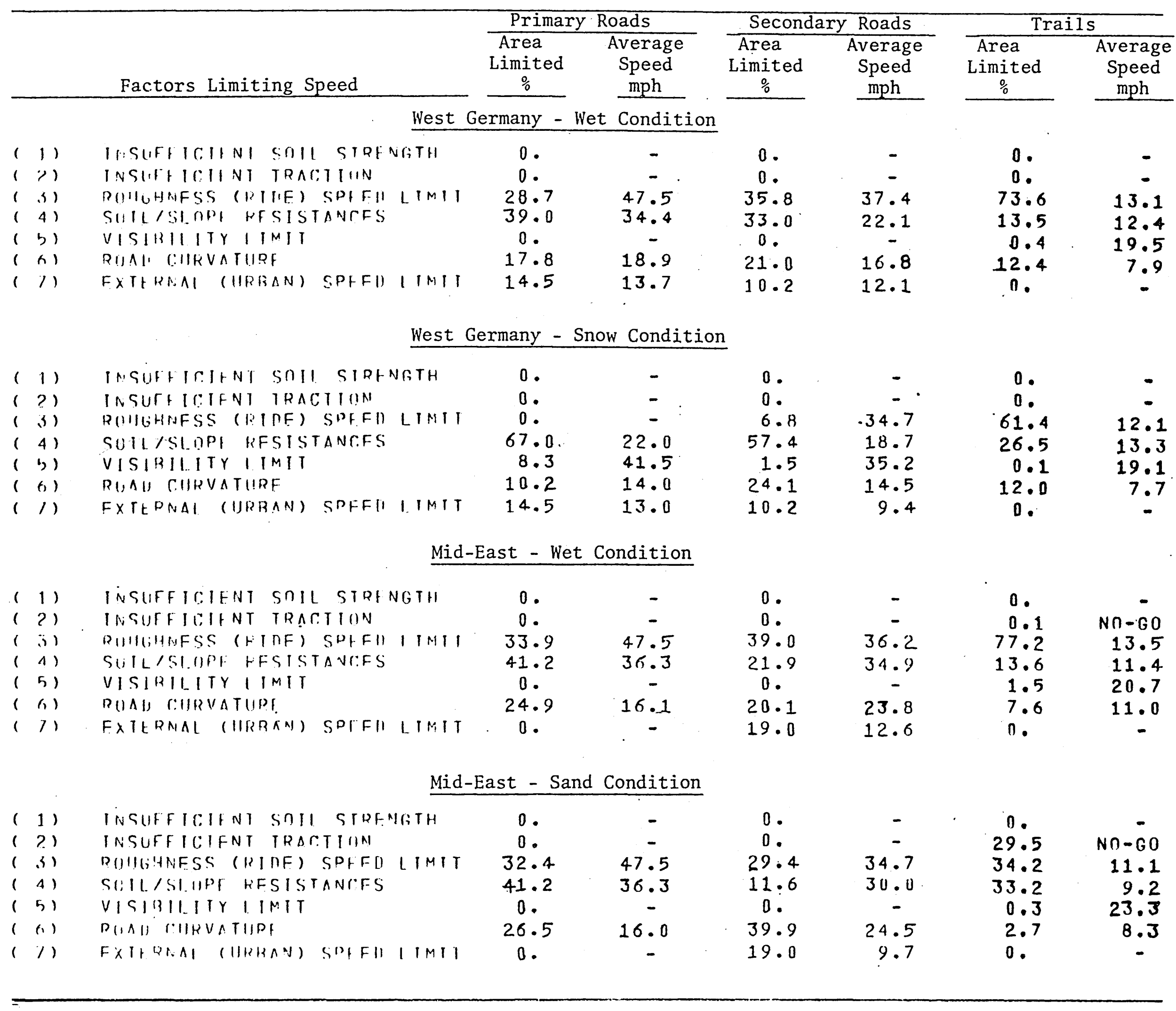


Table E56

Diagnostics Statistics for M656-XM198

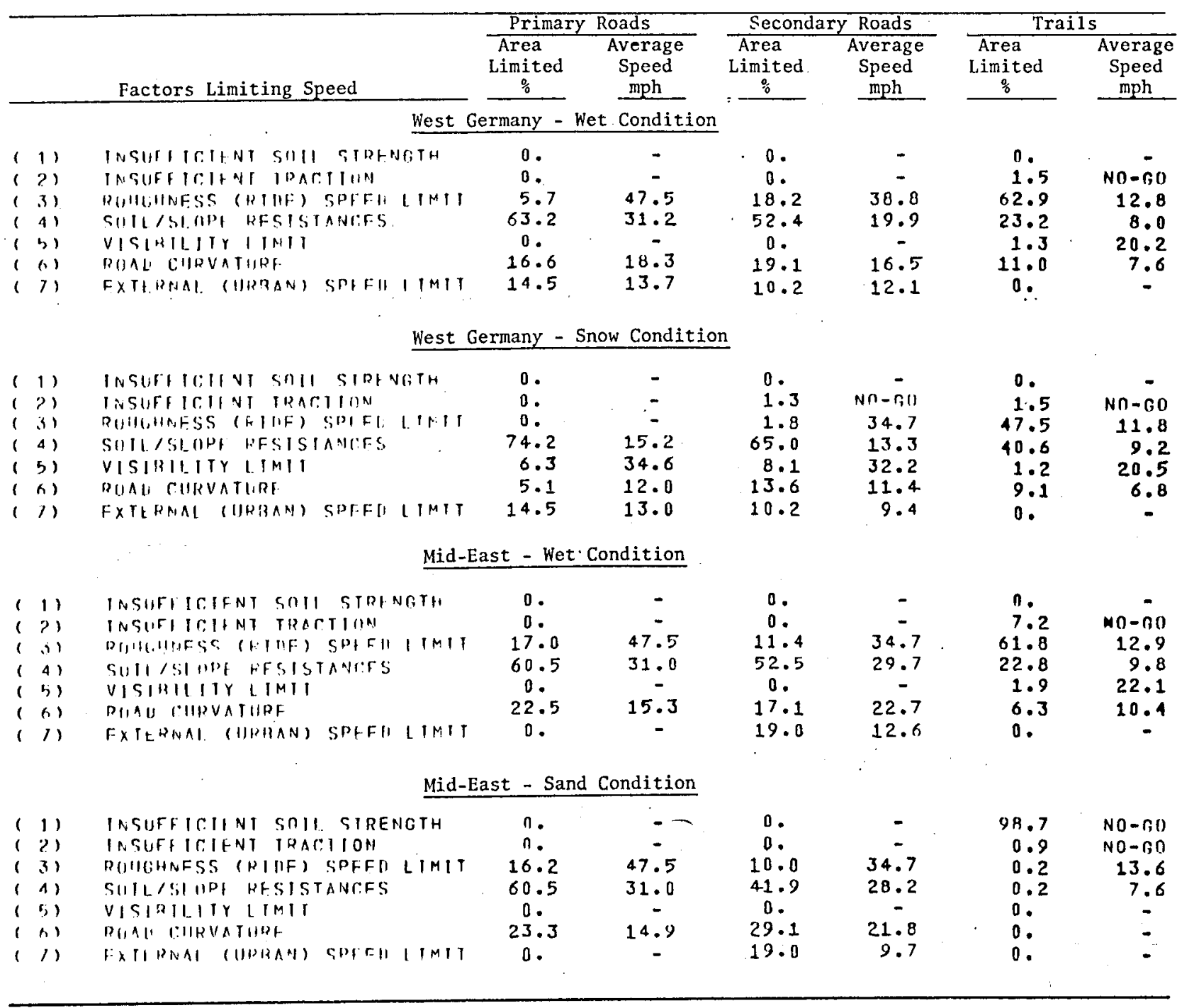


Table E57

Diagnostics Statistics for TDW901-XM204

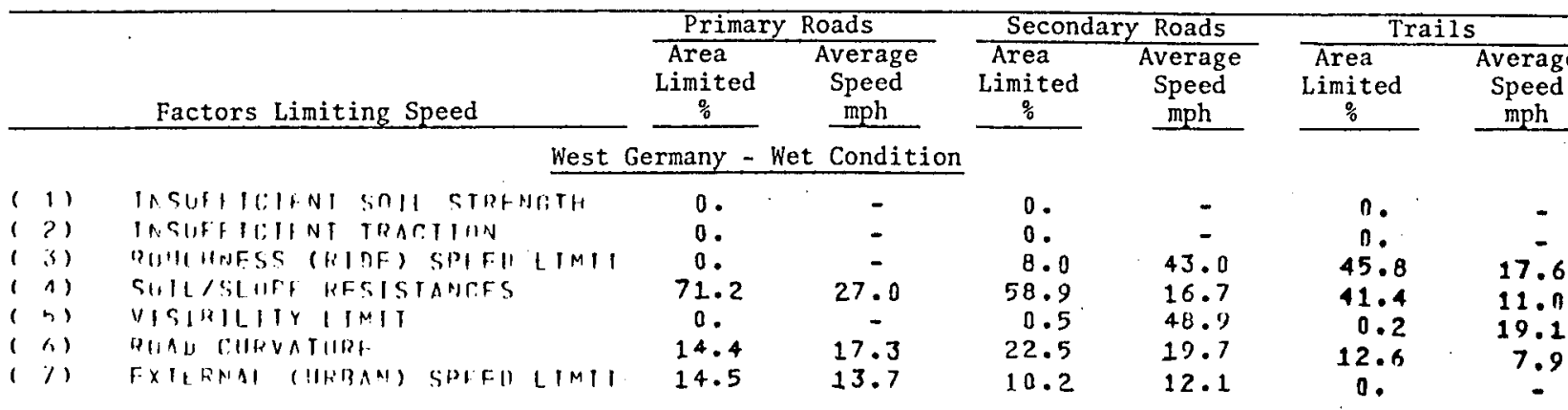

West Germany - Snow Condition

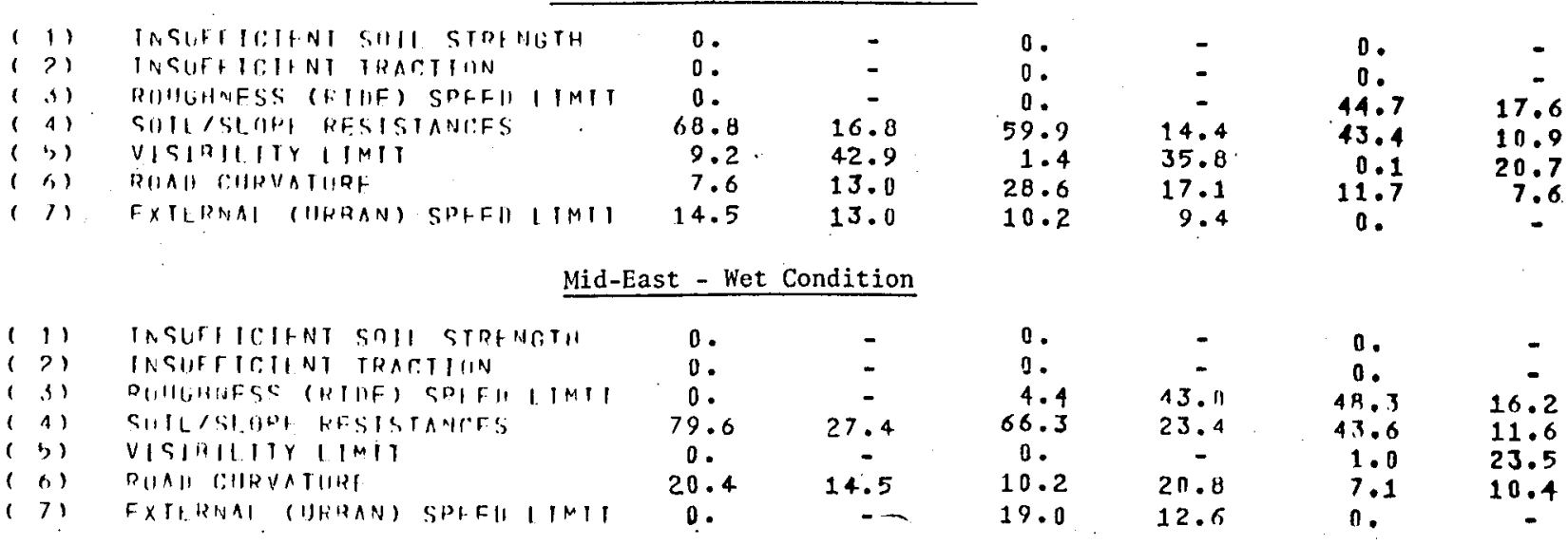

Mid-East - Sand Condition

\begin{tabular}{|c|c|c|c|c|c|c|c|c|c|}
\hline$(1)$ & INSUFFILIFNI SHII. & 1. Sinfa & Neith & D. & - & 0 . & - & 0 . & - \\
\hline$(2)$ & INSUFFICIENT TRAR. & CIIIN & & 0 . & - & 0 . & - & 15.5 & $N \cap-G 0$ \\
\hline 31 & ROHGHNESS (FINE) & SPFFll & LIMII & 0. & - & 0 . & - & 16.0 & 19.3 \\
\hline$(4)$ & SUII/SIOPE RFSIST & TANIFS & & 79.5 & 27.4 & 59.0 & 23.1 & 65.1 & 7.5 \\
\hline 151 & $V|S| B|| I T Y$ IIMAI & & & D. & - & 0. & - & $0 . \overline{3}$ & 24.6 \\
\hline$(t)$ & PIIA\}, COUDVATURE & & & 20.5 & 13.9 & 22.0 & 20.3 & 3.1 & 8.5 \\
\hline 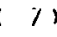 & FXITKNAI (WPNAAS) & SPF. FI & $11 N 11$ & 0. & - & 19.0 & 9.7 & 0 . & - \\
\hline
\end{tabular}


Table E58

Diagnostics Statistics for TDW901-M114A1

\begin{tabular}{|c|c|c|c|c|c|c|c|}
\hline & & Primar & Roads & Second & Roads & & \\
\hline & Factors Limiting Speed & $\begin{array}{c}\text { Area } \\
\text { Limited } \\
\vdots \\
\end{array}$ & $\begin{array}{l}\text { Average } \\
\text { Speed } \\
\text { mph }\end{array}$ & $\begin{array}{c}\text { Area } \\
\text { Limited } \\
:\end{array}$ & $\begin{array}{c}\text { Average } \\
\text { Speed } \\
\text { mph }\end{array}$ & $\begin{array}{c}\text { Area } \\
\text { Limited } \\
\% \\
\end{array}$ & $\begin{array}{c}\text { Average } \\
\text { Speed } \\
\text { mph }\end{array}$ \\
\hline & West & ermany - & $t$ Condit & & & & \\
\hline 111 & $1,51.511: 11018$ : & 0 . & - & 0. & - & $n$. & - \\
\hline 191 & 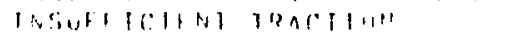 & 0 . & - & 0 . & - & i. & - \\
\hline.$(4)$ & 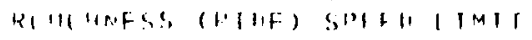 & 0 . & - & 8.0 & 43.0 & 42.2 & 17.6 \\
\hline$(4)$ & 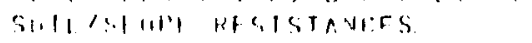 & 75.2 & 22.3 & 63.9 & 14.0 & 45.5 & 9.1 \\
\hline$(3)$ & VIsIG 11 ir limi & 0 & - & 0.5 & 48.9 & 0.8 & 21.7 \\
\hline$(n)$ & DUAD I:HKVAIIIBP & 10.4 & 15.6 & 18.4 & 19.3 & 11.5 & 7.6 \\
\hline 1,1 & 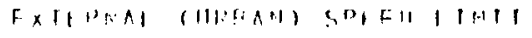 & 14.5 & 13.7 & 10.2 & 12.1 & 0. & 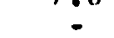 \\
\hline
\end{tabular}

West Germany - Snow Condition

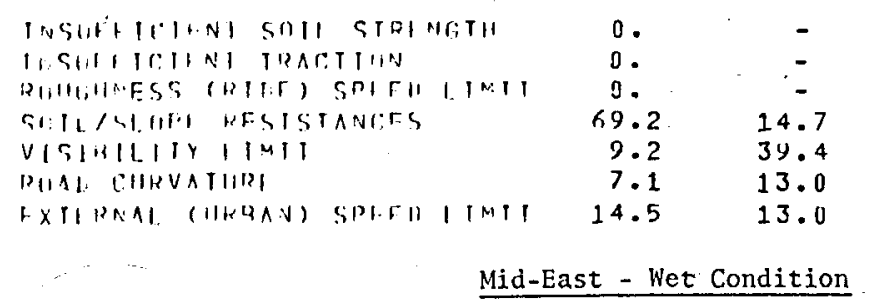

-
-
14.7
13.4
13.0
13.0

Mid-East - Wet Condition

\section{0.}

0.

62.7

9.2

18.0

10.2

-
-
12.4
36.6
13.9
9.4

$\begin{array}{rr}n . & - \\ 42.7 & 17.1 \\ 45.2 & 9.1 \\ 0.8 & 22.7 \\ 11.3 & 7.5 \\ n . & -\end{array}$

\begin{tabular}{|c|c|}
\hline 11 & 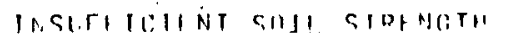 \\
\hline 7 & II. SUIFFICIINI TOARIIIIN \\
\hline$\$ 1$ & DHIL,HESS (FIIF) BDIFII INII \\
\hline 4) & 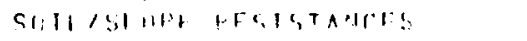 \\
\hline b) & VISIMUIITY IIMHI \\
\hline (i) & $R\|A\| B: H W \cup A T \| R P$ \\
\hline 71 & FXTERAAL (URBAN) SDFFH IIMII \\
\hline
\end{tabular}

$\begin{array}{cc}0 . & - \\ 0.0 & - \\ 0.0 & 23.0 \\ 02.8 & - \\ 0.0 & 13.5 \\ 17.2 & -\end{array}$

0 .

Mid-East - Sand Condition

\begin{tabular}{|c|c|}
\hline$(1)$ & 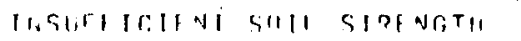 \\
\hline ;) & 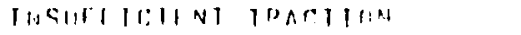 \\
\hline औ & DUHLUWFSS (BIMF) SPHFH IIMII \\
\hline 4) & 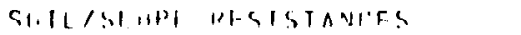 \\
\hline (1) & VISI:RILIIY IIMII \\
\hline (1) & 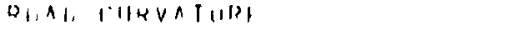 \\
\hline 11 & 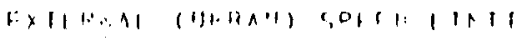 \\
\hline
\end{tabular}

$\begin{array}{cc}0 . & -7 \\ 0.0 & - \\ 0.0 & - \\ 82.1 & 23.0 \\ 0.0 & - \\ 17.6 & 13.0 \\ 0 . & -\end{array}$

0.
0.
0.9
$65 \cdot 9$
0.9
$15 \cdot 1$
$19 \cdot 0$

-
-
19.0
19.4
4.7

$\begin{array}{rr}0 . & \\ 0.1 & N n-50 \\ 41.7 & 15.9 \\ 50.9 & 9.9 \\ 1.6 & 21.5 \\ 5.8 & 9.7 \\ 0 . & -\end{array}$

$\begin{array}{rr}0 . & - \\ 20.3 & N O-G U \\ 13.6 & 19.3 \\ 63.2 & 5.8 \\ 0.4 & 24.3 \\ 2.4 & 8.4 \\ 0 . & \end{array}$


Table E59

Diagnostics Statistics for TDW901-XM198

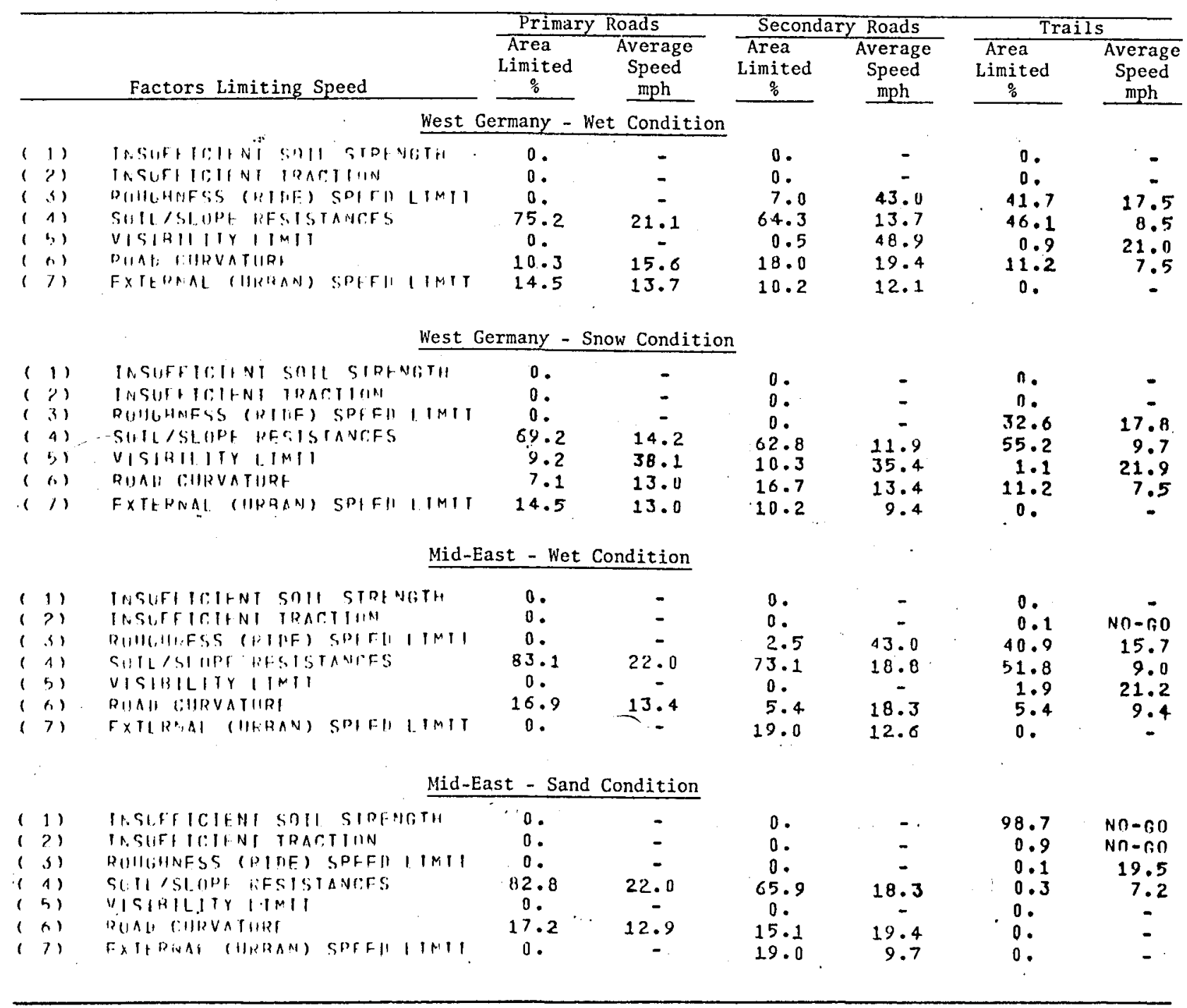


Table E60

Diagnostics Statistics for TDW901-FH70

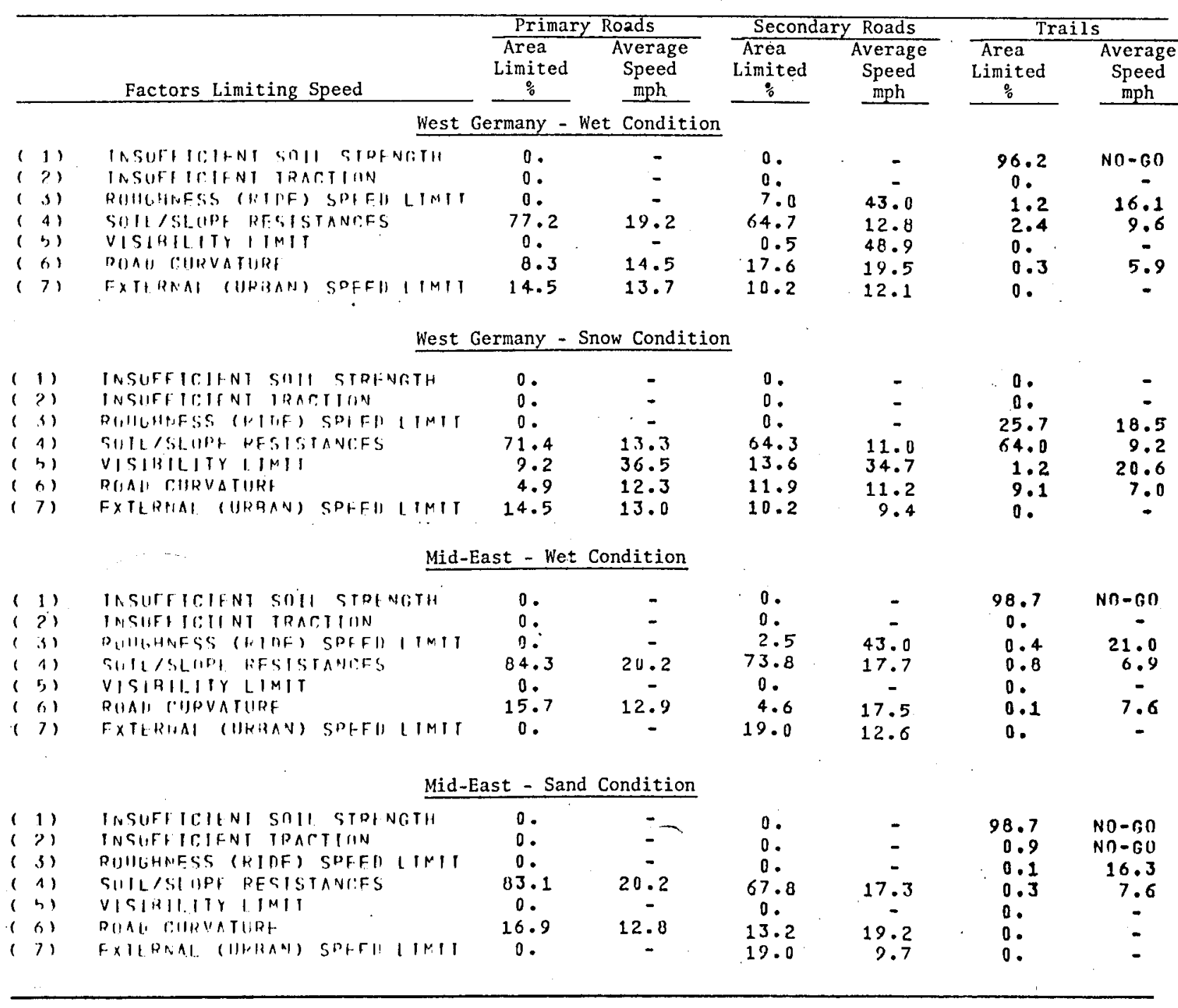


Table E61

Diagnostics Statistics for TDW901-XM(130 mm)

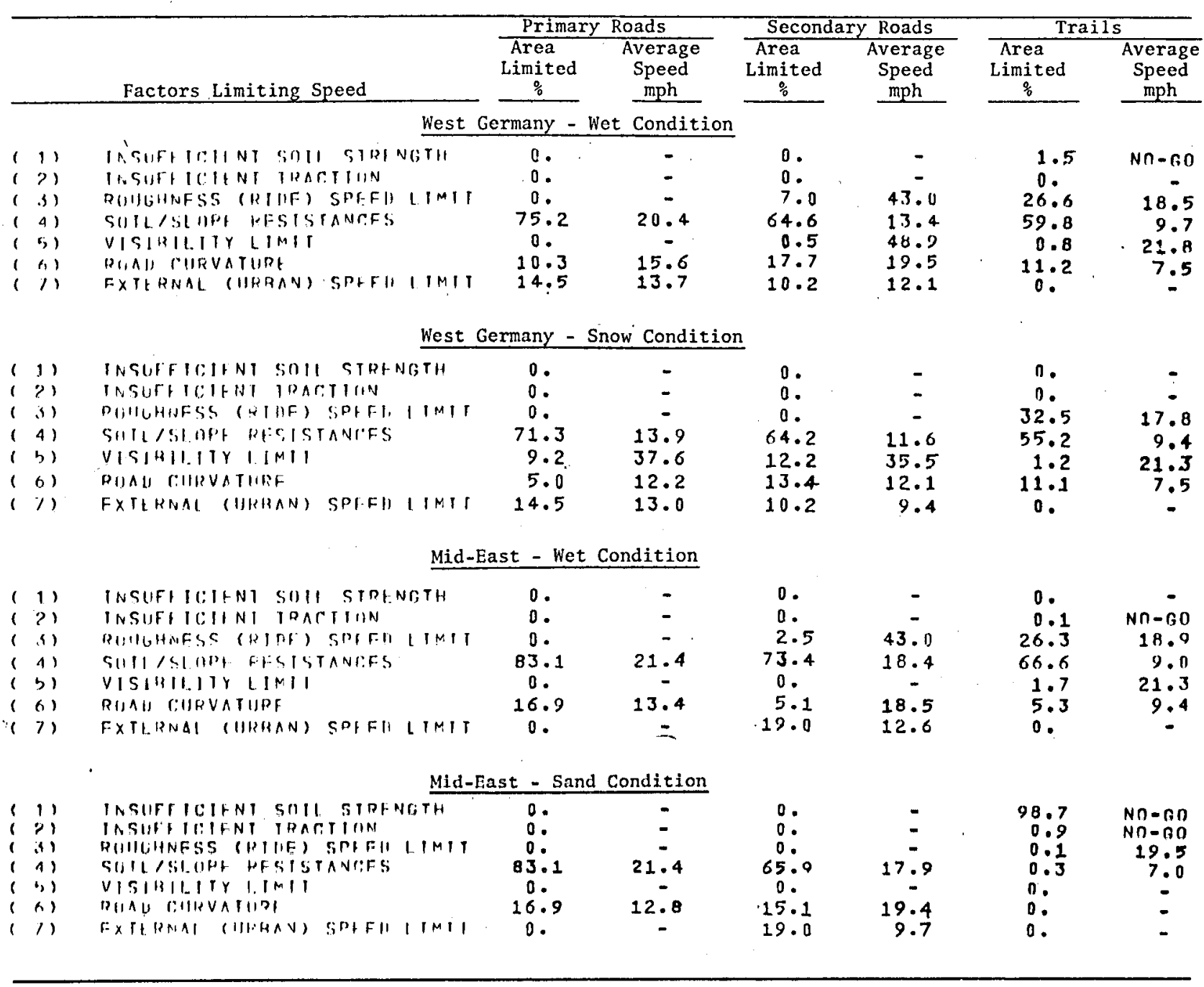


Tab1e E62

Diagnostics Statistics for M520E1-XM204

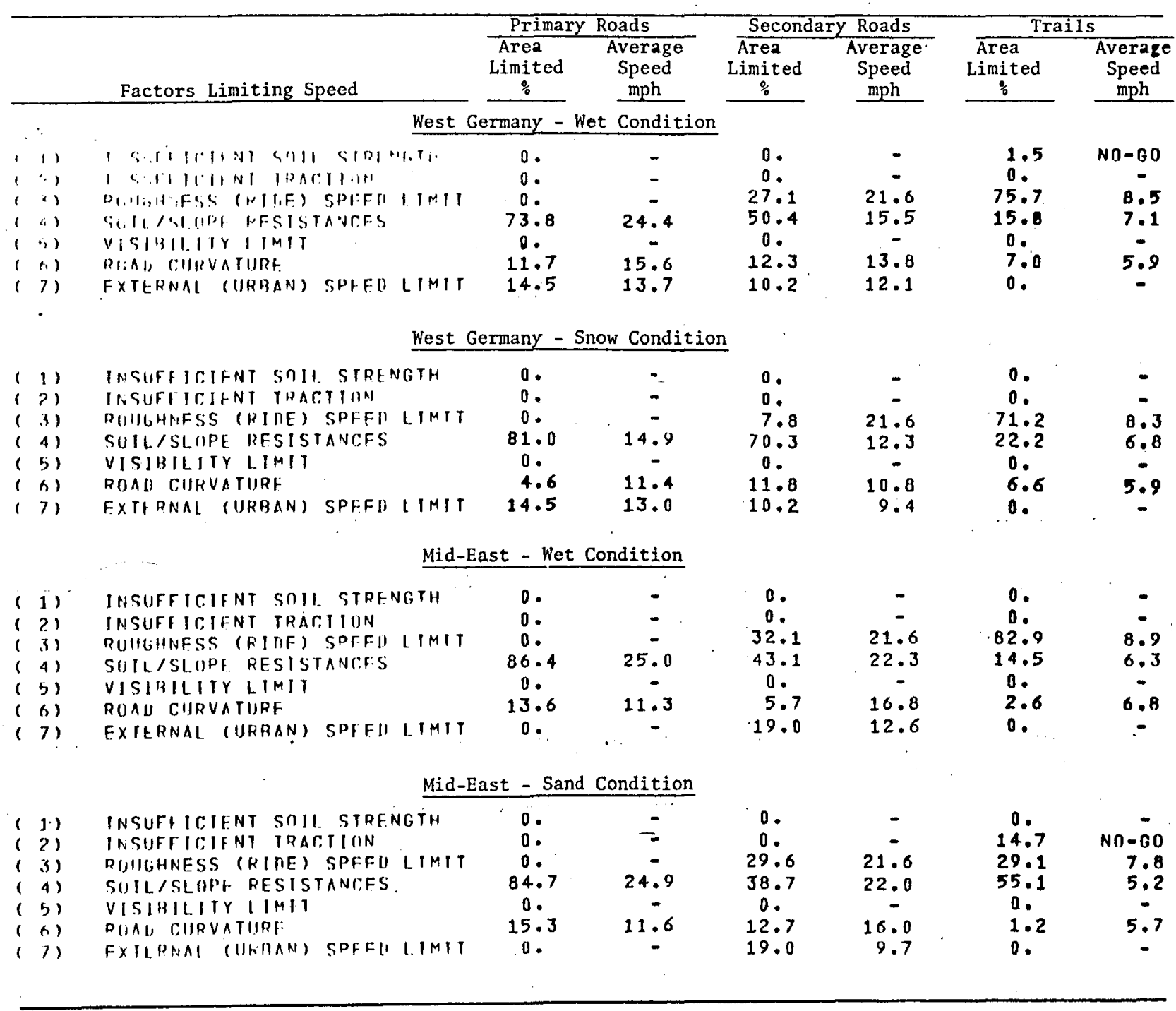


Table E63

Diagnostics Statistics for M520E1-XM198

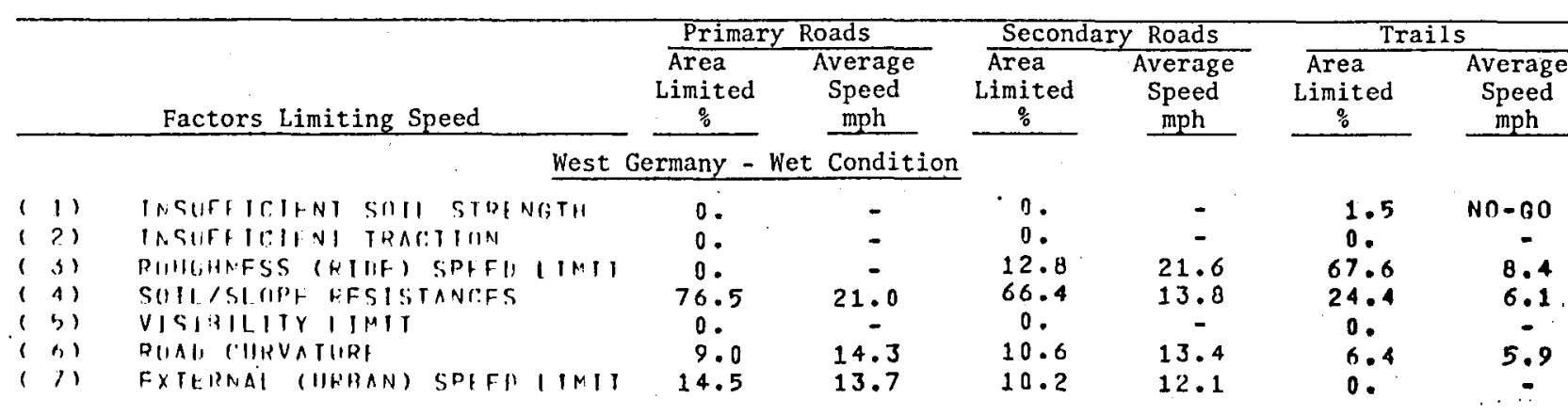

West Germany - Snow Condition

\begin{tabular}{|c|c|c|c|c|c|c|c|c|}
\hline$(1)$ & INSUFFIEIFNI SOII & STRENGTH & 0 . & - & 0 . & - & a. & - \\
\hline$(?)$ & IMSURFIRIENT TRAC & SION & o. & - & 0. & - & 0. & - \\
\hline$(.5)$ & RUHGHNFSS (HIIIE) & SPFFII IIMIT & D. & - & 7.4 & 21.6 & 56.1 & 8.0 \\
\hline$(4)$ & SOIL/SIIIPF RFSIST & TANCES & 81.3 & 11.7 & 74.2 & 9.8 & 38.3 & 6.1 \\
\hline$(3)$ & VISIRII.ITY IIMIT & & 0. & - & 0.4 & 28.5 & 0 . & - \\
\hline$\left(\begin{array}{l}1 \\
(1)\end{array}\right.$ & PUAU R.IIRVATURF & & 4.2 & 11.3 & 7.8 & $10 \cdot 0$ & 5.6 & 5.7 \\
\hline$(7)$ & FXTERNAI (URחAN) & SPFFU LIMTT & 14.5 & 13.0 & $10 \cdot 2$ & 9.4 & B. & - \\
\hline
\end{tabular}

Mid-East - Wet Condition

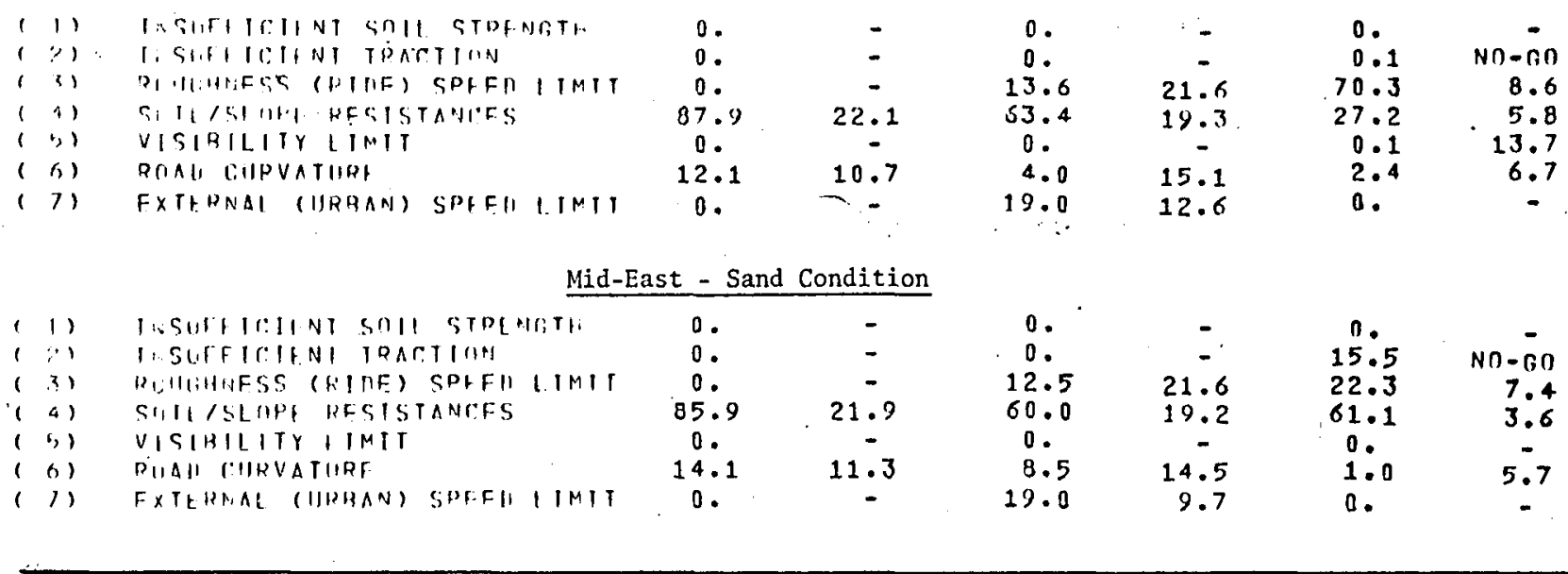


Table E64

Diagnostics Statistics for M520E1-FH70

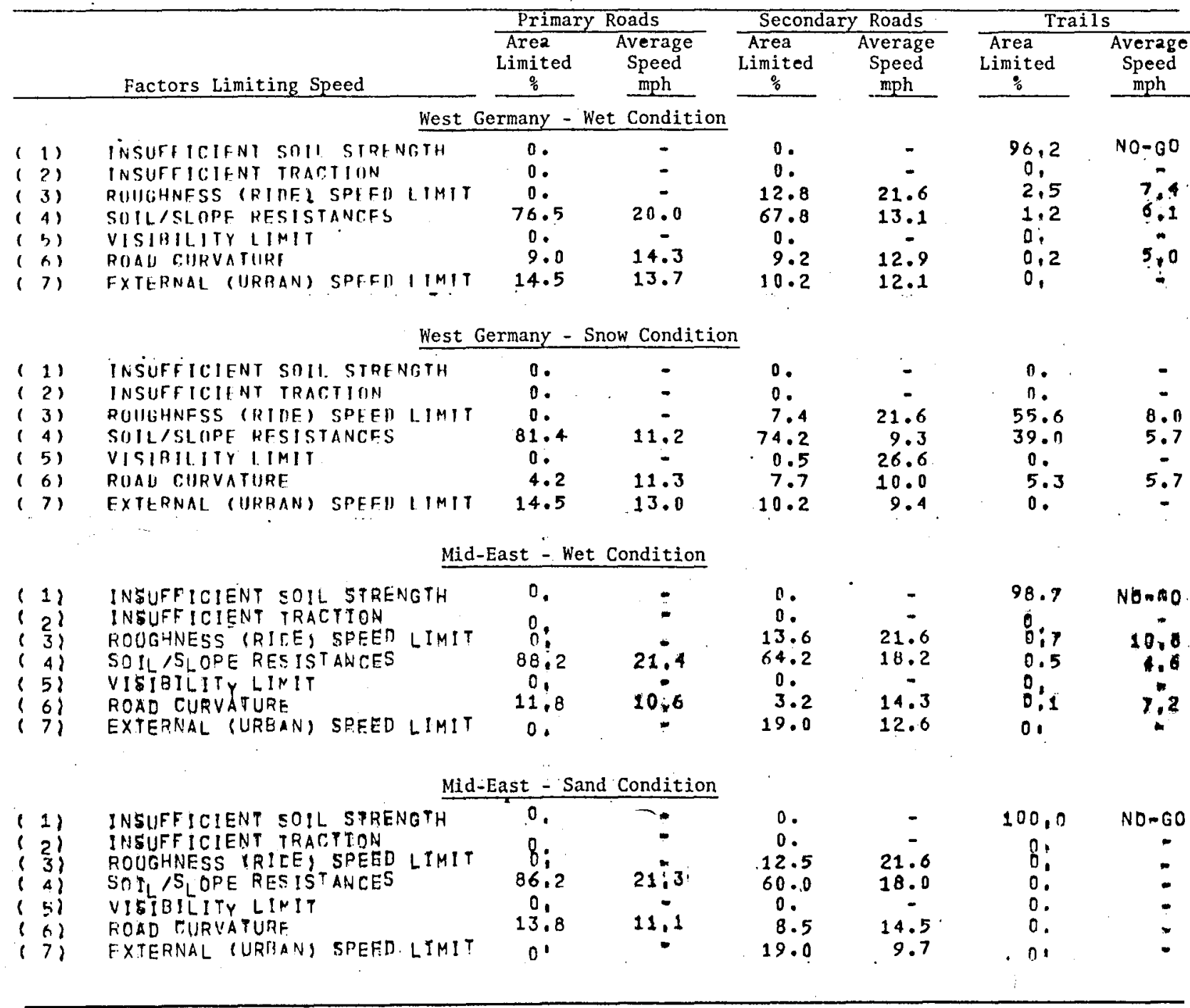


Table E65

Diagnostics Statistics for M125E1 XM198

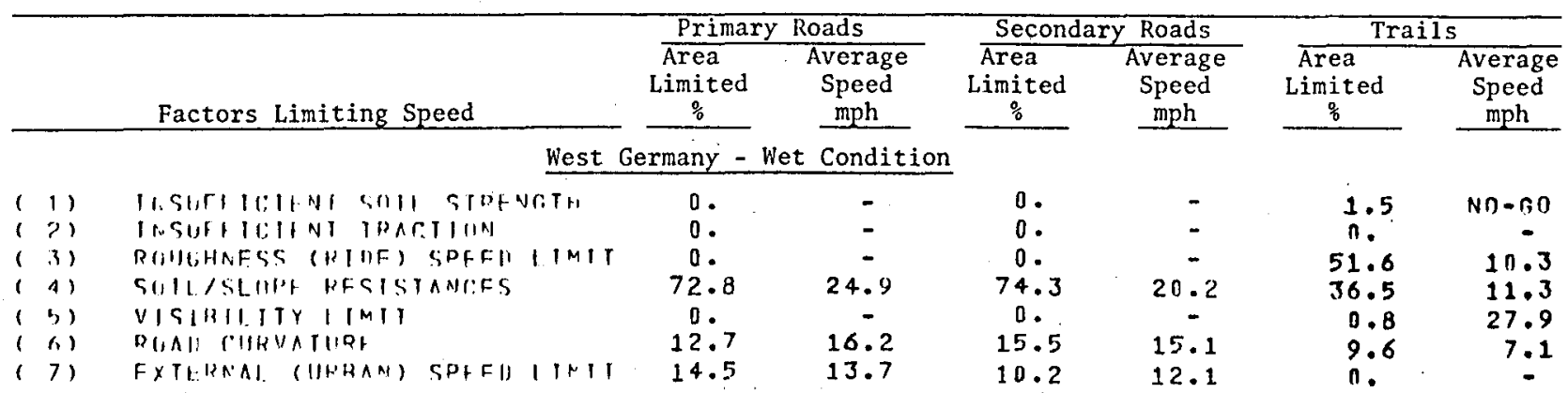

\section{West Germany - Snow Condition}

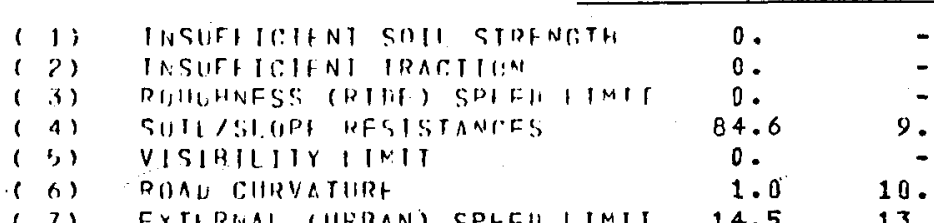

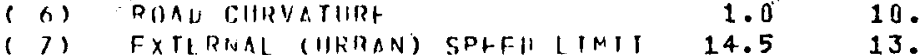

Mid-East - Wet Condition

\begin{tabular}{|c|c|}
\hline$(1)$ & INSURFICITNT SOII SIRTNRIII \\
\hline$(p)$ & IRSUFFICIHNT IHACTIIIN \\
\hline 3) & DUHG,HAFSS (HIIIF) SDFFH ITMIT \\
\hline 4) & 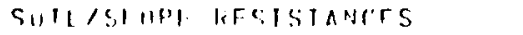 \\
\hline$(b)$ & $V I S I R\|I I Y, I I M\|$ \\
\hline$(6)$ & RUAU COIRVATURH \\
\hline 71 & $F \times T I B N A \mid$ (IIHBAN) SPFFU IIMII \\
\hline
\end{tabular}

$\begin{array}{cc}0 . & - \\ 0 . & - \\ 0.5 & - \\ 81.7 & 26.5 \\ 0.0 & - \\ 18.3 & 13.5 \\ 0 . & -\end{array}$

$\begin{array}{rr}0.3 & - \\ 1.3 & N n=-1: 1 \\ 0.0 & - \\ 42.6 & 8.2 \\ 1.1 & 32.6 \\ 4.8 & 9.2 \\ 10.2 & 9.4\end{array}$

$\begin{array}{rr}0 . & \\ 1.5 & \text { NO- } \\ 24.4 \\ 68.7 & 0.2 \\ 0.4 & 6.5 \\ 5.0 & 24.1 \\ 0 . & 5.9 \\ & \end{array}$

Mid-East - Sand Condition

\begin{tabular}{|c|c|c|c|c|c|c|c|c|c|c|}
\hline & & & & & & & & & & \\
\hline 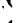 & 1 & INSUA TR:THA SOI & STDF & NETII & 0. & - & 0. & - & 98.7 & $N \cap-G O$ \\
\hline 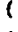 & $? 1$ & IMSIJFFICIFNI TRAC & :TIIIN & & o. & - & 0 . & - & 0.9 & NO $-2,0$ \\
\hline 1 & (1) & Q(1)CHNFSS $(1.1 \| F)$ & SPFFI & $\operatorname{IIMIT}$ & 0 . & - & 0 . & - & 0.1 & 13.3 \\
\hline ( & 11 & SOIL/SI.OPF RESTSI & AnI:Fs & & 81.7 & 26.5 & 46.6 & 22.9 & 0.3 & 7.6 \\
\hline ( & 51 & VISIRILITY LIMIT & & & 0. & - & 0 . & - & o. & - \\
\hline ( & () & DQAAI GHEVATLIPE & & & 18.3 & 12.9 & 34.4 & 24.2 & 0 . & - \\
\hline 1 & 71 & $5 \times 16181.11 \quad(11+18 \wedge N)$ & SPFFH & $11 \times 11$ & 0 . & - & 19.0 & 9.7 & 0 . & - \\
\hline
\end{tabular}


Table E66

Diagnostics Statistics for M548E1-XM2204

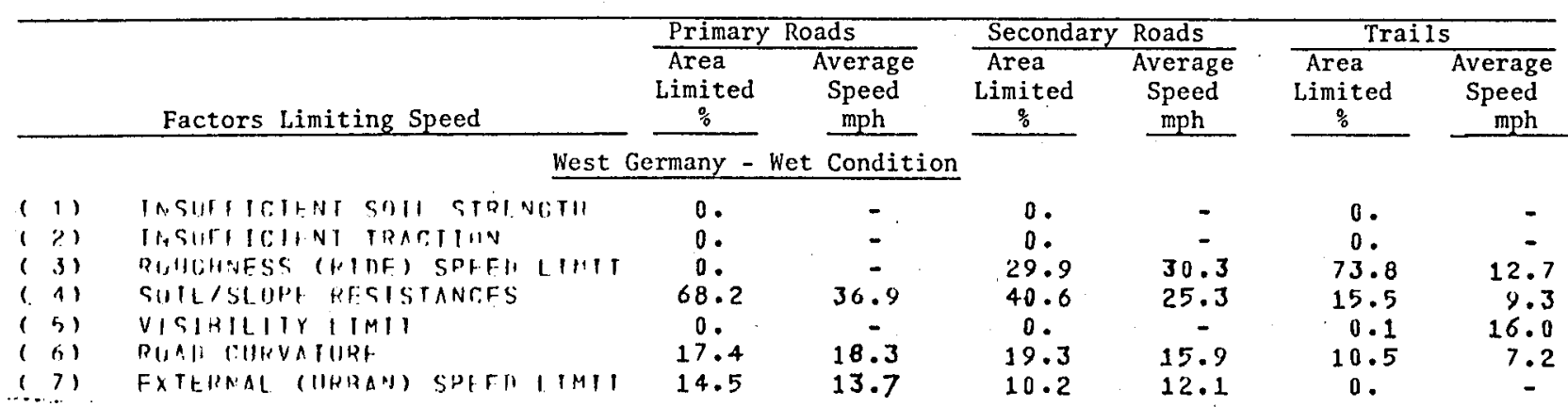

West Germany - Snow Condition

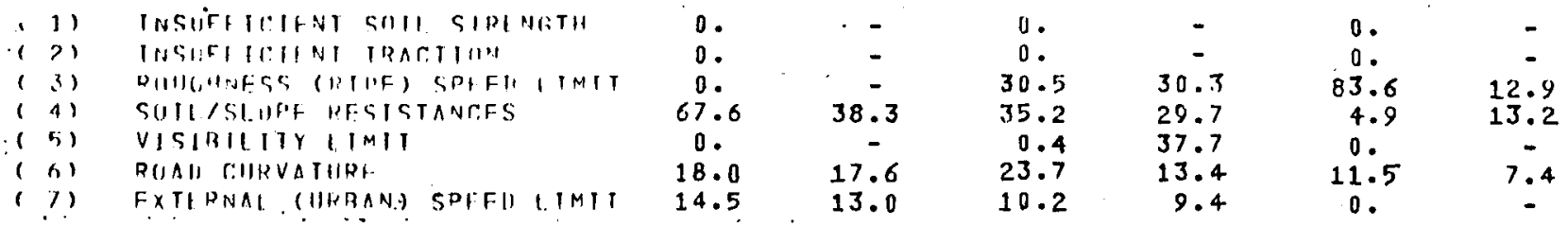

Mid-East - Wet Condition

\begin{tabular}{|c|c|c|c|c|c|c|c|c|c|}
\hline 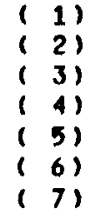 & $\begin{array}{l}\text { INSUFFICIENT SOIL } \\
\text { INSUFFICIENT IRAC } \\
\text { ROUGHNES (RIDE) } \\
\text { SOILISLOPE RESIST } \\
\text { VISIBILITY LIMIT } \\
\text { ROAD CURVATURE } \\
\text { EXTERNAL (URBAN) }\end{array}$ & $\begin{array}{l}\text { L STREI } \\
\text { CTION } \\
\text { SPFED } \\
\text { TANCES }\end{array}$ & $\begin{array}{l}\text { NGTH } \\
\text { LIMIT }\end{array}$ & $\begin{array}{c}0 . \\
0 . \\
03.4 \\
73.4 \\
0.0 \\
26.6 \\
0 .\end{array}$ & $\begin{array}{c}= \\
39.6 \\
16.6 \\
=\end{array}$ & $\begin{array}{r}0 . \\
0 . \\
39.4 \\
27.0 \\
0.0 \\
14.6 \\
19.0\end{array}$ & $\begin{array}{c}- \\
30.3 \\
37.3 \\
21.4 \\
12.6\end{array}$ & $\begin{array}{l}0.0 \\
0.1 \\
80.1 \\
13.9 \\
0.6 \\
5.4 \\
0.1\end{array}$ & $\begin{array}{r}\text { NO-BO } \\
13.6 \\
7.2 \\
18.7 \\
9.3 \\
-.\end{array}$ \\
\hline
\end{tabular}

Mid-East - Sand Condition

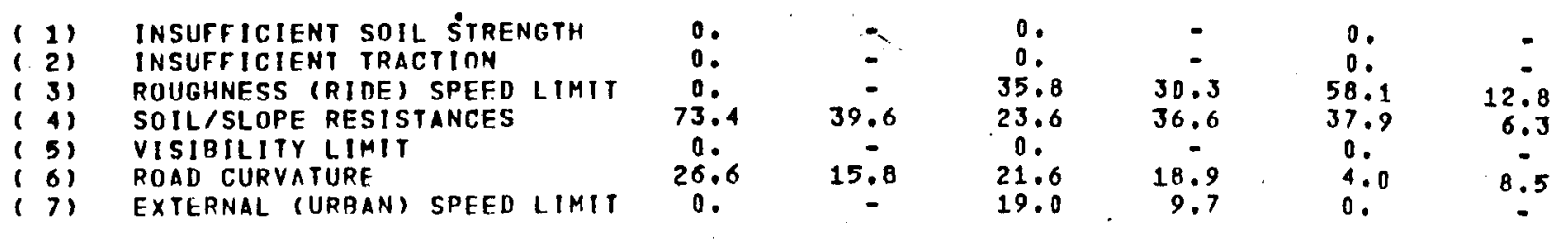


Table E67

Diagnostics Statistics for M548E1-XM198

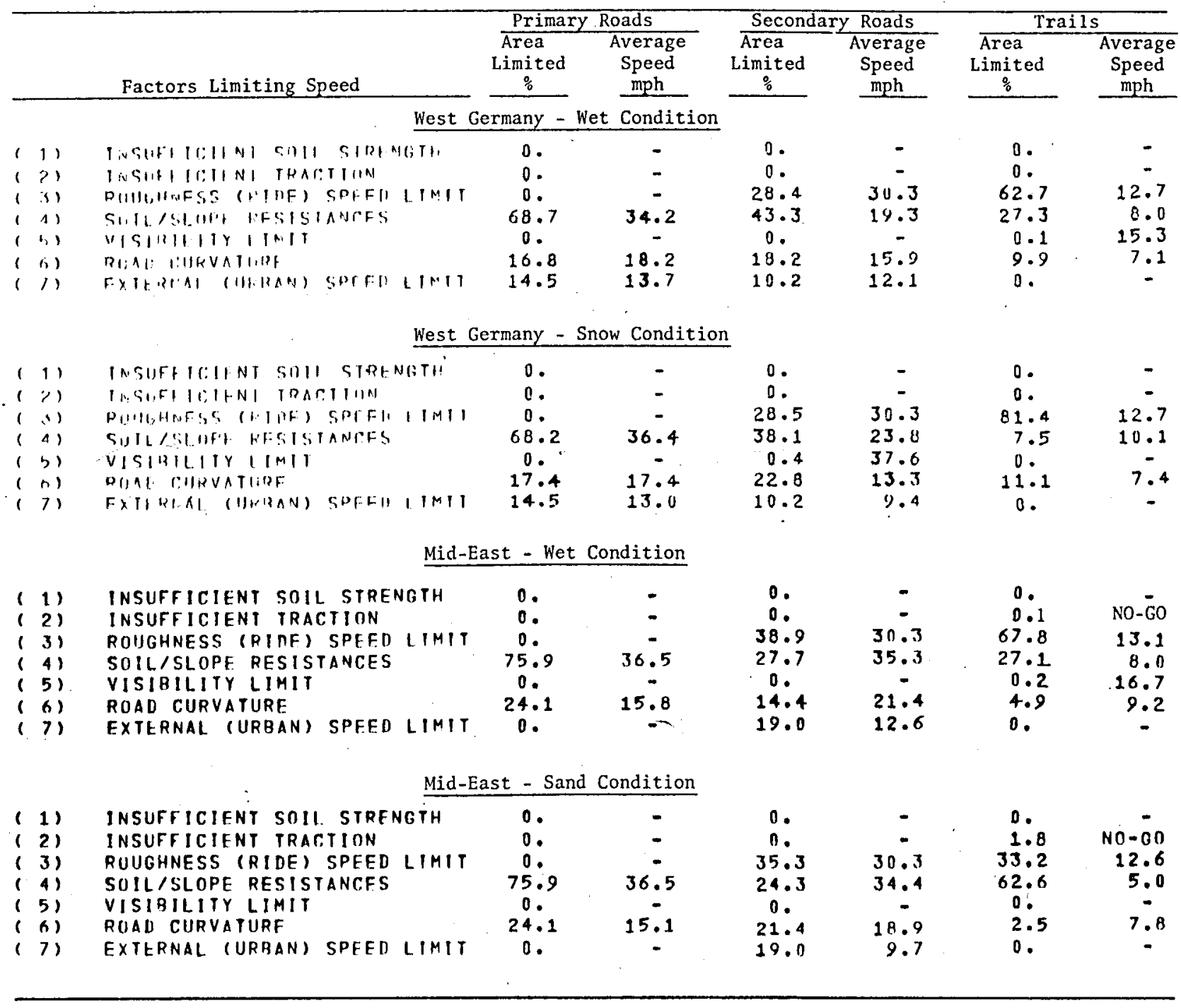


Table E68

Diagnostics Statistics for M548E1-FH70

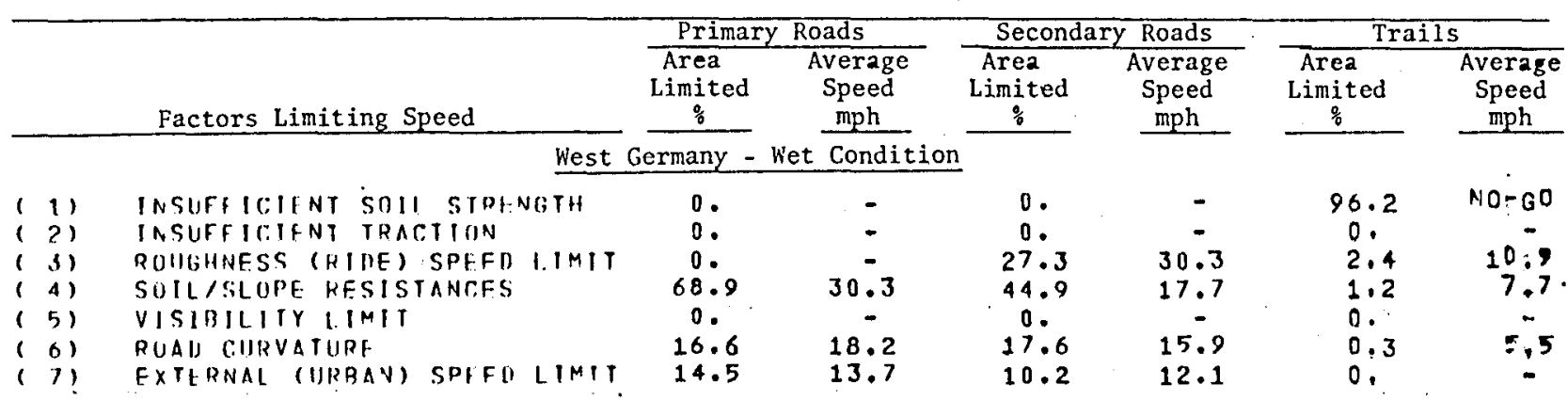

West Germany - Snow Condition

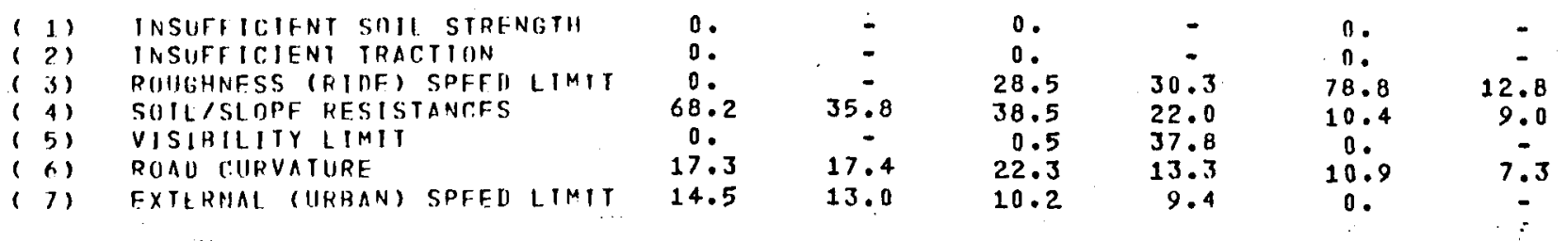

Mid-East - Wet Condition

\begin{tabular}{|c|c|c|c|c|c|c|c|c|c|}
\hline 1) & INSUFFICIFNT SOIL & L STREA & NGTH & $n \cdot$ & - & 0. & - & 98.7 & NOMGO \\
\hline 2) & INSUFFICIFNT TRAR & L, TION & & 0 . & - & 0. & - & 0. & - \\
\hline 3) & ROUGHNESS (RIDE) & SPEFD & $I I M I T$ & 0. & $\begin{array}{ll}- & -1\end{array}$ & 36.2 & 30.3 & 0.3 & 15,2 \\
\hline 4) & SOIL/SLOPF RESIS & TANCFS & & 76.5 & 35.5 & 30.4 & 34.1 & 0.9 & 5,4 \\
\hline b) & VISIBILIIY LIMII & & & 0. & $\therefore$ & 0. & - & 0. & $=$ \\
\hline 6) & ROAD CUIRVATURF & corcu & & 23.5 & 15.6 & $.14 \cdot 4$ & 21.4 & 0.1 & 7.1 \\
\hline 71 & EXTKRNAL (URRAN ) & SPEFI & LIMII & 8. & - & 19.0 & 12.6 & 0. & - \\
\hline
\end{tabular}

Mid-East - Sand Condition

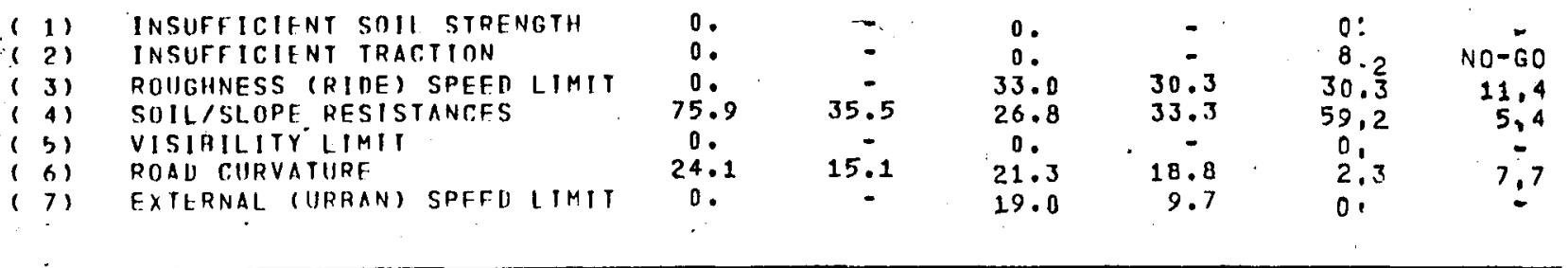


Table E69

Diagnostics Statistics for UET-XM198

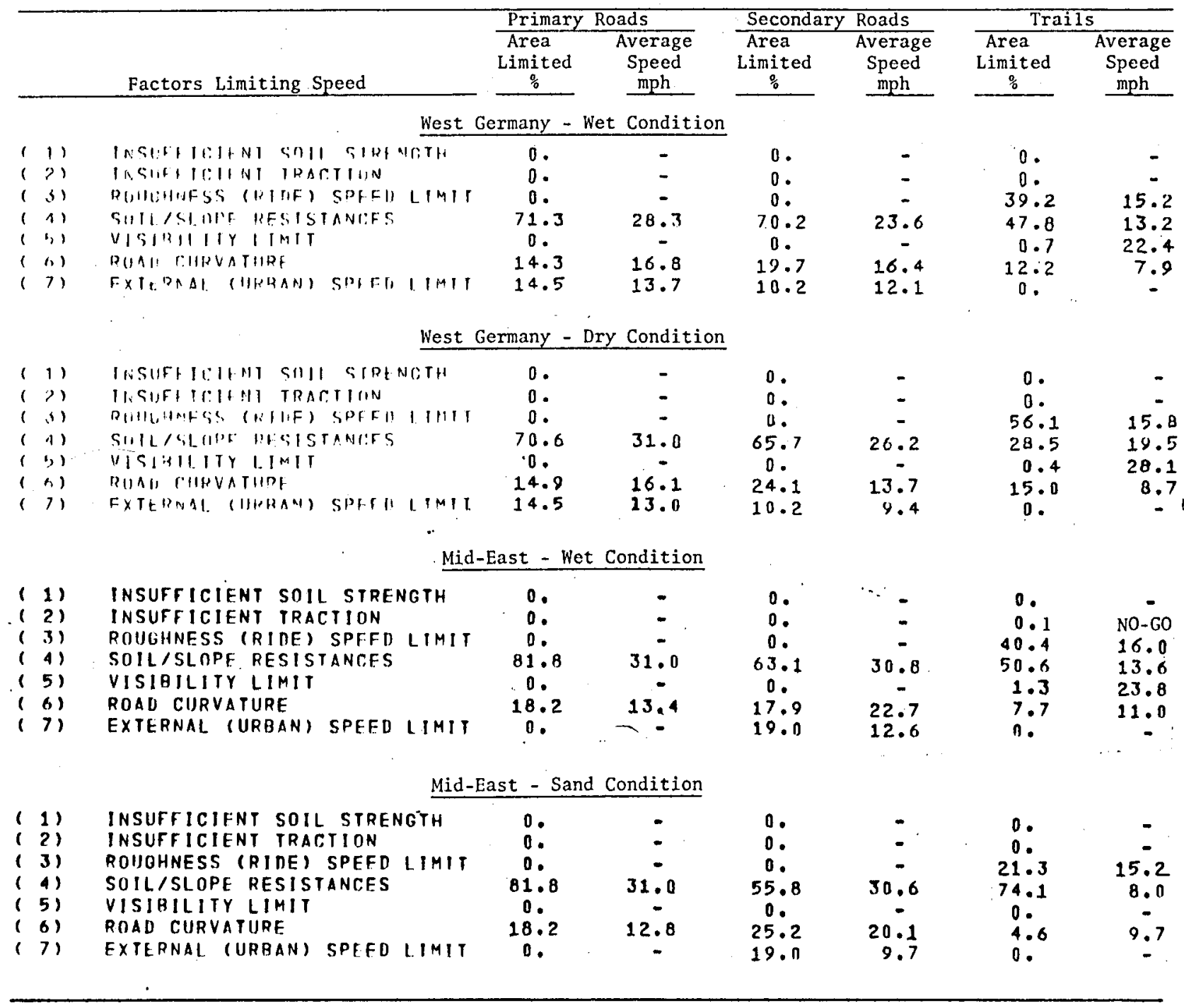


Table E70

Diagnostics Statistics for UET-FH70

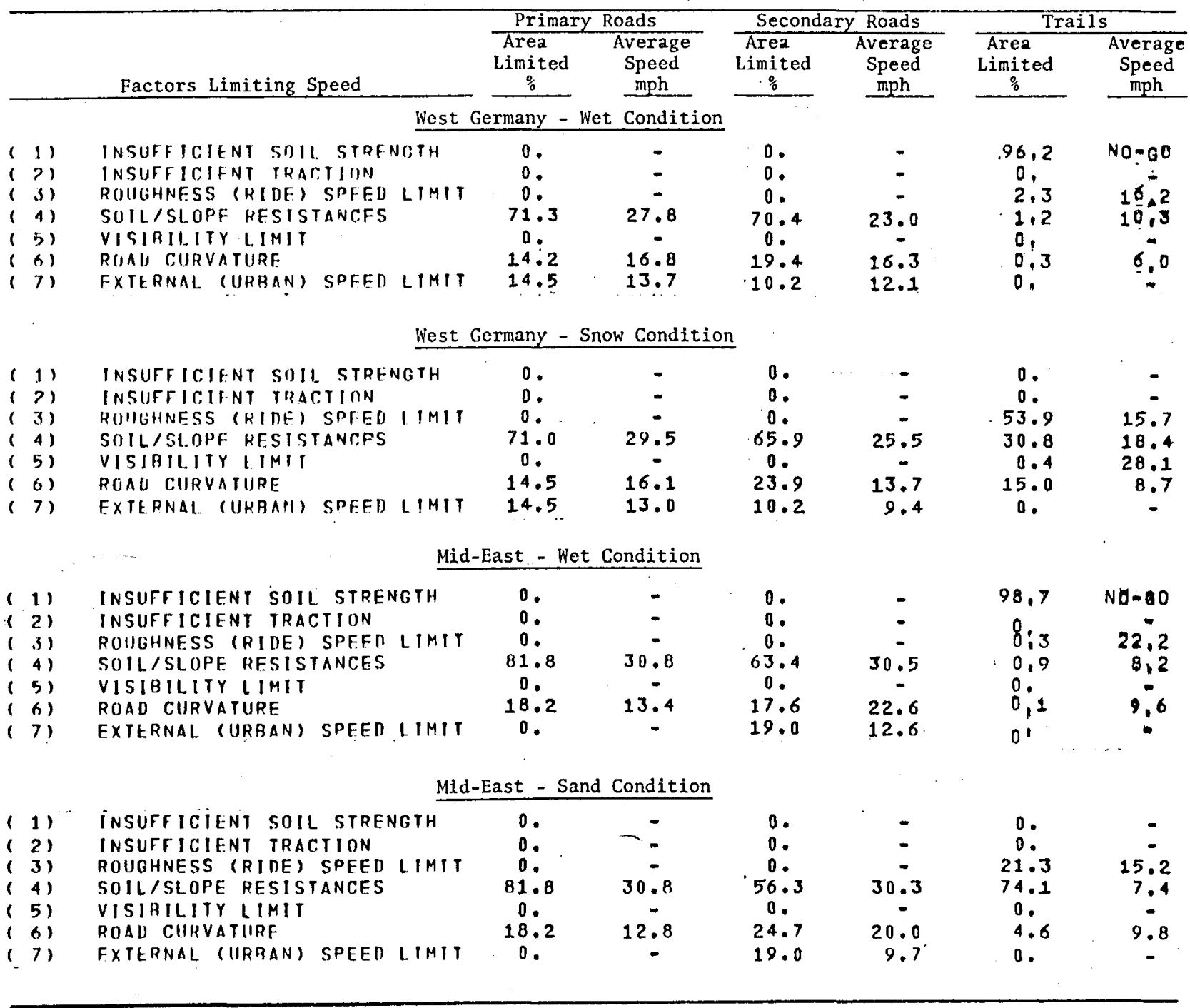


Table E71

Diagnostics Statistics for ASV-XM204

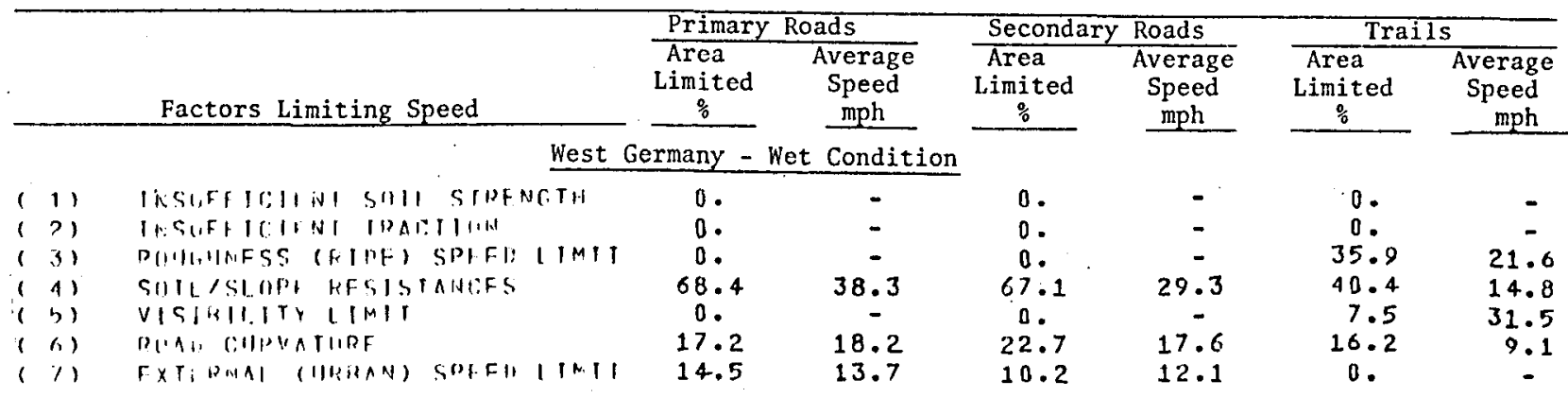

West Germany - Snow Condition

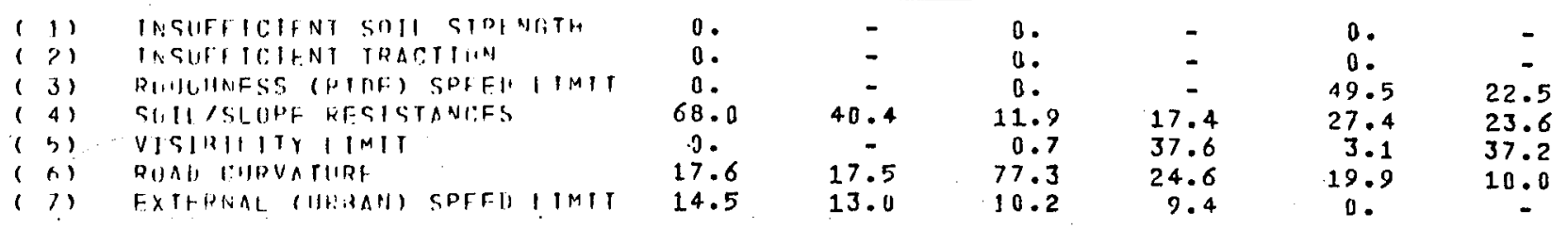

Mid-East - Wet Condition

\begin{tabular}{|c|c|c|c|c|c|c|c|c|c|}
\hline 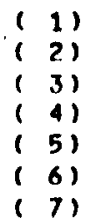 & $\begin{array}{l}\text { INSUFFICIENT SOIL } \\
\text { INSUFFICIENT TRAC } \\
\text { ROUGHNESS (RINE) } \\
\text { SOIL/SLOPE RESIST } \\
\text { VISIBILITY LIMIT } \\
\text { ROAD CURVATURE } \\
\text { EXTERNAL (URBAN) }\end{array}$ & $\begin{array}{l}\text { STREN } \\
\text { CTION } \\
\text { SPEED } \\
\text { TANCES }\end{array}$ & $\begin{array}{l}\text { NGTH } \\
\text { ITMIT }\end{array}$ & $\begin{array}{c}0 . \\
0 . \\
0 . \\
75.5 \\
0.0 \\
24.5 \\
0 .\end{array}$ & $\begin{array}{c}- \\
- \\
40.5 \\
15.9\end{array}$ & $\begin{array}{l}0 . \\
0 . \\
0 . \\
56.2 \\
0.0 \\
24.8 \\
19.0\end{array}$ & $\begin{array}{c}- \\
- \\
41.2 \\
- \\
25.8 \\
12.6\end{array}$ & $\begin{array}{r}0 . \\
0.1 \\
37.0 \\
40.0 \\
9.6 \\
13.3 \\
0 .\end{array}$ & $\begin{array}{r}- \\
N O-G 0 \\
22.4 \\
14.7 \\
30.4 \\
13.7 \\
-\end{array}$ \\
\hline
\end{tabular}

Mid-East - Sand Condition

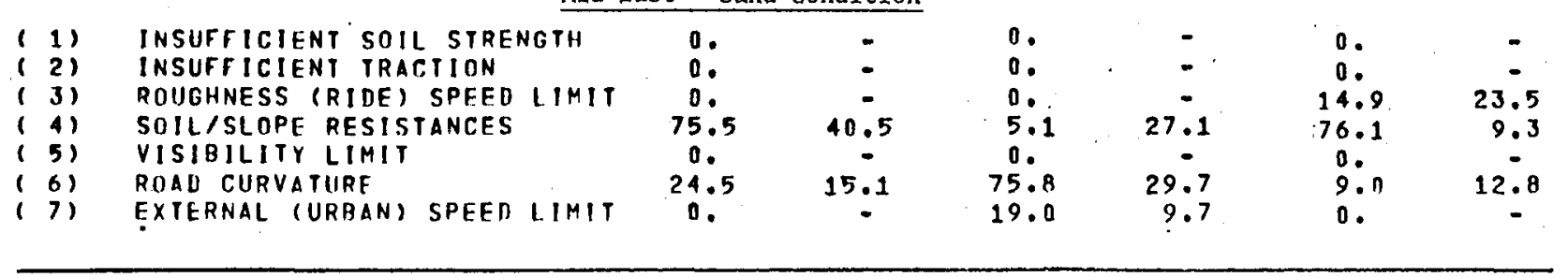


Table E72

Diagnostics Statistics for ASV-XM198

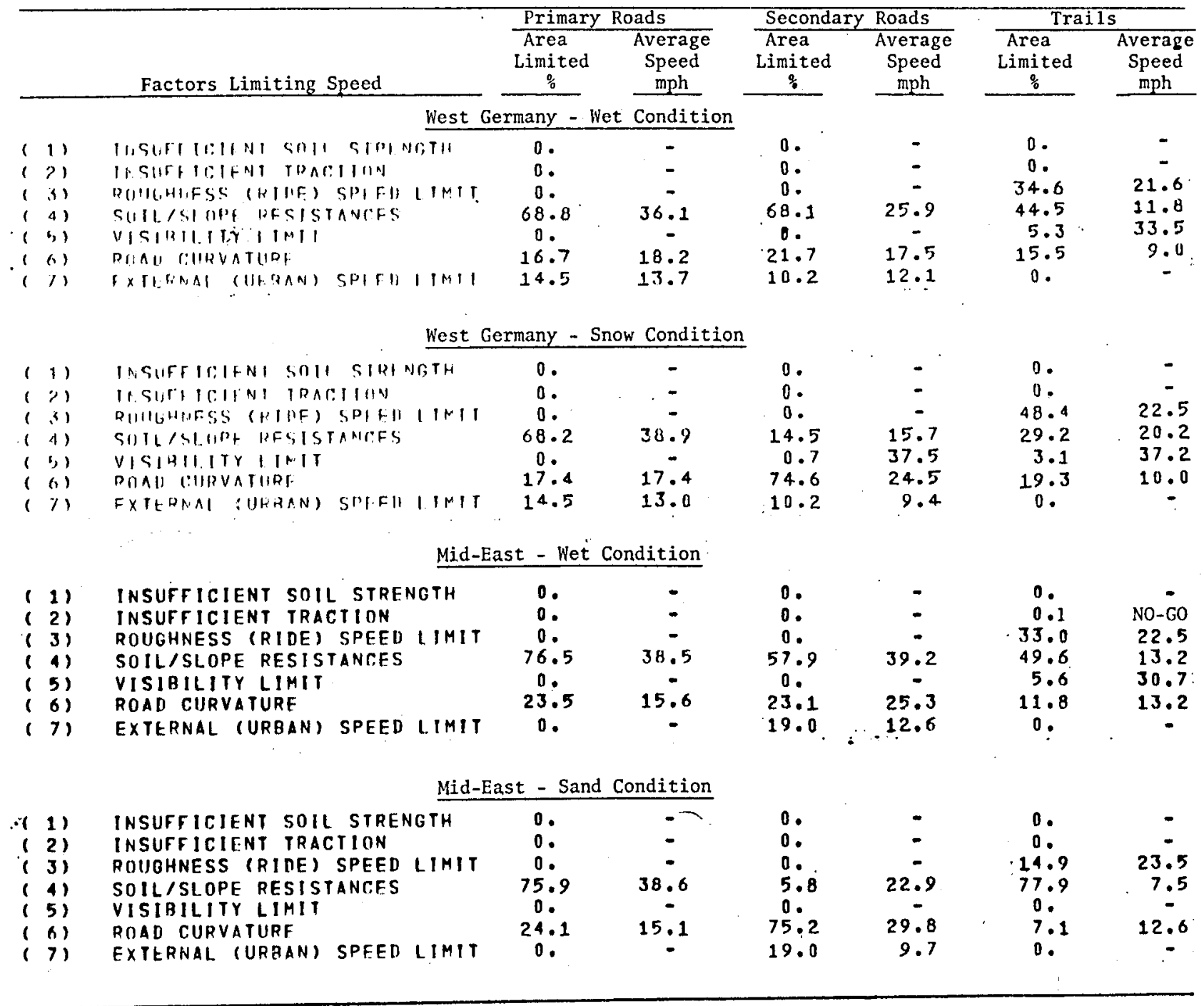


Table E73

Diagnostics Statistics for ASV-FH70

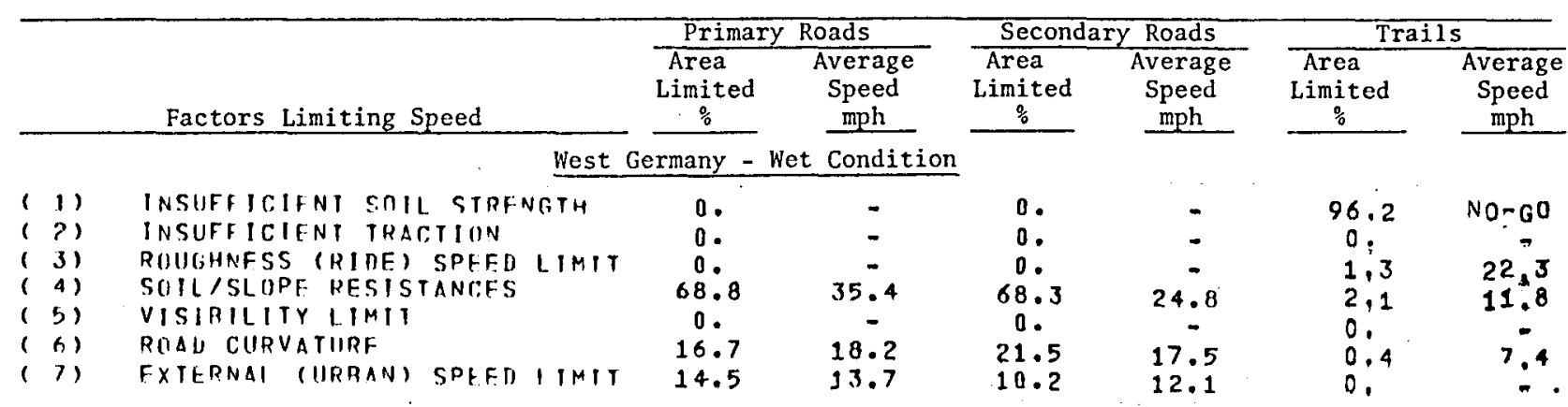

West Germany - Snow Condition

\begin{tabular}{|c|c|c|c|c|c|}
\hline 11 & INSUFFICILNT SOII. & STRE' & NGTH & 0 . & $\rightarrow$ \\
\hline 2) & INSUFFIC,IFNI TRAC. & SIIIN & & D. & - \\
\hline 3) & R()UUHNESS (RIDF) & SPFFD & I. IMIT & a. & - \\
\hline 4) & SOIL/SLOPF RESIST & ANCES & & 68.2 & 38.3 \\
\hline ( 5$)$ & VISIHILIIY LIMII & & & a. & - \\
\hline 6) & ROAL CURVATIIRF & & & 17.3 & 17.4 \\
\hline 7) & FXTERNAL (URBAN) & SPFEI & IIMIT & 14.5 & $13 . n$ \\
\hline
\end{tabular}

Mid-East - Wet Condition

\begin{tabular}{|c|c|c|c|}
\hline$(1)^{\prime}$ & INSUFFICIENT SOII & STREI & VGTH \\
\hline (2) & INSUFFICIENT TRAR & 酒 I & \\
\hline 3) & RUUGHNFSS (RINE) & SPEED & LIMIT \\
\hline 4) & SUIL/SLUPF RESIS & TANCEFS & \\
\hline $\begin{array}{l}\text { 5) } \\
\text { 6). }\end{array}$ & $\begin{array}{l}\text { VISIBILITY IIMIT } \\
\text { ROAD CURVATURF }\end{array}$ & & \\
\hline 7) & EXTERNAL (URBAN) & SPFFD & LIMIT \\
\hline
\end{tabular}

\begin{tabular}{|c|c|}
\hline 0. & - \\
\hline 0 . & 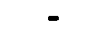 \\
\hline 0. & - \\
\hline $\begin{array}{c}76.5 \\
0 .\end{array}$ & $\begin{array}{c}37.8 \\
-\end{array}$ \\
\hline 23.5 & 15.6 \\
\hline
\end{tabular}

$\begin{array}{rr}0 . & - \\ 0 . & - \\ 14.7 & 14.8 \\ 0.7 & 37.5 \\ 74.4 & 24.5 \\ 10.2 & 9.4\end{array}$

$\begin{array}{rr}0 . & - \\ 0 . & - \\ 48.4 & 22.5 \\ 29.3 & 19.3 \\ 3.1 & 37.2 \\ 19.2 & 9.9 \\ 0 . & -. .\end{array}$

Mid-East - Sand Condition

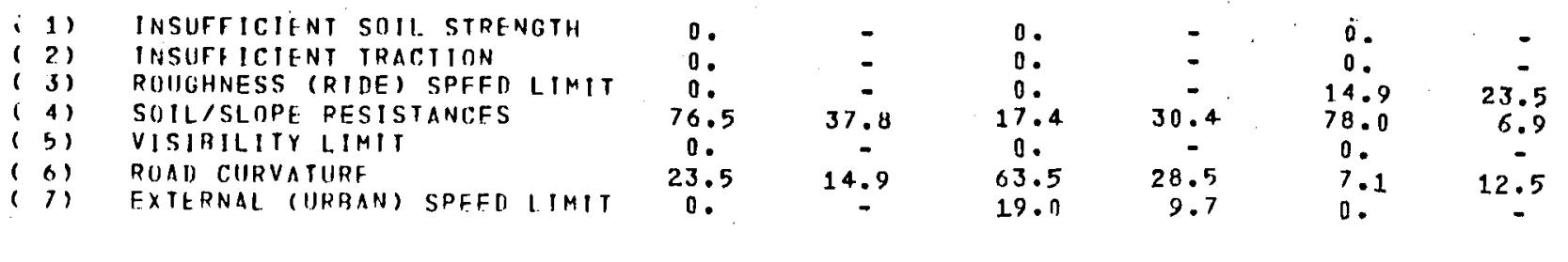


Table E74

Diagnostics Statistics for M109A1

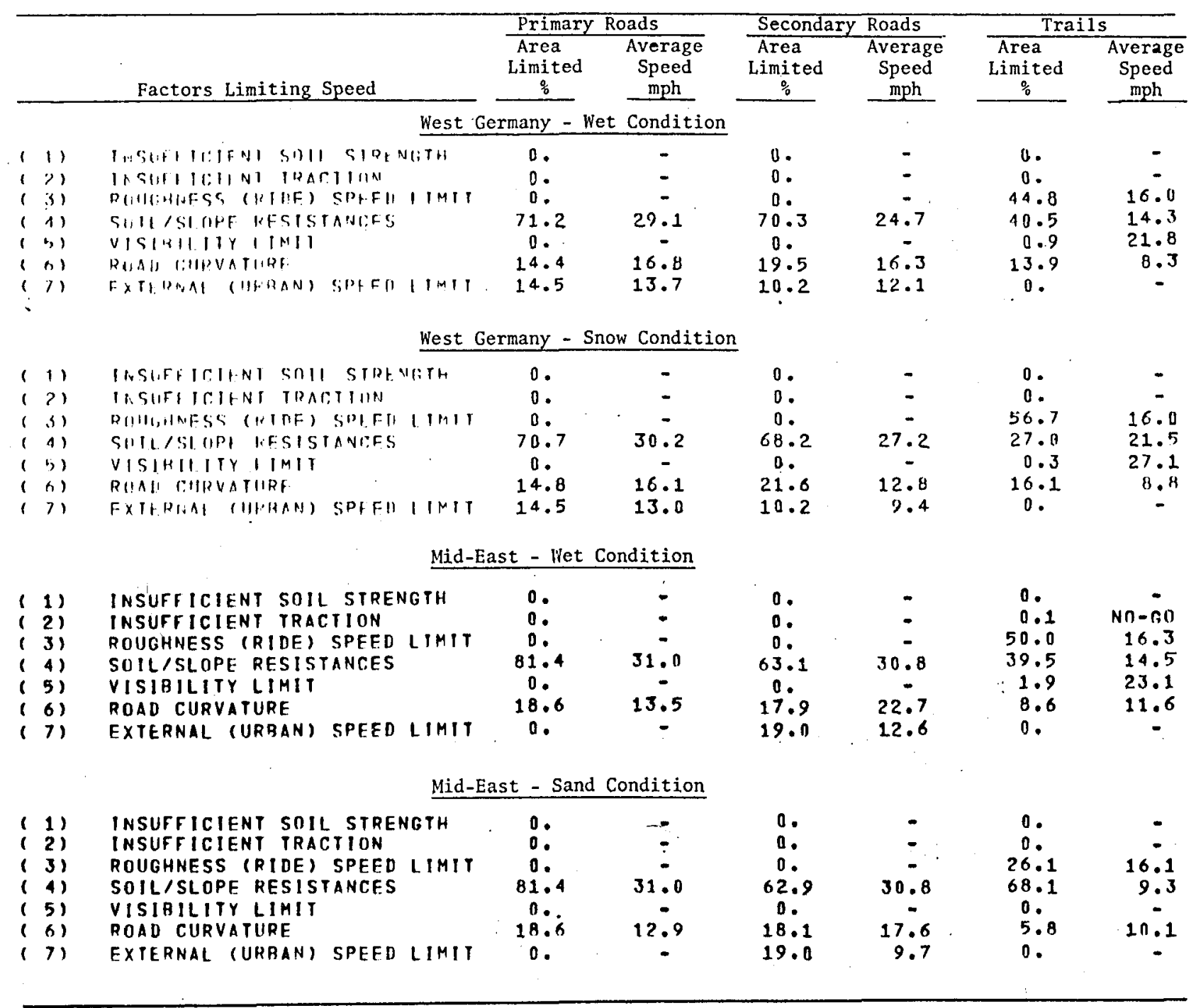


Table E75

Diagnostics Statistics for M107.

\begin{tabular}{|c|c|c|c|c|c|c|c|}
\hline & & Primary & oads & Seconda & Roads & Tra & \\
\hline & Factors Limiting Speed & $\begin{array}{c}\text { Area } \\
\text { Limited } \\
\% \\
\end{array}$ & $\begin{array}{c}\text { Average } \\
\text { Speed } \\
\text { mph } \\
\end{array}$ & $\begin{array}{c}\text { Area } \\
\text { Limited } \\
\% \\
\end{array}$ & $\begin{array}{c}\text { Average } \\
\text { Speed } \\
\text { mph } \\
\end{array}$ & $\begin{array}{c}\text { Area } \\
\text { Limited } \\
\frac{0}{6} \\
\end{array}$ & $\begin{array}{c}\text { Average } \\
\text { Speed } \\
\text { mph }\end{array}$ \\
\hline & West $\mathrm{C}$ & ermany - & t Conditi & & & & \\
\hline 111 & TASGH IE:IINT SIII SIR/AGTH & 0 . & - & 0 . & - & i. & - \\
\hline$(7)$ & $|A S U R||C, 1| N \mid$ TPACIIIIAI & 0 . & - & 0 . & - & u. & - \\
\hline (3) & DUHGHNFSS (UINF) SPFFH IIMIT & o. & - & 0 . & - & 43.2 & 16.0 \\
\hline$(4)$ & SHI, SLLHPH POSISTAICFS & 71.2 & 29.0 & 70.4 & 24.2 & 42.1 & 14.0 \\
\hline (引) & VISIAIIIIY IIMII & a. & - & a. & - & 1.0 & 21.2 \\
\hline$(f)$, & RUAB. CIIPVATIIDF & 14.4 & 16.8 & 13.4 & 16.4 & 13.7 & $8 . \pi$ \\
\hline 111 & $F \times 11,2 R: A !(11 K R A N)$ SDFEH I IMI & 14.5 & 13.7 & $10 \cdot 2$ & 12.1 & 0 . & - \\
\hline & West $\mathrm{C}$ & ermany - & Low Condit & & & & \\
\hline$(1)$ & IASUREICIFNI SOHI SIPENGTH & 0 . & - & 0 . & - & . 0 . & - \\
\hline $1 ? 1$ & II:SI.FFICIINI TOACTION & 0 . & - & 0 . & - & 0 . & - \\
\hline$(5)$ & DUMGHAFSS (HIMF) SPFFH IMIT & D. & - & 0 . & - & 56.7 & 16.0 \\
\hline$(4)$ & SHILASLIPF HFSISTANCFS & 70.8 & 30.1 & 68.2 & 27.0 & 27.0 & 21.2 \\
\hline$(5)$ & VISIPIIIYY LIMIJ & 0 . & - & a. & - & 0.3 & 27.1 \\
\hline$(6)$ & DIIAU IOIRVATIIRF & 14.8 & $16 \cdot 1$ & 21.6 & 12.8 & $16 \cdot 0$ & 8.8 \\
\hline$(7)$ & FXIERNAI. (HIFRAN) SDEFII LIMII & 14.5 & 13.0 & $10 \cdot 2$ & 9.4 & 0 . & - \\
\hline & Mid-E & ast - Wet & ondition & & & & \\
\hline (1) & INSUFFICIENT SOIL STRENGTH & 0 . & - & o. & - & 0 . & - \\
\hline$(2)$ & INSUFFICIENT TRACIION & 0. & - & n. & - & 0.1 & NO- GOO \\
\hline$(3)$ & ROUGHNESS (PIDE) SPEED LIMIT & 0 . & - & D. & - & 48.4 & 16.3 \\
\hline$(4)$ & SUIL/SLOPF RESISTANCFS & 81.4 & 30.9 & 63.1 & 30.7 & 41.0 & 14.1 \\
\hline ( 5$)$ & VISIBILITY LIMIT & 0 . & - & 0. & - & 2.0 & 22.5 \\
\hline$(6)$ & ROAD CURVATURF & 18.6 & 13.5 & 17.9 & 22.7 & 8.6 & 11.6 \\
\hline$(7)$ & EXTERNAL (URBAN) SPEFD LIMIT & 0 . & - & 19.0 & 12.6 & o. & - \\
\hline & Mid-E & ast - San & Condition & & & & \\
\hline (1) & INSUFF ICIFNT SOIL STRENGTH & 0 . & - & 0 . & - & 0 . & - \\
\hline$(2)$ & INSUFFICIENT TRACTION & D. & - & 0. & - & 0 . & - \\
\hline$(3)$ & ROUGHNESS (RIDE) SPEED LIMIT & 0. & - & 0 . & - & 26.1 & 16.1 \\
\hline$(4)$ & SUIL/SLOPE RESISTANCES & 81.4 & 30.9 & 62.9 & 30.7 & 68.1 & 9.0 \\
\hline$(5)$ & VISIBILITY LIMIT & o. & - & 0 . & - & 0. & - \\
\hline$(6)$ & ROAD CURVATURF & 18.6 & 12.9 & 18.1 & 17.6 & 5.8 & 10.1 \\
\hline$(7)$ & EXTERNAL (URBAN) SPFFD LIMIT & 0. & - & 19.0 & 9.7 & 0 . & - \\
\hline
\end{tabular}


Table E76

Diagnostics Statistics for M110E2

\begin{tabular}{|c|c|c|c|c|c|c|c|}
\hline & & Primary & ads & Second & Roads & Tra & \\
\hline & Factors Limiting Speed & $\begin{array}{c}\text { Area } \\
\text { Limited } \\
\% \\
\end{array}$ & $\begin{array}{c}\text { Average } \\
\text { Speed } \\
\text { mph } \\
\end{array}$ & $\begin{array}{c}\text { Area } \\
\text { Limited } \\
\% \\
\end{array}$ & $\begin{array}{c}\text { Average } \\
\text { Speed } \\
\text { mph } \\
\end{array}$ & $\begin{array}{c}\text { Area } \\
\text { Limited } \\
\% \\
\end{array}$ & $\begin{array}{c}\text { Average } \\
\text { Speed } \\
\text { mph }\end{array}$ \\
\hline & West & ermany & indit & & & & \\
\hline 111 & 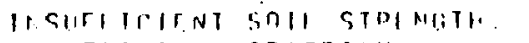 & 0 . & - & 0 . & - & 0 . & - \\
\hline$(2)$ & INSUREIRIHAT TRACTIRMI & 0 . & - & 0 . & - & 0 . & - \\
\hline (3) & DUULAHESS (RIMF) SOFFU ITMIT & 0. & - & 0 . & - & 43.2 & 16.0 \\
\hline$(4)$ & SUII SLUU1 RFSISTANIES & 71.2 & 29.0 & 70.4 & 24.2 & 42.1 & 14.0 \\
\hline ( 5$)$ & $V \mid C I U I I . I T Y$ LIMII & a. & - & 0 . & - & 1.0 & 21.2 \\
\hline$(n)$ & RI,AI: I:URVATHRF & 14.4 & 16.8 & 19.4 & $1 \pi .4$ & 13.7 & 8.3 \\
\hline$(7)$ & 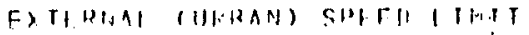 & 14.5 & 13.7 & 10.2 & 12.1 & 0 . & - \\
\hline
\end{tabular}

West Germany - Snow Condition

\begin{tabular}{|c|c|c|c|c|c|c|c|c|}
\hline 1) & IRSUFHIEIIENT S!III & STOENATH & 0 . & - & 0. & - & 0 . & - \\
\hline 2) & INGUFFICIINI THAC. & IIIIN & 0 . & - & 0 . & - & 0. & - \\
\hline 3) & PUH, HUESS (DIUF) & SPFFU I IMII & 0 . & - & 0 . & - & 56.7 & 16.0 \\
\hline 4) & SHILCSLAOPF UFSIST & ANIFS & 70.8 & 30.1 & 68.2 & 27.0 & 27.0 & 21.2 \\
\hline (5) & VISIMUIITY LINIT & & 0 . & - & 0 . & - & 0.3 & 27.1 \\
\hline (h) & R(AA) R:HRVATURF & & 14.8 & 16.1 & 21.6 & 12.8 & 16.0 & 8.8 \\
\hline 小 & FXTH PNAL (IHWRAN) & SPFFIIIINIT & 14.5 & 13.0 & 10.2 & 9.4 & 0 . & - \\
\hline
\end{tabular}

Mid-East - Wet Condition

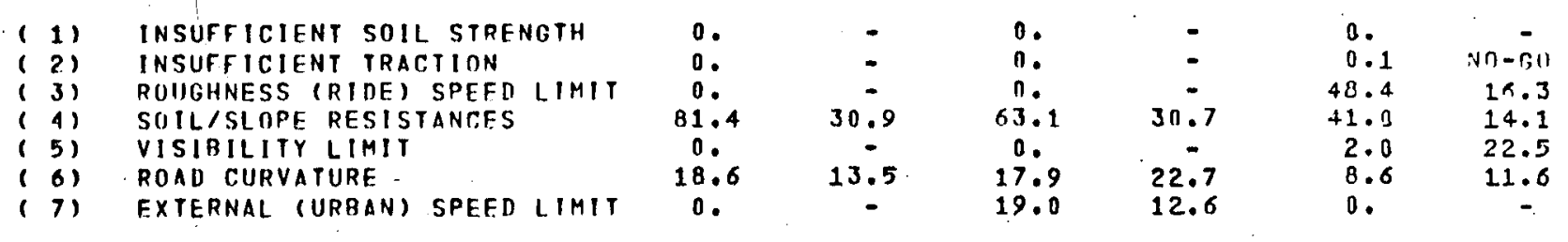

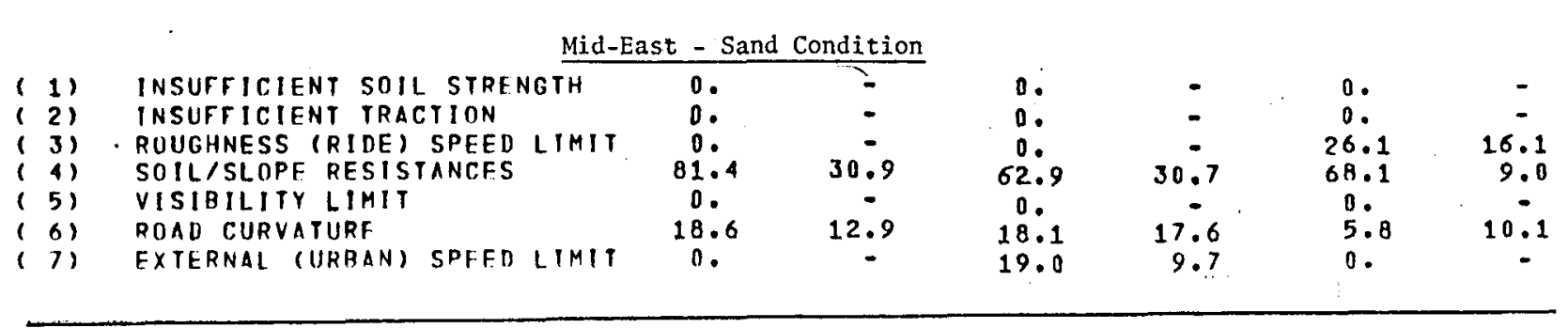


Table E77

Diagnostics Statistics for M113A1

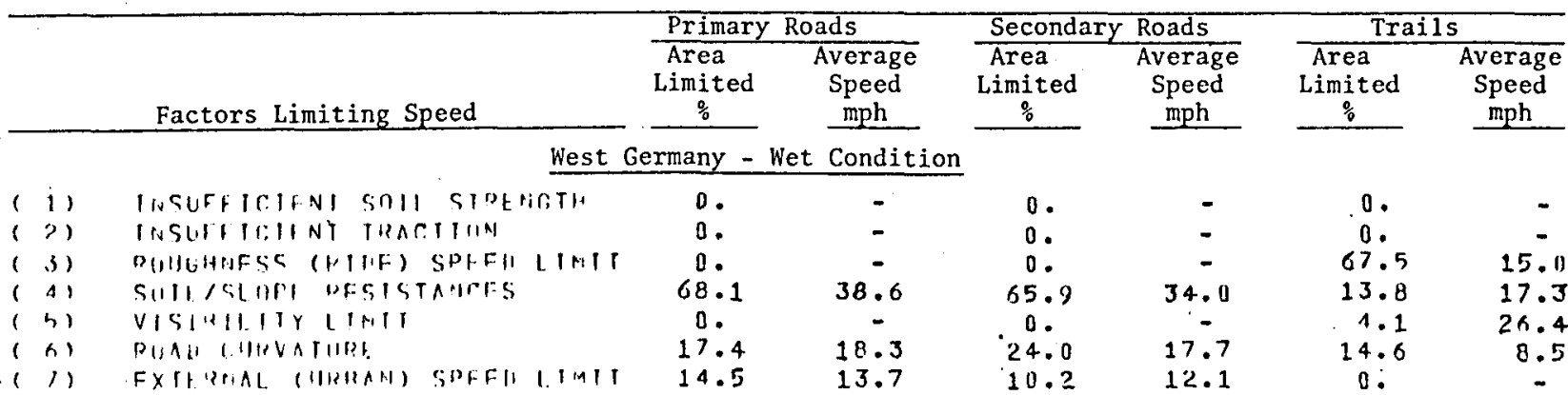

West Germany - Snow Condition

\begin{tabular}{|c|c|c|c|c|c|c|c|c|}
\hline$(1)$ & INSUTFICIENI SOII & STPFNATH & $\pi$. & - & 0. & - & 0 . & - \\
\hline$(2)$ & THSUFI IC:IFNI TRAC: & $110 \times 1$ & 0. & - & 0. & - & 0 . & - \\
\hline 31 & RUIH,WNSS (UIHF) & $S P|F \|| M I \mid$ & 0 . & - & 0. & - & 77.8 & 16.2 \\
\hline A) & SUIL/SLUPF RFSIST & AMCFS & 67.3 & 41.3 & 10.2 & 21.8 & 5.5 & 16.7 \\
\hline$n_{1}$ & VISIMILITY IIMIJ & & a. & - & 0.7 & 37.7 & 0.6 & 31.4 \\
\hline h) & RUAL COUPVATURE & & $18 \cdot 3$ & 17.7 & 78.9 & 24.7 & 16.1 & 9.0 \\
\hline 71 & FXIERNAI (UKRAN) & SPEFI I IMII & 14.5 & 13.0 & 10.2 & 2.4 & 0. & - \\
\hline
\end{tabular}

Mid-East - Wet Condition

\begin{tabular}{|c|c|c|c|c|c|c|c|c|c|}
\hline 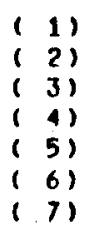 & $\begin{array}{l}\text { INSUFFICIENT SOIL } \\
\text { INSUFFICIENI TRAC } \\
\text { ROUGHNESS (RIDE) } \\
\text { SUIL/SLOPE RESIST } \\
\text { VISIBILITY LIHIT } \\
\text { ROAD CURVATURE } \\
\text { EXTERNAL (URAAN) }\end{array}$ & $\begin{array}{l}\text { L STRE } \\
\text { CTION } \\
\text { SPFFD } \\
\text { TANCFS }\end{array}$ & $\begin{array}{l}\text { NGTH } \\
\text { LIMIT }\end{array}$ & $\begin{array}{c}0 . \\
0 . \\
0.0 \\
73.4 \\
0.0 \\
26.6 \\
0 .\end{array}$ & $\begin{array}{c}- \\
41.8 \\
16.6\end{array}$ & $\begin{array}{l}0 . \\
0 . \\
0 . \\
55.4 \\
0 . \\
25.6 \\
19.0\end{array}$ & $\begin{array}{c}- \\
\vdots \\
41.3 \\
26.0 \\
12.6\end{array}$ & $\begin{array}{r}0 . \\
1.4 \\
64.3 \\
17.4 \\
6.7 \\
10.1 \\
0 .\end{array}$ & $\begin{array}{c}-\overline{-} \\
N O-B O \\
15.9 \\
15.4 \\
27.4 \\
12.5\end{array}$ \\
\hline
\end{tabular}

Mid-East - Sand Condition

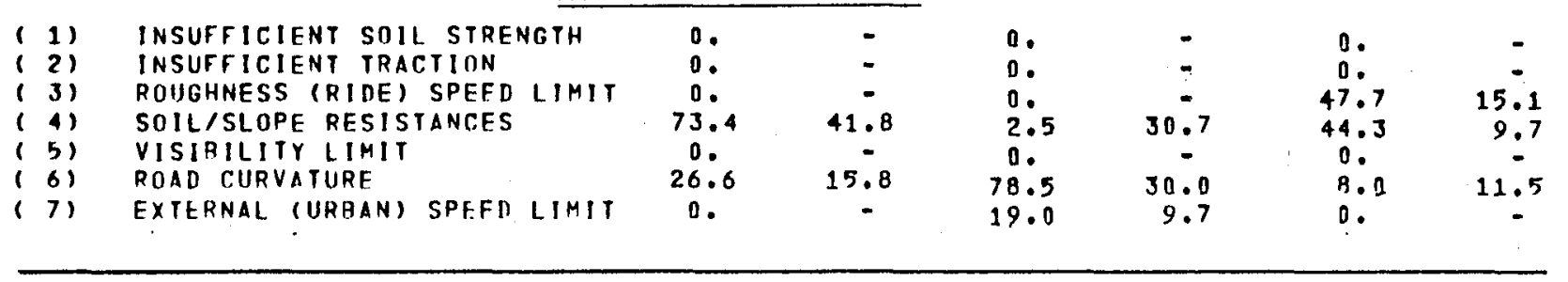


Table E78

Diagnostics Statistics for M60A2

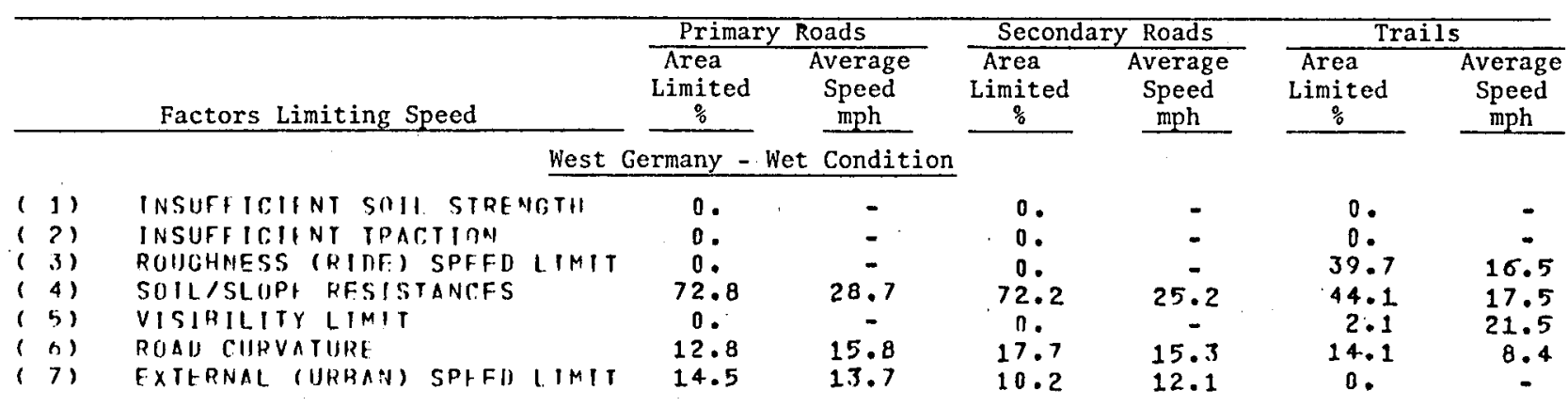

West Germany - Snow Condition

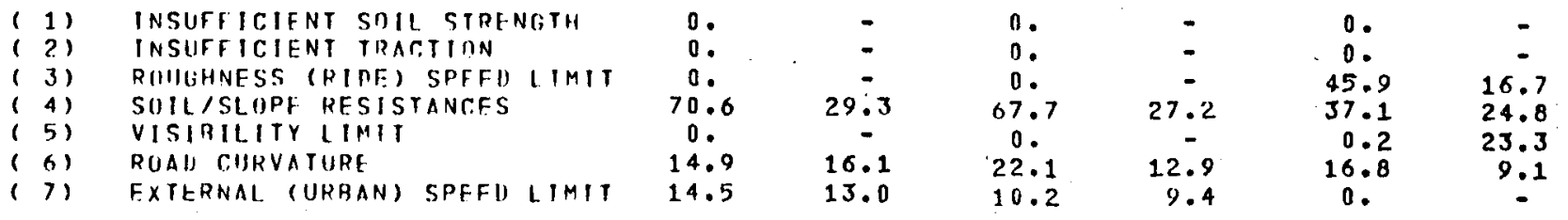

Mid-East : Wet Condition

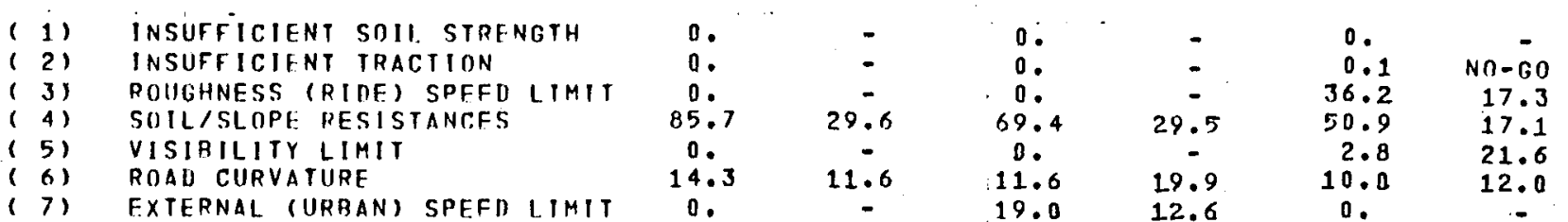

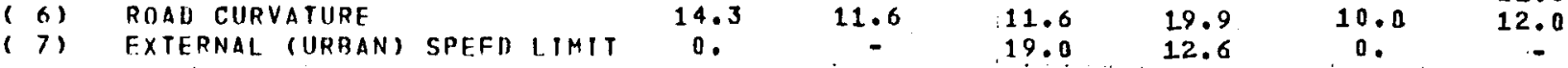

Mid-East - Sand Condition

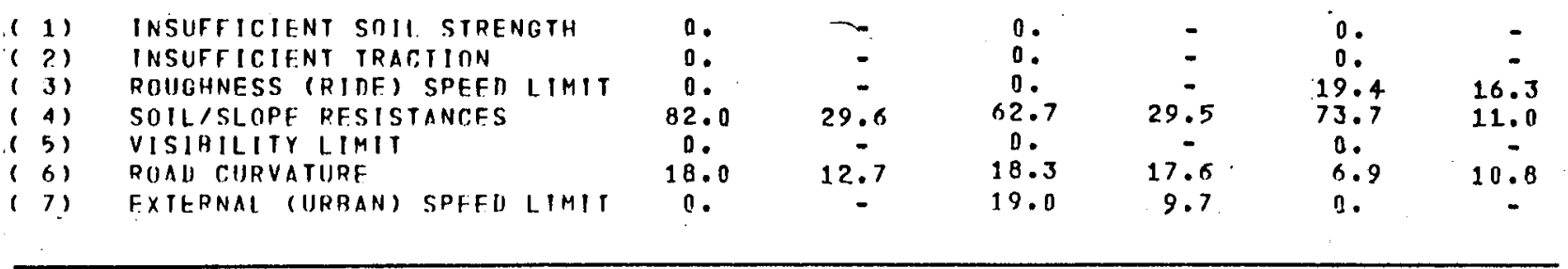


Table E79

Diagnostics Statistics for M548E1

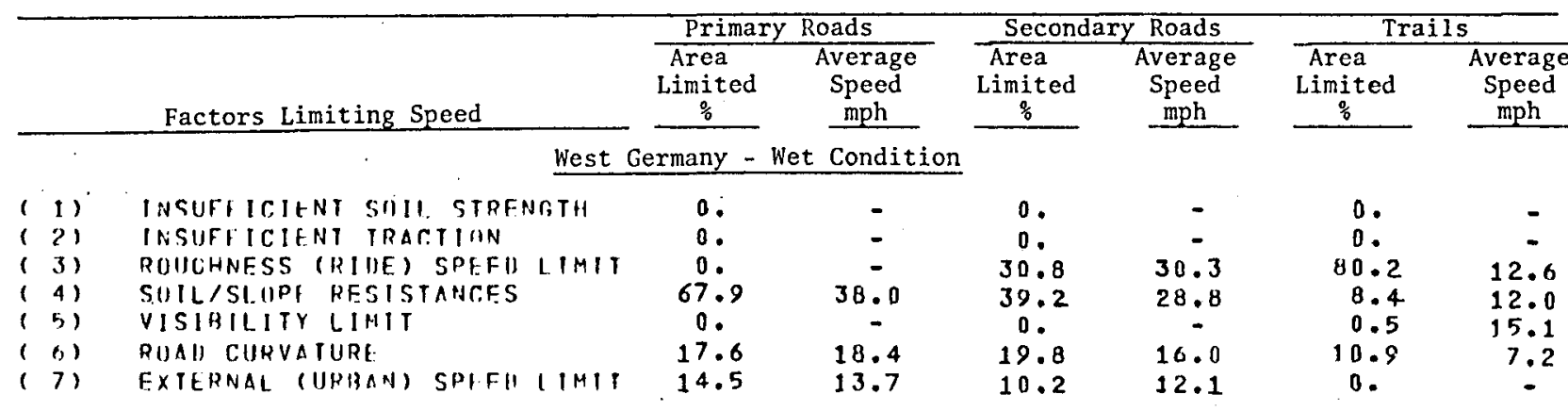

West Germany - Snow Condition

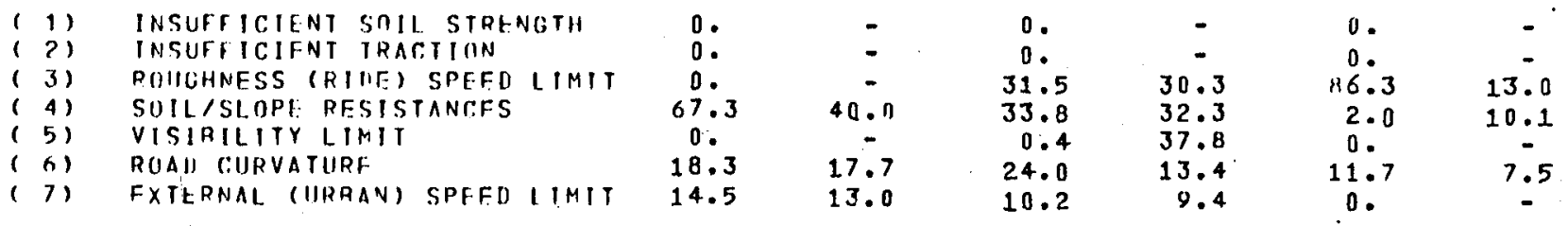

Mid-East - Wet Condition

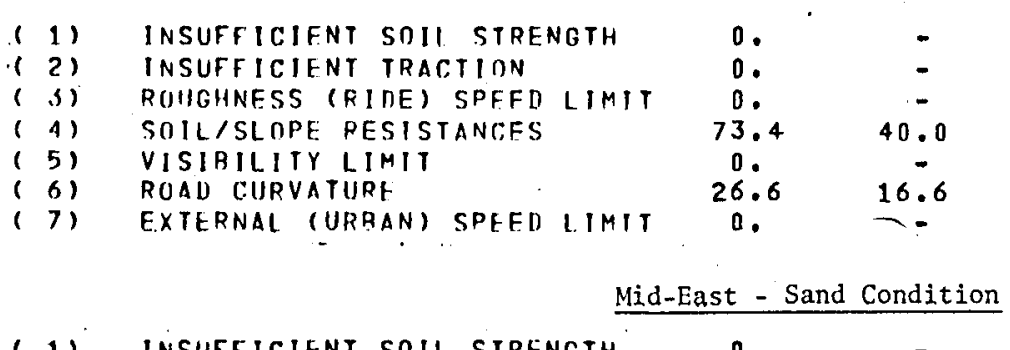

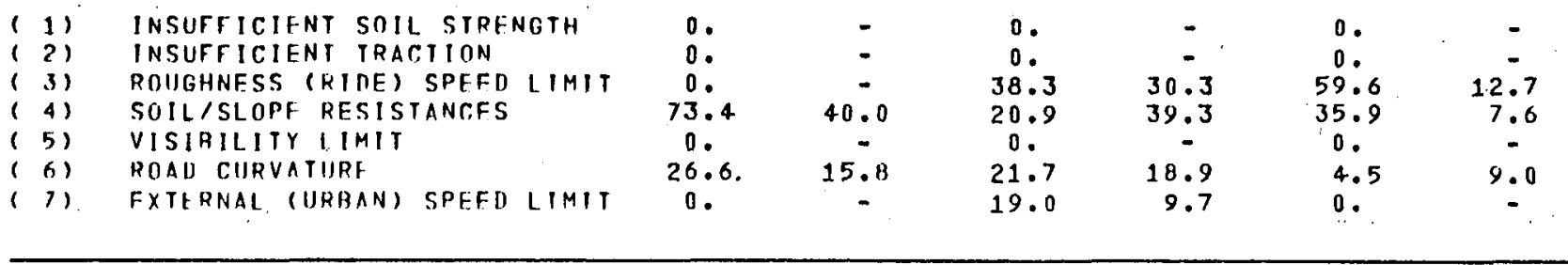


Table E80

Diagnostics Statistics for M577Al

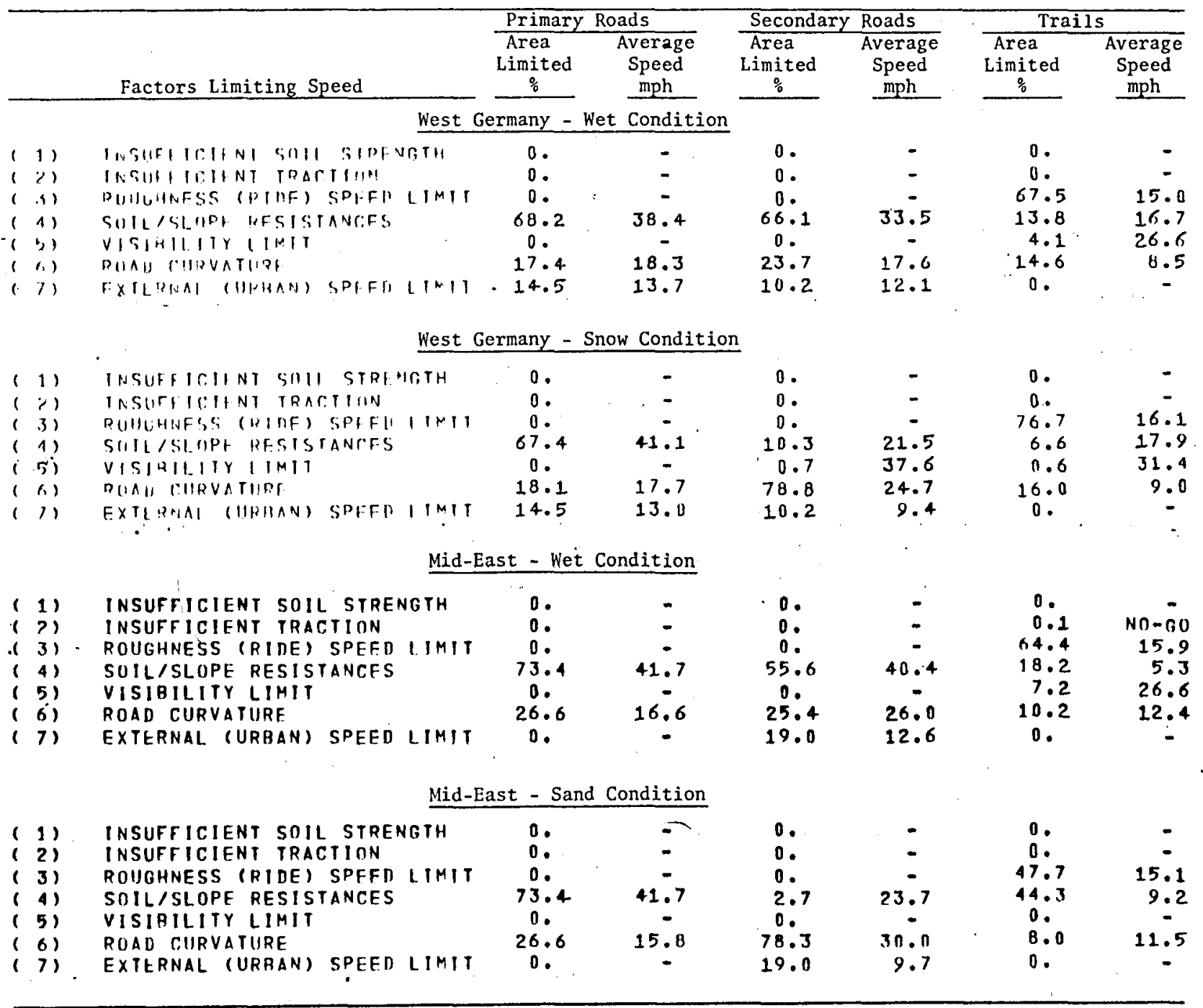


Table E81

Diagnostics Statistics for XM723

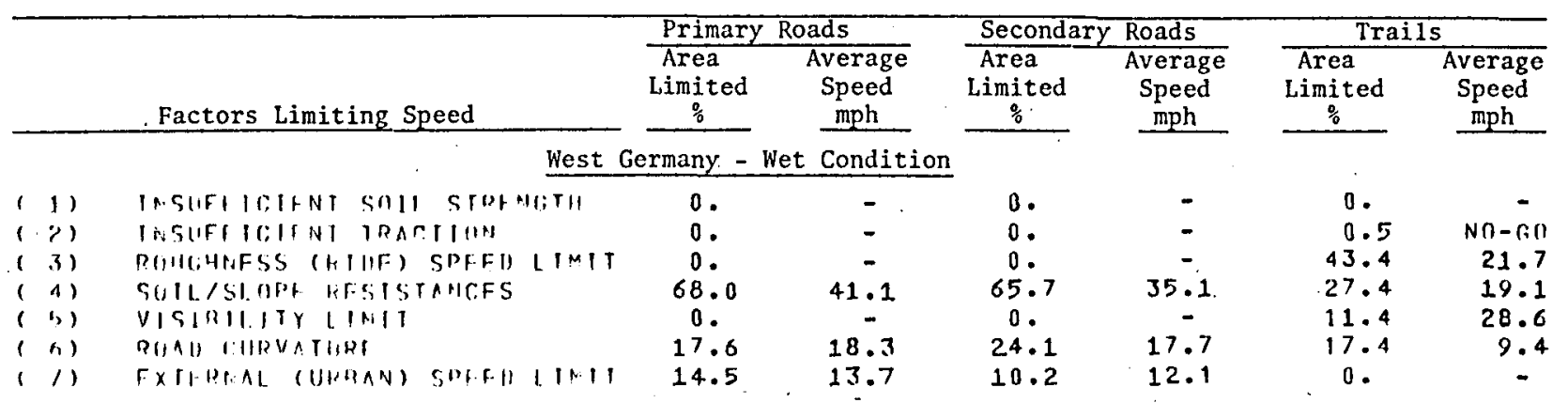

West Germany - Snow Condition

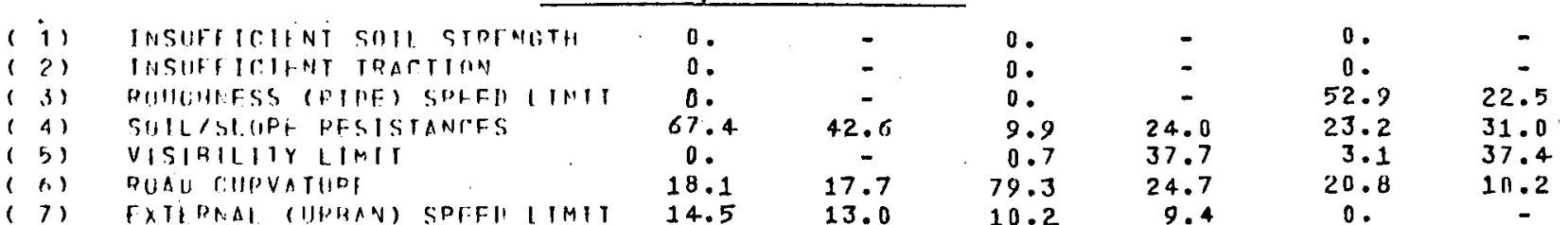

Mid-East - Wet Condition

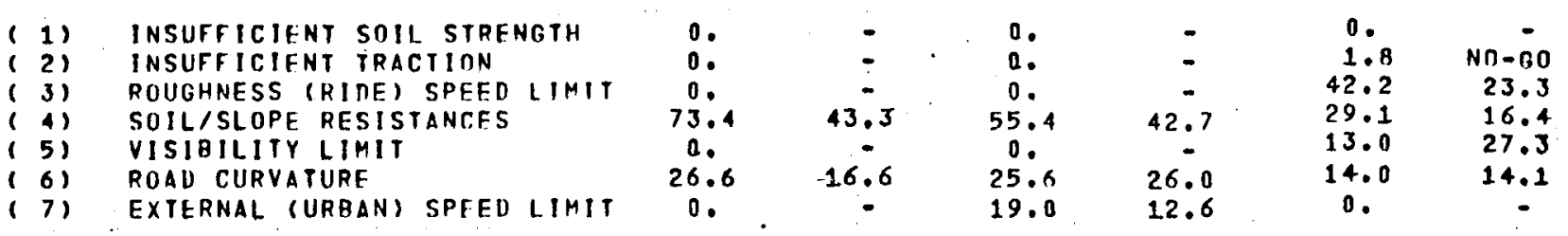

Mid-East - Sand Condition

\begin{tabular}{|c|c|c|c|c|c|c|c|c|c|}
\hline 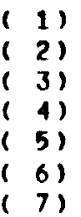 & $\begin{array}{l}\text { INSUFFICIENT SOIL } \\
\text { INSUFFICIENT TRAC } \\
\text { ROUGHNESS (RIDE) } \\
\text { SOILISLOPE RESIST } \\
\text { VISIBILITY LIMIT } \\
\text { ROAD CURVATURE } \\
\text { FXTERNAL (URAAN) }\end{array}$ & $\begin{array}{l}\text { STREN } \\
\text { TION } \\
\text { SPEED } \\
\text { TANCES }\end{array}$ & $\begin{array}{l}\text { NGTH } \\
\text { LIMIT }\end{array}$ & $\begin{array}{l}0 . \\
0 . \\
0.0 \\
73.4 \\
0.0 \\
26.6 \\
0 .\end{array}$ & $\begin{array}{c}\overline{-} \\
43.3 \\
15.8\end{array}$ & $\begin{array}{l}0 . \\
0 . \\
0 . \\
2.5 \\
0.0 \\
78.5 \\
19.0\end{array}$ & $\begin{array}{c}- \\
29.5 \\
30.0 \\
9.7\end{array}$ & $\begin{array}{c}0 . \\
0.0 \\
23.8 \\
64.9 \\
0.0 \\
11.3 \\
0 .\end{array}$ & $\begin{array}{c}= \\
22.4 \\
12.6 \\
13.4\end{array}$ \\
\hline
\end{tabular}


Table E82

Diagnostics Statistics for XMI

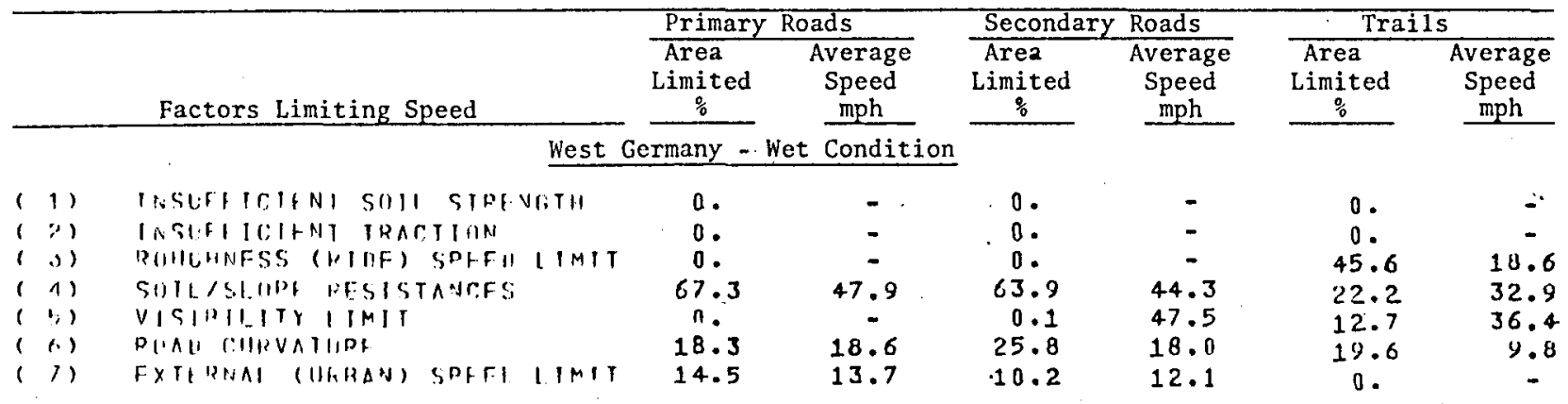

West Germany - Snow Condition

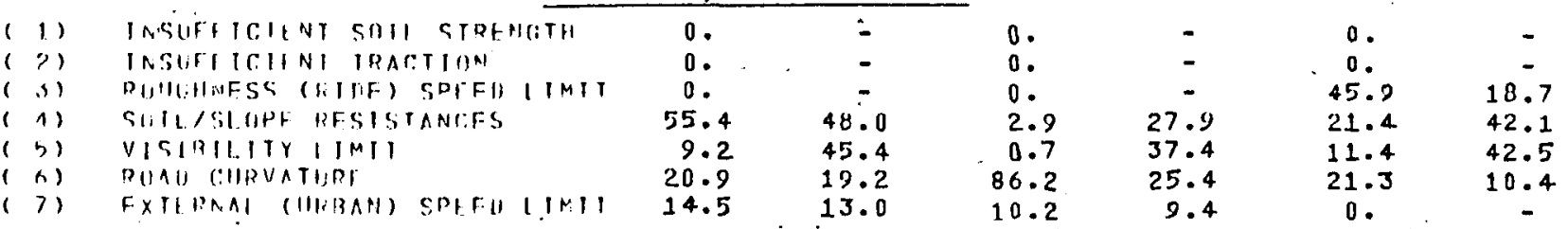

Mid-East - Wet Condition

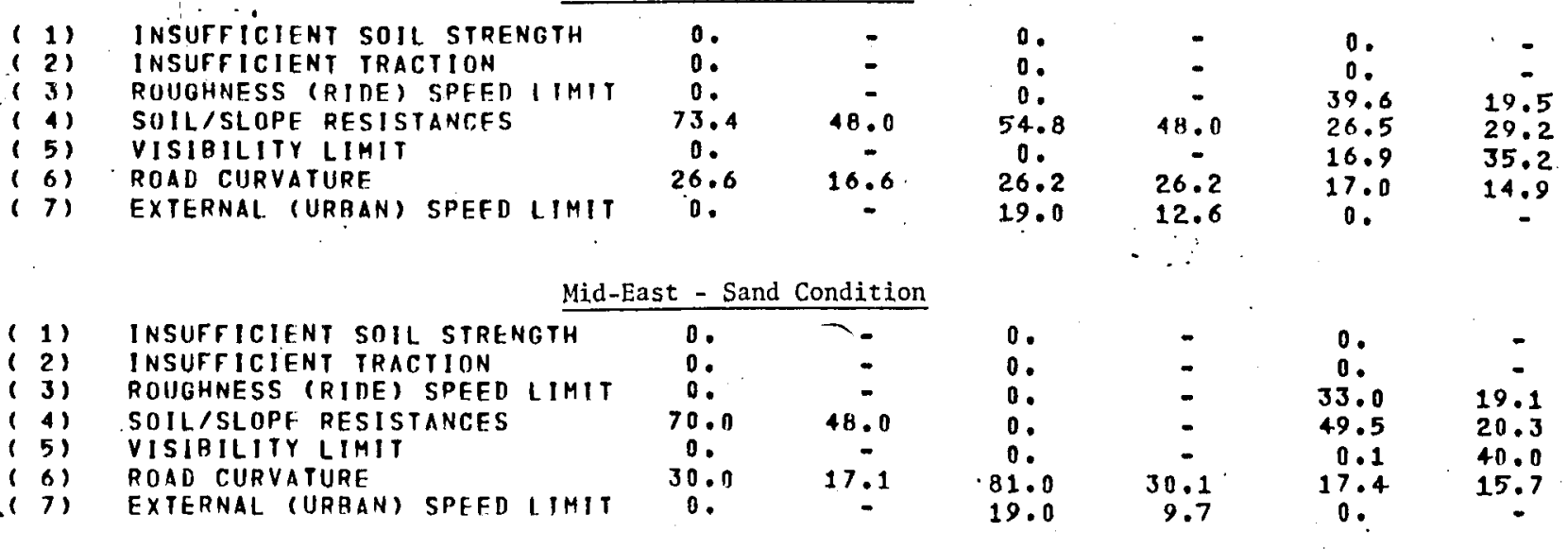


Table E83

Diagnostics Statistics for ASV

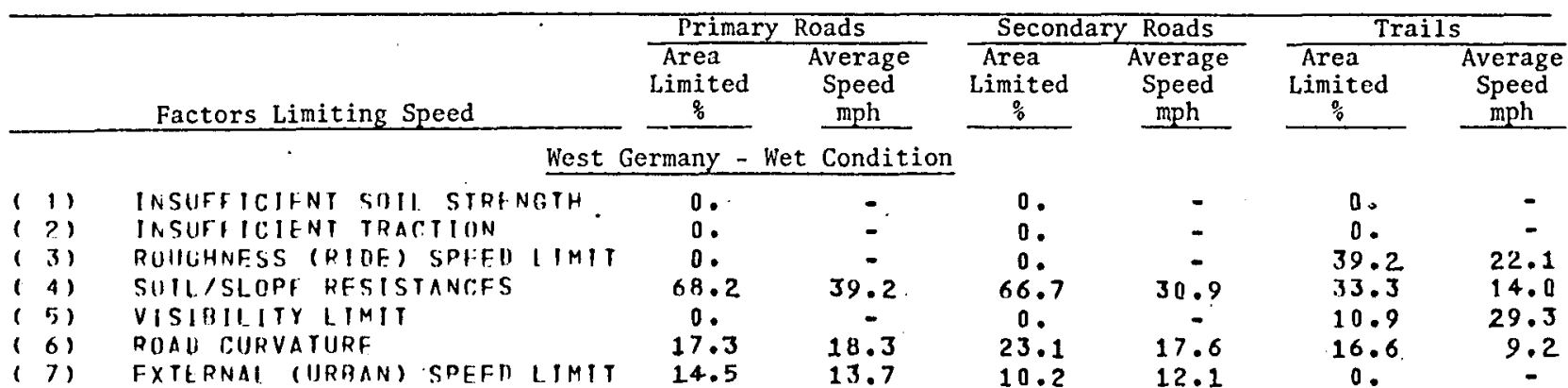

West Germany - Snow Condition

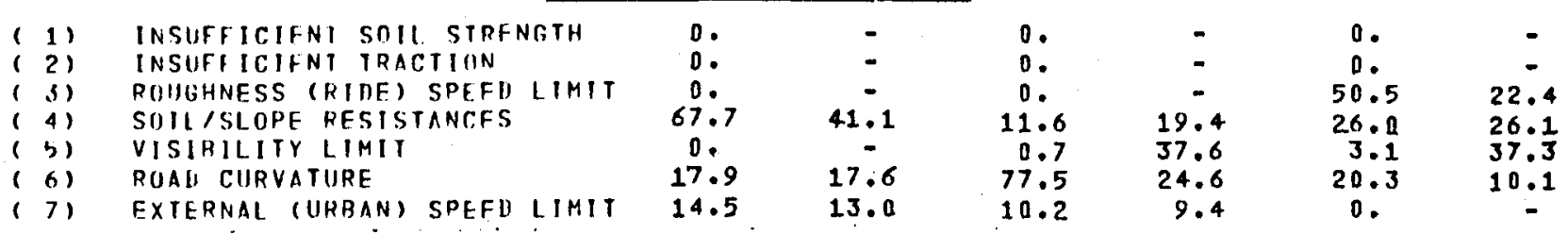

Mid-East - Wet Condition

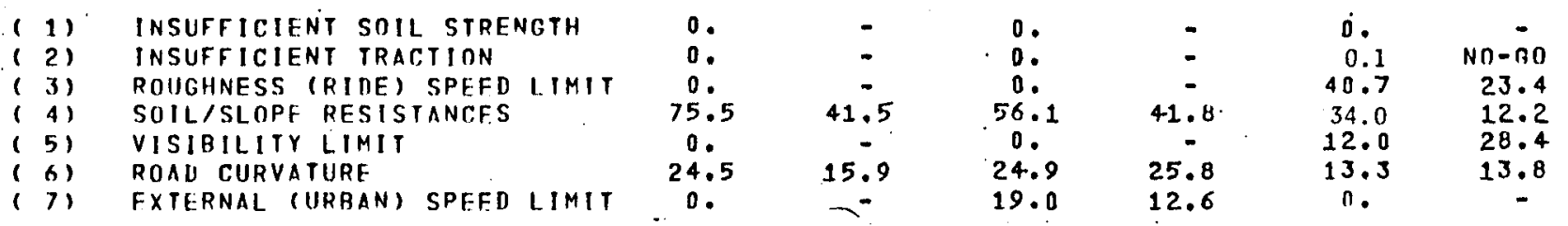

Mid-East - Sand Condition

\begin{tabular}{|c|c|c|c|c|c|c|c|c|c|}
\hline (1) & INSUFFICIENT SOIL & L STREA & NGTH & 0. & - & 0 & & 0. & - \\
\hline$(2)$ & INSUFFICIENT TRAC & $\operatorname{CTION}$ & & 0. & - & 0 . & - & 0 . & - \\
\hline$(3)$ & ROIIGHNESS (RIDE) & SPEED & LIMIT & 0. & - & o. & - & 19.7 & 21.5 \\
\hline$(4)$ & SOIL/SLOPE RESIST & TANCFS & & 75.5 & $41 \cdot 5$ & 3.1 & 25.3 & 71.1 & 10.1 \\
\hline$(5)$ & VISIRILITY LIMIT & & & 0. & - & 0. & - & 0. & - \\
\hline$(6)$ & ROAD CURVATUIRE & & & 24.5 & 15.1 & 77.9 & 29.9 & 9.2 & 13.0 \\
\hline$(7)$ & EXTERNAL (URBAN) & SPFFD & LIMIT & 0. & - & 19.0 & 9.7 & 0 . & - \\
\hline
\end{tabular}


Table E84

Diagnostics Statistics for M561

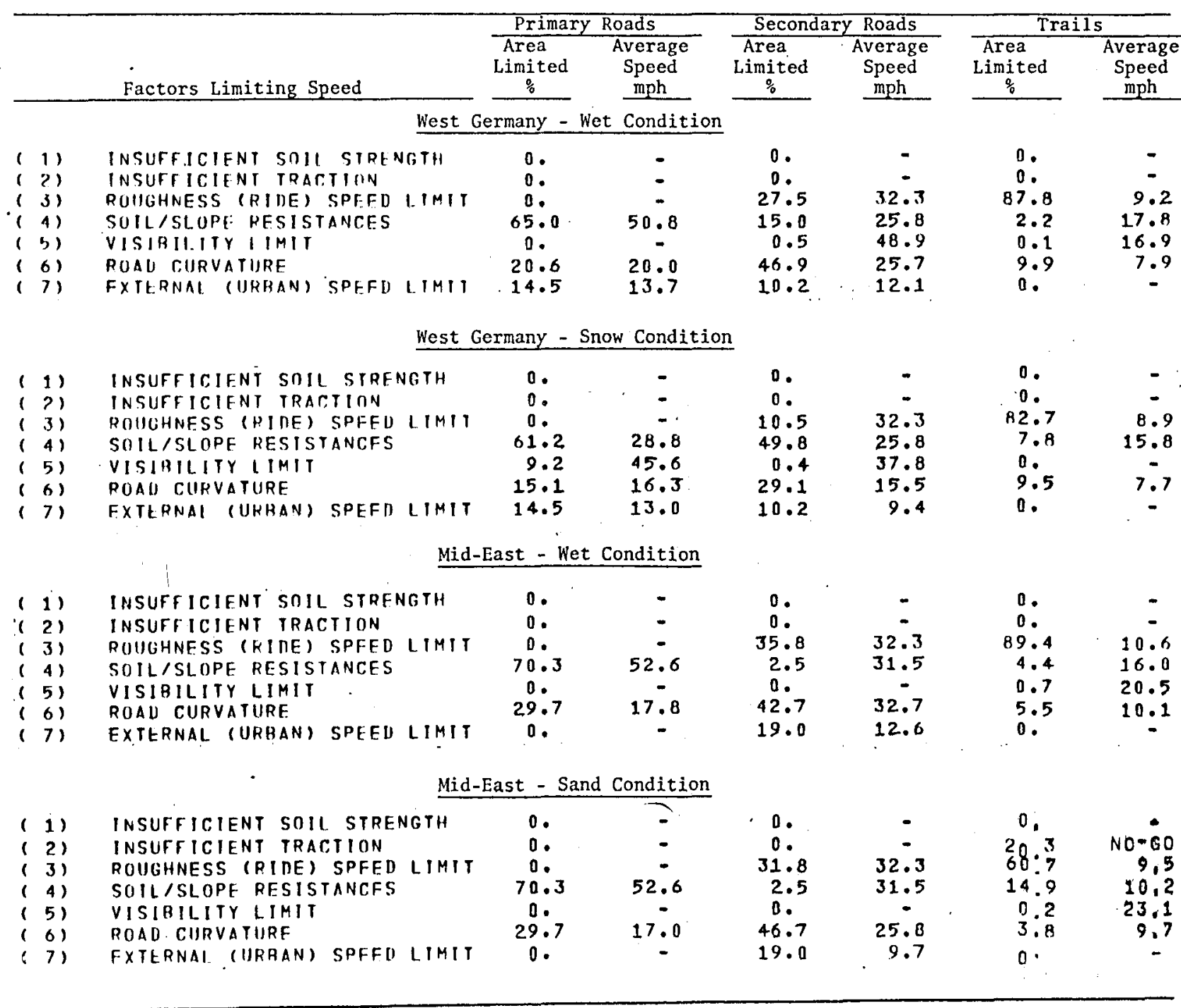


Table E85

Diagnostics Statistics for M813

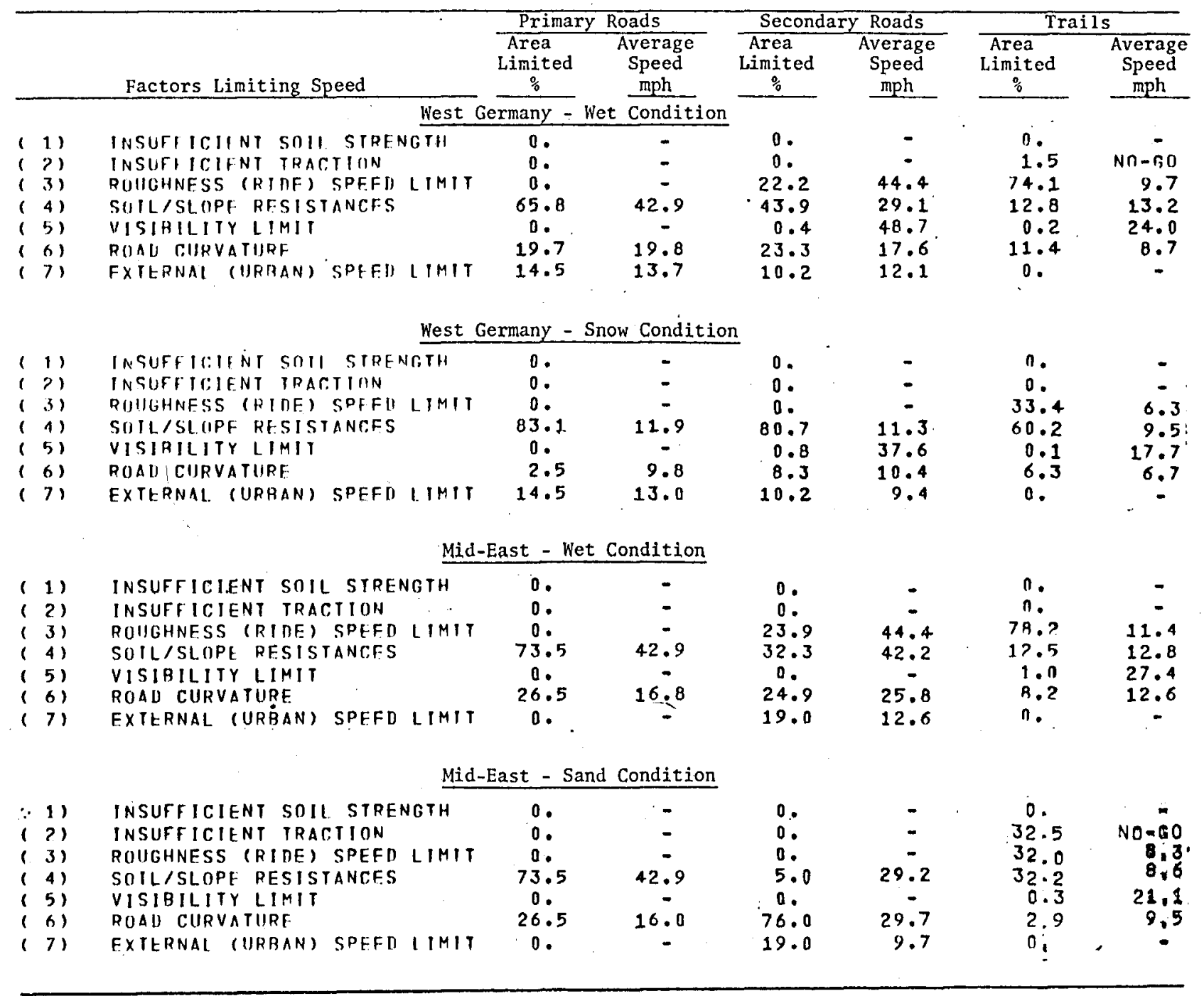


Table E86

Diagnostics Statistics for UET

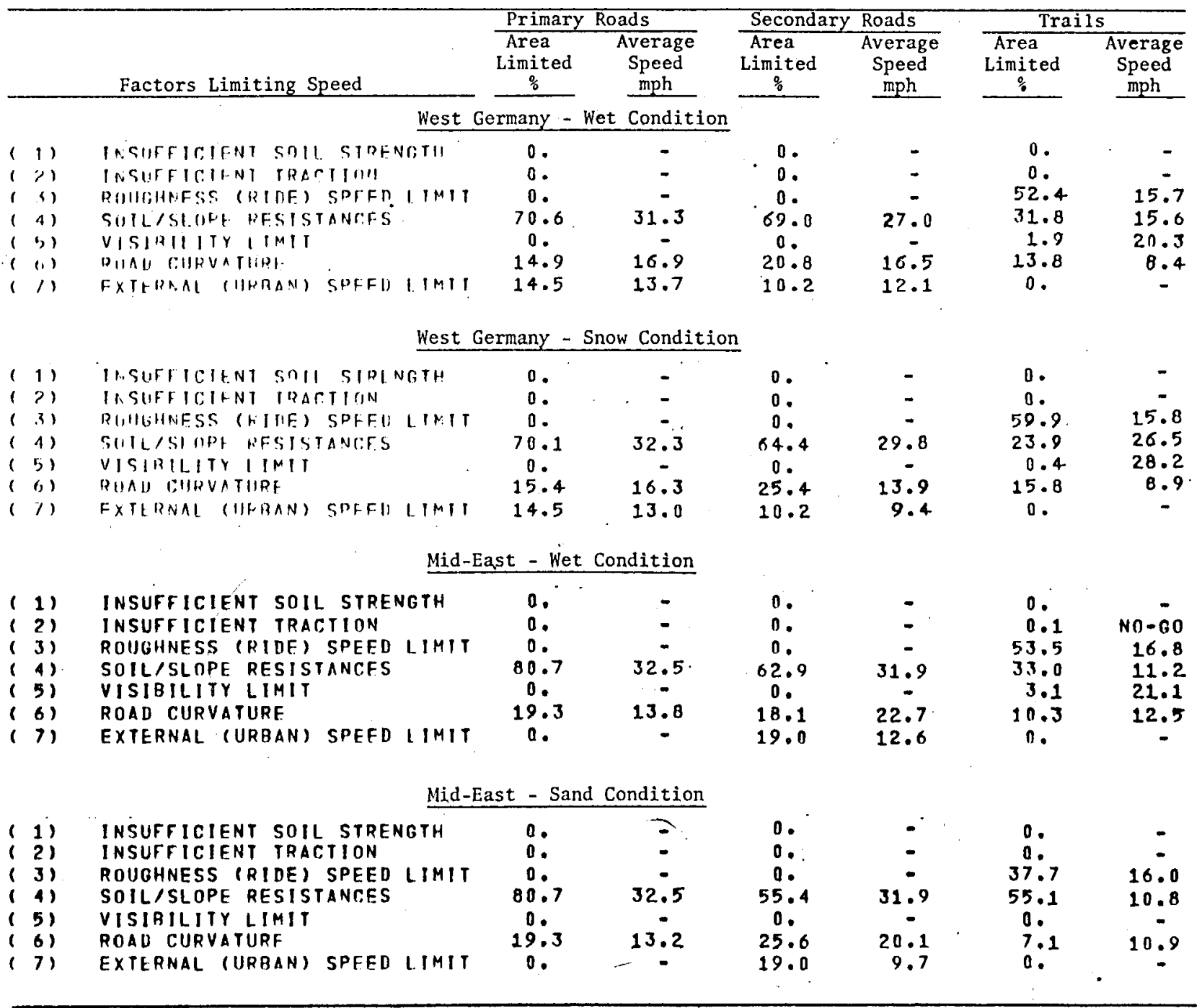


Table $\mathrm{E87}$

Diagnostic Statistics for Study Vehicles

Factors Limiting Speed

Wet Condition Germany

\begin{tabular}{|c|c|}
\hline \multicolumn{2}{|c|}{ Mid-East } \\
\hline$t$ & S.2n \\
\hline $\begin{array}{c}\text { Average } \\
\text { Specd, } \\
\text { imited } \\
\text { mph }\end{array}$ & $\begin{array}{l}\text { Average } \\
\text { Speed, } \\
\text { mpin }\end{array}$ \\
\hline
\end{tabular}
$561-\overline{M 102}$

(1) INSUFFICIENT SOIL STRENGTH
(2) INSUFFICIENT TRACTION FOR SLOPES
(3) OBSTACLE INTERFERENCE OR INSUFF ICIENT OBSTACLE TRACTION
(4) COMBINATION OF TERRAIN FACTORS
(5) ROUGHNSS (RIDE) SPEED LIMTT
(6) SOTL/SLOPE RESISTANCES
(7) VISIBILITY LIMIT
(8) MUNEUVR PROBEMS
(9) VEGETETION RESISTANCES
(10) OBSTACLE CROSSING SPEED AND AC/DC BETWEEN OBSTACLES

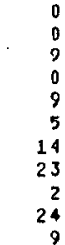

0.9
0.8
0.2
0.9
9.6
5.6
14.3
23.9
2.0
24.2
9.5

$$
\begin{array}{r}
\text { - } \\
N 0=60 \\
N O-60 \\
N O-G 0 \\
15.4 \\
11.9
\end{array}
$$

AND AC/DC BETWEEN OBSTACLES

11.5
10.7

$\begin{array}{r}9.5 \\ \times M 204 \\ \hline\end{array}$

(1) INSUFFICIENT SOIL STRENGTH

i.

INSUFFICIENT TRACTION FOR SLOPES

OBSTACLE INTERFERENCE OR INSUH

ROUCHNESS (RIDE) SPEED LIMIT

MANEUYER PROBLEMS

VEGETATION RESISTANCES

OBSTACLE CROSSING SPEED AND AC/DC BETWEEN OBSTACLES

EXTERNAL (URBAN) SPEED LIMI

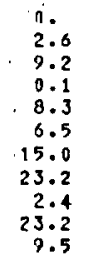

NO-GO
NO-GO
N $0-60$
15.4
3.9
11.3
0.3
6.5
8.5
8.5
14.7

\section{Area Average}

Limited Speed,

Arca Speed,

Limited

min

\section{$\underline{\text { MS61-XM198 }}$}

INSUFFICIENT SOIL STRENGTH

INSUFFICIENT TRACTION FOR SLOPES

OBSTACLE INTERFERENCE OR INSUFS

ROUGHNESS (RIDE) SPEED LIMIT

SOIL/SLOPE RESISTANCES

VISIBILITY LIMIT

MANEUVER PROBLEMS
VEGETATION RESISTANCES

VEGETATION RESISTANCES

EXTERNAL (URBAN) SPEED LIMIT

$\begin{array}{rr}0 . & \\ 0.0 & \text { NO.CO } \\ 9.2 & 0 . \\ 0.0 & 15.9 \\ 12.0 & 9.4 \\ 10.5 & 9.4 \\ 1.2 & 12.5 \\ 23.2 & 10.3 \\ 4.1 & 10.0 \\ 24.2 & 8.6 \\ 9.5 & 14.7\end{array}$

0.
0.3
10.3
0.3
22.7
3.5
23.3
16.2
2.4
11.8
3.4

-
$N 0-60$
$N 0-60$
10.9
7.8
11.6
11.0
6.0
6.5
14.9

$43.7 \quad N O-60$

$12.9 \quad$ NO-GO
$9.1 \quad$ NO-GO
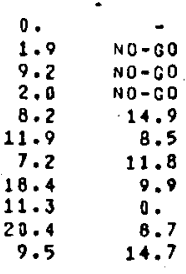

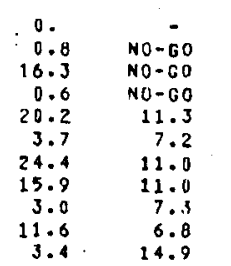

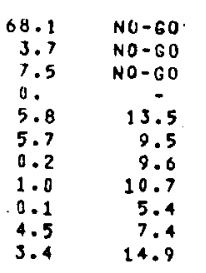

INSUFFICIENT SOIL STRENGTH

NO.GO

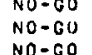

NO-GO

NO $0-60$
14.2
6.9

6.9
10.2

11.

$\begin{array}{rl}19.4 & 0 . \\ 5.8 & 5.2\end{array}$

$\begin{array}{rr}11.5 & 7.9 \\ 9.5 & -14.7\end{array}$

$\begin{array}{rr}0 . & \\ 54.8 & - \\ 9.2 & N O-60 \\ 9.2 & \text { NO-G0 } \\ 2.3 & \text { NO-60 } \\ 0.7 & 13.7 \\ 4.1 & 8.1 \\ 1.9 & 12.2 \\ 9.0 & 1.2 \\ 4.0 & 3.2 \\ 4.1 & 8.00 \\ 9.5 & 14.7\end{array}$

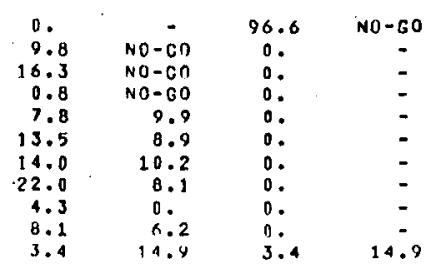

(1) INSUAFICIENT SOIL STRENGTH
(2) INSUFFICIENT TRACTION FOR SLOPES
(3) OBSTACLE INTERFERENCE OR INSUFF ICIENT OBSTACLE TRACTION
(4) COMBINATON OF TERRAI FACTRS
(5) ROUGHNES (RIDE) SPEED LIMIT
(6) SOIL/SLOPE RESISTANCES
(7) VISIBILITY LIMIT
(8) MANEUVER PROBLEMS
(9) VEGETATION RESISTANCES
(10) OBSTACLE CROSSING SPEED AND AC/DC BETWEEN OBSTACLES
(11) EXTERNAL (URBAN) SPEED LIMIT

M3SA2-M102

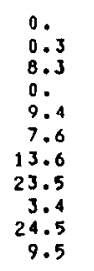

NO-60
NO-60
-
14.9
11.1
12.5
10.4
11.1
7.8
14.7

0.0
4.2
8.3
3.0
3.5
30.0
3.3
14.4
10.1
13.7
9.5

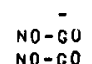

0.
0.
13.9
0.9
30.8
3.1
20.5
16.6
1.7
10.0
3.4

-
$N O-60$
11.2
11.2
8.6
12.3
10.4
13.9
3.4
14.9

$\begin{array}{ll}41.3 & \text { NO } \\ 250 & -60 \\ 25.9 & \text { NO }\end{array}$

4.9
9.9

$\begin{array}{rr}4.0 & \text { NO }-60 \\ 5.2 & 12.9\end{array}$

$0.7 \quad 11.4$

$\begin{array}{ll}3.4 & 10.2 \\ 1.0 & 3.3 \\ 0.6 & 3.4\end{array}$

(Continued)

(Sheet 1 of 11) 
Table E87 (Continued)

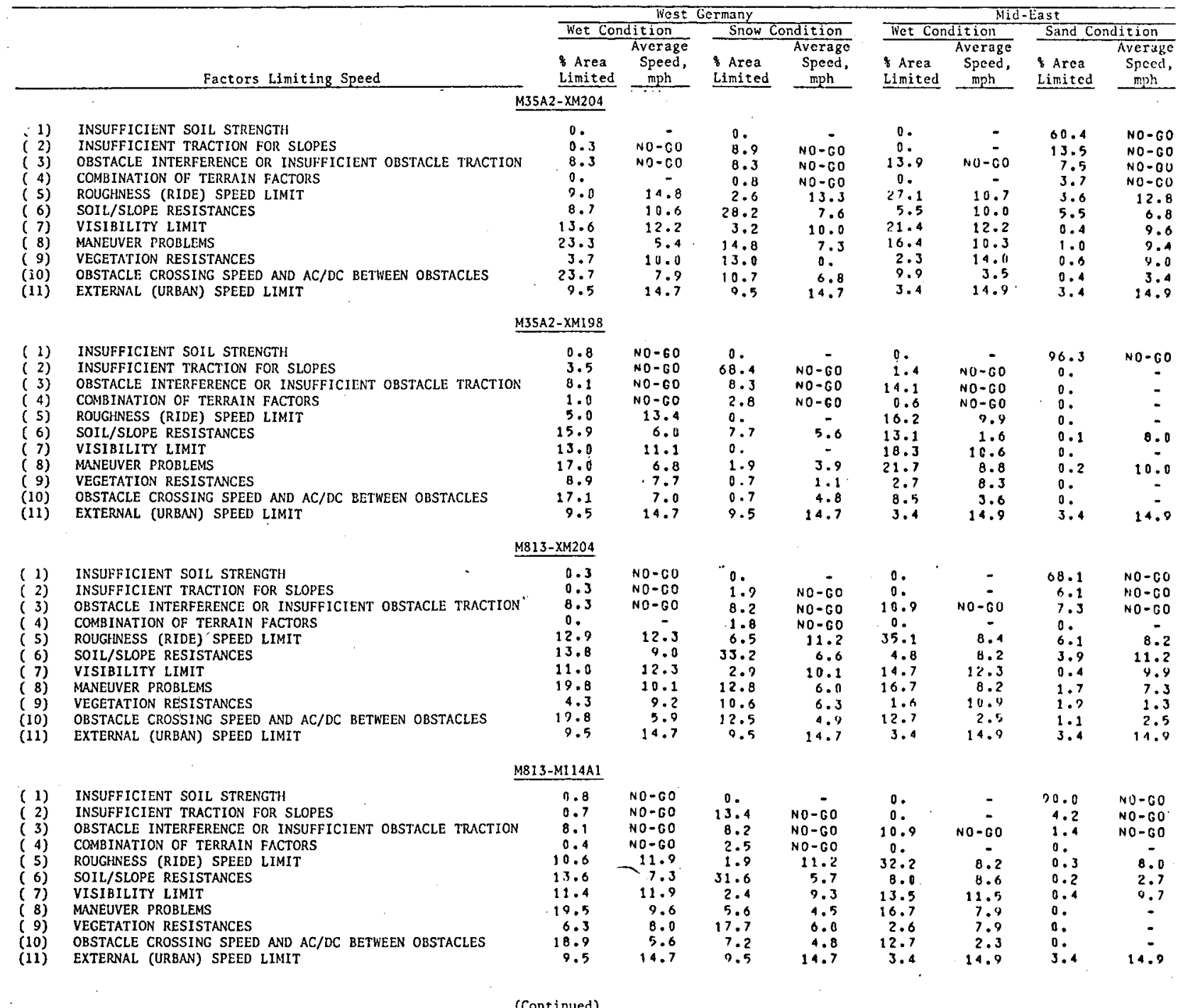


Table E87 (Continued)

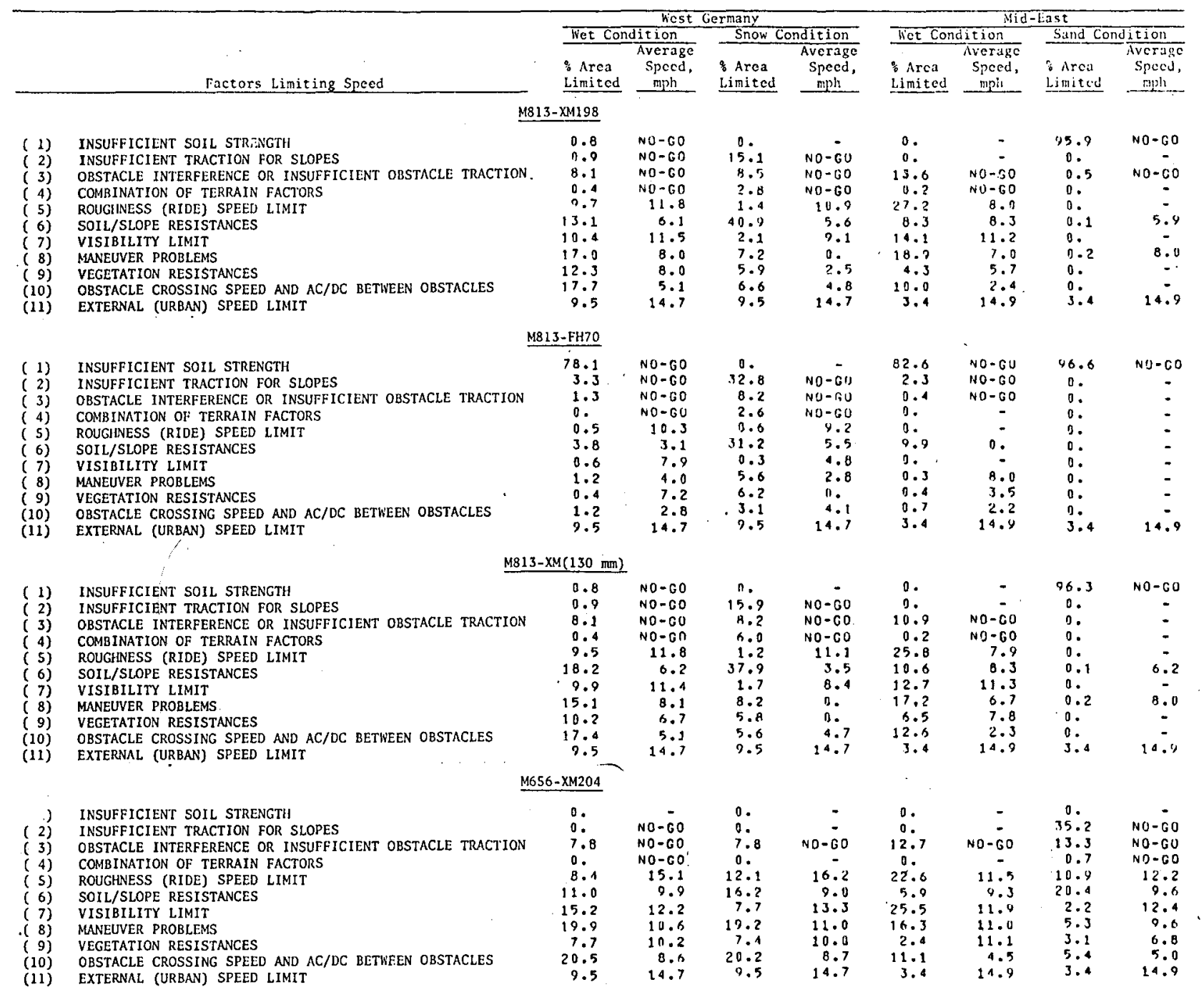

(Cont inued)

(Sheet 3 of 11 ) 
Table E87 (Continued)

\begin{tabular}{|c|c|c|c|c|c|c|c|c|c|}
\hline & & & West & $\frac{\operatorname{cormany}}{c}$ & & & Wid & Eist & \\
\hline & & Wet Con & ndition & Snow C & ndition & Wot Con & dition & Sand Co & dition \\
\hline & & & & & & & & & \\
\hline & Factors Limiting Speed & $\begin{array}{l}\text { Area } \\
\text { Limited }\end{array}$ & $\begin{array}{c}\text { Speed, } \\
\text { mph }\end{array}$ & $\begin{array}{l}\text { Arra } \\
\text { Limited }\end{array}$ & $\begin{array}{l}\text { Speed, } \\
\text { mph }\end{array}$ & $\begin{array}{l}\text { Arca } \\
\text { Limited }\end{array}$ & $\begin{array}{c}\text { Speed, } \\
\text { mph }\end{array}$ & $\begin{array}{l}8 \text { Arca } \\
\text { Limited }\end{array}$ & $\begin{array}{c}\text { Speed, } \\
\text { mphh }\end{array}$ \\
\hline & & $6-X M 198$ & & & & & & & \\
\hline & INSUFFICIENT SOIL STRENGTH & 0 & - & 0. & - & 0. & $\cdot$ & 82.6 & NO-CO \\
\hline (2) & INSUFFICIENT TRACTION FOR SLOPES & 2.9 & NOCOO & 4.2 & NO-GO & 0.0 & NO-GO & 1.4 & NO-GO \\
\hline (3) & OBSTACLE INTERFERENCE OR INSUFFICIENT OBSTACLE TRACTION & 7.8 & NO -60 & 7.8 & NO-GO & 13.3 & NO-CU & 0.9 & NO-GO \\
\hline (4) & COMBINATION OF TERRAIN FACTORS & 0.9 & $\mathrm{NO}-\mathrm{CO}$ & 1.5 & NUTGO & 0.7 & NO- $-6 O$ & o. & \\
\hline (5) & ROUGINESS (RIDE) SPEED LIMIT & 5.6 & 14.3 & 5.3 & 14.1 & 17.4 & 11.2 & 3.6 & 10.1 \\
\hline (6) & SOIL/SLOPE RESISTANCES & 12.4 & 0. & 20.6 & 7.4 & 8.9 & 5.1 & 7.3 & 8.5 \\
\hline (7) & VISIBILITY LIMIT & 14.3 & 11.4 & 8.0 & 22.2 & 22.5 & 11.0 & 0.4 & 9.9 \\
\hline (8) & MANEUVER PROBLEMS & 18.5 & 8.4 & 17.2 & 6.3 & 18.9 & 9.2 & 0.3 & 8.9 \\
\hline (9) & VEGETATION RESISTANCES & 11.6 & 6.2 & 10.5 & 0. & 1.6 & 7.1 & & - \\
\hline (10) & OBSTACLE CROSSING SPEED AND AC/DC BETWEEN OBSTACLES & 10.6 & 7.8 & 15.4 & 8. 0 & 9.2 & 4.7 & 0.1 & 3.7 \\
\hline (11) & EXTERNAL (URBAN) SPEED LIMIT & 9.5 & 14.7 & 9.5 & 14.7 & 3.4 & 14.9 & 3.4 & 14.9 \\
\hline & & $11-\mathrm{XM} 204$ & & & & & & & \\
\hline & INSUFFICIENT SOIL STRENGTII & 0. & - & o. & - & 0 . & - & 0 . & - \\
\hline 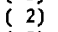 & INSUFFICIENT TRACTION FOR SLOPES & 0. & & 0. & & 0 . & & 17.1 & NO-GO \\
\hline 3) & OBSTACLE INTERFERENCE OR INSUFFICIENT OBSTACLE TRACTION & 5.1 & NOGOG & 4.9 & NO-GO & 2.2 & NO $-G O$ & 8.0 & NO-GO \\
\hline (4) & COMBINATION OF TERRAIN FACTORS & 0. & - & 0. & & 0. & NO-GO & 3.8 & NO-GO \\
\hline ( 5$)$ & ROUGHESS (RIDE) SPEED LIMIT & $4: 4$ & 15.8 & 6.9 & 16.4 & 12.7 & 12.9 & 6.9 & 14.0 \\
\hline (6) & SOIL/SLOPE RESISTANCES & 22.6 & $7 \cdot 3$ & 22.7 & 7.7 & 20.1 & 8.3 & 42.4 & 6.6 \\
\hline (7) & VISIBILITY LIMIT & 26.2 & 12.7 & 9.2 & 14.8 & 22.6 & 12.1 & 2.9 & 12.1 \\
\hline (8) & MNEUUER PROBLEMS & 21.2 & 10.5 & 20.4 & 11.1 & 15.5 & 10.7 & 7.9 & 9.5 \\
\hline (9) & VEGETATION RESISTANCES & 10.9 & 9.7 & 14.7 & 11.3 & 4.9 & 9.2 & 4.4 & 5.0 \\
\hline (10) & OBSTACLE CROSSING SPEED AND AC/DC BETWEEN OBSTACLES & 10.2 & 8.6 & 11.7 & 9.1 & 18.4 & 6.0 & 3.3 & 8.7 \\
\hline (11) & EXTERNAL (URBAN) SPEED LIMIT & 9.5 & 14.7 & 9.5 & 14.7 & 3.4 & 14.9 & 3.4 & 14.9 \\
\hline & & 1 -M114A1 & & & & & & & \\
\hline & INSUFFICIENT SOIL STRENGTH & 0. & - & 0 . & - & o. & - & 43.7 & NO $=60$ \\
\hline (2) & INSUFFICIENT TRACTION FOR SLOPES & 0.2 & NOGO & . & & 0. & No-GO & $\$ 1.4$ & NOEO GO \\
\hline (3) & OBSTACLE INTERFERENCE OR INSUFFICIENT OBSTACLE TRACTION & 5.1 & NO-GO & 5.0 & NO $=G O$ & 2.4 & NO $0-G 0$ & 6.1 & NO $=60$ \\
\hline ( 4) & COMBINATION OF TERRAIN FACTORS & 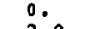 & 10 & 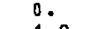 & & 0. & & 0.4 & No -60 \\
\hline ( 5$)$ & ROUGHNESS (RIDE) SPEED LIMIT & 2.9 & 15.8 & 4.9 & 15.9 & 11.1 & 12.6 & $4 \cdot 3$ & 14.1 \\
\hline ( 6) & SOIL/SLOPE RESISTANCES & 28.4 & 6.2 & 24.0 & 6.1 & 26.1 & 7.1 & 23.7 & 4.9 \\
\hline ( 7$)$ & VISIBILITY LIMIT & 14.4 & 12.1 & 10.2 & 14.3 & $20 \cdot 0$ & 11.5 & 0.3 & 9.5 \\
\hline ( 8$)$ & MANEUVER PROBLEMS & 18.6 & 9.4 & 17.2 & 10.2 & 13.0 & 9.6 & 2.9 & 9.0 \\
\hline (9) & VEGETATION RESISTANCES & 11.8 & 9.4 & 18.9 & 10.7 & 8.7 & 6.4 & 2.5 & 6.0 \\
\hline (10) & OBSTACLE CROSSING SPEED AND AC/DC BETWEEN OBSTACLES & 8.9 & 8.6 & 10.3 & 8.7 & 15.2 & 6.7 & 1.3 & 8.6 \\
\hline (11) & EXTERNAL (URBAN) SPEED LIMIT & 9.5 & 14.7 & 9.5 & 14.7 & 3.4 & 14.9 & 3.4 & 14.9 \\
\hline & & $1-\mathrm{x} M 198$ & & & & & & & \\
\hline & INSUFFICIENT SOIL STRENGTH & 0 . & 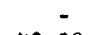 & 0 . & - & o. & 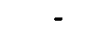 & 82.6 & NO-GU \\
\hline & INSUFF & 0.3 & No-go & 0 . & & n & $N 0-G O$ & 1.4 & NO-GO \\
\hline 3) & RFERENCE OR INSUFFICIENT OBSTACLE TRACTION & 5.0 & NO-GO & 5.1 & NO-GO & 2.4 & NO-GO & 0.2 & NO-CO \\
\hline 4) & COMBINATION OF TERRAIN FACTOR: & 0.7 & NO-GO & 0. & & 0. & & & \\
\hline 5) & $D$ LIMIT & 2.6 & 16.2 & 4.5 & 15.8 & 9.7 & 12.7 & 2.1 & 12.1 \\
\hline 6) & SOIL/SLOPE RESISTANCES & 27.5 & 5.8 & 22.3 & 5.5 & 26. & 6. & 9.8 & 0.7 \\
\hline ( 7 ) & VISI & 13.8 & 11.8 & 10.4 & 14.0 & 16. & 11.3 & 0.2 & 10.0 \\
\hline 8) & MANE & 15.6 & 8.4 & 16.5 & 8.5 & 17.4 & 8.4 & 0.2 & 10.9 \\
\hline (9) & VEGET & 16.8 & 7.8 & 21.7 & 9.2 & 8.0 & 4.9 & 0 . & \\
\hline (10) & ED AND $\wedge$ & 8.2 & 8.5 & 10.0 & 8.6 & 14.4 & 6.4 & 0.1 & 7.8 \\
\hline & EXTERNAL (URBAN) SPEED LIMIT & 9.5 & 14.7 & 9.5 & 14.7 & 3.4 & 14.9 & 3.4 & 14.9 \\
\hline
\end{tabular}


Table E87 (Continucd)

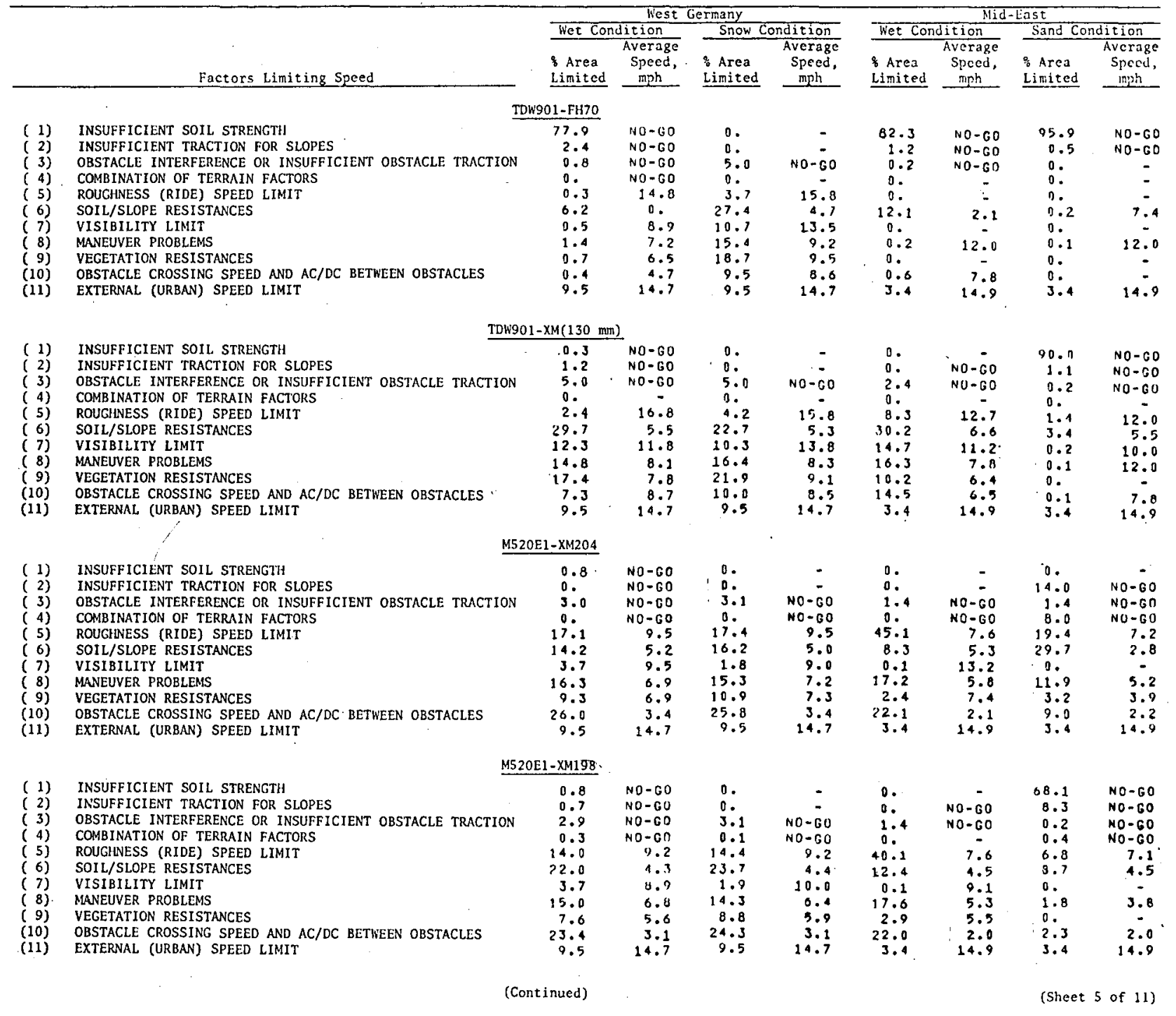


Table E87 (Continued)

\begin{tabular}{|c|c|c|c|c|c|c|c|c|c|}
\hline & \multirow[b]{4}{*}{ Factors Limiting Speed } & \multicolumn{4}{|c|}{ West Germany } & \multicolumn{4}{|c|}{ Mid-Lust } \\
\hline & & \multirow{2}{*}{\multicolumn{2}{|c|}{ Wet Condition }} & \multirow{2}{*}{\multicolumn{2}{|c|}{ Snow Condition }} & \multirow{2}{*}{\multicolumn{2}{|c|}{ Wet condition }} & \multirow{2}{*}{\multicolumn{2}{|c|}{ Sind Condition }} \\
\hline & & & & & & & & & \\
\hline & & $\begin{array}{ll}8 \text { Arca } \\
\text { Limited }\end{array}$ & $\begin{array}{c}\text { Speed, } \\
\text { mph }\end{array}$ & $\begin{array}{l}\text { :Area } \\
\text { Limited }\end{array}$ & Speed, & Arca & Spced, & $\therefore$ Arca & specd, \\
\hline \multicolumn{10}{|c|}{ M520E1-FH70 } \\
\hline ( 1$)$ & INSUFFICIENT SOIL STRENCTH & 17.9 & NO-GO & 0. & - & $82 ; 3$ & No-60 & 96 ;6 & NO-BO \\
\hline c & INSUFFICIENT TRACTION FOR SLOPES & $2 \cdot 3$ & NO 0.60 & 0. & - & & NO -60 & $0: 0$ & 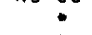 \\
\hline ( 3 ) & OBSTACLE INTERTERENCE OR INSUFFICIENT OBSTACLE TRACTION & 0.7 & NO-GO & 2.0 & NO-GO & $\frac{1}{0} ; 2$ & NO-GO & 0 & - \\
\hline (4) & COMBINATION OF TERRAIN FACTORS & 0.1 & NO-GO & 0.5 & NO-GO & $0:$ & $\therefore$ & $0 \vdots$ & • \\
\hline (5) & ROUGHNESS (RIDE) SPEED LIMIT & 5,1 & 7.9 & 13.4 & $9 \cdot 1$ & $i_{1}^{2}$ & $7 \%$ & $0 ;$ & - \\
\hline ( 7$)$ & $\begin{array}{l}\text { SOIL/SLOPE RESISTANCES } \\
\text { VISIBILITY LIMIT }\end{array}$ & $\begin{array}{l}2.1 \\
0.3\end{array}$ & $\begin{array}{l}2.1 \\
8.1\end{array}$ & $\begin{array}{r}28.4 \\
2.0\end{array}$ & 3.9 & $11 ; 3^{3}$ & $2: 6$ & : & $:$ \\
\hline ( 8$)$ & $\begin{array}{l}\text { MANEUVER PROBLEMS } \\
\text { MNA }\end{array}$ & 1.8 & 4.7 & 16.4 & 4.3 & $0: 3$ & 6.0 & 0 & - \\
\hline ( 9$)$ & VEGETATION RESISTANCES & 0.3 & 1.1 & 5.0 & 5.4 & & & : & $\dot{\epsilon}$ \\
\hline (10) & OBSTACLE CROSSING SPEED AND AC/DC BETWEEN OBSTACLES & 1.5 & 2.3 & 24.7 & 3.1 & $1 ; 0$ & $2: 1$ & & - \\
\hline (11) & EXTERNAL (URBAN) SPEED LIMIT & 9.5 & 14.7 & 9.5 & 14.7 & $3: 4$ & $14 \% 9$ & $3 ; 4$ & $14 ; 9$ \\
\hline \multicolumn{10}{|c|}{ M12SE1-XM198 } \\
\hline ( 1$)$ & INSUFFICIENT SOIL STRENGTH & 0.8 & NO-GD & 0. & _- & 0 . & & 82.6 & nOGgO \\
\hline ( 2) & INSUFFICIENT TRACTION FOR SLOPES & 0.7 & NOGO GO & 4.2 & NO-60 & 0. & NO-GO & 7.9 & NO-GO \\
\hline ( 3$)$ & OBSTACLE INTERFERENCE OR INSUFFICIENT OBSTACLE TRACTION & 7.1 & NO -60 & 7.3 & NO-GO & 10.2 & NO-GO & 0.2 & NO-GO \\
\hline (4) & COMBINATION OF TERRAIN FACTORS & 0. & NO -60 & 2.3 & NO-GO & 0. & & 0. & $=$ \\
\hline ( 5$)$ & ROUGINESS (RIDE) SPEED LIMIT & 6.0 & 12.5 & 2.3 & 11.4 & 18.8 & 8.6 & 0 . & - \\
\hline ( 6$)$ & SOIL/SLOPE RESISTANCES & 27.0 & 0.9 & 37.1 & 0. & 18.2 & 7.7 & 5.5 & 7.5 \\
\hline ( 7$)$ & VISIBILITY LIMIT & 8.2 & .11 .8 & 2.8 & 10.7 & 12.7 & 11.8 & 0.2 & 0.9 \\
\hline ( 8$)$ & MANEUVER PROBLEMS & 15.1 & 7.7 & 12.2 & 5.1 & 18.8 & 7.3 & 0.2 & 8.8 \\
\hline (9) & VEGETATION RESISTANCES & 8.9 & 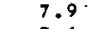 & 11.3 & 5.8. & 5.0 & 7.4 & 0 . & - \\
\hline (10) & OBSTACLE CROSSING SPEED AND AC/DC BETWEEN OBSTACLES & 16.7 & 5.6 & 11.1 & 3.3 & 12.9 & 3.1 & o. & - \\
\hline (11) & EXTERNAL (URBAN) SPEED LIMIT & 9.5 & 14.7 & 9.5 & 24.7 & 3.4. & 14.9 & 3.4 & 14.9 \\
\hline \multicolumn{10}{|c|}{ M548E1-XM204 } \\
\hline (1) & INSUFFICIENT SOIL STRENGTH & o. & - & 0 . & - & o. & - & n. & - \\
\hline ( 2) & INSUFF ICIENT TRACTION FOR SLOPI & 1.2 & NOGO & $0 \cdot$ & & 0.2 & & $\therefore$ & \\
\hline ( 3$)$ & OBSTACLE INTERFERENCE OR INSUFFICIENT OBSTACLE TRACTION & 9.9 & NO -60 & 9.5 & NO-GO & 12.3 & NO $=60$ & 11.8 & $N n-60$ \\
\hline (4) & COMBINATION OF TERRAIN FACTORS & 0.6 & NO-GO & 0. & NO-GO & & & 0. & \\
\hline ( 5$)$ & ROUGHNESS (RIDE) SPEED LIMIT & 8.5 & 14.6 & 16.1 & 16.0 & 25.0 & 11.5 & 29.2 & 12.7 \\
\hline (6) & SOIL/SLOPE RESISTANCES & 12.1 & 7.3 & 2.4 & 10.8 & 7.1 & 6.3 & 21.3 & 4.7 \\
\hline ( 7$)$ & VISIBILITY LIMIT & 12.1 & 12.3 & 8.4 & 15.0 & 16.7 & 11.9 & 1.0 & 11.1 \\
\hline ( 8$)$ & MANEUVER PROBLEMS & 19.1 & 9.8 & 22.2 & 12.2 & 16.8 & 9.3 & 15.3 & 7.7 \\
\hline (9) & VEGETATION RESISTANCES & 5.2 & 5.2 & 4.7 & 10.9 & 2.5 & 9.8 & 4.1 & 9.2 \\
\hline (10) & OBSTACLE CROSSING SPEED AND AC/DC BETWEEN OBSTACLES & 21.7 & 8.3 & 27.3 & 8.6 & 15.9 & 6.6 & 13.9 & 0.7 \\
\hline (11) & EXTERNAL (URBAN) SPEED LIMTT & 9.5 & 14.7 & 9.5 & 14.7 & 3.4 & 14.9 & 3.4 & 14.9 \\
\hline \multicolumn{10}{|c|}{ M548E1-XM198 } \\
\hline (1) & INSUFFICIENT SOIL STRENGTH & 0. & - & o. & - & 0 . & • & n. & - \\
\hline ( 2) & INSUFFICIENT TRACTION FOR SLOPES & 1.2 & NO -60 & 0 . & & & & 0. & \\
\hline (3) & OBSTACLE INTERFERENCE OR INSUFF ICIENT OBSTACLE TRACTION & 9.9 & NO-GO & 9.5 & NO-GO & 0.2 & NO-GO & 11.8 & NO-GO \\
\hline (4) & COMBINATION OF TERRAIN FACTORS & 0.6 & NO-GO & o. & NO-GO & f: $:^{3}$ & -5 & 1.8 & NO- 80 \\
\hline ( 5$)$ & ROUGHNESS (RIDE) SPEED LIMIT & 7.4 & 14.4 & 14.5 & 16.0 . & 17 & 11.4 & 15.9 & 12.1 \\
\hline ( 6$)$ & SOIL/SLOPE RESISTANCES & 15.7 & 5.5 & 5.0 & 6.3 & $14: 3^{3}$ & 6.2 & 39.9 & 3.9 \\
\hline ( 7$)$ & VISIBILITY LIMIT & 10.2 & 12.4 & 8.0 & 14.9 & 12.9 & 11.9 & 1.0 & 11,1 \\
\hline ( 8$)$ & MANEUVER PROBLEMS & $19 . \mathrm{J}$ & 8.6 & 20.8 & 11.2 & 21.6 & 8.6 & 13.0 & 8.0 \\
\hline (9) & VEGETATION RESISTANCES & 8.0 & 6.3 & 7.9 & 9.2 & 3.4 & 7.2 & 2.9 & 5.7 \\
\hline (10) & OBSTACLE CROSSING SPEED AND AC/DC BETWEEN OBSTACLES & 18.2 & 7.6 & 24.8 & 7.8 & 14.2 & 6.1 & $10 \cdot 3$ & 6.0 \\
\hline (11) & EXTERNAL (URBAN) SPEED LIMIT & 0.5 & 14.7 & 9.5 & 14.7 & 3.4 & 14.9 & 3.4 & 14.9 \\
\hline
\end{tabular}


Table E87 (Cont inued)

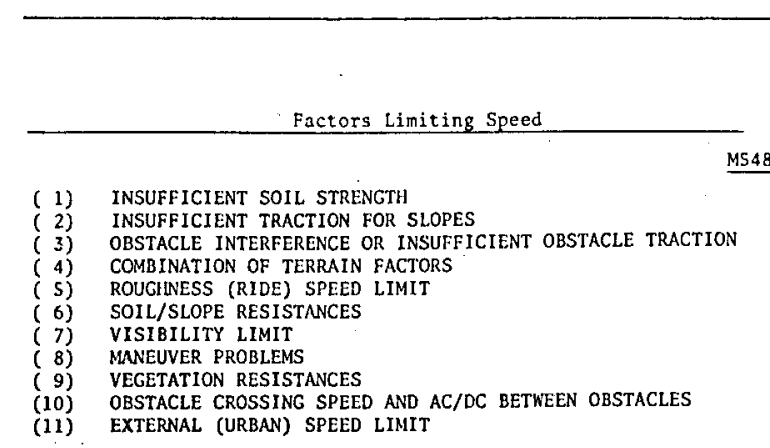

\begin{tabular}{|c|c|c|c|}
\hline Wet Con & dition & Snow $\mathrm{C}$ & idition \\
\hline $\begin{array}{l}\text { \& Area } \\
\text { Limited }\end{array}$ & $\begin{array}{c}\text { Average } \\
\text { Speed, } \\
\text { mph }\end{array}$ & $\begin{array}{l}\text { Area } \\
\text { Linited }\end{array}$ & $\begin{array}{l}\text { Averago } \\
\text { Speed, } \\
\text { mph }\end{array}$ \\
\hline
\end{tabular}

\begin{tabular}{|c|c|c|c|}
\hline$\overline{e t} \overline{C o n}$ & iifit & Sand Co & tition \\
\hline & & & Avera \\
\hline Are & Spcod, & $\begin{array}{l}8 \text { Area } \\
\text { Limited }\end{array}$ & Speced \\
\hline
\end{tabular}

MS48E1-FH70

\begin{tabular}{|c|c|c|c|c|c|c|c|}
\hline $\begin{array}{r}78.1 \\
0.3 \\
1.0 \\
0.8 \\
0.5 \\
6.5 \\
0.1 \\
1.3 \\
1.0 \\
0.8 \\
9.5\end{array}$ & $\begin{array}{r}N n-r, \theta \\
N n=r, 0 \\
N n-G 0 \\
N n-r, 0 \\
12.4 \\
2.9 \\
9.5 \\
3.2 \\
7.6 \\
7.3 \\
14.7\end{array}$ & $\begin{array}{r}n . \\
n: \\
0.5 \\
n: \\
14 . n \\
7.0 \\
8.1 \\
20.4 \\
7.3 \\
24.3 \\
9.5\end{array}$ & $\begin{array}{r}- \\
\text { Nn-r. } \\
\text { Nn-r. } \\
15.9 \\
6.2 \\
14.8 \\
11.3 \\
8.9 \\
8.1 \\
14.7\end{array}$ & $\begin{array}{c:c}82 & 6 \\
0 & 3 \\
0 & 3 \\
0 ! & 8 \\
0 & 12 \\
12 & 1 \\
0 & 1 \\
0 & 1 \\
0 & 1 \\
0 & 6 \\
3 & 4\end{array}$ & $\begin{array}{r}\text { NO } 60 \\
\text { No-60 } \\
\text { NO-G0 } \\
\text { NO-60 } \\
\vdots \vdots 3 \\
30 \\
10 \\
7 \vdots 0 \\
14: 9\end{array}$ & $\begin{array}{r:c}0 & \\
6 & 7 \\
11 & 0 \\
1 & 2 \\
14 & 8 \\
36 & 2 \\
0 & 2 \\
11 & 3 \\
4 & 7 \\
9 & 8 \\
3 & 4\end{array}$ & $\begin{array}{r}\text { NO-6O } \\
N O-60 \\
N O-60 \\
12 ; 5\end{array}$ \\
\hline
\end{tabular}

(1) INSUFFICIENT SOIL STRENGTH
(2) INSUFICIENT TRACTION FOR SLOPES
(3) OBSTACLE INTERFERENCE OR INSUFFICIENT OBSTACLE TRACTION
(4) COMBINATION OF TERRAIN FACTORS
(5) ROUGLESS (ORDE) SPEED LIMIT
(6) SOIL/SLOPE RESISTANCES
(7) VISIBILITY LIMIT
(8)
(9ANEUER PROBLEMS
(10) VEGETATION RESISTANCES
(11) OBSTACLE CROSSING SPEED AND AC/DC BETWEEN OBSTACLES
(11) EXTERAL (URBAN) SPEED LIMIT

ET-XM198
1) INSUFFICIENT SÓIL STRENGTH
INSUFFICIENT TRACTION FOR SLOPES
OBSTACLE INTERFERENCE OR INSUFFICIENT OBSTACLE TRACTION ROUCHNES (RIDE) SPEED LIMT
SOIL/SLOPE RESISTANCES
MANEULITY LIMIT
9) VEGETATION RESISTANCE
(10) OBSTACLE CROSSING SPEED AND AC/DC BETWEEN OBSTACLES

0.8
0.8
8.0
0.0
2.2
17.4
14.9
16.5
16.5
14.1
8.5

NO -80

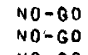

NO-GO

17.8
8.4

13.6
9.5
10.0
6.4

$\begin{array}{lr}0 . & - \\ 0 . & - \\ 8.0 & N O-G 0 \\ 0.0 & N 0-60 \\ 5.0 & 18.4 \\ 13.2 & 14.2 \\ 16.0 & 18.5 \\ 20.4 & 12.4 \\ 10.1 & 12.0 \\ 17.8 & 7.0 \\ 7.5 & 14.7\end{array}$

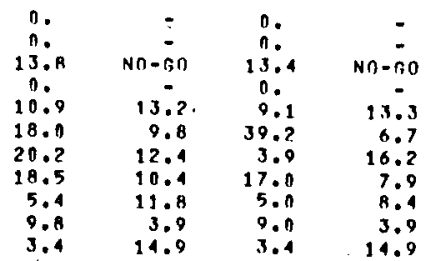

UET-FH70

INSUFFICIENT SOIL STRENGTH

$7 i .9 \quad$ Nn-

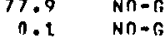

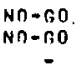

\section{$-$}

\begin{tabular}{|c|c|c|c|}
\hline 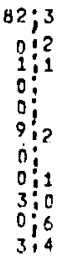 & $\begin{array}{r}\text { NO- } 60 \\
\text { NO-60 } \\
\text { NO-60 } \\
\vdots \\
5: 4 \\
4 \vdots 5 \\
8 \\
4: 1 \\
14.9\end{array}$ & $\begin{array}{r:r}0 & \vdots \\
0 & 7 \\
12 & 7 \\
8 & 5 \\
40 & 5 \\
3 & 9 \\
17 & 4 \\
4 & 3 \\
9 & 3 \\
3 & 4\end{array}$ & $\begin{array}{r}\vdots \\
N O-60 \\
\vdots \\
13: 0 \\
65: 1 \\
16: 2 \\
8: 1 \\
7 \% \\
7 \% \\
149\end{array}$ \\
\hline
\end{tabular}

(2) INSUFFICIENT TRACTION FOR SLOPES

3) OBSTACLE INTERFERENCE OR INSUFFICIENT OBSTACLE TRACTION COMBINATION OF TERRAIN FACTORS

ROUGHNESS (RIDE) SPEED LIMIT

SOIL/SLOPE RESISTANCES

MANEUUER PROBIEMS

9) VEGETATION RESTSTANCES

(10) OBSTACLE CROSSING SPEED AND AC/DC BETWEEN OBSTACLES EXTERNAL (URBAN) SPEED LIMIT

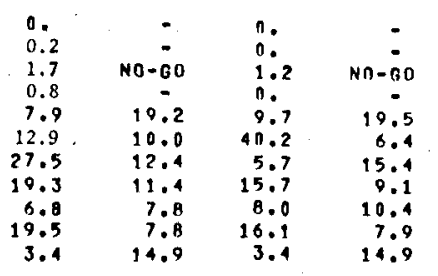

(Sheet 7 of 11) 
Table E87 (Cont inued)

\begin{tabular}{|c|c|c|c|c|c|c|c|c|c|}
\hline \multirow{3}{*}{\multicolumn{2}{|c|}{ Factors Limiting Speed }} & \multirow{2}{*}{\multicolumn{4}{|c|}{$\begin{array}{l}\text { West Germany } \\
\end{array}$}} & \multicolumn{4}{|c|}{ Mid-East } \\
\hline & & licet Condition & & \multicolumn{2}{|c|}{ Snow Condition } & \multicolumn{2}{|c|}{ Ret Condition } & \multicolumn{2}{|c|}{ Sand Condition } \\
\hline & & $\begin{array}{l}8 \text { Area } \\
\text { Limited }\end{array}$ & $\begin{array}{c}\text { Specd, } \\
\text { mph }\end{array}$ & $\begin{array}{l}8 \text { Area } \\
\text { Limited }\end{array}$ & $\begin{array}{l}\text { Speed, } \\
\text { riph }\end{array}$ & $\begin{array}{l}\text { \& Arca } \\
\text { Limited }\end{array}$ & $\begin{array}{c}\text { Speed, } \\
\text { mph }\end{array}$ & $\begin{array}{l}\text { Area } \\
\text { Limited }\end{array}$ & $\begin{array}{l}\text { Spced, } \\
\text { mph }\end{array}$ \\
\hline \multicolumn{10}{|c|}{ ASV-XM198 } \\
\hline & INSUFFICIENT SOIL STRENGTII & $n$. & - & 0 . & - & 0. & - & n. & - \\
\hline (2) & INSUFFICIENT TRACTION FOR SLOPES & 1.7 & No-60 & $\because$. & & 0.2 & NCI-GO & & \\
\hline (3) & OBSTACLE INTERFERENCE OR INSUFFICIENT OBSTACLE TRACTION & 3.7 & NOM- & 3.7 & NO-GO & 1.7 & $n n-60$ & 1.2 & NO -60 \\
\hline (4) & COMBINATION OF TERRAIN FACTORS & 0.8 & NO- 60 & I. 2 & NO $-S_{0}$ & 0.8 & - & $\pi$. & - \\
\hline (5) & ROUGINESS (RIDE) SPEED LIMIT & 1.1 & 25.3 & 4.5 & 26.6 & 7.0 & 19.3 & 8.2 & 20.5 \\
\hline (6) & SOIL/SLOPE RESISTANCES & 16.8 & 6.7 & 3.8 & 8.7 & 20.9 & 8.1 & 44.3 & 5.3 \\
\hline (7) & VISIBILITY LIMIT & 17.5 & 13.8 & 19.1 & 20.1 & 24.0 & 12.6 & 4.2 & 16.2 \\
\hline (8) & MANEUVER PROBLEMS & 18.0 & 4.2 & 24.3 & 12.3 & 17.9 & 9.9 & 15.5 & 7.9 \\
\hline (9) & VEGETATION RESISTANCES & 16.9 & 0.2 & 12.8 & 12.5 & 0.3 & 8.8 & 8.3 & 9.2 \\
\hline (10) & OBSTACLE CROSSING SPEED AND AC/DC BETWEEN OBSTACLES & 13.3 & 8. 7 & 19.2 & 9.7 & 17.9 & 7.5 & 14.8 & 7.3 \\
\hline (11) & EXTERNAL (URBAN) SPEED LIMIT & 7.5 & 14.7 & 9.5 & 14.7 & 3.4 & 14.9 & 3.4 & 14.9 \\
\hline \multicolumn{10}{|c|}{ ASV-FH70 } \\
\hline (1) & INSUFFICIENT SOIL & 77.9 & NO-GO & n. & - & $82 ; 3$ & No- 60 & ni & - \\
\hline ( 2) & INSUFFICIENT TRACTION FOR SLOI & $n+1$ & NOEG & n. & & 0,5 & No. 60 & $n !$ & \\
\hline (3) & OBSTACLE INTERFERENCE OR INSUFF ICIENT OBSTACLE TRACTION & 0.9 & NO-GO & 3.7 & $2 n-6,00$ & $0 ; 2$ & No. -60 & $1 !^{2}$ & NO -80 \\
\hline (4) & COMBINATION OF TERRAIN FACTORS & 0. & $\therefore$ & 0.2 & $N 0-G O$ & : & - & $0 i_{n}$ & 20.5 \\
\hline ( 5$)$ & ROUGHNESS (RIDE) SPEED LIMIT & 0.1 & 74.5 & 4.5 & 26.5 & $0 i$ & - & $8: 2$ & $20: 5$ \\
\hline ( 6$)$ & SOIL/SLOPE RESISTANCES & 6.5 & $4 . \pi$ & 7.3 & A. 2 . & $12 ; 5$ & $4 ; 9$ & $44 ; 4$ & 49 \\
\hline (7) & VISIBILITY LIMIT & 0.8 & 10.1 & 18.7 & 20.0 & $0 ! 2$ & $14 ! 1$ & $4 ! 3$ & $16 ! 2$ \\
\hline ( 8$)$ & MANEU & 2.3 & 5.6 & 24.1 & 12.1 & 0,2 & $6: 4$ & $15: 4$ & $7: 4$ \\
\hline (9) & VEGETA & $1 . n$ & 7.0 & 12.9 & 12.2 & $0 ?$ & 2 & $8 \vdots 5$ & $8: 6$ \\
\hline (10) & ED AND AC/DC BETWEEN OBSTACLES & ח. A & 7.3 & 19.2 & $10 \cdot 0$ & $0 ; 6$ & $8 ; 5$ & $14 i^{6}$ & $7: 3$ \\
\hline (11) & EXTERNAL (URBAN) SPEED LIMIT & 9.5 & 14.7 & 9.5 & 14.7 & $3 i 4$ & $14: 9$ & $3 ; 4$ & $14 i 9$ \\
\hline \multicolumn{10}{|c|}{ M109A1 } \\
\hline & INSUFFICIENT SOIL STRENGTH & 0. & - & 0 . & - & a. & - & 0. & • \\
\hline (2) & INSUFF & 0.8 & NO-GO & & & & & $n$. & \\
\hline 3) & 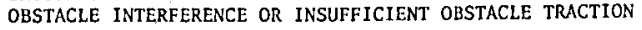 & 1.3 & NO-BO & 1.4 & NO-GO & 0.8 & NOEGO & 0. & - \\
\hline 4) & COMB & 0.5 & NO-CO & 0.2 & NOS $-5, O$ & o. & - & o. & \\
\hline 5) & ROUGl & 1.1 & 18.5 & 3.8 & 20.2 & 5.1 & 15.8 & 8.1 & 15.5 \\
\hline 6) & TANCES & 15.1 & 8.9 & 10.8 & 13.6 & 15.7 & $10 . ?$ & 41.3 & 7.1 \\
\hline 7) & & 15.2 & 12.5 & 10.8 & 17.5 & 3 & 12.1 & 5.0 & 15.4 \\
\hline 8) & & 22.7 & 9.1 & 23. & 11 & 20.7 & 10.3 & 16.0 & 8.5 \\
\hline 9) & vi & 11.2 & 7.7 & 5. & 11. & 4 & 10.6 & 6.6 & 10.1 \\
\hline (10) & BETWEEN OBSTACLES & 22.7 & 9.9 & 34.0 & 11 . & 25.3 & R.3 & 19.6 & 4.2 \\
\hline (11) & EXTERNAL (URBAN) SPEED LIMIT & 9.5 & 14.7 & 9.5 & 14.7 & 3.4 & 14.9 & 3.4 & 14.9 \\
\hline \multicolumn{10}{|c|}{$\underline{\mathrm{M} 107}$} \\
\hline & INSUFFICIENT SOIL STRENG & 0 . & - & 0 . & - & 0. & - & 0. & - \\
\hline (2) & INSUFFICIEN & 0.9 & No & 0. & & 0. & & 0. & \\
\hline (3) & FFICIENT OBSTACLE TRACTION & 3.0 & 30 & 3.0 & NO-GO & 6.6 & NO -60 & 6.3 & NO-GO \\
\hline (4) & СOMBI & 0.7 & 0 & 0.2 & No- & $\therefore$. & $15.8-2+3$ & & \\
\hline ( 5$)$ & SPEED LIMIT & 1.2 & 18.7 & 3.8 & 20 & 5.1 & $15 . \mathrm{A}$ & 7.7 & 15.5 \\
\hline (6) & TANCES & 25.0 & 5 & & 13 & 14.9 & 11 & 40.5 & 7.3 \\
\hline ( 7$)$ & VISIBI & & 12 & 10 & 17 & 3 & 11 & 4.9 & 15.4 \\
\hline ( 8$)$ & MANEU & 22.4 & 8.6 & 24 & 11 & 20.3 & 1 & 16.4 & 9.3 \\
\hline (9) & VEGETATION RESISTANCES & 11.2 & 9.0 & 4 & 10 & 5.5 & 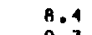 & 6.1 & 9.2 \\
\hline (10 & OBSTACLE CROSSING SPEED AND A & 20.7 & 10.7 & 32.1 & 12 & 19.9 & 9.3 & 14.7 & 9.3 \\
\hline (11) & EXTERNAL (URBAN) SPEED LIMIT & 9.5 & 14.7 & 9.5 & 14.7 & 3.4 & 14.9 & 3.4 & 14.9 \\
\hline
\end{tabular}

(Continued)

(Sheet 8 of 11) 
Table E87 (Continued)

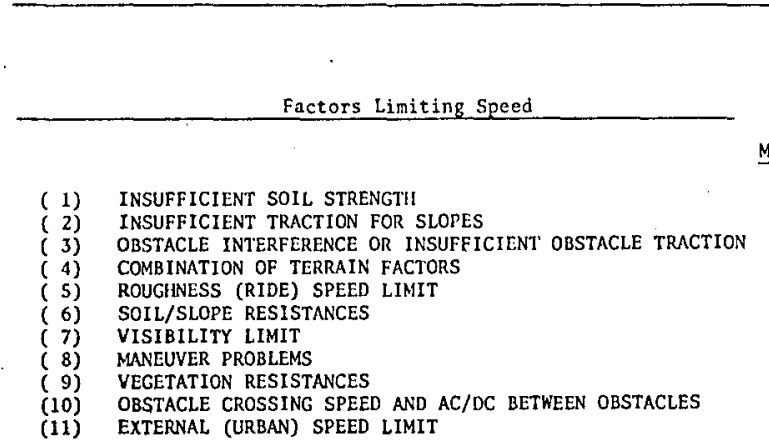

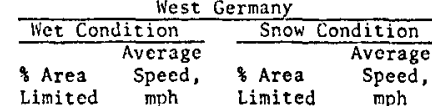

\begin{tabular}{|c|c|c|}
\hline \multicolumn{3}{|c|}{ Mid-East } \\
\hline Wet Conditi & Sar & \\
\hline mphr & & $\mathrm{mph}$ \\
\hline
\end{tabular}

MI10E2

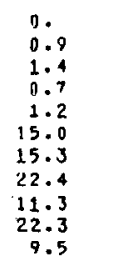

-
$N O-G 0$
$N 0-60$
$N 0-60$
18.7
8.4
12.3
8.6
8.9
10.0

0.
0.0
1.4
0.2
3.8
10.8
10.8
24.8
4.9
33.6
9.5

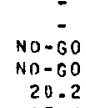
17.5
11.5
10.9

$10.8 \quad 13.4$

AC/DC BETWEEN OBSTACLES

M113AI

(1) INSUFF ICIENT SOIL STRENGTH

STRENGTH

OBSTACLE INTERFERENCE OR INSUFFICIENT OBSTACLE TRACTION

COMBINATION OF TERRAIN FACTORS

ROUGHNESS (RIDE) SPEED

SOIL/SLOPE RESISTANCES

VISIBILTTY LIMIT

VEGETATION RESISTANCES

OBSTACLE CROSSING SPEED AND AC/DC BETWEEN OBSTACLES

EXTERNAL (URBAN) SPEED LIMIT

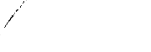

INSUFFICIENT SOIL STRENGTH

INSUFFICIENT / TRACTION FOR SLOPES

OPSTACIE TRACTION ROUGYYSS (RIDE) SPEN FACTORS

SOIL/SLOPE RESISTANCES

VISIBILITY LIMIT

MANEUVER PROBLEMS

VEGETATION RESISTANCES

(10) OBSTACLE CROSSING SPEED AND AC/DC BETWEEN OBSTACLES

(1) INSUFFICIENT SOIL STRENGTI

(3) INSUFFICIENT TRACTION FOR SLOPES

(4) COMBINATION OF TERRAIN FACTORS

(5) ROUGHNESS (RIDE) SPEED LIMIT

(6) SOIL/SLOPE RESISTANCES

(8) MANEUYRR PRODLEMS

(9) VEGETATION RESISTACES

(10) OBSTACLE CROSSING SPEED AND AC/DC BETWEEN OBSTACLES

(11) EXTERNAL (URBAN) SPEED LIMIT

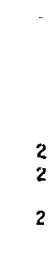

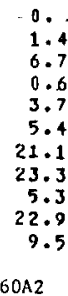

$0.0 \quad$ Nom

$\begin{array}{ll}0.9 & \\ 0.9 & \text { NO } \\ 4.1 & \text { NO } \\ 0.8 & \text { NO }\end{array}$

. 0.8

15.9
19.0
23.3

9.3
16.3
9.5

$-$

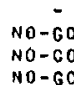

$N 0-60$
16.4

11.4

13.9
10.5
7.8

8.3
14.7

11.5
14.7

0.
0.0
0.8
0.0
5.1
14.9
24.3
20.7
5.5
25.3
3.4

$:$
Nn-GO
-
15.8
10.8
11.9
11.03
8.4
8.2
14.9

$\begin{array}{ll}0 . & -10 \\ 0.5 & \text { NOE }\end{array}$

$7.7 \quad 15.5$

$\begin{array}{rr}4.9 & 15.4 \\ 16.4 & 8.3\end{array}$$$
\text { a. }
$$

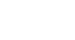

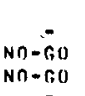

18.5
3.4

$548 \mathrm{E} 1$

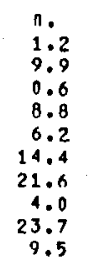

$$
\begin{array}{r}
- \\
N O-G 0 \\
N 0-60 \\
N 0-60 \\
14.8 \\
9.0 \\
12.1 \\
10.2 \\
7.3 \\
9.1 \\
14.7
\end{array}
$$

(Continued)
13.3
9.2
11.8

11.8
$11.5:$

1.1
6.1
14.9

$\begin{array}{rr}0 . & \\ 90.1 & N 0-60 \\ 90.1 & 0 \\ 24.8 & 19.2 \\ 24.0 & 7.8 \\ 4.7 & 14.8 \\ 15.9 & 10.7 \\ 3.5 & 12: 7 \\ 14.6 & 5.5 \\ 3.4 & 14.9\end{array}$

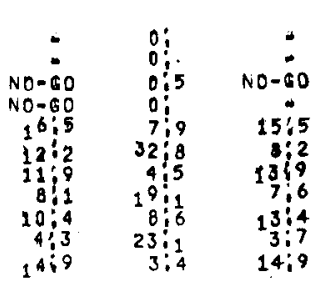

$$
\begin{array}{rr}
0.0 & = \\
0.5 & \text { NO-60 } \\
0.5 & N 0-60 \\
17.5 & 16.0 \\
1.8 & 11.2 \\
8.6 & 15.2 \\
21.8 & 12.4 \\
3.3 & 11.5 \\
28.1 & 9.8 \\
9.5 & 14.7
\end{array}
$$

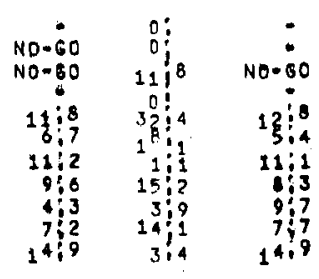

(Sheet 9 of 11) 
Table E87 (Continued)

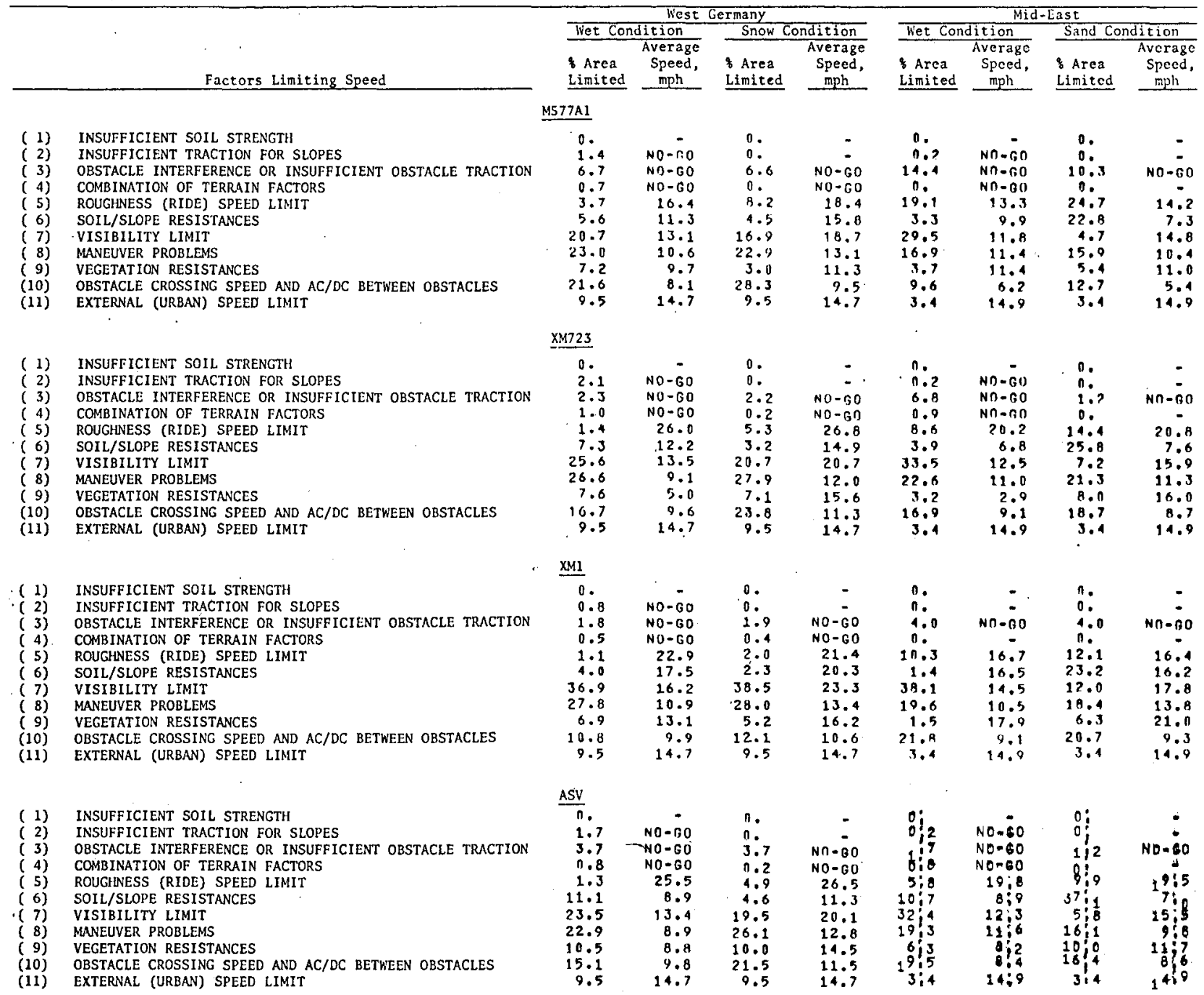


Table E87 (Concluded)

\begin{tabular}{|c|c|c|c|c|c|c|c|c|c|}
\hline & \multirow[b]{4}{*}{ Factors Limiting Speed } & \multicolumn{4}{|c|}{ West Germany } & \multicolumn{4}{|c|}{ Mid-East } \\
\hline & & \multirow{2}{*}{\multicolumn{2}{|c|}{ Wet Condition }} & \multirow{2}{*}{\multicolumn{2}{|c|}{ Snow Condition }} & \multirow{2}{*}{\multicolumn{2}{|c|}{ Wot Condition }} & \multirow{2}{*}{\multicolumn{2}{|c|}{ Sand Condition }} \\
\hline & & & & & & & & & \\
\hline & & $\begin{array}{l}\text { Area } \\
\text { Limited } \\
\end{array}$ & $\begin{array}{c}\text { Speed, } \\
\text { mph }\end{array}$ & $\begin{array}{l}8 \text { Area } \\
\text { Limited } \\
\end{array}$ & $\begin{array}{c}\text { Speed, } \\
\text { mph }\end{array}$ & $\begin{array}{l}\text { Area } \\
\text { Limited } \\
\end{array}$ & $\begin{array}{c}\text { Spced, } \\
\text { mph }\end{array}$ & $\begin{array}{l}\text { Area } \\
\text { Limited } \\
\end{array}$ & $\begin{array}{c}\text { Spoed, } \\
\text { mph }\end{array}$ \\
\hline & & \multicolumn{8}{|l|}{ M561 } \\
\hline \multirow{12}{*}{$\begin{array}{l}1) \\
\text { (2) } \\
(3) \\
(4) \\
(5) \\
(6) \\
(7) \\
(8) \\
(9) \\
(10) \\
(11)\end{array}$} & INSUFFICIENT SOIL STRENGTH & n. & - & 0 . & - & $0 ;$ & - & $0 ;$ & \\
\hline & INSUFFICIENT TRACTION FOR SLOPES & $\therefore$ & Nonco & 0. & 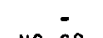 & 0 & M & $24 ; 8$ & No.60 \\
\hline & OBSTACLE INTERFERENCE OR INSUFFICIENT OBSTACLE TRACTION & 9.2 & NOCGO & 9.2 & Nn- $r_{0} 0$ & $16 ; 3$ & NO-60 & $16 ! 3$ & No 0.00 \\
\hline & COMBINATION OF TERRAIN FACTORS & o. & - & n. & - & $0 \vdots$ & & $1 ; 5$ & NO -60 \\
\hline & ROUGHNESS (RIDE) SPEED LIMIT & 12.9 & 15.5 & 15.7 & 16.0 & $30: 2$ & $11: 6$ & $26: 2$ & $13 ; 5$ \\
\hline & SOIL/SLOPE RESISTANCES & 1.1 & 15.4 & 5.3 & 14.1 & $0_{i}^{-}$ & & 7,3 & $12: 6$ \\
\hline & VISIBILITY LIMIT & 14.3 & 12.6 & 7.2 & $14 . n$ & $20: 4$ & $11: 9$ & $1: 2$ & 1008 \\
\hline & MANEUVER PROBLEMS & 24.7 & 10.9 & 23.1 & 11.9 & $16 ; 4$ & $11: 2$ & $B ; \overline{0}$ & $11: 7$ \\
\hline & VEGETATION RESISTANCES & 1.2 & 14.1 & 2.5 & 9.7 & $0 ; 9$ & $14 ; 3$ & $2 ; 1$ & isii 1 \\
\hline & OBSTACLE CROSSING SPEED AND AC & 27.0 & 9.7 & 27.5 & 10.1 & $12 ! 3$ & $7 ! 2$ & $9 ; 2$ & $2: 3$ \\
\hline & EXTERNAL (URBAN) SPEED LIMIT & 9.5 & 14.7 & 9.5 & 14.7 & $3 i 4$ & $14: 9$ & $3: 4$ & $14 i 9$ \\
\hline & & \multicolumn{8}{|l|}{$\underline{M 813}$} \\
\hline (1) & INSUFFICIENT SOIL STRENGTH & 0.2 & -60 & n. & - & $0 ;$ & $*$ & $0 ;$ & \\
\hline ( 2) & INSUFFICIENT TRACTION FOR SLO & $0 \cdot 1$ & $N=-60$ & n. & & $0 ;$ & & $47: 8$ & NO-6O \\
\hline (3) & OBSTACLE INTERFERENCE OR INSUFFICIENT OBSTACLE TRACTION & 7.7 & $N n=6,0$ & 7.7 & NO-GO & $10 ! 0$ & NO-GO & $10 ; 0$ & No. 60 \\
\hline (4) & COMBINATION OF TERRAIN FACTORS & n. & & 0.1 & NO-GO & $0: 0$ & $\therefore$ & $5: 1$ & NO-GO \\
\hline ( 5$)$ & ROUGHNESS (RIDE) SPEED LIMIT & 14.9 & 12.5 & 7.5 & 11.5 & $37 ! 1$ & $8: 6$ & $14 \vdots 6$ & $10: 2$ \\
\hline (6) & SOIL/SLOPE RESISTANCES & 7.6 & 9.6 & 34.6 & 7.4 & $2 ; \overline{9}$ & $7 ; 8$ & $9 i^{3}$ & $8\}^{0}$ \\
\hline ( 7) & VISIBILITY LIMIT & 10.7 & 12.6 & $3 \cdot 0$ & 10.7 & $14: 4$ & $12: 8$ & 0,4 & 19 it \\
\hline ( 8$)$ & MANEUVER PROBLEMS & 19.9 & 10.7 & 13.0 & 8.1 & $16 ; 8$ & $8\} 3$ & $4 ! 6$ & $8 ! ?$ \\
\hline (9) & VEGETATION RESISTANCES & 7.0 & 9.2 & 9.3 & 6.8 & $1 j^{6}$ & $10 ; 7$ & $1: 5$ & $3 i 9$ \\
\hline (10) & OBSTACLE CROSSING SPEED AND AC/DC BETWEEN OBSTACLES & 22.4 & .6 .5 & 15.3 & 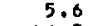 & $13: 7$ & $\therefore$ & $3: 3$ & $3 i 2$ \\
\hline \multirow[t]{2}{*}{ (ii) } & EXTERNAL (URBAN) SPEED LIMIT & 9.5 & 14.7 & 9.5 & 14.7 & $3 ; 4$ & $14: 9$ & $3 ; 4$ & $14 ; 9$ \\
\hline & & 1 & & & & & & & \\
\hline & JNSUFFICIENT SOCL STRENGTH & & - & 0. & - & n. & - & 0. & \\
\hline (2) & INSUFFICIENT TRACTION FOR SLOPES & 1.2 & NO-GO & 0. & 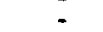 & 0. & & n: & - \\
\hline 3) & OBSTACLE INTERFERENCE OR INSUFFICIENT OBSTACLE TRACTION & 5.2 & NO-GO & 5.0 & NO-GO & 9.5 & NO-GO & 9.7 & NOS-BO \\
\hline 4) & COMBINATION OF TERRAIN FACTORS & 0.1 & NO-GO & 0. & NOEGO & 0.2 & NO-GO & 0. & - \\
\hline 5) & (RIDE) SP & 2.9 & 18.2 & 6.9 & 18.8 & 13.7 & 13.5 & 14.4 & 13.5 \\
\hline (0) & PE RESISTANCES & 17.4 & 7.7 & 12.6 & 20.6 & 9.9 & 10.7 & 30.5 & 8.8 \\
\hline (7) & visI & 28.9 & 13.7 & 24.5 & 19.4 & 31.5 & 12.1 & 6.6 & 15.8 \\
\hline (8) & ROBLEMS & 22.6 & 10.2 & 27.2 & 13.0 & 17.0 & 11.5 & 14.6 & 11.6 \\
\hline (9) & VEGETATION & 7.8 & 5.4 & 8.2 & 14.4 & 4.4 & 5.8 & 10.5 & 13.1 \\
\hline 10 & DC BETWEEN OBSTACLES & 4.3 & 9.5 & 5.9 & 11.0 & 10.4 & 10.0 & 10.3 & 10.8 \\
\hline & EXTERNAL (URBAN) SPEED -LIMIT & 9.5 & 14.7 & 9.5 & 14.7 & 3.4 & 14.9 & 3.4 & 14.9 \\
\hline & & & & & & & & & \\
\hline
\end{tabular}


Table t:88

Speed Profile for Group 1

off-Road

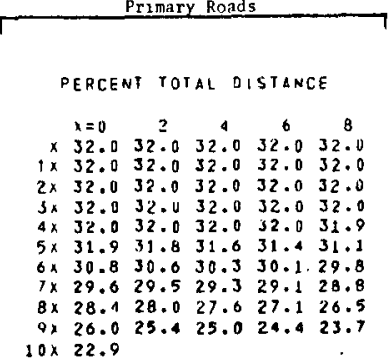

$10 \times 22.9$

percent total uigtance

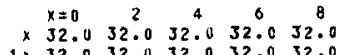

$2 \times 32.032 .032 .0 \quad 32.0 \quad 32.0$

$3 \times 32.032 .032 .032 .032 .0$

$6 \times 31.331 .130 .930 .830 .0$

$7 \times 30.430 .3 \quad 30.129 .929 .8$

$\begin{array}{llllll}8 \times & 29.1 & 28.6 & 28.1 & 27.5 & 26.9 \\ 9 x & 26.2 & 25.6 & 25.1 & 24.5 & 23.7\end{array}$

$10 \times 22.9$

PERCENT TOTAL DISTANCE

$\begin{array}{rrrrr}x=0 & 2 & 4 & 6 & 8 \\ \times \quad 32.0 & 32.0 & 32.0 & 32.0 & 32.0\end{array}$

$\begin{array}{llllll}2 \times & 32.0 & 32.0 & 32.0 & 32.0 & 32.0 \\ 3 \times & 32.0 & 32.0 & 32.0 & 32.0 & 32.0\end{array}$

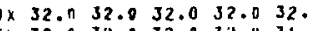

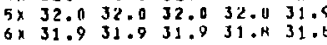

$\begin{array}{llllll}2 \times & 31.8 & 51.8 & 31.8 & 31.7 & 31.0\end{array}$

$\begin{array}{rrrrrr}8 \times & 31.4 & 31.3 & 31.1 & 31.0 & 30.8 \\ 8 \times & 30.4 & 20.0 & 28.3 & 27.1 & 26.0 \\ 10 \times & 25.0 & & & & \end{array}$

PERCENT TOTAL DISTANCE

$\begin{array}{rrrrr}x=11 & 2 & 4 & 6 & 8 \\ \times \quad 32.0 & 32.0 & 32.0 & 32.0 & 32.0\end{array}$

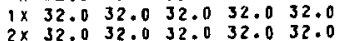
$\begin{array}{llllll}3 x & 32.0 & 32.0 & 32.0 & 32.0 & 32.0 \\ 1 \times & 32.0 & 32.0 & 32.0 & 32.0 & 32.0\end{array}$

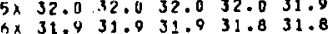
$\begin{array}{llllll}7 x & 31.8 & 31.8 & 31.7 & 31.6 & 31.5 \\ 8 \times & 31.5 & 31.2 & 31.1 & 30.9 & 30.7\end{array}$ $8 \times 31.3 \quad 31.2 \quad 31.130 .930 .7$ $10 \times 24,0$

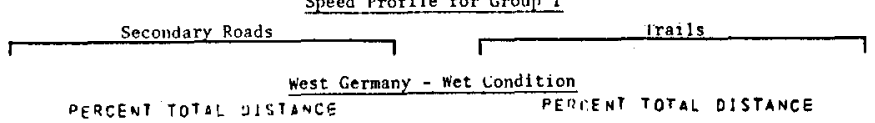

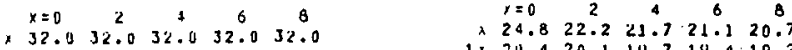

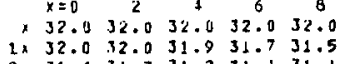

$2 \times 31.431 .3 \quad 3: .231 .-131.1$

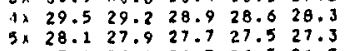

ox 726.126 .926 .726 .526 .3

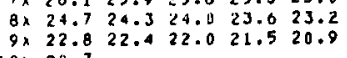

\section{west Germany. Snow Condition}

percent total Distance PERTENT TOTAL DISTAHCE

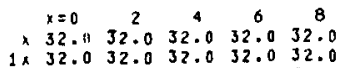

$2 \times 31.831 .731 .631 .531 .4$

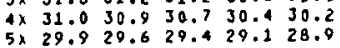

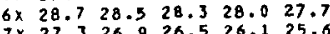

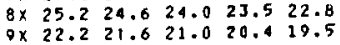

$10 \times 38.8$

$$
\text { Mid }
$$

NERTENT TOTAL DISTANCE

$\begin{array}{rrrrrr} & x=0 & 2 & 4 & 6 & 8 \\ \times & 32.11 & 32.0 & 32.0 & 32.0 & 32.0 \\ 1 \times & 32.0 & 32.0 & 32.0 & 32.0 & 32.0 \\ 2 x & 32.0 & 2.0 & 32.0 & 31.0 & 35.0\end{array}$

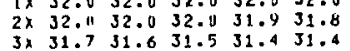

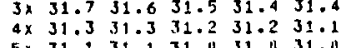

$\begin{array}{llllll}5 \times & 31.1 & 31.1 & 31.0 & 31.21 & 31.0 \\ 6 \times & 31.0 & 30.9 & 30.0 & 31.5 & 30.3\end{array}$

$\begin{array}{cccccc}7 x & 39.1 & 76.9 & 29.9 & 24.4 & 27.1 \\ 8 \times & 78.5 & 27.8 & 29.11 & 20.3 & 25.7\end{array}$

$\begin{array}{rr}8 \times & 88.5 \\ 9 x & 25.1 \\ 10 \times & 22.7\end{array}$

$\begin{array}{llllll}12 & 20.4 & 20.5 & 19.7 & 19.4 & 19.2 \\ 2 \times & 19.0 & 18.8 & 18.0 & 18.4 & 10.1\end{array}$

$\begin{array}{llllll}31 & 1.8 & 17.6 & 17.4 & 17.1 & 16.8 \\ 12 & 10.4 & 16.0 & 5.7 & 15.4 & 15.8\end{array}$

5x 15.414 .814 .614 .514 .0

$\begin{array}{ccccccc}6 x & 14.2 & 14.0 & 15.9 & 13.7 & 13.6 \\ 7 x & 13.4 & 13.3 & 13.1 & 13.0 & 12.0\end{array}$

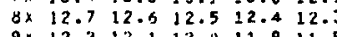

Dercent total distance

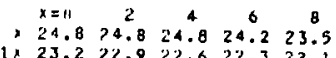

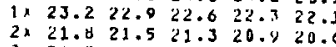

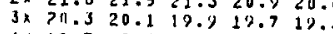
$\begin{array}{llllll}4 \times & 18.7 & 18.2 & 17.7 & 17.2 & 16.0 \\ 5 \times & 16.5 & 16.2 & 16.0 & 15.7 & 15.5\end{array}$ $\begin{array}{llllll}5 \times & 16.5 & 16.2 & 16.0 & 15.7 & 15.5 \\ 6 x & 15.3 & 15.1 & 15.0 & 14.8 & 14.7\end{array}$ $\begin{array}{rrrrrr}8 \times & 13.6 & 13.5 & 13.3 & 13.2 & 13.8 \\ 9 \times & 13.11 & 12.9 & 12.8 & 12.5 & 13.1 \\ 10 \times & 11.8 & & & & \end{array}$ East - wet Condition

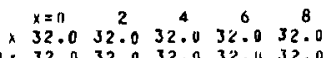

$\begin{array}{llllll}2 x & 32.0 & 32.0 & 31.9 & 31.0 & 31.9 \\ 3 x & 31.0 & 31.5 & 31.4 & 31.4 & 31 .\end{array}$

$\begin{array}{llllll}4 \times & 31.3 & 31.2 & 31.2 & 31.1 & 31.1 \\ 5 \times & 31.1 & 31.0 & 31.0 & 31.2 & 30.6\end{array}$

$\begin{array}{llllll}6 \times & 30.3 & 311.1 & 29.9 & 29.6 & 29.4 \\ 3 \times & 24.1 & 28.8 & 26.4 & 27.9 & 27.4\end{array}$

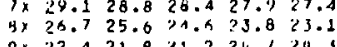

$n \times$
$10 \times 19.6$

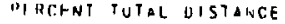

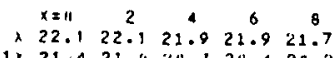

$1 \times 21.4 \quad 21.020 .120 .420 .2$

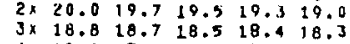

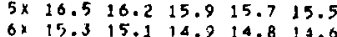
$\Rightarrow$ 14.5 14.4 14.2 14.813 .9 $9 \times 13.8 \quad 13.6 \quad 13.5 \quad 13.413 .2$ $\begin{array}{ll}9 \times & 13.1 \\ \ln x & 9.1\end{array}$

Pepcent rotal distanee

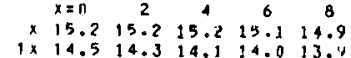
$\begin{array}{llllll}2 \times & 13.8 & 13.7 & 13.6 & 13.5 & 13.4 \\ 3 x & 13.3 & 13.2 & 13.2 & 13.0 & 12.0\end{array}$ 512.4 ox $11.811 .8 \quad 11.711 .0111 .5$

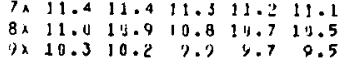

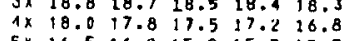

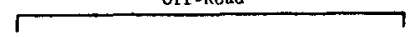

PERCENT IUIAL DISTALGE

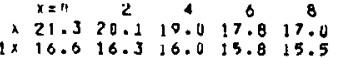
$2 x \quad 15.315 .014 .7 \quad 14.4 \quad 14.2$ $\begin{array}{lllllll}3 \times & 14.0 & 13.7 & 3.6 & 13.4 & 13.2 \\ 4 \times & 13.1 & 12.9 & 12.8 & 12.7 & 12.5\end{array}$ $\begin{array}{lllllll}5 \times & 12.4 & 12.3 & 12.2 & 12.1 & 12.0\end{array}$ $\begin{array}{lllllll}7 \times & 11.3 & 11.2 & 11.0 & 10.9 & 10.8 \\ 8 \times & 10.6 & 10.4 & 10.3 & 10.9 & 8.8\end{array}$ $\begin{array}{rrrrrr}8 \times & 10.6 & 10.4 & 10.3 & 10.1 & 8.8 \\ 9 \times & 3.0 & 1.8 & 1.3 & 1.3 & 0.9\end{array}$

$10 \times n, 0$

PERLENT TOTAL DISTANCE

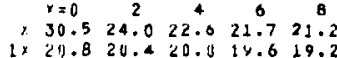

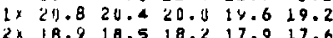

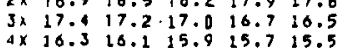
$5 \times 15.415 .215 .114 .914 .7$ 7x 14.814 .414 .2 14.0 13.8 $\begin{array}{rrrrrr}8 \times & 12.8 & 12.6 & 12.5 & 12.3 & 12.0 \\ 9 x & 11.7 & 3.7 & 2.1 & 1.5 & 1.2\end{array}$

perlent tural distance

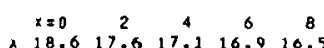

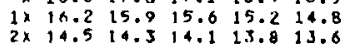
$\begin{array}{llllll}3 \times & 13.4 & 13.3 & 13.1 & 13.0 & 12.8\end{array}$ $\begin{array}{lllllll}5 x & 12.0 & 11.8 & 11.9 & 11.6 & 11.5 \\ 6 x & 11.3 & 11.2 & 11.1 & 11.5 & 10.8\end{array}$ $7 \times 10.710 .5110 .410 .210 .1$

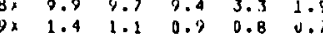
$\begin{array}{cc}10 \times & 0.0 \\ 10 & 0\end{array}$

IERLENT rotal Distance

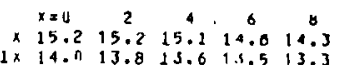

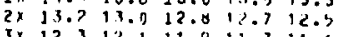

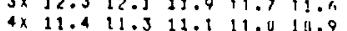

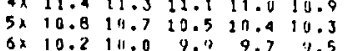
$\begin{array}{llllll}7 x & 0.4 & 0.2 & 0.1 & 8.7 & 9.7 \\ 8 x & 8.5 & 1.2 & 0.1 & 8.9 & 9.7\end{array}$ $\begin{array}{lllllll}8 \times & 8.5 & 1.2 & 8.2 & 8.7 & 9.7 \\ 8 x & 2.7 & 1.7 & 1.3 & 1.0 & 0.5 \\ 10 x & 0.7 & & & & \end{array}$ 
Table Es9

Speed Proftle for Group 2

Primary Roads

Pergent total distance

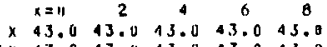

$2 x \quad 43.043 .043 .0433043$.

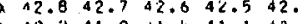

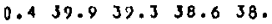

$5 \times 37.2 \quad 36.5 \quad 35.9 \quad 35.334 .8$

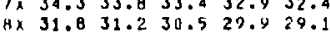

$9 \times 28.3$
$10 \times 24.6$

percent total distance

$\begin{array}{rrrrr}x=0 & 2 & 4 & 6 & 8 \\ \times 16.9 & 10.9 & 16.9 & 15.0 & 14.8\end{array}$

$1 \times 14.314 .0013 .312 .211 .4$

$3 \times 9.49 .29 .19 .80 .0$

$4 \times \quad 8.8 \quad 8.7 \quad 8.6 \quad 8.6 \quad 8.5$

$\begin{array}{lllllll}5 x & 8.4 & 8.4 & 8.3 & 0.3 & 8.2 \\ 6 x & 8.2 & 8.1 & 8.1 & 8.0 & 8.0\end{array}$

$\begin{array}{llllll}7 \times & 7.9 & 7.9 & 7.8 & 7.0 & 9.0 \\ 8 \times & 7.5 & 7.7 & 7.6 & 7.6 & 7.6\end{array}$

$\begin{array}{cc}8 \times & 7.5 \\ 10 \times & 7.4\end{array}$

PERCENT TOTAL DISTANCE

$\begin{array}{rrrccc} & x=11 & 2 & 4 & 6 & \theta \\ x & 41.0 & 41.0 & 41.0 & 41.0 & 41.0 \\ 1 \times & 41.0 & 41.0 & 41.0 & 41.0 & 41.0\end{array}$

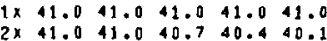
$\begin{array}{ccccccc}2 x & 41.0 & 41.0 & 40.7 & 40.4 & 40.1 \\ 3 \times & 39.9 & 39.7 & 39.5 & 39.3 & 39.1\end{array}$ $\begin{array}{llllll}4 \times & 39.0 & 38.8 & 38.7 & 38.6 & 38.5 \\ 5 \times & 38.4 & 38.3 & 38.1 & 37.9 & 37.8\end{array}$ 37.237.

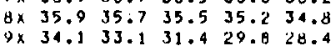
$10 \times 27.2$

PERGENI TOTAL DISIANCE

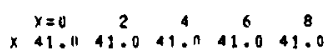

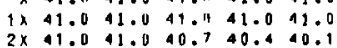
$2 \times \quad 31.0411 .040 .7440 .440 .1$ $4 \times 30.098 .838 .7 \quad 38.0 \quad 38.5$ $\begin{array}{lllllll}5 \times & 38.4 & 38.2 & 38.11 & 37.9 & 37.7 \\ 6 \times & 37.5 & 37.4 & 37.3 & 37.1 & 37.0\end{array}$ $\begin{array}{cccccc}6 \times & 37.5 & 31.4 & 37.3 & 37.1 & 37.0 \\ 7 \times & 36.8 & 36.0 & 36.5 & 30.3 & 36.1\end{array}$

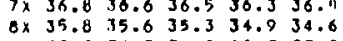
$0 \times 33.8 \quad 32.7 \quad 30.929 .3 \quad 27.9$

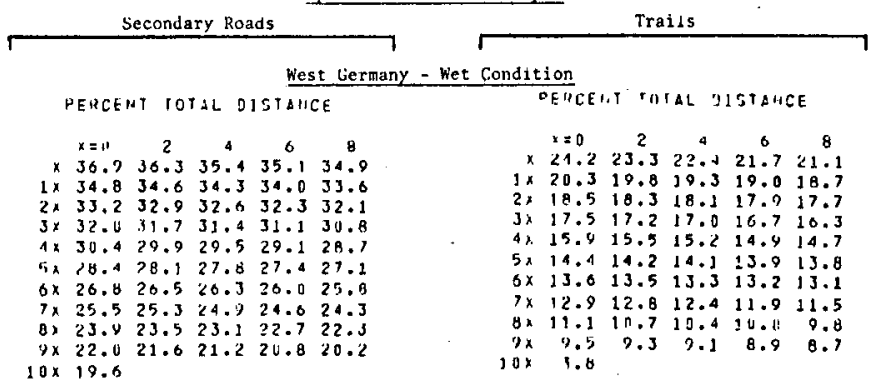

West Cermany - Snow Condition

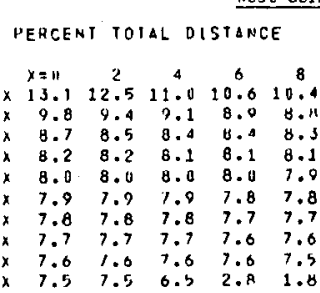

\section{Mid-East - Wet Condition}

PERCERT TOTAL DISTANCE

pertent total ulstance

$\begin{array}{rrrrr}x=0 & 2 & 4 & 6 & 8 \\ \times 34.7 & 34.4 & 41.4 & 34.1 & 34.4\end{array}$

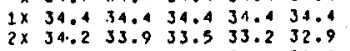

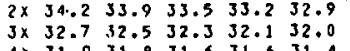

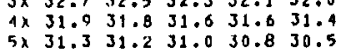

$\begin{array}{llllll}5 x & 31.3 & 31.2 & 31.0 & 30.8 & 30.5 \\ 8 \times & 30.2 & 30.0 & 29.7 & 29.4 & 20.1\end{array}$

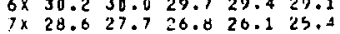

$\begin{array}{rrrrrr}8 \times & 24.1 & 21.2 & 23.7 & 23.2 & 22.8 \\ 7 \times & 22.2 & 0.7 & 4.0 & 2.9 & 2.2\end{array}$

Mis-

Dent:ENt total oISTANCE

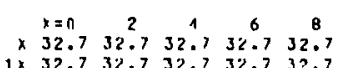

2k $32.4 \quad 32.1 \quad 31.9 \quad 31.3 \quad 31$.

$4 \times 31.11311 .430 .830 .730 .6$

$6.28 .928 .628 .3 \quad 27.727$.

$\begin{array}{llllll}7 \times & 26.5 & 25.3 & 21.3 & 23.1 & 22.6 \\ 0 \times & 21.9 & 21.2 & 20.6 & 20.1 & 19.7\end{array}$

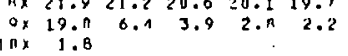

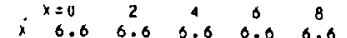

$1 \times \quad 0.0 \cdot 0.0 \quad 0.8 \quad 0.060 .0$

$\begin{array}{llllll}2 \times & 0.6 & 0.0 & 0.6 & 0.6 & 0.0 \\ 3 \times & 0.6 & 0.6 & 0.0 & 0.5 & 0.5\end{array}$

$\begin{array}{llllll}4 \times & 6.5 & 6.5 & 0.5 & 0.5 & 0.5 \\ 5 \times & 8.5 & 0.5 & 0.5 & 0.5 & 6.5 \\ x \times & 0.4 & 0.4 & 0.5 & 0.4 & 0.4\end{array}$

$\begin{array}{llllll}3 x & 0.4 & 0.4 & 0.4 & 0.5 & 0.5 \\ 7 x & 0.4 & 0.3 & 0.4 & 0.4 & 3.4\end{array}$

$\begin{array}{llllll}4 \times & 0.4 & 6.3 & 6.2 & 5.1 & 6.1 \\ 8 x & 6.0 & 6.0 & 5.9 & 5.9 & 5.1 \\ 9 x & 5.8 & 5.8 & 4.2 & 2.3 & 1.0\end{array}$ $0 \times 1$

pericent total doistance

$x=1122040$ $1421.02 \pi .920 .313 .810 .4$ ix $18 . ? 17.917 .718 .318 .4$ $1 \times 17.010 .8 \quad 16.5 \quad 16.215 .8$ $5 x \quad 15.515 .2 \quad 15.014 .614 .6$ कx 13.7 15.0 13.513 .013 .0 \&x 13.112 .912 .512 .1131 .2 $\begin{array}{lll}0 \times & 8.7\end{array}$

\section{st Sand Condition}

fepcenar rotal pisiance

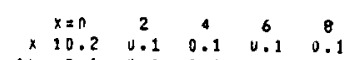
$\begin{array}{llllll}1 \times & 0.1 & 0.1 & 0.1 & 0.1 & 0.1 \\ 2 \times & 0.1 & 0.1 & 0.1 & 0.1 & 0.1 \\ 3 & 0.1 & 0.1 & 0.1 & 0.1 & 0.1\end{array}$ $\begin{array}{llllll}3 \times & 0.1 & 0.1 & 0.1 & 0.1 & 0.1 \\ 12 & 0.1 & n .1 & 0.1 & 0.1 & 0.1\end{array}$ $\begin{array}{llllll}5 x & 0.1 & 0.1 & 0.1 & 0.1 & 0.1 \\ 3 x & 0.1 & 4.1 & 0.1 & 0.1 & 0.1\end{array}$ $\begin{array}{llllll}7 x & 0.1 & 0.1 & 0.1 & 0.1 & 0.1 \\ \theta x & 0.1 & 0.1 & 0.1 & 0.1 & 0.1\end{array}$ $\begin{array}{cccccc}0 x & 0.1 & 1.1 & 0.1 & 0.1 & 0.1 \\ 0 x & 0.1 & 1.1 & 0.1 & 0.1 & 0.1 \\ 10 x & 0.1 & & & & \end{array}$

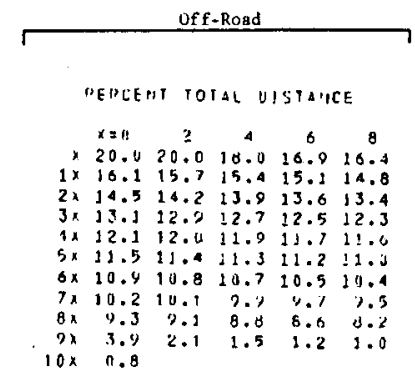

Depcent toral uistance

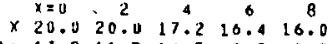
$\begin{array}{llllll}2 \times & 8.9 & 11.7 & 10.5 & 9.7 & 9.2 \\ 3 \times & 7.8 & 8.5 & 8.3 & 8.1 & 7.9\end{array}$ $\begin{array}{cccccc}3 \times & 7.8 & 7.6 & 7.5 & 7.4 & 7.3 \\ 1 \times & 7.2 & 7.2 & 7.1 & 7.0 & 7.0\end{array}$ $\begin{array}{lllllll}5 x & 0.2 & 6.8 & 0.8 & 0.7 & 6.0 \\ 6 x & 0.5 & 0.4 & 0.8 & 0.7 & 0.0\end{array}$

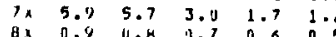
$\begin{array}{llllll}8 x & 0.9 & 4.8 & 3.7 & 0.0 & 0.5 \\ 9 x & 11.5 & 0.4 & 0.4 & 0.4 & 0.4\end{array}$

lox 0.4

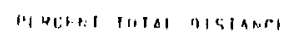

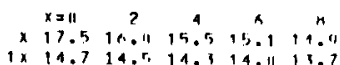

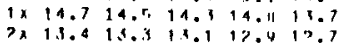

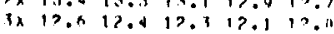
$\begin{array}{lllllll}4 x & 11.4 & 11.7 & 11.5 & 11.3 & 11.1 \\ 5 x & 10.9 & 11.7 & 10.5 & 11.9 & 10.1\end{array}$

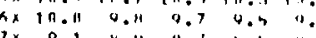

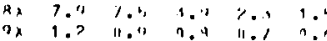

$\ln x$ 0.n

Deprent tomal gistance

$\begin{array}{rrrrr}x=17 & 2 & 4 & 6 & 8 \\ \times 15.015 & 15.0 & 1.2 & 10.3 & 0.2\end{array}$

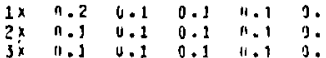
4x 0.1 a.1 0.1 (1) $\begin{array}{llllll}5 x & 7.1 & 0.1 & 0.1 & 11.1 & 9.1 \\ 6 x & 0.1 & 0.1 & 0.1 & 10.1 & 0.1\end{array}$ $\begin{array}{cccccc}8 \times & 0.1 & 0.1 & 0.1 & 10.1 & 1.1 \\ 0 \times & 0.1 & 0.1 & 0.1 & 0.1 & y .1\end{array}$ 


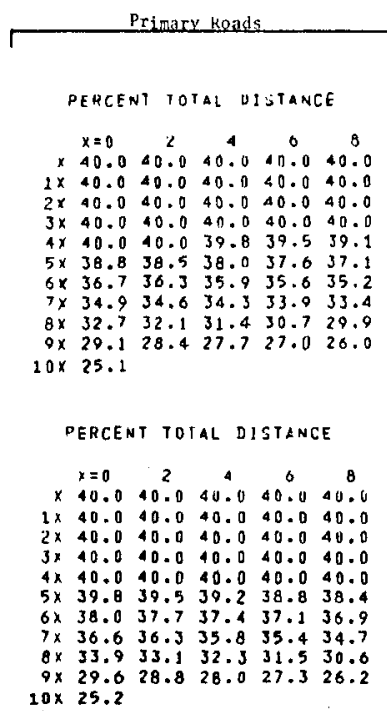

percent total distance

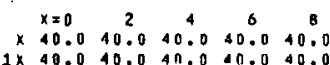

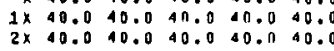
$4 \times 40.040 .40 .040 .040 .0$ $5 \times 40.0 \quad 39.939 .739 .439 .1$

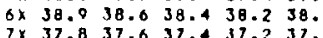

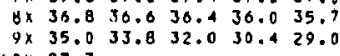
$10 \times 27.7$

PERCEH! TOTAL DISTANCE

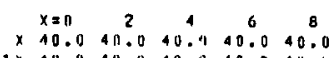

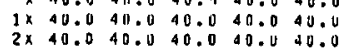
$3 \times 40.040 .040 .046 .040 .0$ $\begin{array}{cccccc}5 \times & 40.4 & 30.8 & 39.5 & 39.3 & 39.0 \\ 6 \times & 38.8 & 38.5 & 38.3 & 38.1 & 3 \% .9\end{array}$ $8 \times 36.636 .430 .35 .035$. $9 \times 34.6 \quad 33.531 .6 \quad 29.928 .4$ $9 \times 34.6$
$10 \times 27.2$
Table E90
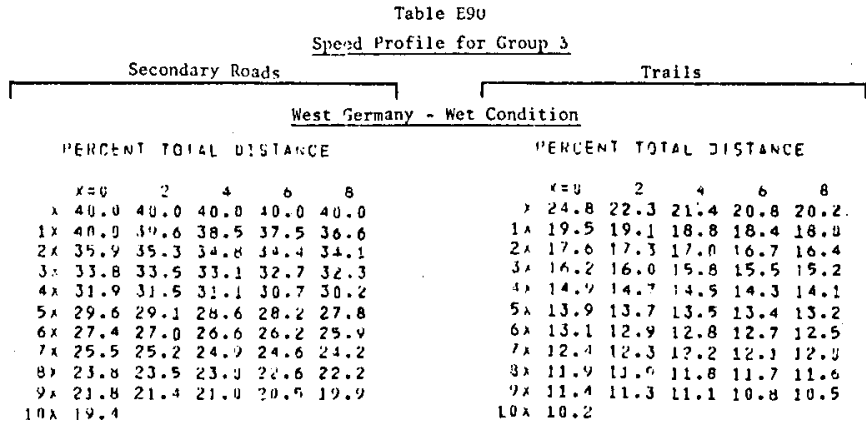

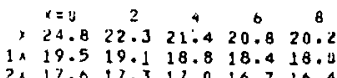

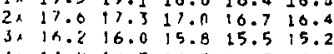

$$
\begin{aligned}
& 5 \times 13.914 .7 \text { 13.5 } 14.515 .2 \\
& \begin{array}{llllll}
6 \times & 13.1 & 12.9 & 12.8 & 12.7 & 12.5
\end{array} \\
& \begin{array}{llllll}
7 \times & 12.4 & 12.3 & 12.2 & 12.9 & 12.3 \\
3 x & 11.9 & 11.0 & 11.8 & 11.7 & 11.0
\end{array} \\
& \begin{aligned}
1) \times & 11.0 \\
10 \times & 10.2
\end{aligned}
\end{aligned}
$$$$
\text { "ERLENT TOTAL JISTANCE }
$$

\section{West Germany - Snow Cond 1 tion}

\section{percent total distance Deqcent iotal distahee}

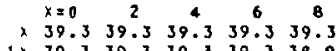

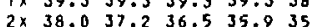

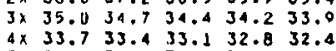

130.8

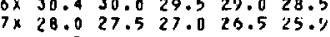

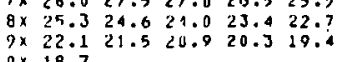

Mid-Eas

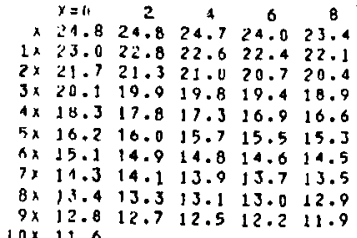

percent total DISTANCE

Condition

$x$
$2 \pi$

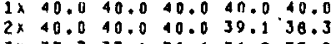

$4 \times \quad 35.5 \quad 35.2 \quad 35.0 \quad 34.235 .8$

$5434.3 \quad 34.2 \quad 34.0 \quad 33.9 \quad 33.7$

$8 \times$

$\begin{array}{llllll}9 x & 30.3 & 29.3 & 26.4 & 27.6 & 26.9 \\ 9 \times & 20.2 & 25.6 & 25.1 & 24.6 & 24.9\end{array}$

$10 \times 23.5$

Mid-

percent tOTAL DISTAMCE

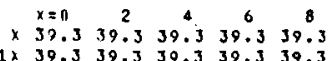

$\begin{array}{llllll}12 & 39.3 & 39.3 & 39.3 & 39.3 & 39.3 \\ 2 x & 39.3 & 38.8 & 38.1 & 37.3 & 36.7\end{array}$

$3 \times 36.534 .3540 .13 .033 .7$

$5 \times 33.6 \quad 33.5 \quad 33.3 \quad 33.0 \quad 32.7$

$\begin{array}{llllll}6 \times & 32.3 & 32.0 & 31.7 & 31.4 & 31.1 \\ 1 \times & 30.7 & 30.3 & 21.8 & 29.2 & 28.8\end{array}$

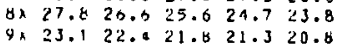

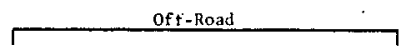

percent total jolstance

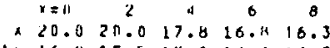
2) 14.9133 .513 .414 .15 .3 $\begin{array}{llllll}3 x & 12.6 & 12.3 & 12.4 & 11.9 & 12.9\end{array}$

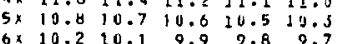
$\begin{array}{rrrrrr}7 \times & 9.5 & 9.4 & 9.3 & 9.1 & 9.4 \\ 8 \times & 8.9 & 8.7 & 8.5 & 8.4 & 8.2\end{array}$ $\begin{array}{llllll}3 \times & 3.4 & 2.7 & 8.5 & 8.4 & 8.0 \\ 0 \times & 0.5 & & & & \end{array}$

$10 \times 0.8$

PERCENT TOTAL DISTLNCE

$\begin{array}{rlrrr} & x=0 & 2 & 4 & 0\end{array}$

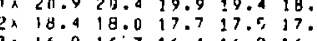
$4 \times 15.915 .0 \quad 15.415 .215 .0$ 6x $14.8 \quad 14.6 \quad 14.3$ 14. 1313. 1x 12.612 .312 .111 .411 .7 3. 11.411 .5 11.1 10.6 10.5 $10 \times 1.0$

Perchot lutal uistance

$\begin{array}{rrrrr}x=11 & 2 & 4 & 6 \\ \times 18.4 & 18.4 & 15.7 & 15.1 & 8\end{array}$ $\begin{array}{llllll}1 \times & 14.5 & 14.2 & 13.9 & 13.7 & 13.9 \\ 2 \times & 13.2 & 13.0 & 12.8 & 12.7 & 12.5\end{array}$ $4 \times 11.7 \quad 11.5 \quad 11.4$ t1. 3111. $\begin{array}{rrrrrr}5 x & 11.1 & 11.0 & 110.8 & 10.7 & 10.6 \\ 6 x & 10.5 & 10.3 & 10.2 & 10.1 & 9.9\end{array}$ $\begin{array}{llllll}32 & 9.7 & 9.5 & 9.4 & 9.2 & 9.0 \\ 02 & 8.8 & 8.7 & 4.5 & 9.2 & 9.0\end{array}$

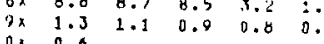

PEREEN toTAL DISTAUCE

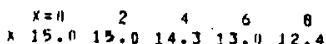

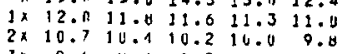
$\begin{array}{llllll}3 x & 9.0 & 7.4 & 0.3 & 4.1 & 0.0 \\ 4 x & 8.5 & 8.7 & 8.0 & 8.5 & 8.0\end{array}$ $\begin{array}{lllllll}5 x & 7.7 & 0.7 & 8.11 & 7.4 & .8 \\ 6 x & 7.7 & 7.2 & 1.5 & 7.4 & 1.2\end{array}$ $\begin{array}{llllll}8 \times & 7.1 & 7.01 & 5.9 & 5.9 & 5.5 \\ 3 \times & 9.5 & 0.0 & 5.9 & 5.5 & 2.5\end{array}$ 


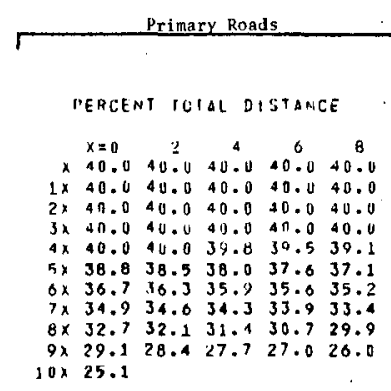

percent total Distance

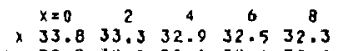

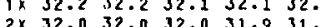

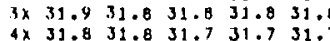

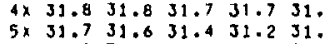

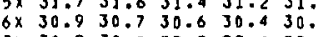
$\begin{array}{llllll}7 \times & 30.2 & 30.0 & 29.9 & 29.6 & 29.3 \\ 8 \times & 28.8 & 28.3 & 27.9 & 27.3 & 26.7 \\ 9 \times & 26.0 & 25.5 & 24.9 & 24.4 & 23.6\end{array}$

Percent total ulsiance

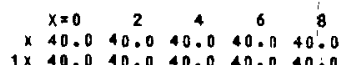

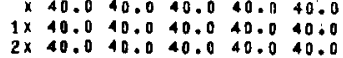

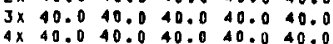
$5 \times 40.0 \quad 39.939 .7 \quad 39.439 .1$ 3x 38.938 .638 .438 .238 .0 $\begin{array}{llllll}8 \times & 36.8 & 36.0 & 36.4 & 36.0 & 35.7 \\ 9 \times & 35.0 & 33.8 & 32.0 & 30.4 & 29.0\end{array}$ $10 \times 27.7$

PERCENT TOTAL oJstance

$x=0 \quad 2 \quad 4 \quad 8$ $1 \times 40.040 .0 \quad 40.0 \quad 40.01140 .0$ $2 \times 40.040 .010 .0 \quad 40.0 \quad 40.0$

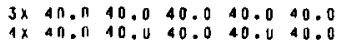
$5 \times 40.039 .8 \quad 39.5 \quad 39.3 \quad 39.0$

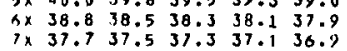

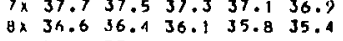
$0 \times 34.6$
$11 \times 27.2$
Table E91

\begin{tabular}{|c|c|}
\hline Secondary Roads & iroup 4 \\
\hline \multicolumn{2}{|c|}{ west Germany - wet Condition } \\
\hline PERCENT TOTAL DISTAHCE & PERCENT TOTAL WISIAHCE \\
\hline$y=0 \quad 2^{2} \quad{ }^{4} \quad 0$ & $\therefore=0$ \\
\hline 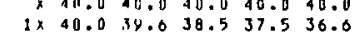 & 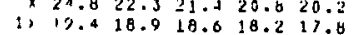 \\
\hline $2 \times 35.9 \quad 15.3 \quad 34.8 \quad 34.434 .1$ & $\because 17.517 .215 .910 .015 .3$ \\
\hline $\begin{array}{llllll}3 x & 33.8 & 33.5 & 33.1 & 32.7 & 32.3\end{array}$ & $3,: 0.1 / 5.9$ th. \\
\hline 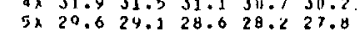 & $\Rightarrow 13.613 .413 .313 .113 .0$ \\
\hline$A \times 27.127 .0260 .026 .225 .4$ & $0,12.0 \quad 12.6 \quad 12.512 .412 .2$ \\
\hline $7 \times 25.325 .224 .924 .024 .2$ & $\therefore: 2.1 \quad 11.811 .5 \quad 11.1 \quad 10.7$ \\
\hline 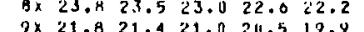 & 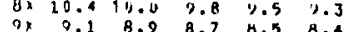 \\
\hline $10 \times 19.4$ & $10 \times 8.20000 .10 .30 .4$ \\
\hline
\end{tabular}

\section{West Germany - Snow Condition}

percent total distance

percent total distance

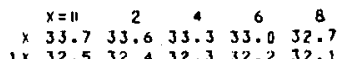
$\begin{array}{llllll}1 \times & 32.5 & 32.4 & 32.3 & 32.2 & 32.1 \\ 2 \times & 31.9 & 31.7 & 31.5 & 31.5 & 31.4\end{array}$ $3 x 31.331 .2 \quad 31.130 .430 .6$ $\begin{array}{llllll}4 \times & 30.3 & 30.0 & 29.7 & 29.5 & 29.2 \\ 5 \times & 29.0 & 28.8 & 28.6 & 28.4 & 28.2\end{array}$ $6 \times 27.929 .027 .320 .920 .5$ $7 \times \quad 26.1 \quad 25.725 .3 \quad 24.9 \quad 24.4$ $\begin{array}{llllll}4 x & 2.3 .9 & 23.3 & 22.8 & 22.3 & 21.7 \\ 9 x & 21.1 & 20.0 & 20.1 & 19.5 & 18.7\end{array}$ $\begin{aligned} 9 \times & 21.1 \\ 10 \times & 18.0\end{aligned}$

\section{Mid-East - Wet Condition}

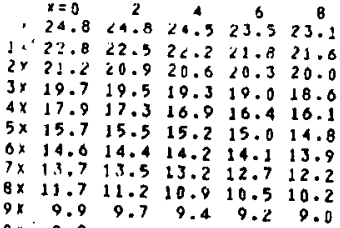

percent total ulstance

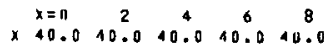

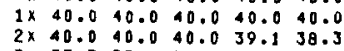
$3 \times 47.740 .0 \quad 40.0636 .238 .0$ $4 \times 35.5 \quad 35.2 \quad 35.0 \quad 34.7 \quad 34.5$ $5 \times 34.3 \quad 34.2 \quad 34.033 .933 .7$ $\begin{array}{llllll}5 \times & 33.6 & 33.3 & 33.1 & 32.8 & 32.5 \\ 7 \times & 32.2 & 32.0 & 31.7 & 31.3 & 30.9\end{array}$ $\begin{array}{llllll}8 \times & 31.3 & 28.3 & 28.4 & 27.6 & 26.9 \\ 8 \times & 26.2 & 25.6 & 25.1 & 24.6 & 24.1\end{array}$

$$
\text { Mid-E }
$$

DeRTENT TOTAL Distance

PERIENI TUTAL DISTANCE

$\begin{array}{rrrrr}x=11 & 2 & 4 & 6 & 0 \\ \times & 39.3 & 39.3 & 30.3 \quad 39.3 & 39.3\end{array}$ $\begin{array}{llllll}1 \times & 39.3 & 39.3 & 39.3 & 39.3 & 39.3 \\ 2 \times & 39.3 & 38.8 & 38.1 & 37.3 & 36.7\end{array}$

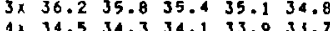
$5 x \quad 33.6 \quad 33.5 \quad 33.3 \quad 33.0 \quad 32.7$ $\begin{array}{llllll}4 \times & 32.3 & 32.0 & 31.9 & 31.4 & 31.1 \\ 7 \times & 30.7 & 30.3 & 22.8 & 29.2 & 28.6\end{array}$ $\begin{array}{rrrrrr}A \times & 27.8 & 26.6 & 25.6 & 24.7 & 23.8 \\ 9 \times & 33.1 & 22.4 & 21.8 & 21.3 & 20.8 \\ 10 \times & 20.1\end{array}$

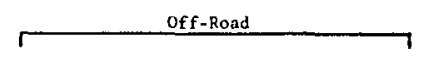

perCENT idTAL DISTANCE

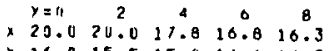
$\begin{array}{llllll}1 \times & 16.0 & 15.5 & 15.0 & 14.0 & 14.3 \\ 2 \times & 14.0 & 13.8 & 13.5 & 13.5 & 14.3 \\ 4 x & 11.6 & 13.4 & 13.5 & 13.0 & 13.0\end{array}$ $4 x \quad 12.7 \quad 12.413 .212 .011 .8$ 5. $10.410 .511 .3 \quad 11.211 .1$ $6 \times 10.3 \quad 10.1 \quad 10.0 \quad 0.99 .7$

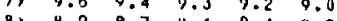
$\begin{array}{rrrrrr}8 \times & 8.9 & 8.7 & 4.6 & 8.4 & 8.2 \\ 9 x & 3.6 & 2.0 & 1.4 & 1.1 & 0.9\end{array}$

$10 \times 0.0$

PERCENT TOLAL DISTANCE

$\begin{array}{rrrrrr}x=1 & 2 & 4 & 6 & 8 \\ \times \quad 26.7 & 22.5 & 21.4 & 20.7 & 20.1\end{array}$ 14 19.7 19.3 18.920 .518 .1 $3 \times 16.5 \quad 16.2 \quad 16.0 \quad 15.815 .6$

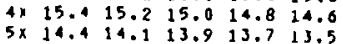

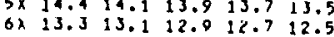
8x 11.211 .0110 .811 .80 .811 .4 $9 x \quad 0.1 \quad 2.6 \quad 1.7 \quad 1.3 \quad 1.0$

Percent rotal histance

$\begin{array}{rl}x=0 & 2 \\ \times 10.016 .215 .0 \quad 0 & 8\end{array}$ $\begin{array}{llllll}1 \times & 14.4 & 14.2 & 13.9 & 13.7 & 14.7 \\ 2 \times & 13.3 & 13.2 & 13.9 & 12.7 & 13.5 \\ 4 x & 13.0 & 2.4 & 13.0 & 12.9 & 12.7\end{array}$ $3 \times 12.6 \quad 12.412 .0232 .112 .0$ $\begin{array}{llllll}4 x & 11.0 & 11.7 & 11.6 & 11.5 & 11.3 \\ 5 x & 11.2 & 11.1 & 11.0 & 10.8 & 10.7\end{array}$ $7 \times \quad 0.5 \quad 0.4 \quad 0.3 \quad 10.1 \quad 0.9$ $\begin{array}{llllll}7 \times & 9.8 & 9.0 & 9.4 & 9.2 & 9.0 \\ 8 \times & 8.9 & 8.8 & 3.3 & 1.9 & 1.0 \\ 9 \times & 1.1 & 0.9 & 1.7 & 0.7 & 0.0\end{array}$ $\begin{array}{cccccc}9 \times & 2.1 & 0.9 & 0.7 & 0.7 & 0.6\end{array}$

percent total oistance

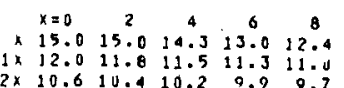
$\begin{array}{rrrrrr}2 \times & 10.6 & 10.4 & 10.2 & 9.9 & 0.7 \\ 3 \times & 9.5 & 9.4 & 9.2 & 9.1 & 8.9\end{array}$ $\begin{array}{llllll}4 x & 8.8 & 8.0 & 8.5 & 8.4 & 8.3 \\ 3 x & 8.1 & 8.0 & 7.8 & 7.6 & 2.3 \\ 6 x & 1.3 & 0.4 & 0.7 & 0.0 & 0.5 \\ 3 x & 0.5 & 0.4 & 0.4 & 0.4 & 0.3\end{array}$ $\begin{array}{llllll}7 x & 0.5 & 0.4 & 0.4 & 0.4 & 0.3 \\ 0 x & 0.3 & 0.3 & 0.3 & 0.3 & 0.3\end{array}$ $\begin{array}{llllll}8 x & 0.3 & 0.3 & 0.3 & 0.3 & 0.3 \\ 9 x & 0.3 & 0.3 & 0.2 & 0.2 & 0.3\end{array}$ 
Table E92

Time for Study Prime Mover-Towed Artillery Combinations to Complete Selected Artillery Mission

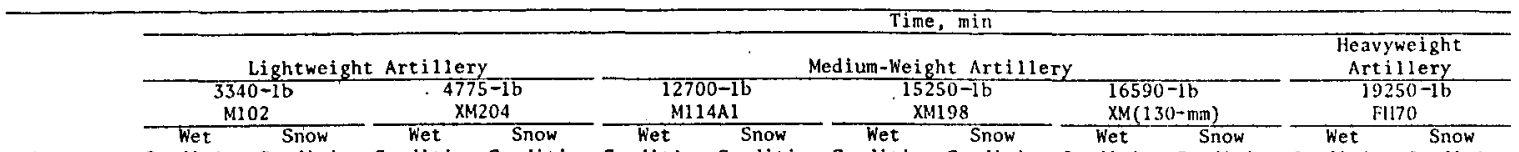

Prime Mover Condition Condition Condition Condition Condition Condition Condition Condition Condition Condition Condition Condition

\begin{tabular}{|c|c|c|c|c|c|c|c|c|}
\hline \multirow[b]{2}{*}{ M561 } & \multirow{3}{*}{$\begin{array}{r}5.9 \\
59.0\end{array}$} & \multirow{3}{*}{$\begin{array}{r}6.9 \\
11.2\end{array}$} & \multirow[b]{2}{*}{6.2} & \multicolumn{5}{|c|}{ ssion $1-2.06$ Miles } \\
\hline & & & & 7.3 & & & 60.8 & 67 \\
\hline M35A2 & & & 59.1 & 11.6 & & & 60.1 & 72 \\
\hline M813 & & & 59.1 & 12.7 & 59.6 & 15.1 & 59.8 & 16 \\
\hline M656 & & & 6.0 & 7.4 & & & 60.1 & 9 \\
\hline TDW901 & & & 7.9 & 8.5 & 10.3 & 9.3 & 61.9 & 9 \\
\hline M520E 1 & & & 61.4 & 10.3 & & & 62.3 & 12 \\
\hline M125E1 & & & & & & & 60.6 & 4 \\
\hline M548E: & & & 6.1 & 6.0 & & & 7.8 & 6 \\
\hline UET & & , & & & & & 7.1 & 6 \\
\hline ASV & & & 5.8 & 5.6 & & & 6.6 & 5 \\
\hline
\end{tabular}

\begin{tabular}{|c|c|c|}
\hline & & 174.6 \\
\hline & & 173.7 \\
\hline \multirow[t]{2}{*}{174.1} & 21.2 & 174.1 \\
\hline & & 78.4 \\
\hline \multirow[t]{6}{*}{60.6} & 9.0 & 63.3 \\
\hline & & 176.2 \\
\hline & & 173.8 \\
\hline & & 60.3 \\
\hline & & 18.3 \\
\hline & & 15.9 \\
\hline
\end{tabular}

Mission $3-3.07$ Miles

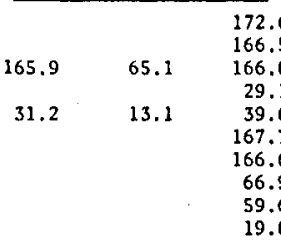

Mission $4-2.80$ Miles

$\begin{array}{rrrr} & & 185.5 & 128.8 \\ 184.2 & 79.0 & 184.5 & 134.8 \\ 81.4 & & 81.3 & 82.3 \\ & 13.5 & 80.9 & 72.2 \\ & & 186.1 & 14.3 \\ & & 185.4 & 79.3 \\ & & 20.3 & 9.8 \\ & & 14.8 & 8.5 \\ & & 14.8 & 7.7\end{array}$

Mission $5-2.72$ Miles

$\begin{array}{llll}51.1 & 51.0 & 54.6 & 74.0\end{array}$

$\begin{array}{rr}54.6 & 74 \\ 74.4 & 76 \\ 130.7 & 78 \\ 50.1 & 50 \\ 22.0 & 50\end{array}$

$\begin{array}{ll}250.6 & 50.0 \\ & 55.6\end{array}$

$51.4 \quad 49.6$

$49.2 \quad 46.5$

UET

$\begin{array}{lllll}\text { M561 } & & & & \\ \text { M35A2 } & 12.3 & 10.5 & 14.5 & 10.9 \\ \text { M813 } & 16.8 & 14.4 & 22.0 & 15.5\end{array}$

M813

M656

TDW901

M520E

M125E

M548E

UET

$\begin{array}{lrrrr}\text { M561 } & 9.9 & 8.5 & 11.3 & 8.9 \\ \text { M35A2 } & 15.2 & 12.7 & 28.4 & 13.4 \\ \text { M813 } & & & 123.4 & 14.4 \\ \text { M656 } & & & 10.3 & 8.3 \\ \text { TDW901 } & & & 14.4 & 9.3 \\ \text { MS20E1 } & & & 124.7 & 12.7 \\ \text { M12SE1 } & & & 10.9 & 7.9 \\ \text { M548E1 } & & & & \\ \text { UET } & & & 10.3 & 5.3 \\ \text { ASV } & & & & \end{array}$

$248.0 \quad 193.4$

$\begin{array}{llll} & & 248.2 & 199.4 \\ 247.8 & 81.9 & 247.9 & 143.0\end{array}$

$\begin{array}{lrrr}84.5 & 51.5 & 87.7 & 74.9 \\ & & 850.5 & 52.6\end{array}$

$247.9 \quad 81.1$

$\begin{array}{ll}71.2 & 50.2 \\ 53.8 & 47.6\end{array}$

Mission $6-2.52$ Miles

$\begin{array}{rrr}184.5 & 71.6 \\ 124.9 & 78.1\end{array}$

$\begin{array}{llll}124.7 & 20.0 & 124.9 & 78.1 \\ & & 124.7 & 22.6\end{array}$

$126.1 \quad 16.0$

$124.9-18.6$

$25.5 \quad 10.2$

$\begin{array}{ll}18.1 & 8.4 \\ 19.2 & 7.3\end{array}$

Mission $7-2.31$ Miles

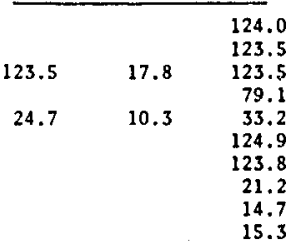

(Continued)

112.0
$250.9 \quad 57.9$

$\begin{array}{llll}29.5 & 11.7 & 37.5 & 12.1 \\ & & 126.1 & 12.0\end{array}$

$\begin{array}{rrrr}60.0 & 18.5 & 60.3 & 76.2 \\ 62.0 & 9.7 & 62.3 & 10.0 \\ & & 62.9 & 12.8 \\ & & 60.2 & 6.3 \\ & & 60.2 & 6.4 \\ & & 59.6 & 5.9\end{array}$

$\begin{array}{rrrr}174.1 & 63.0 & 174.3 & 80.6 \\ 173.6 & 9.9 & 168.4 & 9.9 \\ & & 176.4 & 13.4 \\ & & & \\ & & 168.9 & 9.1 \\ & & 167.6 & 7.1 \\ & & 167.0 & 6.0\end{array}$

$166.1 \quad 69.1 \quad 166.3 \quad 75.7$

$\begin{array}{llll}132.7 & 13.6 & 168.0 & 14.1 \\ & & 167.9 & 19.7\end{array}$

$\begin{array}{ll}166.4 & 51.8 \\ 166.2 & 50.3\end{array}$

$\begin{array}{rr}165.8 & 50.3\end{array}$

$184.5 / 84.4 \quad 183.3 \quad 142.3$

$\begin{array}{rrrr}186.2 & 14.7 & 184.5 & 158.0 \\ & & 184.3 & 19.4\end{array}$

$\begin{array}{rr}183.2 & 10.0 \\ 183.5 & 8.7\end{array}$

$183.1 \quad 7.9$

$\begin{array}{rrrr}248.0 & 143.3 & 284.2 & 145.0 \\ 137.0 & 53.1 & 284.9 & 54.1 \\ & & 287.0 & 59.1 \\ & & & \\ & & 284.8 & 50.3 \\ & & 283.4 & 47.8 \\ & & 282.8 & 46.6\end{array}$

$\begin{array}{rrrr}124.8 & 23.0 & 184.1 & 82.0 \\ 125.4 & 12.2 & 184.8 & 12.5 \\ & & 185.2 & 16.9 \\ & & & \\ & & 184.5 & 10.3 \\ & & 184.1 & 8.5 \\ & & 184.2 & 7.4\end{array}$

$\begin{array}{rrrrr}118.7 & & & & \\ 20.7 & 123.6 & 21.7 & 123.8 & 51.2\end{array}$

$\begin{array}{lllll}10.4 & & & & \\ 10.6 & 124.1 & 10.7 & 124.6 & 11.1\end{array}$

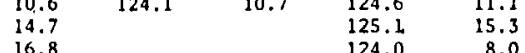

$\begin{array}{rrr}16.8 & 124.0 & 8.0 \\ 8.0 & 123.6 & 6.7\end{array}$

$\begin{array}{ll}123.6 & 6.7 \\ 123.3 & 5.5\end{array}$

(Sheet 1 of 6 ) 
Table t92 (Continued)

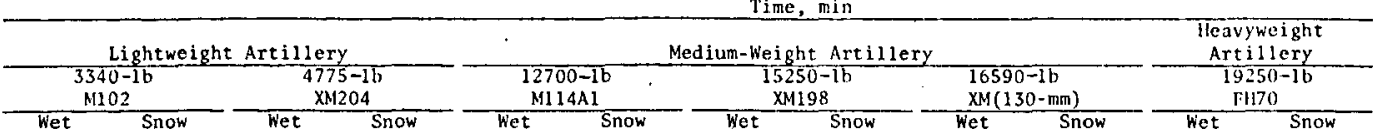

Prime Mover Condition Condition Condition Condition Condition Condition Condition Condition Condition Condition Condition Condition

\section{Mission $8-3.07$ Miles}

\begin{tabular}{|c|c|c|c|c|c|c|c|c|c|c|c|c|}
\hline \\
\hline MS61 & 8.4 & 10.1 & 8.6 & 10.8 & & & 80.5 & 91.1 & & & & \\
\hline M35A2 & 8.0 & 15.2 & 8.1 & 16.2 & & & 9.3 & 98.0 & & & & \\
\hline$M 813$ & & & 8.1 & 16.4 & 8.5 & 85.5 & 8.7 & 102.0 & 8.8 & 102.0 & 68.5 & 102.1 \\
\hline M656 & & & 8.4 & 10.6 & & & 8,9 & 13.3 & & & & \\
\hline TOW901 & & & 9.7 & 12.0 & 11.1 & 12.8 & 11.4 & 13.2 & 11.5 & 13.4 & 71.0 & 13.7 \\
\hline M520E1 & & & 10.7 & 13.6 & & & 11.2 & 15.9 & & & 70.6 & 16.2 \\
\hline M125El & & & & & & & 9.9 & 19.1 & & & & \\
\hline M548E1 & & & 8.9 & 9.4 & & & 9.1 & 9.4 & & & 68.3 & 9.5 \\
\hline UET & & & & & & & 9.0 & 9.5 & & & 68.4 & 9.5 \\
\hline ASV & & & 8.1 & 8.8 & & & 8.2 & 8.8 & & & 67.5 & 8.9 \\
\hline
\end{tabular}

\begin{tabular}{|c|c|c|c|c|c|c|c|c|c|c|c|c|}
\hline \multirow{2}{*}{$\begin{array}{l}\text { M561 } \\
\text { M35A2 }\end{array}$} & 16.9 & 10.6 & 18.8 & 11.6 & & & 69.3 & 71.9 & & & & . \\
\hline & 70.7 & 19.6 & .72 .9 & 61.5 & & & 73.2 & 75.1 & & & & \\
\hline M813 & & & 66.2 & 15.5 & 72.2 & 82.3 & 72.3 & 81.6 & 72.4 & 81.9 & 67.3 & 76.0 \\
\hline M650 & & & 11.0 & 10.3 & & & 81.1 & 18.6 & & & & \\
\hline TDW9Ol & & & 14.3 & 11.0 & 57.0 & 12,2 & 85.8 & 12.9 & 73.5 & 18.8 & 68.5 & 13.8 \\
\hline M520E I & & & 70.0 & 15.6 & & & 75.3 & 23.6 & & & 70.4 & 24.4 \\
\hline M125El & & & & & & & 72.6 & 24.5 & & & & \\
\hline $\mathrm{M} 48 \mathrm{E} 1$ & & & 18.1 & 9.7 & & & 25.6 & 10.3 & & & 68.5 & 10.9 \\
\hline UET & & & & & & & 20.3 & 8.6 & & . & 66.7 & 8.7 \\
\hline ASV & & & 17.0 & 7.3 & & & 21.0 & 7.7 & & & 66.8 & 7.9 \\
\hline
\end{tabular}

\begin{tabular}{|c|c|c|c|c|c|c|c|c|c|c|c|c|}
\hline \multirow[b]{2}{*}{ M561 } & \multicolumn{11}{|c|}{ Mission $10-2.50$ Miles } & \multirow[b]{4}{*}{74.4} \\
\hline & 11.1 & 12.0 & 64.8 & 12.3 & & & 171.6 & 68.1 & & & \multirow[b]{3}{*}{118.4} & \\
\hline M35A2 & 11.2 & 15.2 & 12.0 & 68.7 & & & 170.9 & 73.5 & & & & \\
\hline$M 813$ & & & 117.8 & 17.0 & 118.3 & 72.1 & 119.6 & 74.1 & 120.7 & 74.2 & & \\
\hline M656 & & & 10.8 & 11.8 & & & 66.7 & 13.3 & & & & \\
\hline TDW901 & . & & 12.3 & 12.2 & 15.0 & 13.0 & 16.6 & 13.3 & 119.0 & 13.4 & 119.3 & 13.7 \\
\hline M520E1 & & & 119.6 & 14.9 & & & 120.5 & 16.6 & & & 120.3 & 17.1 \\
\hline M125E1 & & & & & & & 118.5 & 18.8 & & & & \\
\hline M548E1 & & & 11.4 & 10.9 & & & 14.2 & 10.9 & & & 118,1 & 11.0 \\
\hline UET & & & & & & & 12.2 & 10.6 & & & 117.8 & 10.6 \\
\hline ASV & & & 11.1 & 9.8 & & & 12.2 & 9.9 & & & 117.3 & 10.0 \\
\hline
\end{tabular}

ASV $11.1 \quad 9.8 \quad$ Mission $11-2.80$ Miles

\begin{tabular}{|c|c|c|c|c|c|c|c|c|c|c|c|c|}
\hline M561 & 68.2 & 10.5 & 69.3 & 69.9 & & & 206.0 & 255.7 & & & & \\
\hline M35A2 & 46.9 & 133.3 & 52.4 & 133.9 & & & 205.1 & 262.3 & & & & \\
\hline M813 & & & 164.3 & 78.3 & 204.2 & 138.2 & 204.4 & 140.4 & 204.6 & 140.6 & 164.3 & 142.8 \\
\hline M656 & & & 10.8 & 10.7 & & & 195.8 & 130.6 & & & & \\
\hline TDW901 & & & 13.9 & 12.2 & 54.4 & 14.6 & 96.4 & 17.1 & 207.4 & 74.3 & 167.3 & 22.1 \\
\hline M520E 1 & & & 181.0 & 15.2 & & & 207.5 & 76.7 & & & 168,0 & 80,3 \\
\hline M125El & & & & & & & 205.4 & 137.5 & & & & \\
\hline M548E1 & & & 69.2 & 8.8 & & & 73.7 & 9.5 & & & 164.9 & 9.7 \\
\hline UET & & & & & : & & 71.0 & 8.8 & & & 163.9 & 8.9 \\
\hline ASV & & & 68.6 & 7.8 & 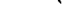 & & 70.8 & 8.0 & & & 163.4 & 8.2 \\
\hline
\end{tabular}

Mission $12-1.64$ Miles

\begin{tabular}{|c|c|c|c|c|c|c|c|c|c|c|c|c|}
\hline M561 & 8.5 & 6.9 & 10.1 & 7.2 & \multirow{3}{*}{124.2} & \multirow{3}{*}{76.7} & \multirow{2}{*}{$\begin{array}{l}175.4 \\
176.6\end{array}$} & 145.9 & \multirow{3}{*}{125.3} & \multirow[b]{3}{*}{150.6} & & \multirow[b]{3}{*}{151.0} \\
\hline$M 35 \wedge 2$ & 12.1 & 10.8 & 16.7 & 12.7 & & & & 151.7 & & & \multirow[b]{2}{*}{124.6} & \\
\hline MS13 & & & 123.1 & 12.3 & & & & 153.2 & & & & \\
\hline M656 & & & 8.9 & 7.0 & \multirow{3}{*}{19.4} & \multirow{3}{*}{8.6} & 83.1 & 9.2 & \multirow{3}{*}{124.1} & \multirow{3}{*}{9.4} & & \multirow{3}{*}{$\begin{array}{r}9.6 \\
14.3\end{array}$} \\
\hline TDW901 & & & 11.9 & 7.6 & & & 25.7 & 9.2 & & & \multirow{2}{*}{$\begin{array}{l}125.3 \\
126.9\end{array}$} & \\
\hline MS20E1 & & & 125.7 & 11.9 & & & 126.4 & 13.7 & & & & \\
\hline M125El & & & & & & & 124.1 & 14.9 & & & & \multirow{2}{*}{7.1} \\
\hline MS48E1 & & & 9.8 & 6.6 & & & 76.7 & 7.1 & & & & \\
\hline UET & & & & & & & 13.2 & 5.9 & & & 124.1 & 5.9 \\
\hline ASV & & & 10.2 & 5.1 & & & 14.2 & 5.5 & & & 124.0 & 5.6 \\
\hline \multicolumn{13}{|c|}{ Mission $13-2.11$ Miles } \\
\hline M561 & 66.3 & 66.8 & 66.3 & 66.9 & & & 108,1 & 151.2 & & & & \\
\hline M35A2 & 66.8 & 68.4 & 69.5 & 68.6 & & & 108.0 & 153.1 & & & & \\
\hline M813 & & & 107.7 & 69.0 & 107.8 & 70.5 & 107.8 & 111.7 & 107.9 & 111.7 & 107.9 & 111.8 \\
\hline M656 & & & 66.4 & 66.9 & & & 108.0 & 67.6 & & & & \\
\hline TDW901 & & & 8.9 & 9.2 & 10.3 & 9.5 & 11.4 & 9.6 & 53.6 & 9.7 & 108.6 & 9.8 \\
\hline M520E1 & & & 108.4 & 13.0 & & & 108.6 & 13.9 & & & 108.7 & 14.1 \\
\hline M125E1 & & & & & & & 108.1 & 69.8 & & & & \\
\hline M548E1 & & & 66.5 & 66.5 & & & 67.4 & 66.5 & & & 108.0 & 66.7 \\
\hline UET & & & & & & & 66.8 & 66.5 & & & 107.9 & 66.6 \\
\hline ASV & & & 7.6 & 7.8 & & & 8.0 & 7.8 & & & 107.8 & 7.8 \\
\hline
\end{tabular}

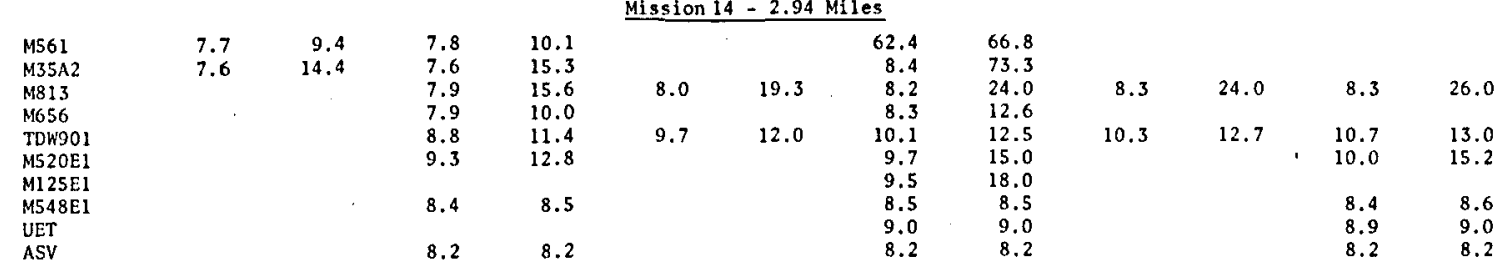

(Continued) 
Table E92 (Continued)

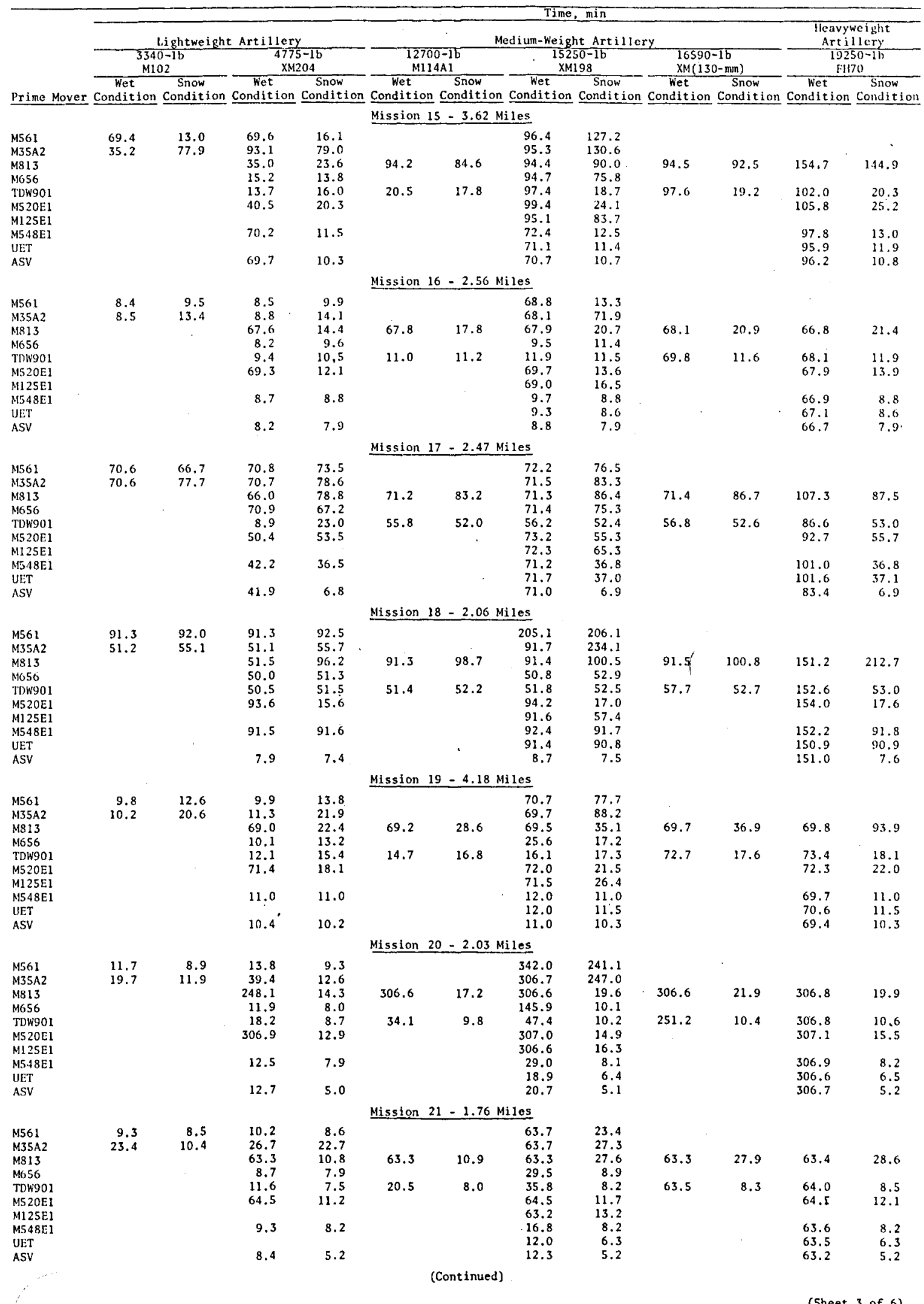

(Sheet 3 of 6 ) 
Table t92 (Cont inued)

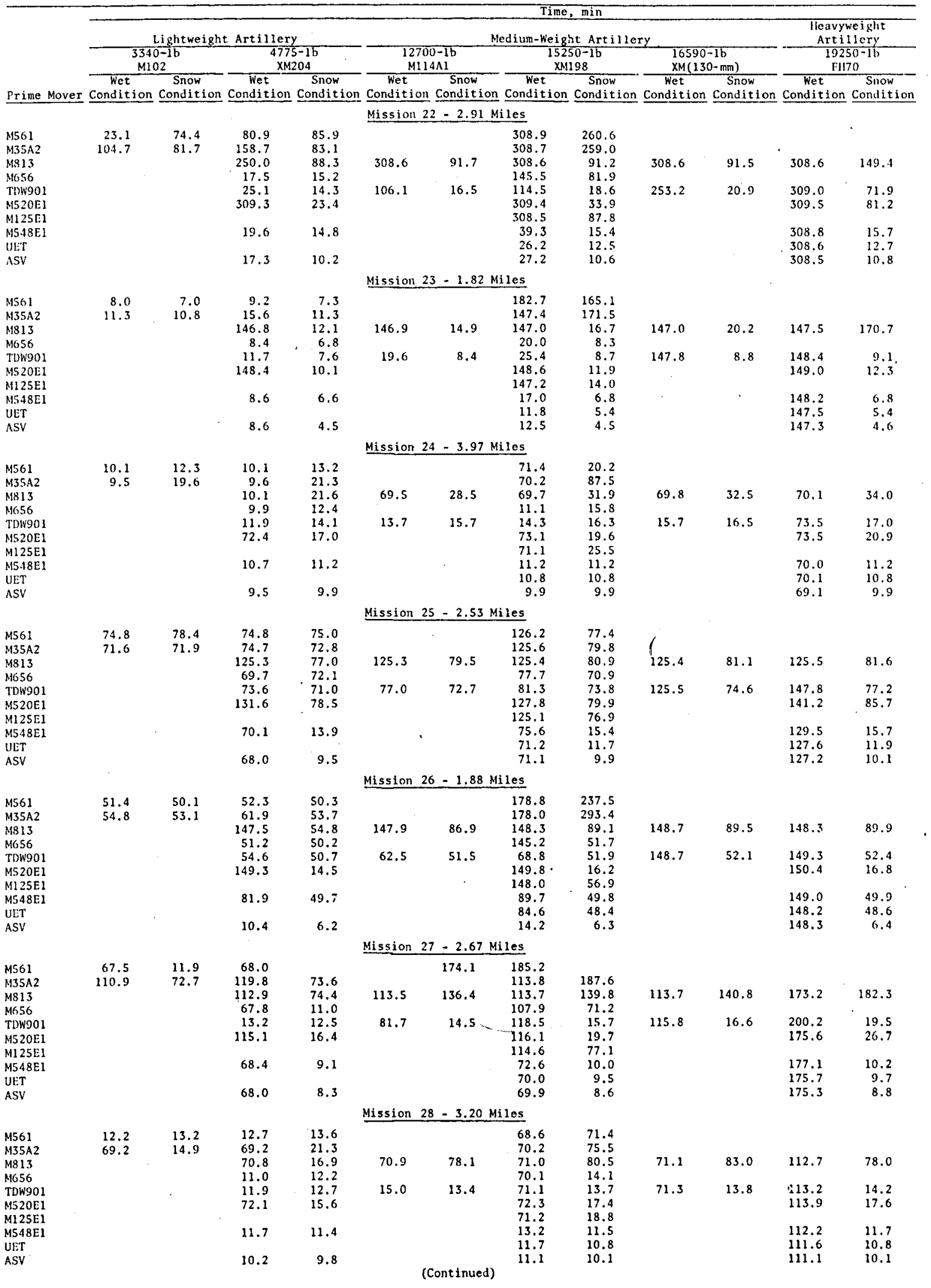


Table E92 (Continued)

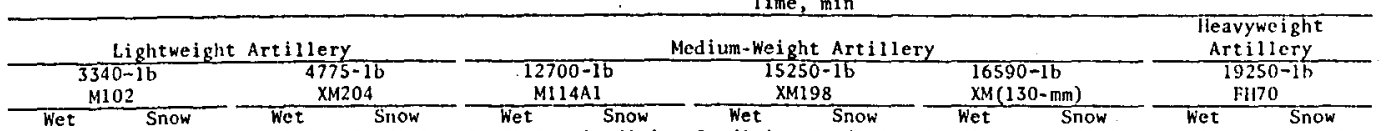

Prime Mover Condition Condition Condition Condition Condition Condition Condition Condition Condition Condition Condition Condition

\begin{tabular}{|c|c|c|c|c|c|c|c|c|c|c|c|c|}
\hline \multicolumn{13}{|c|}{ Mission } \\
\hline $\begin{array}{l}\text { M56L } \\
\text { M35A2 }\end{array}$ & $\begin{array}{l}69.6 \\
69.2\end{array}$ & $\begin{array}{l}69.9 \\
73.9\end{array}$ & $\begin{array}{r}123.0 \\
87.8\end{array}$ & $\begin{array}{r}70.4 \\
128.0\end{array}$ & & & $\begin{array}{l}258.9 \\
257.0\end{array}$ & $\begin{array}{r}188.8 \\
195.5\end{array}$ & & & & \\
\hline $\begin{array}{l}\text { M35A2 } \\
M 813\end{array}$ & 204.0 & $\begin{array}{l}13.9 \\
76.6\end{array}$ & & & 204.7 & 133.3 & 205.8 & $\begin{array}{l}195.5 \\
135.7\end{array}$ & 205.9 & 136.2 & 205.1 & 154.7 \\
\hline Mo56 & & & 70.1 & 68.6 & & & 142.4 & 71.2 & & & & \\
\hline TowQ01 & & & 72.8 & 69.3 & 73.2 & 70.5 & 75.5 & 71.0 & 205.2 & 71.2 & 206.7 & 71.6 \\
\hline$M 520 \mathrm{E} 1$ & & & 206.9 & 73.2 & & & 207.7 & 75.4 & & & 208.2 & 76.3 \\
\hline$M 125 E 1$ & & & & & & & 204.5 & 78.7 & & & & \\
\hline M548:1 & & & 71.8 & 63.9 & & & 73.1 & 66.5 & & & 205.5 & 65.6 \\
\hline UET & & & & & & & 77.4 & 63.5 & & & 204.8 & 63.8 \\
\hline ASV & & & 17.0 & 10.7 & & & 24.8 & 11.9 & & & 181.5 & 12.7 \\
\hline \multicolumn{13}{|c|}{ Mission $30-3.35$ Miles } \\
\hline M561 & 10,3 & 11.7 & 10.5 & 12.2 & & & 34.7 & 85.6 & & & & \\
\hline M35A2 & 27.8 & 15.0 & 27.9 & 15.6 & & & 34.4 & 89.9 & & & & \\
\hline M813 & & & 33.8 & 15.8 & 34.0 & 18.4 & 34.1 & 22.2 & 34.3 & 74.8 & 88.0 & 76.1 \\
\hline M656 & & & 10.3 & 12.0 . & & & 28.4 & 13.8 & & & & \\
\hline TDW901 & & & 11.3 & 13.0 & 29.8 & 13.5 & 30.0 & 13.7 & 35.9 & 13.8 & 89.6 & 14.1 \\
\hline M520E1 & & & 35.4 & 13.9 & & & 35.6 & 15.5 & & & 89.1 & 15.6 \\
\hline M12SE1 & & & & & & & 34.9 & 17.5 & & & & \\
\hline MS48EI & & & 10.6 & 11.2 & & & 11.3 & 11.3 & & & 88.0 & 11.3 \\
\hline UET & & & & & & & 10.9 & 11.3 & & & 88.0 & 11.3 \\
\hline ASV & & & 10.2 & 10.8 & & & 10.5 & 10.9 & & & 87.5 & 10.9 \\
\hline
\end{tabular}

Mission $31-3.65$ Miles

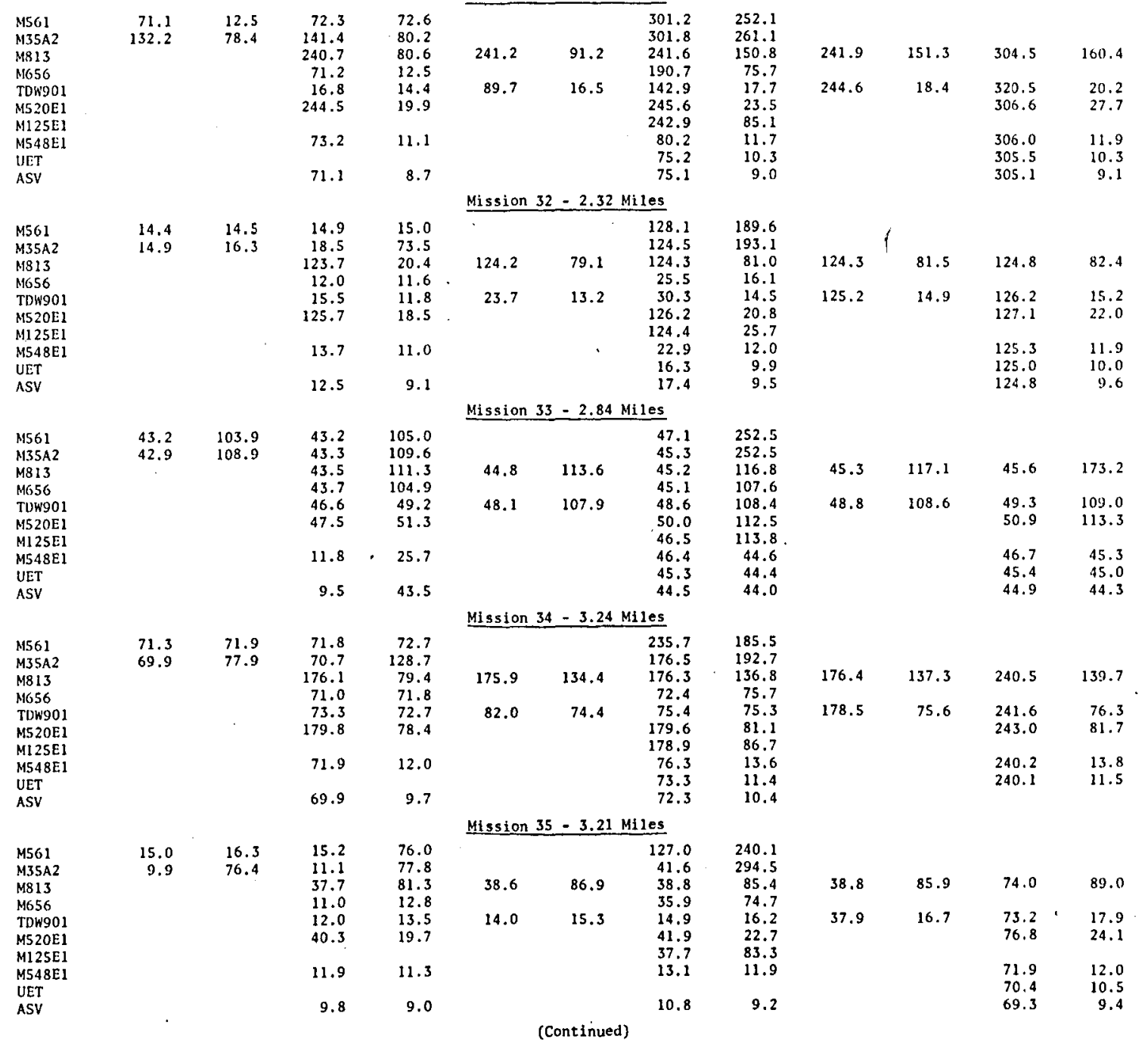


Table E92 (Concluded)

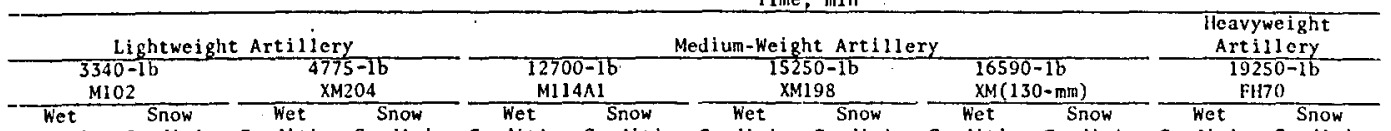

Prime Mover Condition Condttion Condition Condition Condition Condition Condition Condition Condition Condition Condition Condition

Mission $36-2.53$ Miles

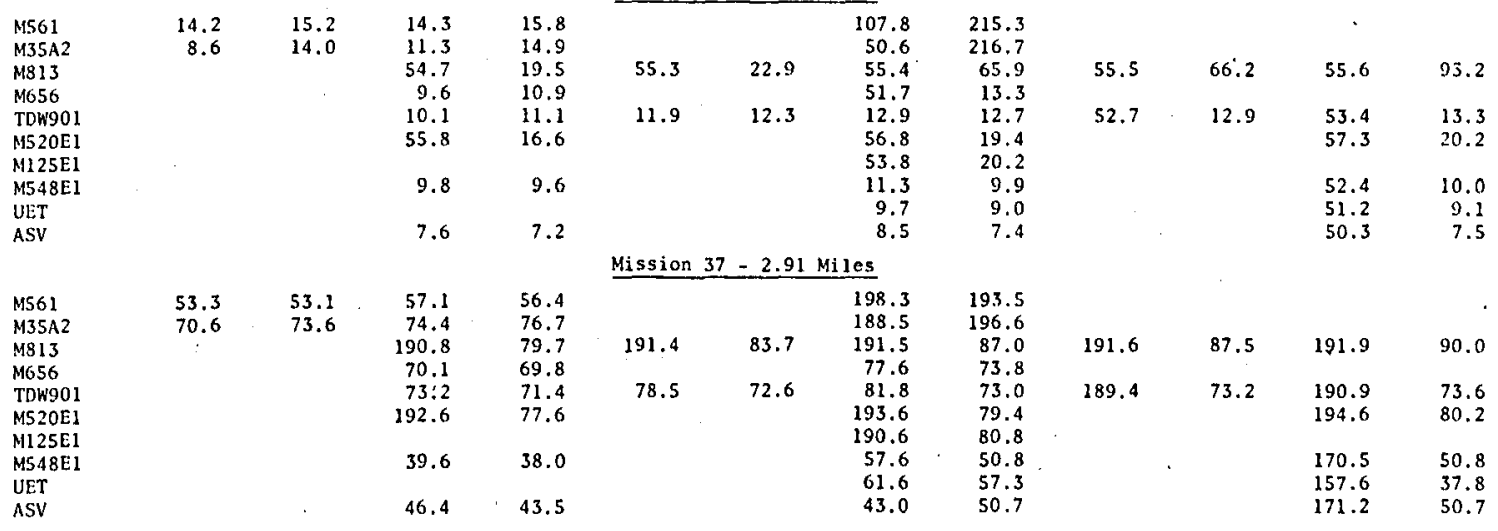


Table E!3

Time (min.) for Study Self-Propelled and Keference Velicle to Complete Selected Artillery Missions

\begin{tabular}{|c|c|c|c|c|c|c|c|c|c|c|c|c|c|c|}
\hline \multirow{2}{*}{$\begin{array}{c}\text { Mission } \\
\text { No. }\end{array}$} & \multirow{2}{*}{$\begin{array}{c}\text { Length } \\
16 \\
\end{array}$} & \multicolumn{3}{|c|}{ Self-Propelled Artillery } & & & & & rence Ve & hicles & & & & \\
\hline & & M109A1 & M107 & M110E2 & M113A! & M60A2 & M548 & M577A1 & $\mathrm{XM723}$ & $\mathrm{XM}$ & ASV & M561 & M813 & UIT \\
\hline & & & & & & & Condit $i$ & & & & & & & \\
\hline 1 & 2.06 & 6.5 & 6.5 & 6.5 & 5.1 & 6.2 & 5.5 & 5.2 & 5.0 & 4.6 & 5.3 & 5.2 & 58.9 & 5.8 \\
\hline 2 & 1.60 & 14.7 & 14.5 & 14.5 & 13.9 & 13.1 & 15.1 & 13.9 & 12.1 & 12.3 & 12.5 & 9.5 & 168.2 & 13.3 \\
\hline 3 & 3.07 & 13.4 & 12.9 & 12.9 & 50.0 & 11.4 & 51.4 & 50.2 & 7.7 & 8.0 & 8.9 & 51.4 & 125.5 & 9.8 \\
\hline 4 & 2.80 & 11.1 & 10.8 & 10.8 & 8.2 & 9.8 & 9.7 & 8.3 & 7.8 & 7.3 & 8.7 & 8.3 & 183.9 & 9.0 \\
\hline 5 & 2.72 & 50.1 & 50.0 & 50.0 & 47.6 & 48.7 & 49.7 & 47.9 & 46.4 & 10.6 & 47.1 & 49.2 & 130.1 & 47.7 \\
\hline 6 & 2.52 & 13.3 & 12.8 & 12.8 & 8.7 & 10.6 & 10.0 & 8.9 & 7.9 & 8.2 & 9.1 & 9.8 & 65.6 & 9.8 \\
\hline 7 & 2.31 & 10.6 & 10.4 & 10.4 & 7.1 & 8.4 & 8.3 & 7.2 & 6.1 & 6.2 & 7.2 & 7.8 & 123.3 & 7.8 \\
\hline 8 & 3.07 & 9.0 & 9.0 & 9.0 & 8.2 & 9.3 & 8.9 & 8.2 & 8.0 & 7.7 & 8.1 & 8.4 & 8.0 & 8.9 \\
\hline 9 & 2.25 & 17.7 & 17.6 & 17.6 & 14.5 & 16.1 & 16.1 & 14.6 & 14.0 & 13.7 & 14.8 & 9.6 . & 65.7 & 15.4 \\
\hline 10 & 2.50 & 12.3 & 11.7 & 11.7 & 63.1 & 14.9 & 63.6 & 63.2 & 62.8 & 10.1 & 63.1 & 10.6 & 71.1 & 63.6 \\
\hline 11 & 2,80 & 60.3 & 69.2 & 69.2 & 66.9 & 68.5 & 67.7 & 67.0 & 66.8 & 66.1 & 67.5 & 7.9 & 163.2 & 67.9 \\
\hline 12 & 1.64 & 10.5 & 10.2 & 10.2 & 7.2 & 50.6 & 8.7 & 7.8 & 9.7 & 47.5 & 8.4 & 6.4 & 65.8 & 7.8 \\
\hline 13 & 2.11 & 7.9 & 66.3 & 8.0 & 65.9 & 66.2 & 66.2 & 66.0 & 7.3 & 7.0 & 7.4 & 66.0 & 107.6 & 66.2 \\
\hline 14 & 2.94 & 9.0 & 9.0 & 9.0 & 8.3 & 9.2 & 8.4 & 8.3 & 8.2 & 8.0 & 8.2 & 7.7 & 7.8 & 9.0 \\
\hline 15 & 3.62 & 70.5 & 70.5 & 70.5 & 68.9 & 70.3 & 69.6 & 68.9 & 68.6 & 67.8 & 69.2 & 9.8 & 33.6 & 69.8 \\
\hline 16 & 2.56 & 8.8 & 8.7 & 8.7 & 8.0 & 8.7 & 8.6 & 8.0 & 7.7 & 7.5 & 7.8 & 8.2 & 67.5 & 8.4 \\
\hline 17 & 2.47 & 42.8 & 42.7 & 42.7 & 41.7 & 42.9 & 41.8 & 41.7 & 41.5 & 41.3 & 41.6 & 35.1 & 64.8 & 42.3 \\
\hline 18 & 2.06 & 8.5 & 8.6 & 8.6 & 49.9 & 9.7 & 91.3 & 49.9 & 7.3 & 6.8 & 7.6 & 91.1 & 50.6 & 49.2 \\
\hline 19 & 4.18 & 11.7 & 11.7 & 11.7 & 10.2 & 11.8 & 10.7 & 10.2 & 10.1 & 9.7 & 10.2 & 9.6 & 68.3 & 11.3 \\
\hline 20 & 2.03 & 12.5 & 12.1 & 12.1 & 7.3 & 9.5 & 8.6 & 7.5 & 6.4 & 6.4 & $7: 8$ & $8.6^{\circ}$ & 247.7 & 8.2 \\
\hline 21 & 1.76 & 9.2 & 8.9 & 8.9 & 6.8 & 7.1 & 8.2 & 6.8 & 5.5 & 5.8 & 6.1 & 8.4 & 63.3 & 6.9 \\
\hline 22 & 2.91 & 17.8 & 17.1 & 17.1 & 13.5 & 14.2 & 15.7 & 13.7 & 11.0 & 11.0 & 12.6 & 19.8 & 249.7 & 13.4 \\
\hline 23 & 1.82 & 8.7 & 8.6 & 8.6 & 5.6 & 7.0 & 6.7 & 5.7 & 5.2 & 5.2 & 6.0 & 6.5 & 146.7 & 6.5 \\
\hline 24 & 3.97 & 10.7 & 10.7 & 10.7 & 9.3 & 11.1 & 10.5 & 9.3 & 9.2 & 9.0 & 9.2 & 9.9 & 9.5 & 10.4 \\
\hline 25 & 2.53 & 69.2 & 69.1 & 69.1 & 68.4 & 67.5 & 69.9 & 68.4 & 66.1 & 66.5 & 66.5 & 78.1 & 128.4 & 67.8 \\
\hline 26 & 1.88 & 10.6 & 10.3 & 10.3 & 38.6 & 10.3 & 80.2 & 38.7 & 7.6 & 7.7 & 8.2 & 49.9 & 147.4 & 79.5 \\
\hline 27 & 2.67 & 68.5 & 68.4 & 68.4 & 66.7 & 67.9 & 67.2 & 66.7 & 66.4 & 65.9 & 67.0 & 10.1 & 110.2 & 67.3 \\
\hline 28 & 3.20 & 11.1 & 11.0 & 11.0 & 10.2 & 10.6 & 11.2 & 10.2 & 9.6 & 9.3 & 9.8 & 12.0 & 70.7 & 10.6 \\
\hline 29 & 2.49 & 70.0 & 69.4 & 69.4 & 121.9 & 20.3 & 123.1 & 122.0 & 67.5 & 14.0 & 68.2 & 69.4 & 147.1 & 121.3 \\
\hline 30 & 3.35 & 10.7 & 10.7 & 10.7 & 10.0 & 10.7 & 10.5 & 10.1 & 10.0 & 9.8 & 10.1 & 10.2 & 33.8 & 10.6 \\
\hline 31 & 3.65 & 72.6 & 72.4 & 72.4 & 69.6 & 71.7 & 71.6 & 69.7 & 68.6 & 68.2 & 69.4 & 10.3 & 183.1 & 70.8 \\
\hline 32 & 2.32 & 13.0 & 12.7 & 12.7 & 10.0 & 10.8 & 11.7 & 10.2 & 10.0 & 8.2 & 10.1 & 13.2 & 71.9 & 10.2 \\
\hline 33 & 2.84 & 9.9 & 9.6 & 9.6 & 7.6 & 9.4 & 8.3 & 7.8 & 7.3 & 8.6 & 8.1 & 6.9 & 42.7 & 8.5 \\
\hline 34 & 3.24 & 71.1 & 71.0 & 71.0 & 69.0 & 70.2 & 70.7 & 69.1 & 68.6 & 67.5 & 68.8 & 70.1 & 123.1 & 69.5 \\
\hline 35 & 3.21 & 11.2 & 11.2 & 11.2 & 10.1 & 11.1 & 11.7 & 10.2 & 9.0 & 8.6 & 9.5 & 14.3 & 37.4 & 11.0 \\
\hline 36 & 2.53 & 8.4 & 8.4 & 8.4 & 8.5 & 8.5 & 9.4 & 8.5 & 6.9 & 6.9 & 7.2 & 13.5 & 54.3 & 8.7 \\
\hline 37 & 2.91 & 58.2 & 61.2 & 61.2 & 31.2 & 25.4 & 30.2 & 32.0 & 32.8 & 68.3 & 55.6 & 62.1 & 190.7 & 38.6 \\
\hline & & & & & & & Condit $i$ & & & & 1 & & & \\
\hline 1 & 2.06 & 6.1 & 6.1 & 6.1 & 5.5 & 6.2 & 6.0 & 5.5 & 5.5 & 5.4 & 5.6 & 6.5 & 11.0 & 5.9 \\
\hline 2 & 1.60 & 7.3 & 7.3 & 7.3 & 7.6 & 6.5 & 9.0 & 7.6 & 6.0 & 5.8 & 6.0 & 9.9 & 11.5 & 6.9 \\
\hline 3 & 3.07 & 9.8 & 9.8 & 9.8 & 50.1 & 9.1 & $\$ 1.8$ & 50.1 & 7.4 & 7.0 & 7.4 & 52.0 & 17.9 & 8.7 \\
\hline 4 & 2.80 & 8.6 & 8.7 & 8.7 & 7.7. & 8.3 & 9.0 & 7.7 & 7.0 & 6.5 & 7.3 & 9.5 & 16.8 & 8.1 \\
\hline 5 & 2.72 & 48.2 & 48.2 & 48.2 & 47.7 & 47.4 & 49.6 & 47.7 & 46.4 & 45.7 & 46.4 & 50.3 & 54.1 & 47.3 \\
\hline 6 & 2.52 & 9.4 & 9.4 & 9.4 & 8.4 & 7.9 & 10.2 & 8.4 & 7.1 & 6.6 & 7.2 & 10.3 & 14.6 & 8.2 \\
\hline 7 & 2.31 & 7.5 & 7.6 & 7.6 & 6.5 & 6.0 & 7.9 & 6.5 & 5.2 & 4.7 & 5.2 & 8.3 & 12.7 & 6.4 \\
\hline 8 & 3.07 & 9.5 & 9.5 & 9.5 & 8.7 & 9.7 & 9.3 & 8.7 & 8.7 & 8.6 & 8.7 & 9.5 & 16.2 & 9.3 \\
\hline 9 & 2.25 & 8.0 & 8.9 & 8.9 & 8.0 & 8.2 & 9.6 & 8.1 & 6.9 & 6.8 & 7.1 & 9.8 & 14.4 & 7.9 \\
\hline 10 & 2.50 & 10.5 & 10.5 & 10.5 & 10.2 & 10.8 & 10.8 & 10.2 & 9.8 & 9.9 & 9.7 & 11.5 & 15.6 & 10.4 \\
\hline 11 & 2.80 & 8.5 & 8.5 & 8.5 & 7.6 & 8.3 & 8.6 & 7.6 & 7.3 & 6.8 & 7.7 & 9.4 & 17.6 & 8.1 \\
\hline 12 & 1.64 & 5.8 & 5.8 & 5.8 & 5.0 & 47.0 & 6.4 & 5.0 & 5.2 & 45.5 & 4.9 & 6.3 & 10.7 & 5.1 \\
\hline 13 & 2.11 & 8.0 & 66.4 & 8.0 & 66.2 & 66.4 & 66.5 & 66.2 & 7.7 & 7.4 & 7.8 & 66.5 & 68.4 & 66.4 \\
\hline 14 & 2.94 & 9.0 & 9.0 & 9.0 & 8.3 & 9.2 & 8.5 & 8.3 & 8.2 & 8.0 & 8.2 & 9.1 & 15.4 & 8.9 \\
\hline 15 & 3.62 & 11.6 & 11.6 & 11.6 & 10.1 & 11.0 & 11.4 & 10.1 & 9.5 & 8.9 & 10.0 & 12.1 & 21.0 & 10.4 \\
\hline 16 & 2.56 & 8.6 & 8.7 & 8.7 & 8.2 & 8.7 & 8.8 & 8.2 & 7.8 & 7.7 & 7.9 & 9.2 & 13.8 & 8.5 \\
\hline 17 & 2.47 & 7.5 & 7.5 & 7.5 & 36.2 & 8.5 & 36.4 & 36.3 & 6.7 & 6.5 & 6.7 & 40.5 & 72.7 & 7.2 \\
\hline 18 & 2.06 & 8.2 & 8.2 & 8.3 & 49.9 & 9.4 & 91.5 & 49.9 & 7.1 & 6.7 & 7.2 & 91.7 & 55.2 & 49.0 \\
\hline 19 & 4.18 & 11.6 & 11.6 & 11.6 & 10.5 & 11.8 & 10.9 & 10.5 & 10.2 & 9.9 & 10.2 & 11.9 & 21.9 & 11.4 \\
\hline 20 & 2.03 & 7.5 & 7.5 & 7.5 & 6.3 & 5.7 & 7.9 & 6.3 & 4.9 & 4.0 & 4.9 & 8.7 & 12.4 & 6.1 \\
\hline 21 & 1.76 & 6.9 & 6.9 & 6.9 & 6.7 & 5.9 & 8.2 & 6.7 & 5.2 & 5.1 & 5.2 & 8.4 & 10.1 & 6.3 \\
\hline 22 & 2.91 & 12.2 & 12.3 & 12.3 & 12.3 & 11.2 & 14.5 & 12.3 & 9.7 & 9.2 & 10.1 & 20.1 & 23.8 & 11.8 \\
\hline 23 & 1.82 & 6.0 & 6.0 & 6.0 & 5.3 & 5.1 & 6.6 & 5.3 & 4.4 & 4.0 & 4.4 & 6.7 & 10.0 & 5.3 \\
\hline 24 & 3.97 & 11.0 & 11.0 & 11.0 & 10.0 & 11.2 & 11.1 & 10.0 & 9.9 & 9.8 & 9.9 & 11.2 & 20.8 & 10.8 \\
\hline 25 & 2.53 & 11.0 & 11.0 & 11.0 & 11.4 & 10.1 & 13.5 & 11.5 & 8.8 & 8.6 & 9,3 & 78.3 & 79.8 & 11.3 \\
\hline 26 & 1.88 & 7.4 & 7.5 & 7.5 & 7.6 & 7.9 & 49.6 & 7.5 & 6.0 & 5.9 & 6.1 & 49.9 & 53.0 & 48.1 \\
\hline 27 & 2.67 & 9.1 & 9.2 & 9.2 & 8.2 & 8.8 & 8.8 & 8.2 & 7.7 & 7.2 & 8.2 & 11.2 & 17.4 & 8.7 \\
\hline 28 & 3.20 & 10.1 & 10.7 & 10.7 & 10.5 & 10.5 & 11.4 & 10.5 & 9.6 & 9.6 & 9.8 & 12.7 & 16.7 & 10.6 \\
\hline 29 & 2.49 & 61.7 & 61.7 & 61.7 & 62.0 & 10.0 & 63.7 & 62.1 & 7.6 & 7.9 & 9.9 & 69.1 & 75.3 & 63.5 \\
\hline 30 & 3.35 & 11.3 & 11.3 & 11.3 & 10.8 & 11.4 & 11.2 & 10.8 & 10.8 & 10.7 & 10.8 & 11.4 & 15.7 & 11.3 \\
\hline 31 & 3.65 & 10.8 & 10.8 & 10.8 & 9.2 & 10.0 & 10.8 & 9.2 & 8.4 & 7.5 & 8.6 & 11.5 & 21.2 & 9.8 \\
\hline 32 & 2.32 & 10.1 & 10.0 & 10.0 & 9.4 & 9.1 & 10.9 & 9.4 & 9.0 & 7.9 & 8.9 & 13.6 & 19.0 & 9.3 \\
\hline 33 & 2.84 & 44.4 & 44.4 & 44.4 & 14.0 & 44.0 & 19.1 & 18.8 & 42.8 & 42.2 & 43.2 & 44.3 & 110.2 & 43,3 \\
\hline 34 & 3.24 & 11.3 & 11.3 & 11.3 & 10.0 & 10.3 & 11.7 & 10.1 & 9.0 & 7.9 & 9.4 & 70.7 & 78.2 & 10.2 \\
\hline $\begin{array}{l}34 \\
35\end{array}$ & 3.21 & 10.0 & 10.1 & 10.1 & 9.9 & 20.1 & 11.2 & 9.9 & 8.6 & 8.8 & 8.8 & 15.6 & 21.5 & 10.0 \\
\hline 36 & 2.53 & 8.1 & 8.1 & 8.1 & 8.6 & 8.3 & 9.4 & 8.6 & 7.1 & 7.4 & 7.2 & 14.6 & 18.9 & 8.5 \\
\hline 37 & 2,91 & 56.2 & 59.4 & 59.4 & 30.9 & 23.6 & 30.2 & 31.6 & 32.0 & 67.2 & 54.2 & 62.7 & 79.2 & 37.4 \\
\hline
\end{tabular}


APPENDIX F: SELECTION OF TACTICAL HIGH AS MOBILITY

LEVEL FOR COMPARING STUDY VEHICLES

1. The percentages of primary and secondary roads, trails, and off-road terrain that make up the network for the 37 missions related to artillery movement (Table 3 ) were compared to the percentages of operating distance for tactical high mobility (Table 18) with the following results:

\section{Mission Network Composite Percentage

\begin{tabular}{ll}
$\begin{array}{l}\text { Primary } \\
\text { Road }\end{array}$ & $\begin{array}{c}\text { Secondary } \\
\text { Road }\end{array}$ Trails Off-Road \\
\hline
\end{tabular}

$\begin{array}{lcccc}\begin{array}{l}\text { Artillery missions } \\ \begin{array}{c}\text { Tactical high mobility } \\ \text { level }\end{array}\end{array} & 15 & 33 & 42 & 10 \\ & 10 & 32 & 8 & 50\end{array}$

2. The values of 10,32 , and 8 were obtained by taking 50 percent of the values shown for primary roads, secondary roads, and trails from the HIMO network shown in Table 3. The comparison apove shows that the artillery missions have about the same percentage of trails and offroad combined $(42+10)$ as the tactical high mobility level $(50+8)$ but the artillery missions have a much larger percentage of trails than does the tactical high mobility levels.

3. To show that the tactical high mobility level was adequate for describing the mobility performance for artillery-related missions, a preliminary definition of "tactical artillery mobility" was defined as follows:
a. 20 percent of primary roads at $\mathrm{V}_{100}$
b. 20 percent of secondary roads at $v_{100}$
c. 40 percent of trails at $\mathrm{v}_{100}$
d. 20 percent of off-road at $V_{90}$

The equations given in Appendix $G$ were then used to determine a tactical artillery mobility rating speed based on the preliminary definitions of "tactical artillery mobility" given above. Tactical artillery 
mobility rating speeds and the tactical high mobility rating speeds for several study vehicles for the wet condition in West Germany are given below.

\section{Study Vehicle}

M109A1

ASV-XM204

TDW901-XM204

M561-XM204

M548-XM204

ASV-XM198

TDW901-XM198

M656-XM198

M548-XM198

ASV-FH 70

TDW901-FH70

M520-FH 70

M548-FH 70
Tactical Artillery Mobility Rating

Speed, mph

13.0

14.2

12.0

4.3

7.4

12.9

10.4

4.9

7.1

0.1

0.1

0.1

0.1
Tactical High. Mobility Rating

Speed, mph

10.1

10.7

9.3

3.1

4.3

9.8

8.2

3.9

4.3

0.1

0.1

0.1

0.1

4. The differences in speed show that the tactical high mobility level is slightly more severe than the tactical artillery mobility level. However, it is felt that the difference in performance between tactical high mobility and tactical artillery mobility do not warrant establishing a tactical artillery mobility definition at this time. Therefore, it is recommended that the tactical high mobility level be used to determine the relative mobility of artillery vehicles until sufficient data are available to more completely define the tactical artillery mobility definition. 


\section{APPENDIX G: COMPUTATION OF MISSION-ORIENTED AVERAGE SPEED BASED \\ ON STATISTICAL MISSION DEFINITION AND VEHICLE PERFORMANCE STATISTICS FOR AN AREA AND CONDITION}

1. The equation for computing the mobility rating speeds from the HIMO report is given as follows:

where

$$
v_{w}=\frac{100}{\frac{P}{V_{C}}\left(1+\frac{L}{100}\right)+\frac{100-P}{V_{R}}}
$$

$$
\begin{aligned}
\mathrm{V}_{\mathrm{w}}= & \begin{array}{l}
\text { mobility rating speed, mph, for a vehicle performing a } \\
\text { mission for a specific area and condition. }
\end{array} \\
\mathrm{P}= & \text { the percentage of expected off-road operating distance. } \\
\mathrm{V}_{\mathrm{C}}= & \text { the speed from the off-road profile, mph, corresponding } \\
& \text { to } \mathrm{C} \text {. } \\
\mathrm{C}= & \text { the percentage of the off-road terrain that should be } \\
& \text { negotiable. } \\
\mathrm{L}= & \text { the percentage of the total time over the route network } \\
& \text { considered as an off-road traverse, that is spend in } \\
& \text { negotiating linear features. (This figure is available } \\
& \text { from the link statistics for each vehicle, area, and } \\
& \text { condition.) } \\
\mathrm{V}_{\mathrm{R}}= & \text { the speed from the on-road speed profile, mph, corresponding } \\
& \text { to R. } \\
\mathrm{R}= & \text { the percentage of the road and trail network that should be } \\
& \text { negotiable. }
\end{aligned}
$$

2. The speed from the on-road profile, $V_{R}$, is not directly available from this study, but can be computed using the speeds from the profiles of the primary and secondary roads and trails as follows:

$$
\mathrm{V}_{\mathrm{R}}=\frac{100-\mathrm{P}}{\frac{\mathrm{P}_{\mathrm{P}}}{\mathrm{V}_{\mathrm{PP}}}+\frac{\mathrm{P}_{\mathrm{S}}}{\mathrm{V}_{\mathrm{SP}}}+\frac{\mathrm{P}_{\mathrm{T}}}{\mathrm{V}_{\mathrm{TP}}}}
$$


where:

$$
\begin{aligned}
\mathrm{P}_{\mathrm{P}}, \mathrm{P}_{\mathrm{S}}, \mathrm{P}_{\mathrm{T}}= & \begin{array}{l}
\text { percentages of the composite on-road and off-road } \\
\text { network that are primary roads, secondary roads, } \\
\\
\text { and trails, respectively. }
\end{array} \\
\mathrm{V}_{\mathrm{PP}}, \mathrm{V}_{\mathrm{SP}}, \mathrm{V}_{\mathrm{TP}}= & \begin{array}{l}
\text { the speeds from the primary road, secondary road, } \\
\text { and trail speed profiles respectively, mph, that } \\
\text { correspond to } \mathrm{R} .
\end{array}
\end{aligned}
$$


In accordance with ER 70-2-3, paragraph $\sigma_{c}(1)(b)$, dated 15 February 1973, a facsimile catalog card in Library of Congress format is reprotuced below.

Randolph, Donald D

Moblitity performance of towed and self-propelled

artillery and related vehicles, by Donald D. Randolph

[and] James H. Robinson. Vicksburg, U. S. Army

Engineer Waterways Experiment Station, 1977.

$1 \mathrm{v}$. (various pagings) 11lus. $27 \mathrm{~cm}$. (U. S.

Waterways Experiment Station. Miscellaneous paper $\mathrm{M}-77-1$ )

Prepared for U. S. Army Training and Doctrine

Command, Fort Monroe, Virginta.

Includes bibliography.

1. Military vehicles. 2. Misston performance.

3. Off-road mobility. 4. On-road mobility.

5. Vehicle performance. I. Robinson, James H.,

joint author. II. U. S. Army Training and Doctrine

Command. (Series: U., S. Waterways Experiment

Station, Vicksburg, Miss. Miscellaneous paper $\mathrm{M}-77-1$ )

TA7.W34m no.M-77-1 
